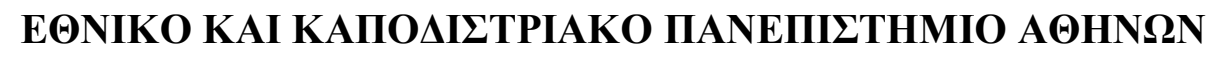

IАТРIКН $\Sigma$ ХАН

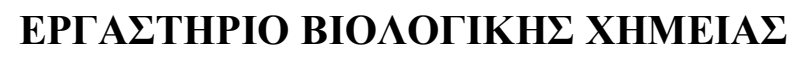

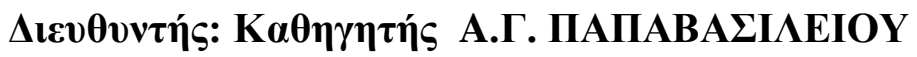

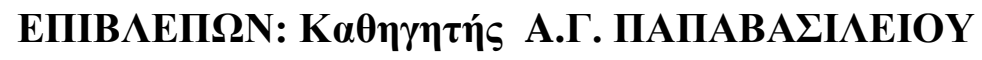

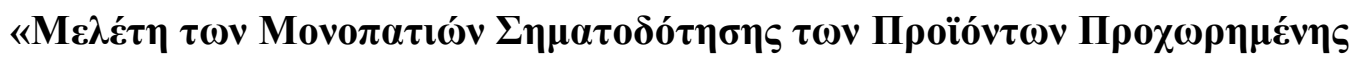

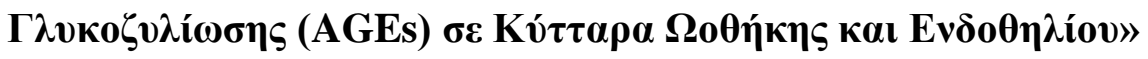

\author{
$\triangle$ ISAKTOPIKH $\triangle$ IATPIBH
}

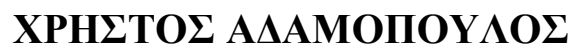

ВІО人ОГОГ

AOHNA 2013

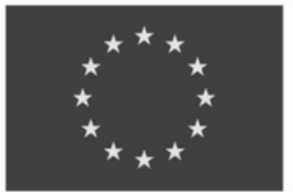

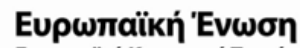

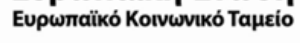

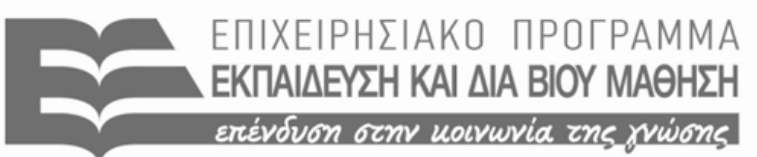

YПOYPГEIO ПAIAEIAL \& OPHEKEYMATON, ПОNITILMOY \& ABAHTILMOY EI $\triangle I K H \quad Y П H P E \Sigma|A \quad \triangle| A X E I P \mid \Sigma H \Sigma$

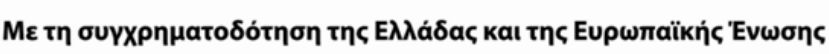

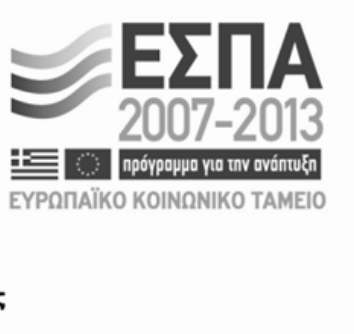




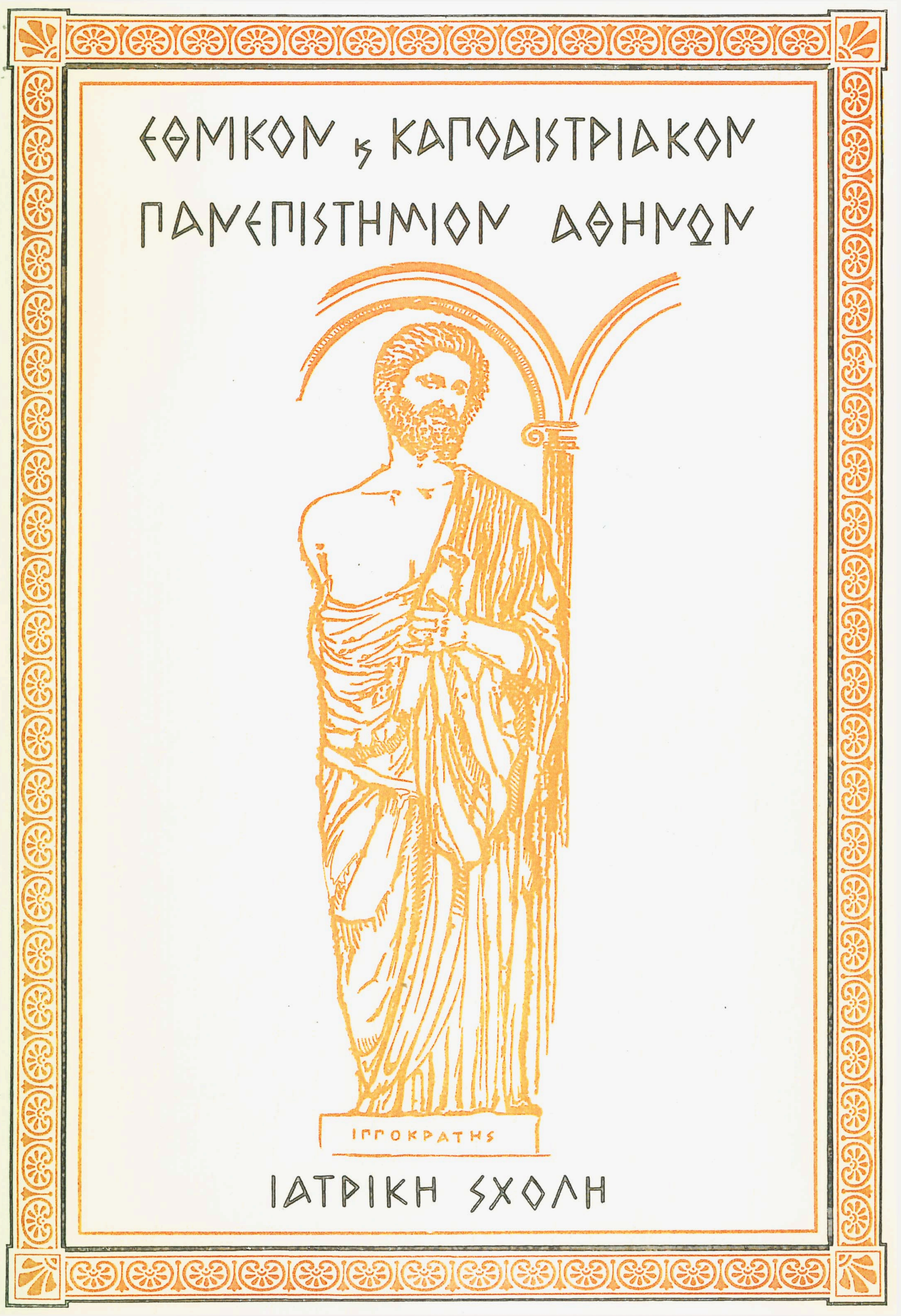




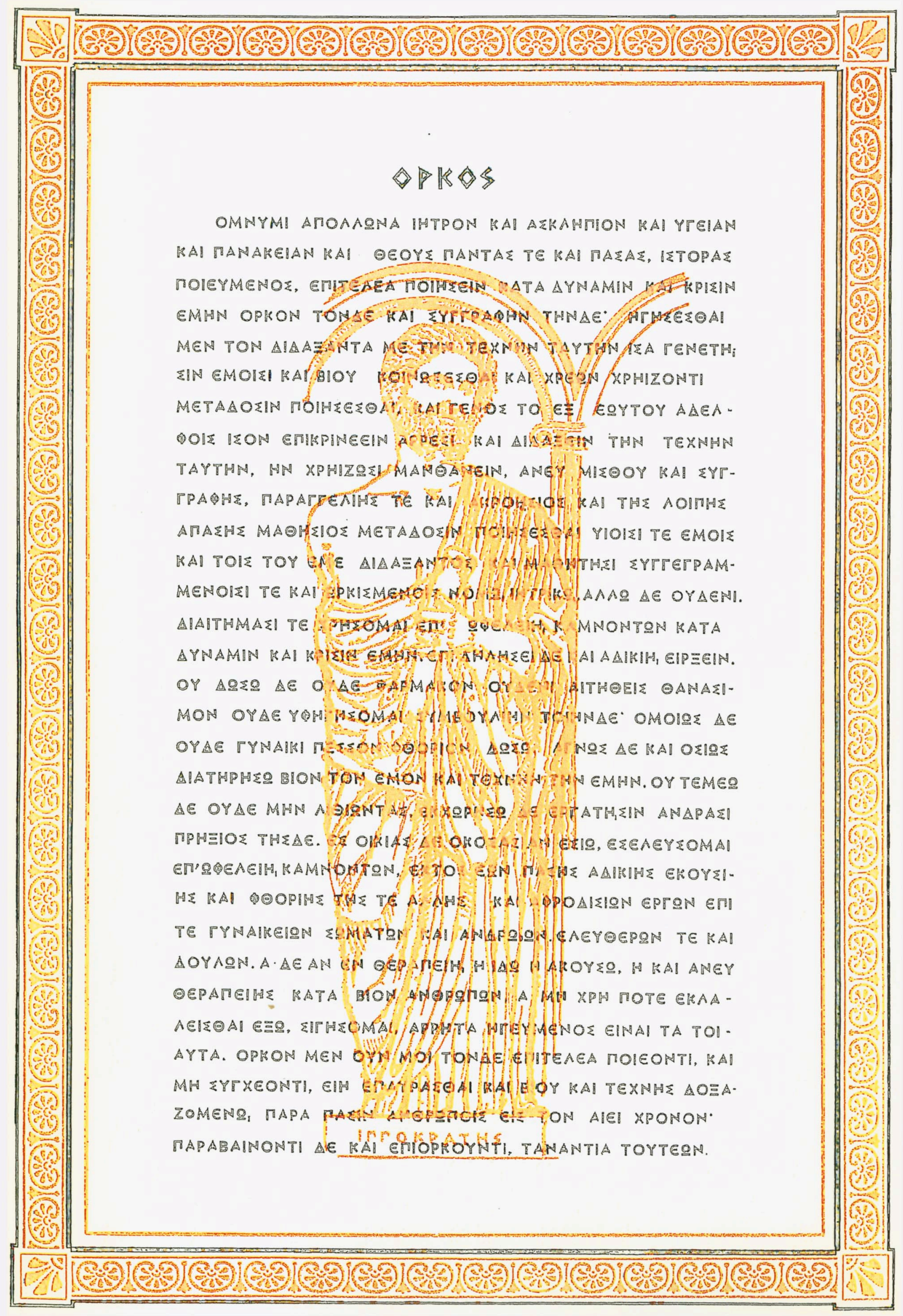




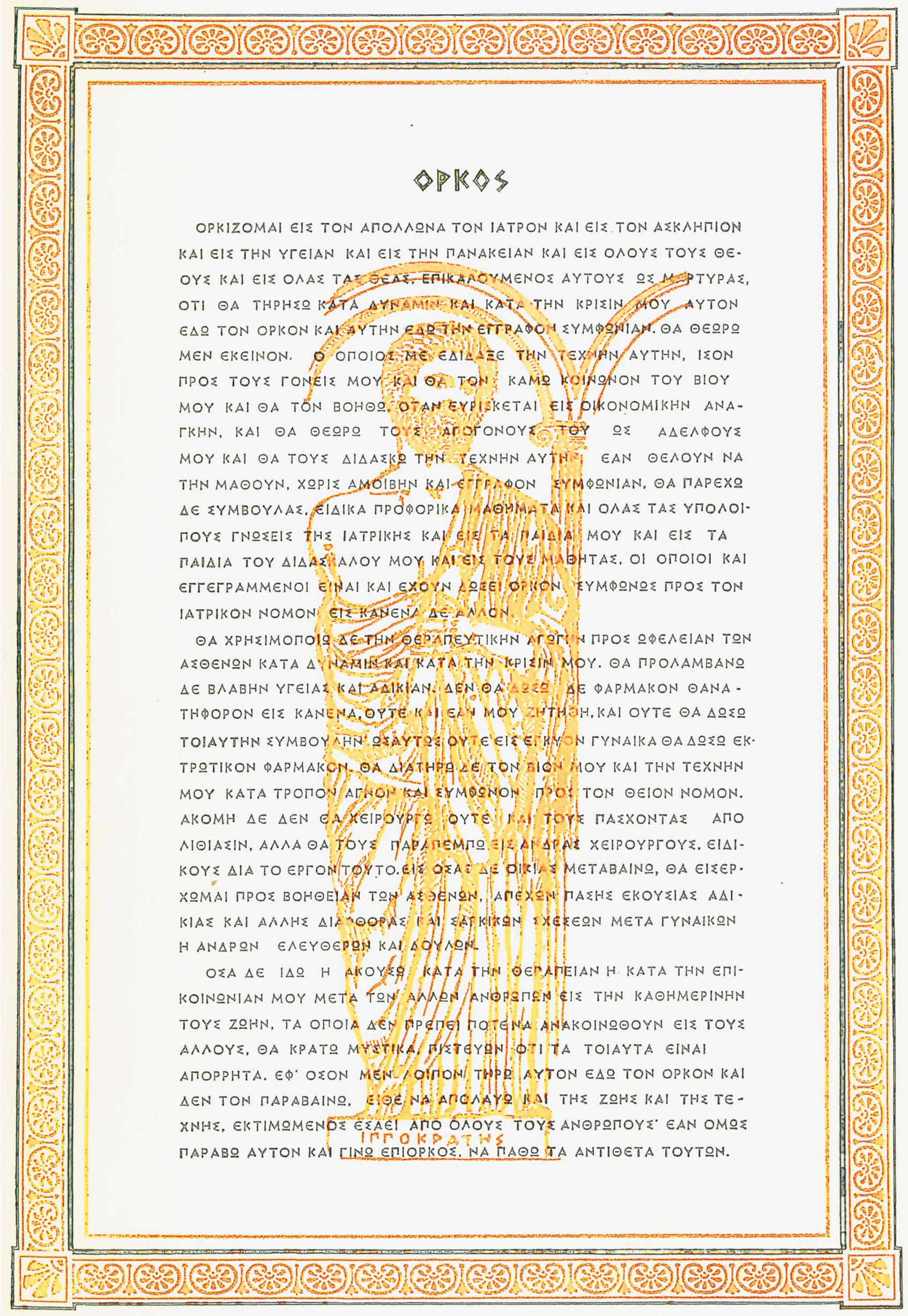




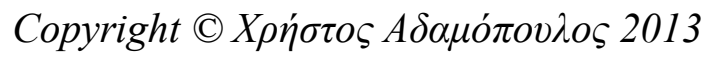

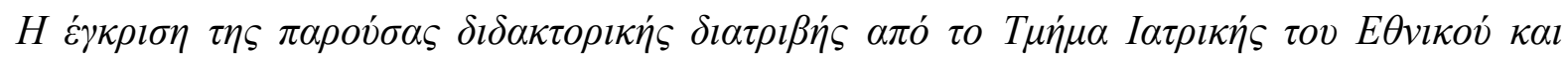

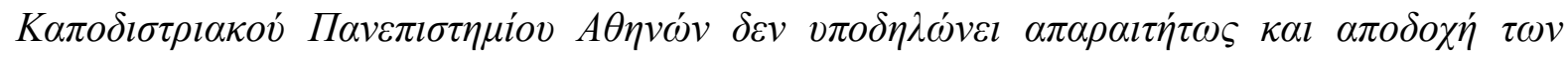

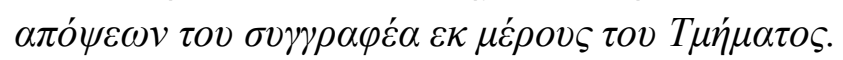

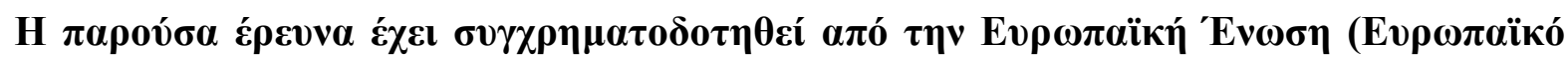

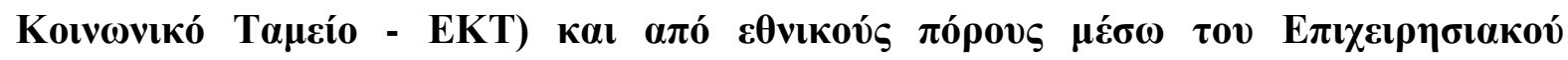

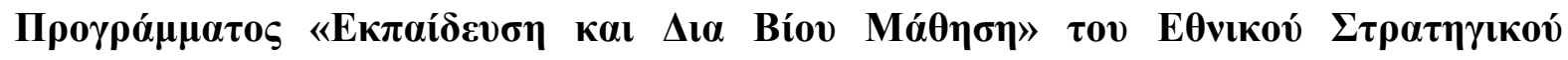

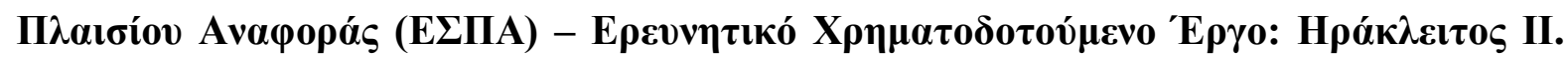

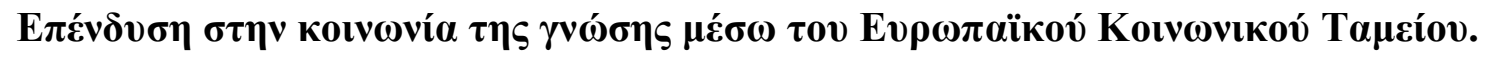

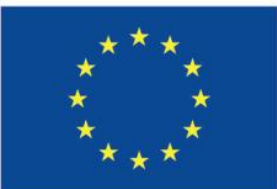

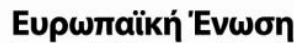

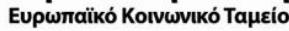

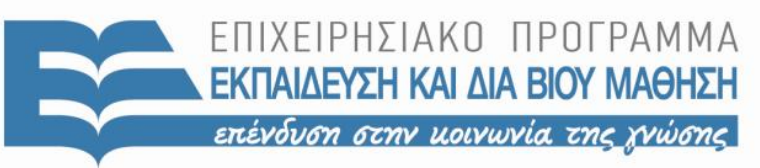

YПOYPГEIO ПАILEIAL \& OPHEKEYMATQN, ПОNITILMOY \& ABAHTILMOY EI $\triangle I K H \quad Y \sqcap H P E \Sigma I A \quad \triangle I A X E I P I \Sigma H \Sigma$

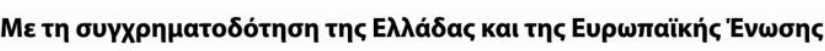

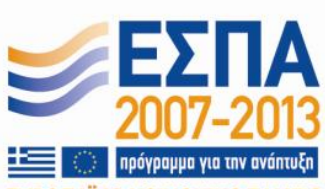

EYPRПAÏKO KOINQNIKO TAMEIO 


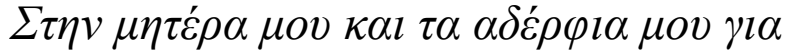

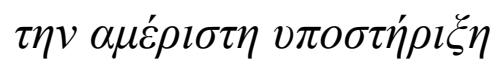

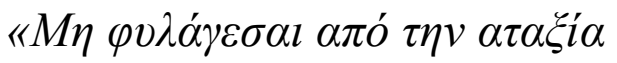

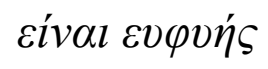

$H \tau \alpha \dot{\xi} \eta \eta \dot{v} v \alpha \imath \alpha \gamma \kappa v \dot{\lambda} \omega \sigma \eta \Phi v \lambda \alpha \dot{\xi} \sigma o v$

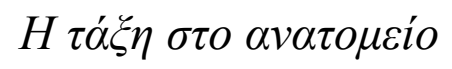

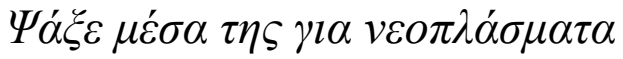
$\gamma \iota \alpha \dot{\alpha} \tau v \pi \alpha \kappa v ́ \tau \tau \alpha \rho \alpha \alpha \rho \imath \theta \mu o v$

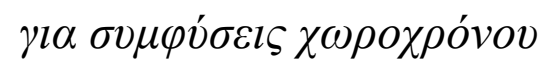

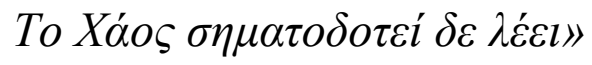

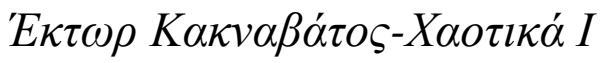


HMEPOMHNIA AITH $\Sigma E \Omega \Sigma$ OPI $\Sigma$ MOY TPIME $\Lambda$ OY $\Sigma \Sigma$ YMBOY $\Lambda$ EYTIKH $\Sigma$ ЕПІТРОПНЕ:

06-02-2008

HMEPOMHNIA OPI $\Sigma$ MOY TPIME $\Lambda O Y \Sigma \Sigma$ YMBOY $\Lambda$ EYTIKH $\Sigma$ EПITPOПH $\Sigma$ :

15-04-2008

ME $\Lambda$ HPIME $\Lambda O Y \Sigma$ ЕПITPOПH $\Sigma:$

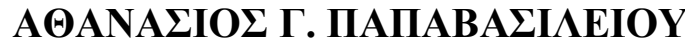

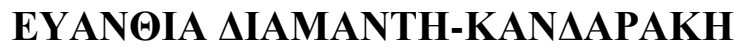

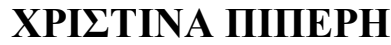

HMEPOMHNIA OPI $\Sigma$ MOY $\Theta E M A T O \Sigma \Delta$ I $\Delta$ AKTOPIKH $\Sigma \Delta$ IATPIBH $\Sigma:$

29-07-2008

HMEPOMHNIA KATA $\Theta E \Sigma H \Sigma \mathrm{A}^{\prime}$ ПPOO $\triangle \mathrm{OY}$ :

29-07-2009

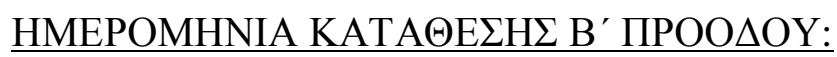

30-07-2010

HMEPOMHNIA KATA $\Theta E \mathrm{H} \Sigma \Gamma^{\prime} \Pi \mathrm{MOO} \Delta \mathrm{OY}:$

02-4-2012

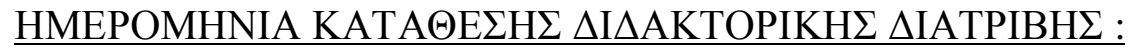

21-06-2013 


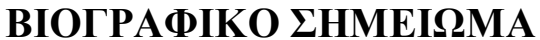

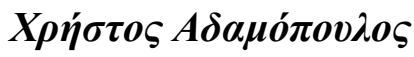

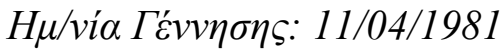

E-mail: chr.adamopoulos@gmail.com

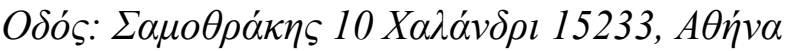

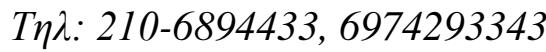

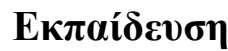

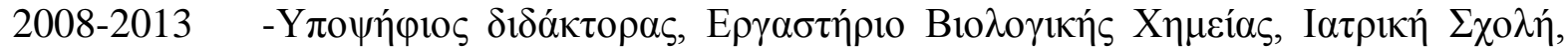

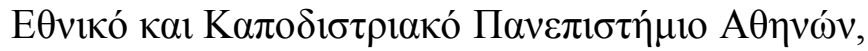

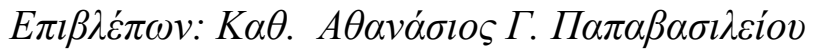

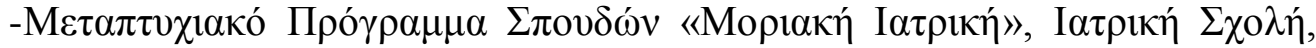

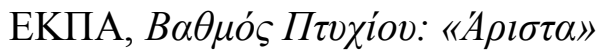

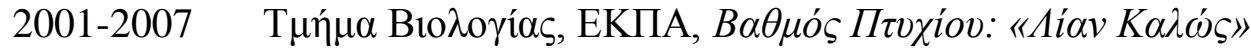

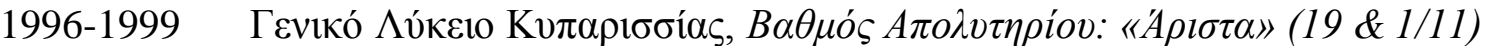

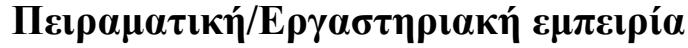

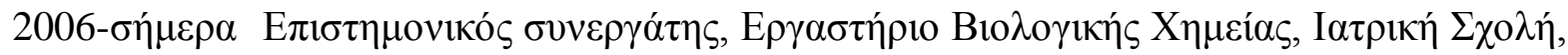
ЕКПА

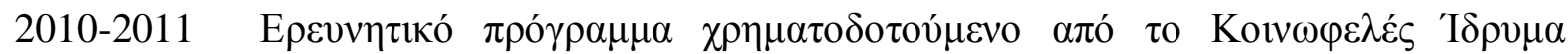

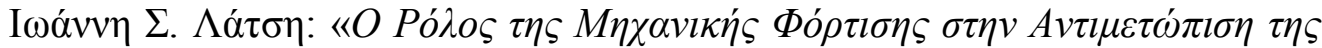

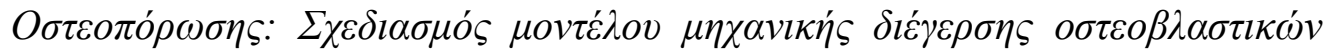

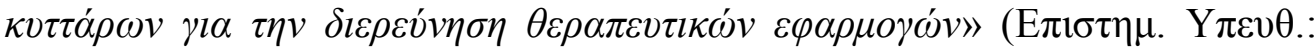
Av. K $\alpha \theta$. E.M $\pi \alpha ́ \sigma \delta \rho \alpha)$

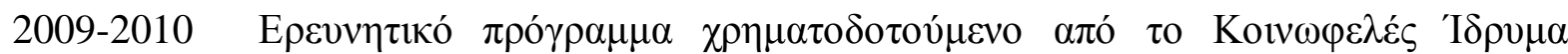

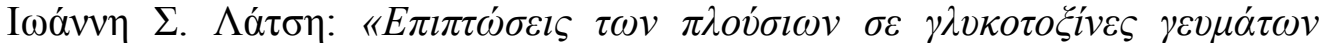

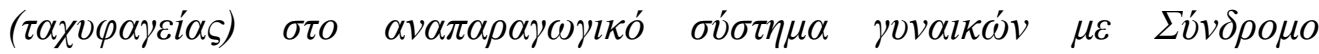

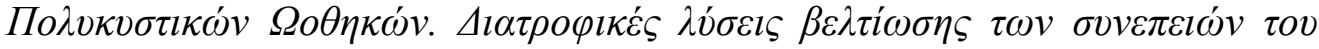
$\sigma v v \delta \rho o ́ \mu o v »(\mathrm{E} \pi \imath \sigma \tau \eta \mu$. Ү $\pi \varepsilon v \theta .:$ K $\alpha \theta$. А.Г. П $\alpha \pi \alpha \beta \alpha \sigma \imath \lambda \varepsilon i ́ o v)$

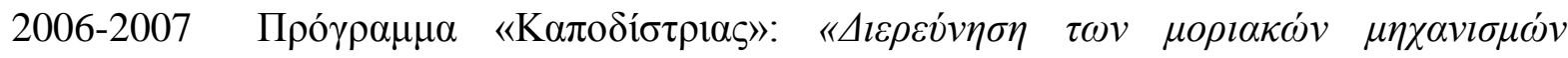

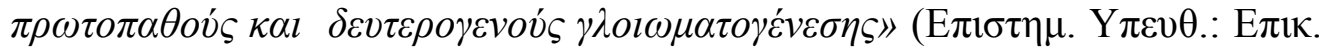

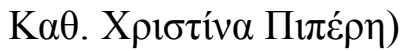

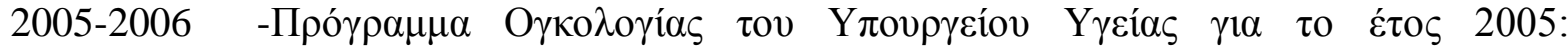

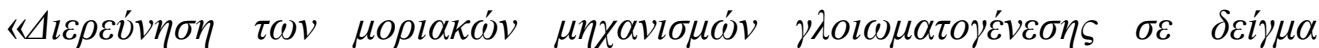

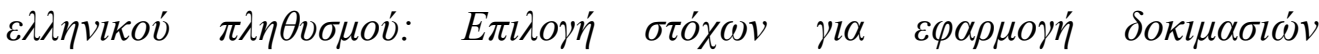

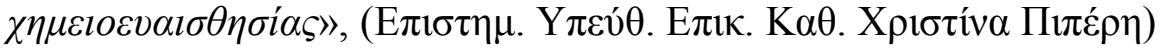

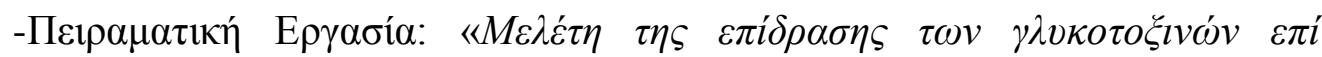

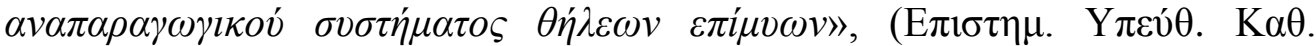

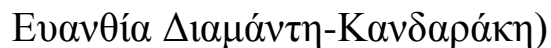

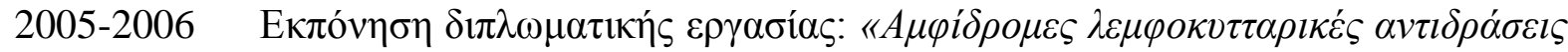

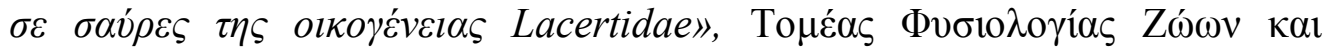

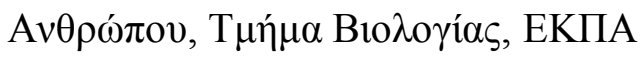




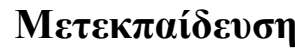

Iov́ 2008 University of California, Davis, School of Medicine, Department of

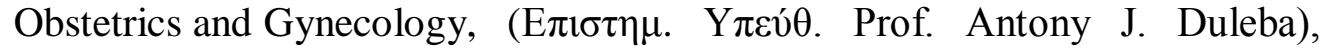

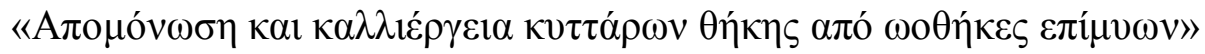

Үлотрофі́єs:

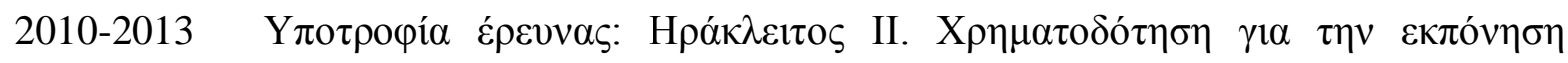

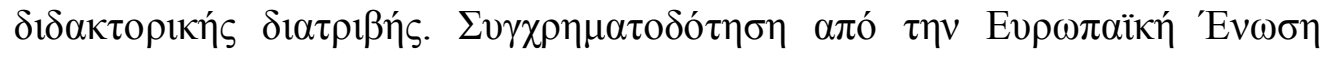

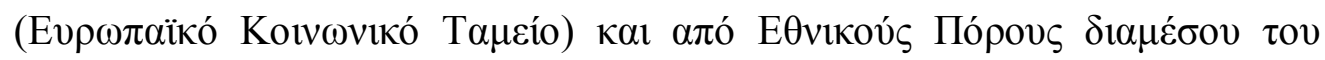

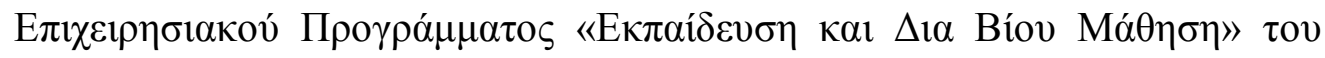

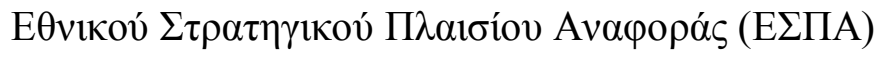

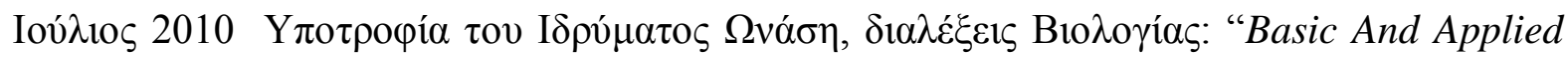

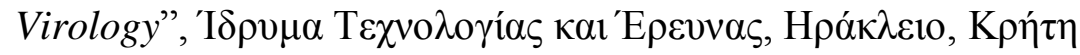

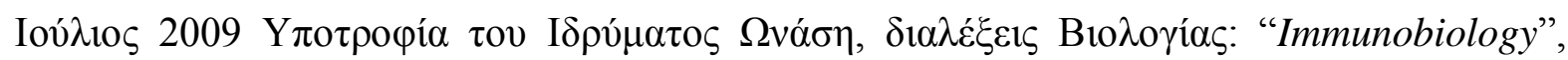

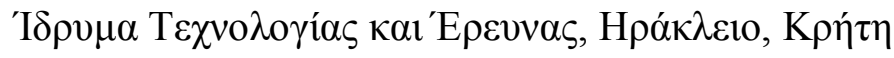

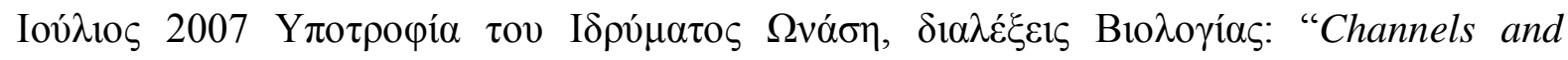

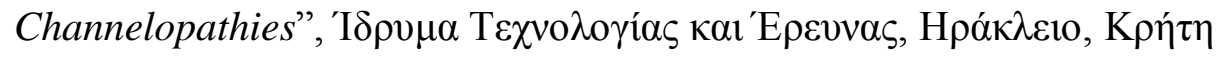

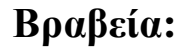

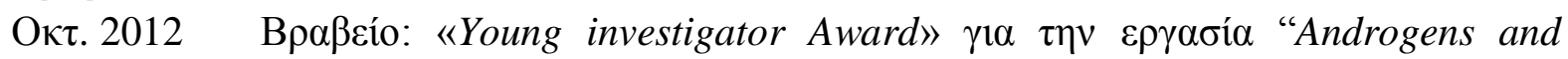
dietary glycotoxins excess may accelerate ovarian tissue ageing by decreasing GLO-I activity: a link to PCOS syndrome", 22nd European Tissue Repair Society Congress, Athens, Greece

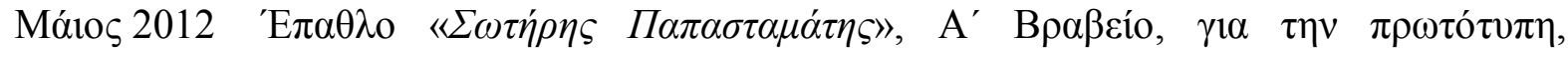

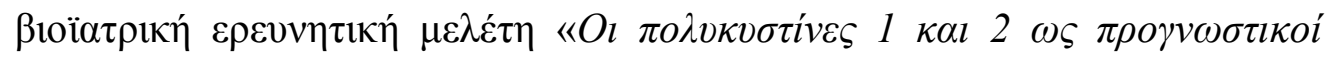

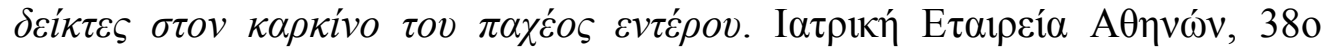

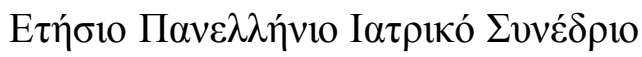

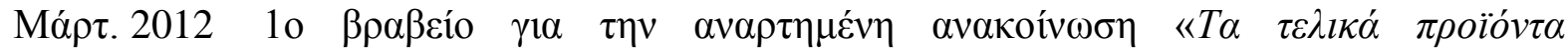

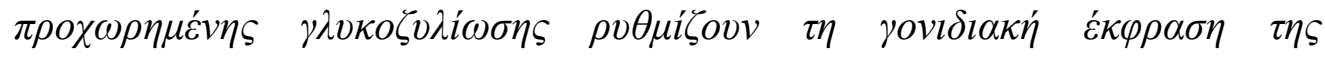

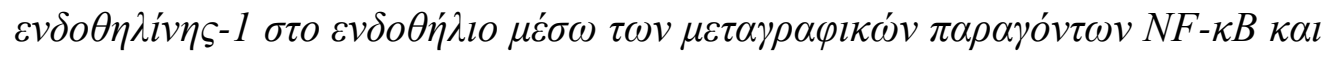

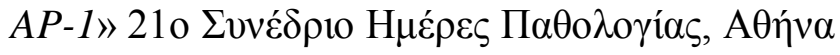

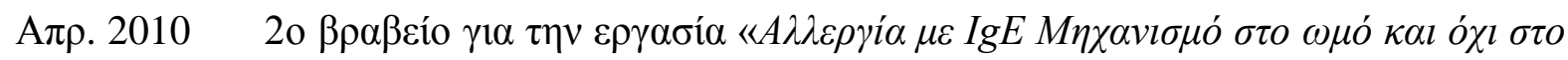

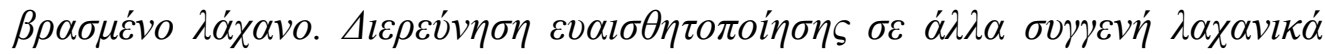

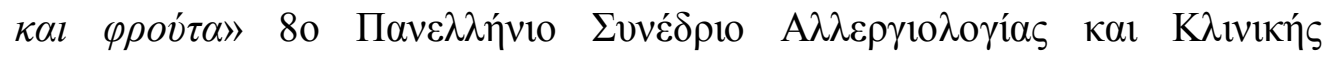

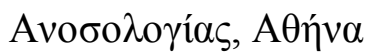

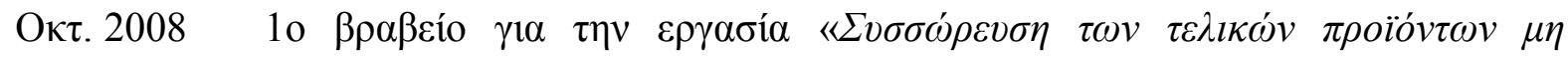

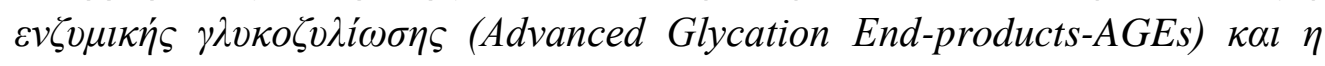

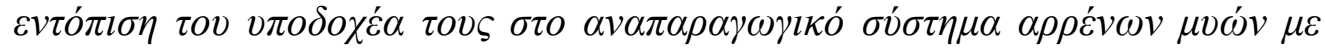

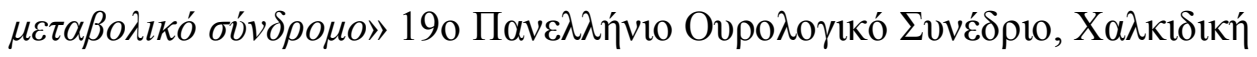

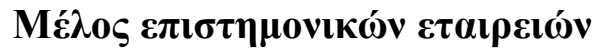

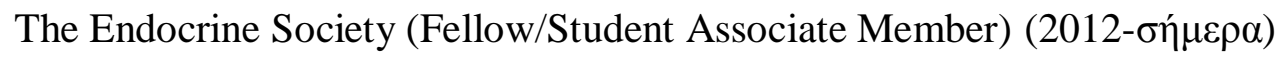




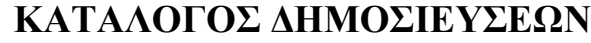 \\ $\Delta \eta \mu 0 \sigma เ \varepsilon v ́ \sigma \varepsilon ı$ :}

1. Role of Histone Lysine Methyltransferases SUV39H1 and SETDB1 in Gliomagenesis: Modulation of Cell Proliferation, Migration, and Colony Formation. Spyropoulou A, Gargalionis A, Dalagiorgou G, Adamopoulos C, Papavassiliou KA, Lea RW, Piperi C, Papavassiliou AG. Neuromolecular Med. 2013 Aug 13. [Epub ahead of print]

2. Sox11 expression in astrocytic gliomas: correlation with Nestin/c-MET/IDH1-R132H expression phenotypes, p-STAT-3 and survival. Korkolopoulou P, Levidou G, El-Habr E, Adamopoulos C, Fragkou P, Boviatsis E, Themistocleous M, Petraki K, Vrettakos G, Sakalidou M, Samaras V, Zisakis A, Saetta A, Chatziandreou I, Patsouris E, Piperi C, Br J Cancer. 2013 May 28;108(10):2142-52.

3. Advanced Glycation End-products induce Endoplasmic Reticulum stress in human aortic endothelial cells. Adamopoulos C, Farmaki E, Spilioti E, Kiaris H, Piperi C, Papavasiliou AG. Clin Chem Lab Med. 2013 Mar 2:1-10.

4. Mechanical stimulation of polycystin-1 induces human osteoblastic gene expression via potentiation of the calcineurin/NFAT signalling axis. Dalagiorgou G., Piperi C., Georgopoulou U., Adamopoulos C., Basdra E.K., Papavassiliou AG. Cell Mol Life Sci. 2013 Jan;70(1):167-80.

5. Polycystic ovary syndrome offspring display increased oxidative stress markers comparable to gestational diabetes offspring. Boutzios G, Livadas S, Piperi C, Vitoratos N, Adamopoulos C, Hassiakos D, Iavazzo C, Diamanti-Kandarakis E. Fertil Steril. 2013 Mar 1;99(3):943-50.

6. Deregulated Chromatin Remodeling in the Pathobiology of Brain Tumors. Spyropoulou A, Piperi C, Adamopoulos C, Papavassiliou AG. Neuromolecular Med. 2012 Nov 1. [Epub ahead of print] Neuromolecular Med. 2013 Mar;15(1):1-24.

7. Phosphorylated 4E-Binding Protein 1 (p-4E-BP1): a novel prognostic marker in human astrocytomas. P. Korkolopoulou, G. Levidou, E. El-Habr, C. Piperi, C. Adamopoulos, V. Samaras, E. Boviatsis, I. Thymara, G. Vretakos, EA. Trigka, S. Sakellariou, N. Kavantzas, E. Patsouris, A. Saetta. Histopathology. 2012 Aug;61(2):293-305.

8. Histone modifications as a pathogenic mechanism of colorectal tumourigenesis. Gargalionis AN, Piperi C, Adamopoulos C, Papavassiliou AG. Int J Biochem Cell Biol. 2012 Aug;44(8):1276-89.

9. Crosstalk between advanced glycation and endoplasmic reticulum stress: emerging therapeutic targeting for metabolic diseases. C. Piperi, C. Adamopoulos, G. Dalagiorgou, E. DiamantiKandarakis, A.G. Papavassiliou, J Clin Endocrinol Metab. 2012 Jul;97(7):2231-42.

10. Expression of Interleukin-8 (IL-8) Receptor CXCR2 and Suppressor of Cytokine Signaling-3 (SOCS-3) in Astrocytic Tumors. Korkolopoulou P, Levidou G, El-Habr EA, Adamopoulos C, Samaras V, Zisakis A, Kavantzas N, Boviatsis E, Fragkou P, Papavassiliou AG, Patsouris E, Piperi C, Mol Med. 2012 May 9;18:379-88.

11. The clinical and prognostic significance of activated Akt-mTOR pathway in human astrocytomas. E. El-Habr, C. Adamopoulos*, G. Levidou, A. Saetta, P. Korkolopoulou, C. Piperi. Neurol Res Int. 2012; 2012: 454957. (*co-authorship) 
12. Strong and positive association of Endothelin-1 with AGEs in PCOS: A causal relationship or a bystander? Christakou C., Economou F., Livadas S., Piperi C., Adamopoulos C., Marinakis E., Diamanti-Kandarakis E. Hormones, 2011 Oct-Dec;10(4):292-7

13. High Incidence of MGMT and RARbeta Promoter Methylation in Primary Glioblastomas: Association with Histopathological Characteristics, Inflammatory Mediators and Clinical Outcome. Piperi C, Themistocleous MS, Papavassiliou GA, Farmaki E, Levidou G, Korkolopoulou P, Adamopoulos C, Papavassiliou AG, Mol Med 2010 Jan-Feb;16(1-2):1-9.

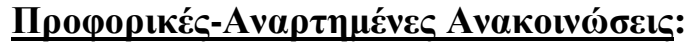

1) "Interference of Advanced Glycation End-products signaling with collagen cross-linking in human endothelium" C. Adamopoulos, C. Piperi, P. Korkolopoulou, G. Dalagiorgou, A. Spyropoulou, A. N. Gargalionis, E. A. Kandaraki, A. G. Papavassiliou, The Endocrine Society's 95th Annual Meeting \& Expo, San Francisco, USA 15-18/06/2013

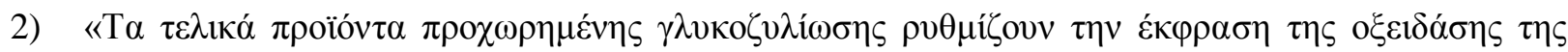

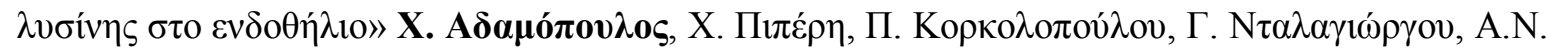

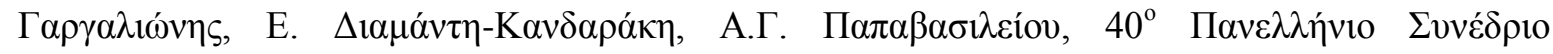

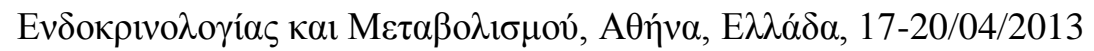

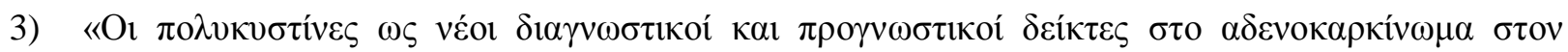

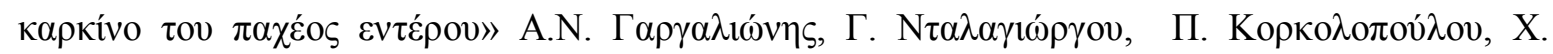

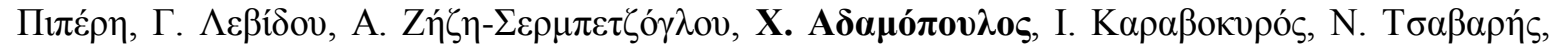

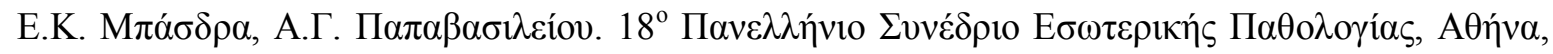
E $\lambda \lambda \alpha \dot{\alpha} \alpha \alpha 18-20 / 10 / 2012$

4) "Induction of endoplasmic reticulum stress by Advanced Glycation End-products in human endothelial cells" C. Adamopoulos, E. Farmaki, H. Kiaris, A. G. Papavassiliou, 22nd European Tissue Repair Society Meeting, Athens, Greece, 4-5/10/2012

5) "Polycystins: mechanosensor molecules with prognostic significance in colorectal cancer" A.N. Gargalionis, G. Dalagiorgou, P. Korkolopoulou, C. Piperi, G. Levidou, C. Adamopoulos, A. ZiziSermpetzoglou, N. Tsavaris, E.K. Basdra, A. G. Papavassiliou, $22^{\text {nd }}$ European Tissue Repair Society Meeting, Athens, Greece, 4-5/10/2012

6) "Androgens and dietary glycotoxins excess may accelerate ovarian tissue ageing by decreasing GLO-I activity: a link to PCOS syndrome" E. Kandaraki, A. Chatzigeorgiou, C. Piperi, E. Palioura, S. Palimeri, C. Adamopoulos, A. G. Papavassiliou, M. Koutsilieris, $22^{\text {nd }}$ European Tissue Repair Society Meeting, Athens, Greece, 4-5/10/2012

7) "Impact of dietary Advanced Glycation End Products (AGEs) modifications on metabolic and hormonal profile in women with polycystic ovary syndrome" S. Livadas, E. Tantalaki, C. Piperi, Anastasios Kollias, C. Adamopoulos, A. Koulouri, C. Christakou, E. Diamanti-Kandarakis, 22nd European Tissue Repair Society Meeting, Athens, Greece, 4-5/10/2012

8) "Polycystins 1 and 2 as novel prognostic markers in colorectal cancer" A.N. Gargalionis, G. Dalagiorgou, P. Korkolopoulou, C. Piperi, G. Levidou, C. Adamopoulos, A. Zizi-Sermpetzoglou, 
N. Tsavaris, E.K. Basdra, A. G. Papavassiliou, 37th ESMO Congress (Abstract), Vienna, Austria, 28/09-02/10/2012

9) "Advanced Glycation End-Products induce endoplasmic reticulum stress in endothelium" C. Adamopoulos, E. Farmaki, H. Kiaris, A. G. Papavassiliou, C. Piperi, $11^{0}$ International Symposium on the Maillard Reaction, Nancy, France, 16-20/09/2012

10) "Advanced Glycation End-Products regulate lysyl oxidase expression in endothelium" C. Adamopoulos, C. Piperi, P. Korkolopoulou, G. Dalagiorgou, A. Spyropoulou, E. DiamantiKandarakis, A. G. Papavassiliou, $11^{0}$ International Symposium on the Maillard Reaction, Nancy, France, 16-20/09/2012

11) "Prognostic implications of interleukin-8 (IL-8)-CXCR axis in astrocytic tumors" P. Korkolopoulou, G. Levidou, E. El-Habr, C. Adamopoulos, P. Fragkou, N. Kavantzas, E. Boviatsis, A. G. Papavassiliou, C. Piperi, E. Patsouris, 10th European Congres of Neuropathology-British Neuropathological Society-Euro CNS, Edinburg, Scotland, UK 31/07-04/08/2012

12) "Transcriptional regulation of endothelin-1 expression by Advanced Glycation End-Products in human aortic endothelium is mediated via NF- $\kappa$ B and AP-1" C. Adamopoulos, C. Piperi, G. Dalagiorgou, A. N. Gargalionis, E. A. Kandaraki, E. Diamanti-Kandarakis, A. G. Papavassiliou, The Endocrine Society's 94th Annual Meeting \& Expo, Houston, USA 23-26/06/2012

13) "Impact of dietary Advanced Glycation End Products (AGEs) modifications on metabolic and hormonal profile in women with polycystic ovary syndrome" S. Livadas, E. Tantalaki, C. Piperi, Anastasios Kollias, C. Adamopoulos, A. Koulouri , C. Christakou, E. Diamanti-Kandarakis, The Endocrine Society's 94th Annual Meeting \& Expo, Houston, USA 23-26/06/2012

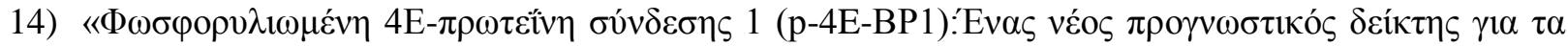

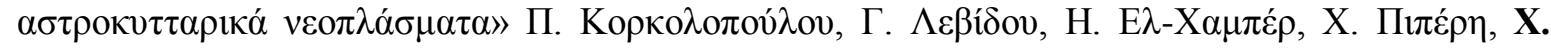

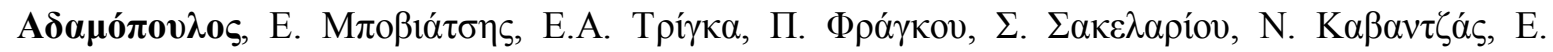

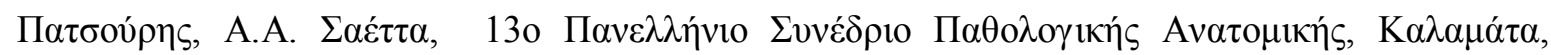
E $\lambda \lambda \alpha \dot{\delta} \alpha, 13-16 / 06 / 2012$

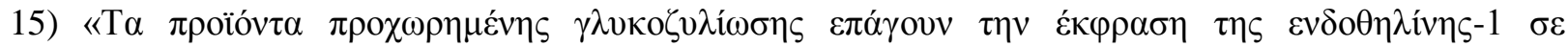

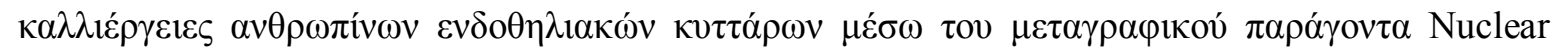

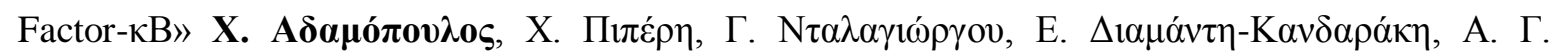

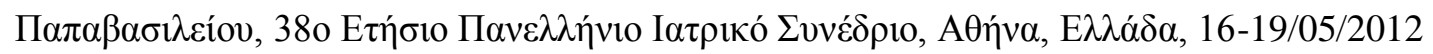

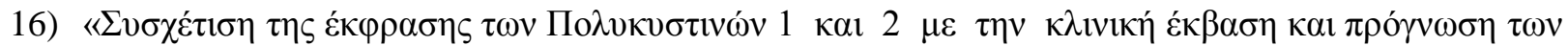

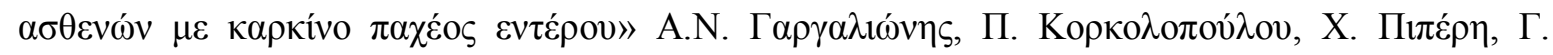

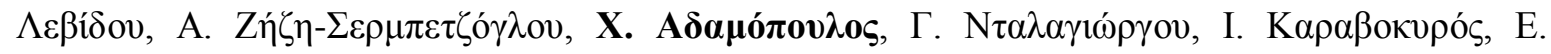

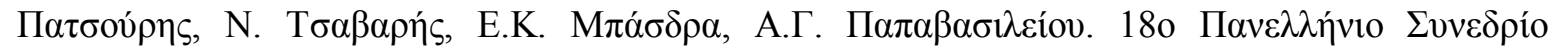

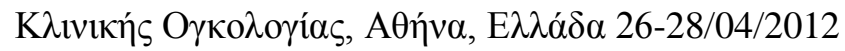

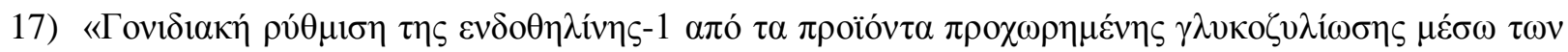

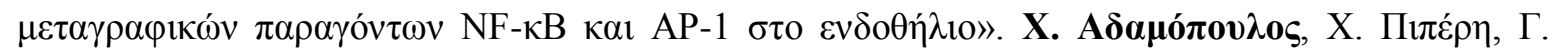

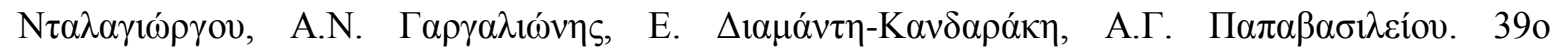

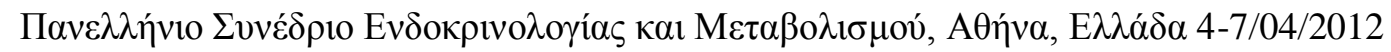




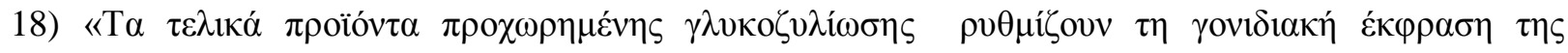

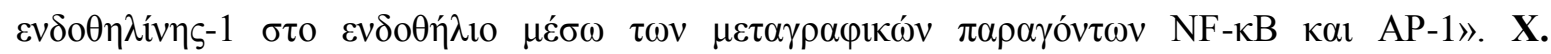

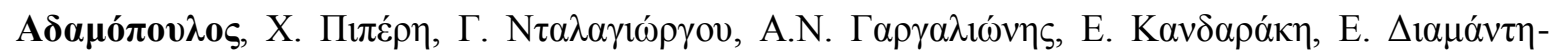

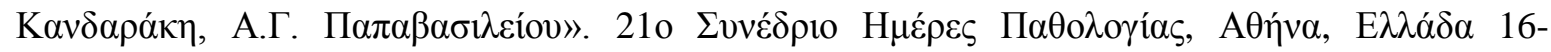
$17 / 03 / 2012$

19) "Prognostic significance of the mTOR pathway in human astrocytomas: a multivariate survival analysis, including IDH1-R132H presence, with emphasis on p-4E-BP1." E. El-Habr, P. Korkolopoulou, G. Levidou, C. Piperi, C. Adamopoulos, V. Samaras, E. Boviatsis, G. Vretakos, I. Thymara, E. Trigka, S. Sakellariou, E. Patsouris, A. Saetta - International Symposium on Clinical and Basic Investigation in Glioblastoma, Valencia, Spain 23-25/06/2011

20) "How do we move teeth after all: the role of Polycystin-1 in mechanically induced alveolar bone remodelling." G. Dalagiorgou, C. Piperi, U. Georgopoulou, C. Adamopoulos, A. Papavassiliou, E. Basdra. EOS 87th Congress of the European Orthodontic Society, Istanbul, Turkey 19-23/06/2011

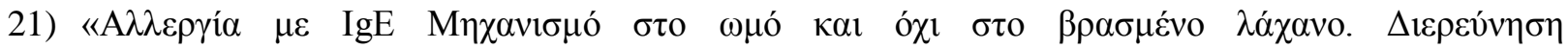

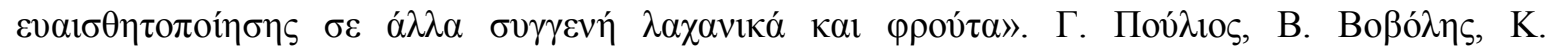

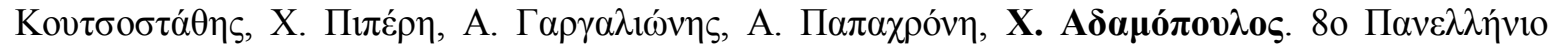

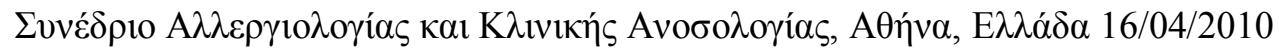

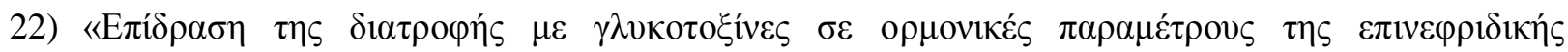

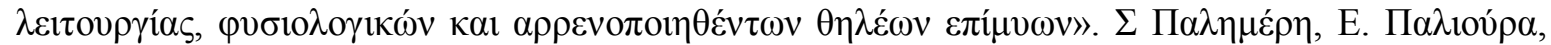

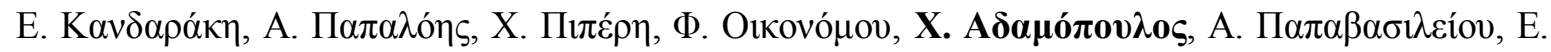

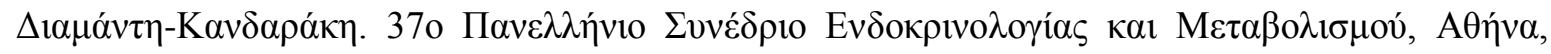
E $\lambda \lambda \alpha ́ \delta \alpha ~ 14-17 / 04 / 2010$

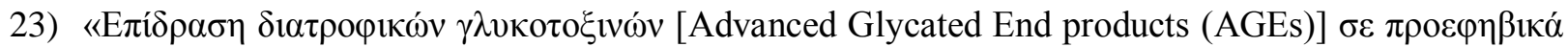

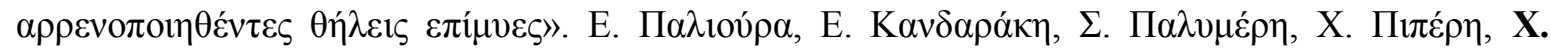

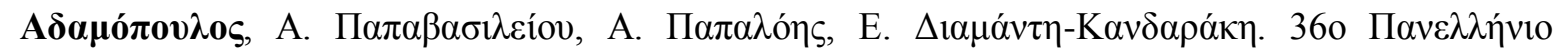

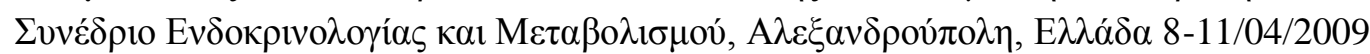

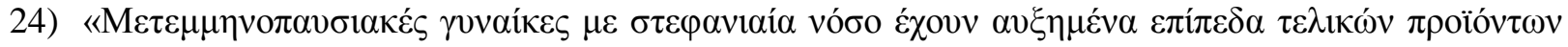

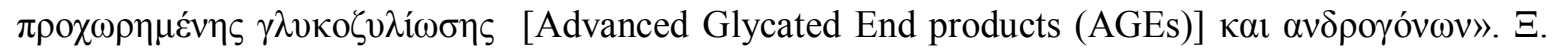

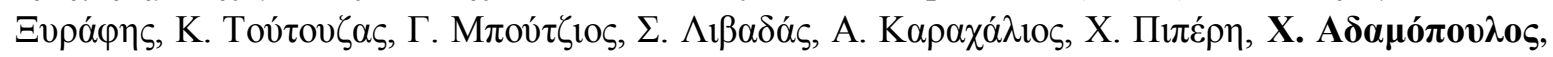

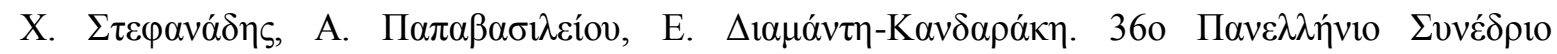

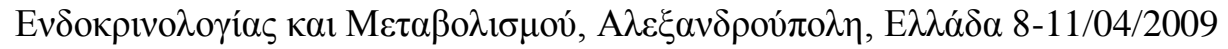

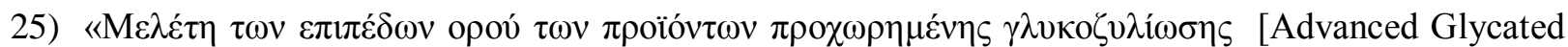

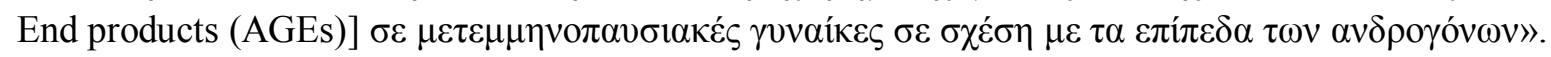

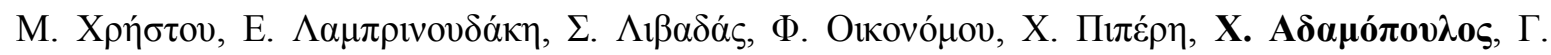

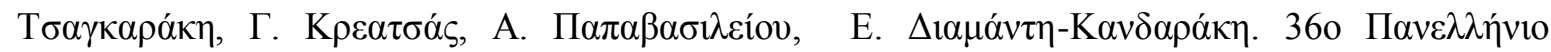

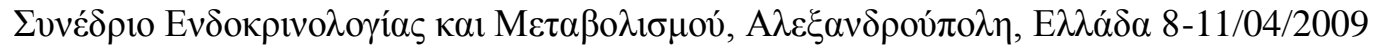

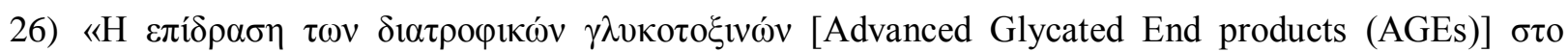

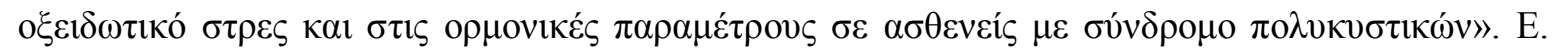

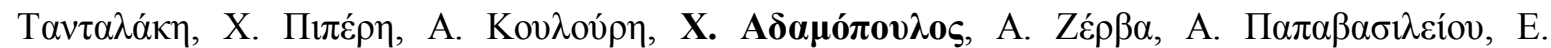




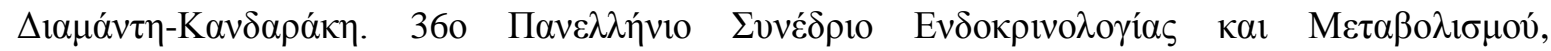
$\mathrm{A} \lambda \varepsilon \xi \alpha \nu \delta \rho \circ \tilde{\pi} \pi \lambda \eta, \mathrm{E} \lambda \lambda \alpha \dot{\alpha} \delta \alpha$ 8-11/04/2009

27) "Serum levels of the Soluble isoform of the Receptor of Advanced Glycosylated End products (sRAGE) are increased in women with PCOS”, C. Piperi, E. Tantalaki, S. Livadas, C. Christakou, X. Xyrafis, C. Adamopoulos, S. Kandarakis, A. Papavasiliou and E. Diamanti-Kandarakis - 10th European Congress of Endocrinology, Berlin, Germany 3-7/5/2008

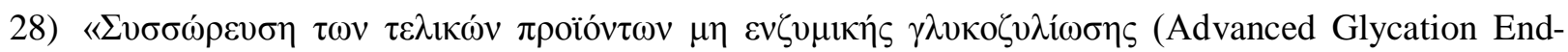

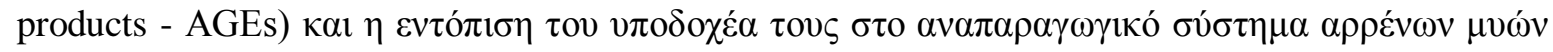

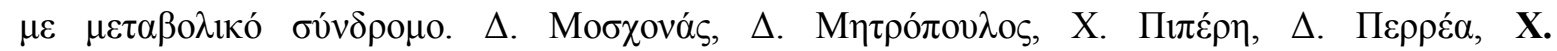

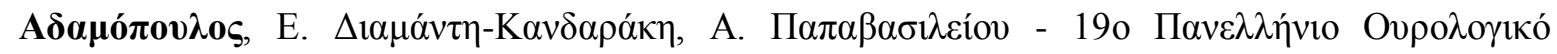
$\Sigma v v \varepsilon ́ \delta \rho ı, ~ X \alpha \lambda \kappa \imath \delta ı \kappa \eta, ~ E \lambda \lambda \alpha ́ \delta \alpha ~ 1-5 / 10 / 2008$

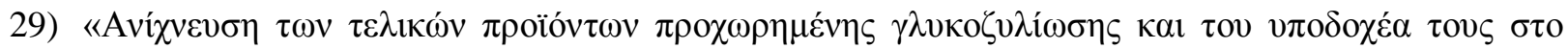

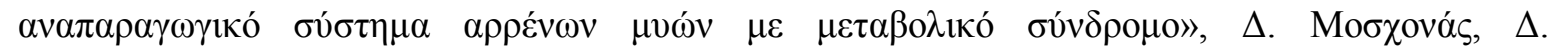

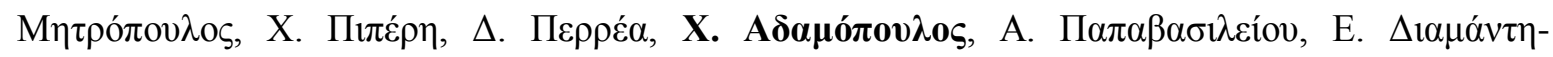

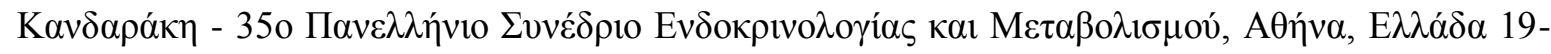
$22 / 3 / 2008$

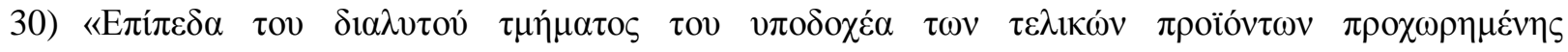

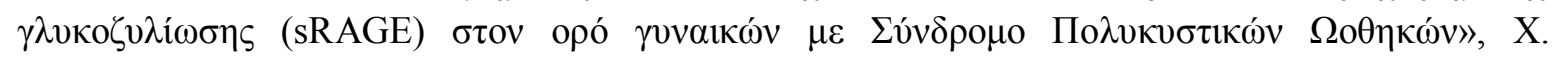

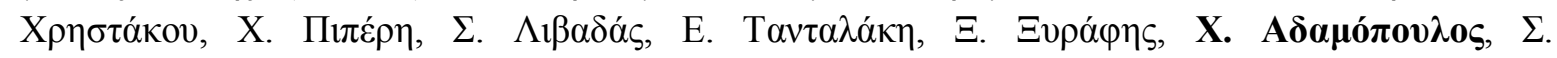

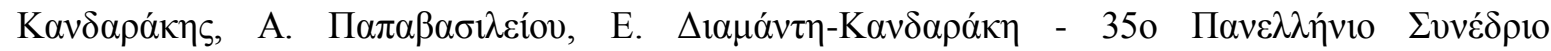

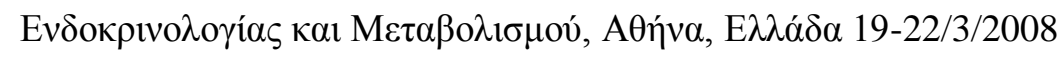

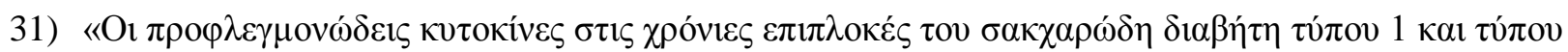

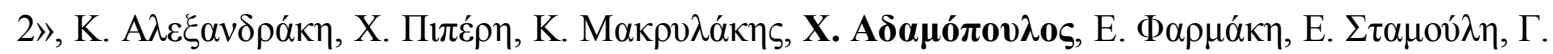

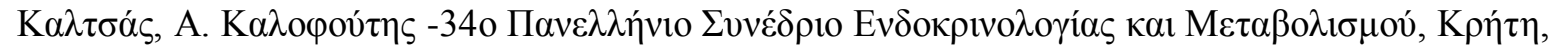
E $\lambda \lambda \alpha \dot{\alpha} \delta \alpha 28-31 / 3 / 2007$

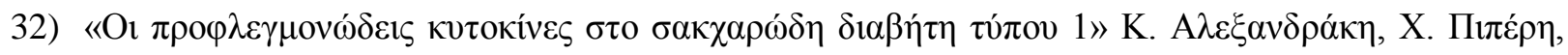

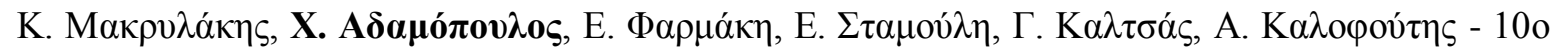

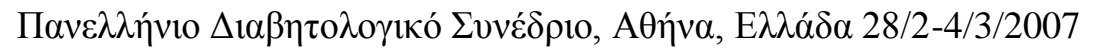

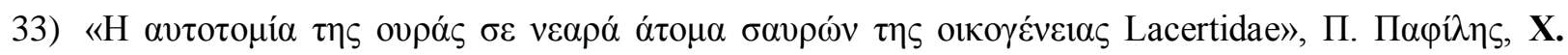

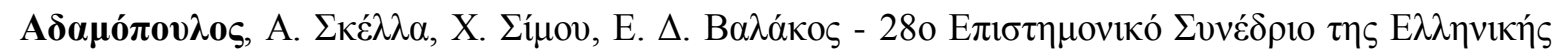

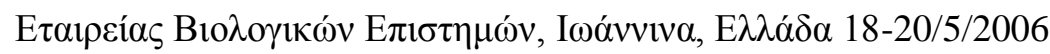




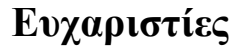

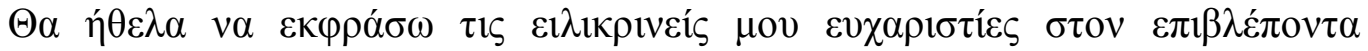

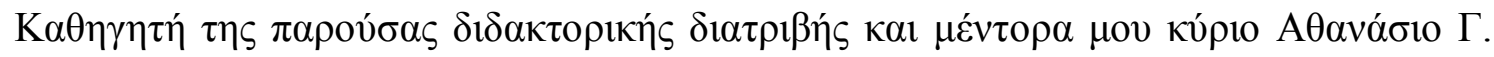

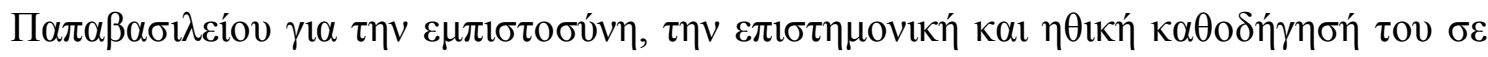

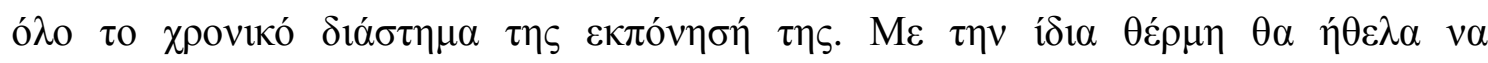

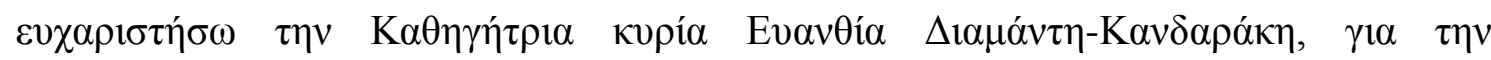

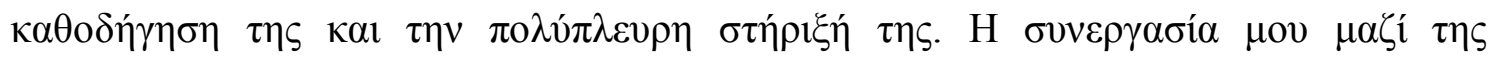

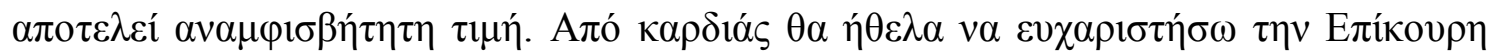

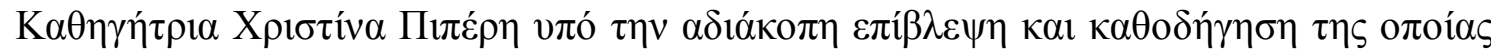

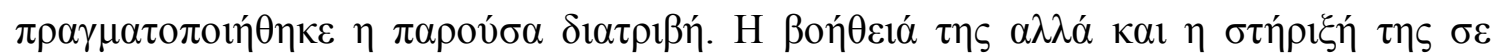

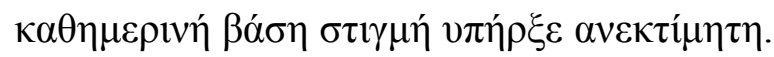

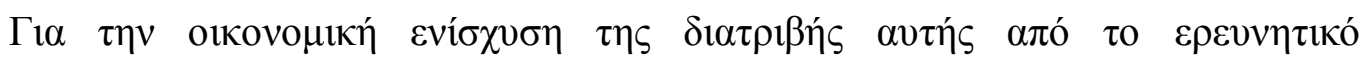

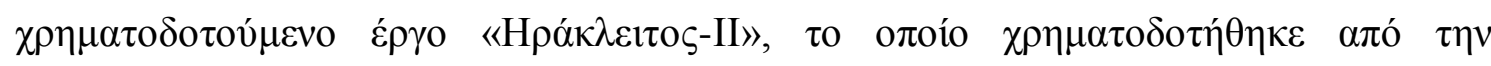

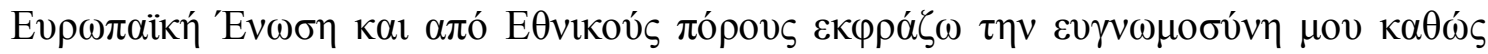

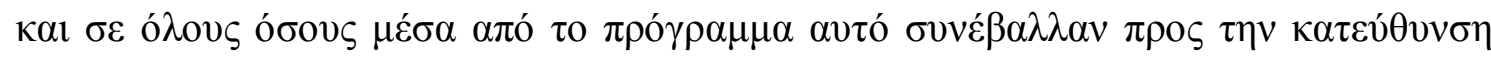
$\alpha v \tau \eta ́$.

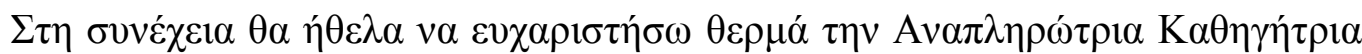

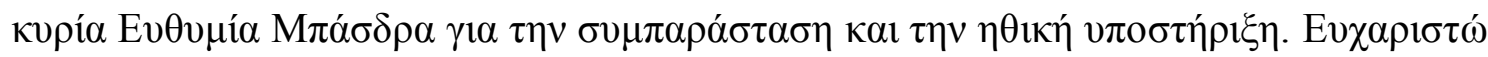

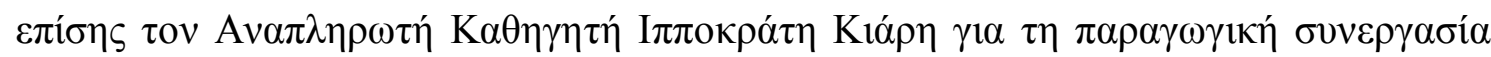

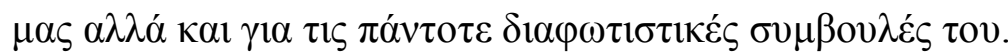

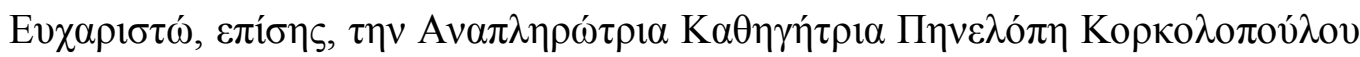

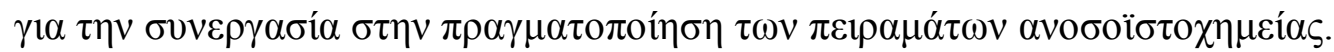

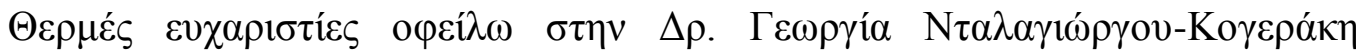

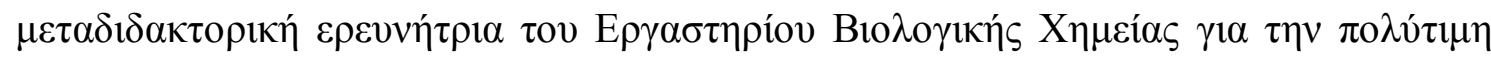

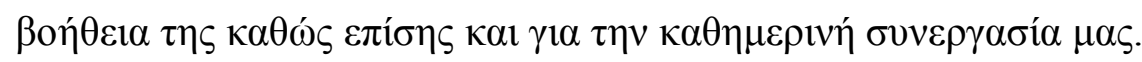

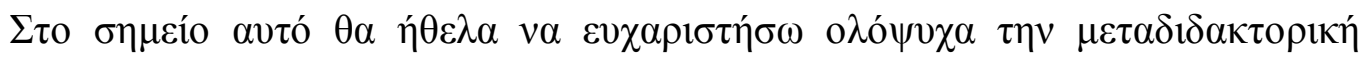

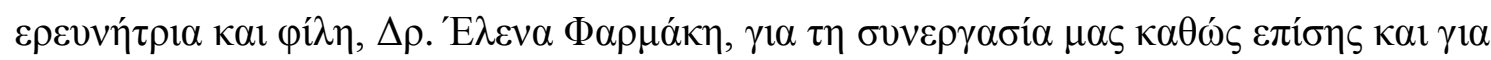

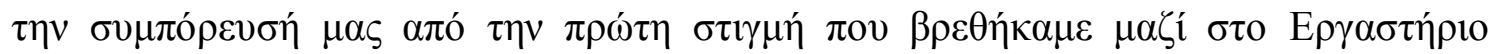

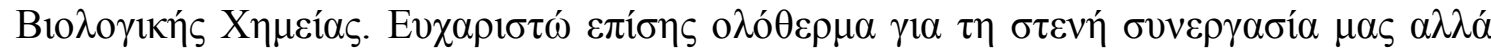

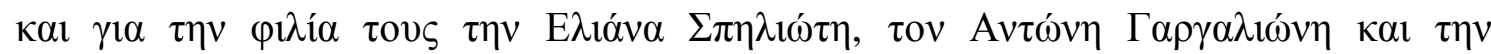

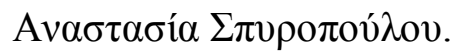

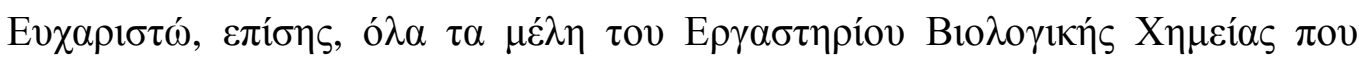

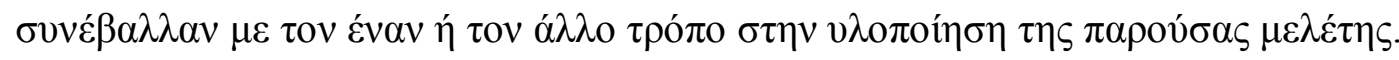




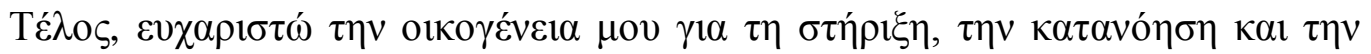

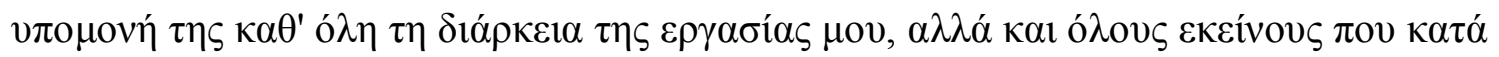

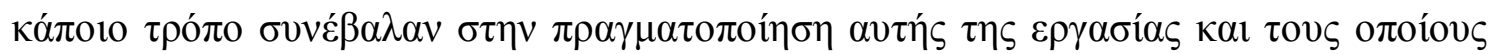

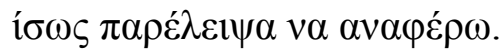




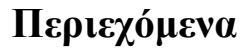

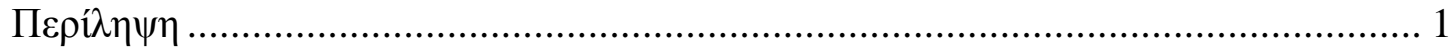

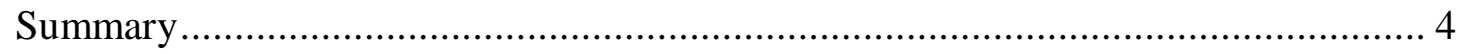

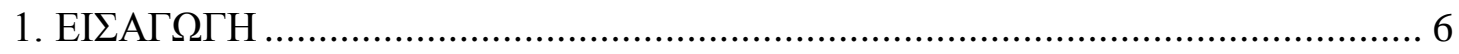

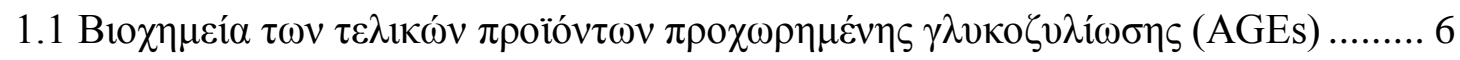

1.1.1 Y $\pi \mathrm{o} \delta \mathrm{o} \chi \varepsilon \dot{\varepsilon} \alpha \varsigma \tau \omega v$ AGEs - RAGE ............................................................. 17

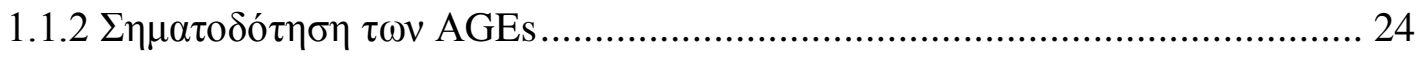

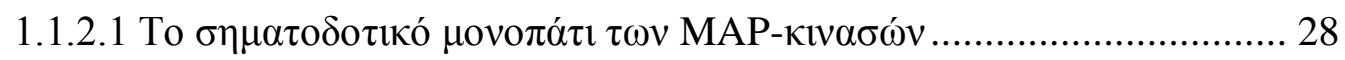

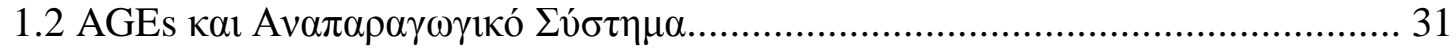

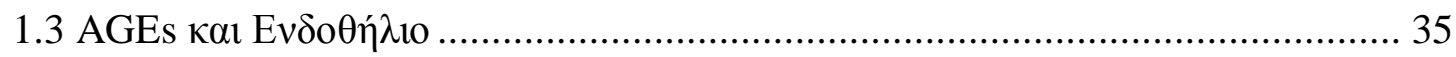

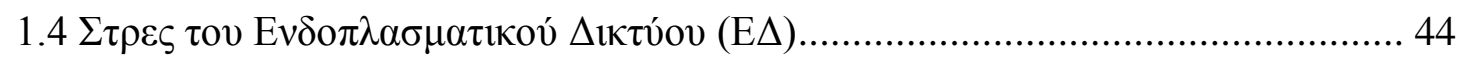

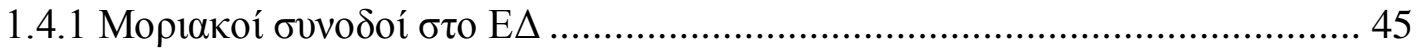

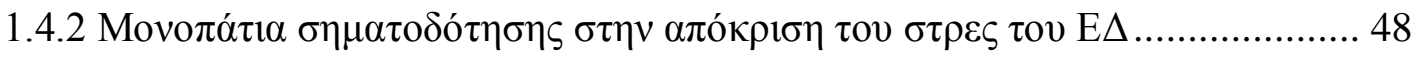

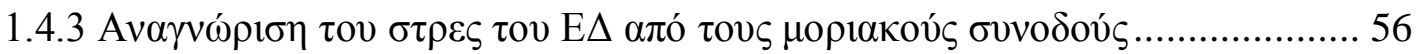

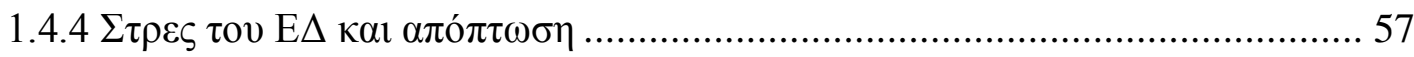

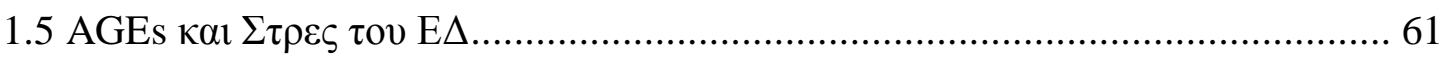

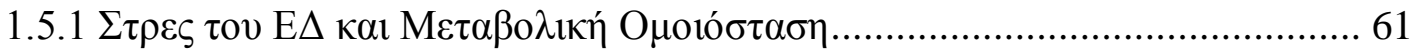

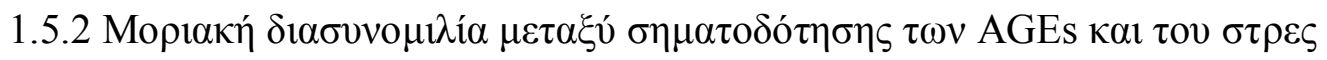

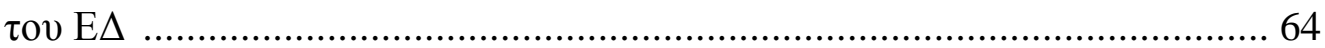

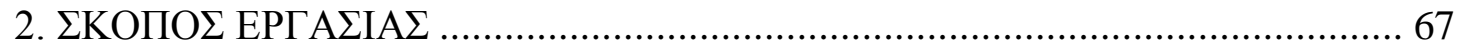

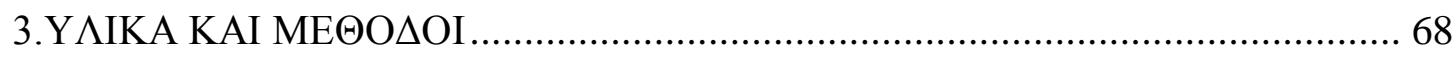

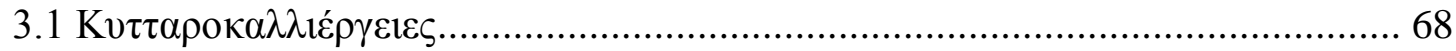

3.2 А

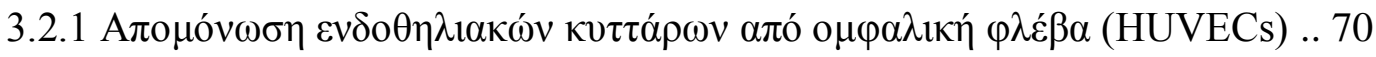

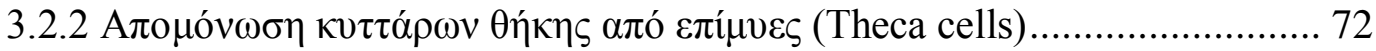

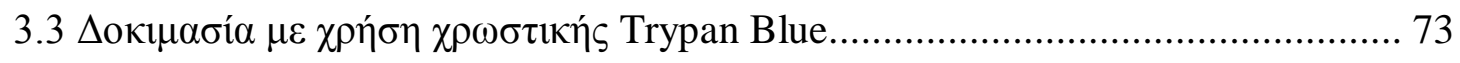

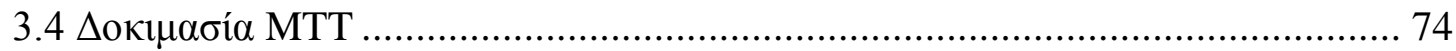

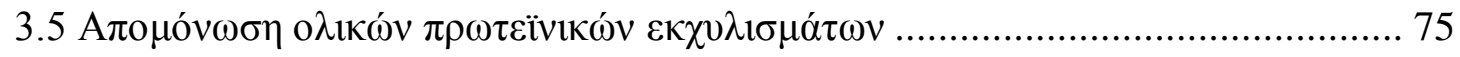

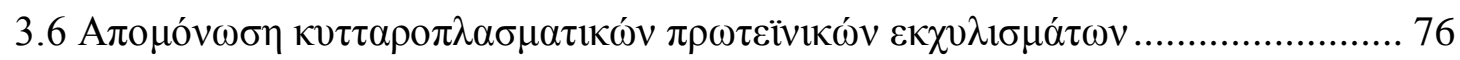


3.7 А

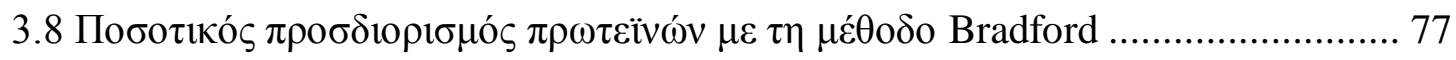

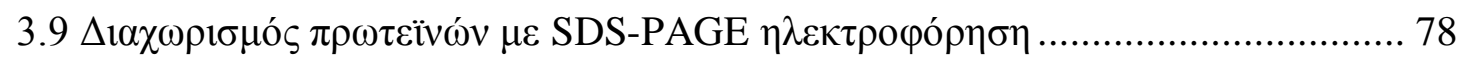

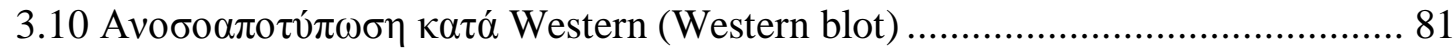

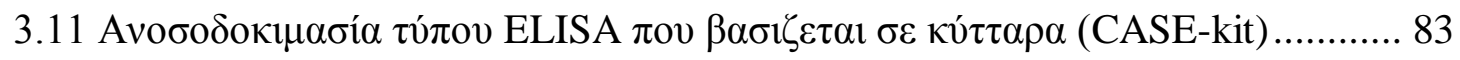

3.12 А

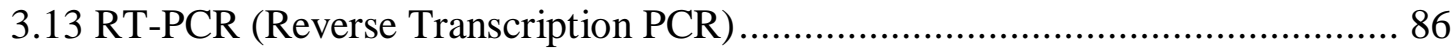

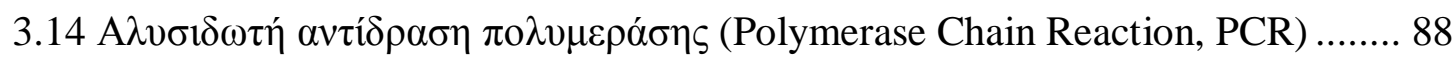

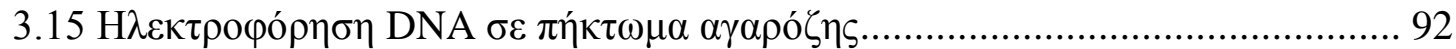

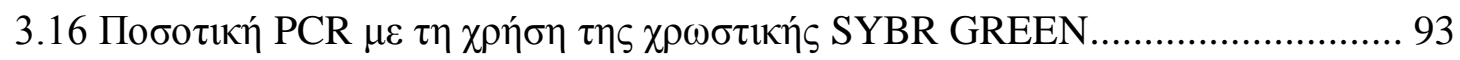

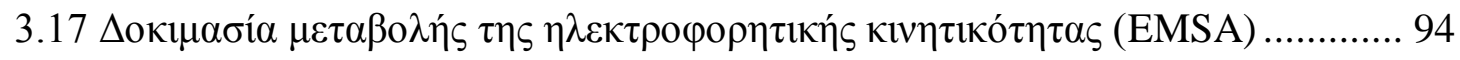

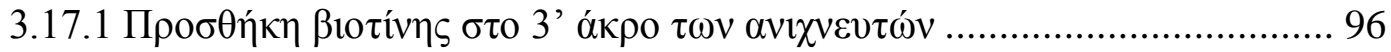

3.17.2 Electrophoretic Mobility Shift Assay (EMSA) ................................. 97

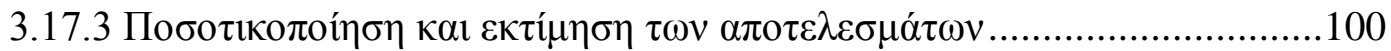

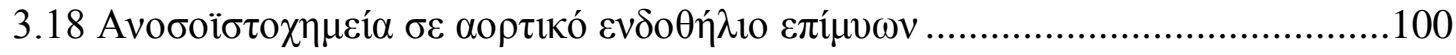

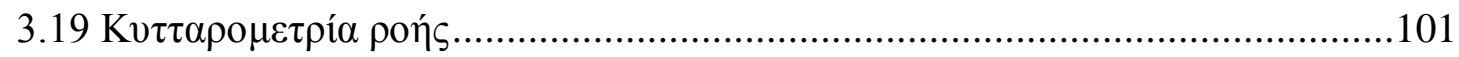

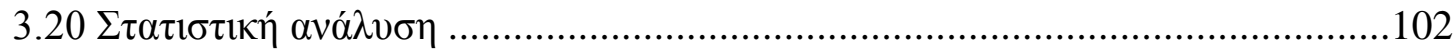

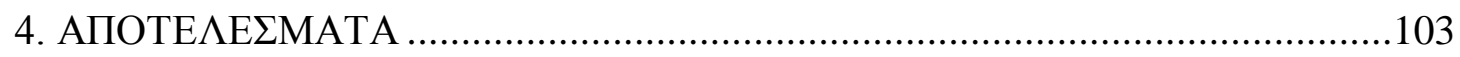

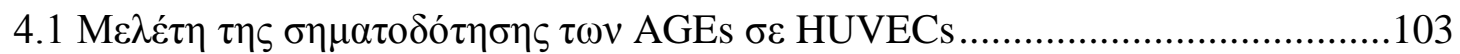

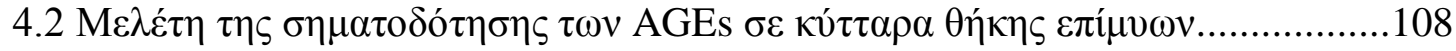

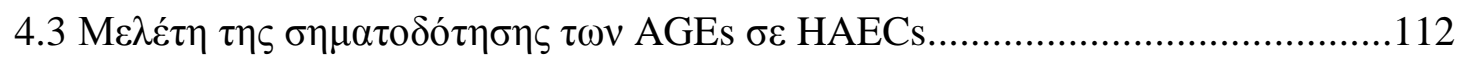

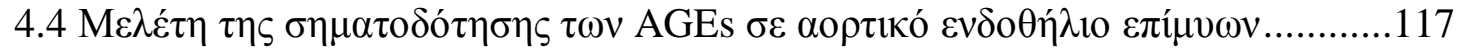

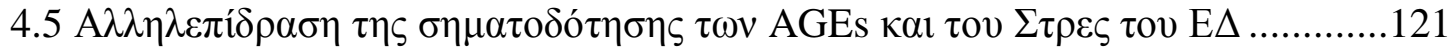

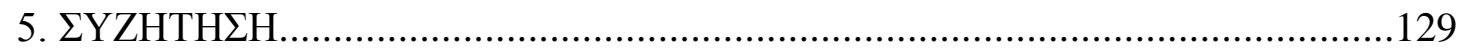

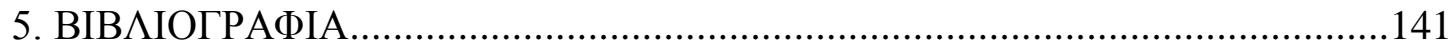




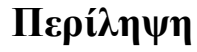

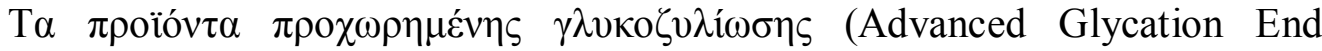

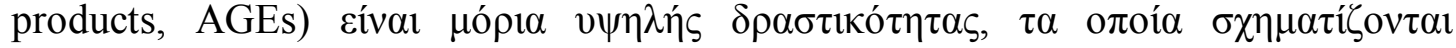

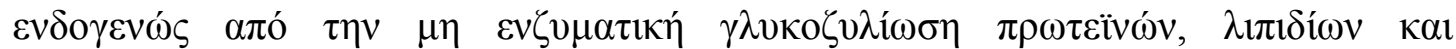

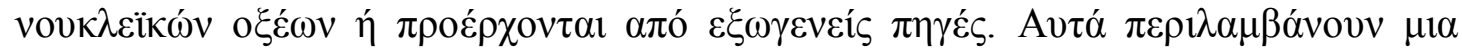

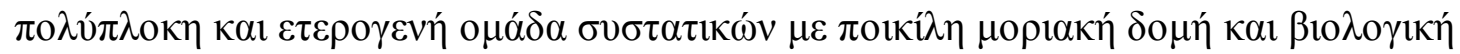

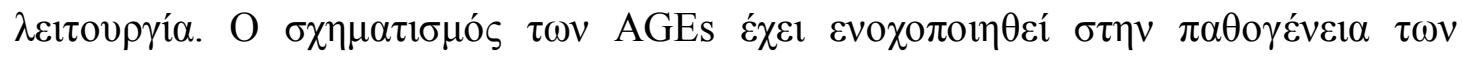

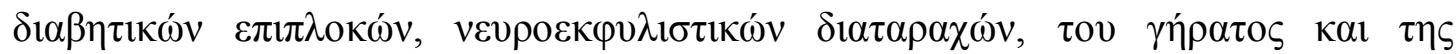

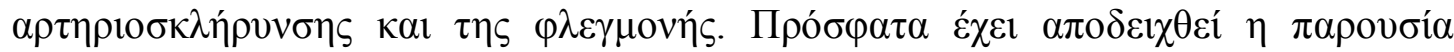

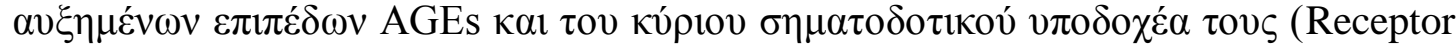

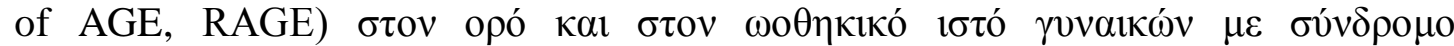

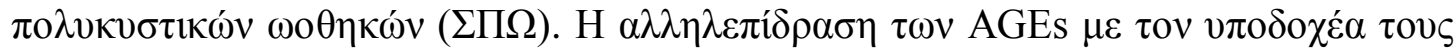

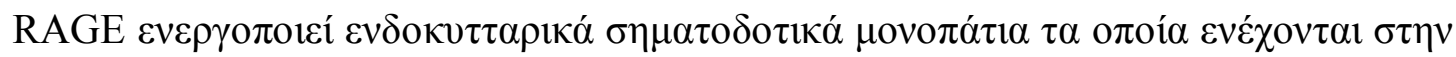

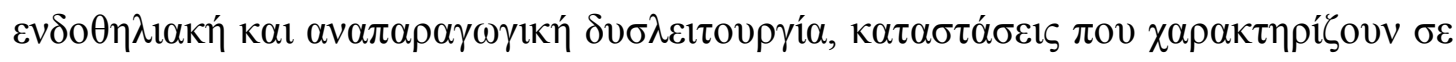

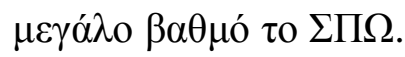

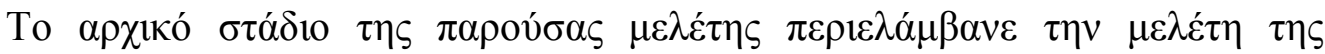

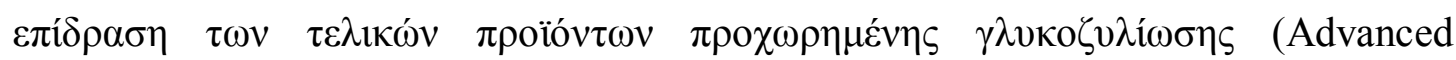

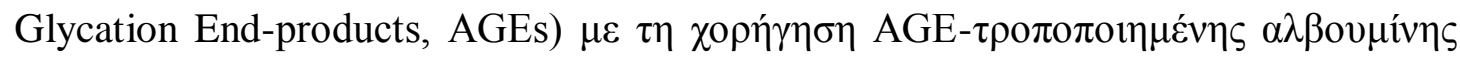

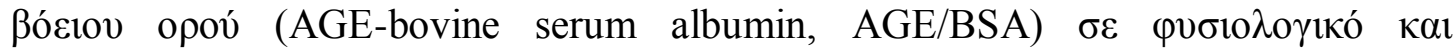

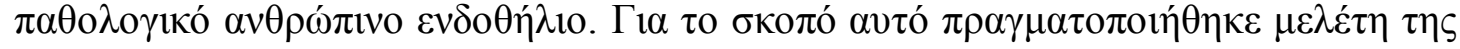

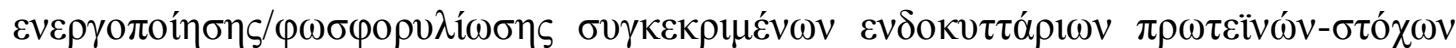

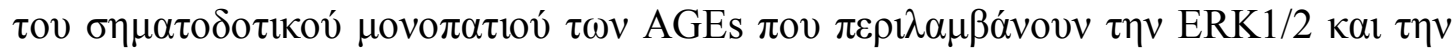

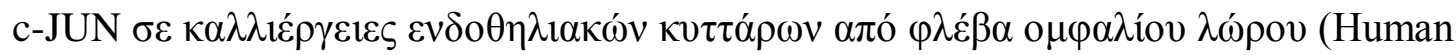

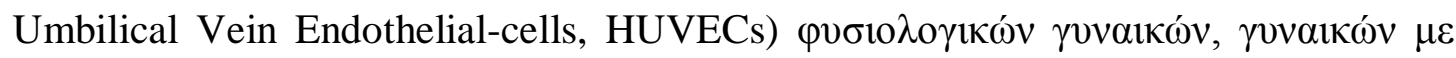

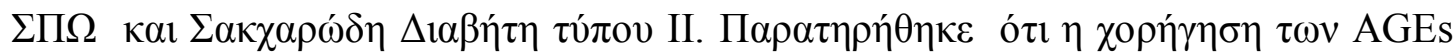

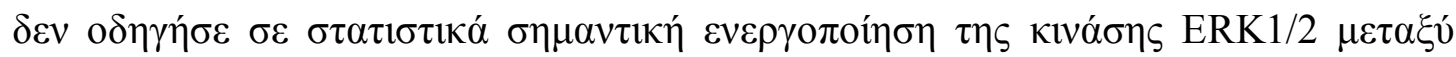

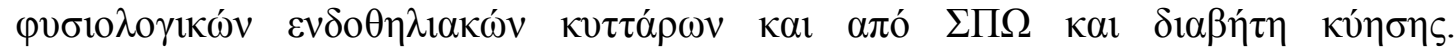

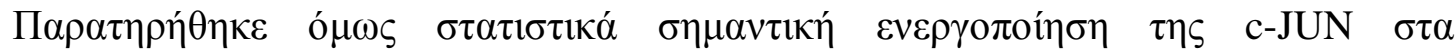

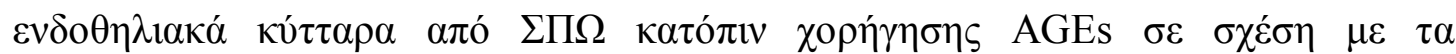

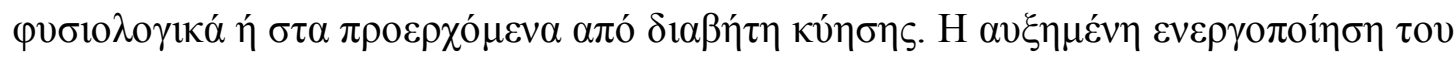

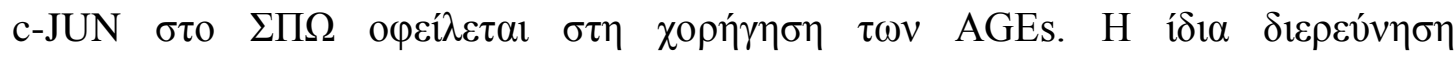

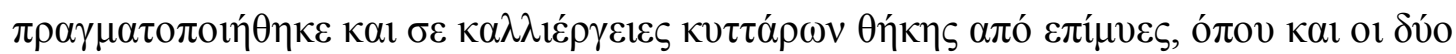




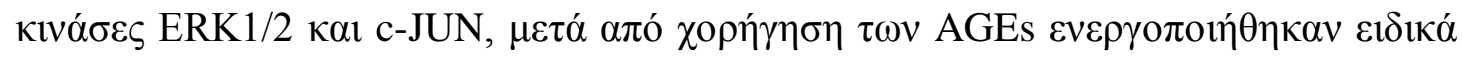
$\mu \varepsilon \dot{\sigma} \omega \varphi \omega \sigma \varphi о \rho v \lambda i ́ \omega \sigma \eta s$.

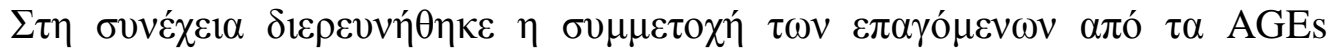

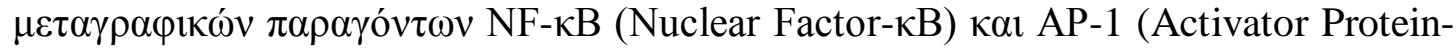

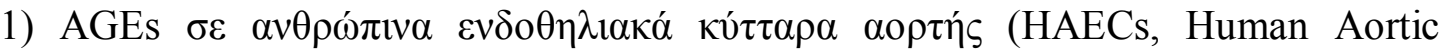

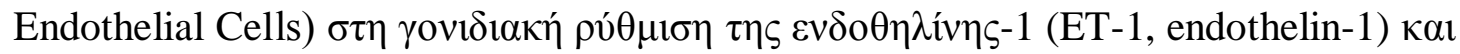

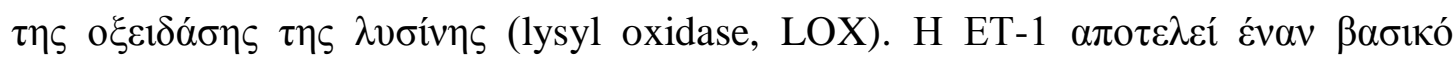

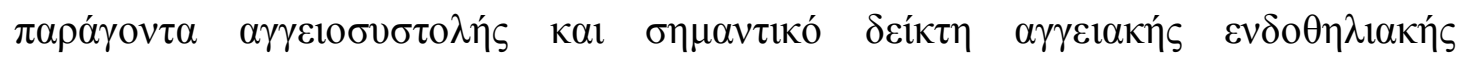

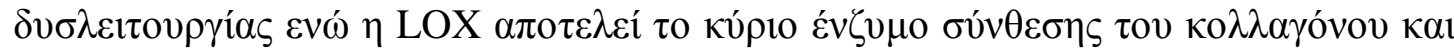

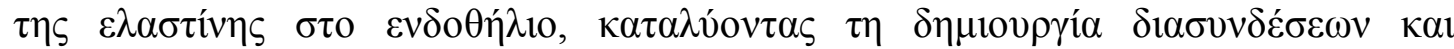

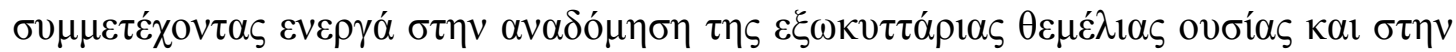

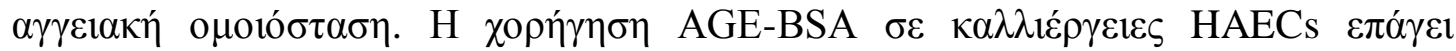

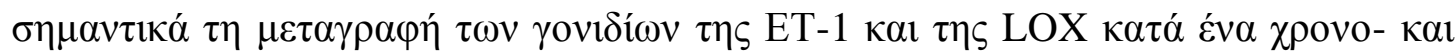

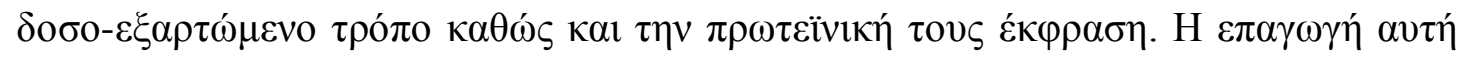

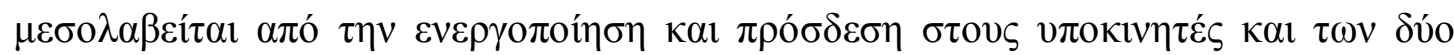

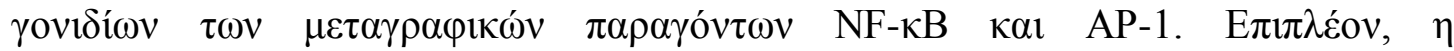

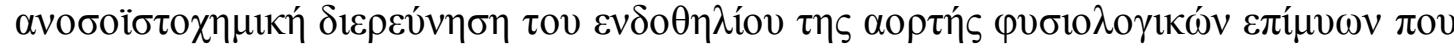

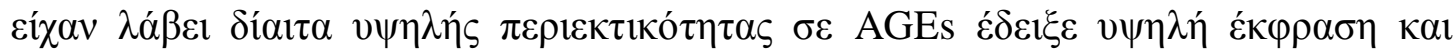

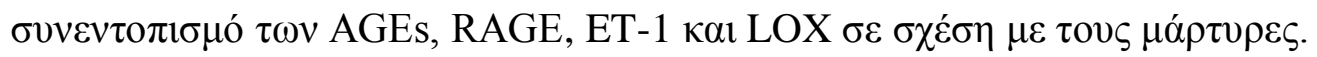

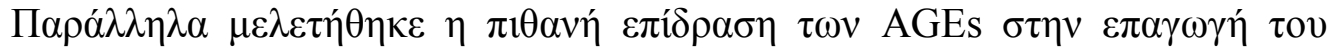

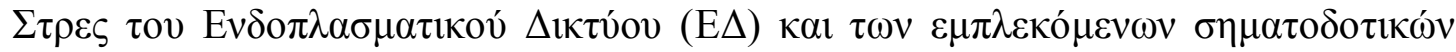

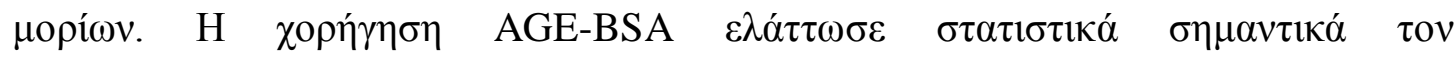

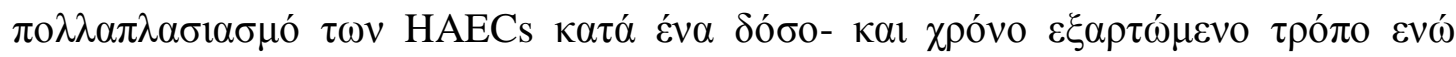

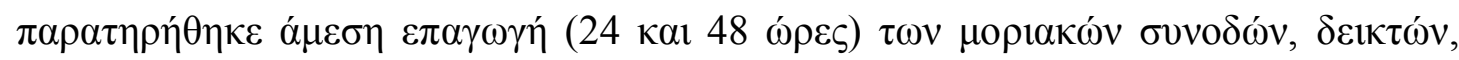

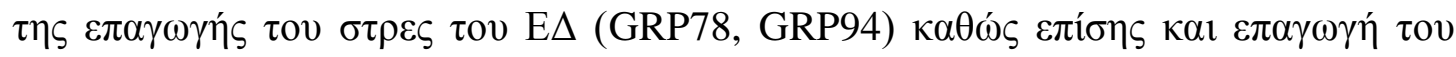

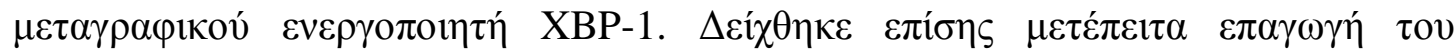

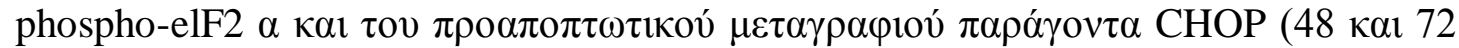
 хрóvovs.

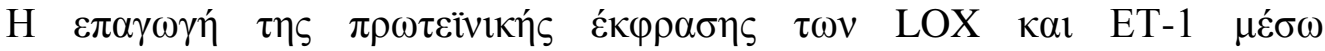

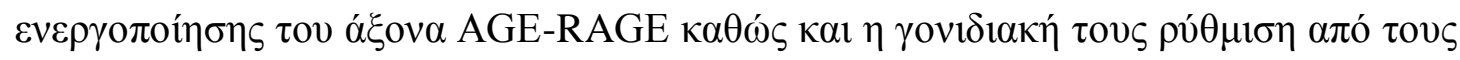




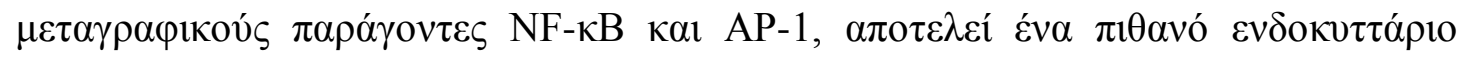

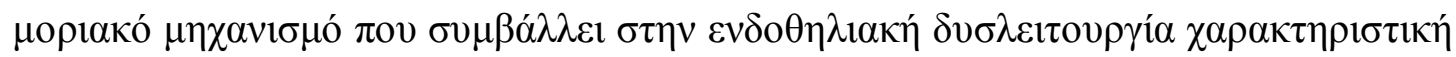

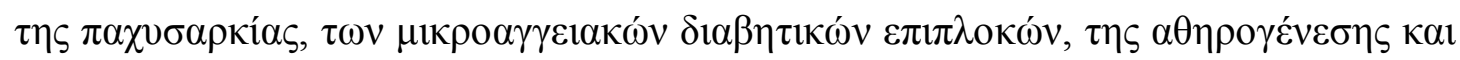

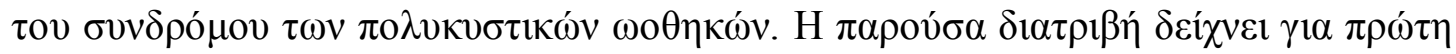

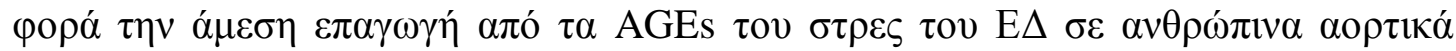

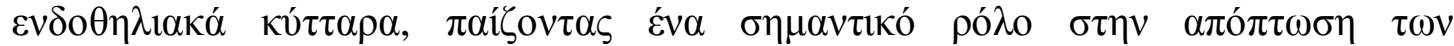
$\varepsilon v \delta \circ \theta \eta \lambda 1 \alpha \kappa \omega ́ v \kappa v \tau \tau \alpha ́ \rho \omega v$. 


\section{Summary}

Advanced glycation products (AGEs) are highly reactive molecules, which are formed from the inherently non-enzymatic glycosylation of proteins, lipids and nucleic acids or derived from exogenous sources. These include a complex and heterogeneous group of components with varying molecular structure and biological function. The formation of AGEs has been implicated in the pathogenesis of diabetic complications, neurodegenerative disorders, aging and atherosclerosis and inflammation. Recently, it has been demonstrated the presence of elevated AGEs levels of and their principal signaling receptor signaling (Receptor of AGE, RAGE) in serum and ovarian tissue of women with polycystic ovary syndrome (PCOS). The interaction of AGEs with their receptor RAGE activates intracellular signaling pathways that are involved in endothelial and reproductive dysfunction, conditions that characterize PCOS.

The first part of this project included the study of AGE effects in normal and pathological human endothelium after treatment with AGE-modified bovine serum albumin (AGE/BSA). The study involved activation/phosphorylation of specific intracellular target proteins of the signaling pathway of AGEs, specifically ERK1/2 and c-JUN, in cultured endothelial cells from umbilical vein (HUVECs) of normal women, women with PCOS and gestastional diabetes. Administration of AGEs resulted in no statistically significant activation of the kinase ERK1/2 between normal endothelial cells from PCOS women and those with gestational diabetes. However, a statistically significant activation of c-JUN was observed in endothelial cells following administration of AGEs compared to normal or derived from gestational diabetes. Increased activation of c-JUN was also observed in PCOS after AGEs treatment. The same investigation was also conducted in cell cultures from rat ovaries (theca cells) where both kinases ERK1/2 and c-JUN were specifically activated after administration of AGEs.

Then we investigated the involvement of the AGEs induced transcription factors NF- $\kappa$ B (Nuclear Factor- $\kappa \mathrm{B}$ ) and AP-1 (Activator Protein-1) AGEs in human aortic endothelial cells (HAECs, Human Aortic Endothelial Cells) in the gene regulation of endothelin -1 (ET-1) and lysine oxidase (LOX). The ET-1 is a key factor 
in vasoconstriction and an important indicator of vascular endothelial dysfunction. LOX on the other hand is the main enzyme implicated in the synthesis of collagen and elastin in the endothelium, catalyzing the formation of crosslinks and participating actively in the reconstruction of the extracellular matrix as well as contributing to vascular homeostasis. The administration of AGE-BSA in HAECs significantly induced gene transcription of ET-1 and LOX in a time-and dose-dependent manner and their protein expression. This induction is mediated by binding and activation of the transcription factors NF-kappaB and AP-1 in the promoters of both genes. Furthermore, immunohistochemical investigation of normal aortic endothelium from rats that had received high in AGEs-diet showed increased expression and colocalization of AGEs, RAGE, ET-1 and LOX in the same areas.

In parallel, the effect of AGEs on the induction of endoplasmic reticulum stress (ER stress) was also investigated in endothelial cells. AGE-BSA administration significantly decreased proliferation of HAECs at a dose- and time-dependent manner, while there was an immediate induction (at 24 and 48 hours) of the molecular chaperons, GRP78, GRP94 which are representative markers of ER stress induction. Furthermore activation of the transcriptional activator, XBP-1 was also observed. In addition a subsequent induction of phospho-elF2 $\alpha$ and of the proapoptotic transcription factor, CHOP (at 48 and 72 hours) was associated with the increased number of apoptotic cells at the same time points.

The elevated protein expression of LOX and ET-1 via activation of AGERAGE axis and gene regulation through the transcription factors NF- $\kappa$ B and AP-1 suggest a potential intracellular molecular mechanism contributing to endothelial dysfunction that characterizes microvascular diabetic complications, obesity, atherogenesis and polycystic ovary syndrome. The present study shows for the first time the direct induction of ER stress by AGEs in human aortic endothelial cells, playing an important role in the apoptosis of endothelial cells. 


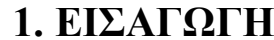

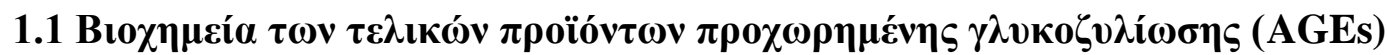

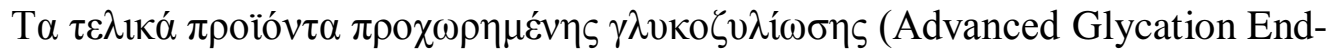

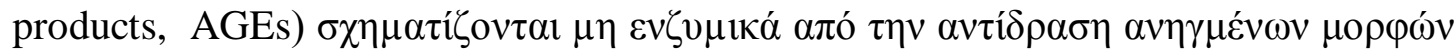

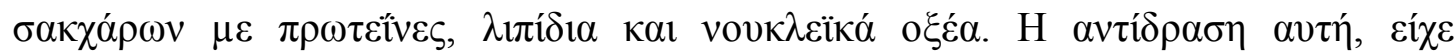

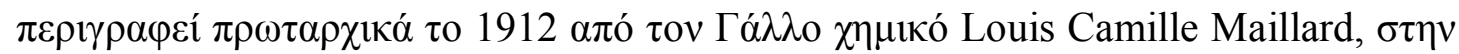

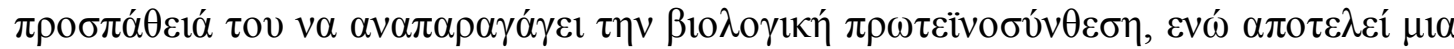

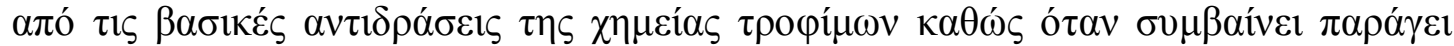

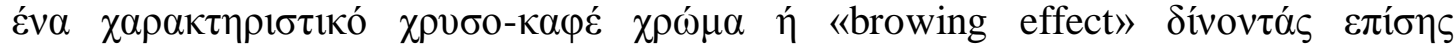

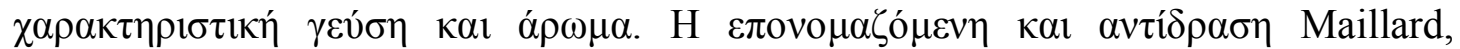

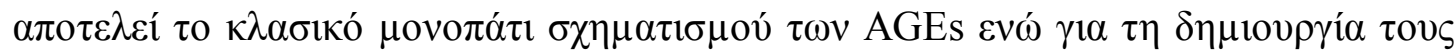

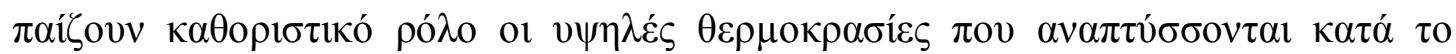
$\mu \alpha \gamma \varepsilon i ́ p \varepsilon \mu \alpha[1]$.

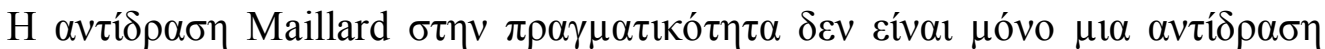

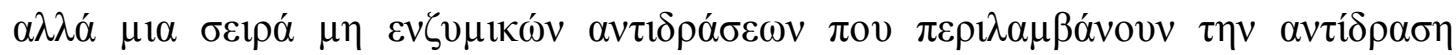

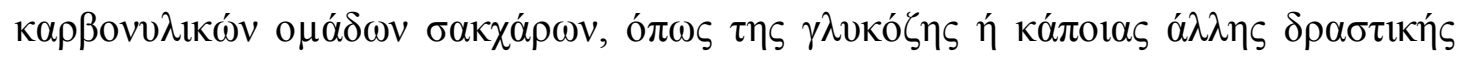

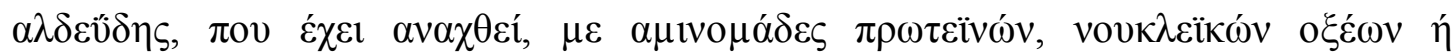

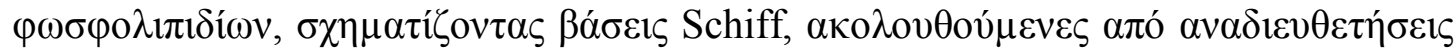

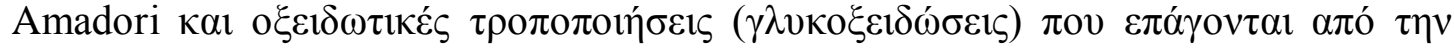

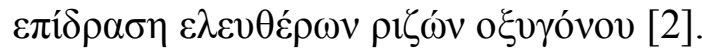

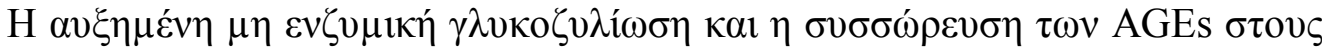

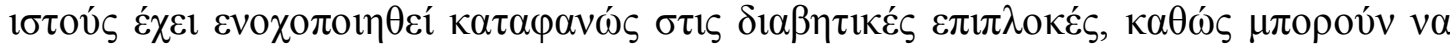

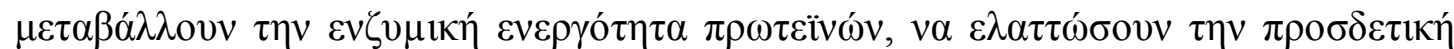

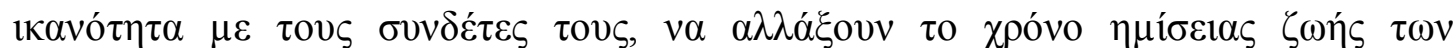

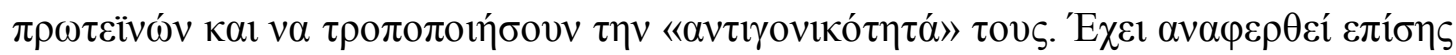

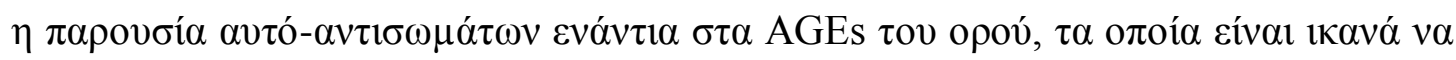

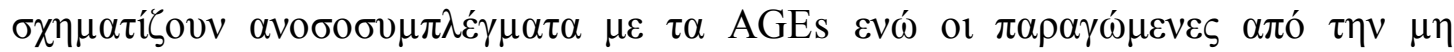

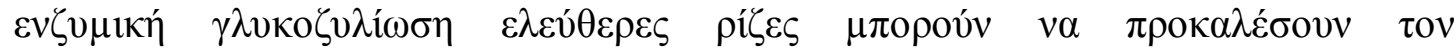




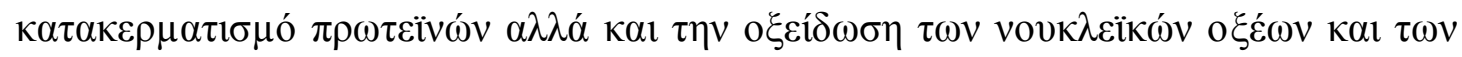
$\lambda \imath \imath 1 \delta i ́ \omega v[2]$.

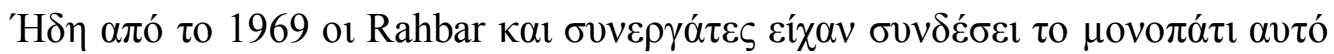

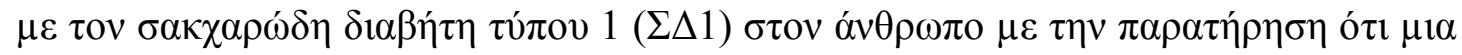

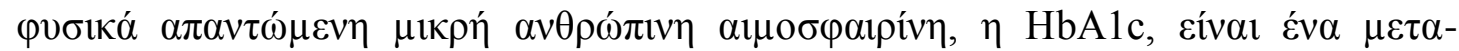

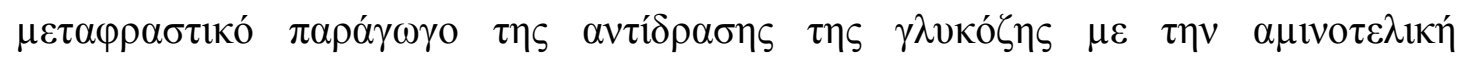

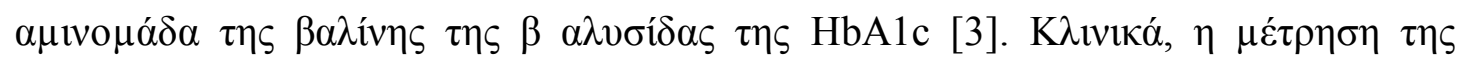

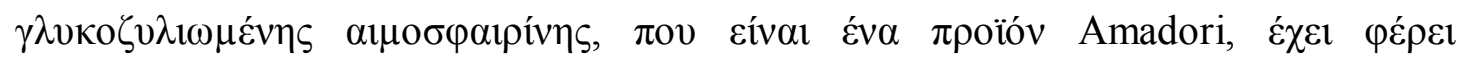

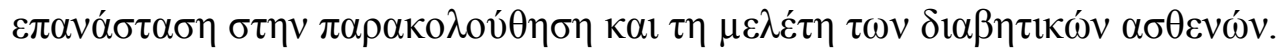

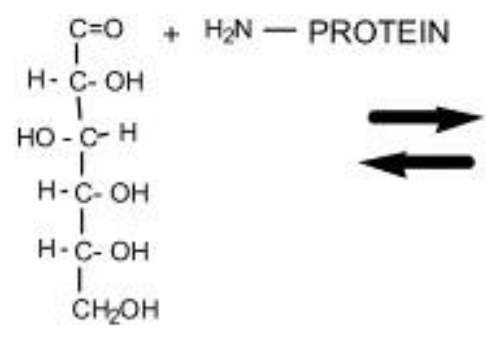

Glucose

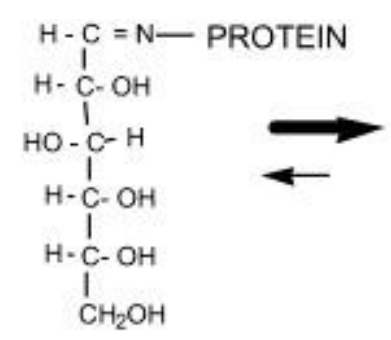

Schiff base<smiles>N#[Po+]c1ccccc1</smiles>

Amadori product

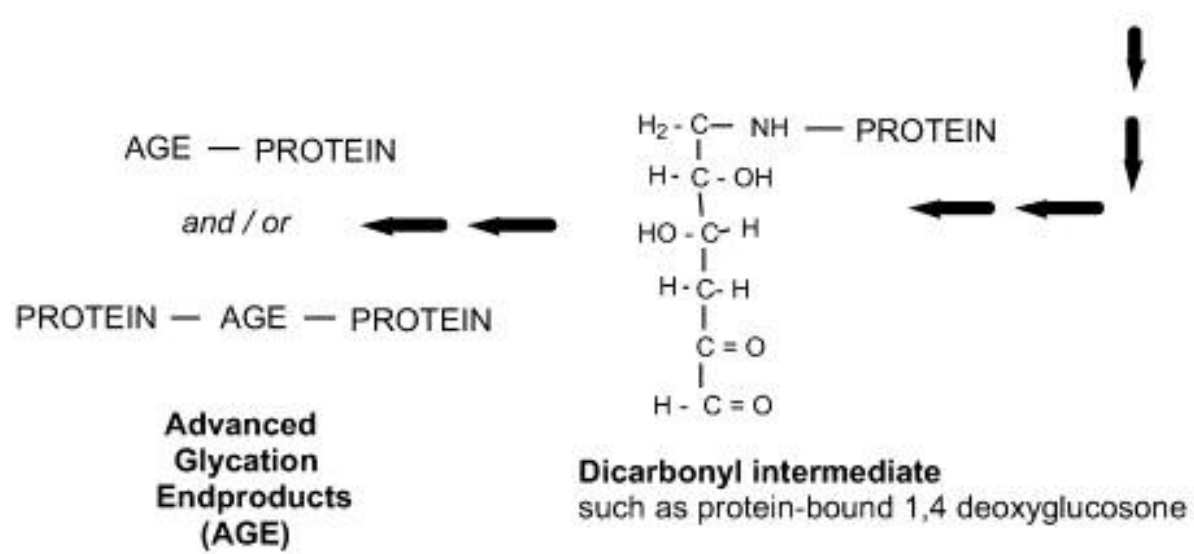

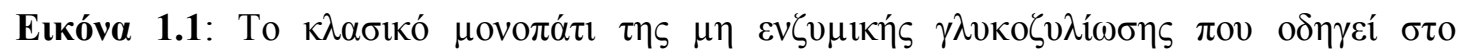

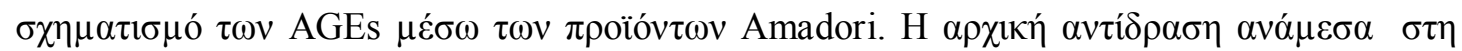

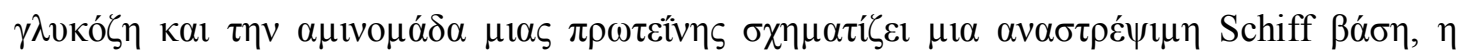

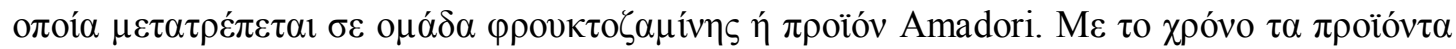

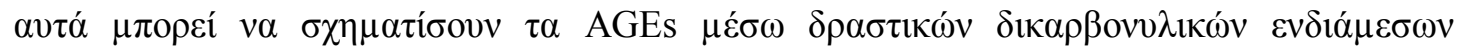

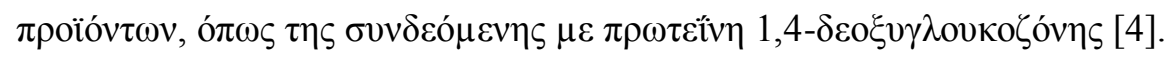




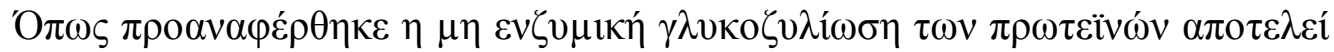

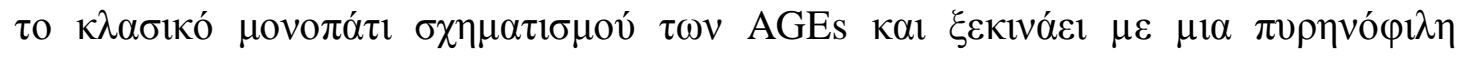

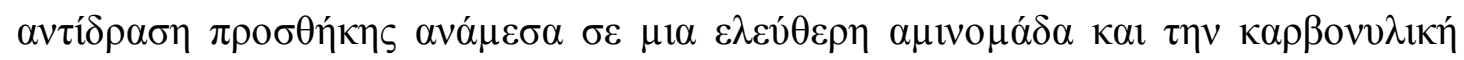

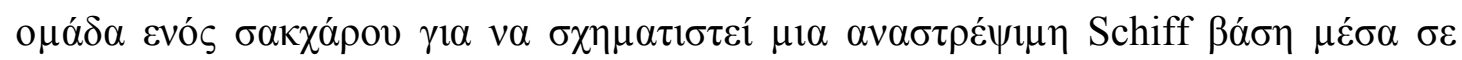

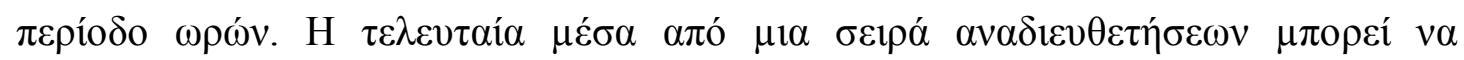

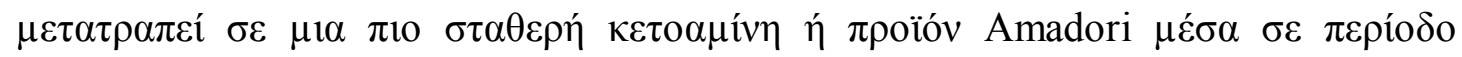

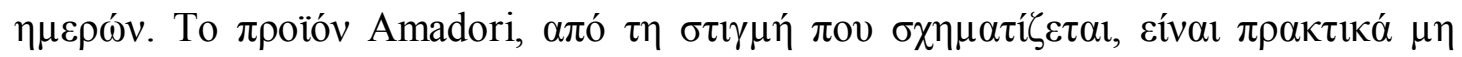

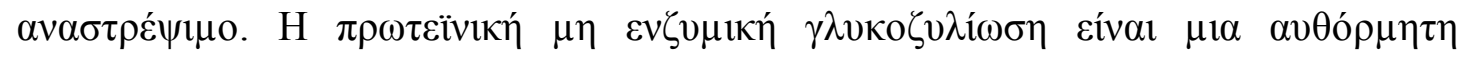

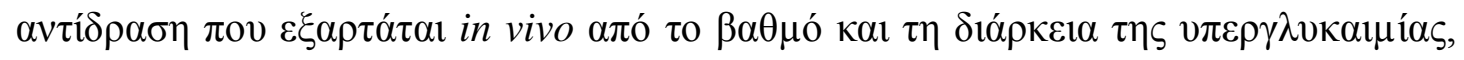

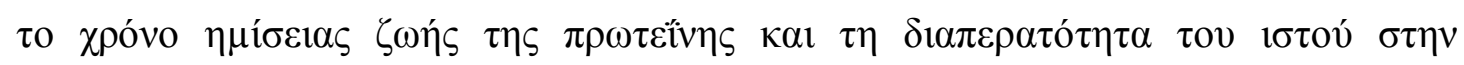

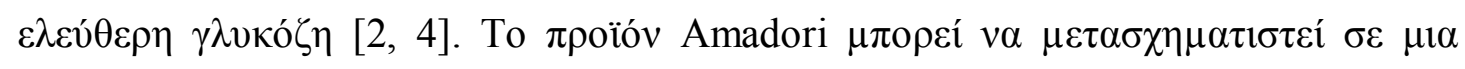

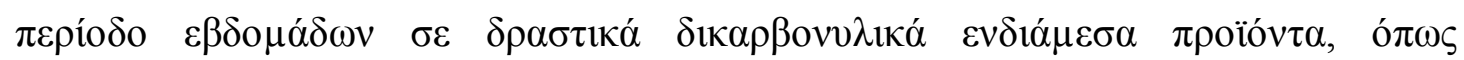

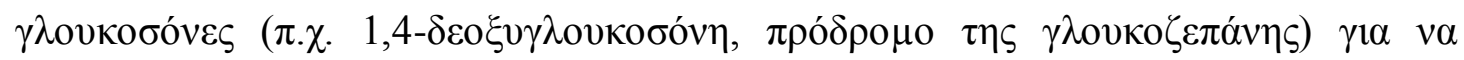

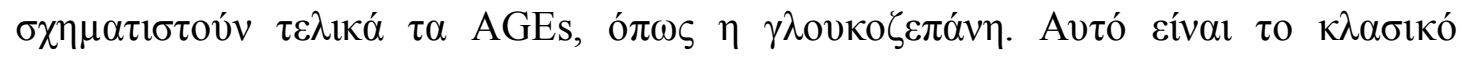

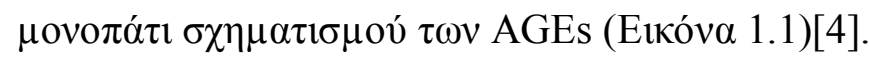

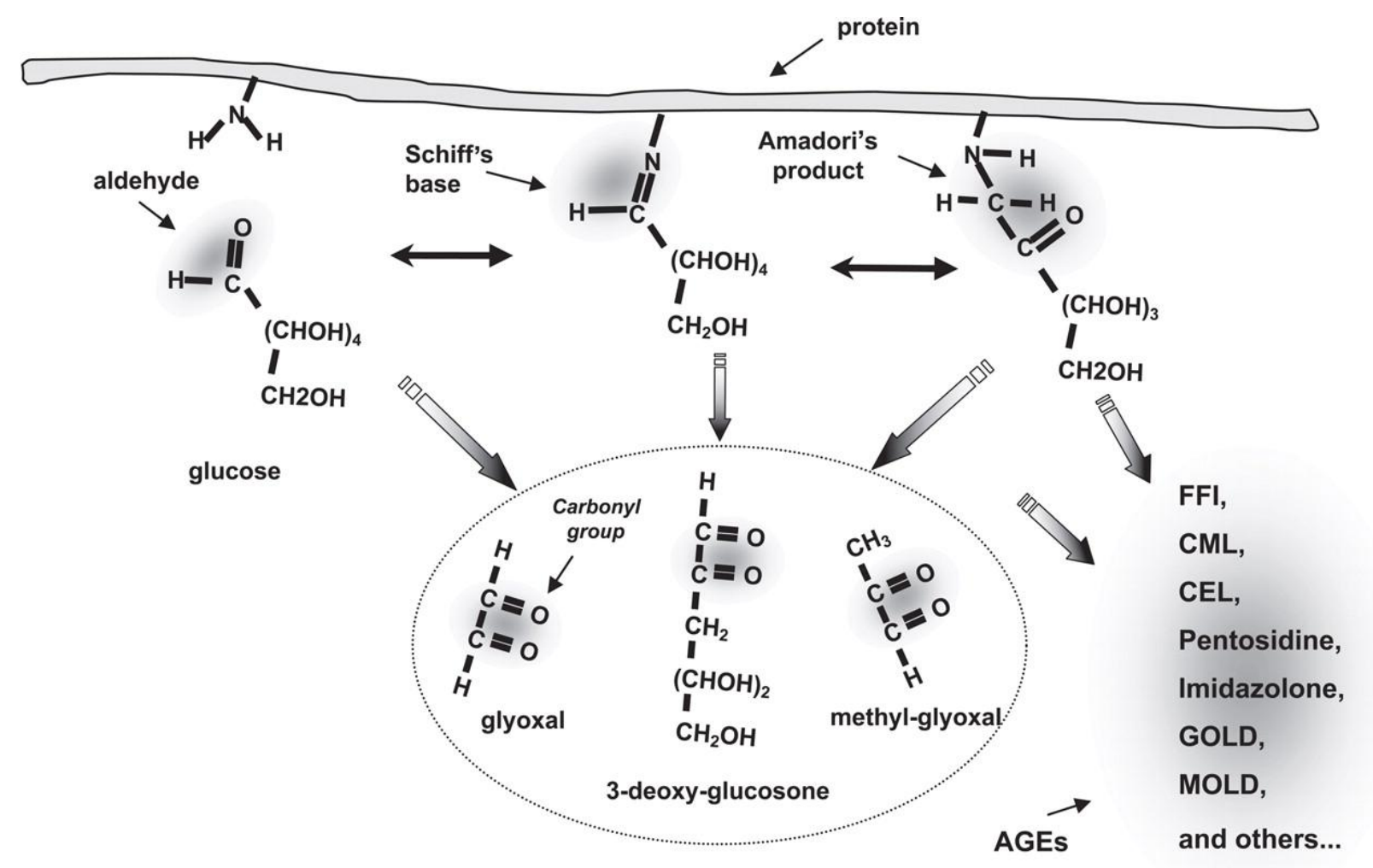

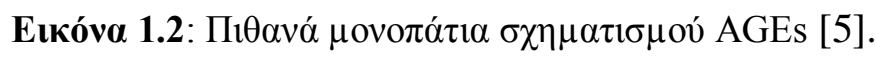




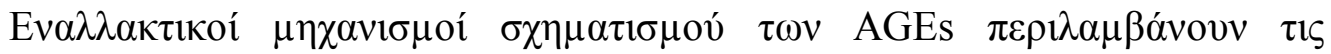

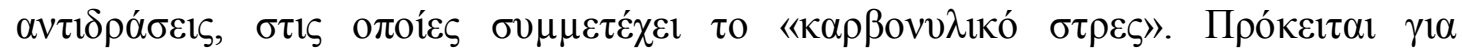

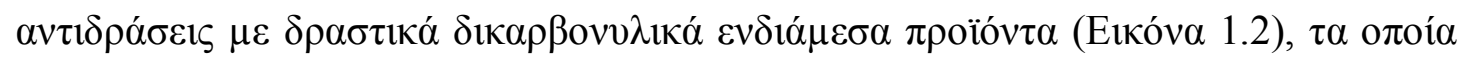

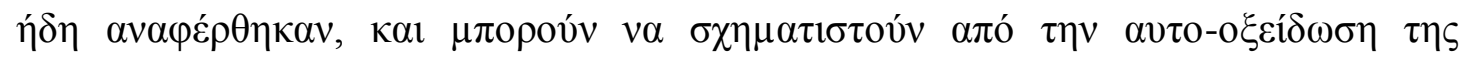

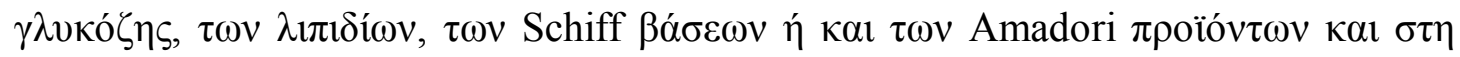

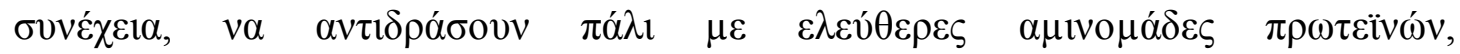

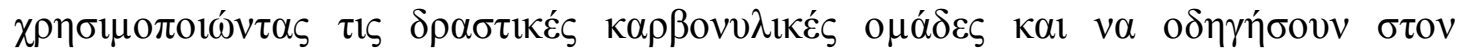

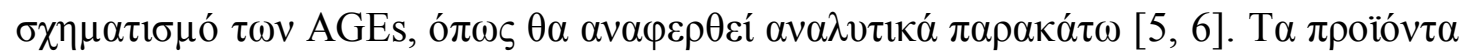

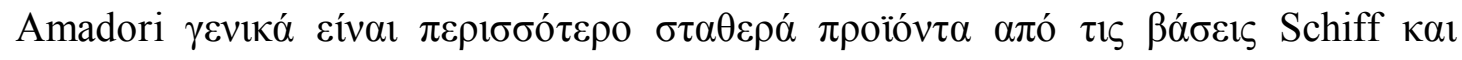

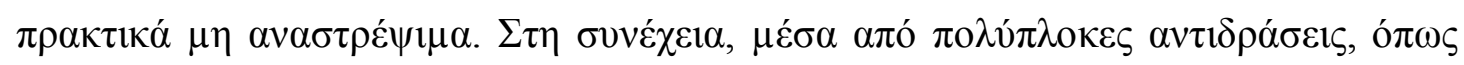

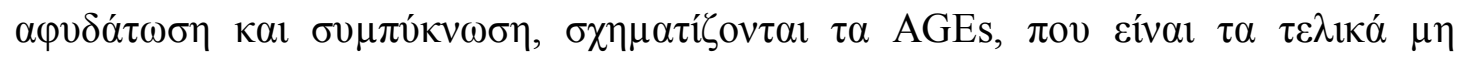

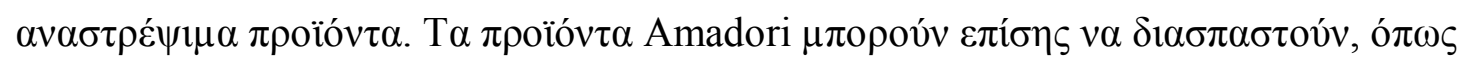

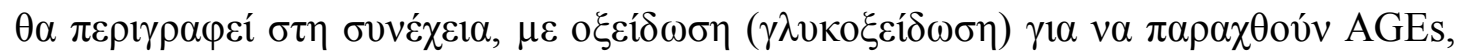
ó $\pi \omega \varsigma \eta \kappa \alpha \rho \beta о \xi v \mu \varepsilon \theta 0 \lambda-\lambda v \sigma i ́ v \eta$ (carboxymethyl-lysine: CML) [4].

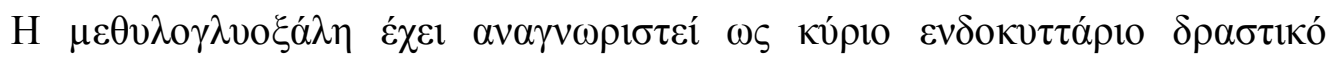

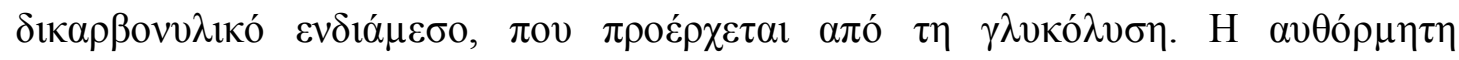

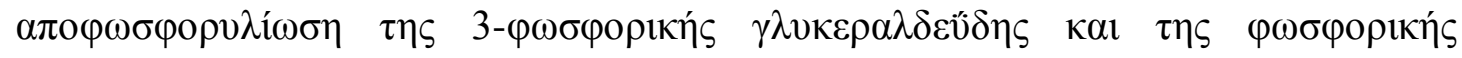

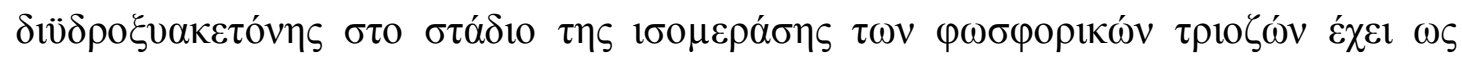

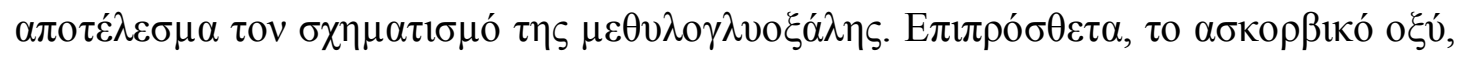

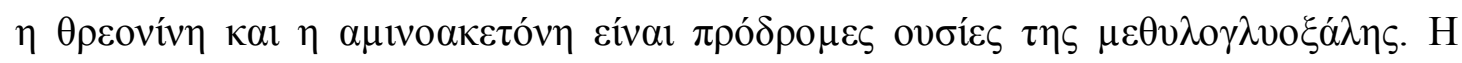

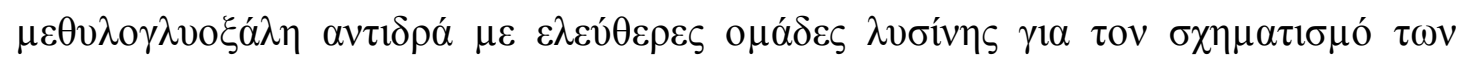

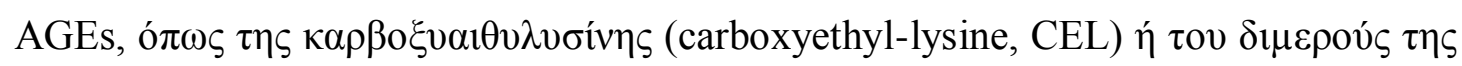

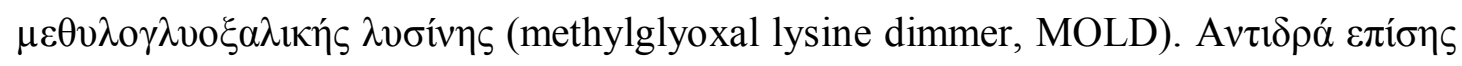

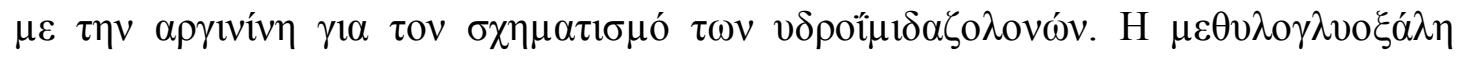

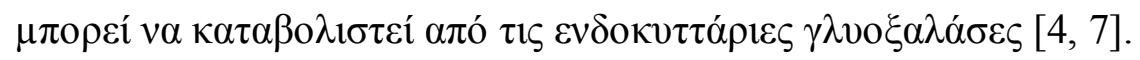

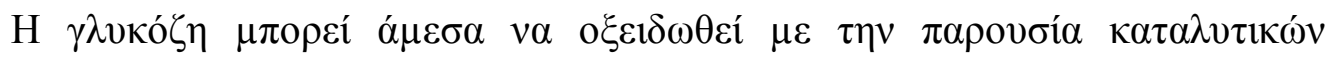

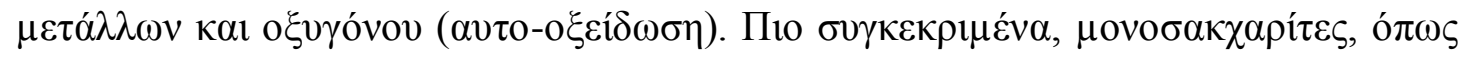

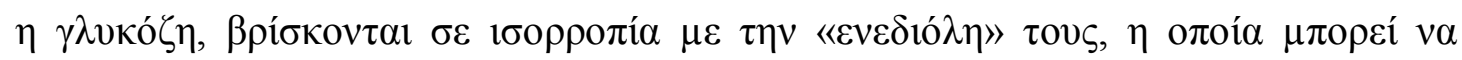

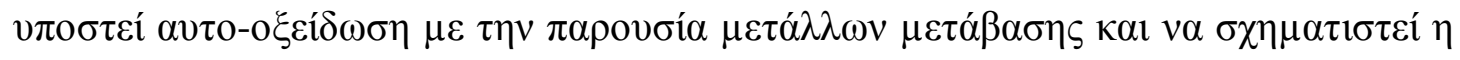

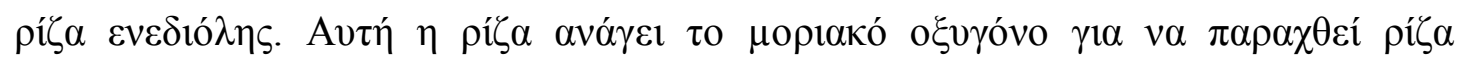

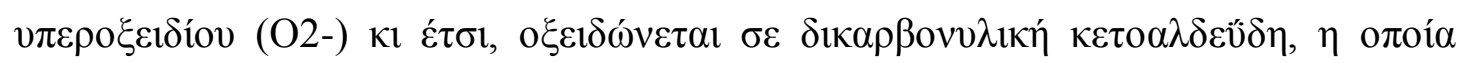




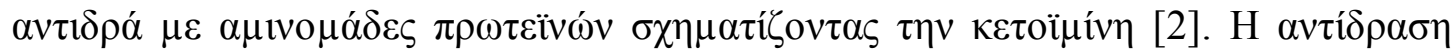

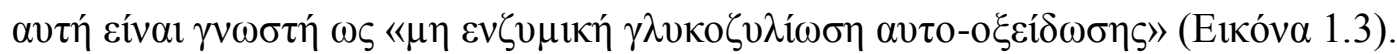

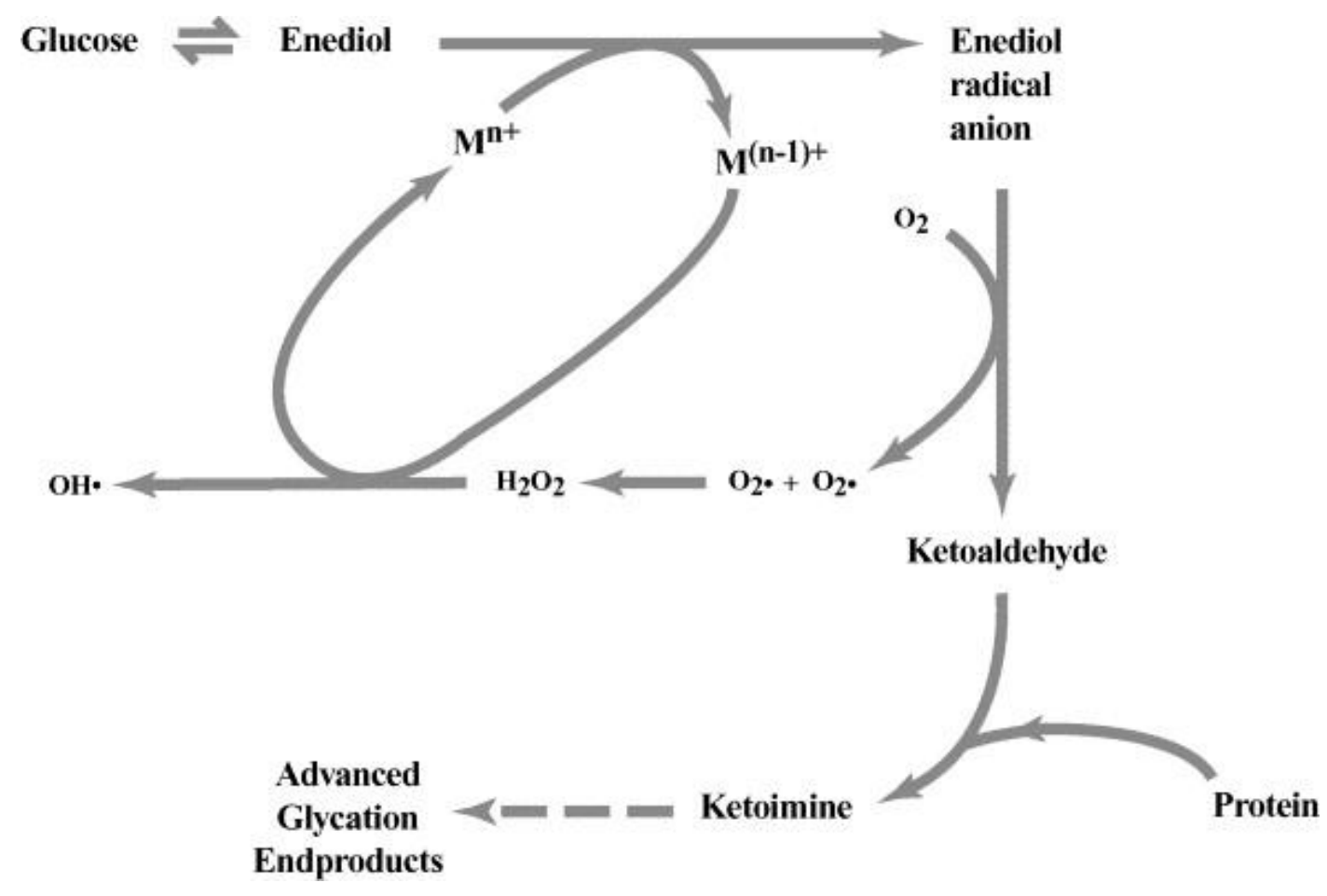

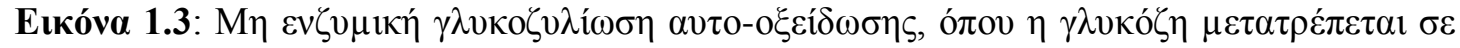

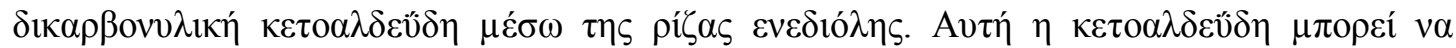

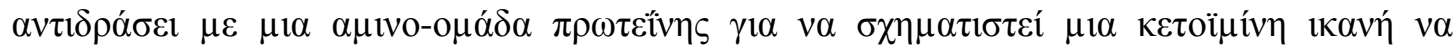

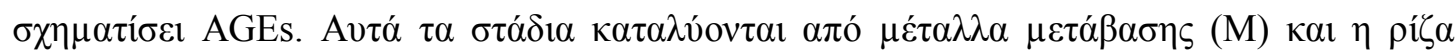

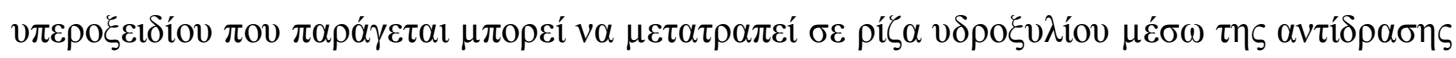
Fenton.

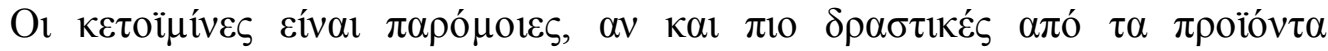

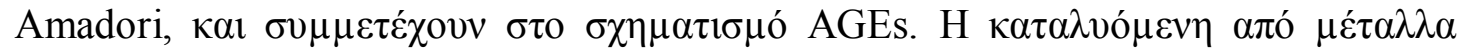

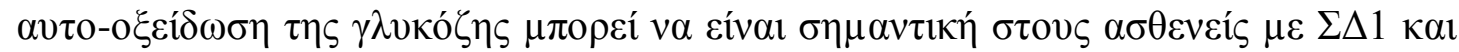

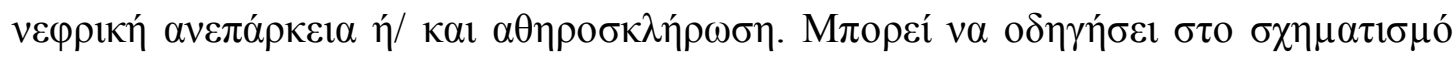

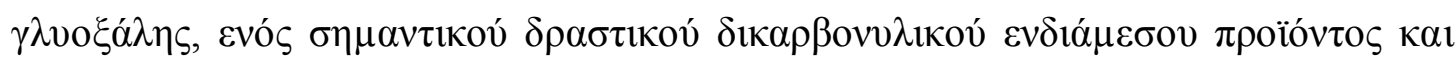

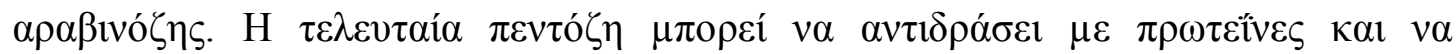

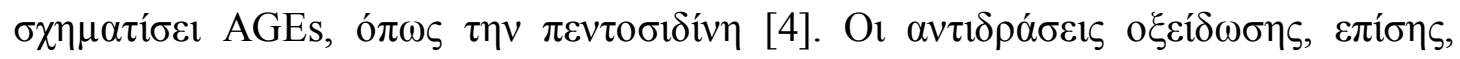

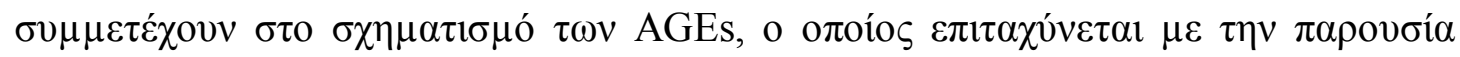

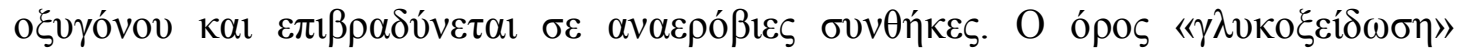

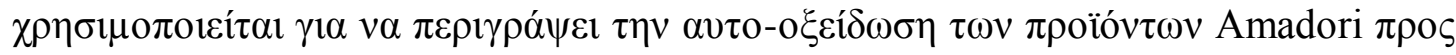

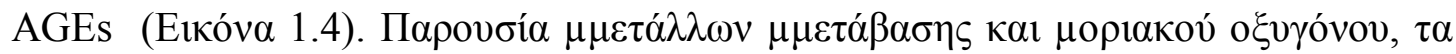




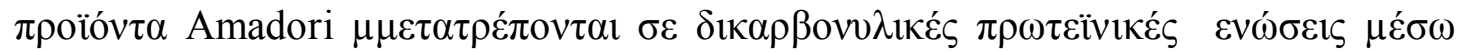

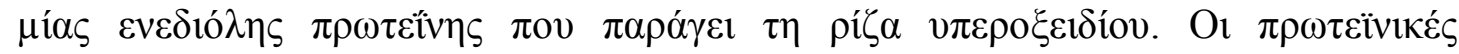

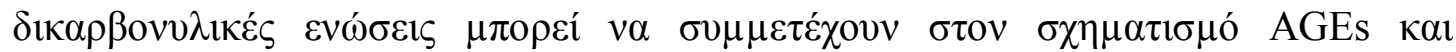

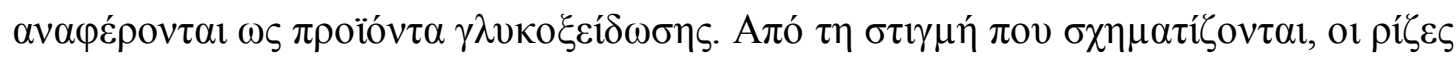

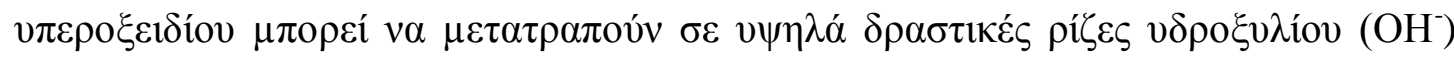
$\mu \varepsilon \dot{\varepsilon} \sigma \omega \tau \eta \varsigma \alpha \nu \tau i ́ \delta \rho \alpha \sigma \eta \varsigma$ Fenton [2].

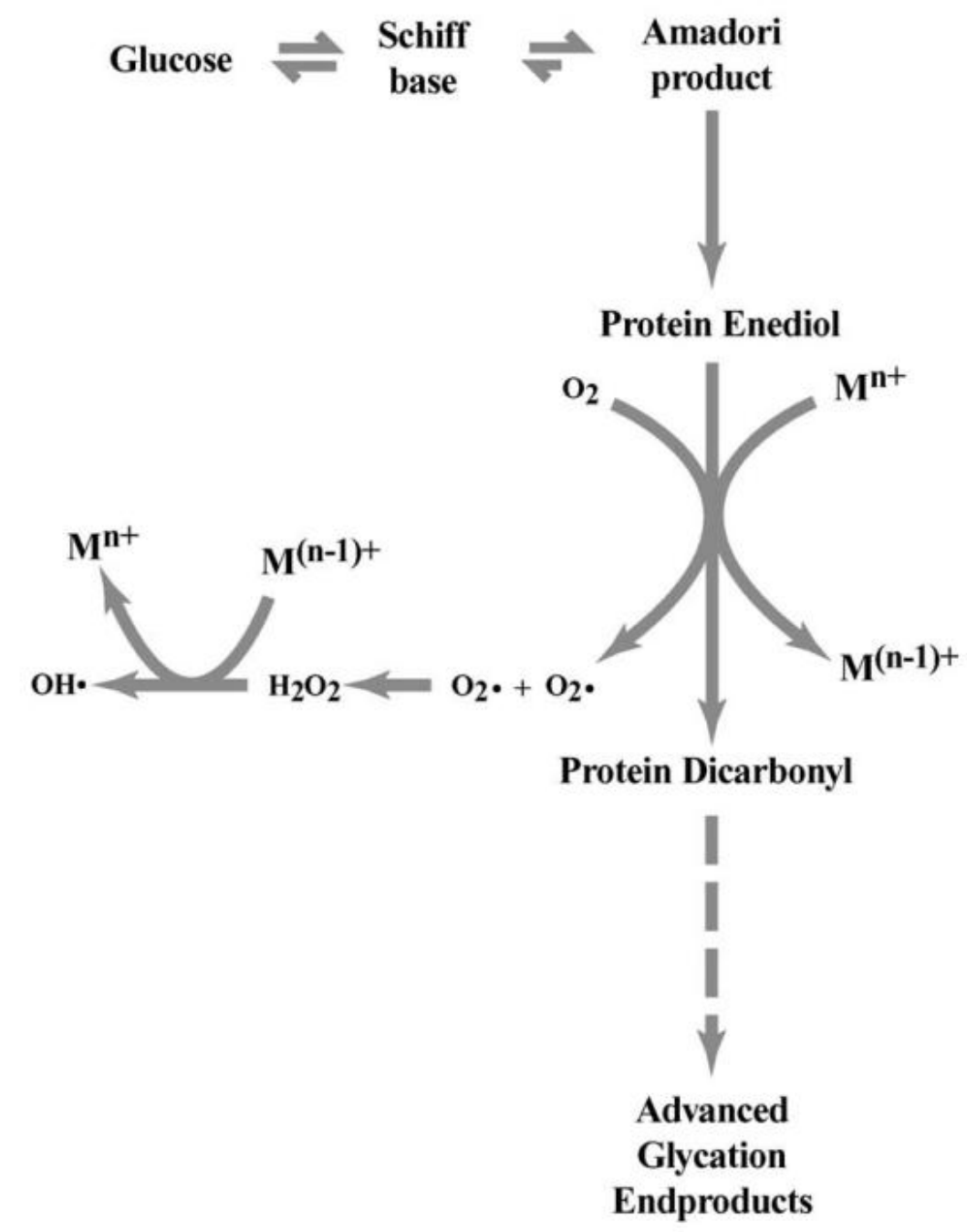

Euкóva 1.4: H $\gamma \lambda$

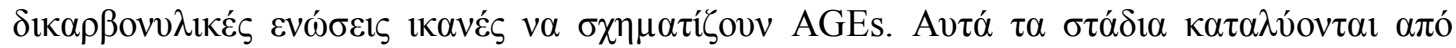

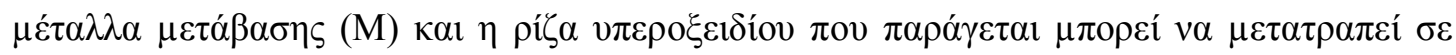

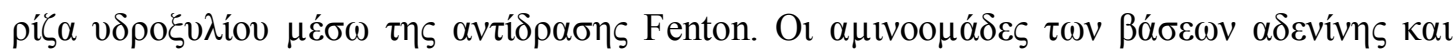

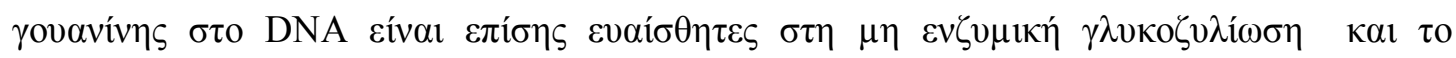

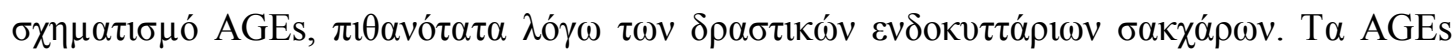

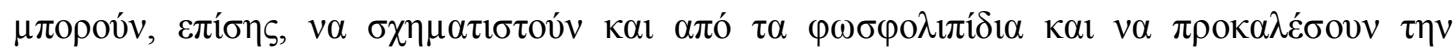




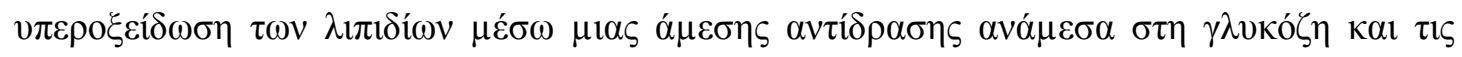

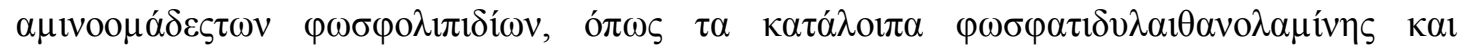

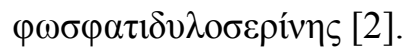

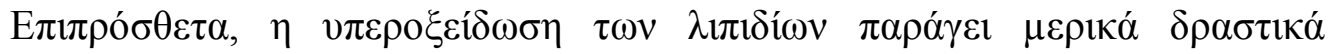

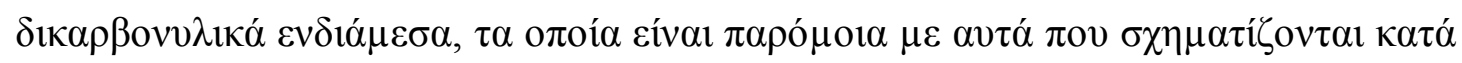

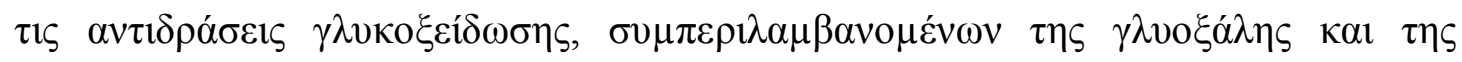

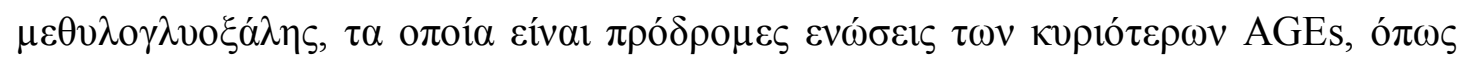

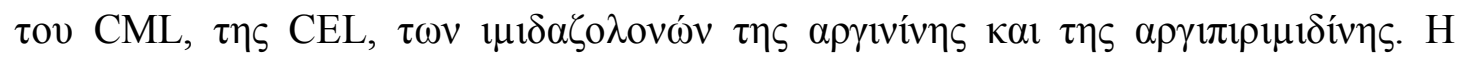

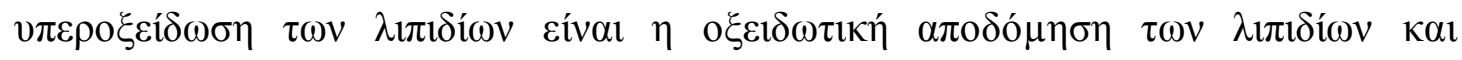

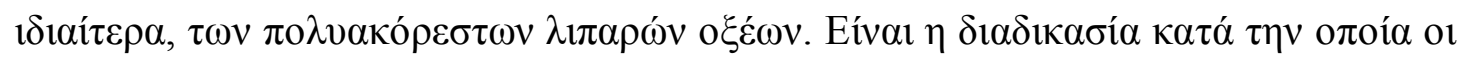

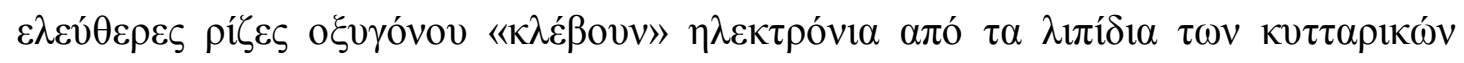

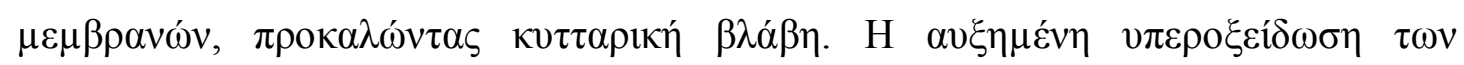

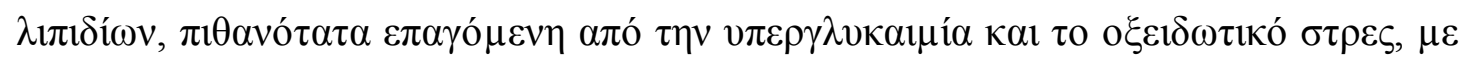

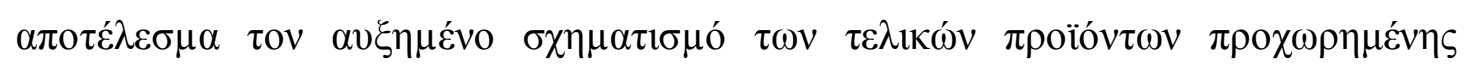

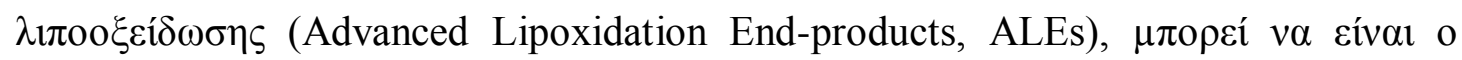

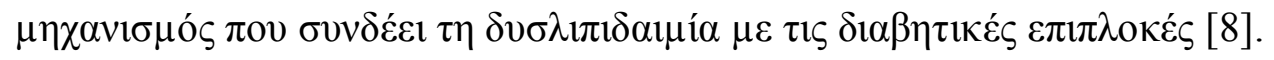

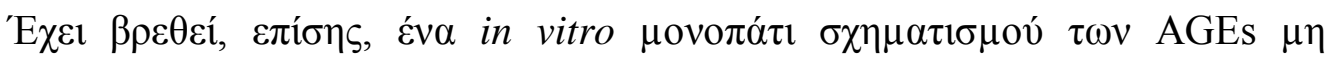

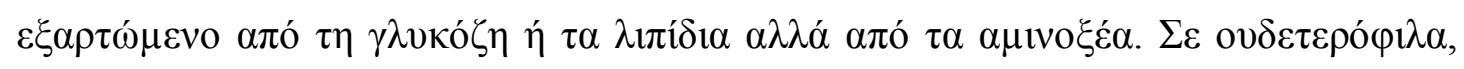

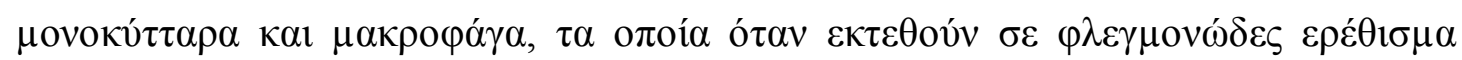

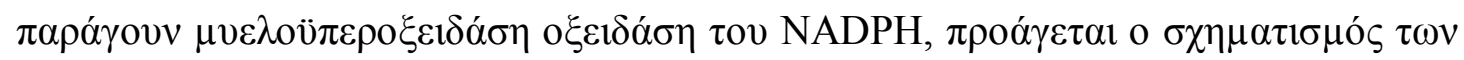

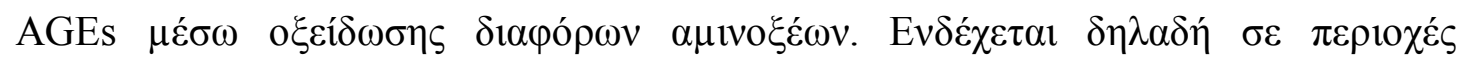

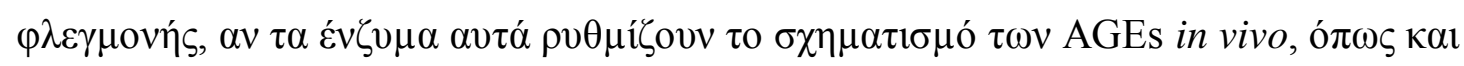

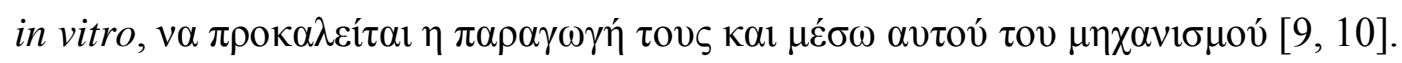

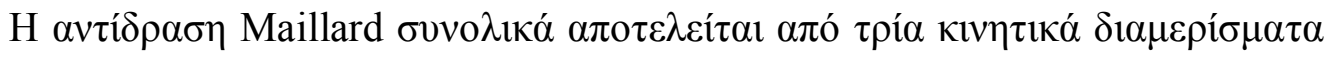

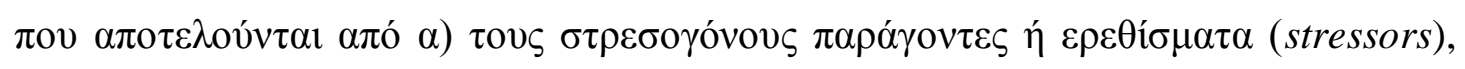

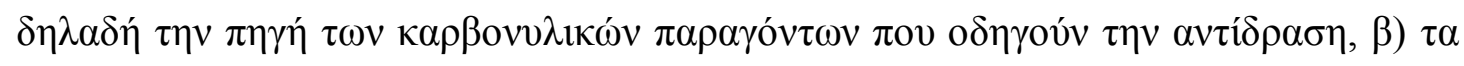

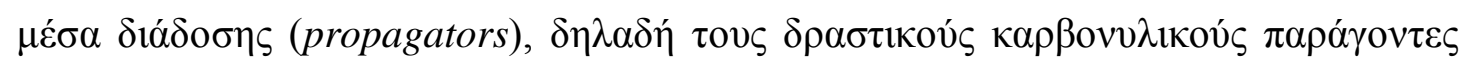

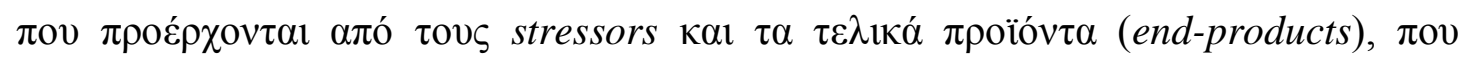

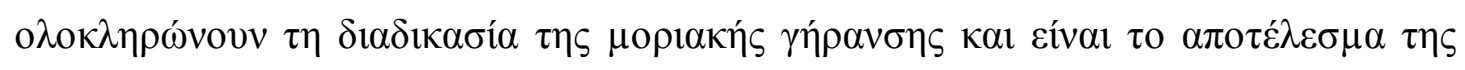

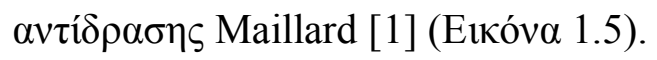




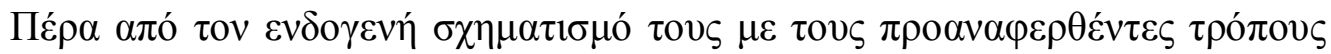

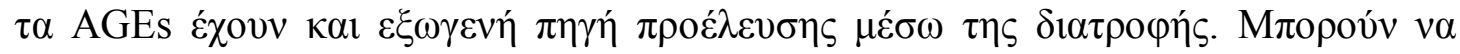

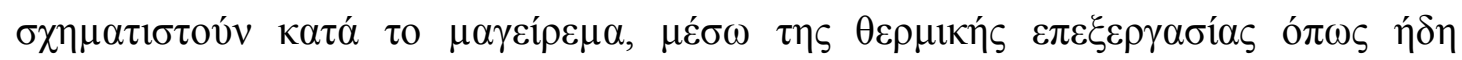

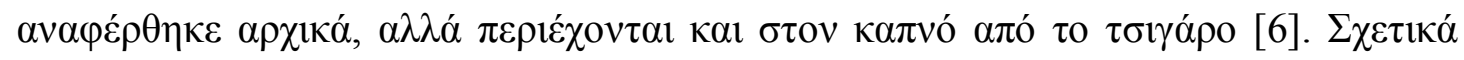

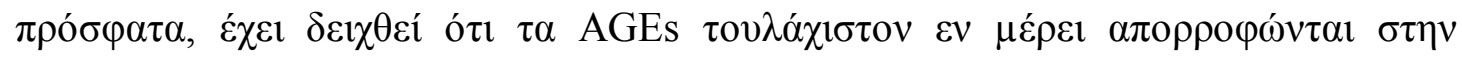

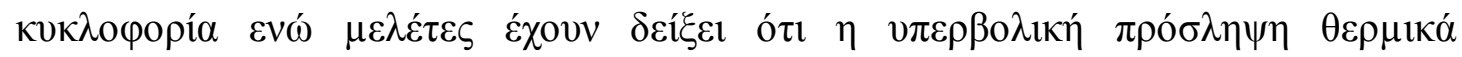

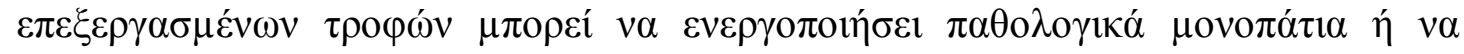

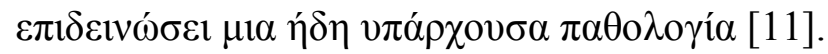

\section{Stressors Propagators Endproducts}

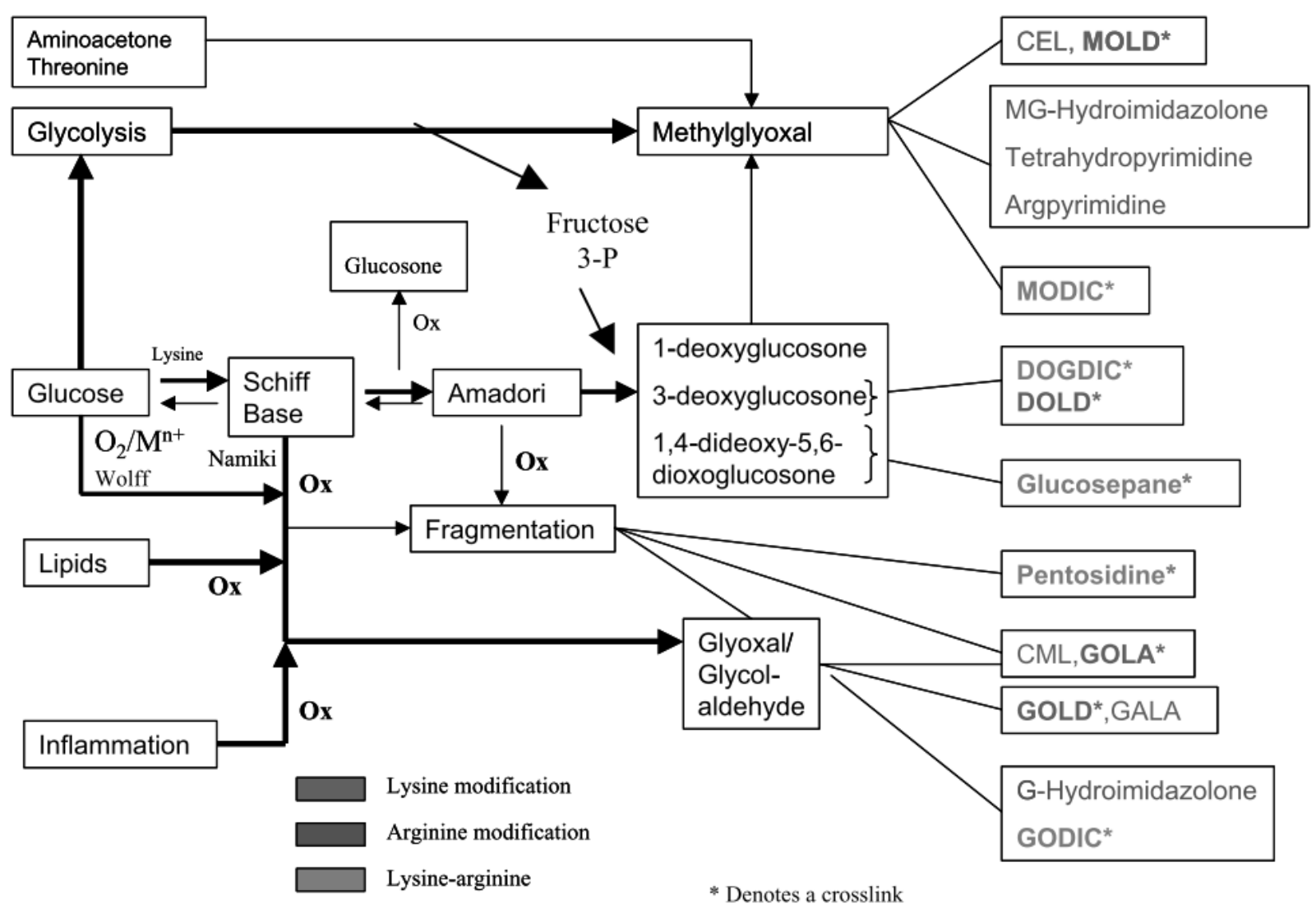

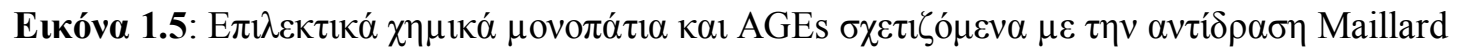
in vivo [1].

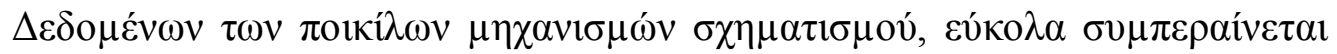

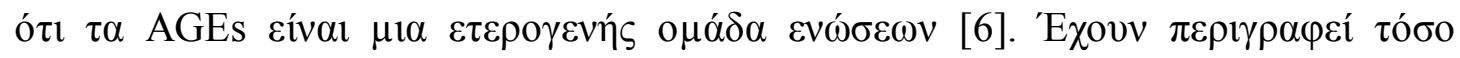

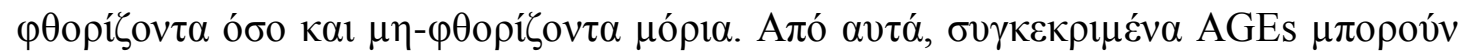




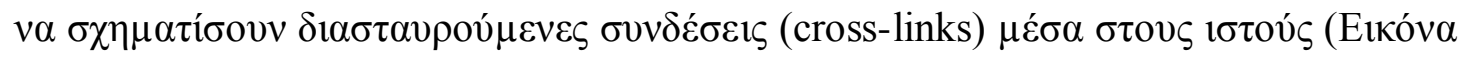
$1.6)$.

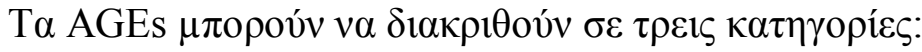

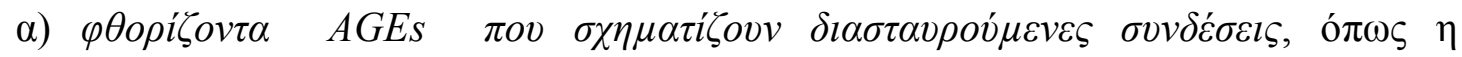

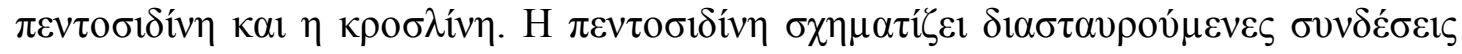

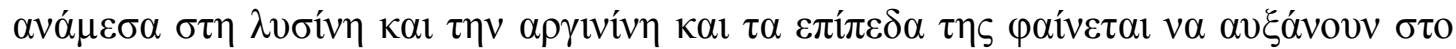

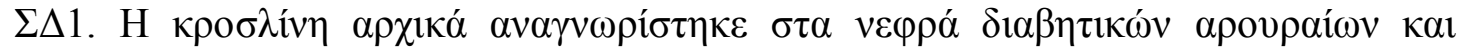

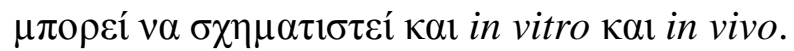

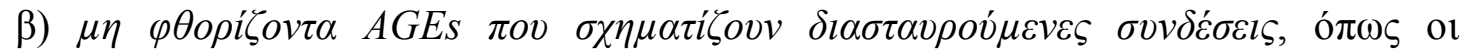

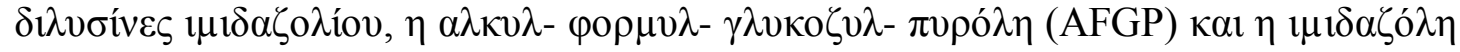

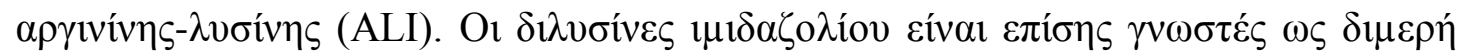

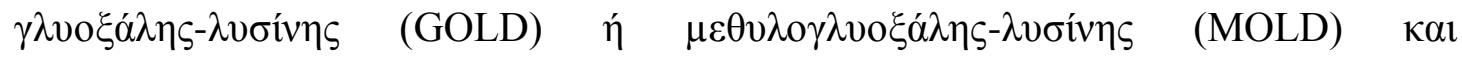

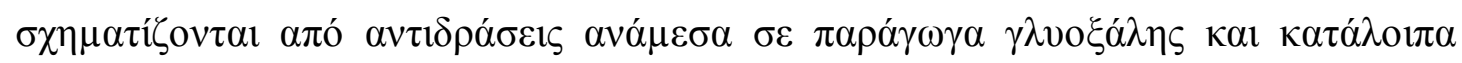
$\lambda v \sigma i ́ v \eta \varsigma \kappa \alpha 1 \alpha v i \chi v \varepsilon v ́ o v \tau \alpha l$ in vivo. O1 $\alpha \lambda \kappa v \lambda-\varphi \circ \rho \mu v \lambda-\gamma \lambda v \kappa o \zeta v \lambda-\pi v \rho o ́ \lambda \varepsilon \varsigma$ (AFGP)

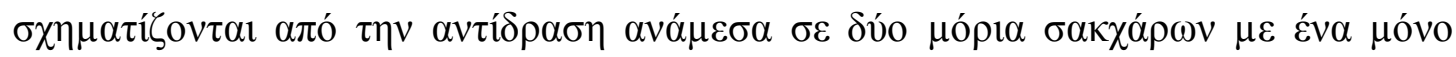

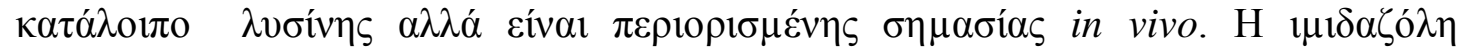

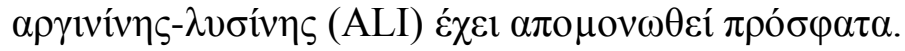

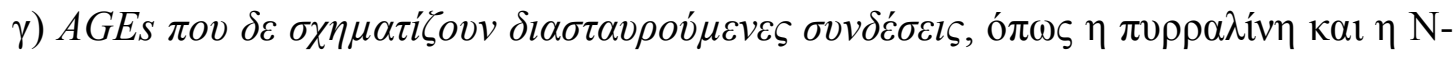

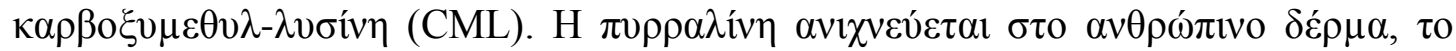

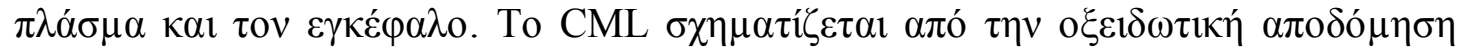

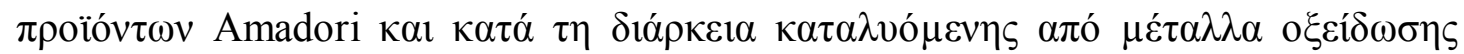

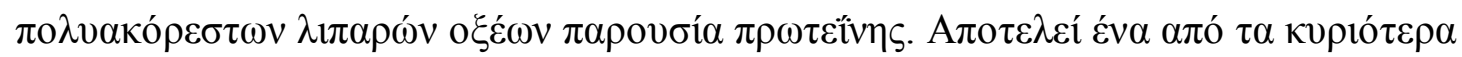

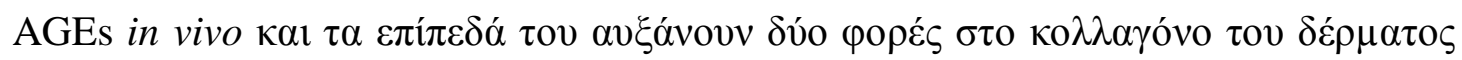

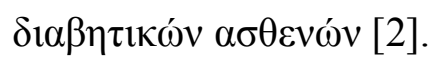

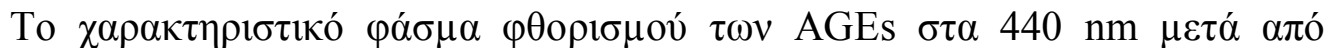

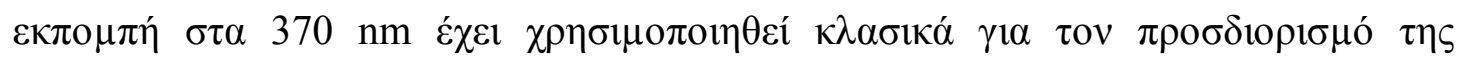

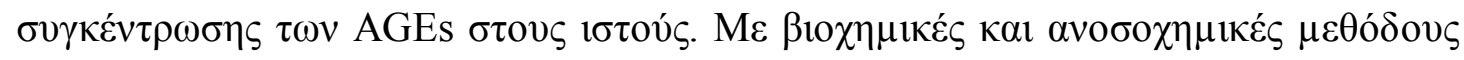

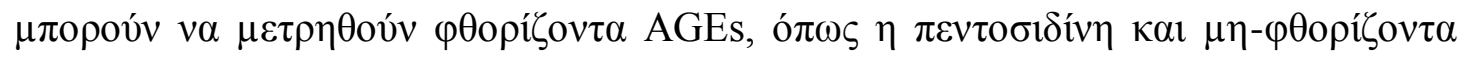

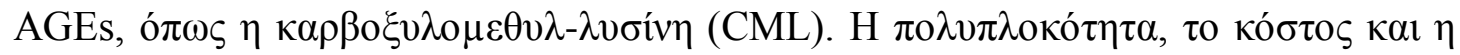

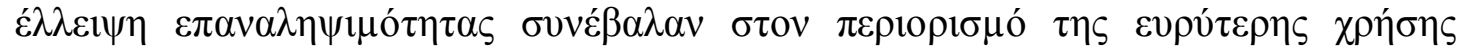




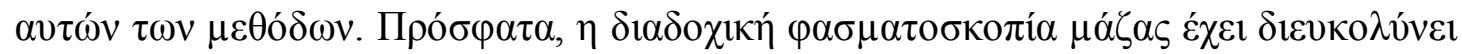

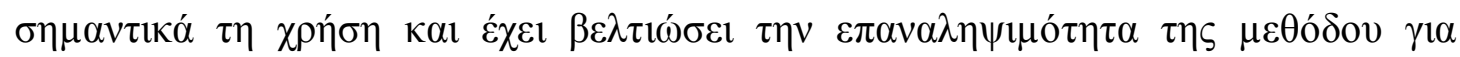

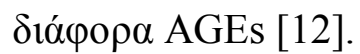

(a) Fluorescent Cross-linking AGEs

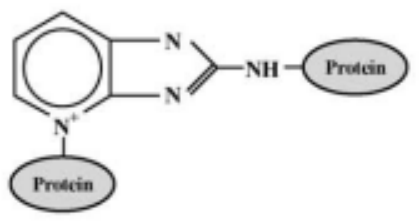

Pentosidine

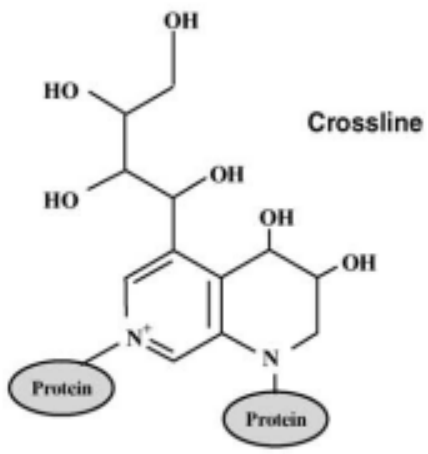

(b) Non-Fluorescent Cross-linking AGEs

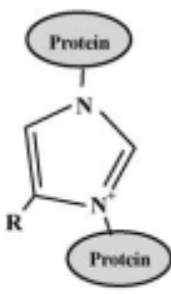

Imidazolium dilysines

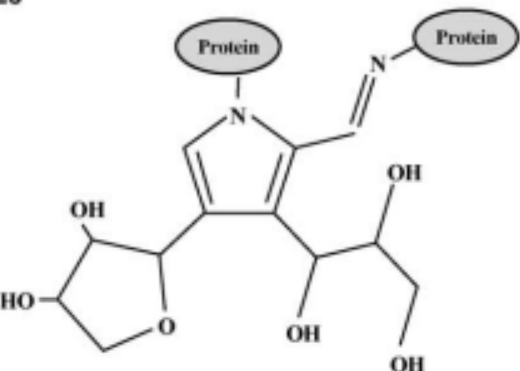

Alkyl formyl glycosyl pyrrole

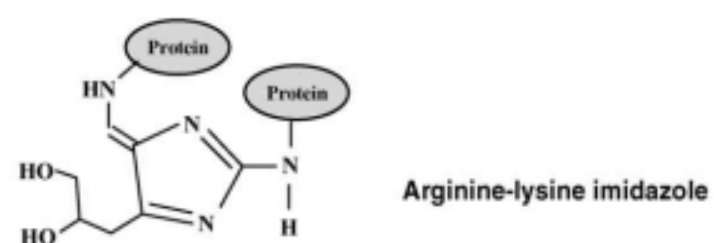

(c) Non-Cross-linking AGEs

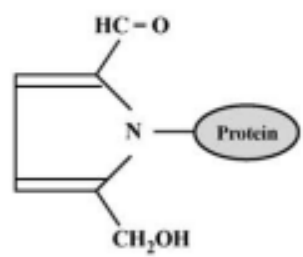

Pyralline

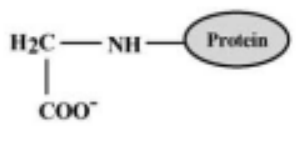

Carboxymethyllysine

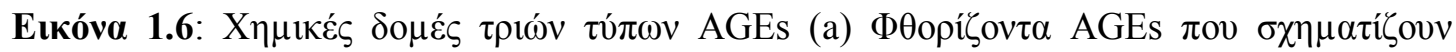

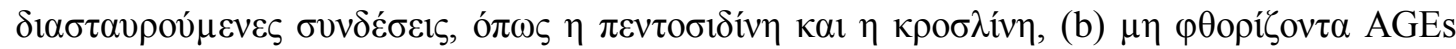

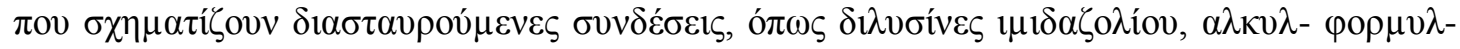

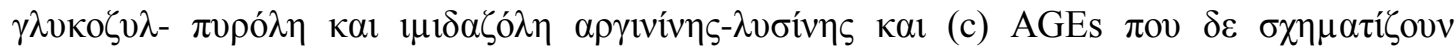

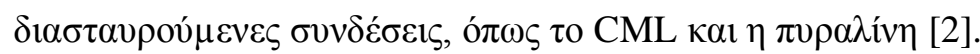




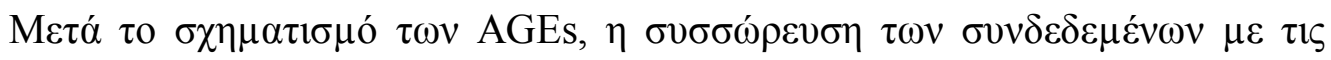

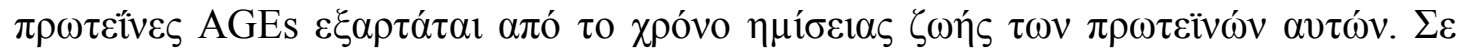

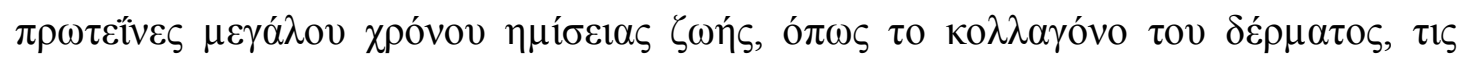

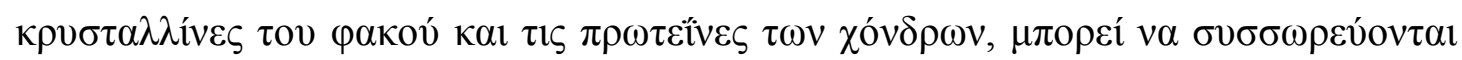

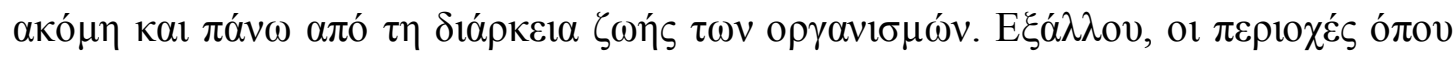

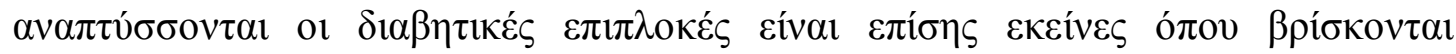

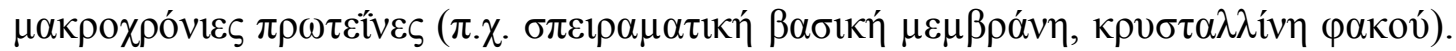

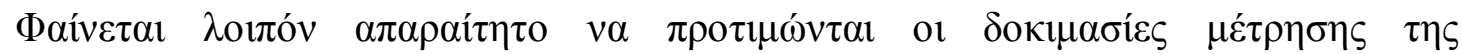

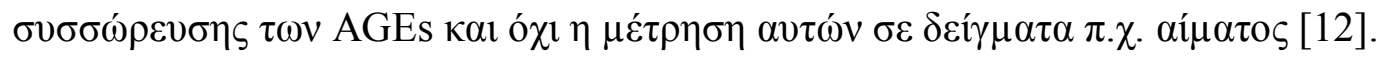

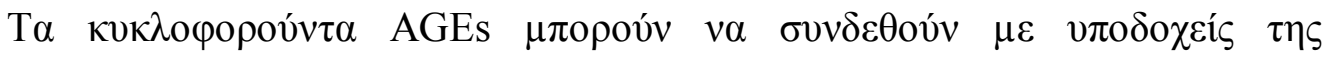

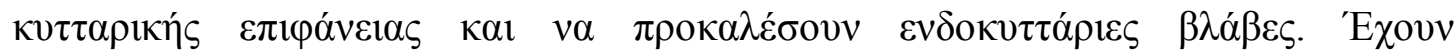

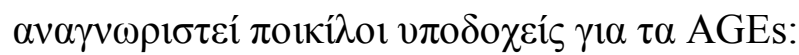

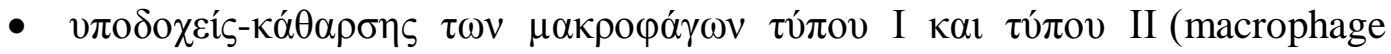
scavenger receptors type I and type II, MSR)

- $\gamma \alpha \lambda \varepsilon \kappa \tau i ́ v \eta-3(\mathrm{AGE}-\mathrm{R} 3)$

- AGER1 (AGE receptor-1)

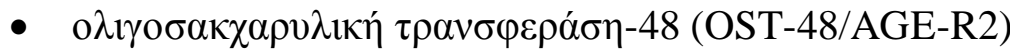

- RAGE (Receptor for AGEs)

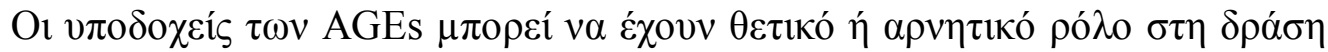

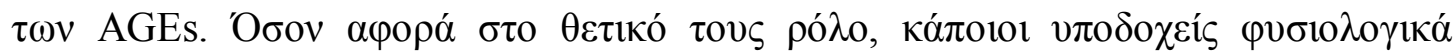

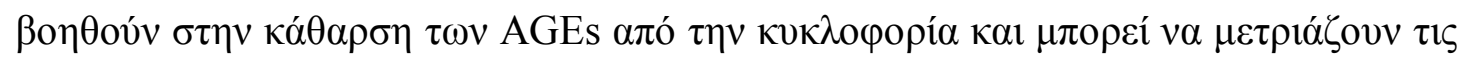

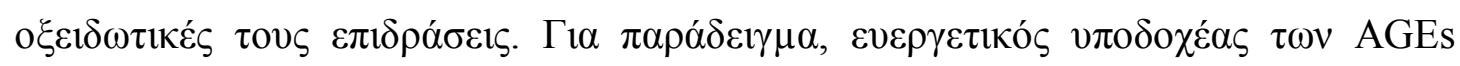

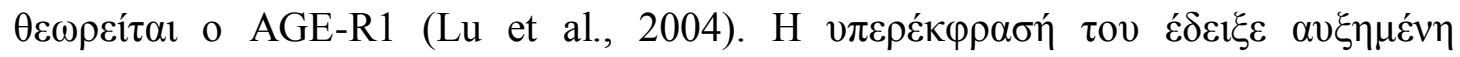

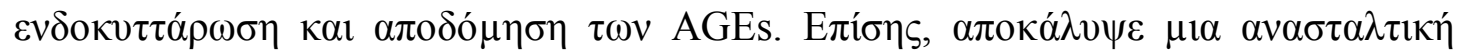

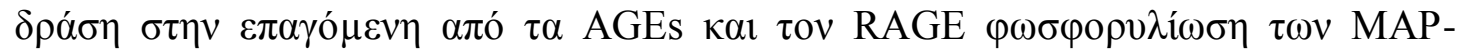

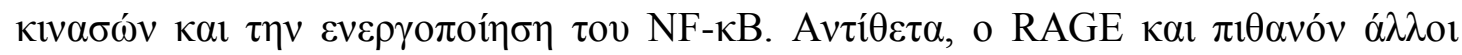

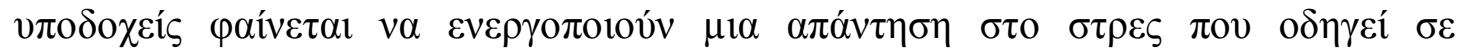

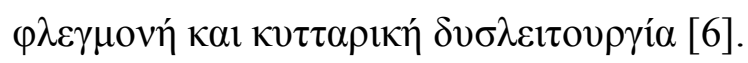




\subsubsection{Y $\pi 0 \delta o \chi \varepsilon ́ \alpha \varsigma \tau \omega v$ AGEs - RAGE}

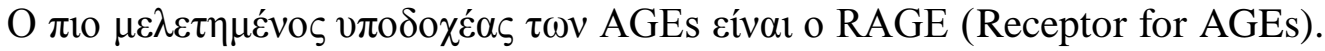

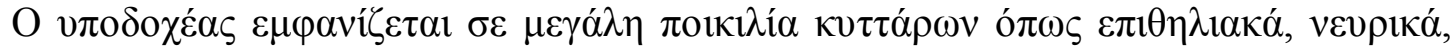

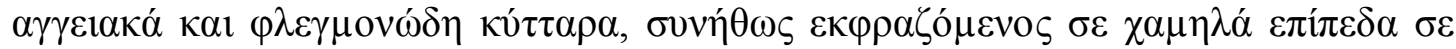

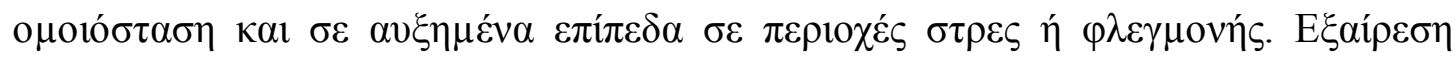

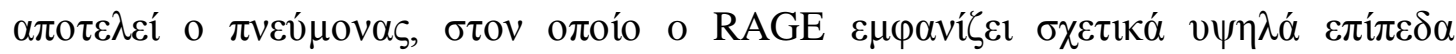

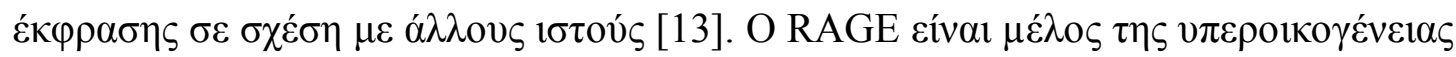

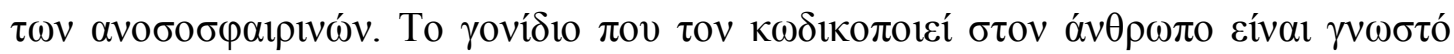

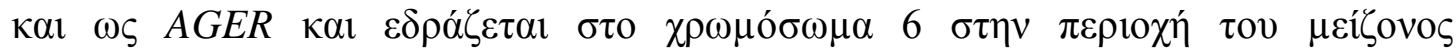

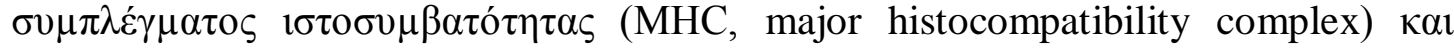

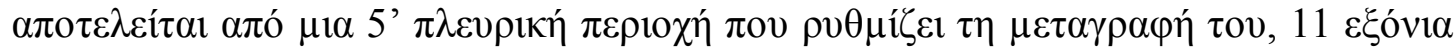

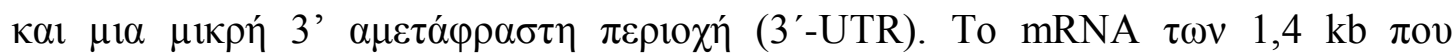

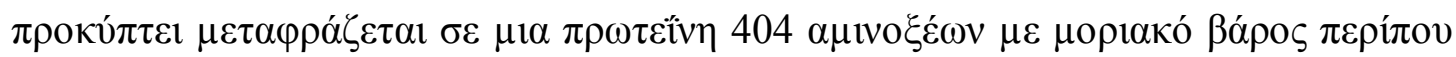

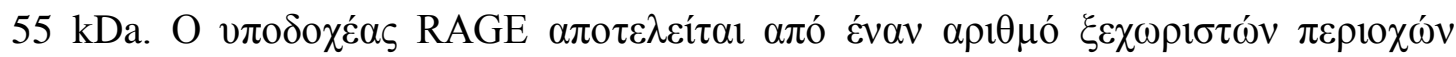

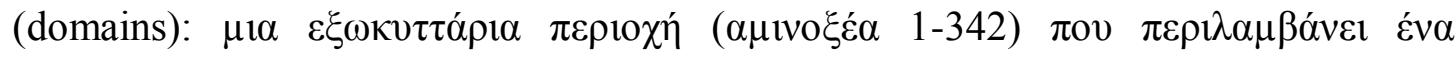

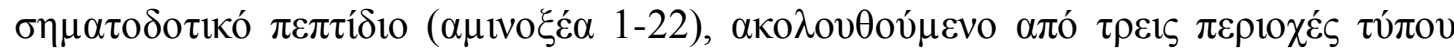

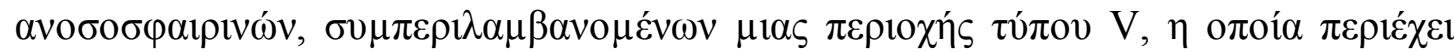

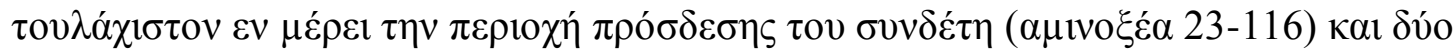

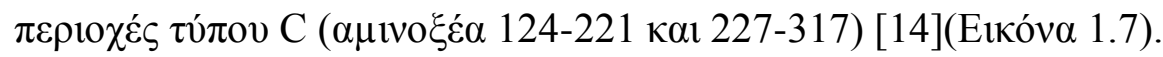

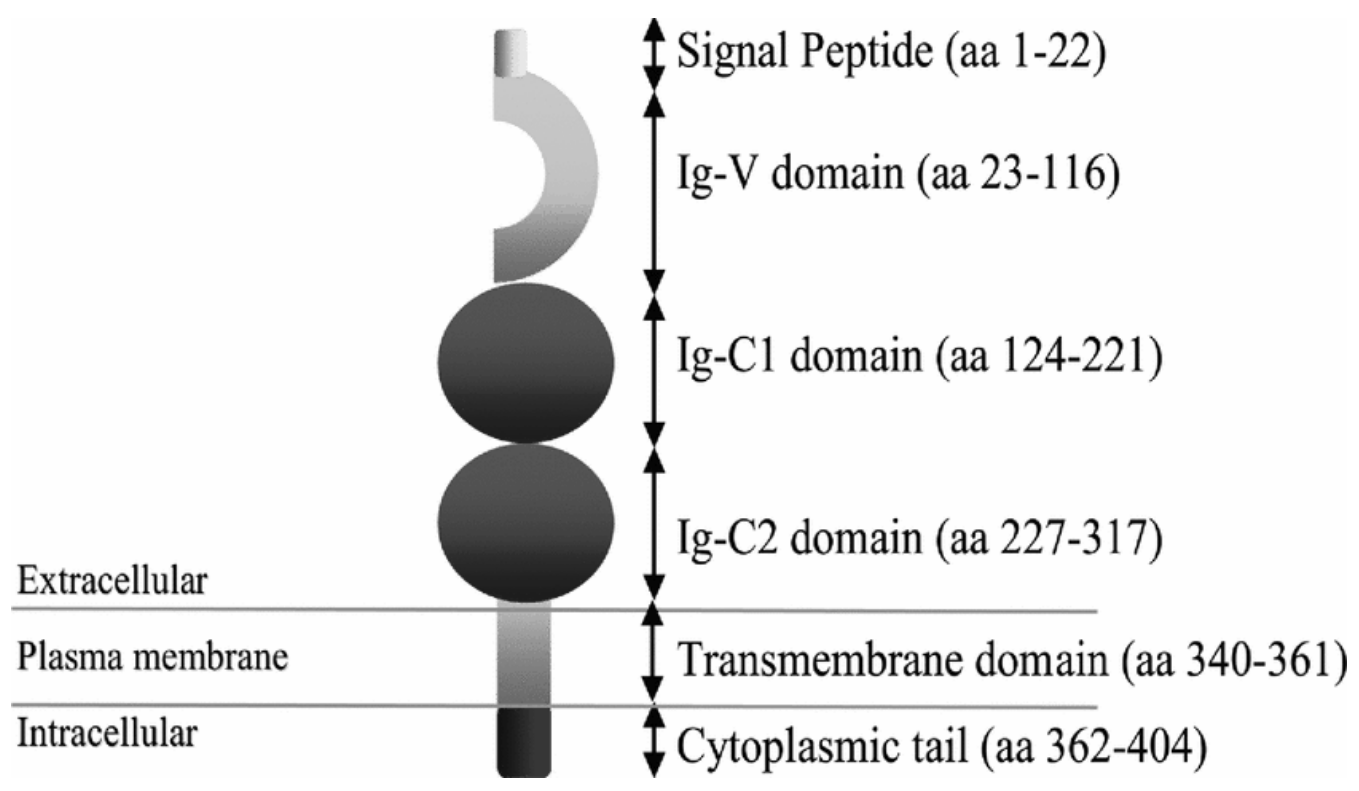

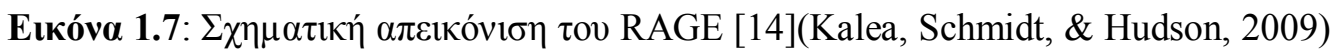




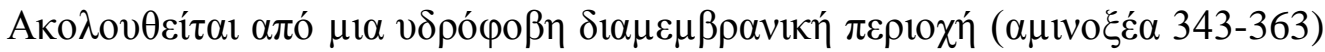

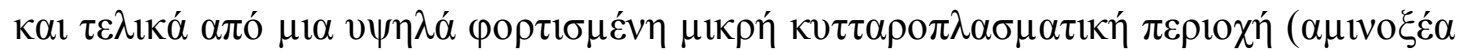

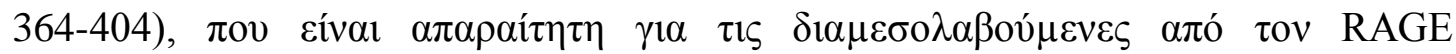

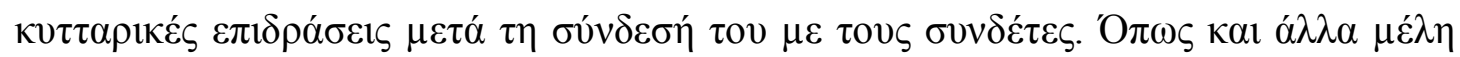

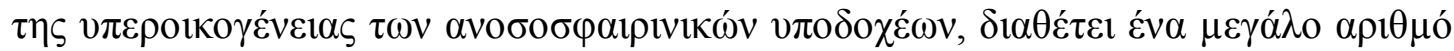

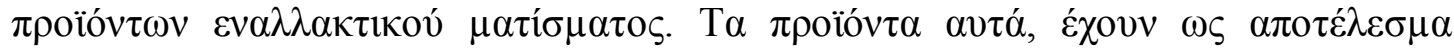

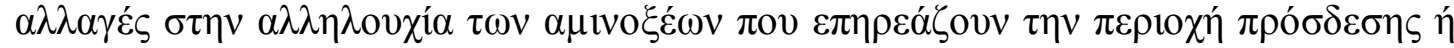

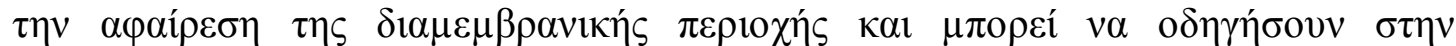

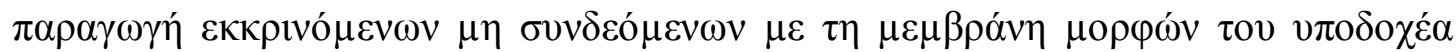
[15-18].

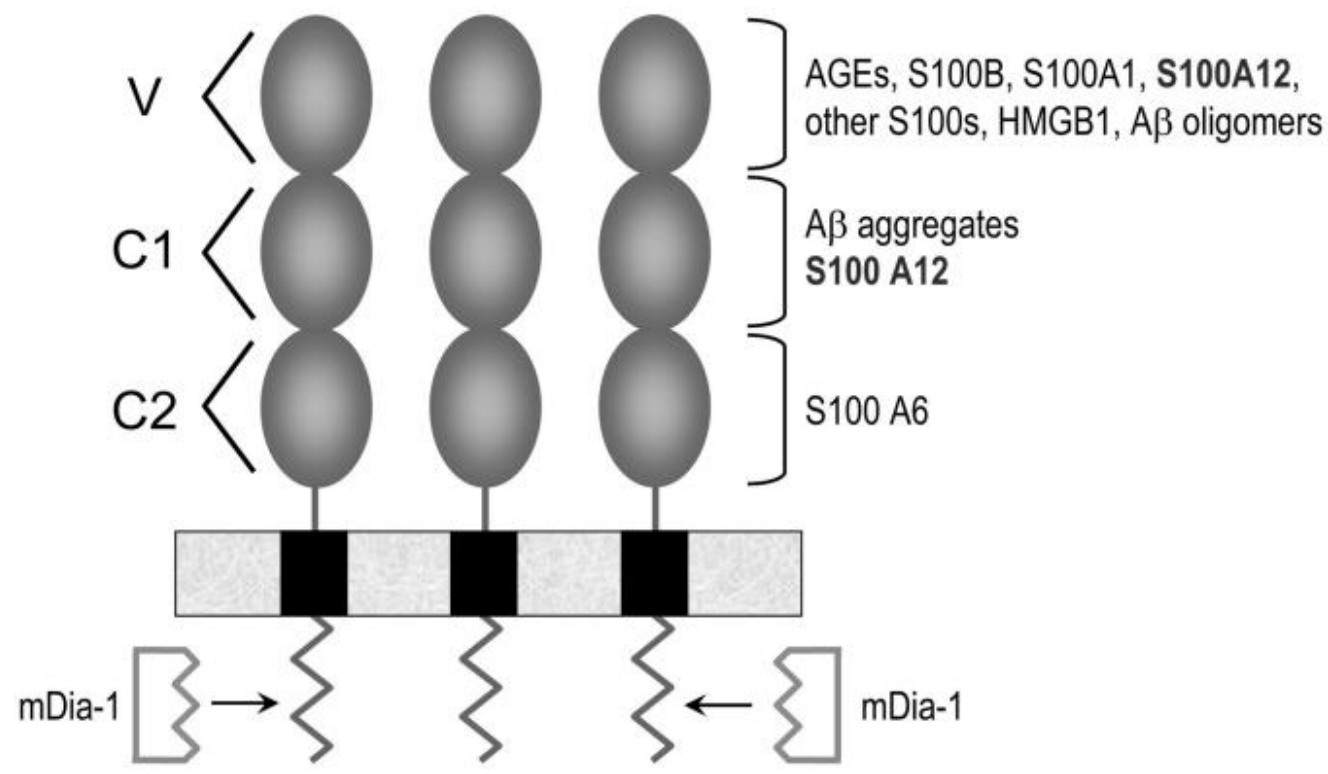

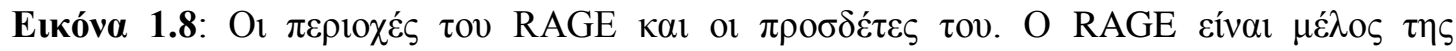

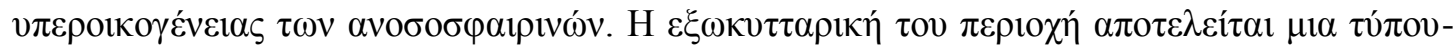

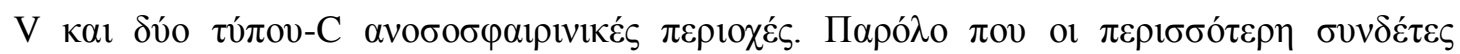

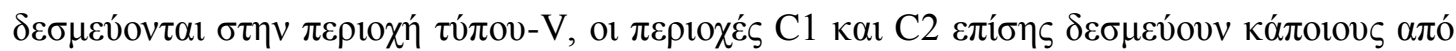

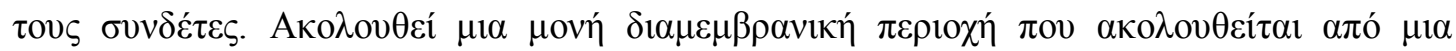

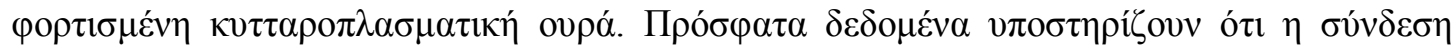

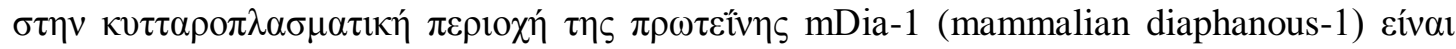

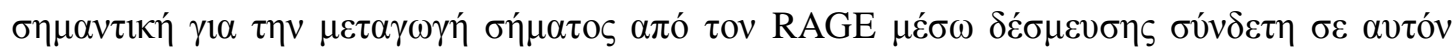
[13].

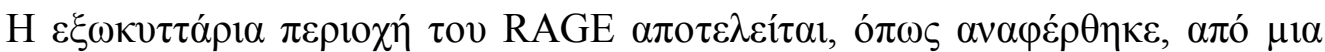

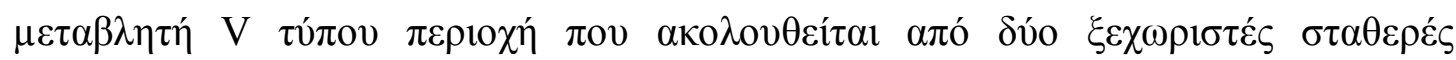




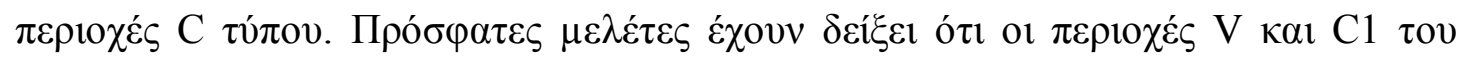

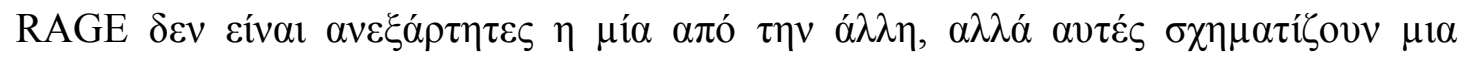

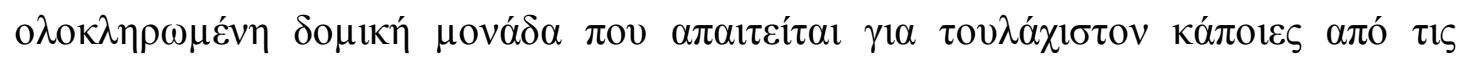

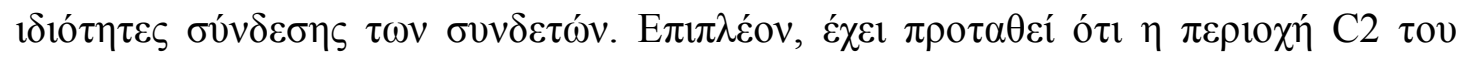

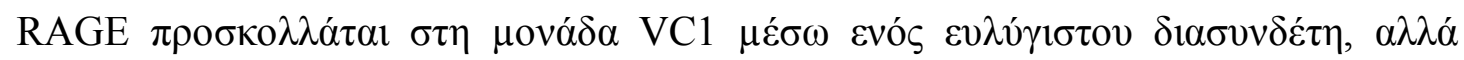

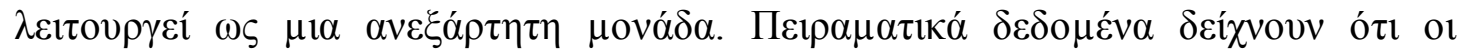

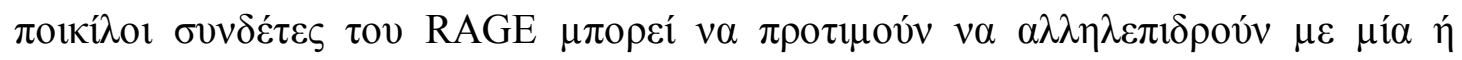

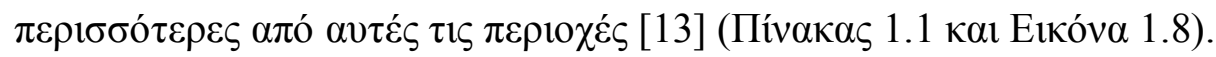

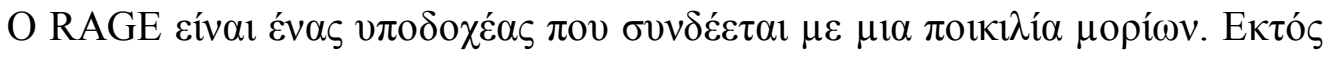

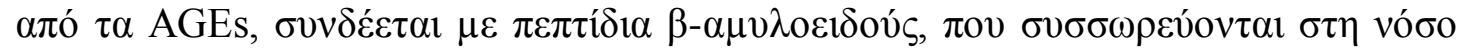

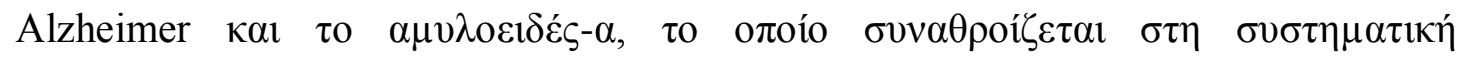

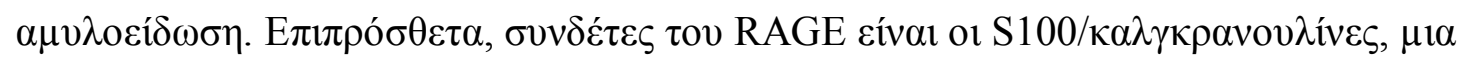

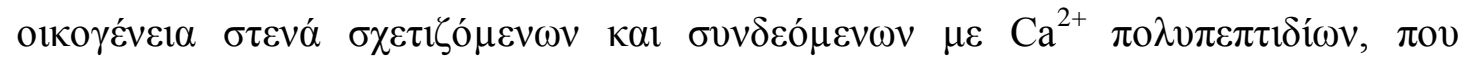

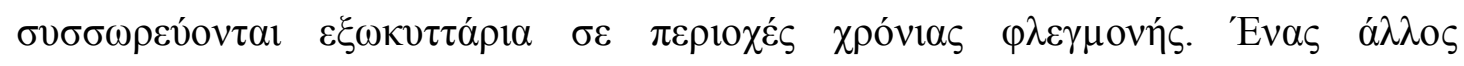

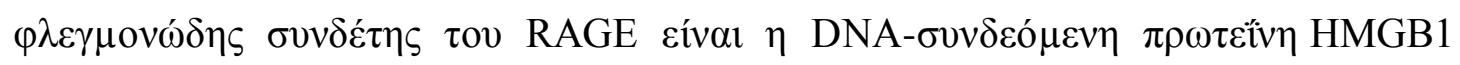

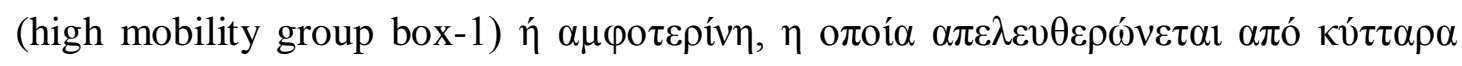

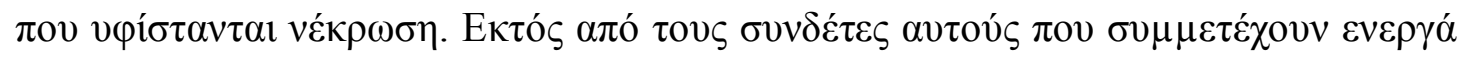
$\sigma \tau \eta \varphi$

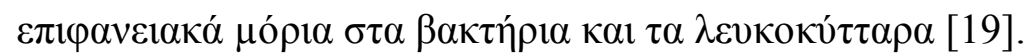

\begin{tabular}{|c|c|c|}
\hline Ligand & Ligand Family & $\begin{array}{l}\text { Extracellular Domain(s) } \\
\text { of RAGE/Interaction }\end{array}$ \\
\hline $\begin{array}{l}\text { CML-AGE } \\
\text { (carboxy- } \\
\text { methyl lysine) }\end{array}$ & AGEs & - V-type domain \\
\hline pronyl glycine & AGEs & - not studied \\
\hline $\begin{array}{l}\text { AGE } \\
\text { peptides }\end{array}$ & AGEs & -V-type domain \\
\hline $\begin{array}{l}\text { AOPPs } \\
\text { Advanced } \\
\text { Oxidation } \\
\text { Protein products }\end{array}$ & AOPPs & - not studied \\
\hline HMGB1 & HMGB1 & - V-type domain \\
\hline $\mathrm{S} 100 \mathrm{~A} 12$ & S100/calgranulins & - V- \& C1- type domains \\
\hline S100B & S100/calgranulins & $\cdot$ V-type domain \\
\hline S100A6 & S100/calgranulins & - C2-type domain \\
\hline $\begin{array}{l}\text { Amyloid- } \beta \\
\text { Oligomers }\end{array}$ & $\begin{array}{l}\text { Amyloid- } \beta \text { \& } \beta \text { - } \\
\text { Sheet fibrils }\end{array}$ & - V-type domain \\
\hline $\begin{array}{l}\text { Amyloid- } \beta \\
\text { Aggregates }\end{array}$ & $\begin{array}{l}\text { Amyloid- } \beta \text { \& } \beta \\
\text { Sheet Fibrils }\end{array}$ & - C1-type domain \\
\hline
\end{tabular}

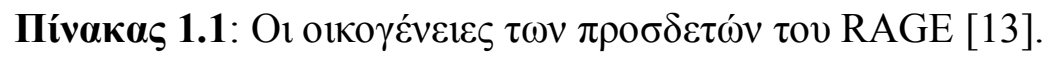




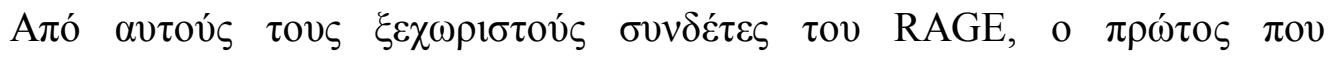

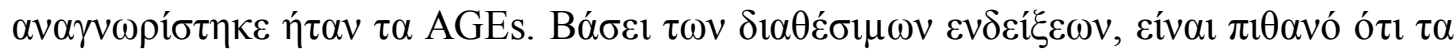

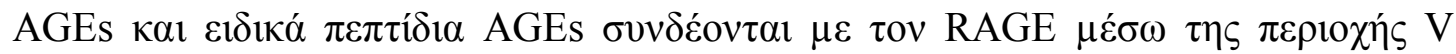

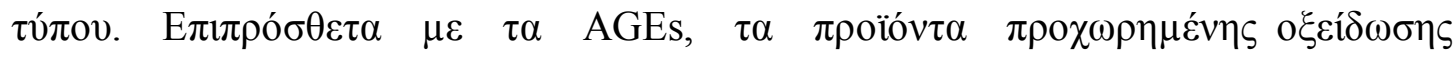

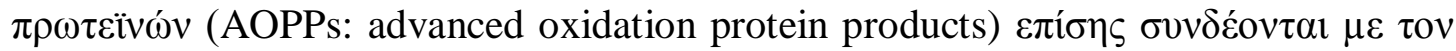

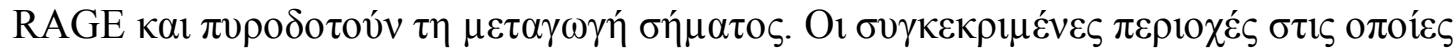

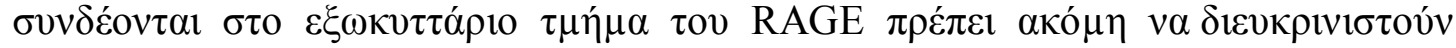
[13].

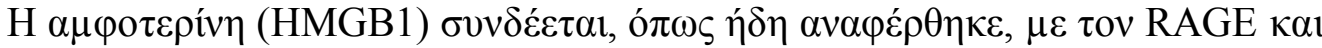

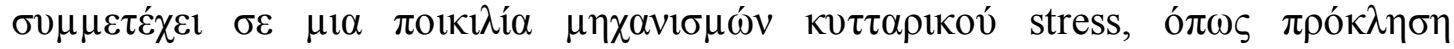

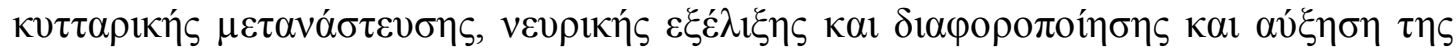

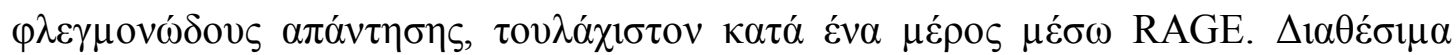

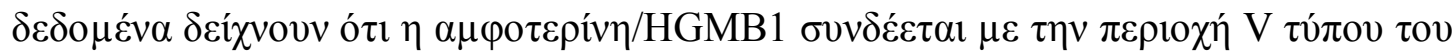

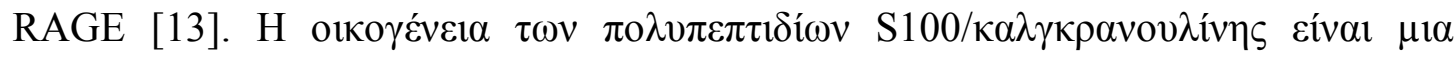

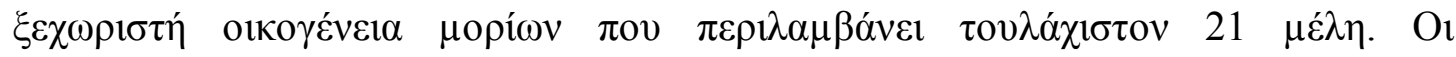

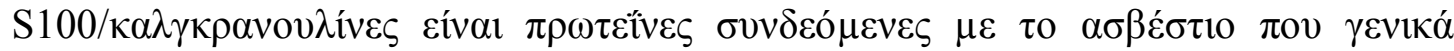

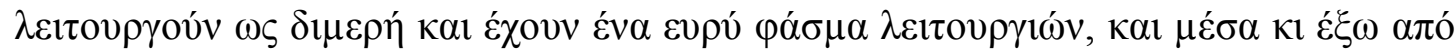

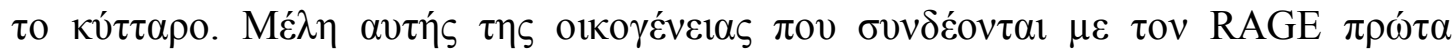

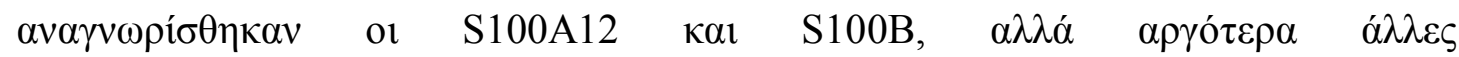

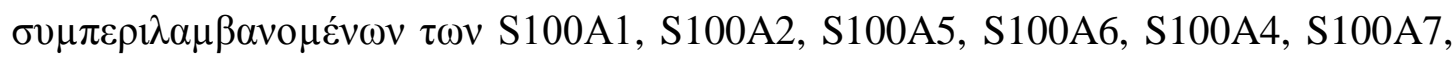

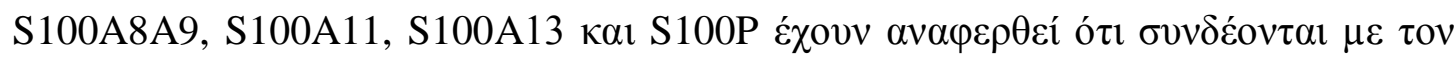

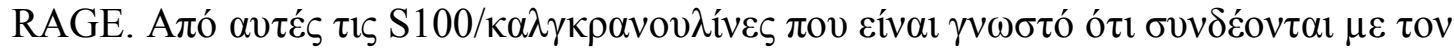
RAGE, or S100B, S100A1, S100A2, S100A5, S100A6, S100A12 $\alpha \lambda \lambda \eta \lambda \varepsilon \pi ı \rho \circ o v ~ \mu \varepsilon$

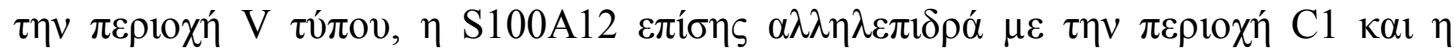

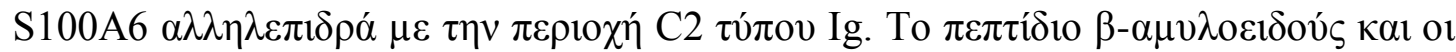

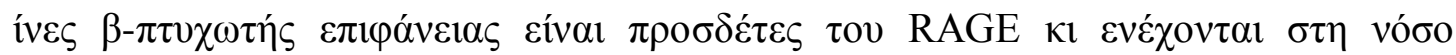

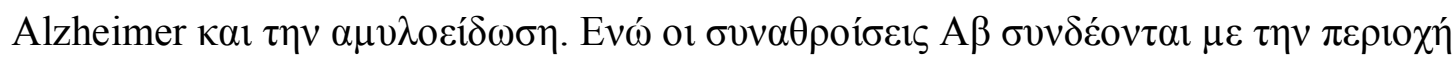

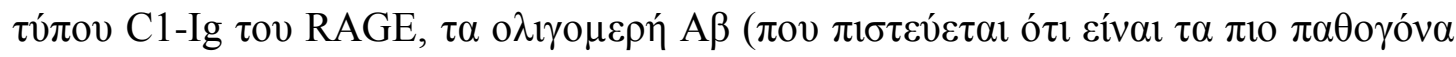

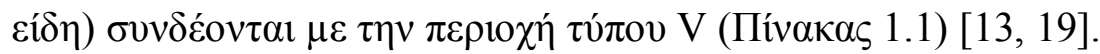

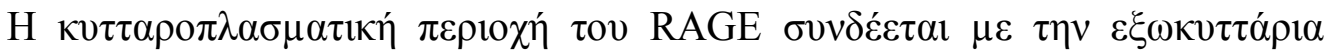

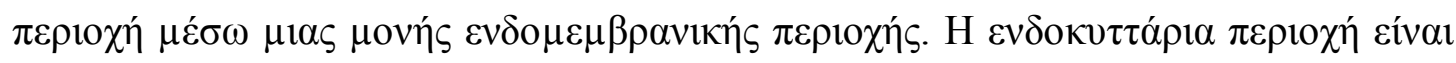

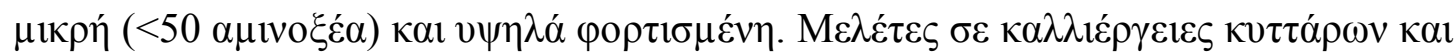




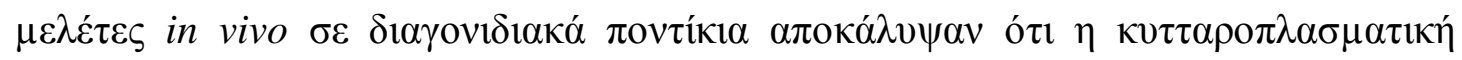

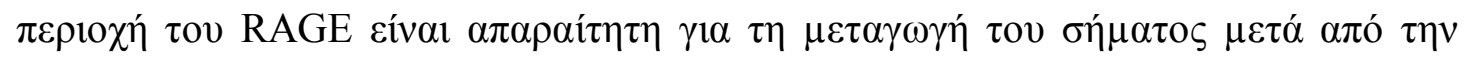

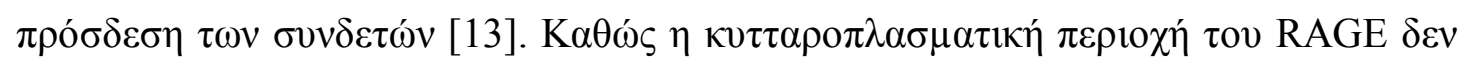

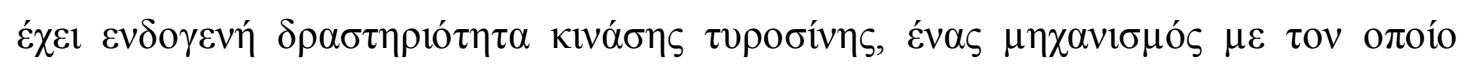

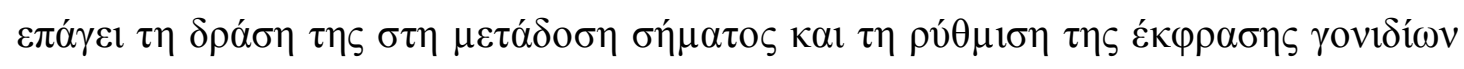

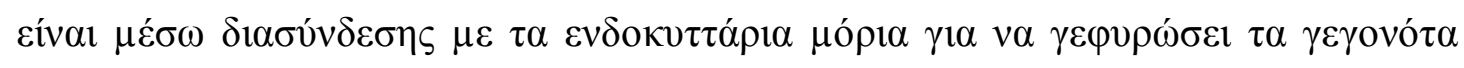

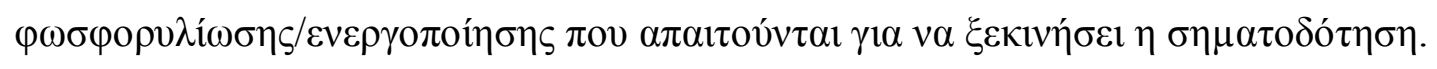

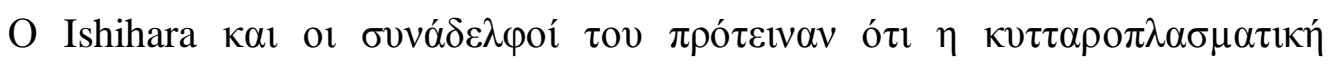

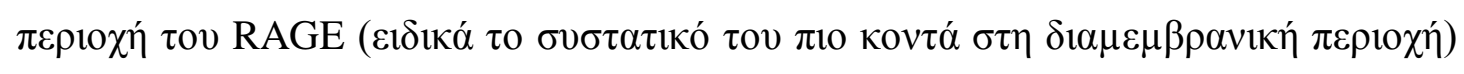

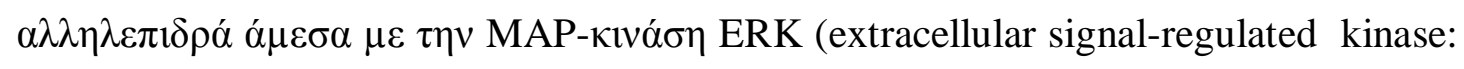

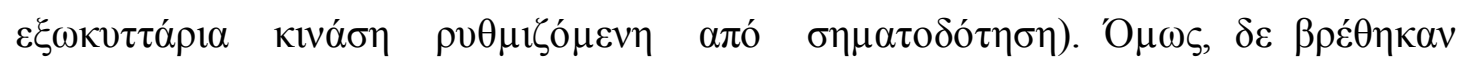

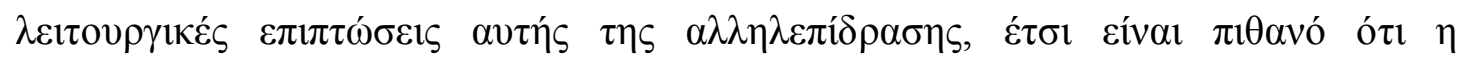

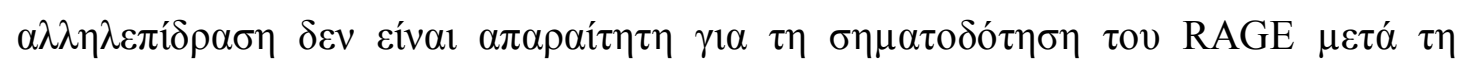
$\sigma u ́ v \delta \varepsilon \sigma \eta \mu \varepsilon \tau 0 \cup \varsigma \pi \rho 0 \sigma \delta \varepsilon \dot{\tau} \varepsilon \varsigma[13,20]$

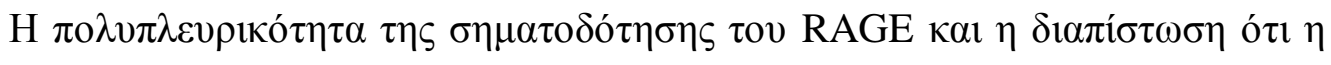

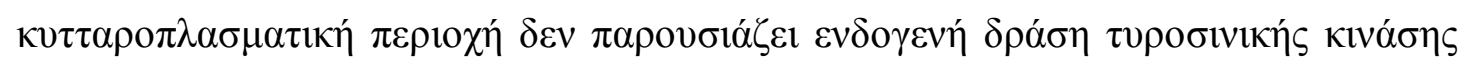

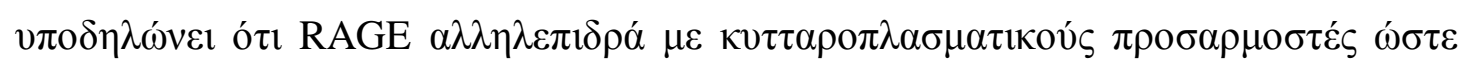

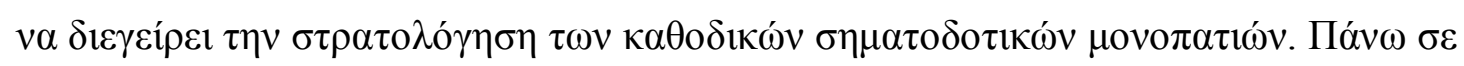

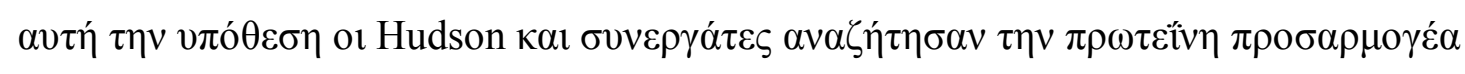

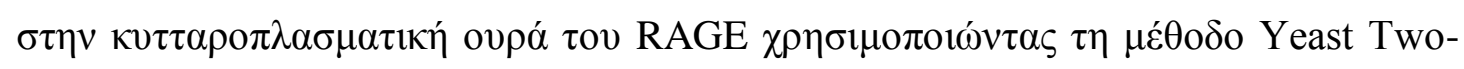

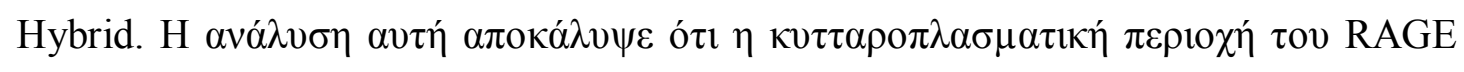

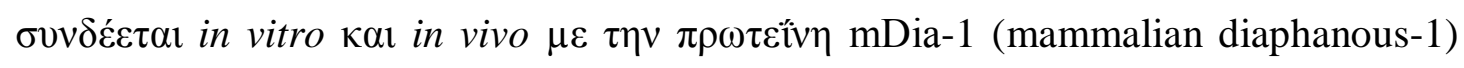

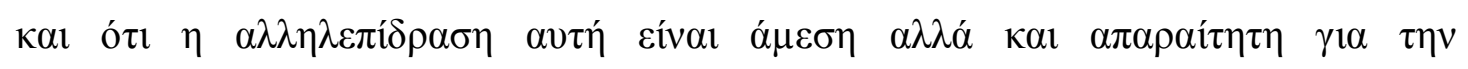

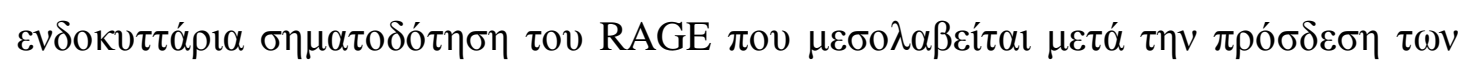

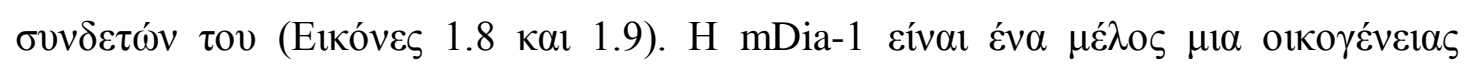

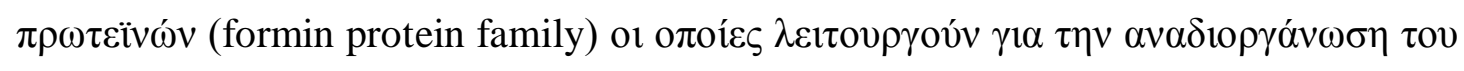

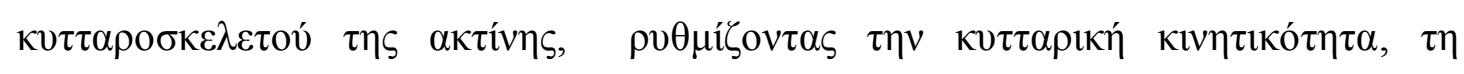

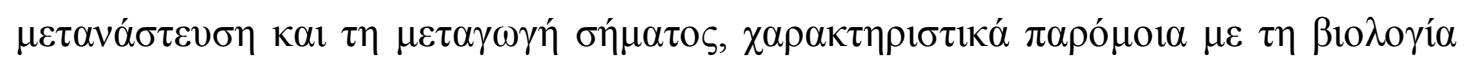

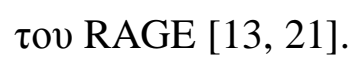

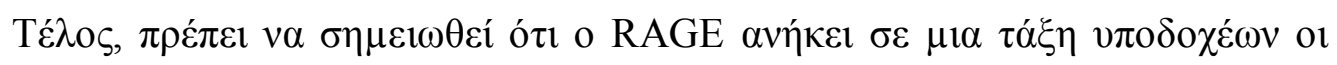

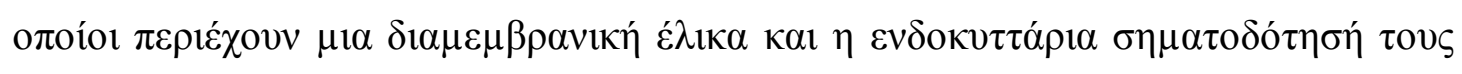




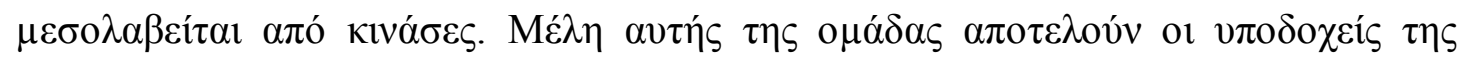

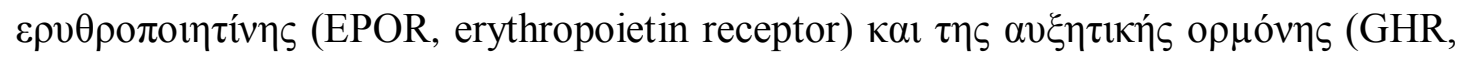

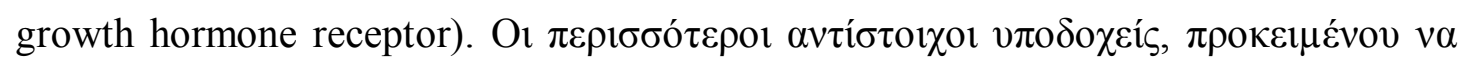

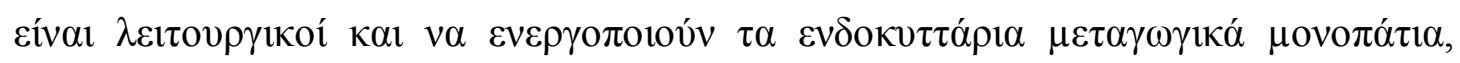

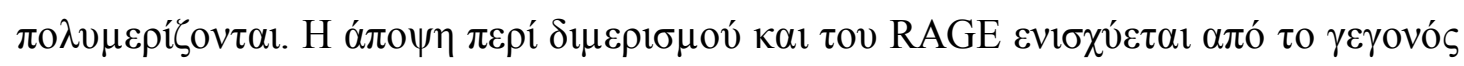

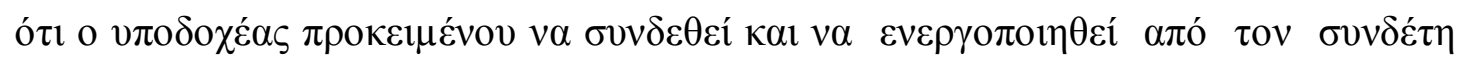

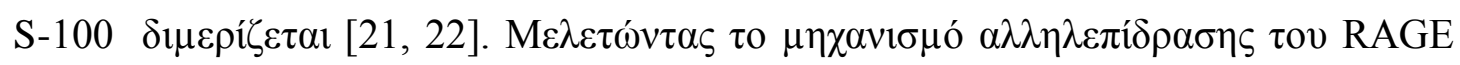

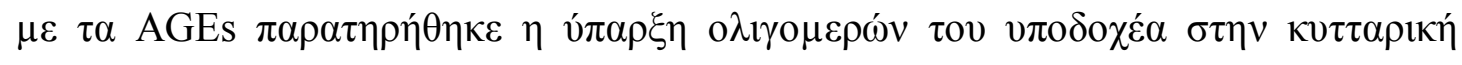

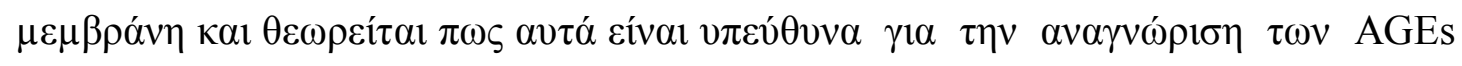

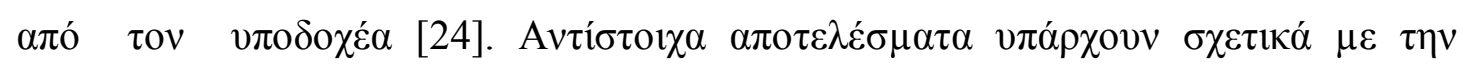

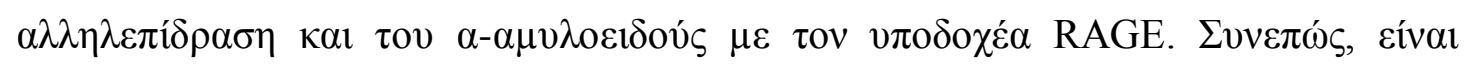

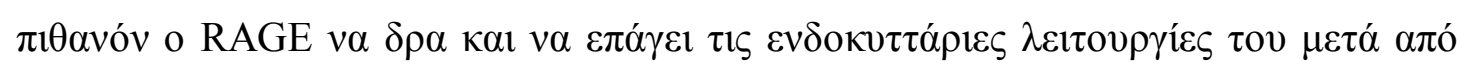

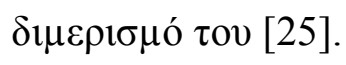

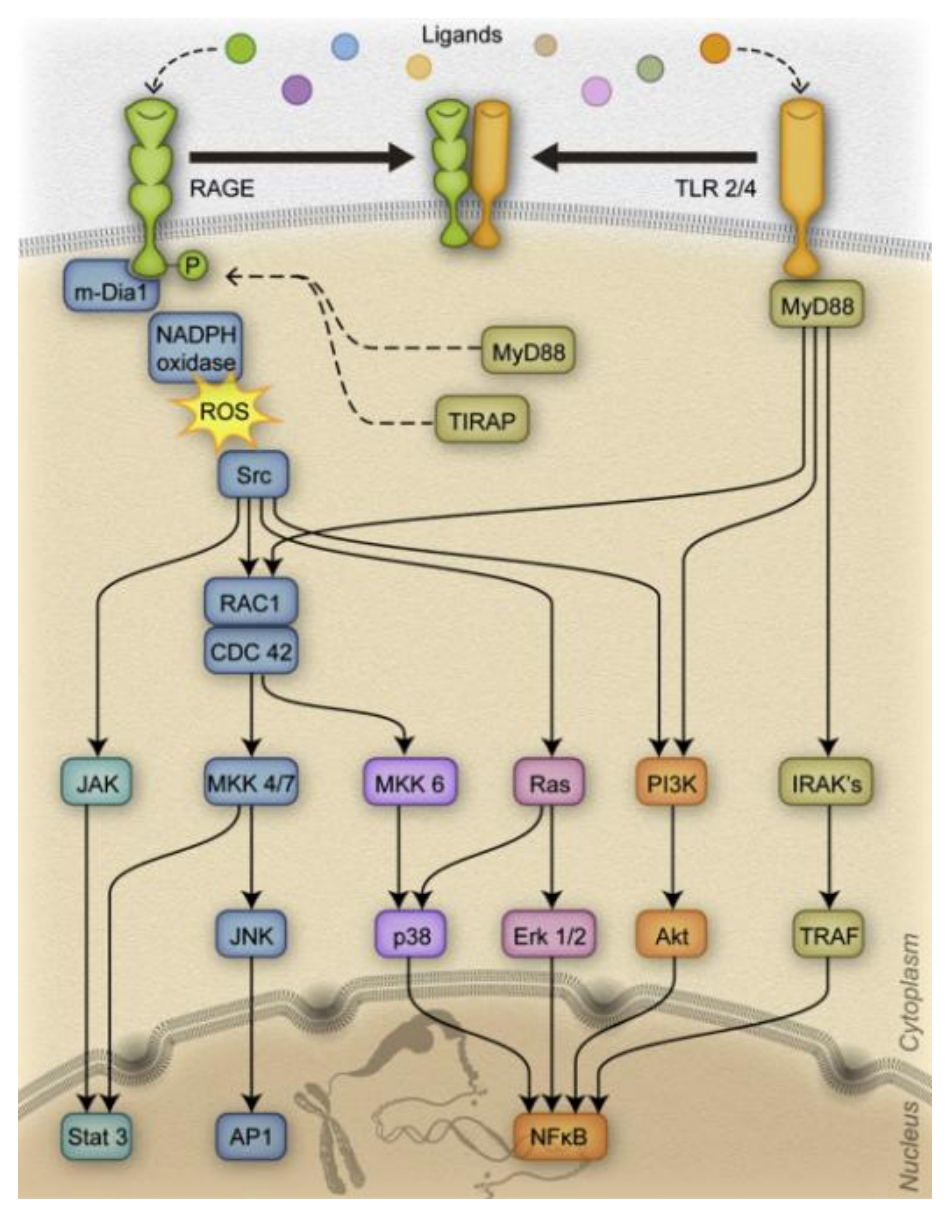

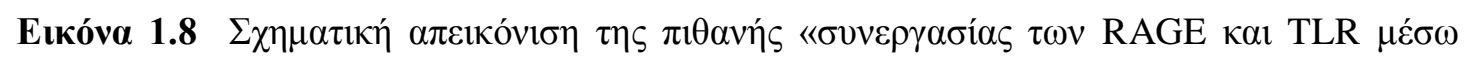

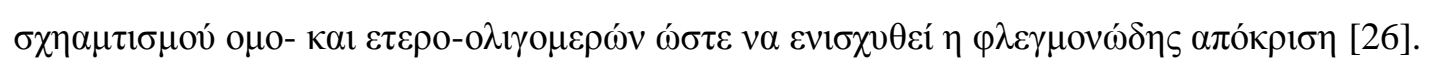




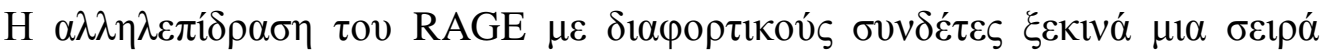

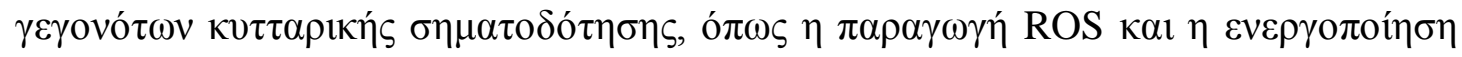

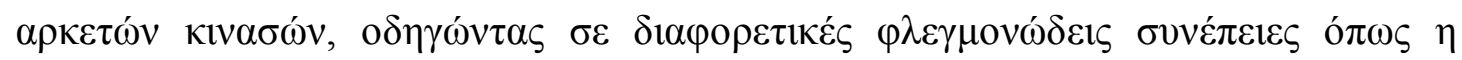

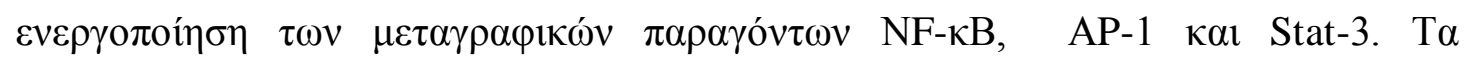

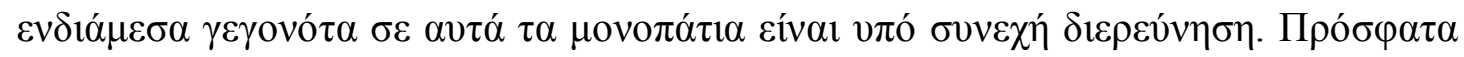

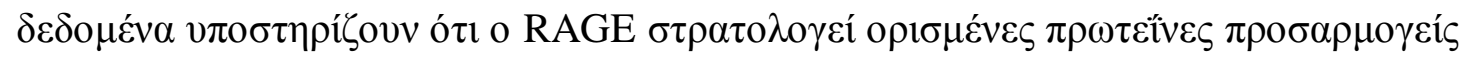

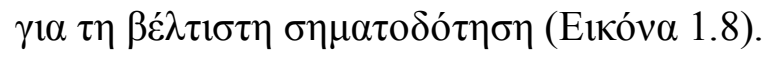

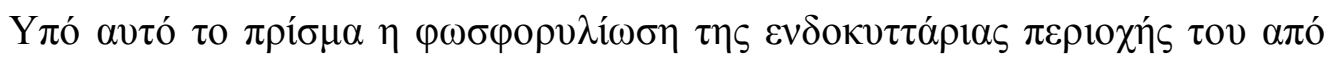

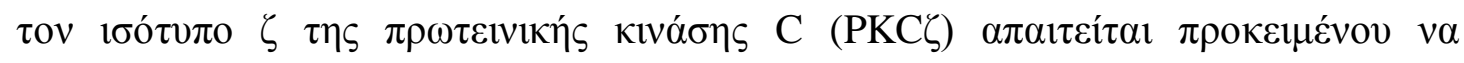

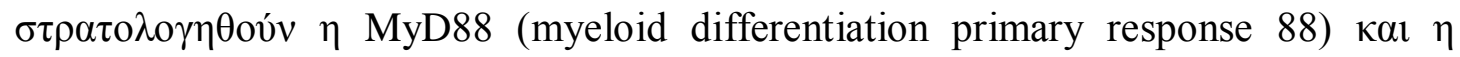
TIRAP (toll-interleukin 1 receptor domain-containing adaptor protein), $\pi \rho \omega \tau \varepsilon i \hat{v} \varepsilon \varsigma \pi \mathrm{ov}$

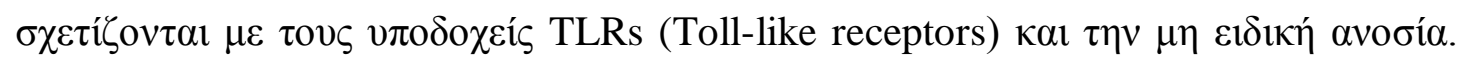

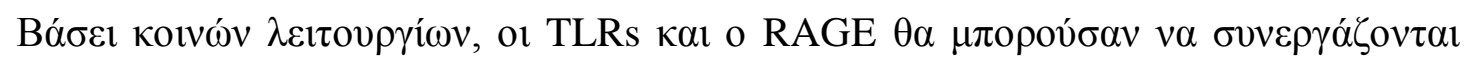

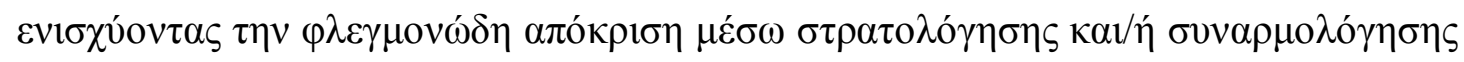

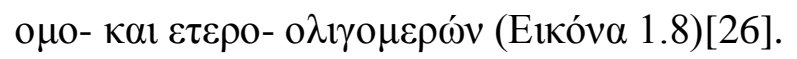

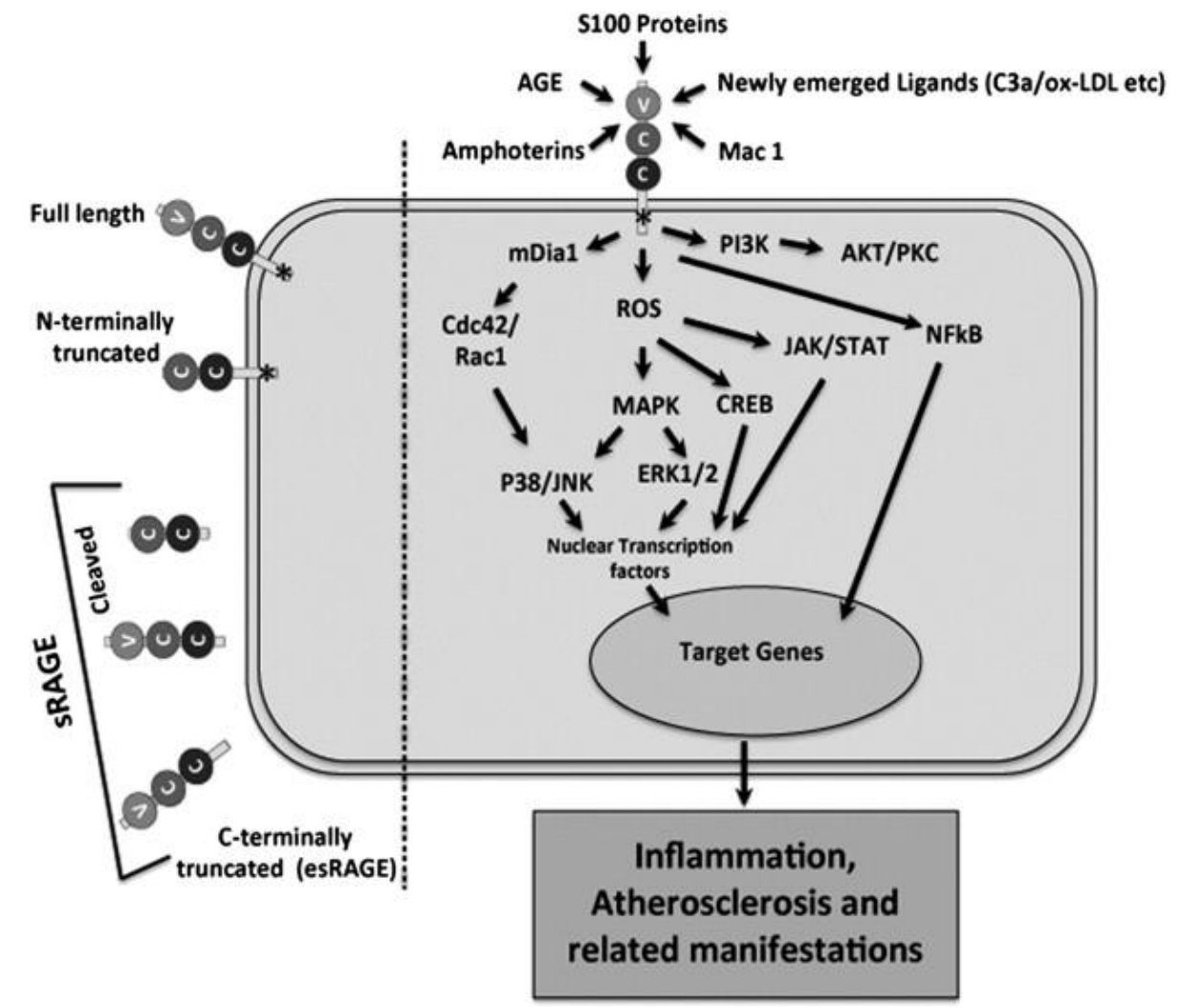

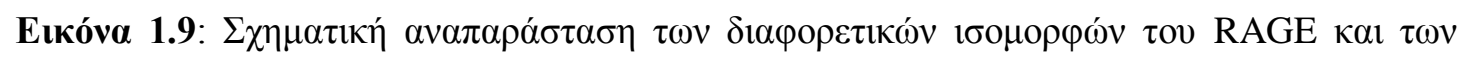

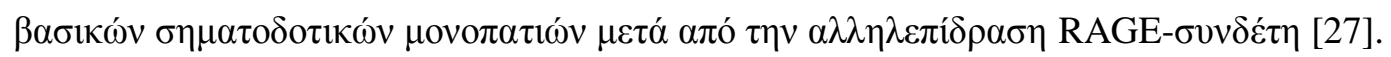




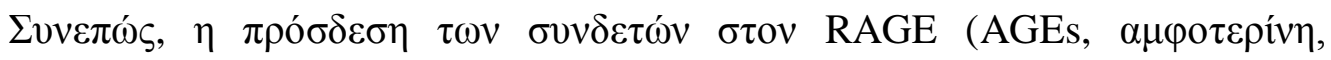

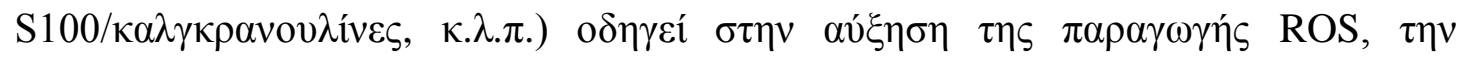

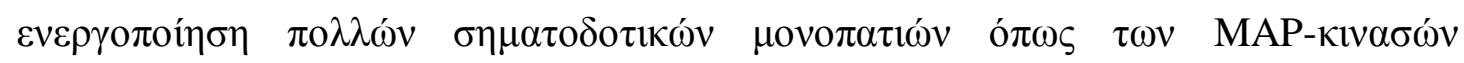
(Mitogen-activated protein kinases), $\tau\rceil \varsigma$ PI3K (Phosphatidylinositide 3-kinase), $\tau$ ov JAK (Janus kinase)/STAT (signal transducer and activator of transcription) $\kappa \alpha \imath \tau o v$

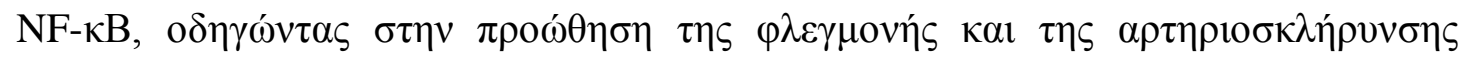
(Eıкóva 1.9)[27].

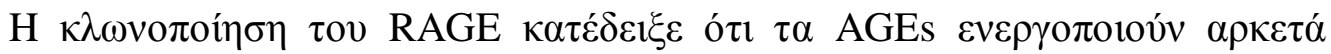

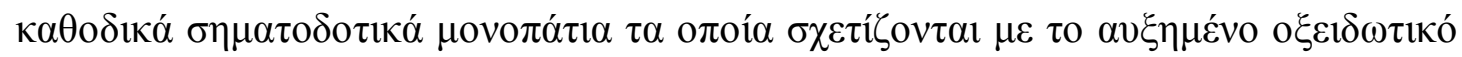

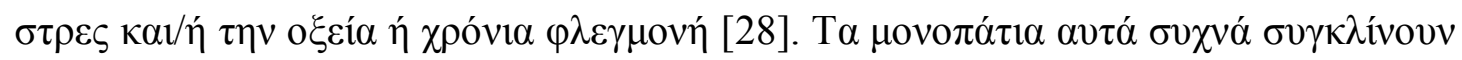

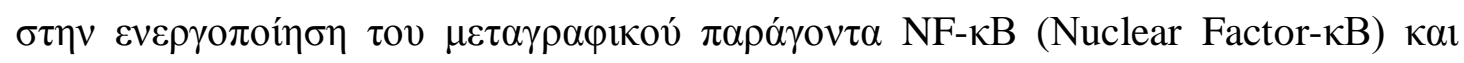

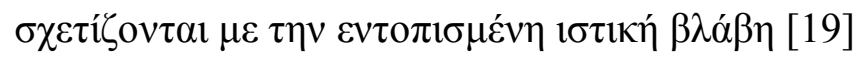

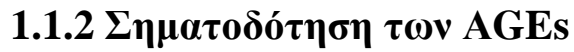

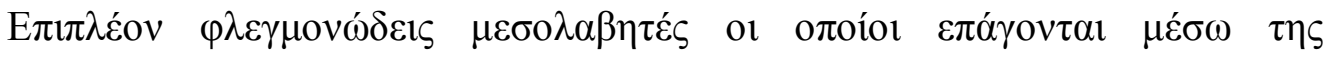

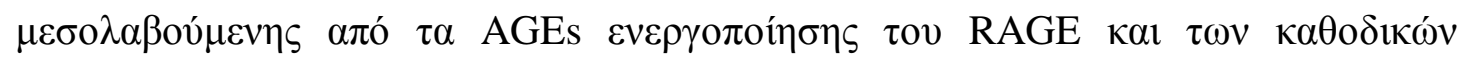

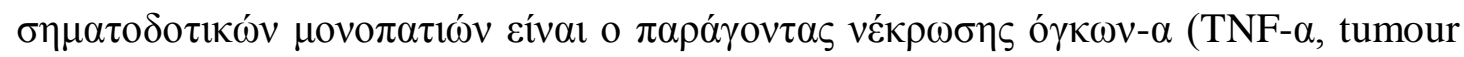

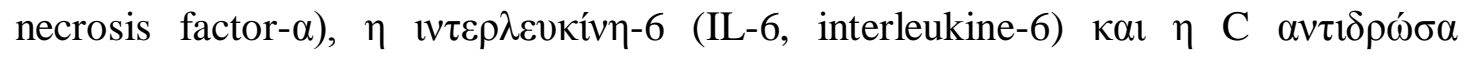

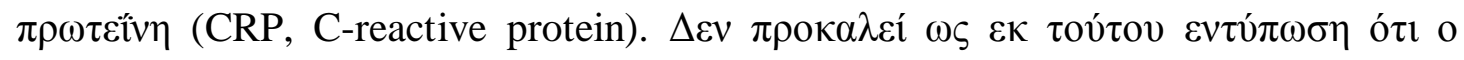

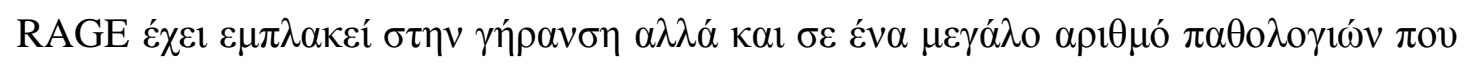

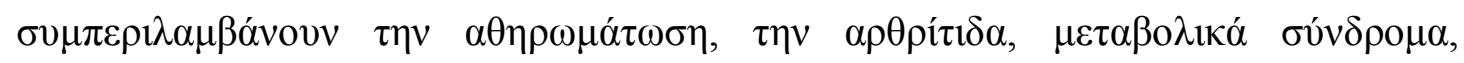

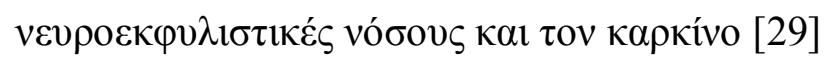

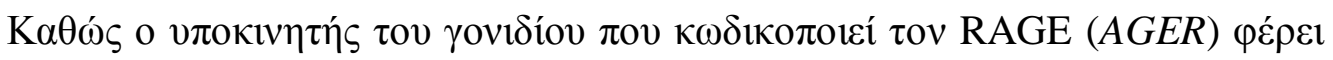

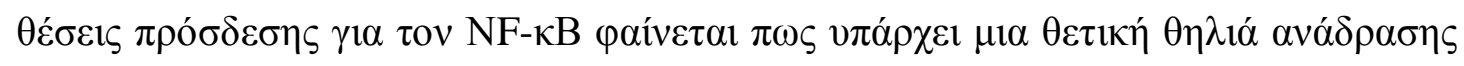

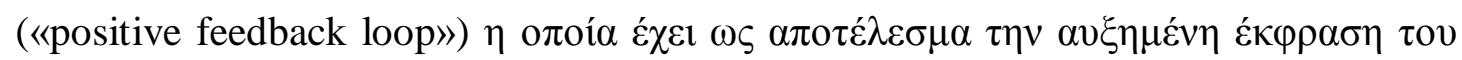

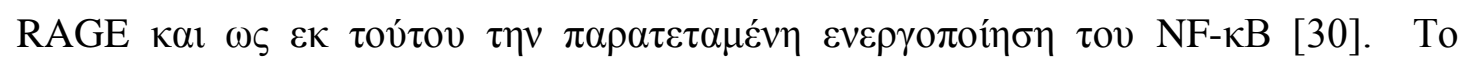

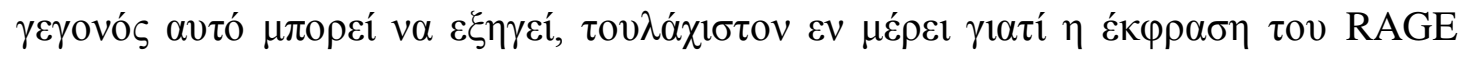

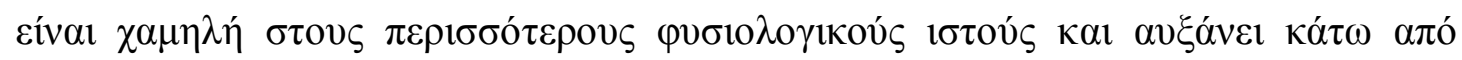

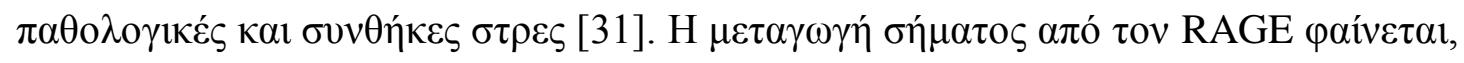

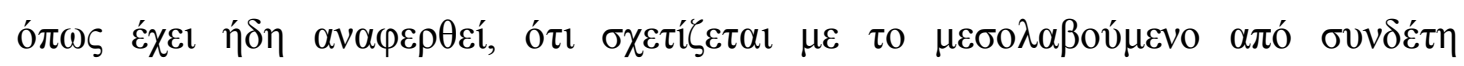




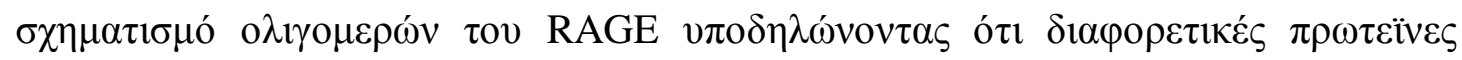

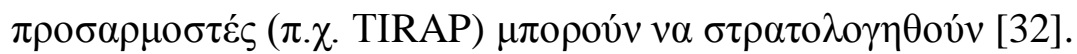

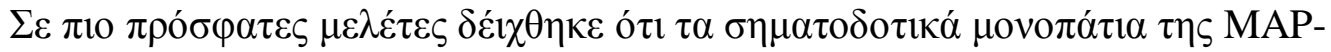

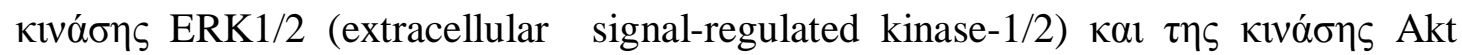

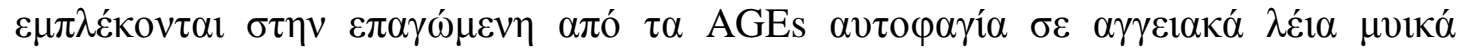

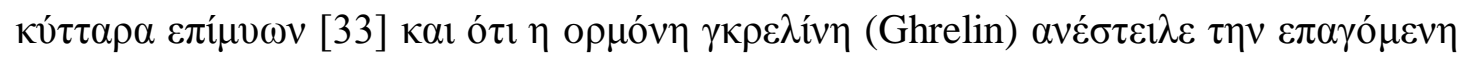

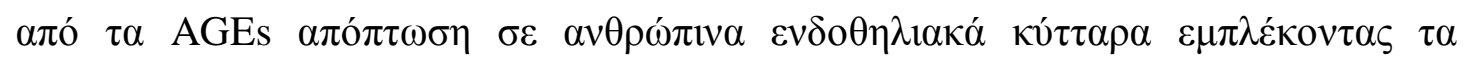

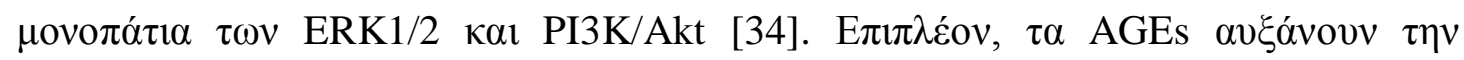

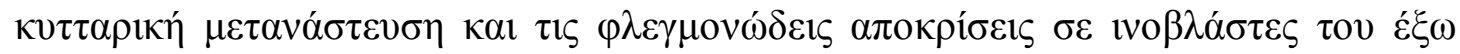

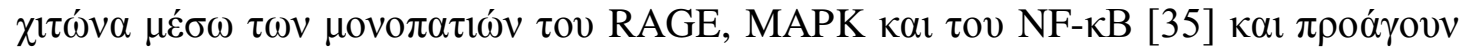

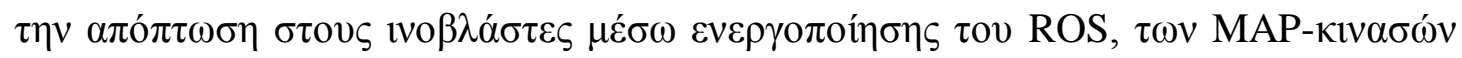

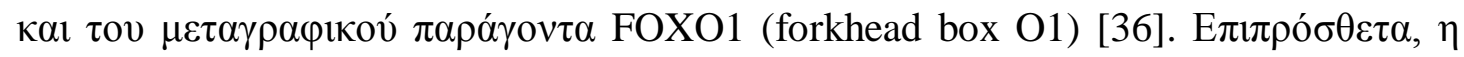

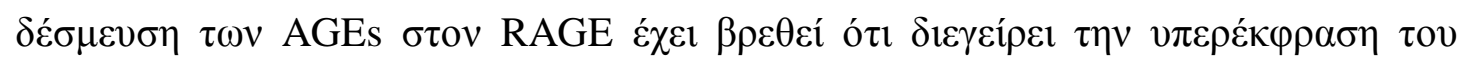

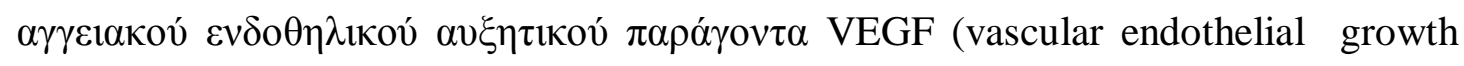

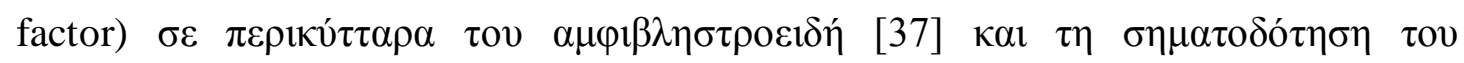

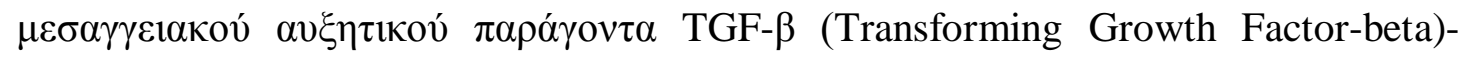

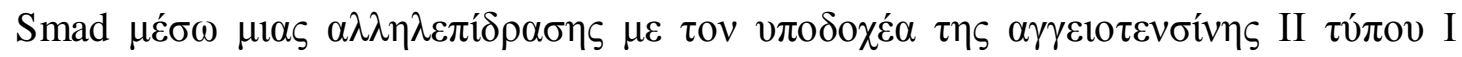

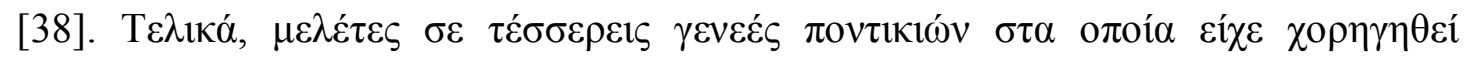


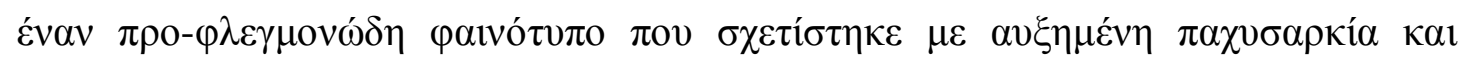

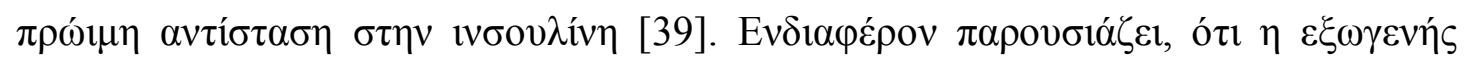

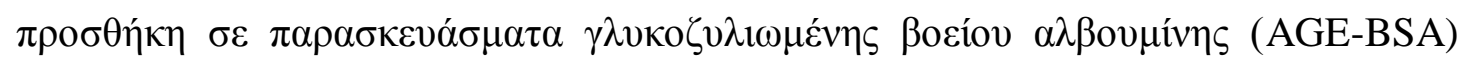

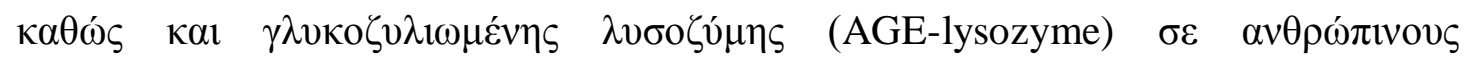

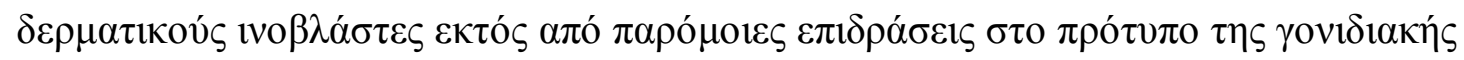

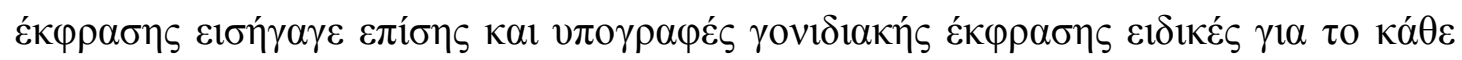

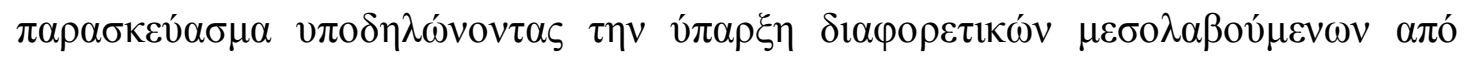

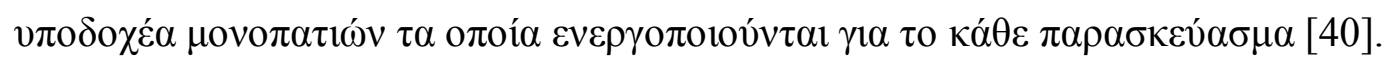

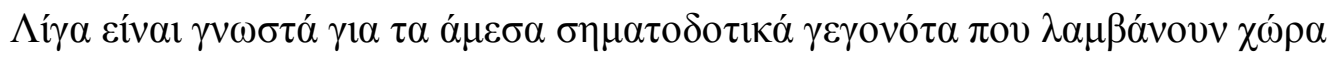

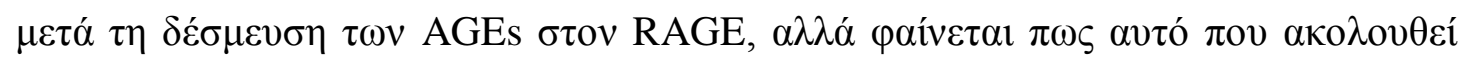

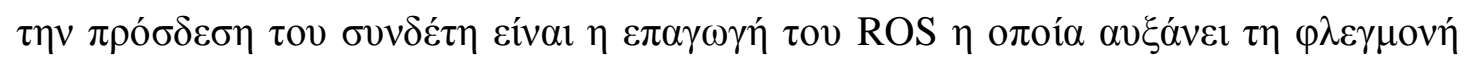

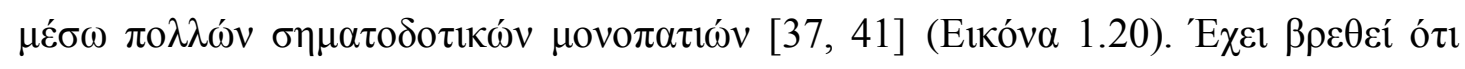

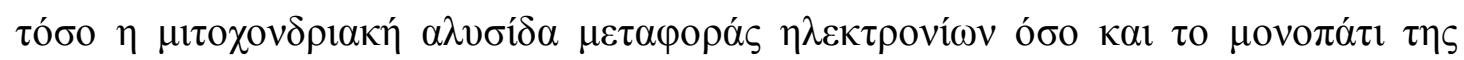




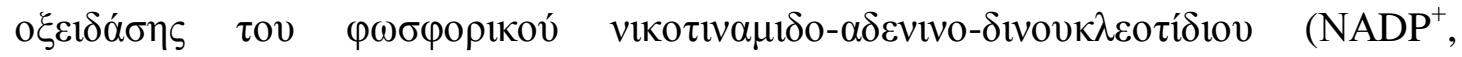

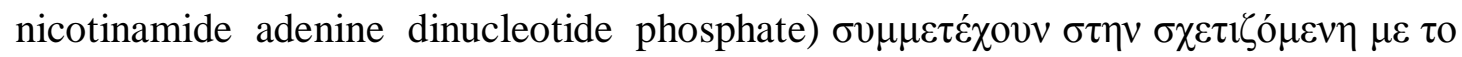

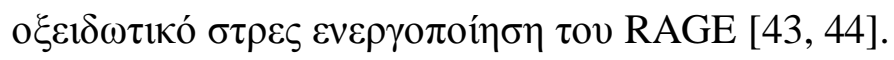

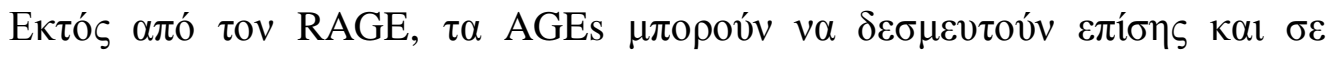

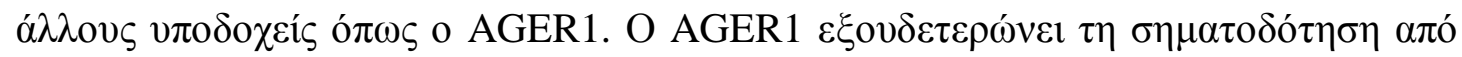

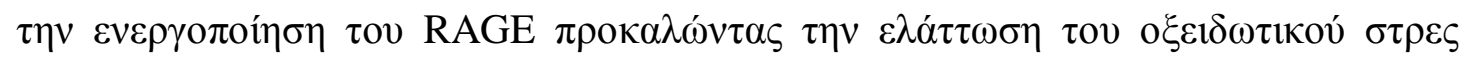

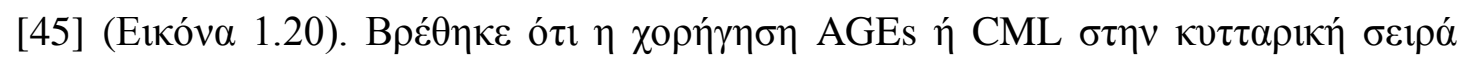

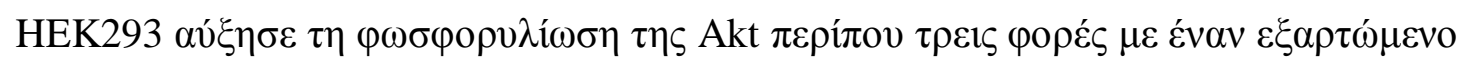

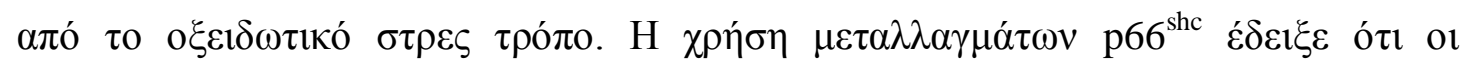

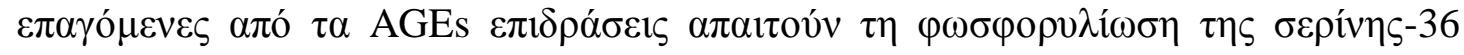

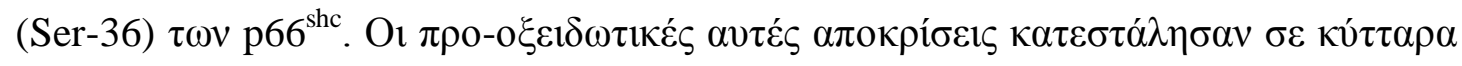

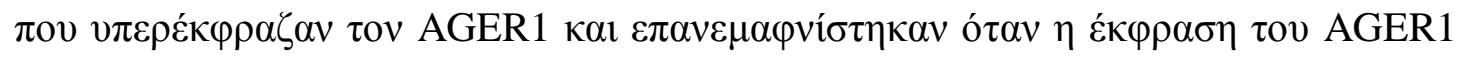

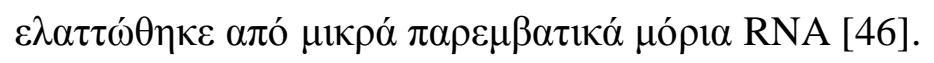

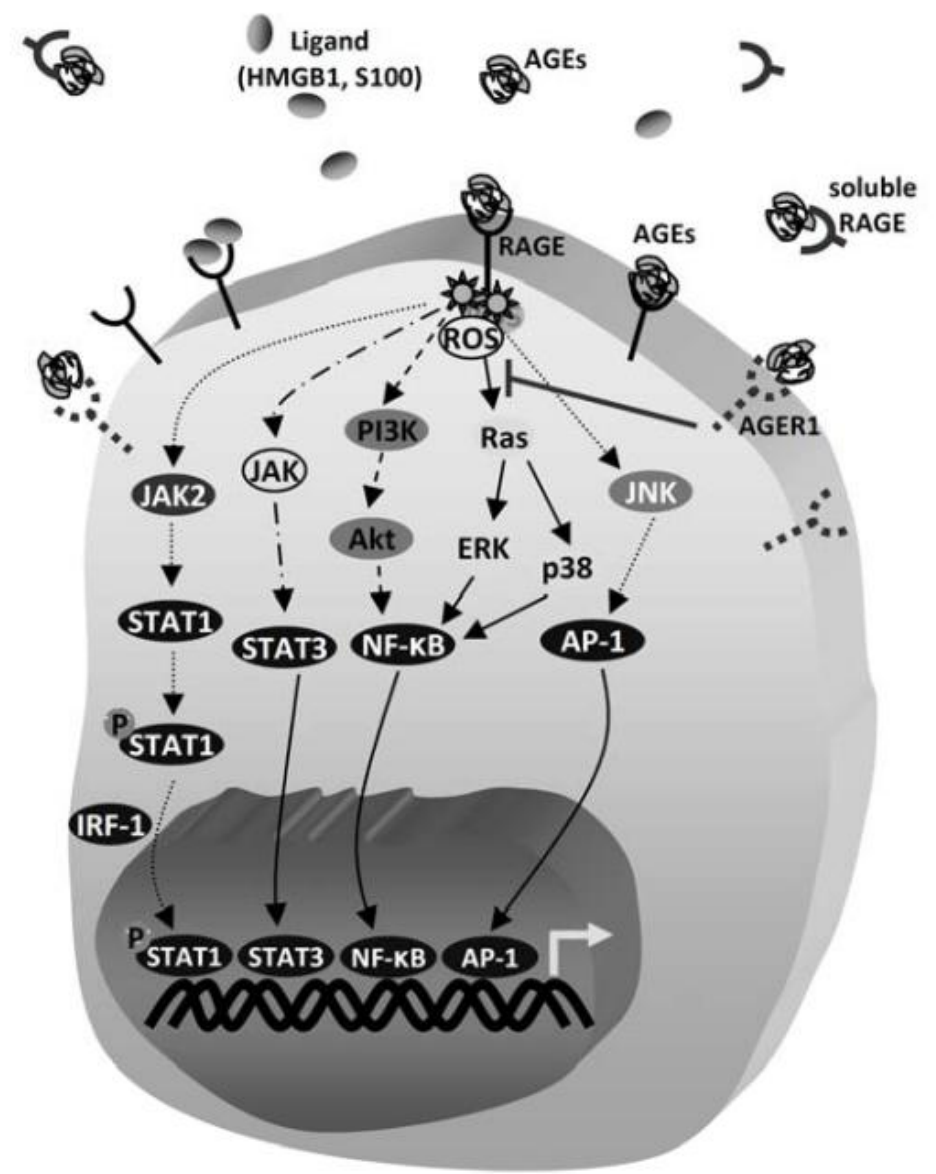

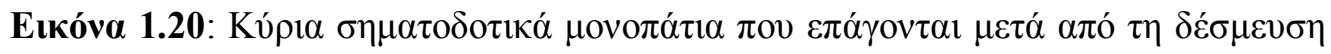
$\tau \omega \nu$ AGEs $\sigma \tau$ R RAGE [42]. 


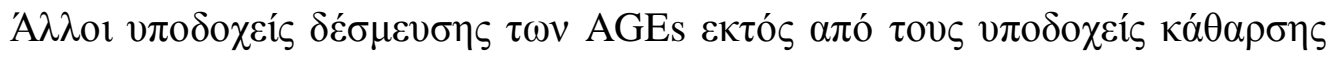

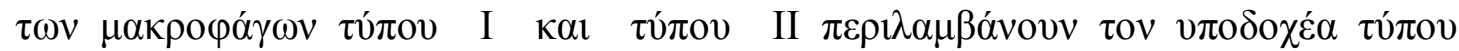
$\lambda \varepsilon \kappa \tau i ́ v \eta \varsigma$ LOX-1 (lectin like oxidized low density lipoprotein receptor-1), $\tau \eta$

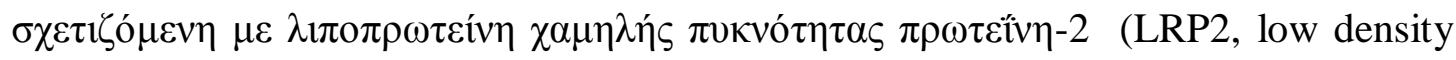

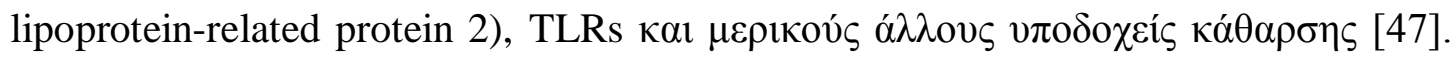

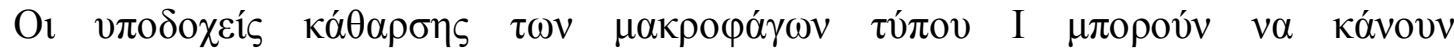

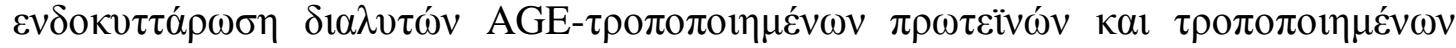

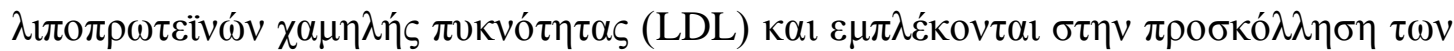

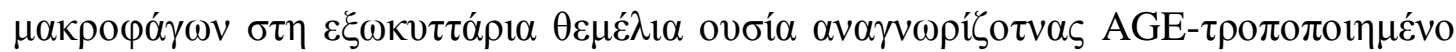

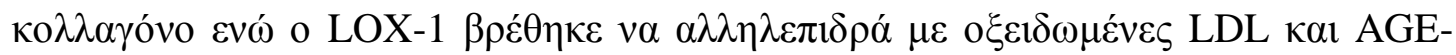

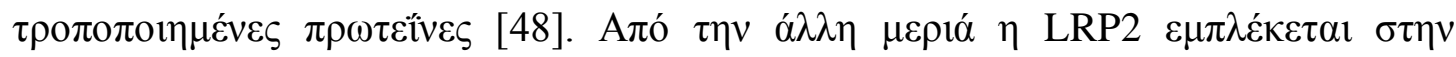

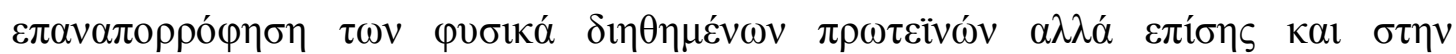

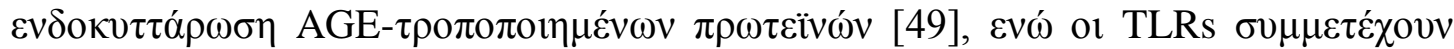

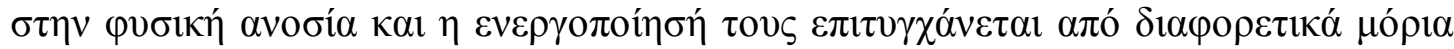

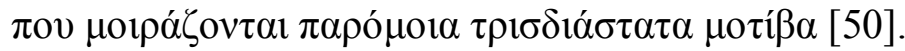

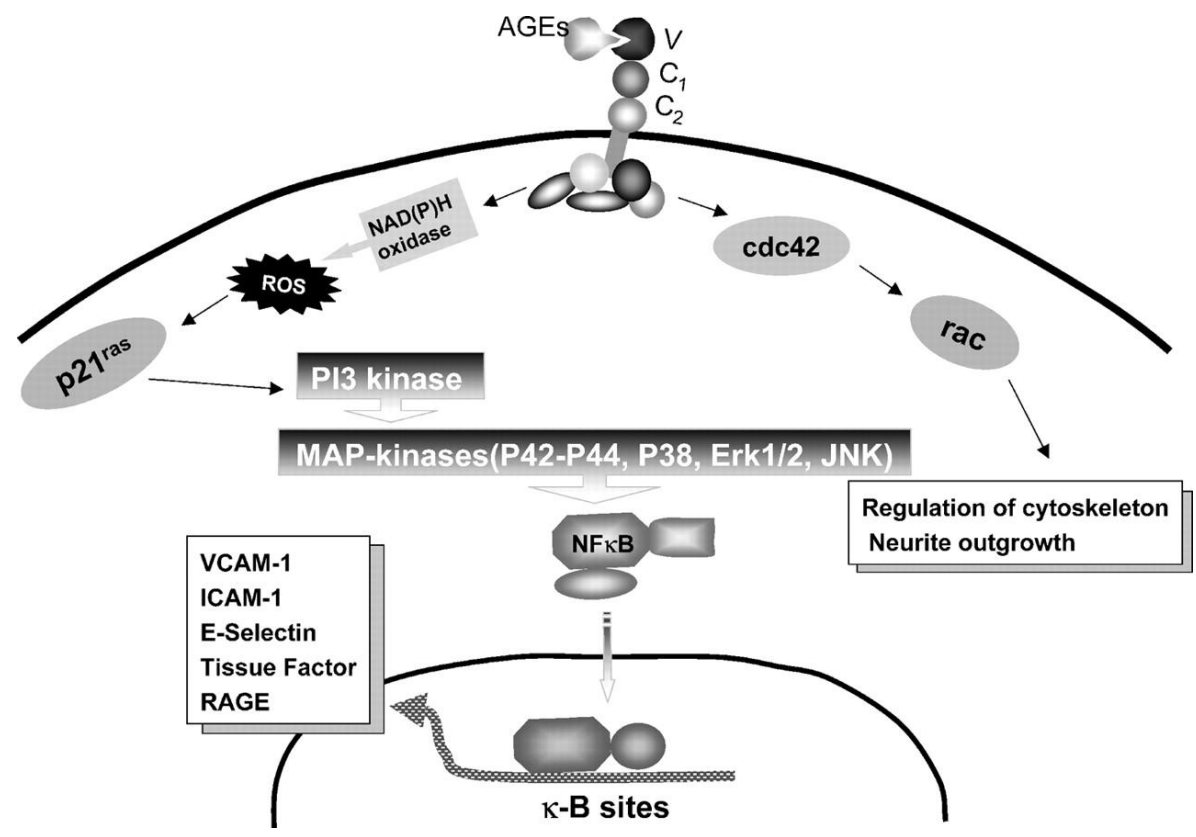

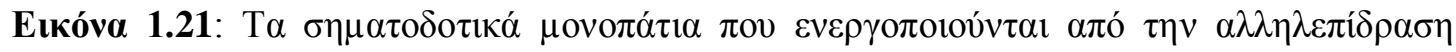
AGE-RAGE.

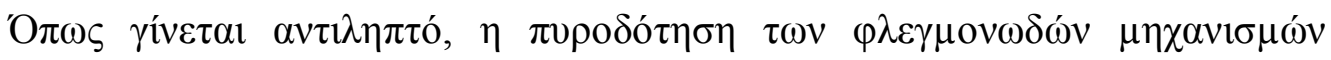
( 


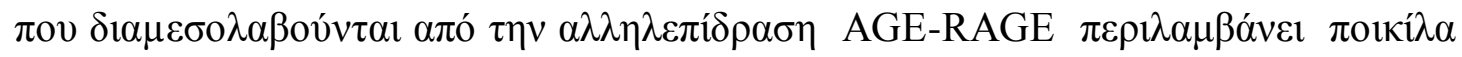

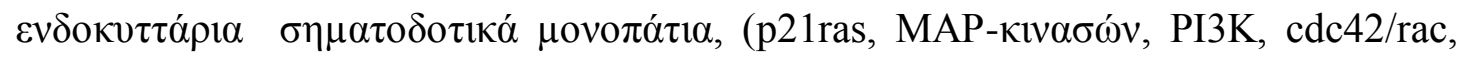

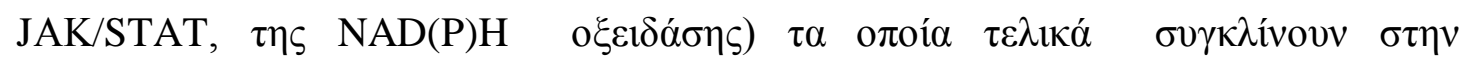

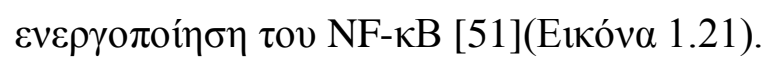

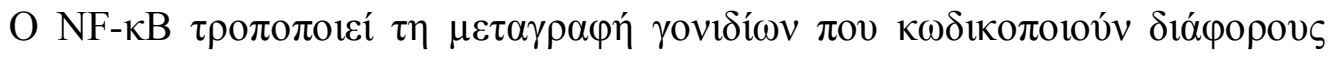

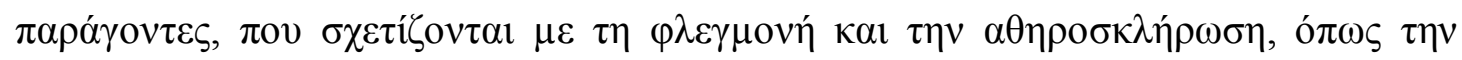

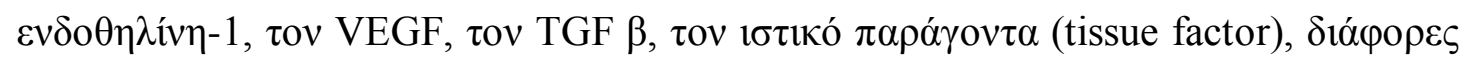
$\varphi \lambda \varepsilon \gamma \mu$

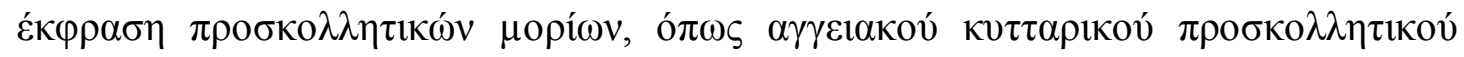

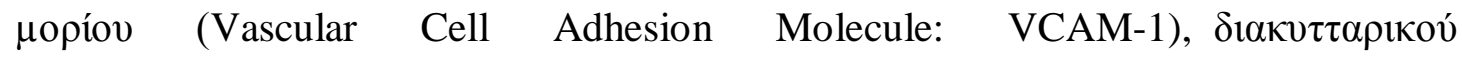

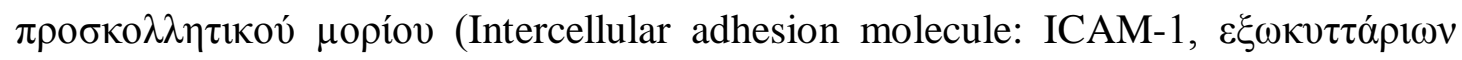

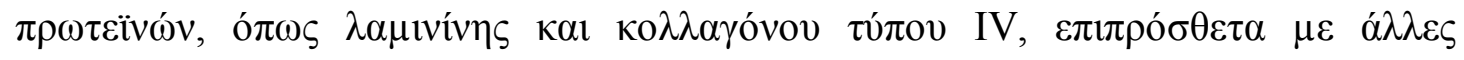

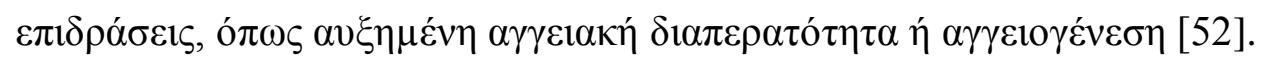

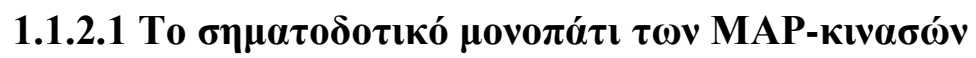

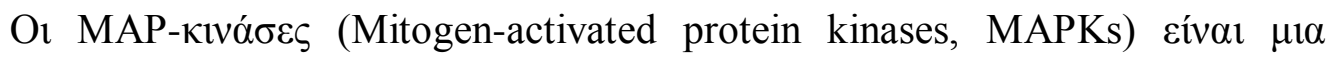

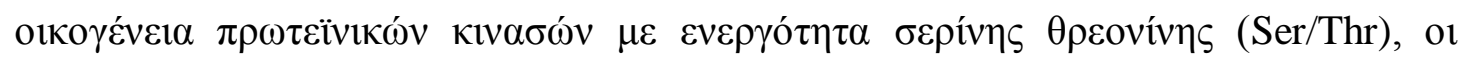

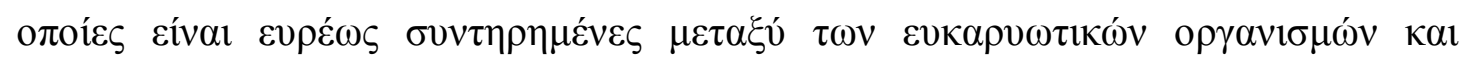

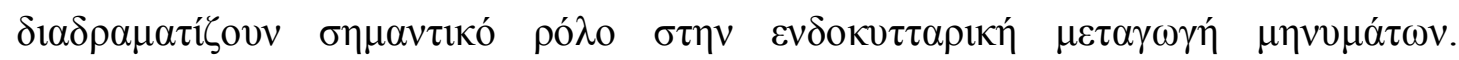

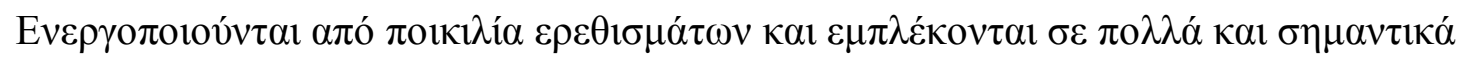

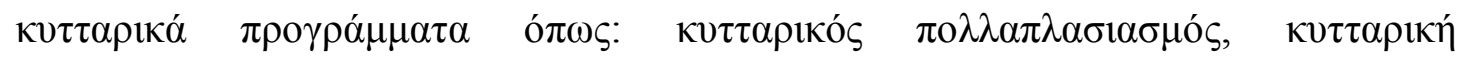

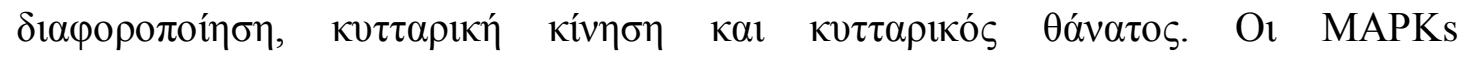

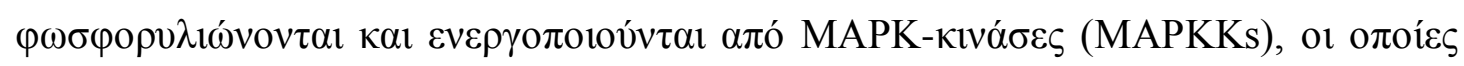

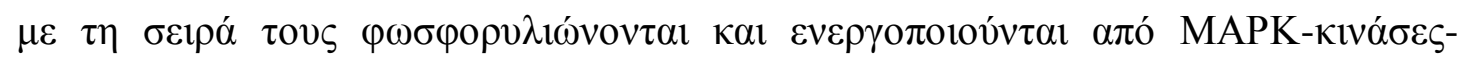

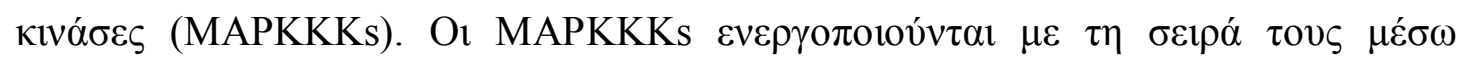

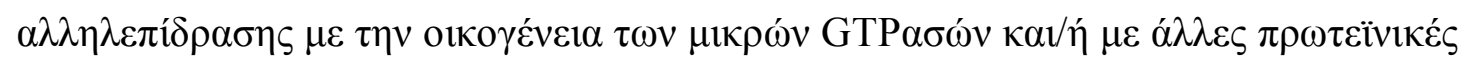

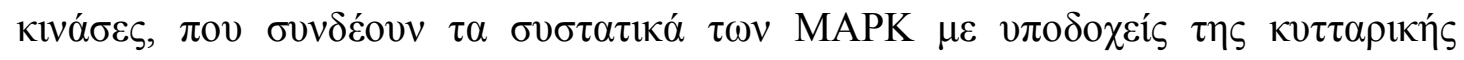

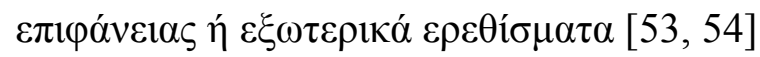

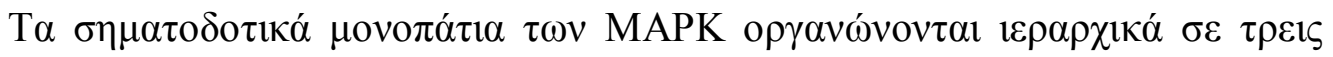

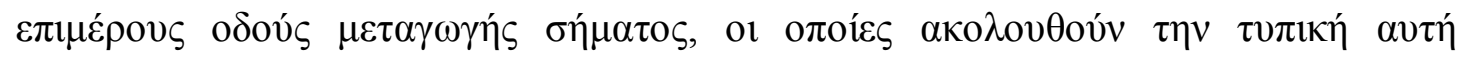




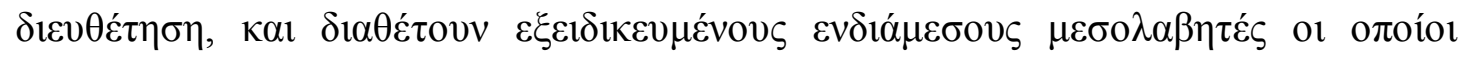

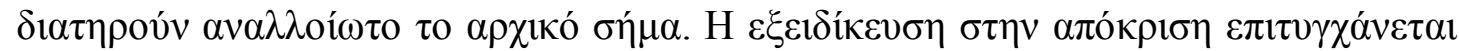

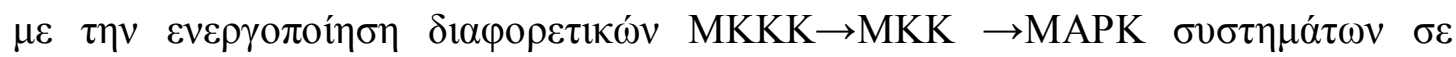

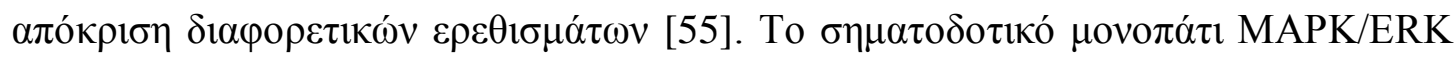

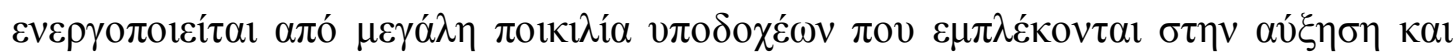

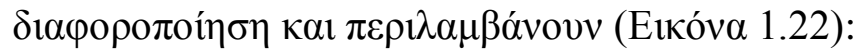

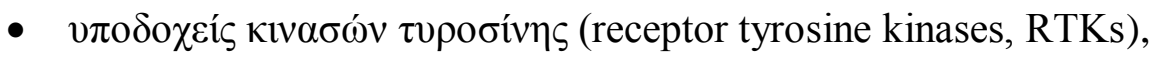

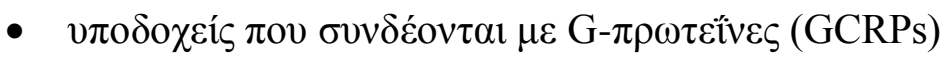

- $\quad$ iv $\tau \varepsilon \gamma \kappa \rho i ́ v \varepsilon \varsigma \&$

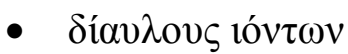

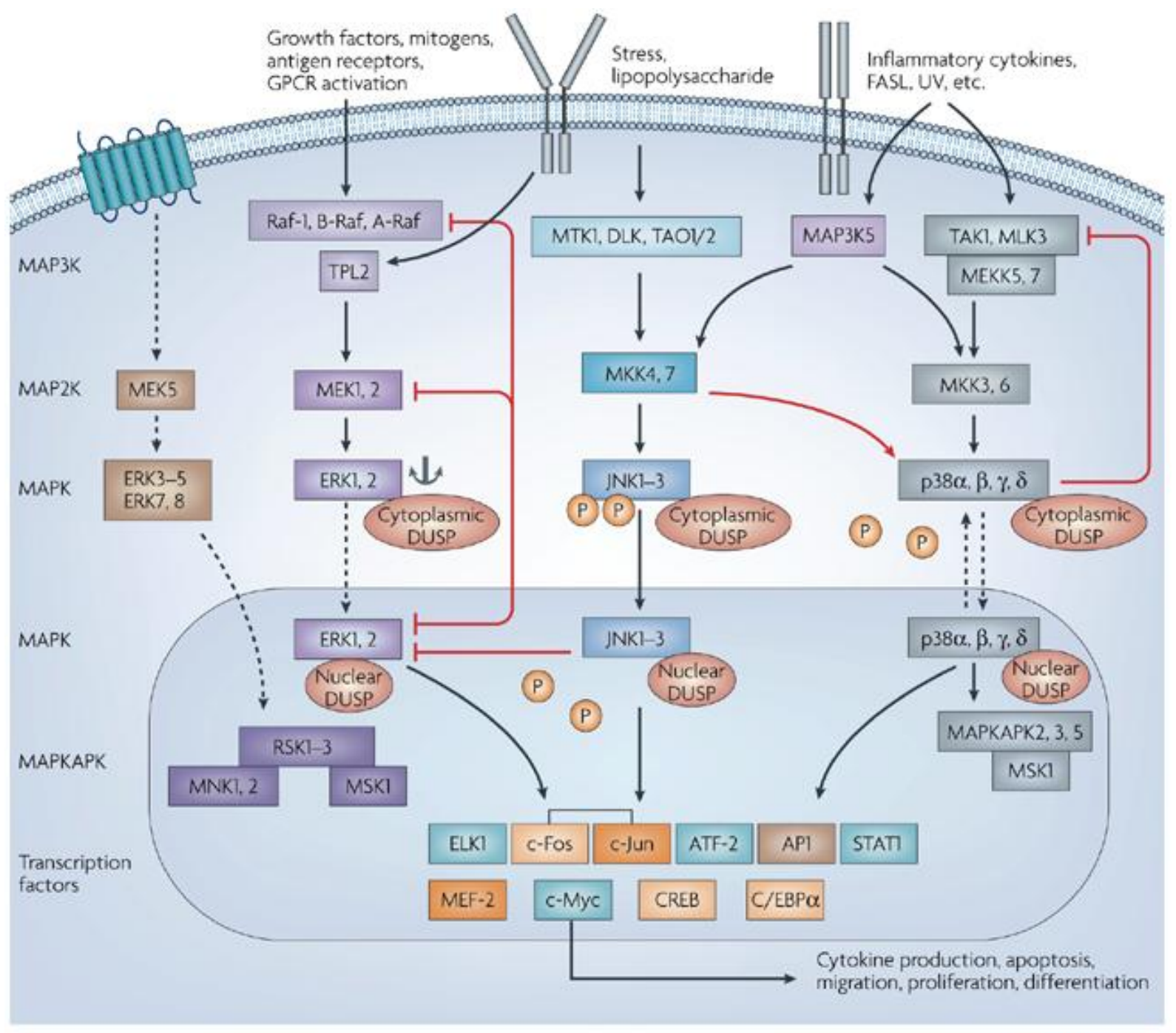

Nature Reviews | Drug Discovery

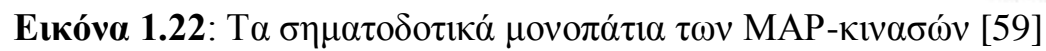




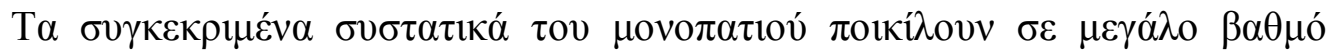

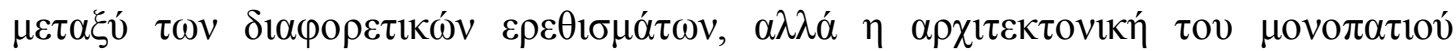

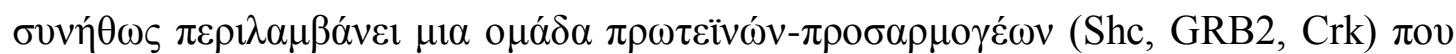

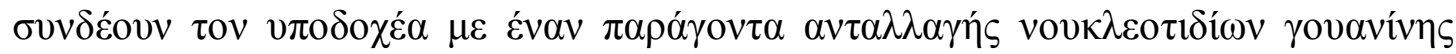

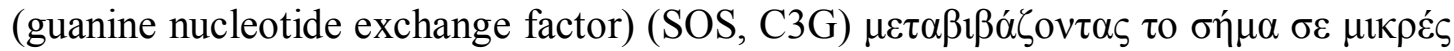

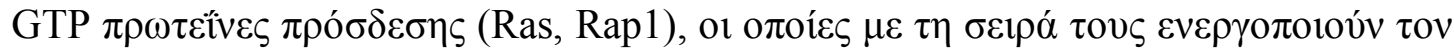

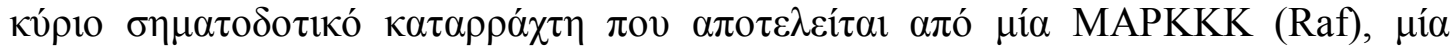

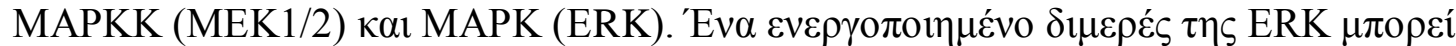

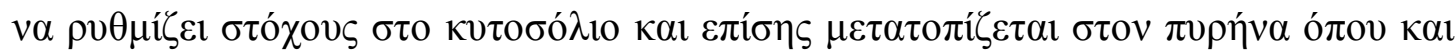

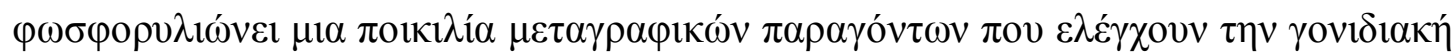

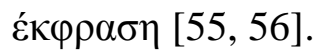

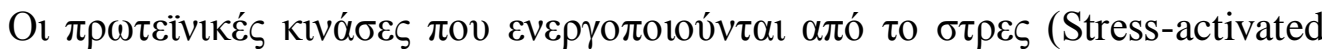

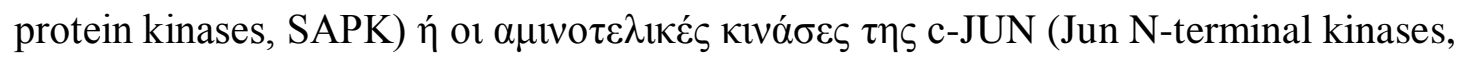

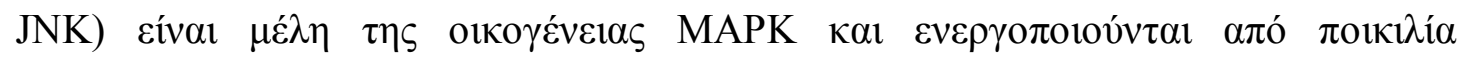

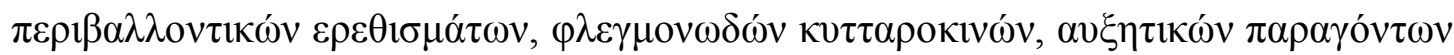

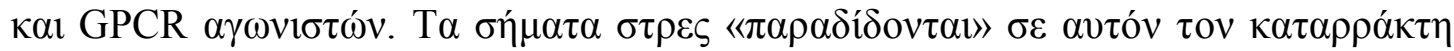

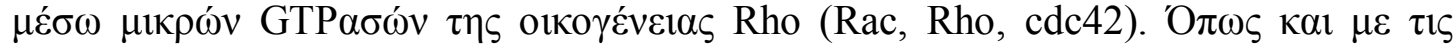

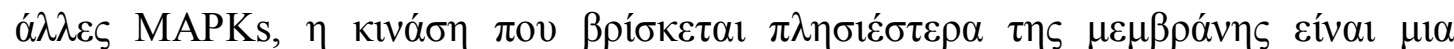

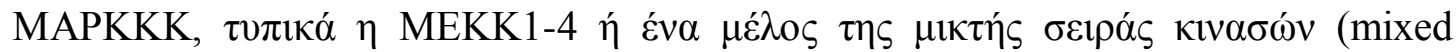

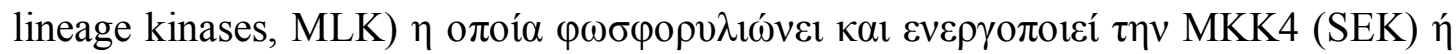

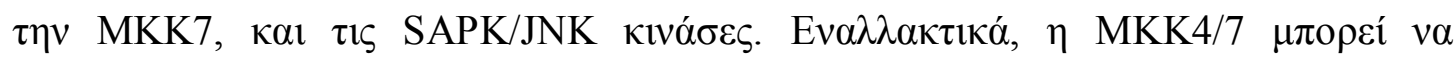

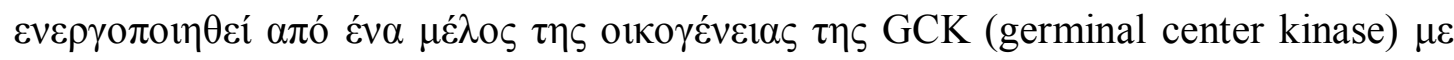

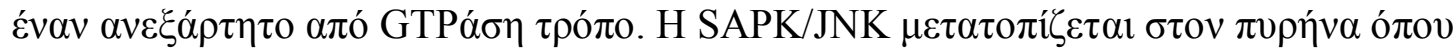

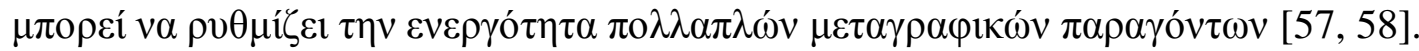

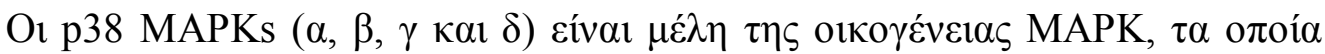

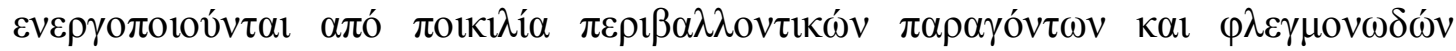

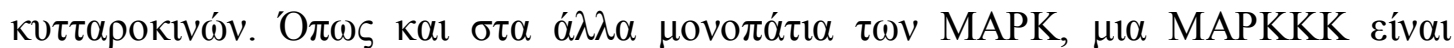

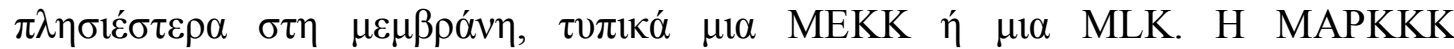

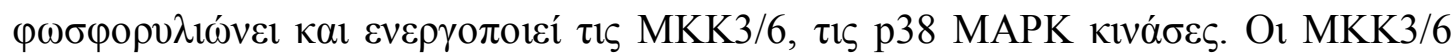

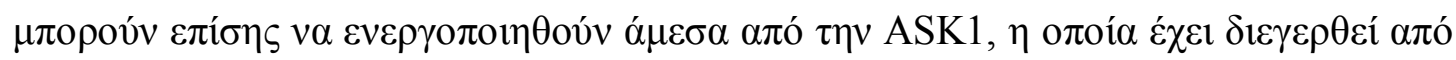

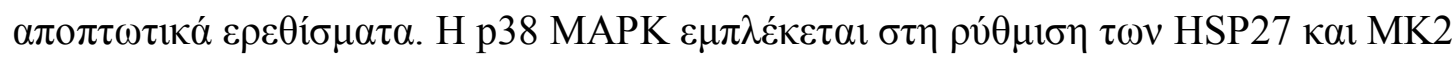

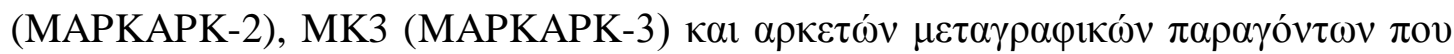




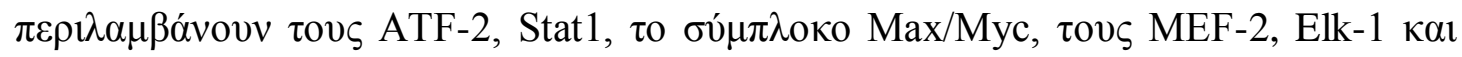

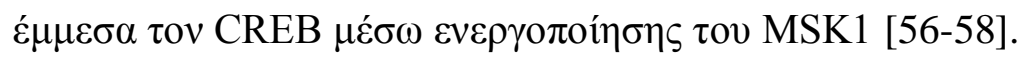

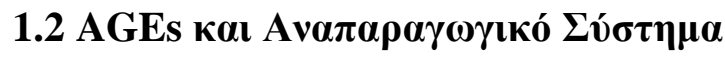

O

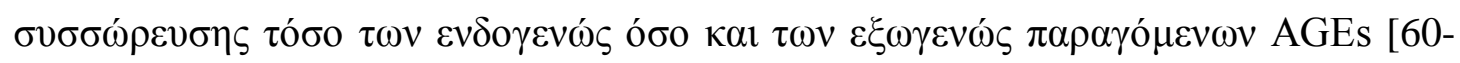
62].

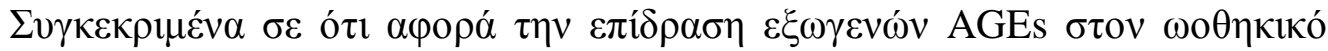

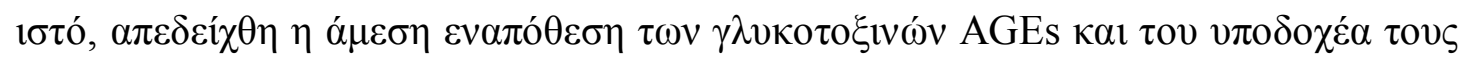

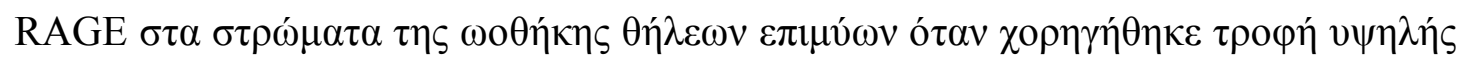

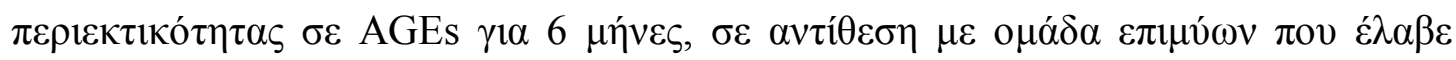

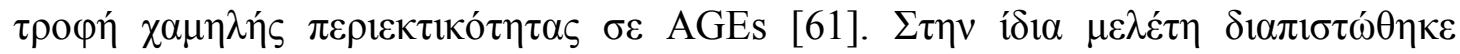

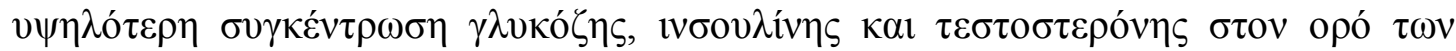

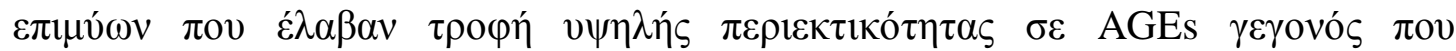

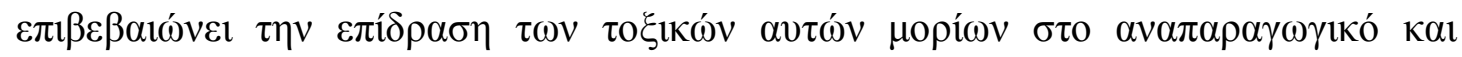

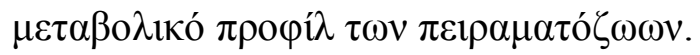

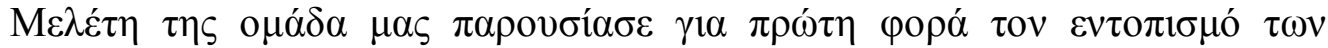

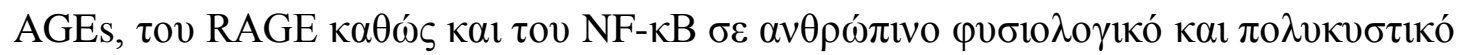

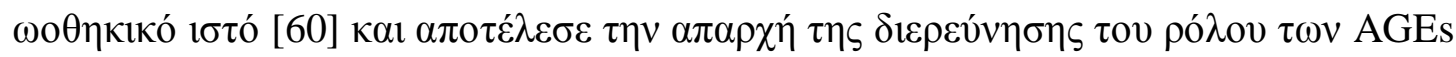

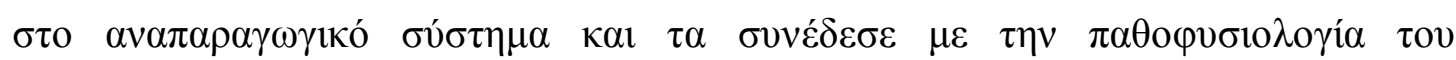

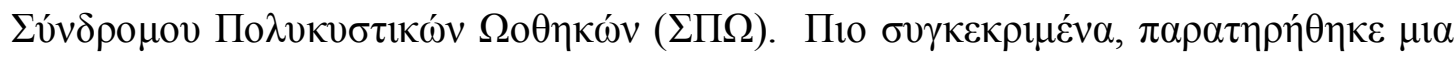

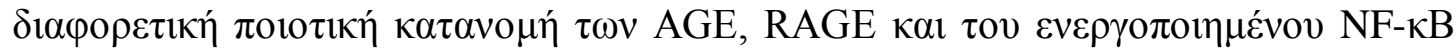

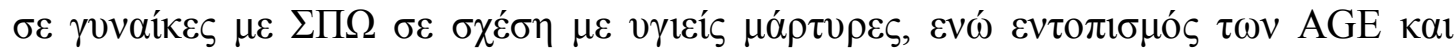

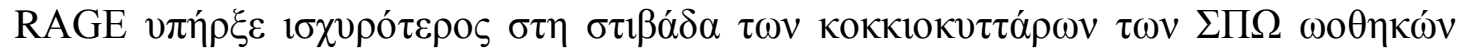

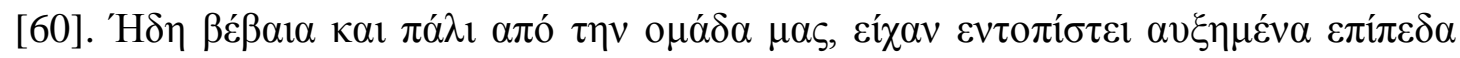

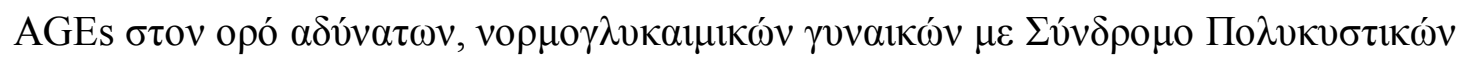
$\Omega \circ \eta \eta \kappa \omega ́ v(\Sigma \Pi \Omega)[63]$.

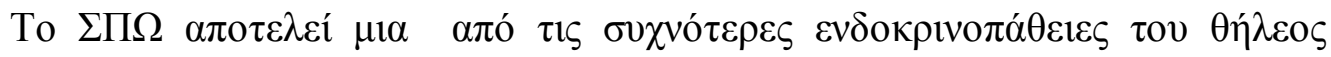

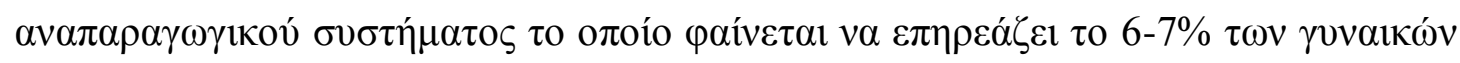

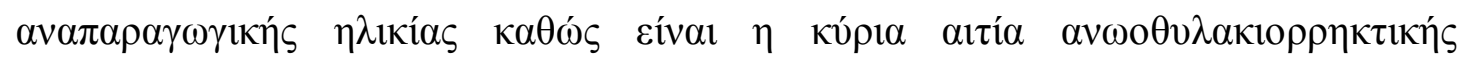




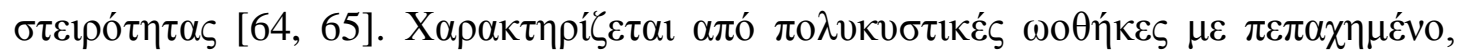

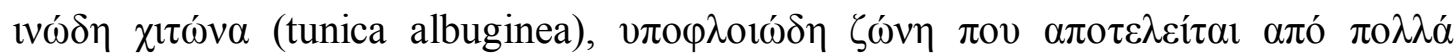

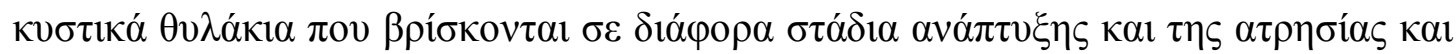

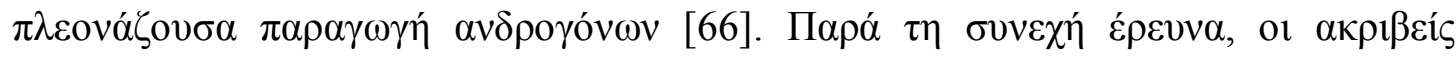

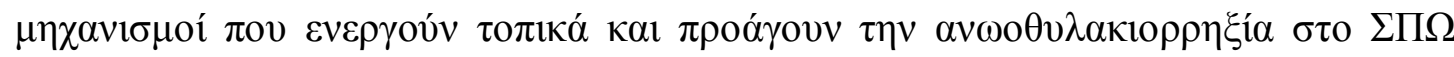

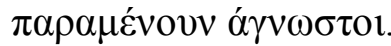

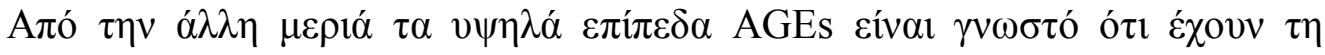

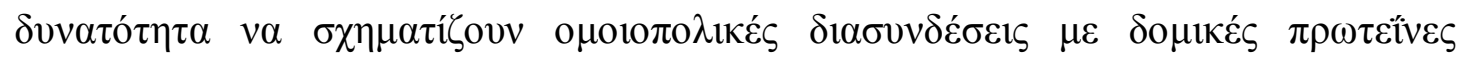

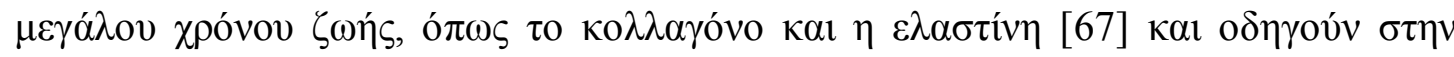

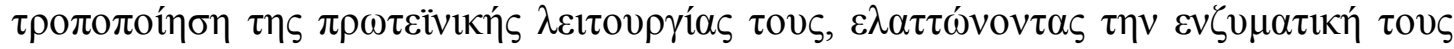

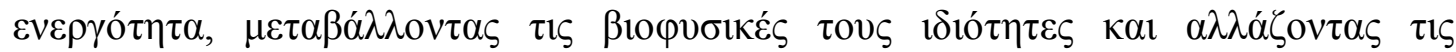

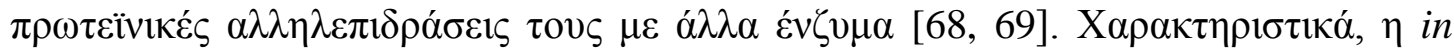

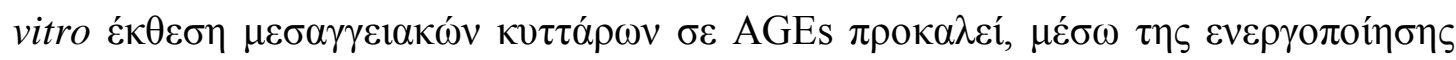

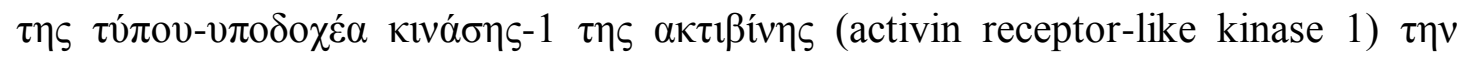

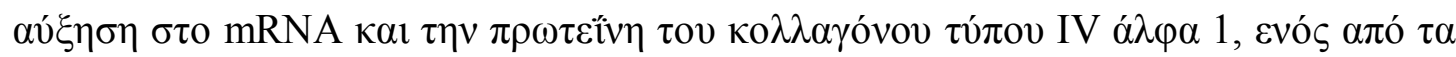

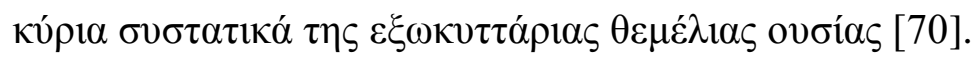

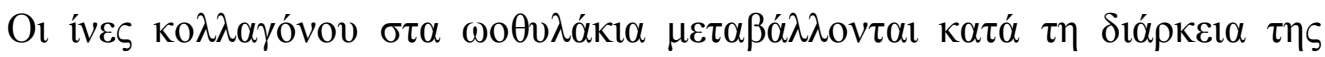

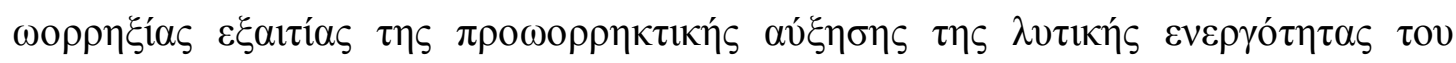

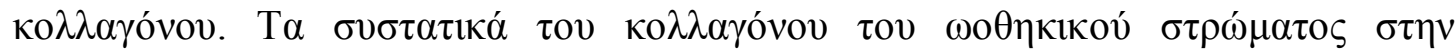

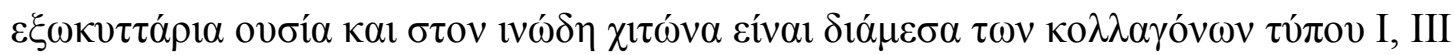

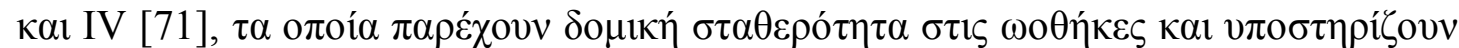

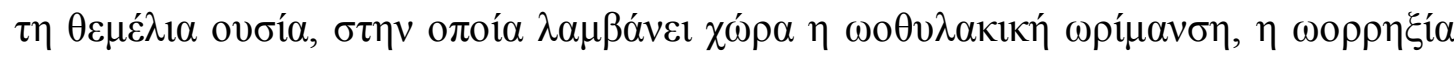

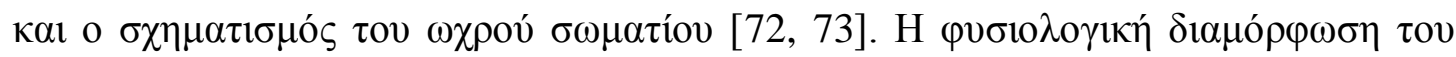

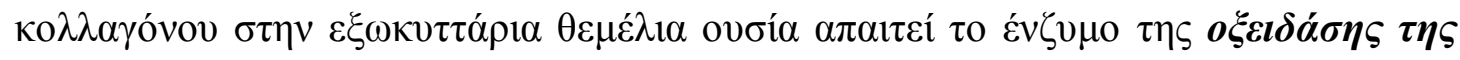

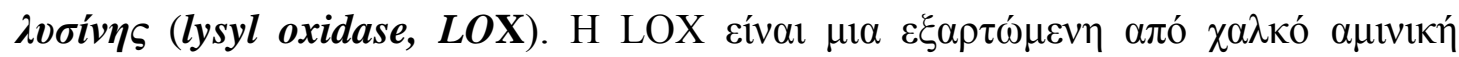

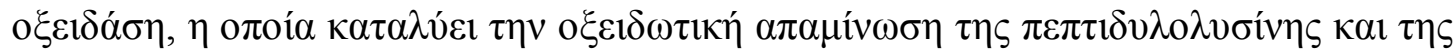

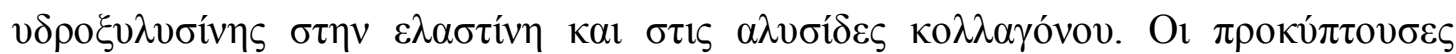

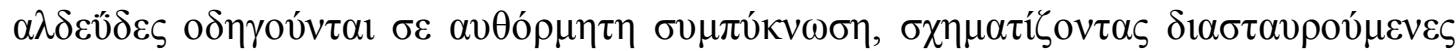

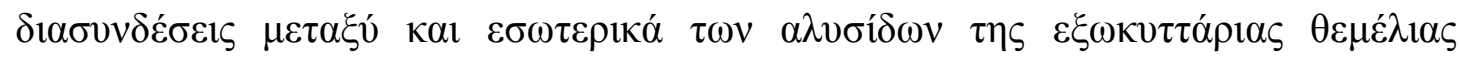

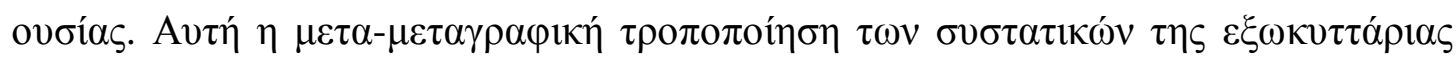

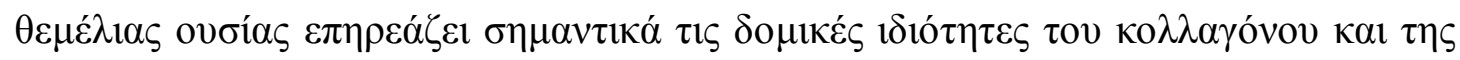

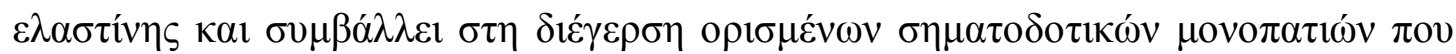




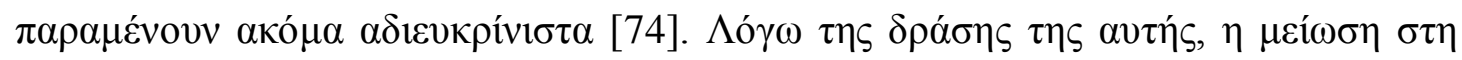

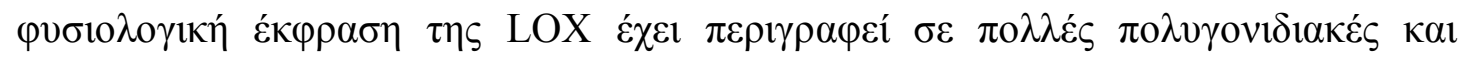

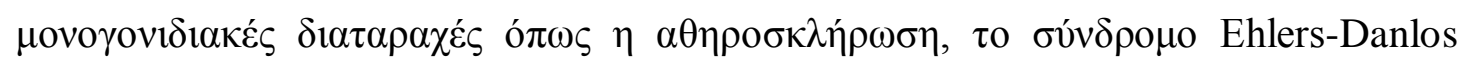

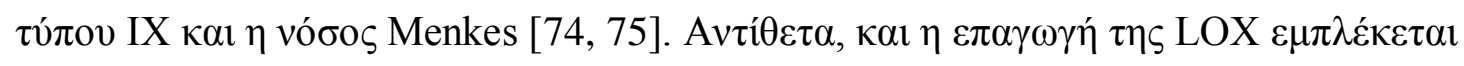

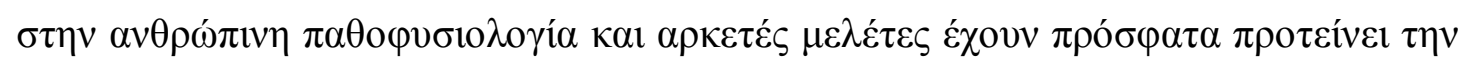

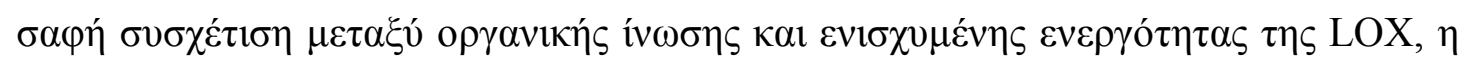

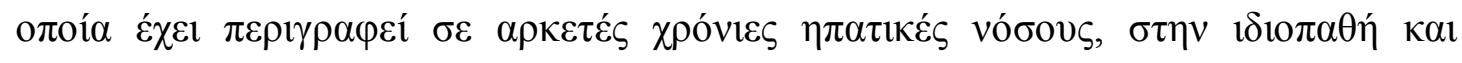

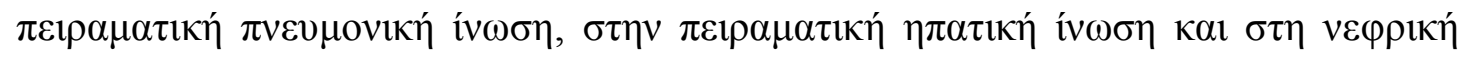

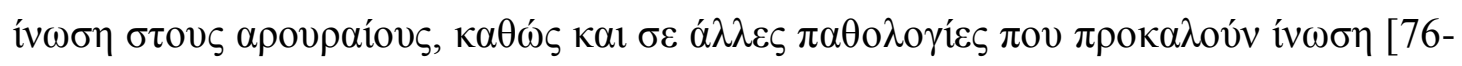
83].

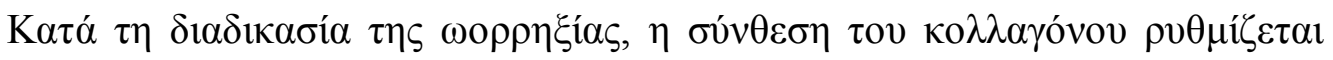

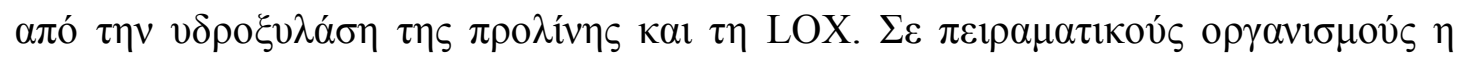

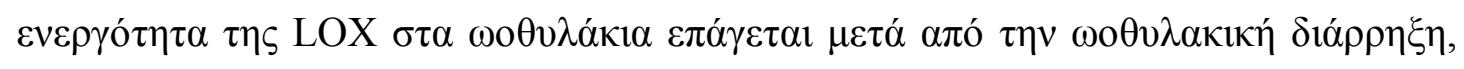

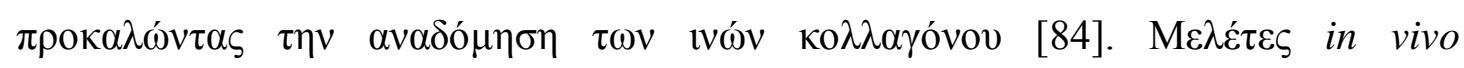

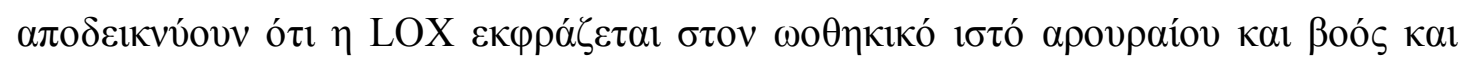

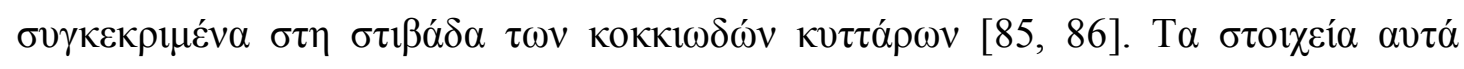

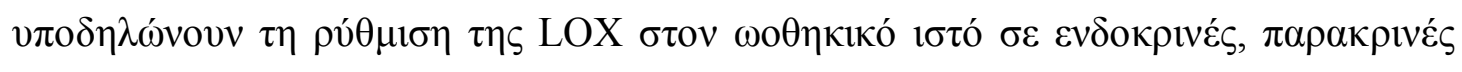

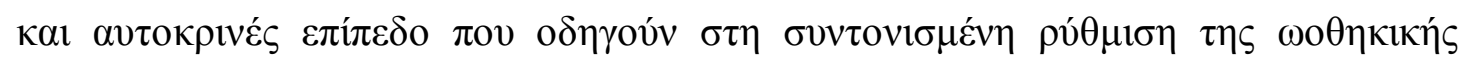

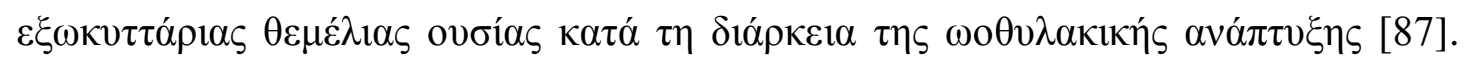

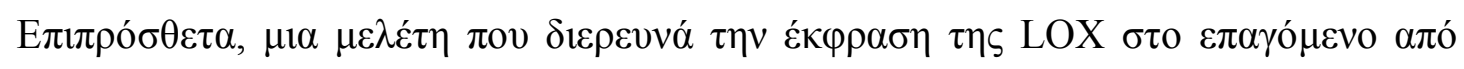

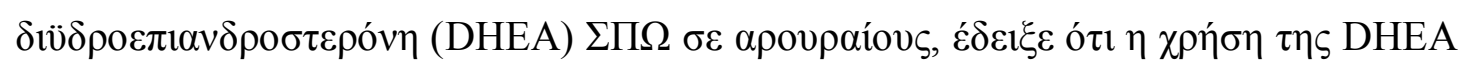

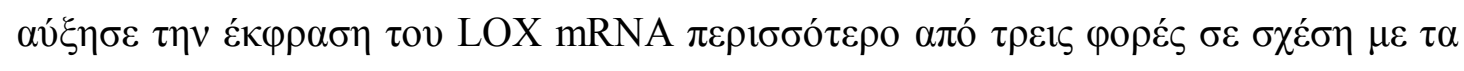

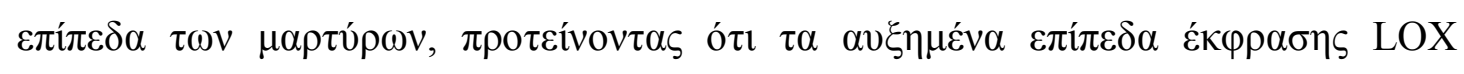

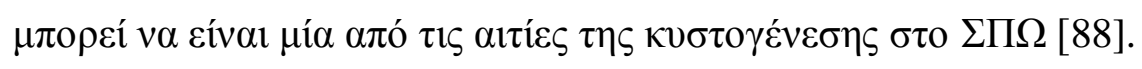

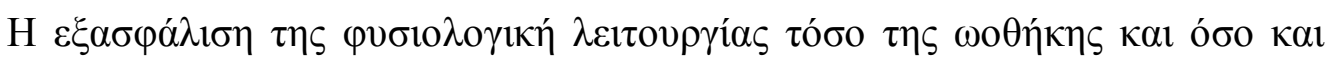

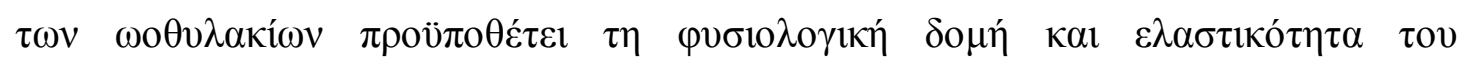

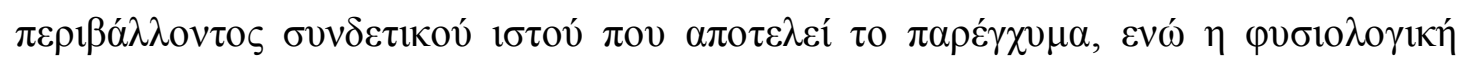

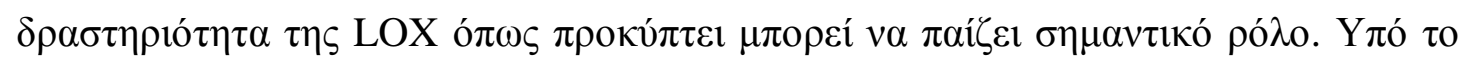

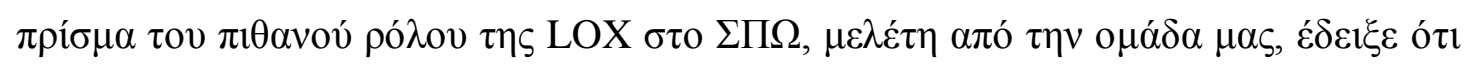

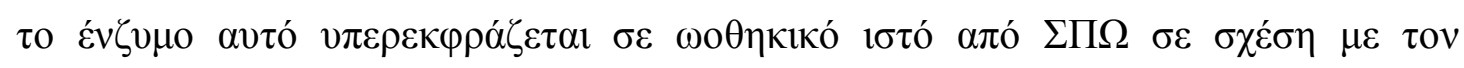

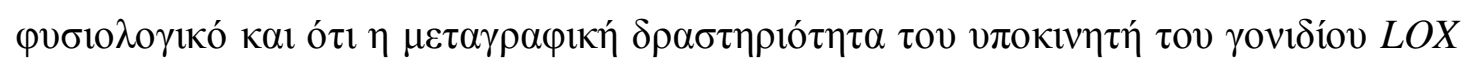

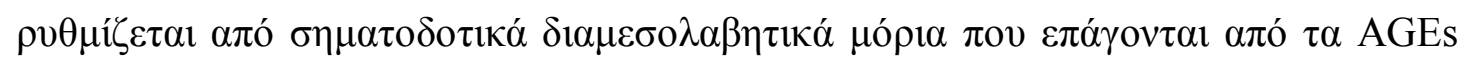




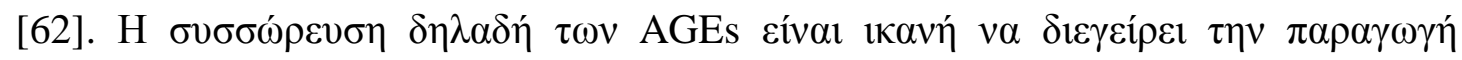

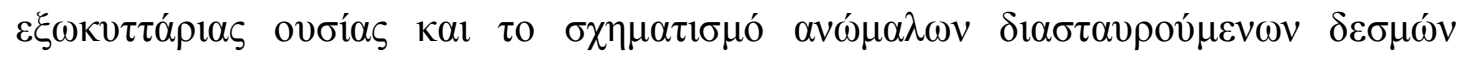

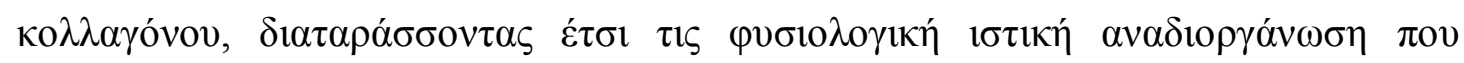

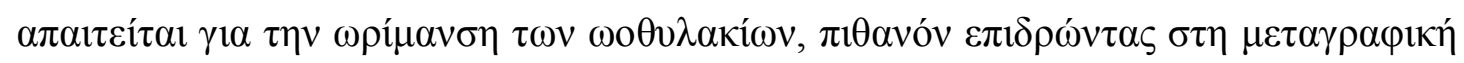
$\delta 1 \alpha \delta 1 \kappa \alpha \sigma i ́ \alpha$ o $\xi \varepsilon 1 \delta \omega \tau 1 \kappa \omega ́ v ~ \pi \alpha \rho \alpha \gamma o ́ v \tau \omega v$ [62].

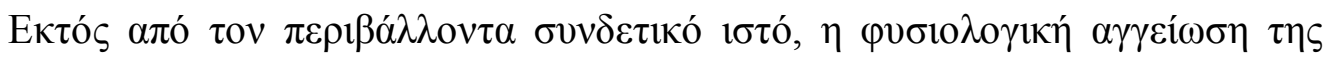


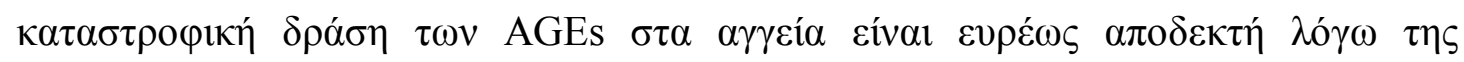

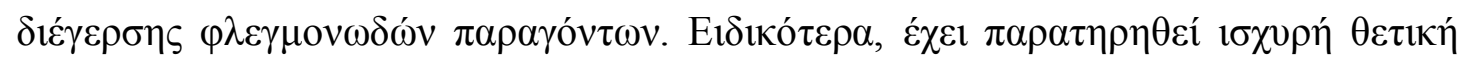

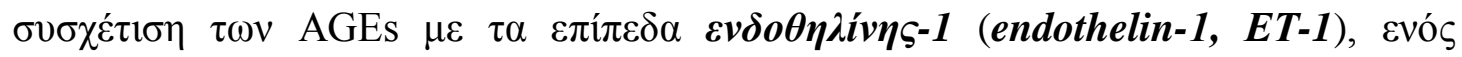

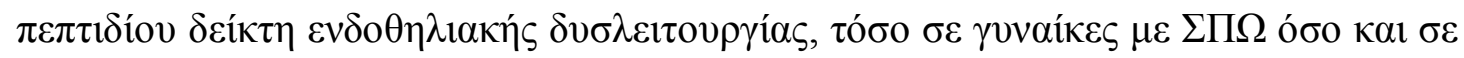

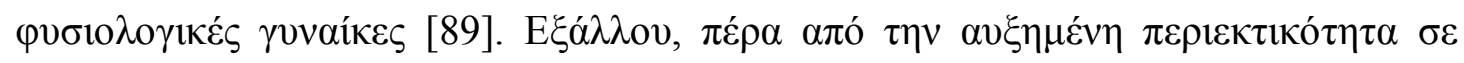

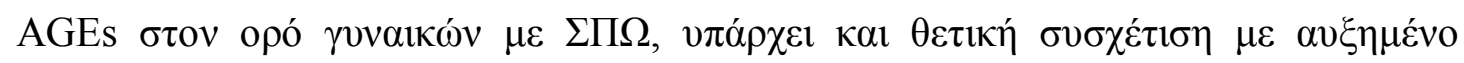

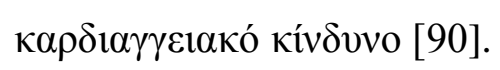

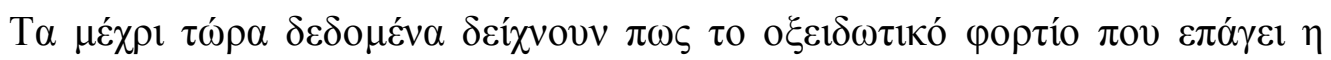

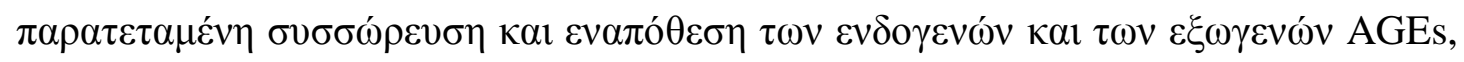

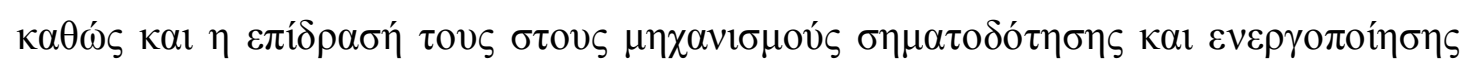

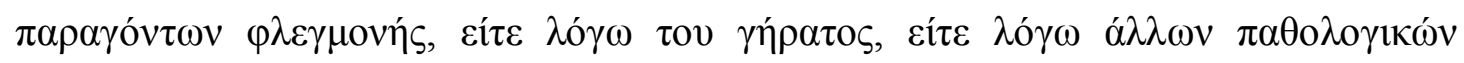

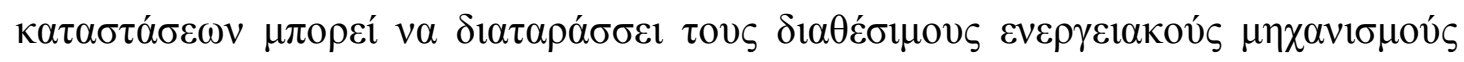

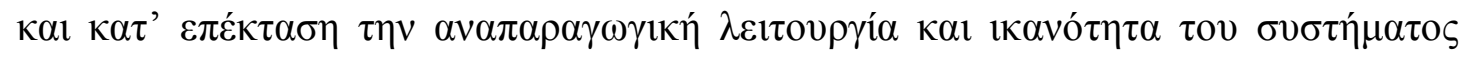
[91]. 


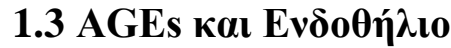

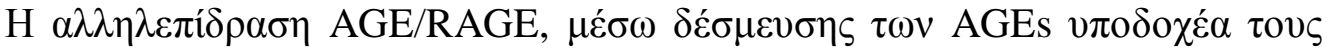

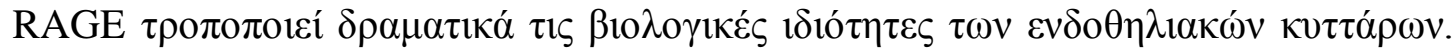

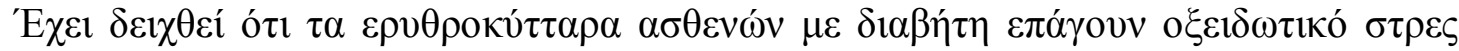

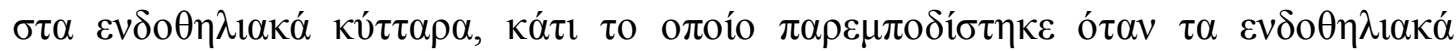

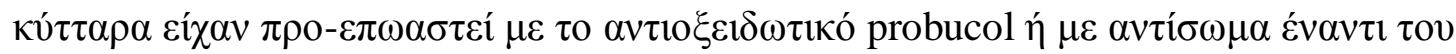

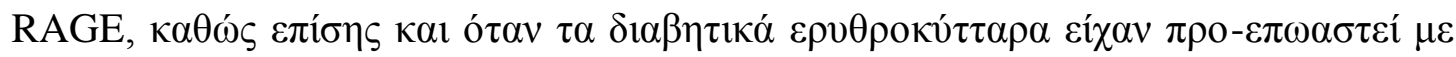

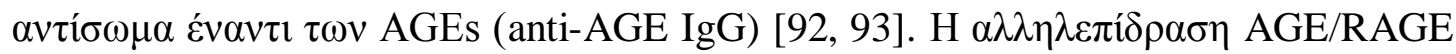

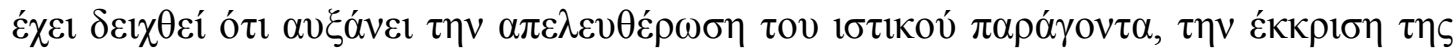

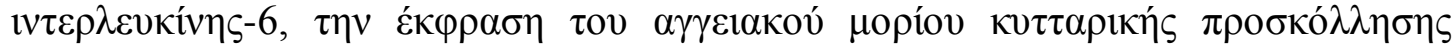

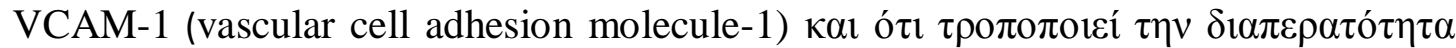

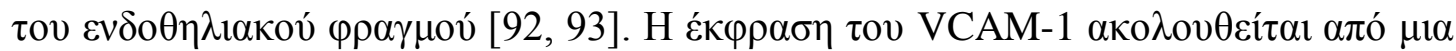

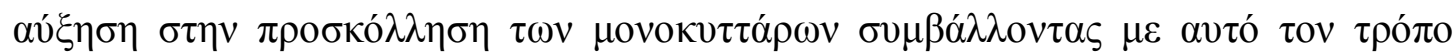

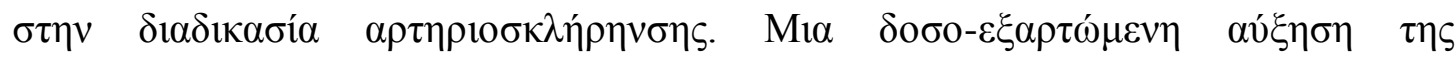

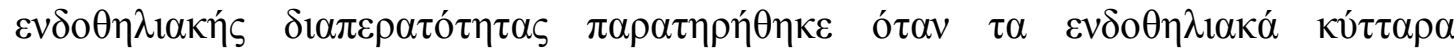

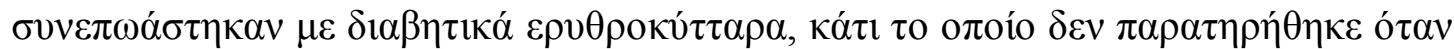

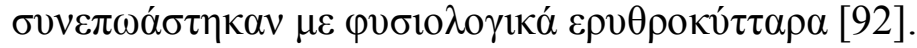

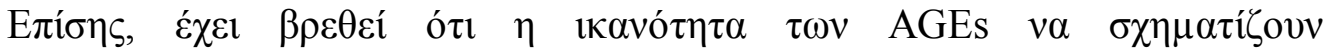

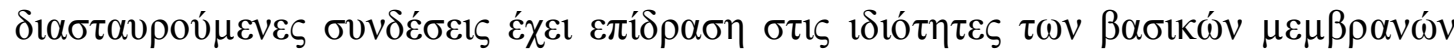

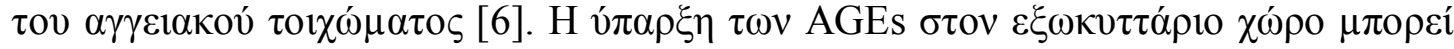

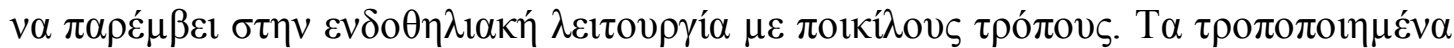

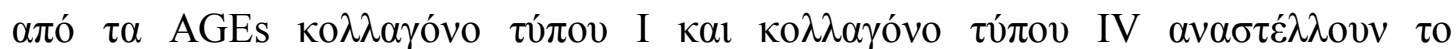

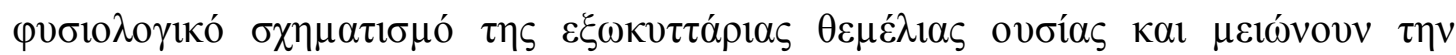

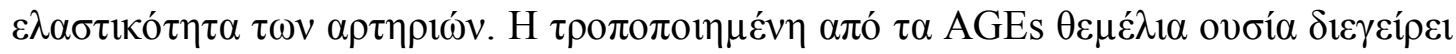

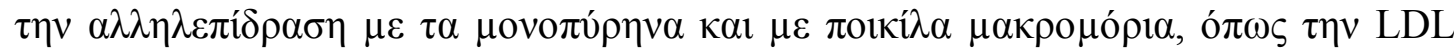

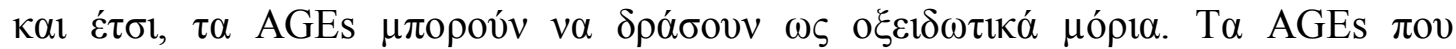

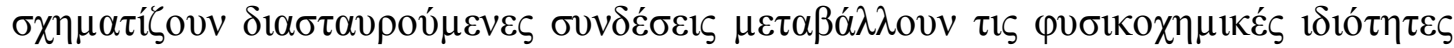

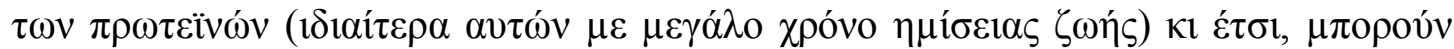

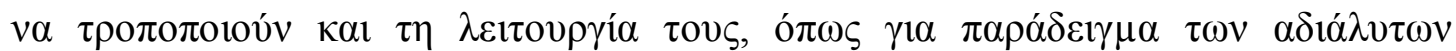

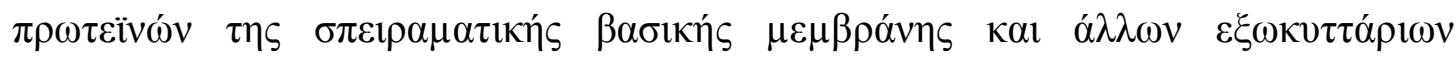

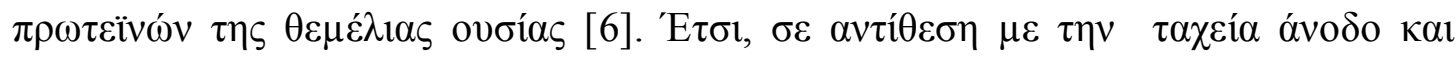




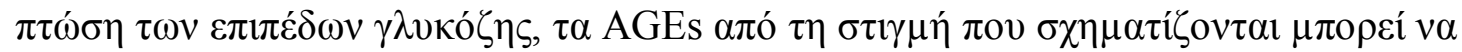

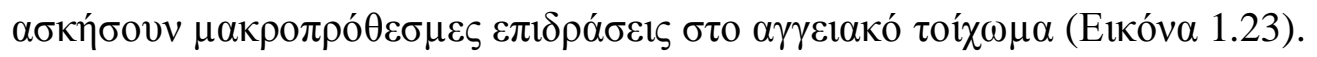

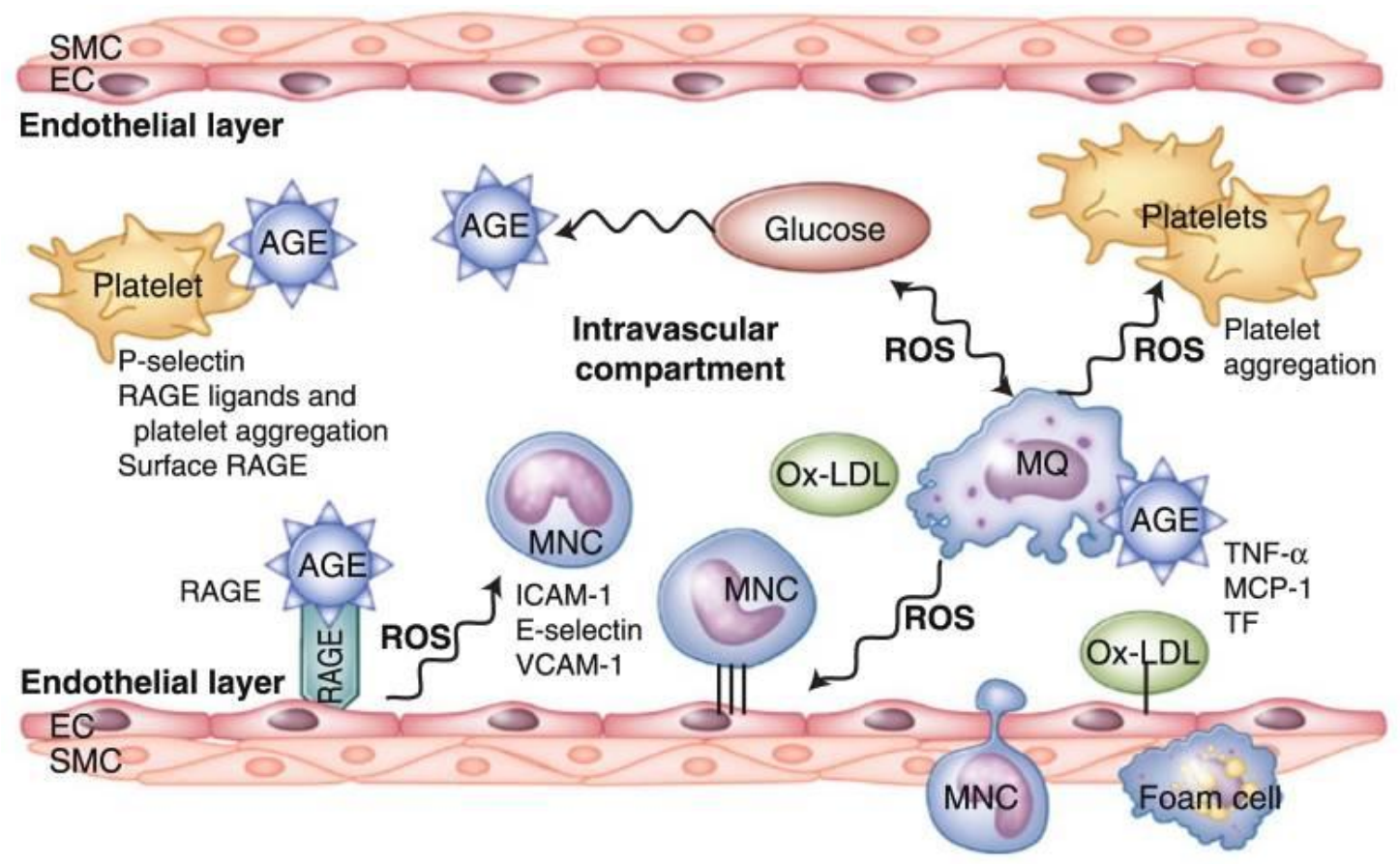

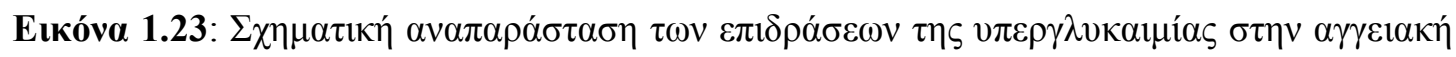

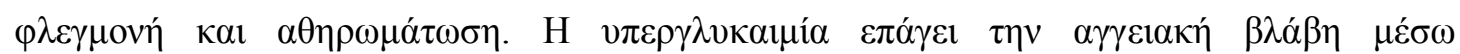

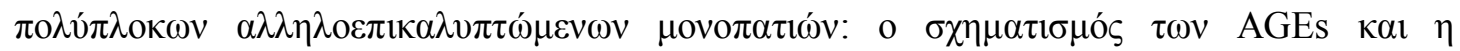

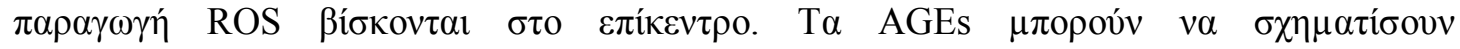

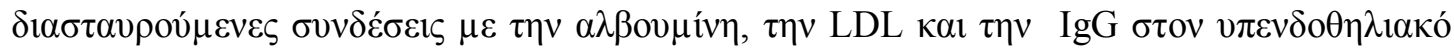

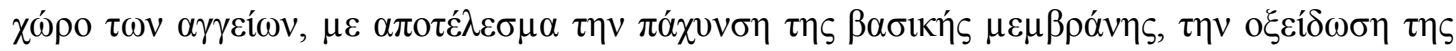

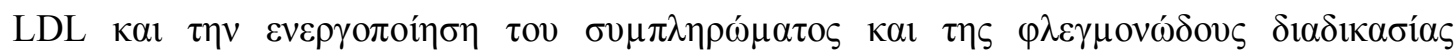
$\alpha v \tau i ́ \sigma \tau o l \chi \alpha$.

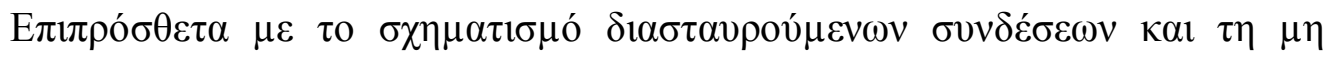

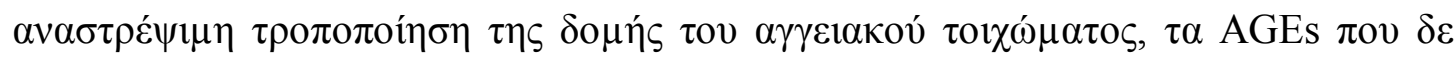

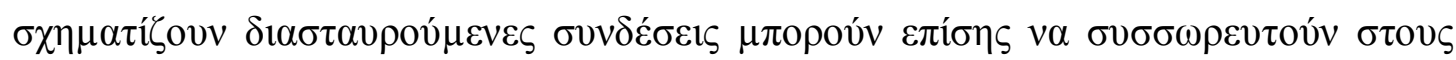

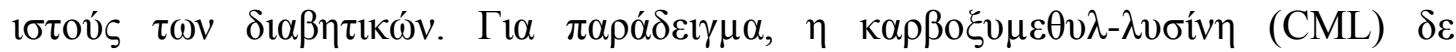

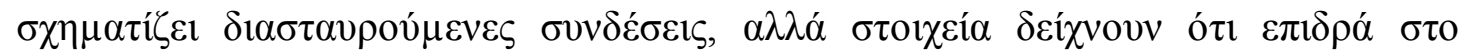

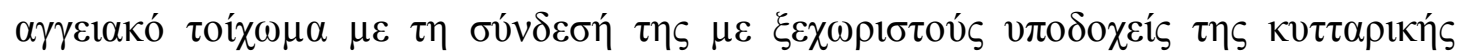




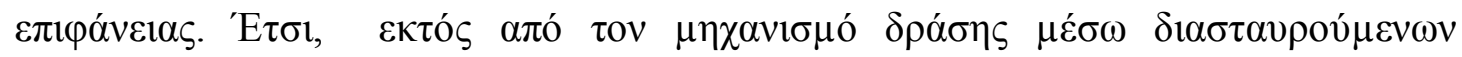

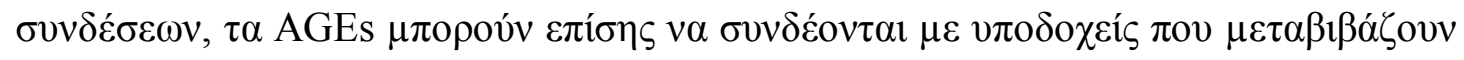

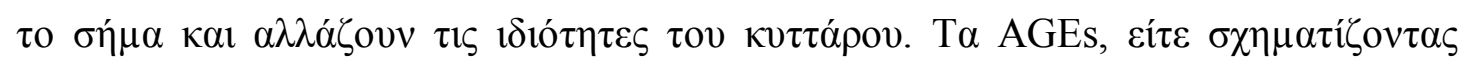

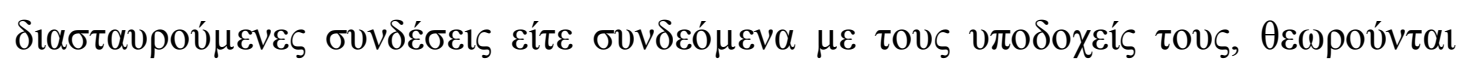

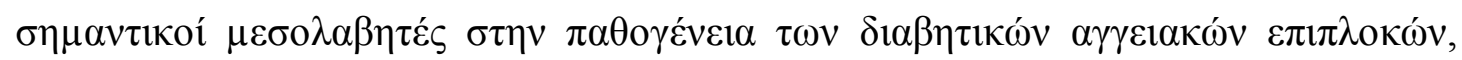

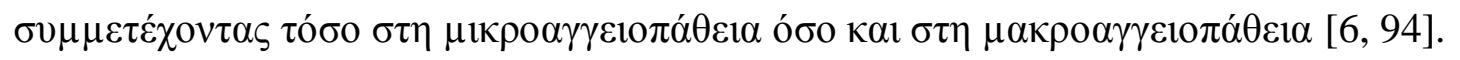

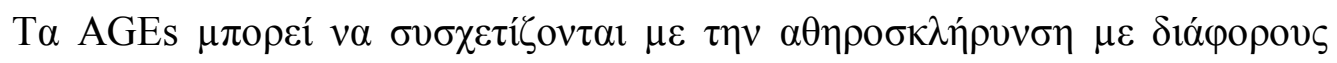

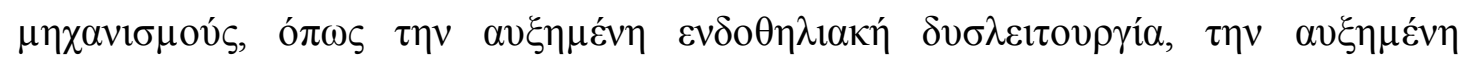

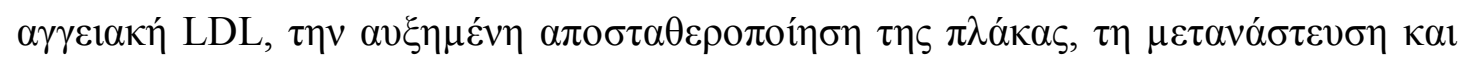

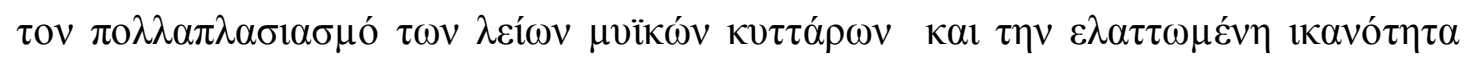

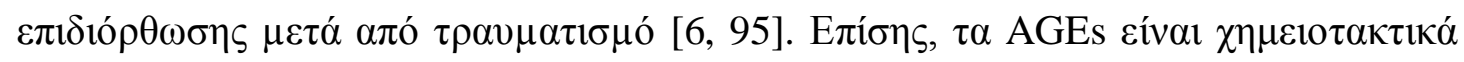

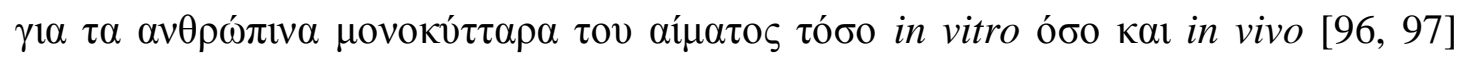

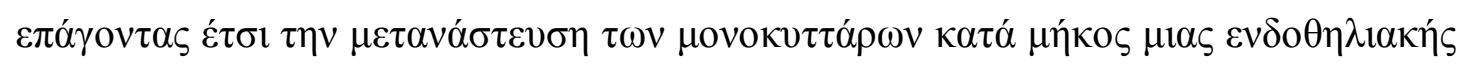

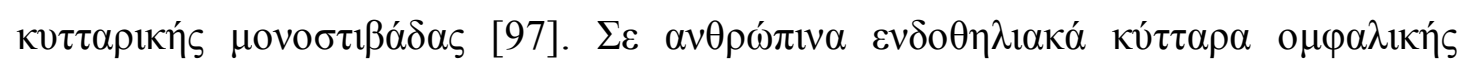

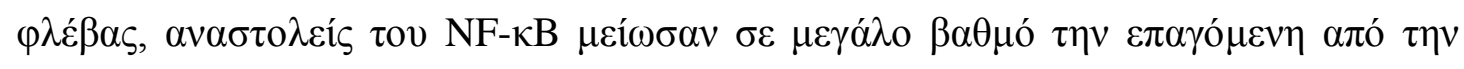

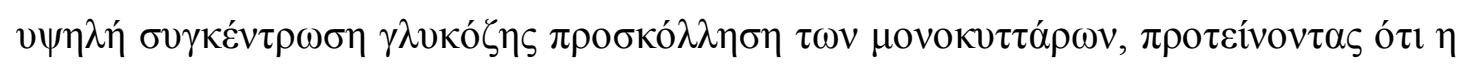

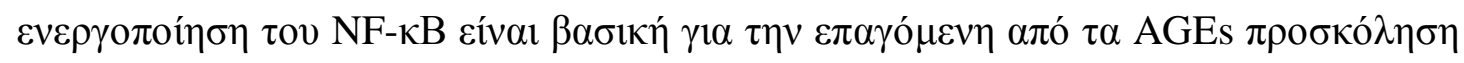

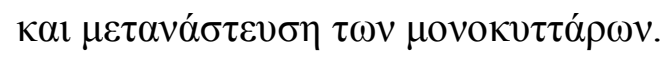

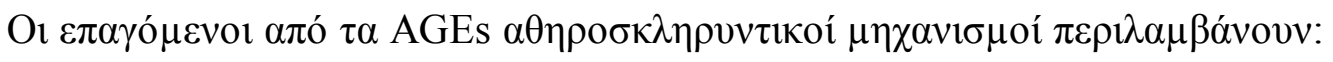

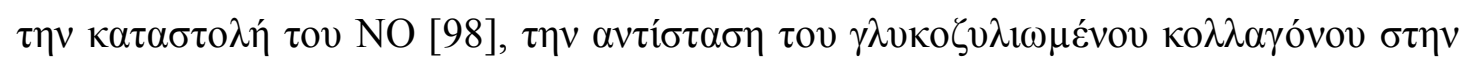

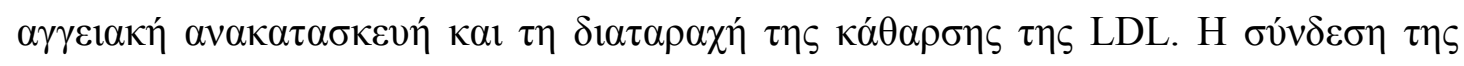

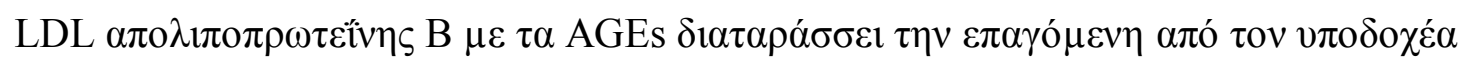

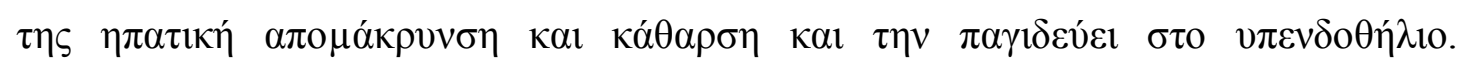

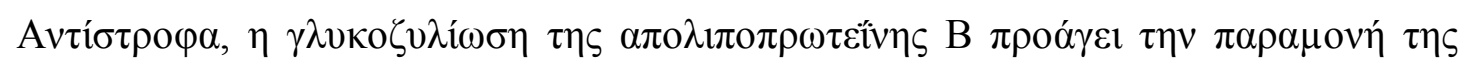

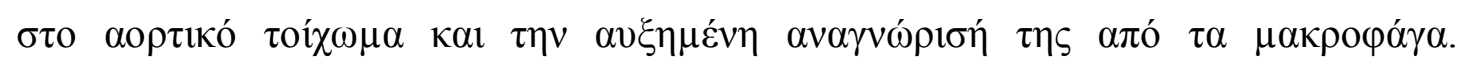

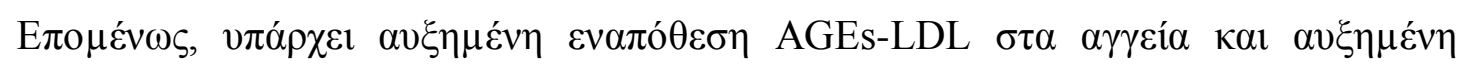

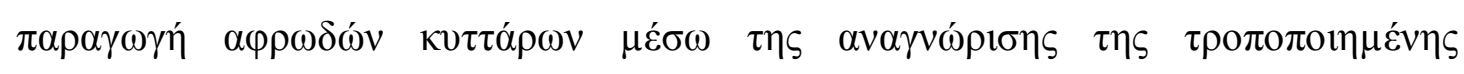

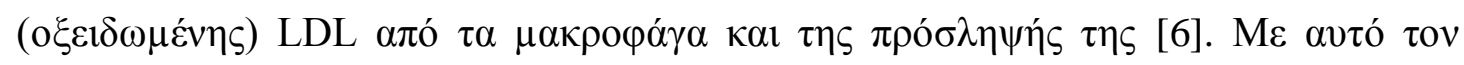

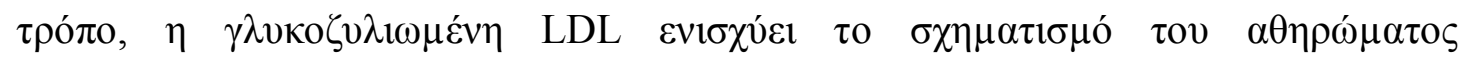

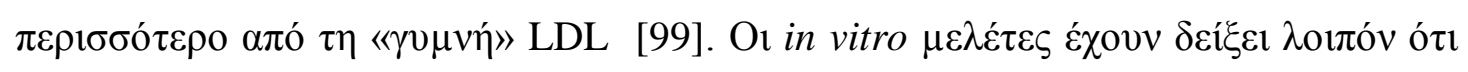

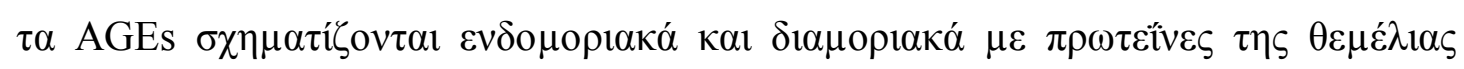

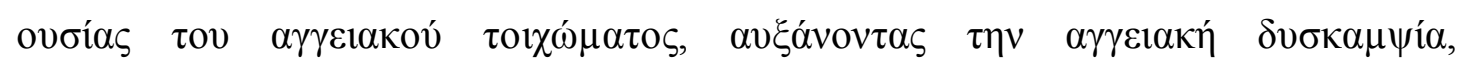




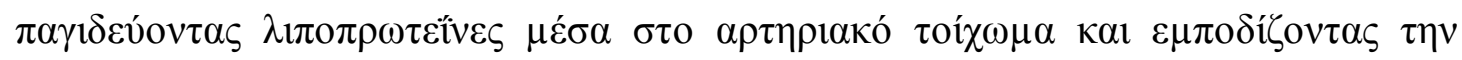

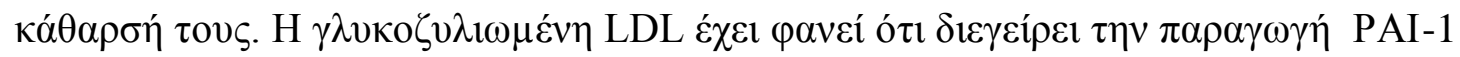

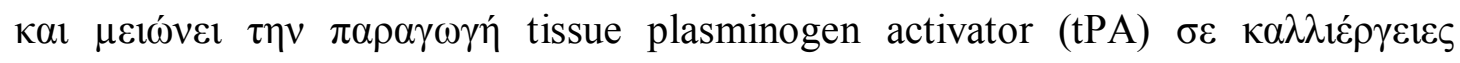

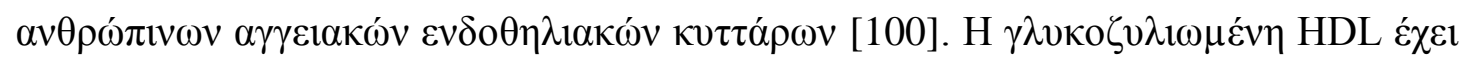

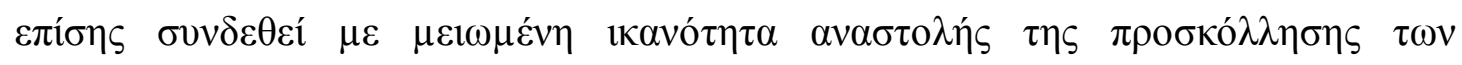

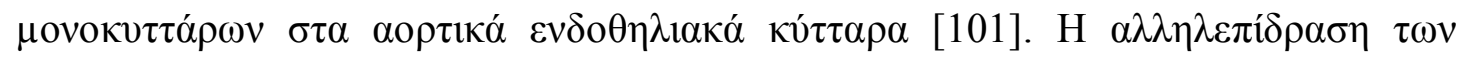

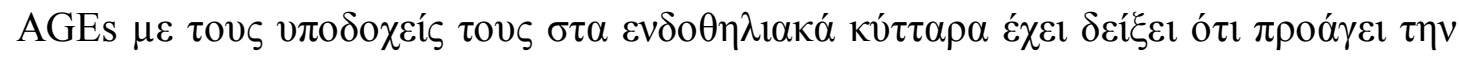

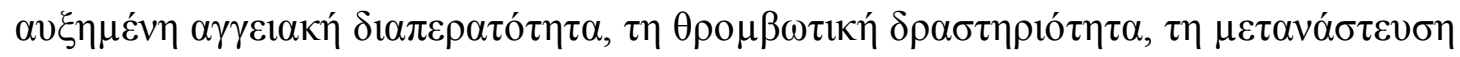

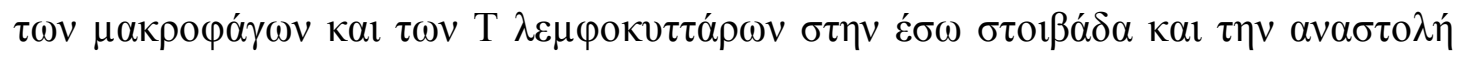

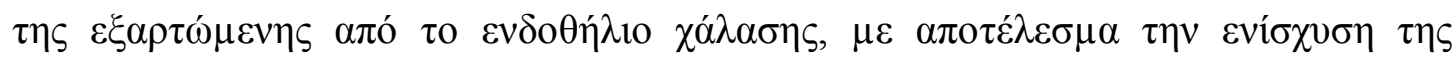
$\alpha \theta \eta \rho о \gamma \varepsilon ́ v \varepsilon \sigma \eta \varsigma$ [98].

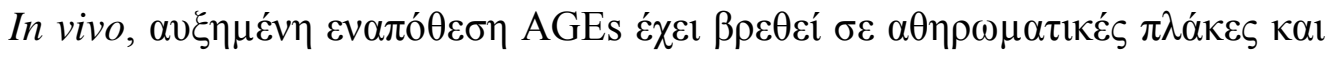

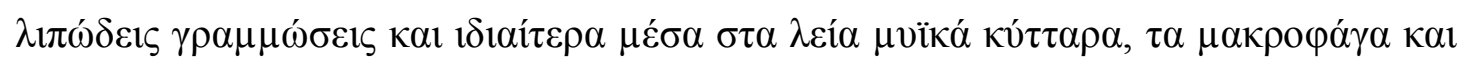

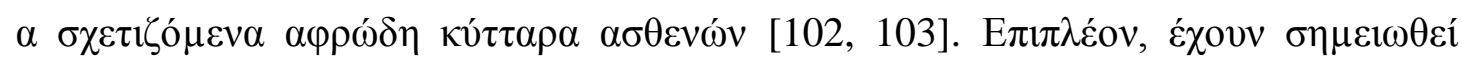

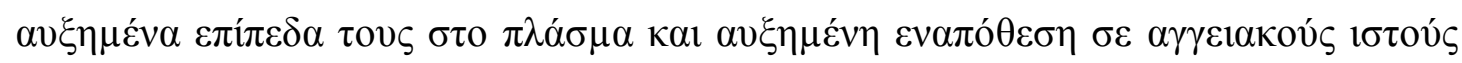

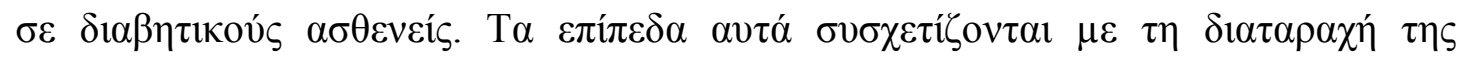

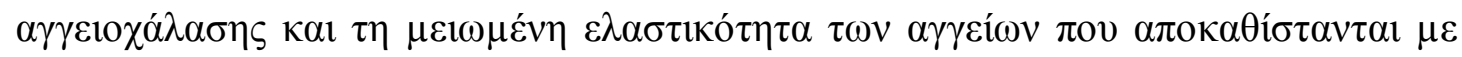

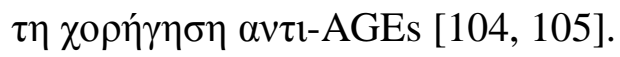

\section{$\underline{E v \delta o \theta \eta \lambda i v \eta-1}$}

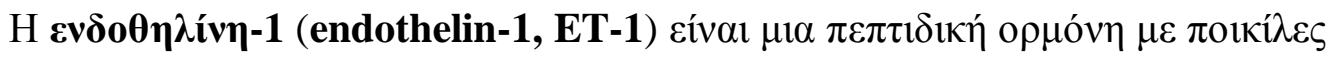

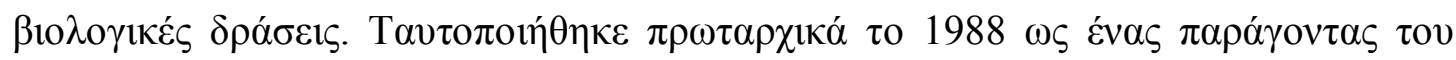

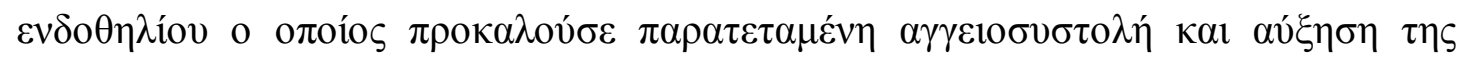

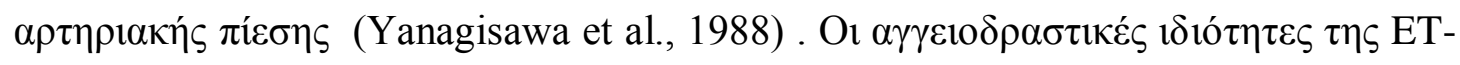

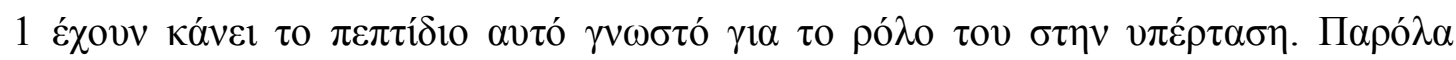

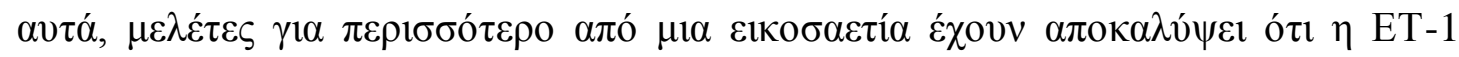

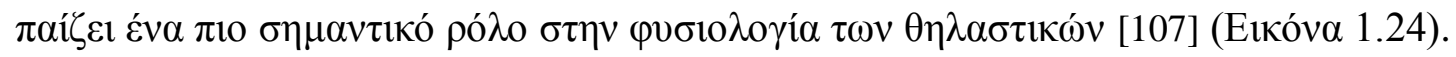

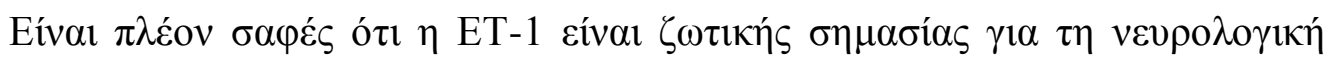

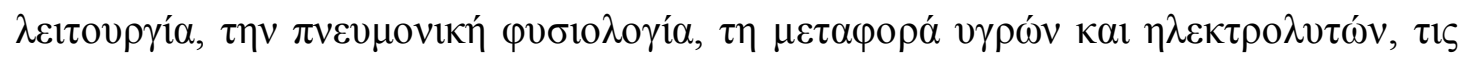

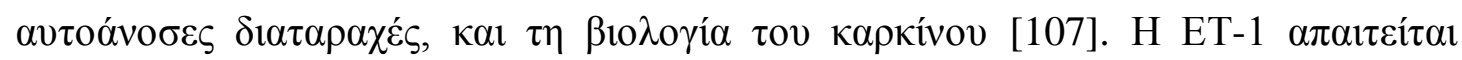




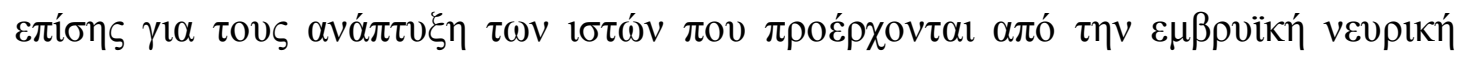

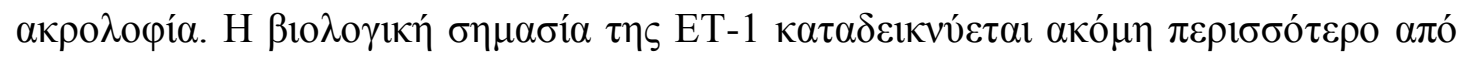

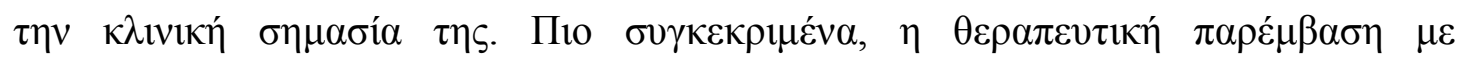

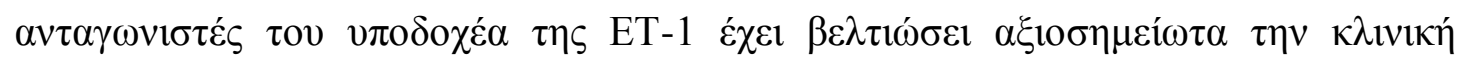

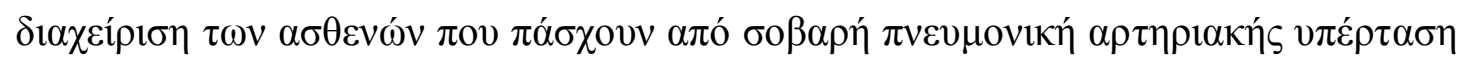

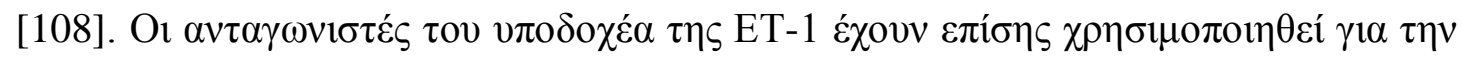

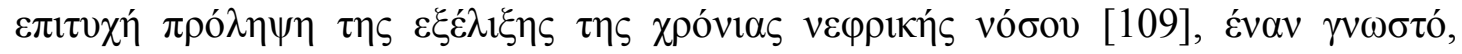

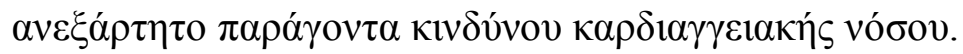

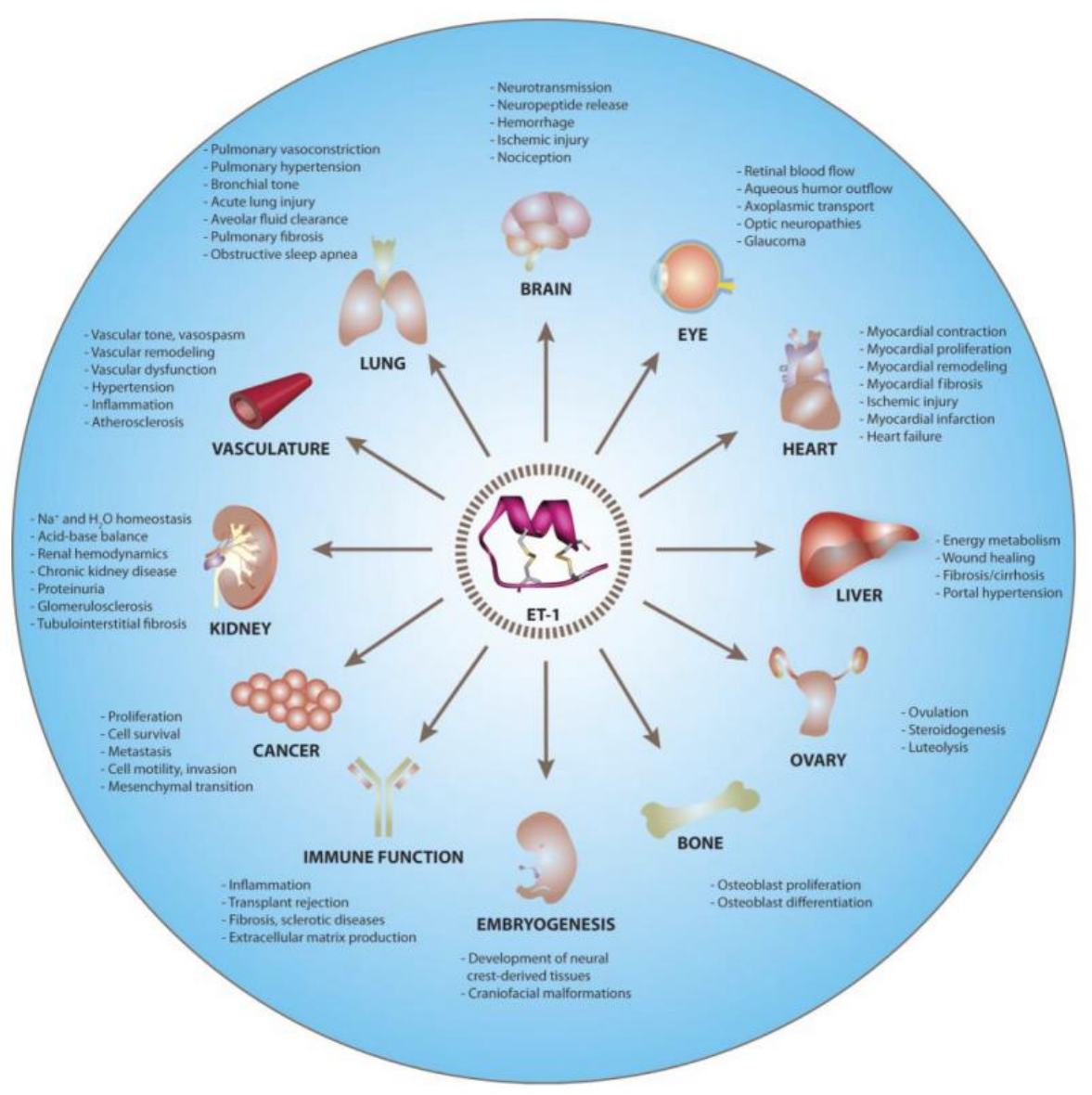

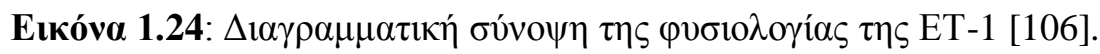

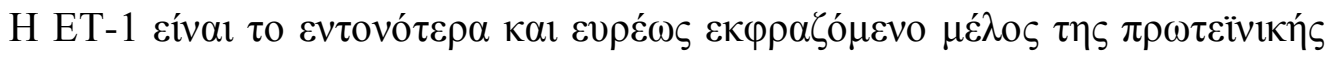

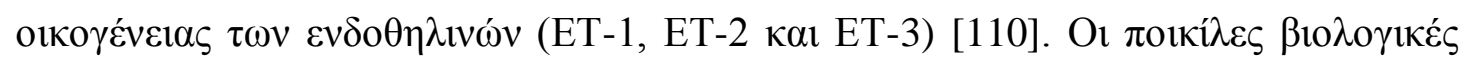

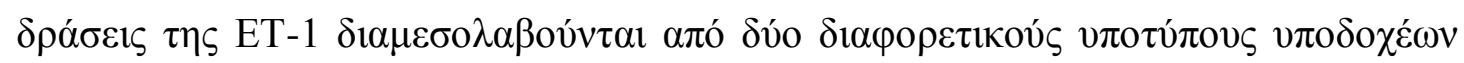

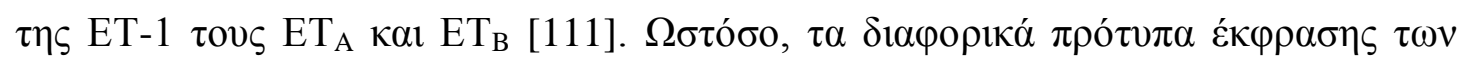




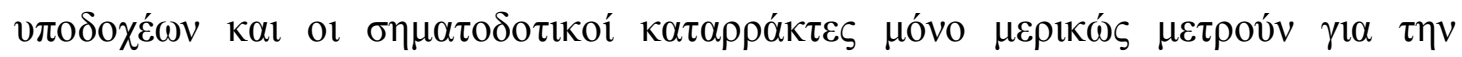

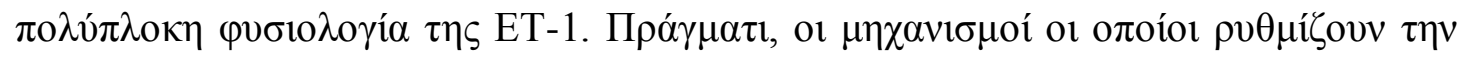

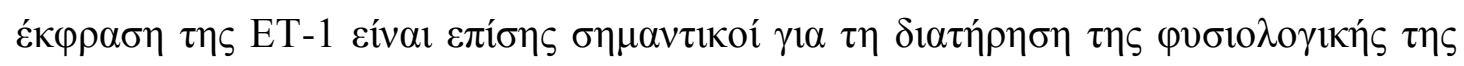

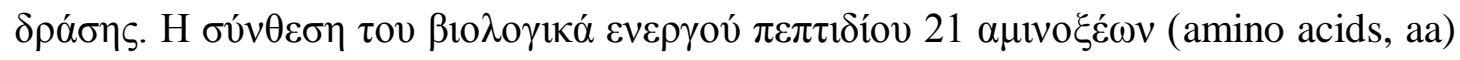

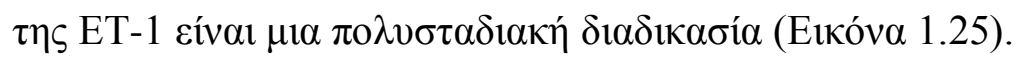

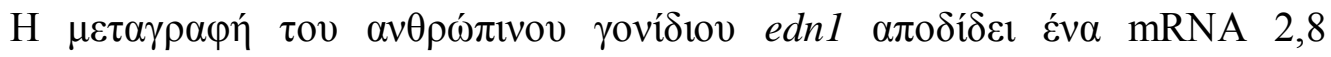

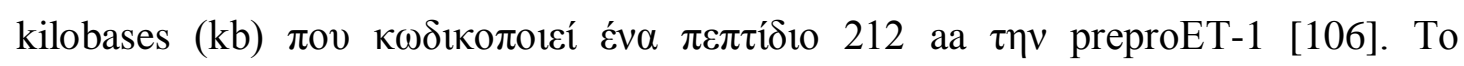

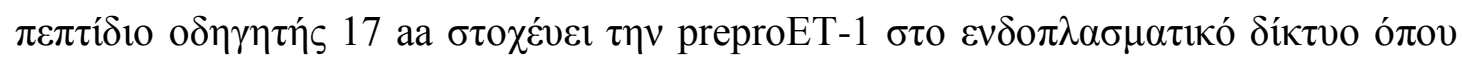

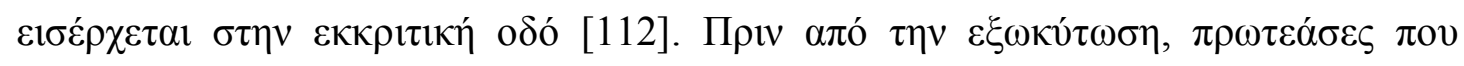

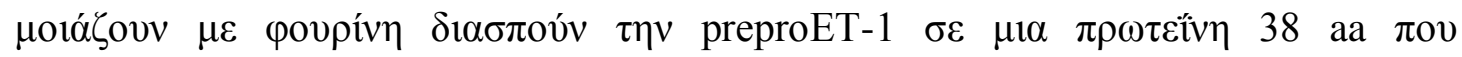

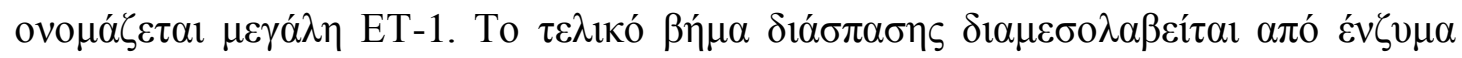

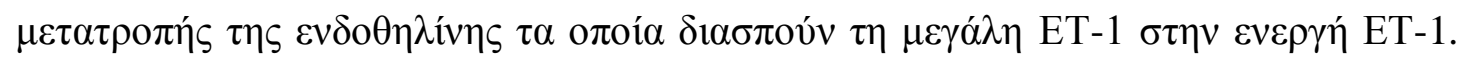

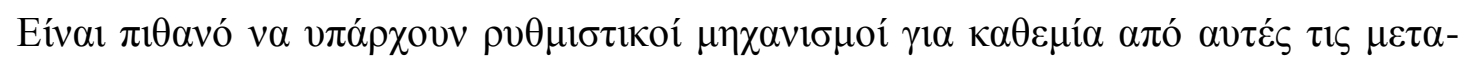

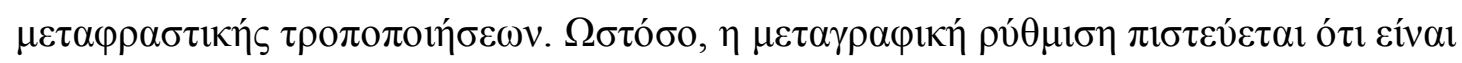

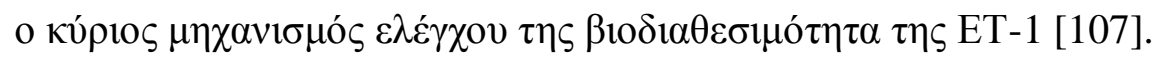

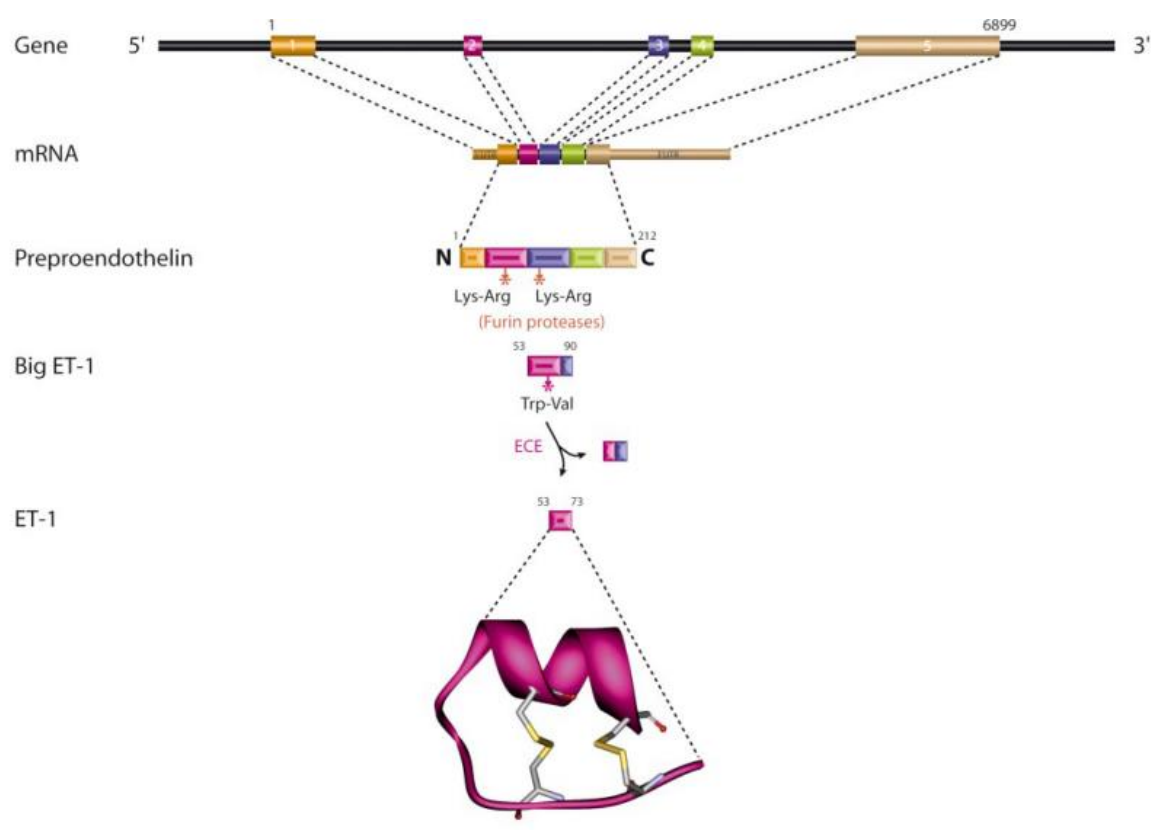

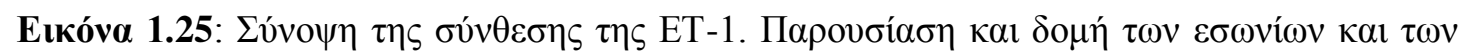

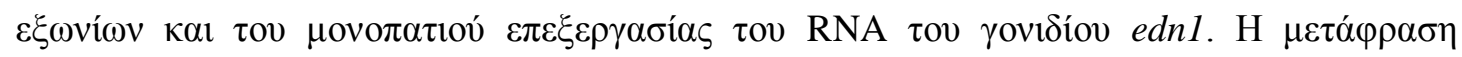

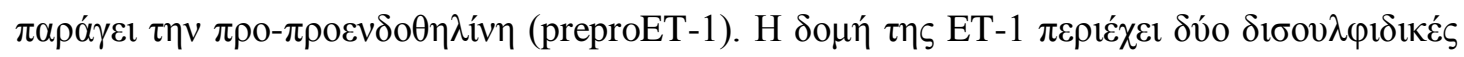

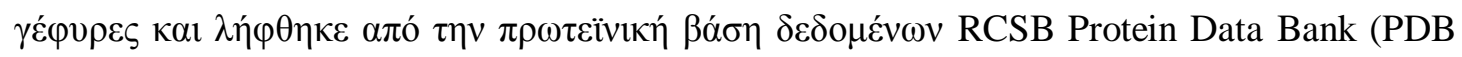
1T7H) [107]. 


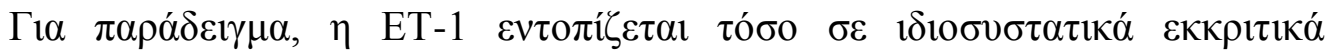

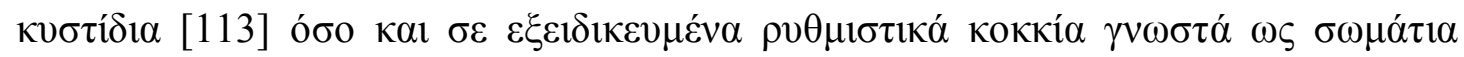

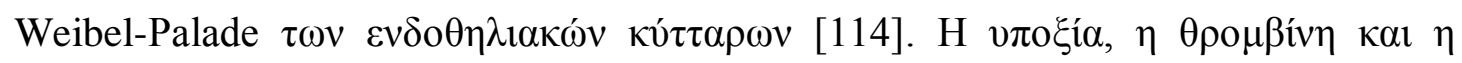

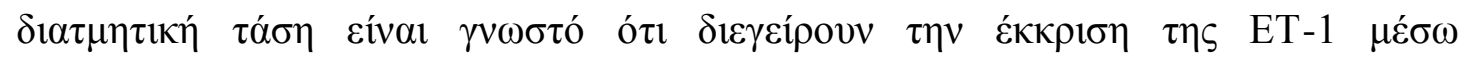

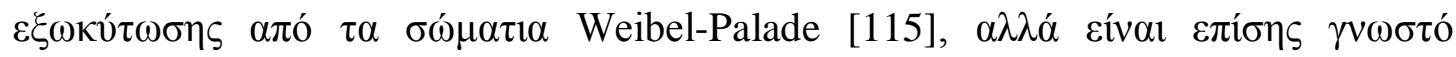

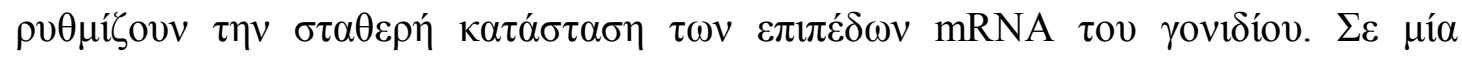

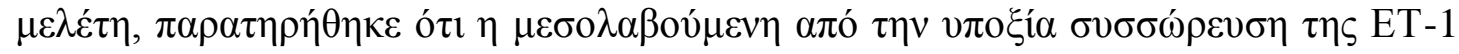

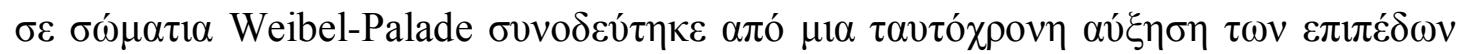

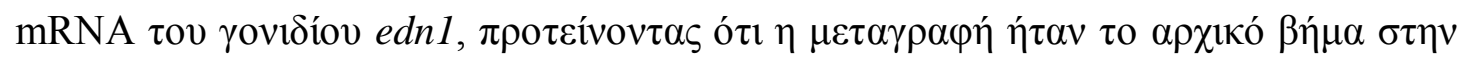

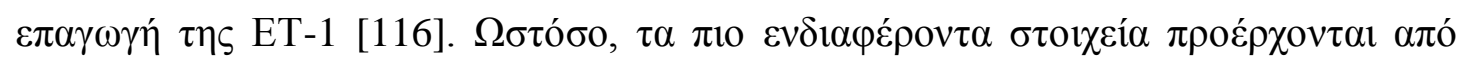

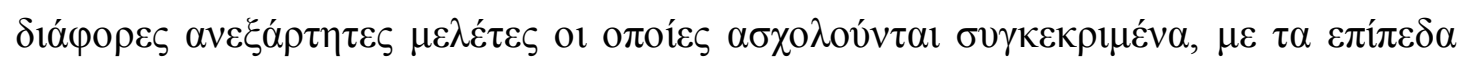

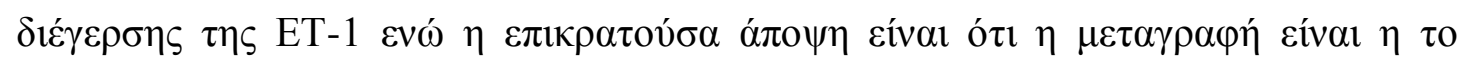

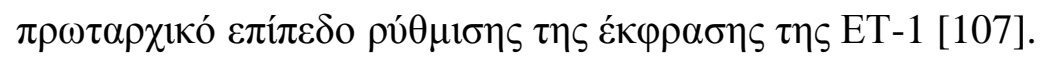

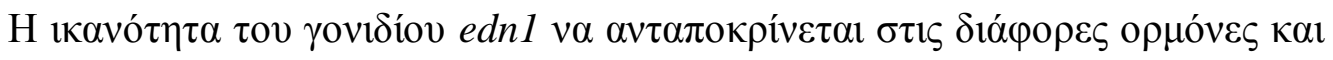

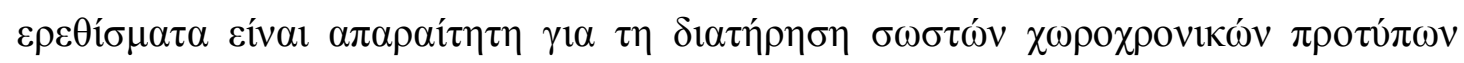

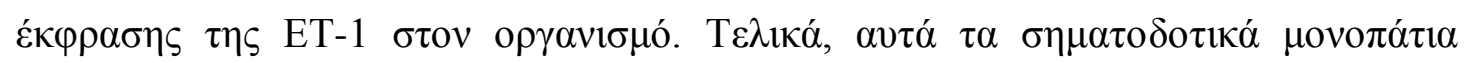

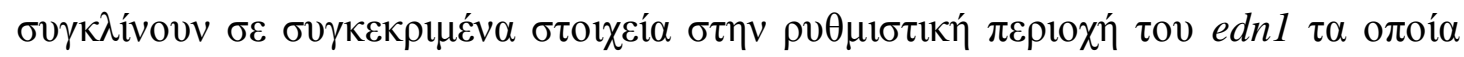

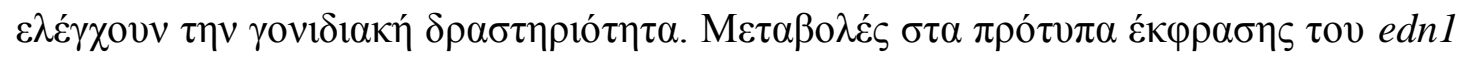

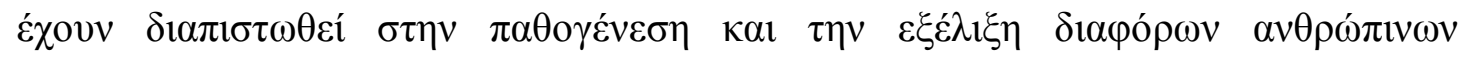

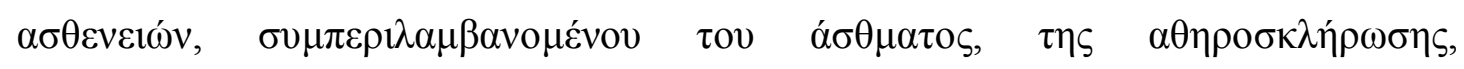

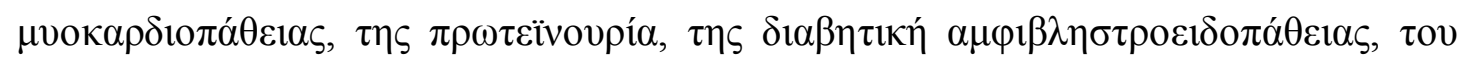

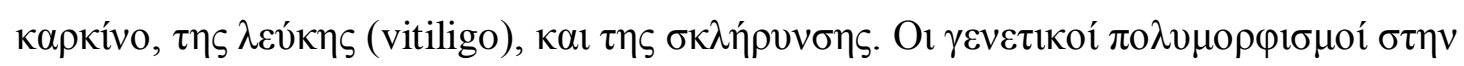

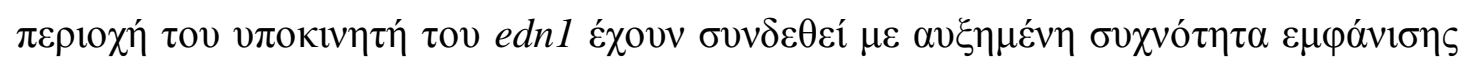

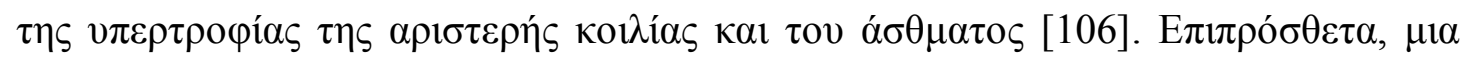

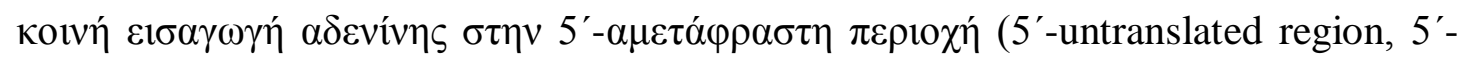

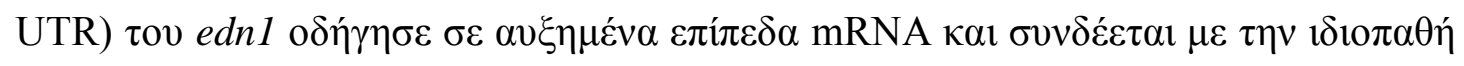

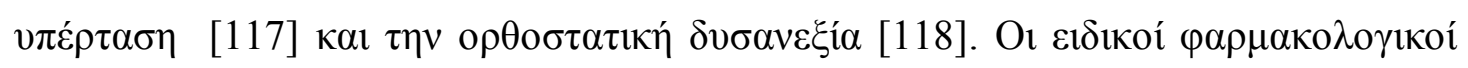

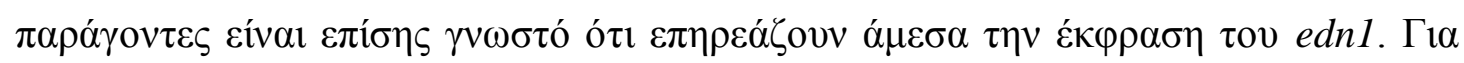

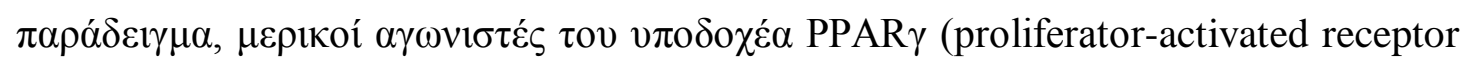

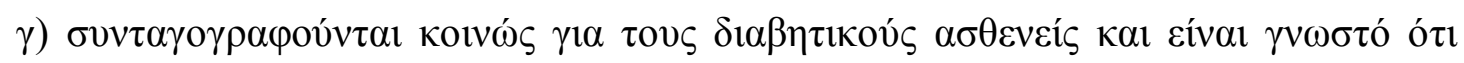

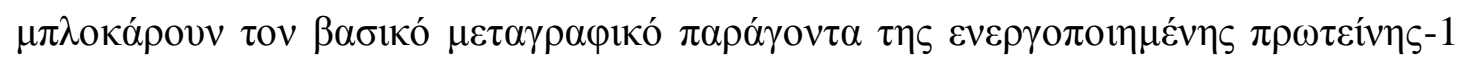

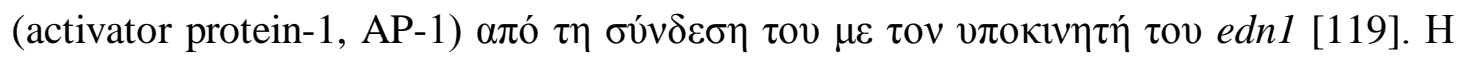




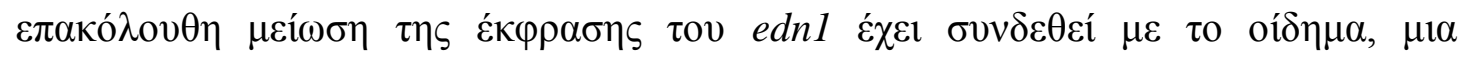

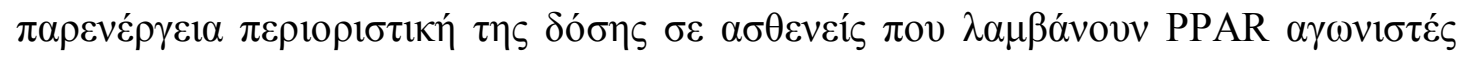

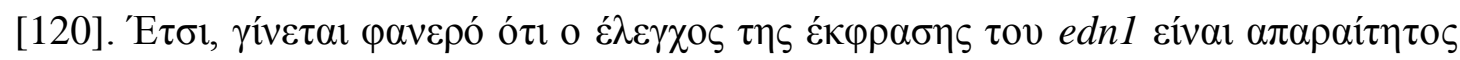

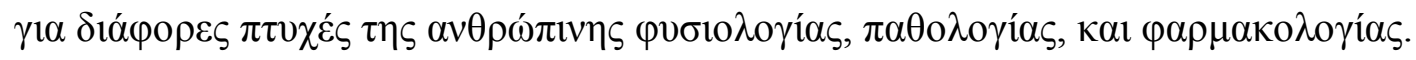

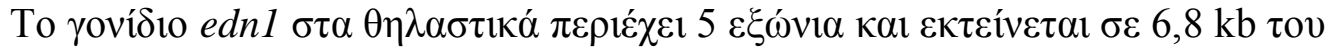

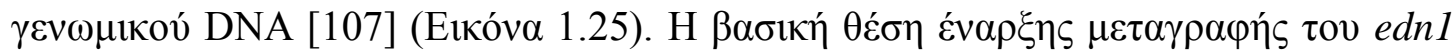

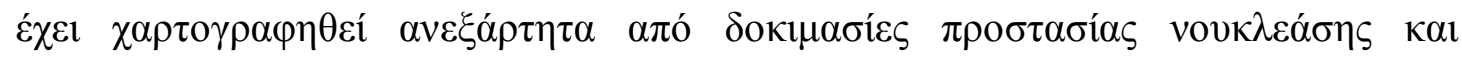

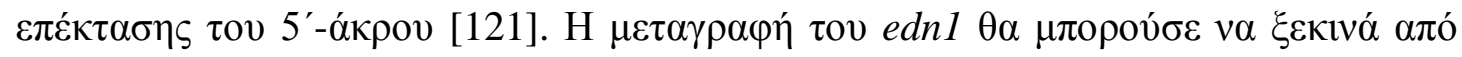

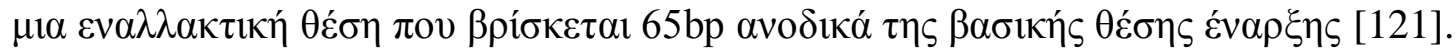

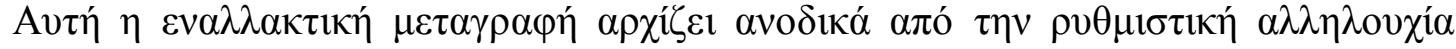

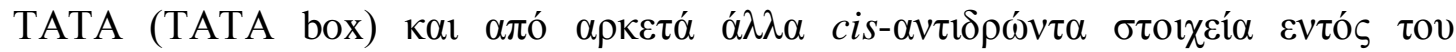

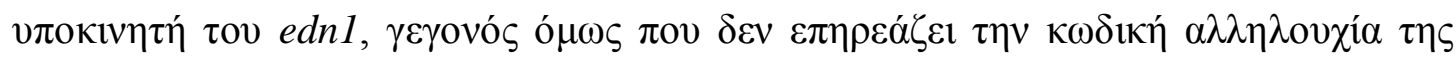

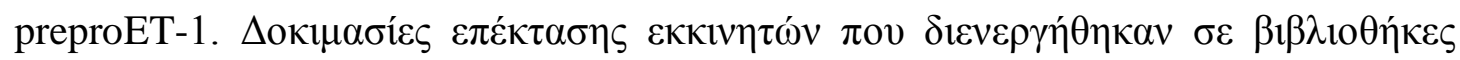

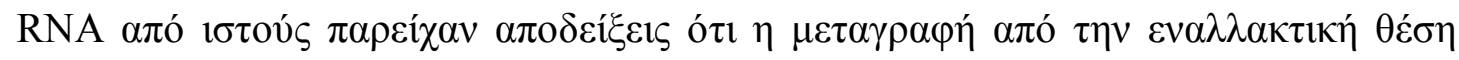

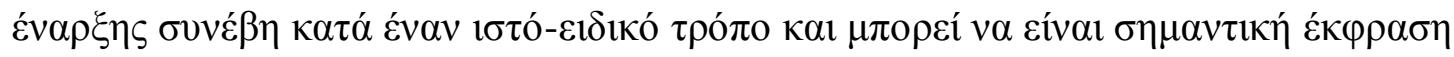

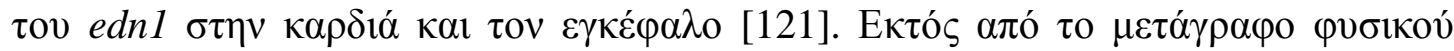

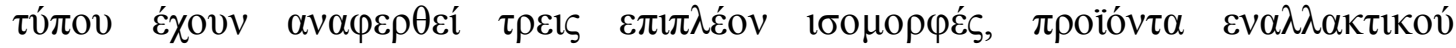

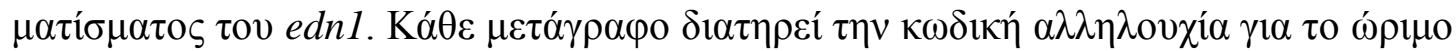

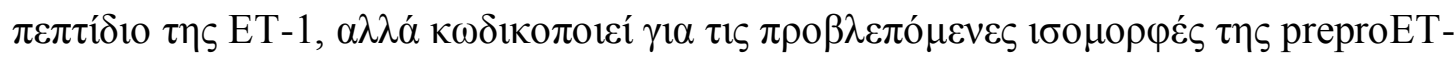

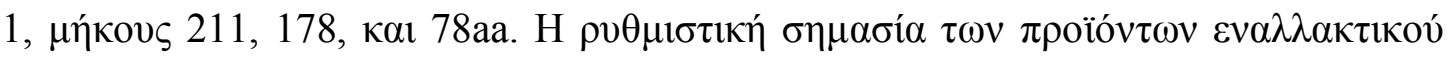

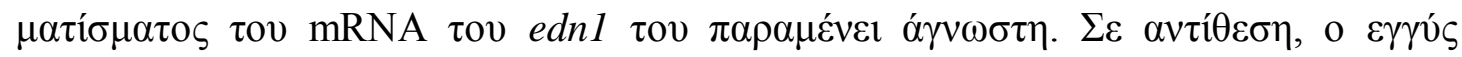

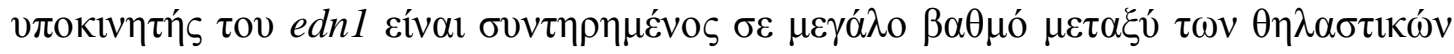

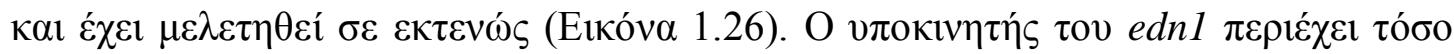

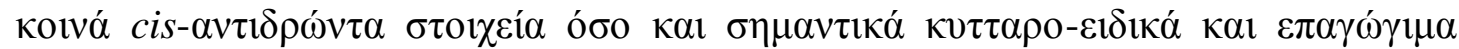

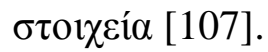

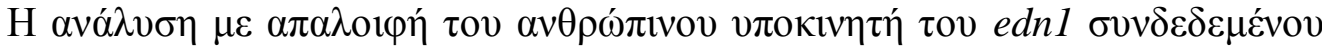

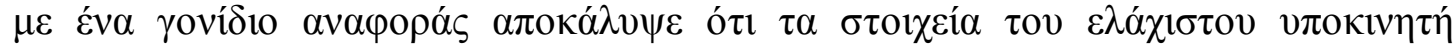

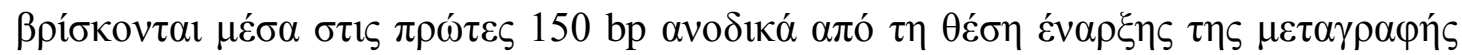

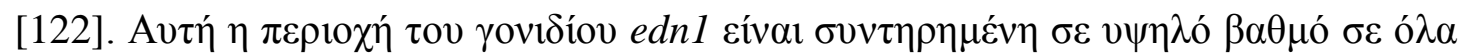

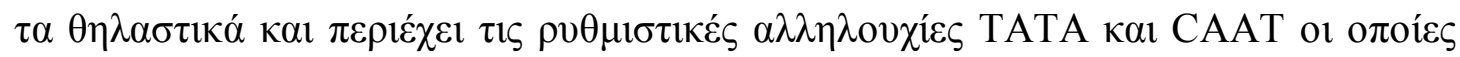

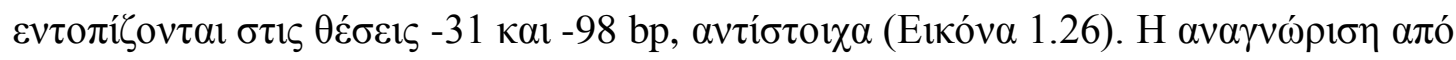

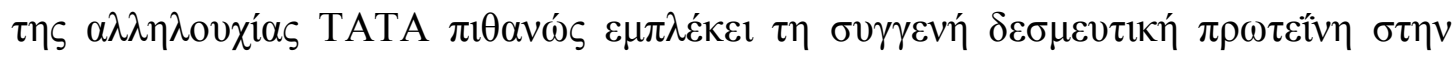




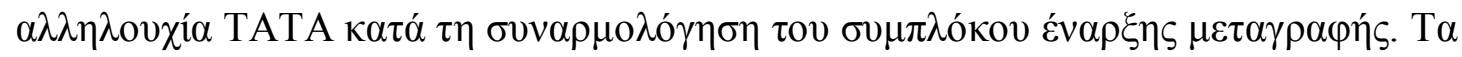

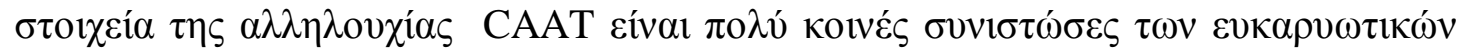

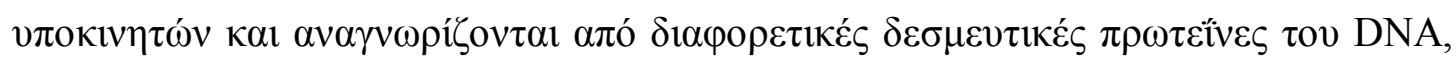

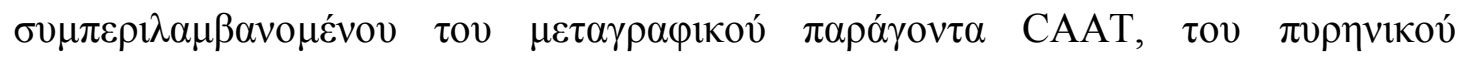

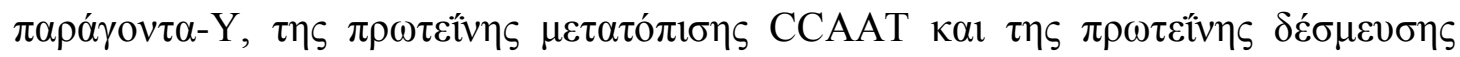

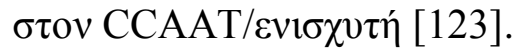

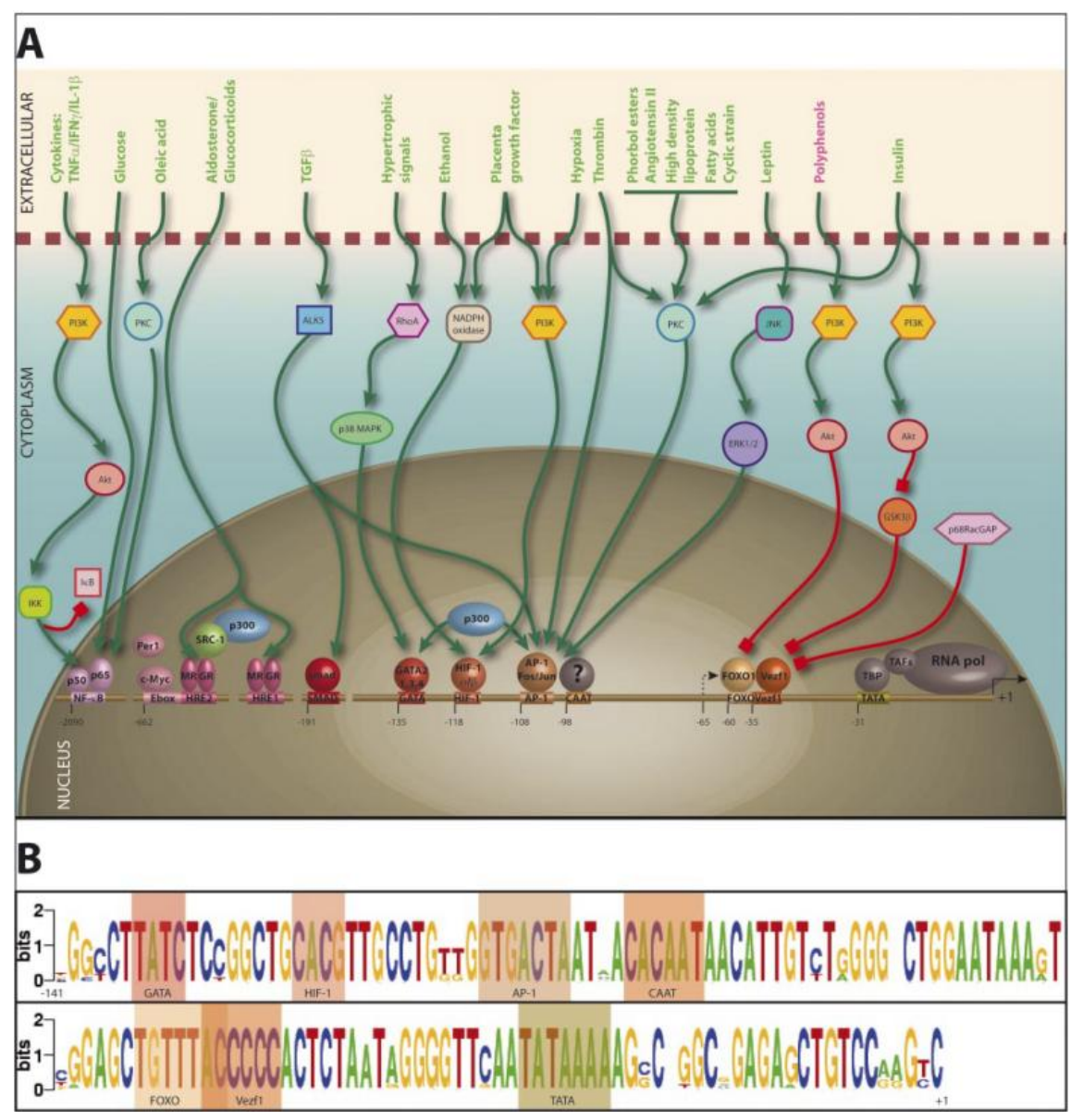

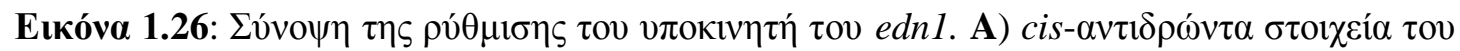

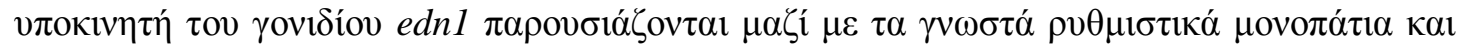

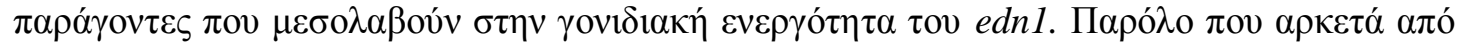

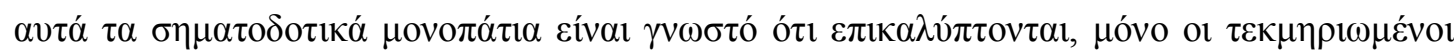

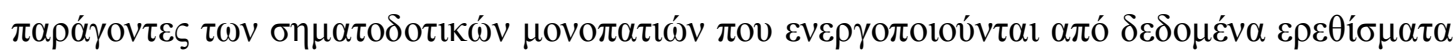

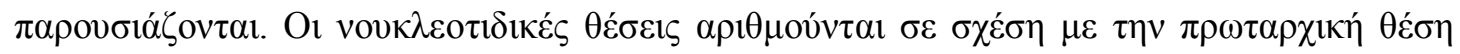

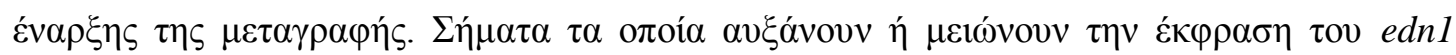

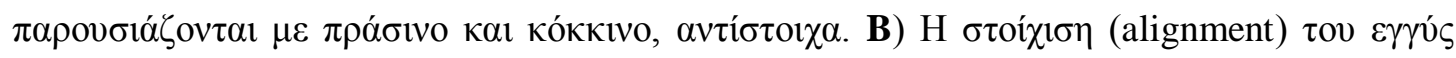

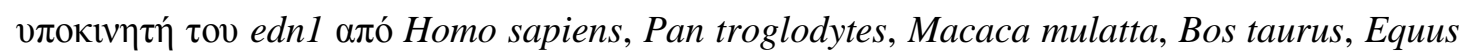
caballus, Canis familiaris, Sus scrofa, Rattus norvegicus кal Mus musculus [107]. 


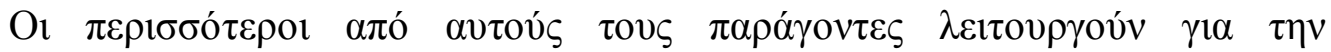

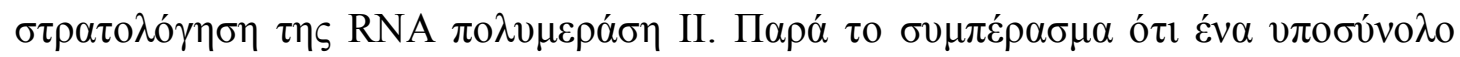

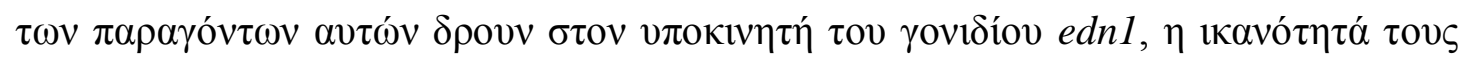

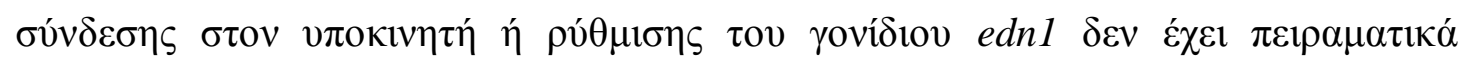

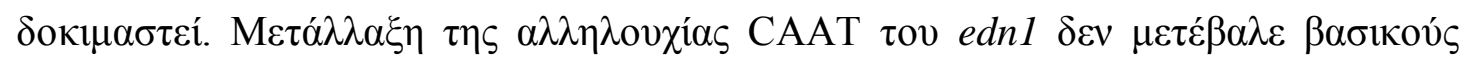

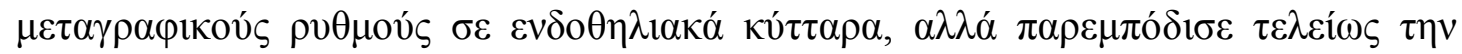

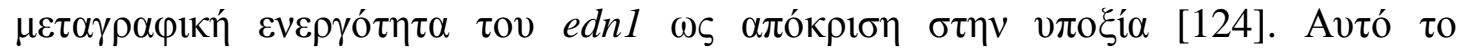

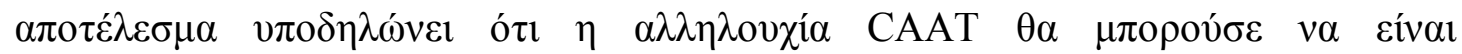

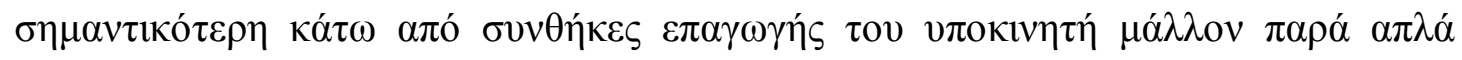

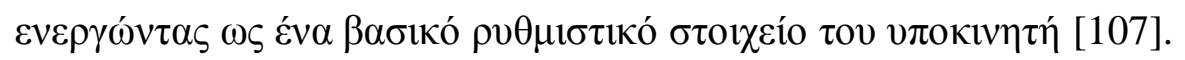

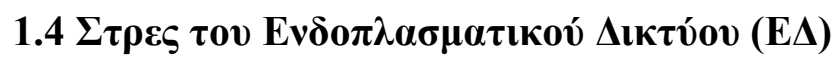

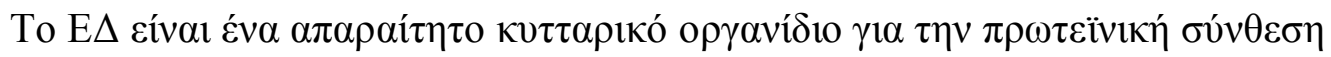

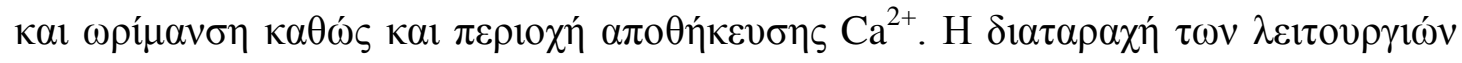

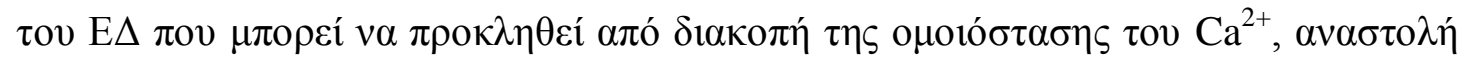

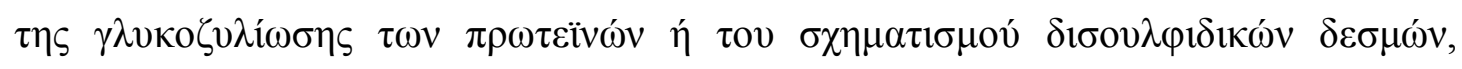

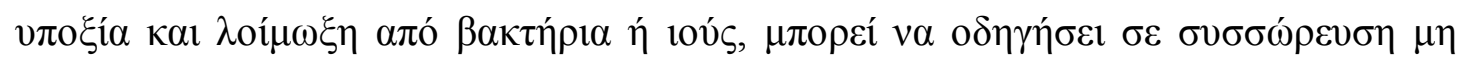

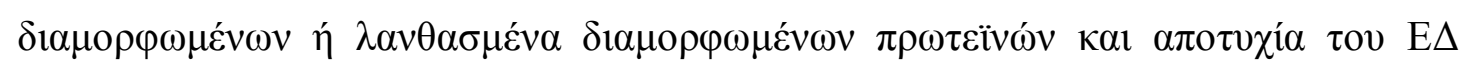

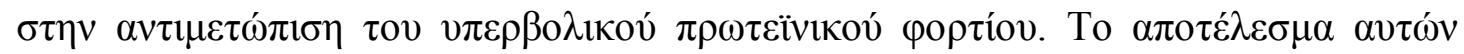

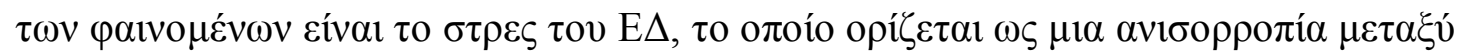

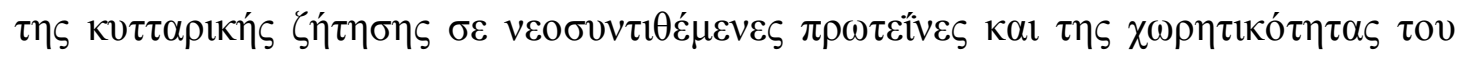

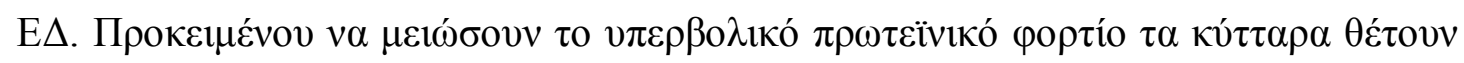

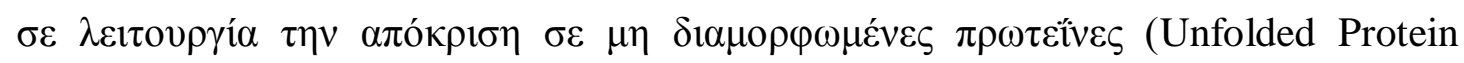

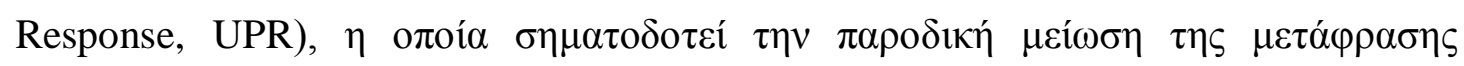

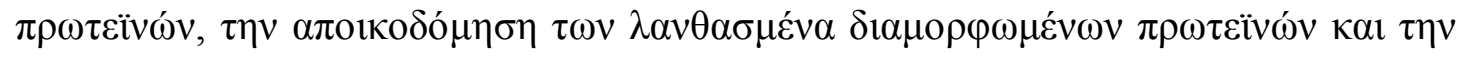

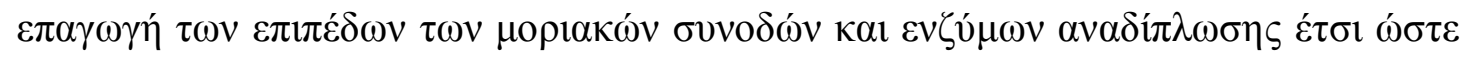

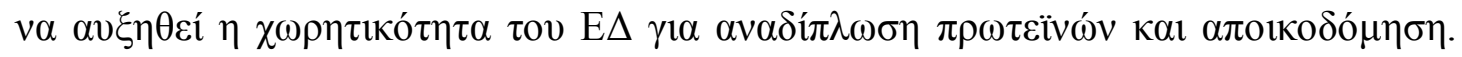

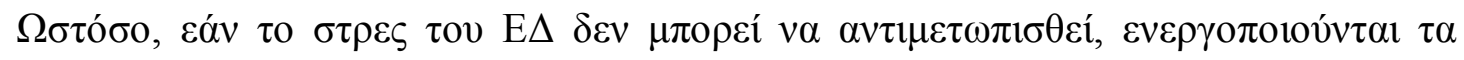

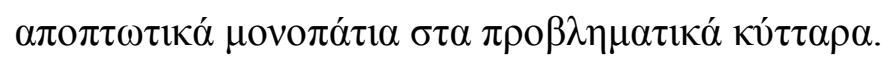




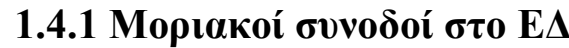

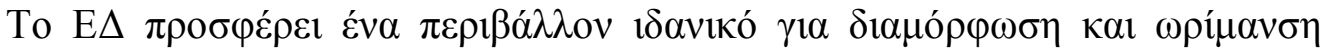

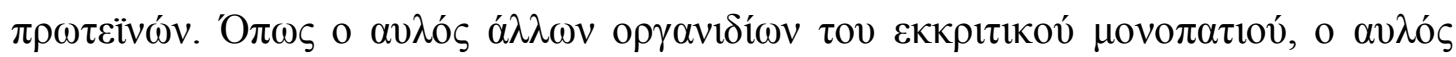

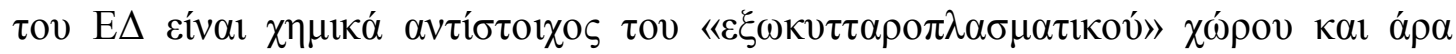

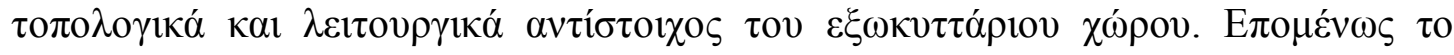

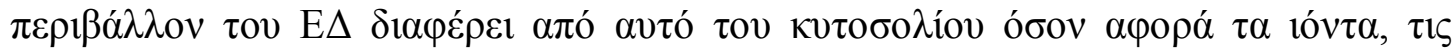

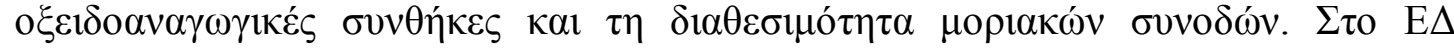

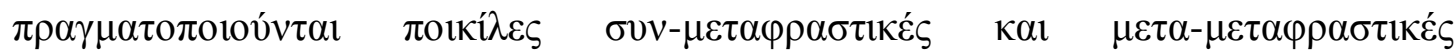

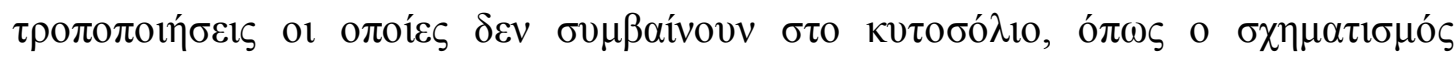

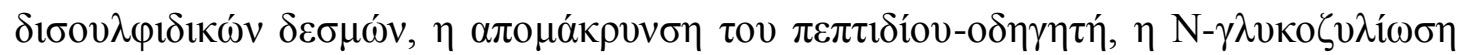

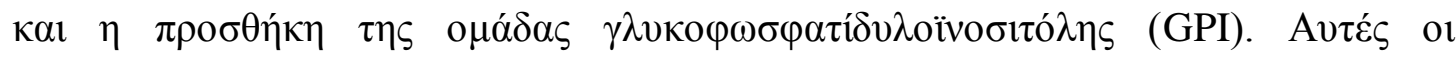

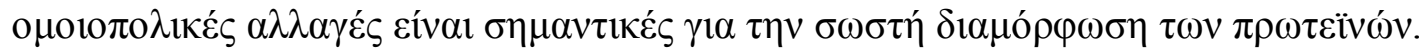

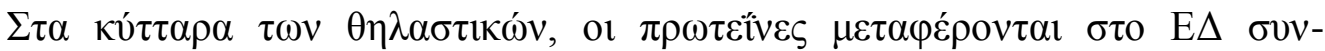

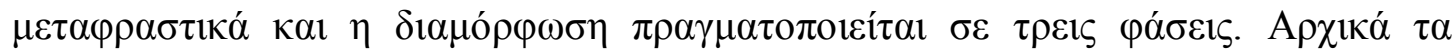

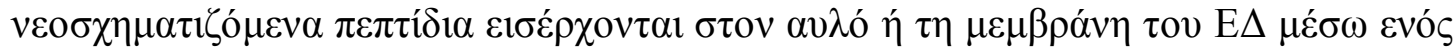

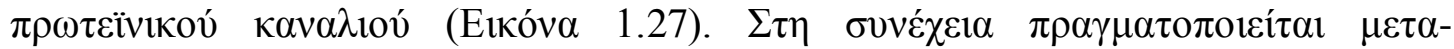

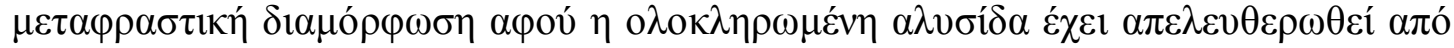

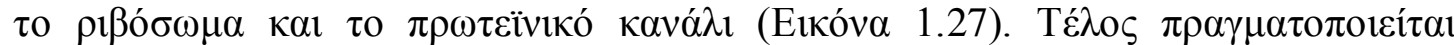

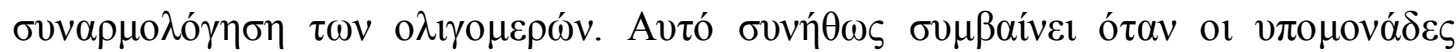

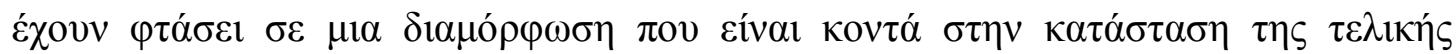

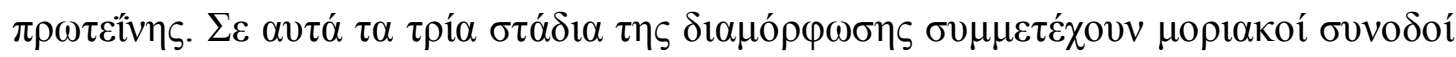

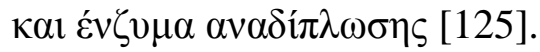

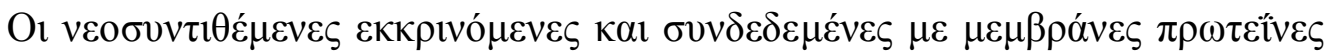

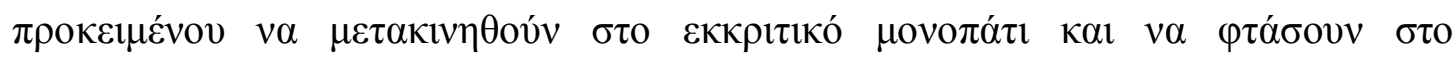

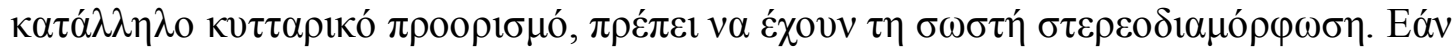

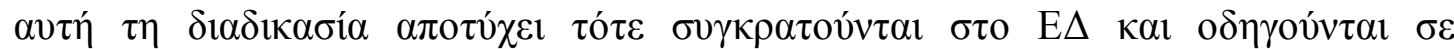

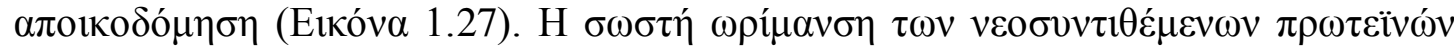

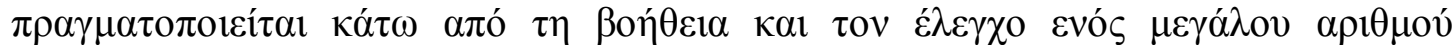

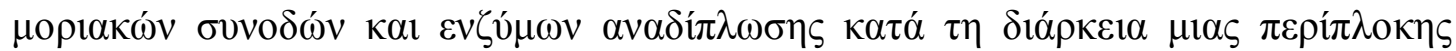

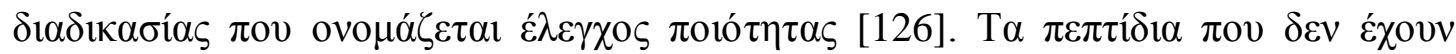

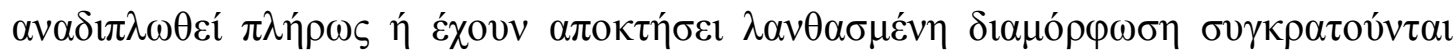




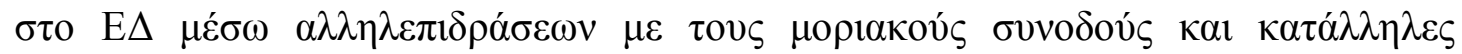

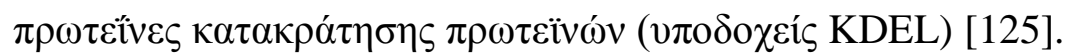

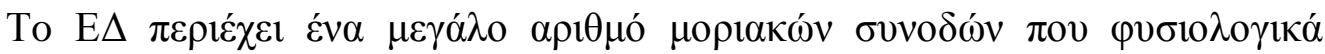

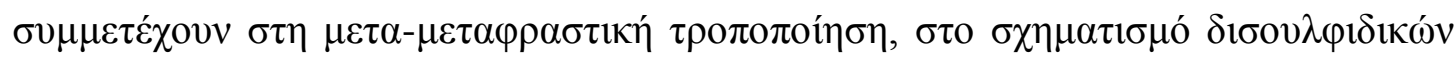

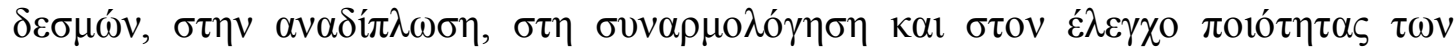

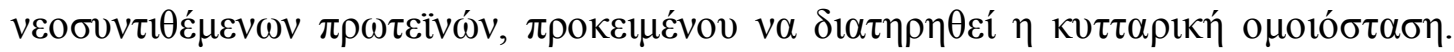

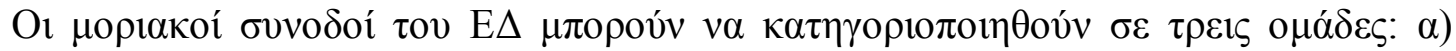

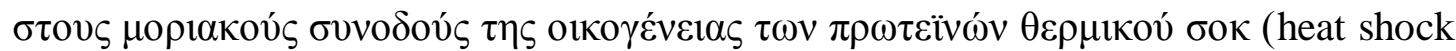

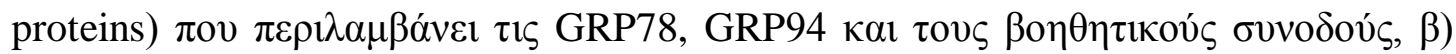

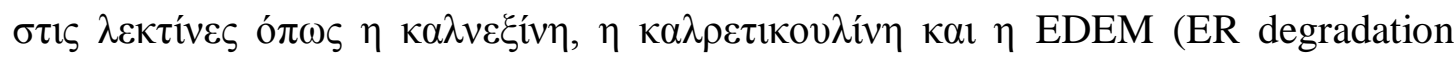

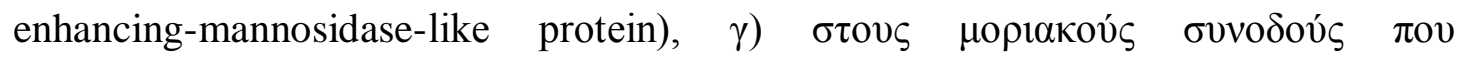

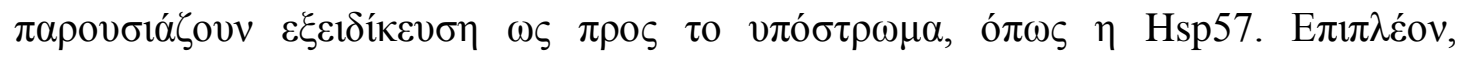

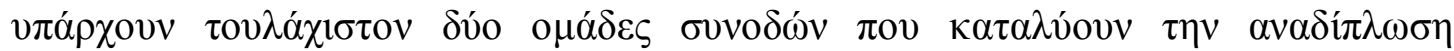

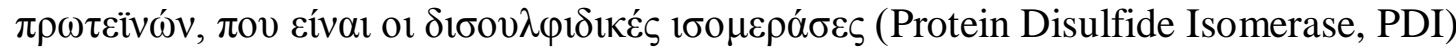
$\kappa \alpha \imath$ or $\pi \varepsilon \pi \tau \imath \delta \nu \lambda-\pi \rho \circ \lambda v \lambda-1 \sigma o \mu \varepsilon \rho \alpha ́ \sigma \varepsilon \varsigma$ (Peptidyl Prolyl Isomerases, PPIs). $\Sigma \tau$ E E $\Delta$

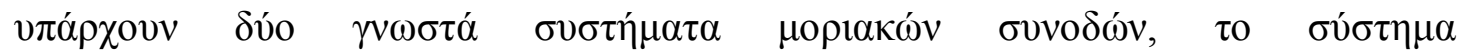

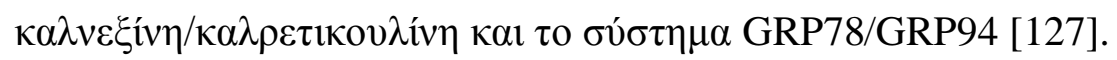

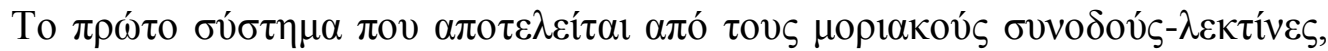

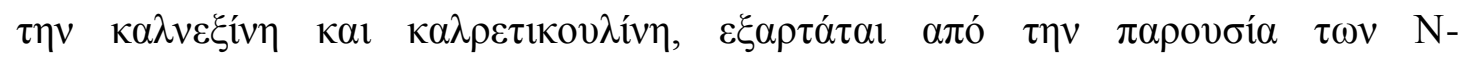

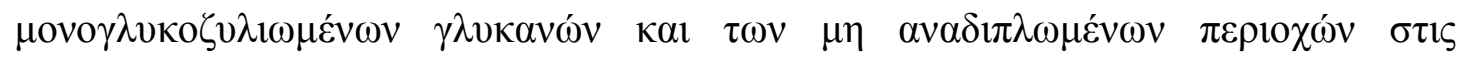

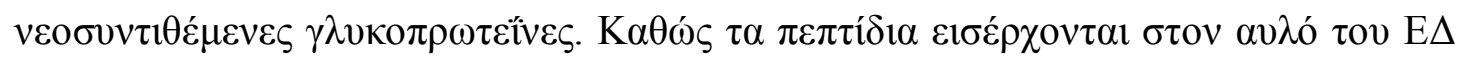

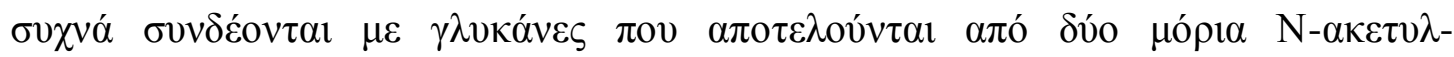

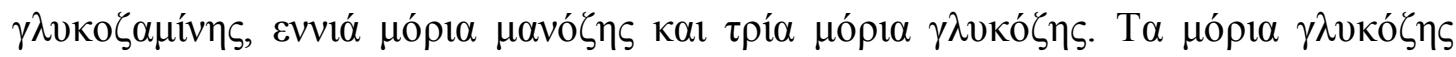

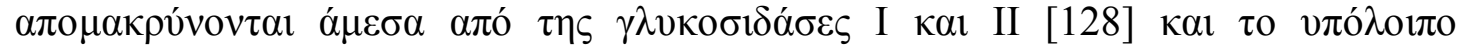

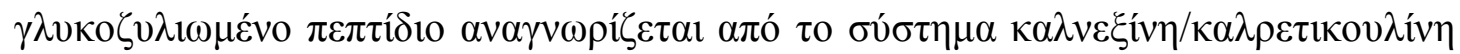

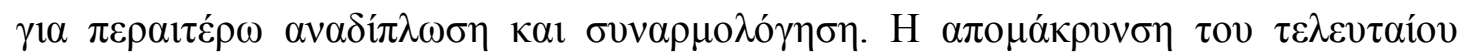

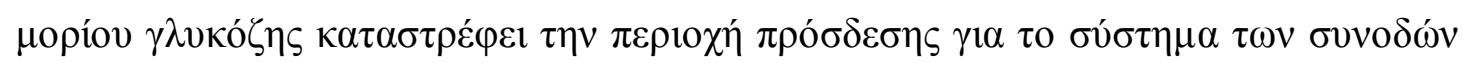

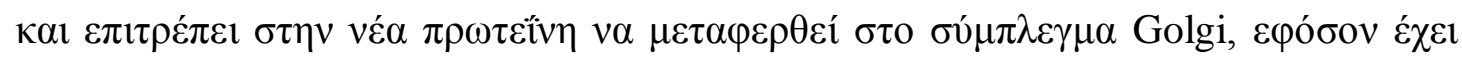

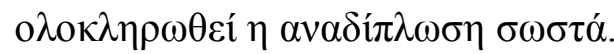

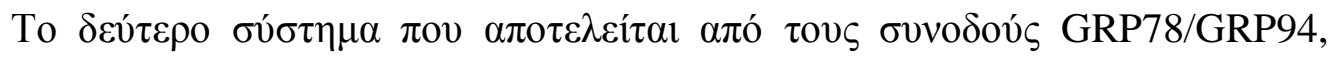

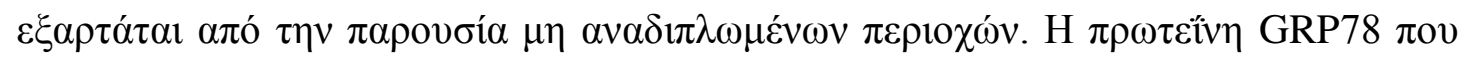

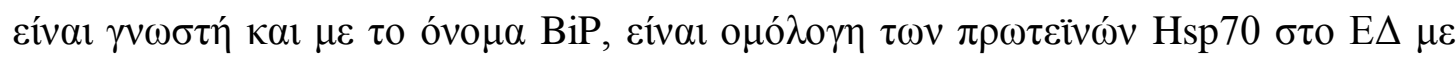




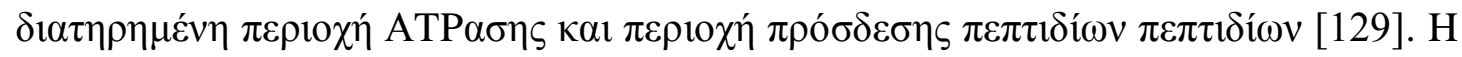

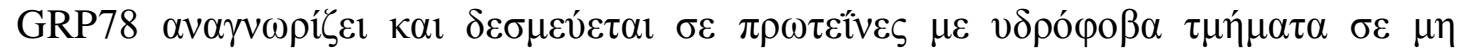

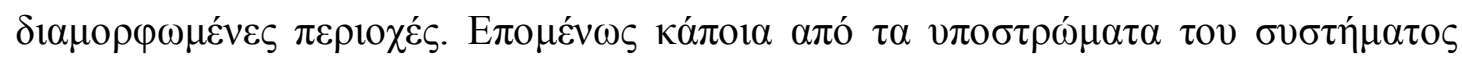

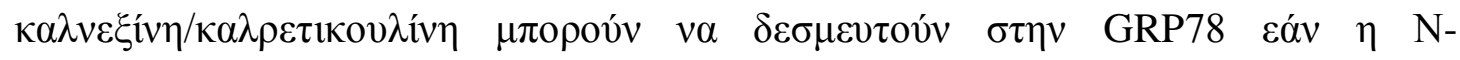

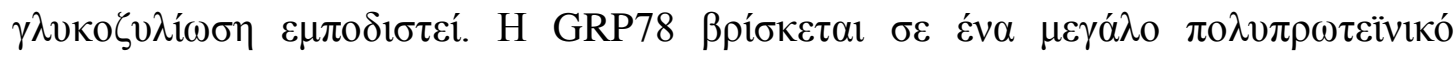

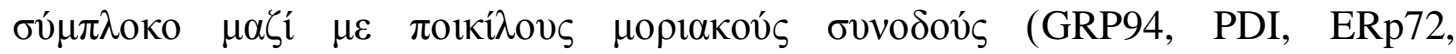

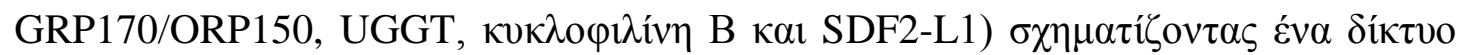

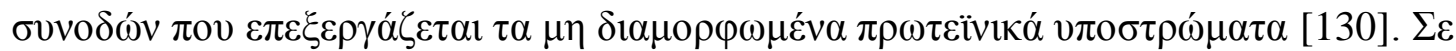

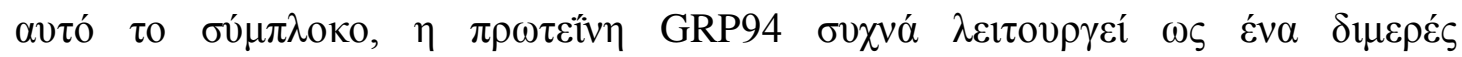

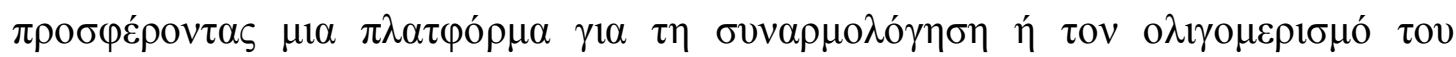

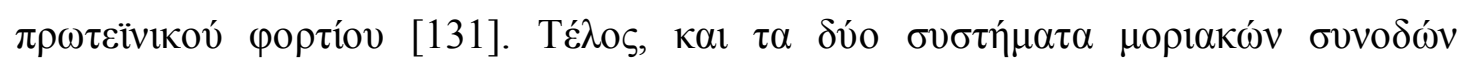

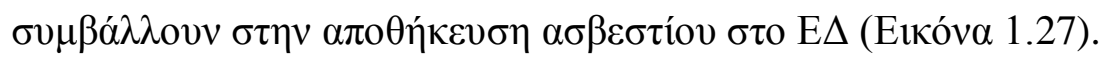

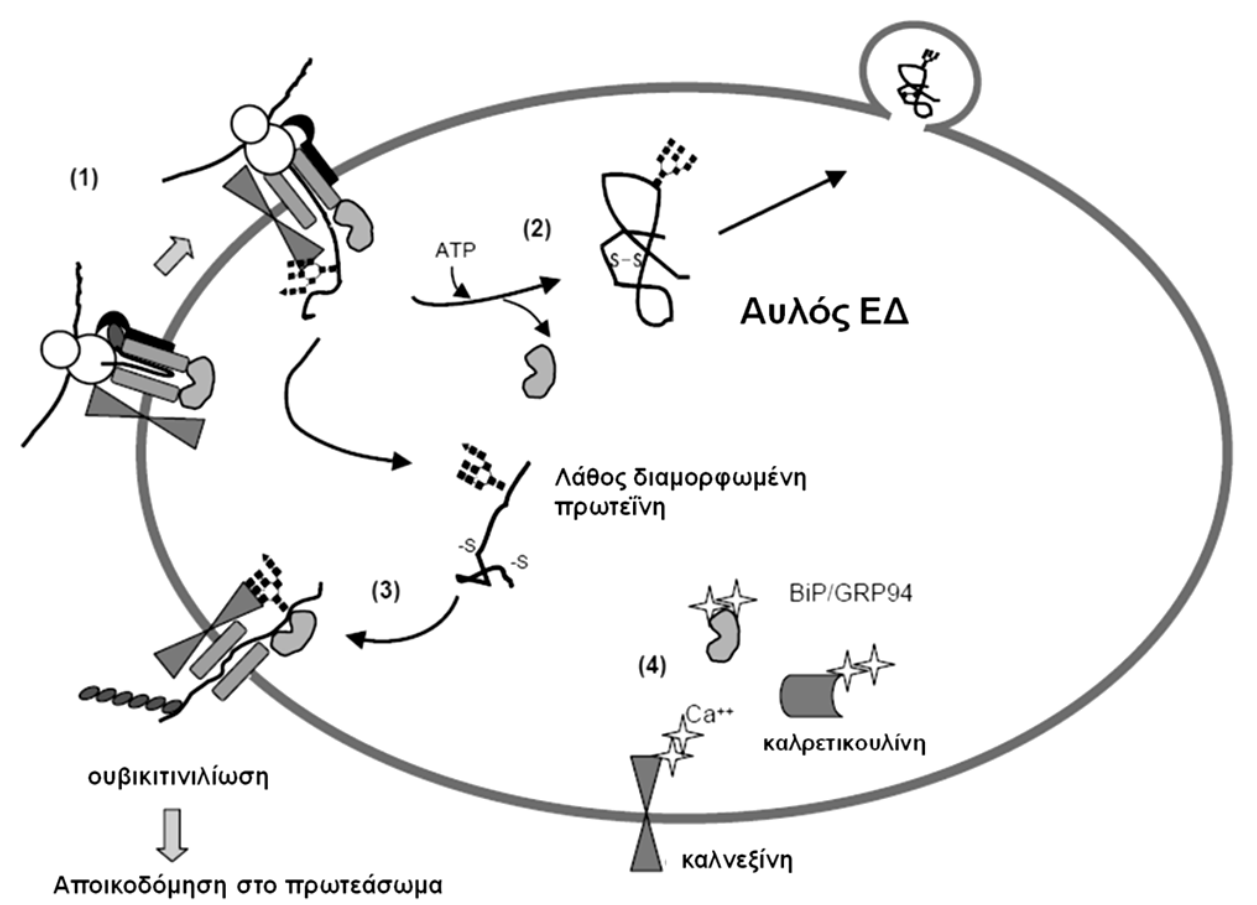

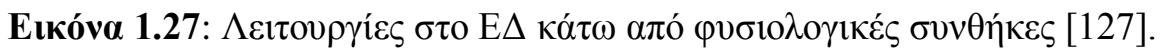

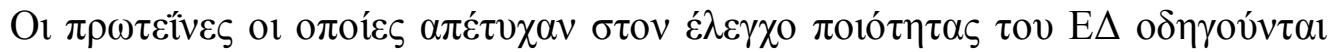

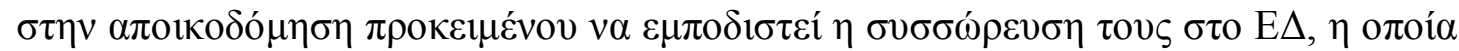

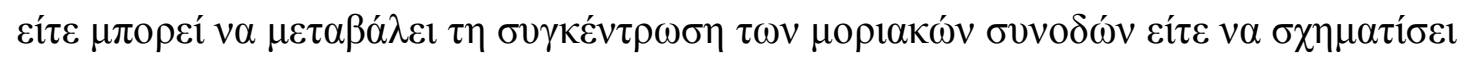

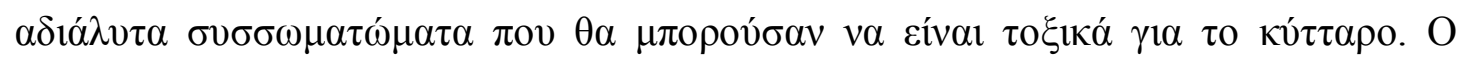

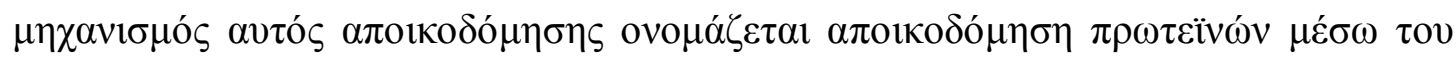

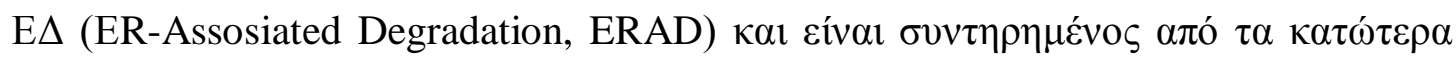




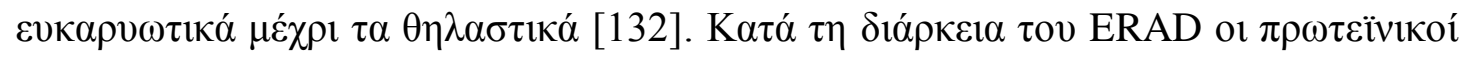

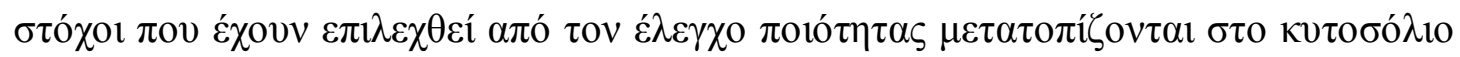

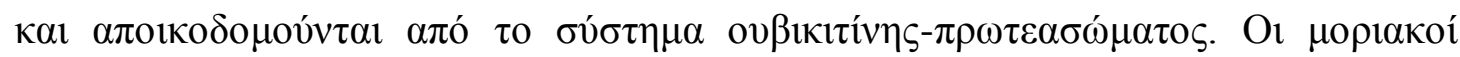

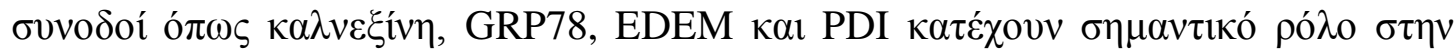

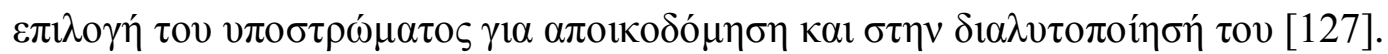

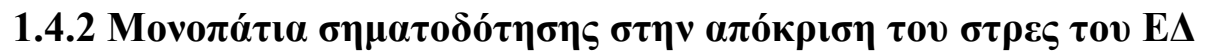

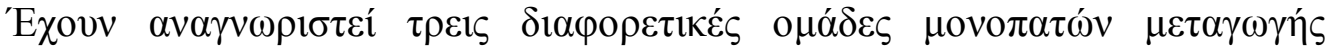

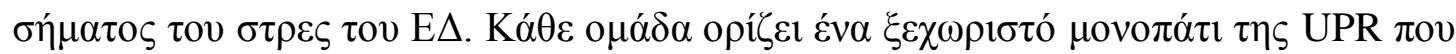

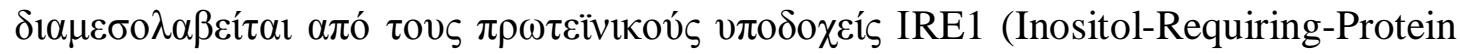
1), ATF6 (Activating Transcription Factor-6) кa PERK (protein kinase RNA, PKR-

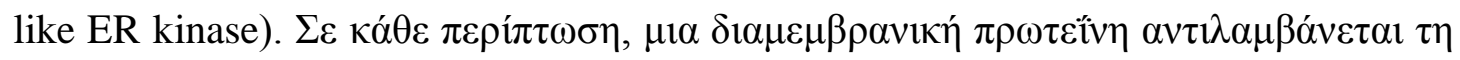

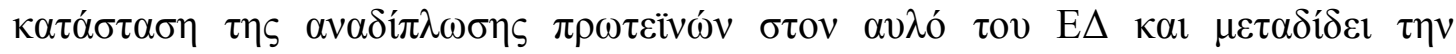

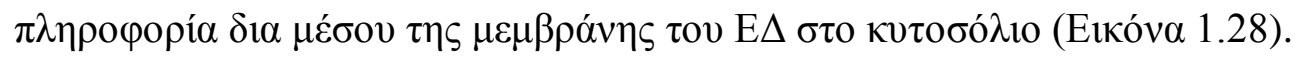

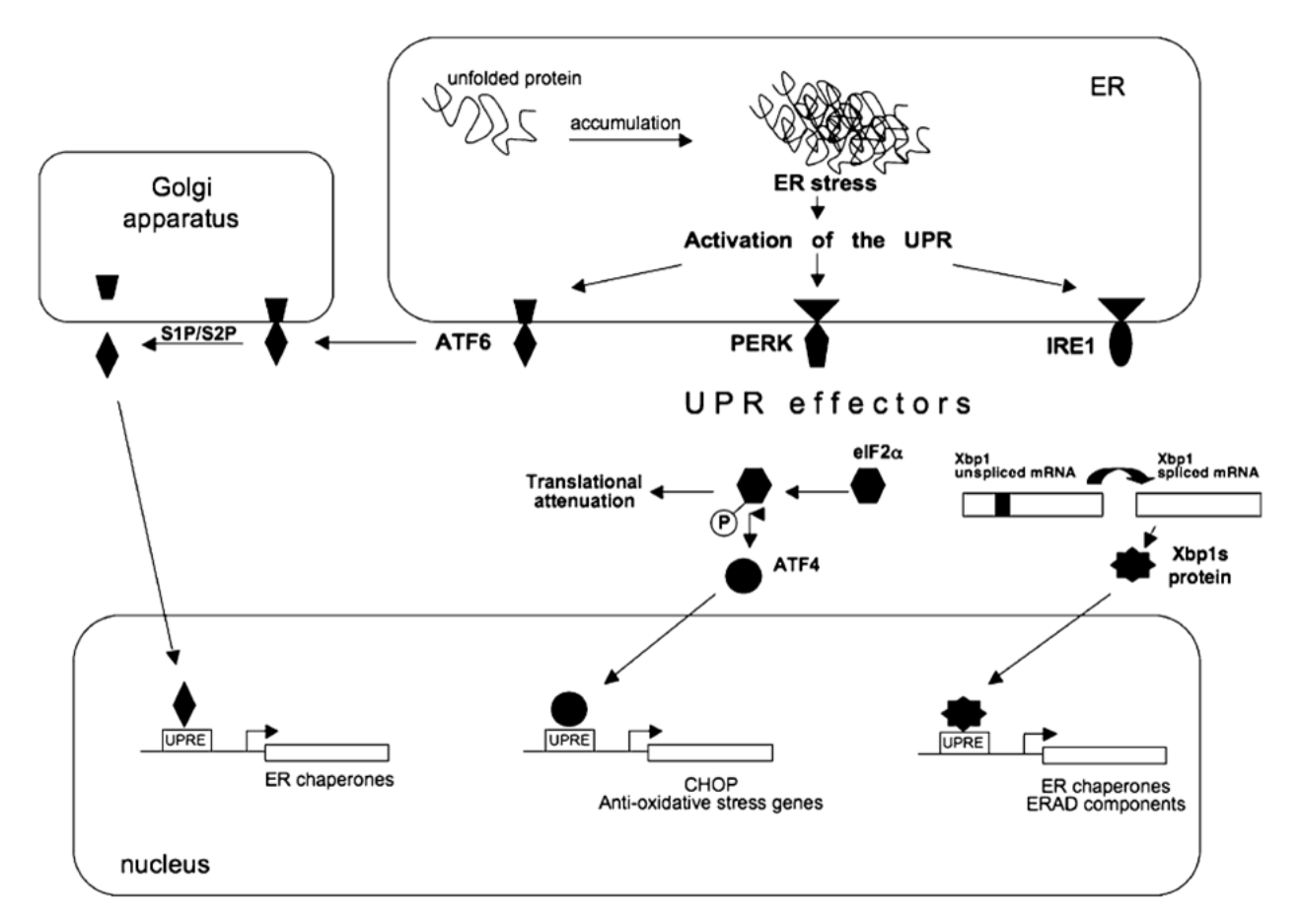

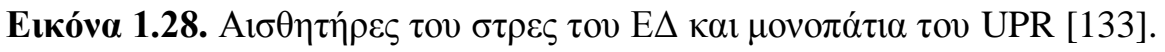




\section{IRE1}

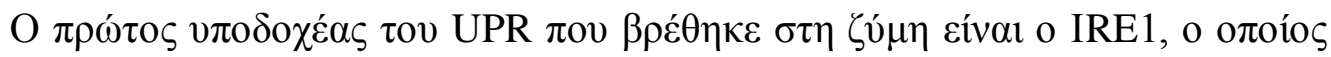

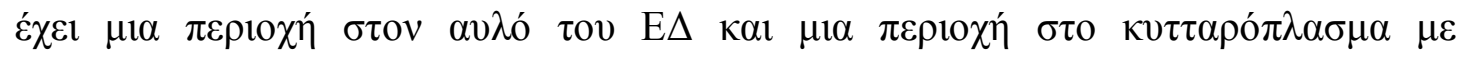

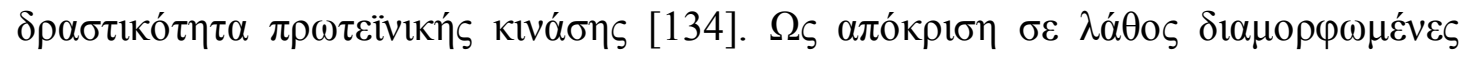

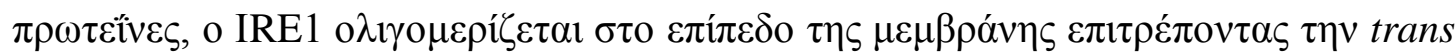

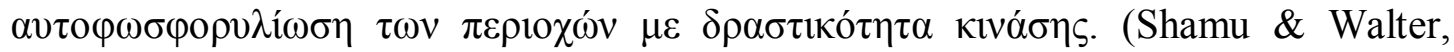
1996).

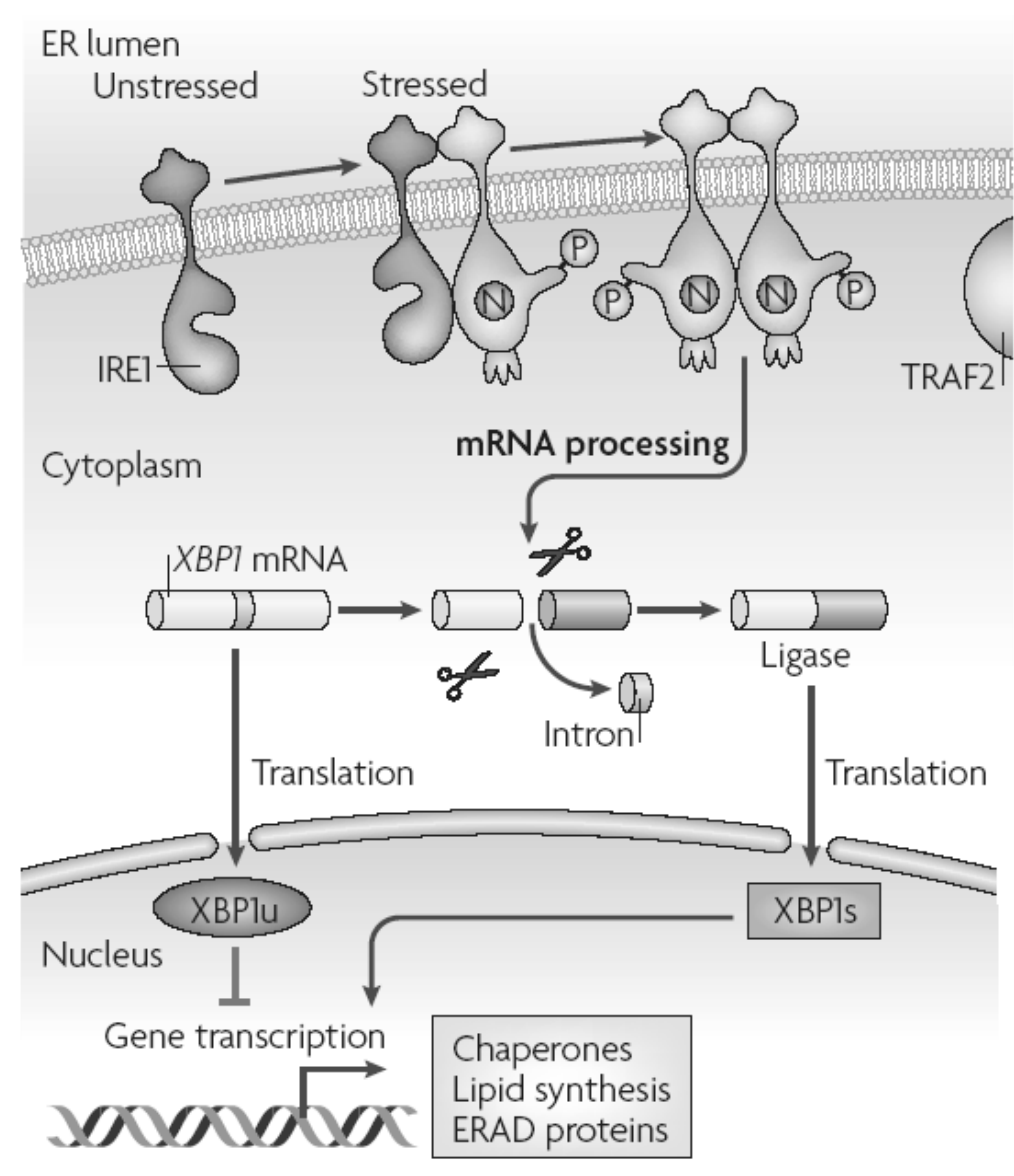

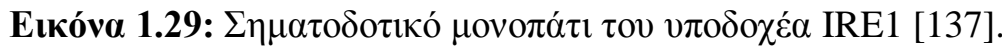

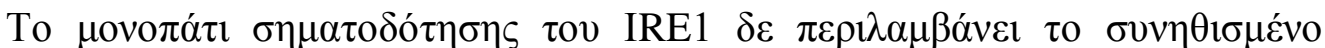

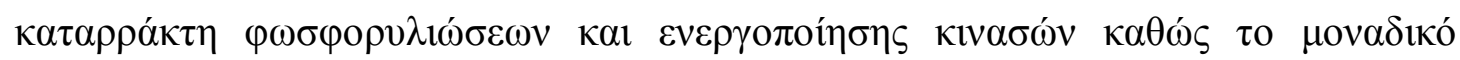

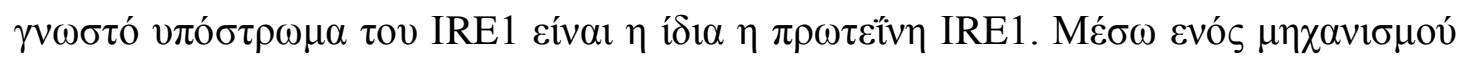

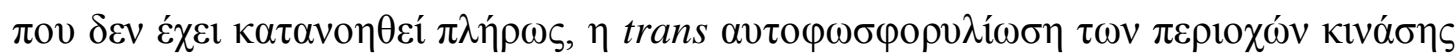

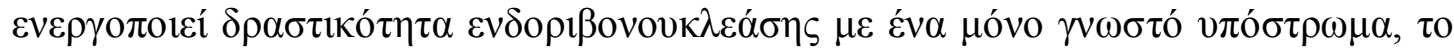

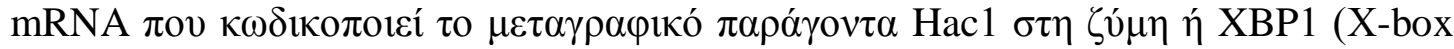




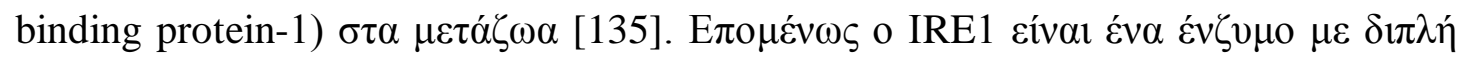

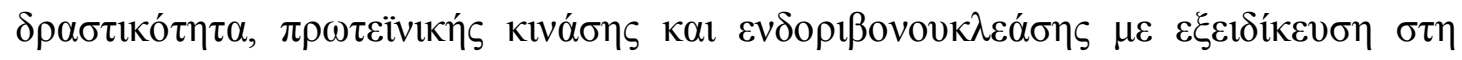

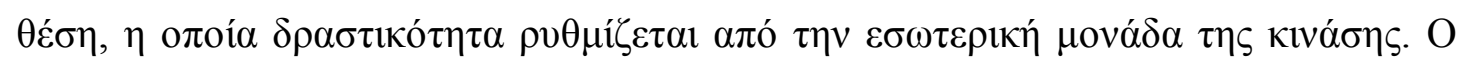

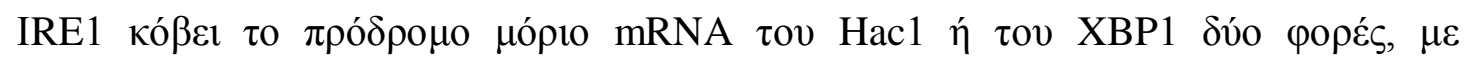

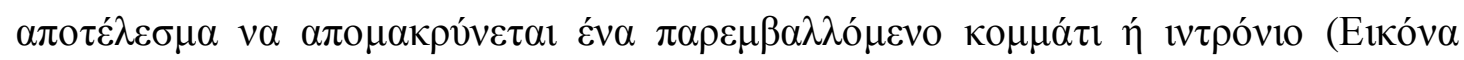

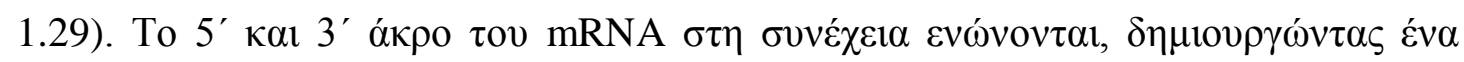

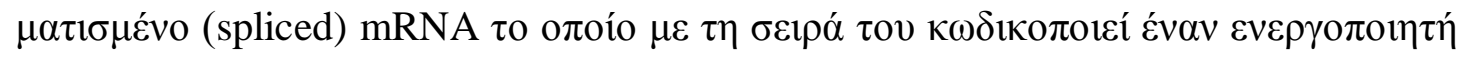

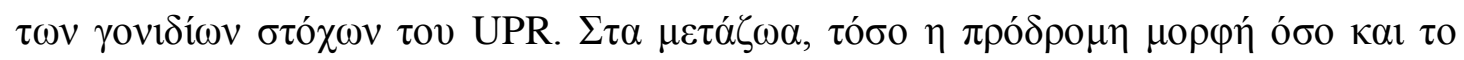

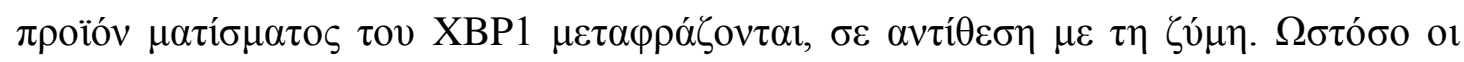

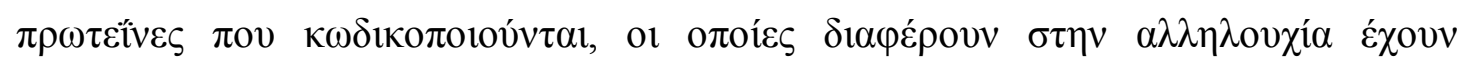

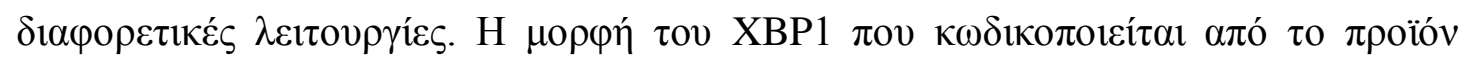

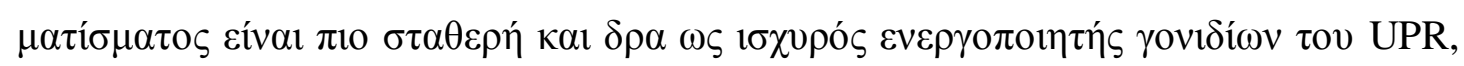

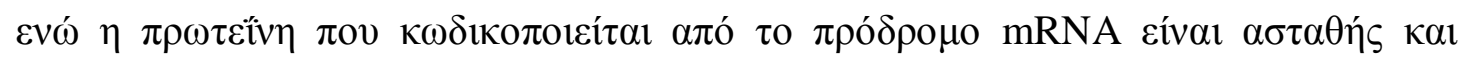

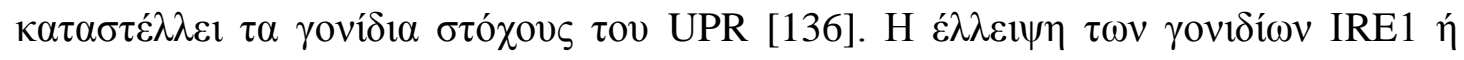

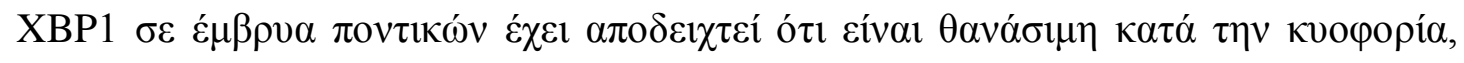

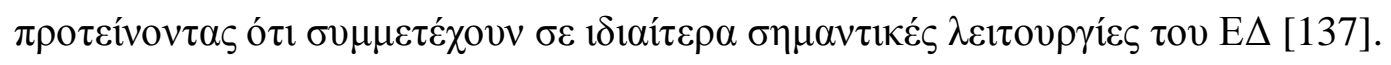

\section{ATF6}

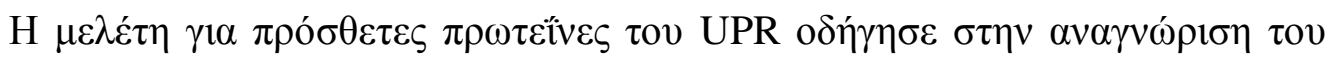

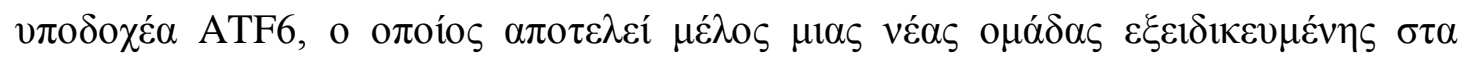

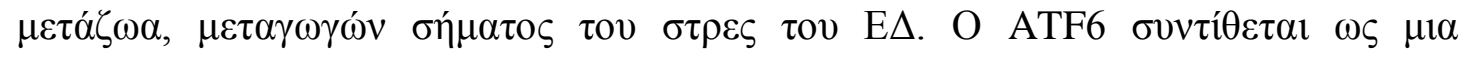

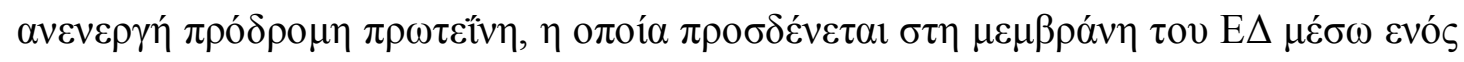

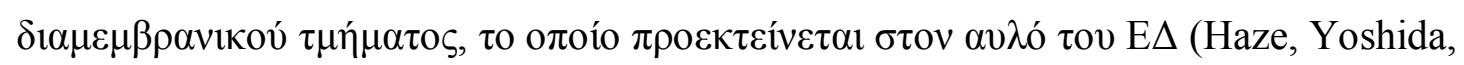

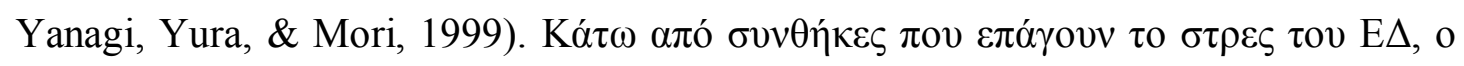

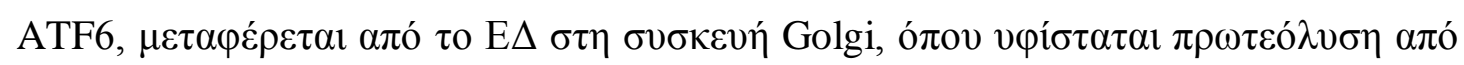

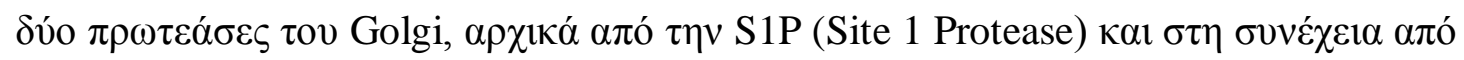

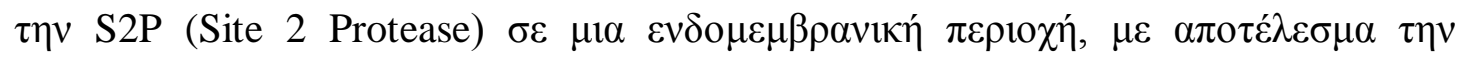

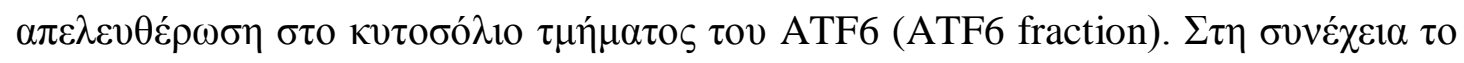

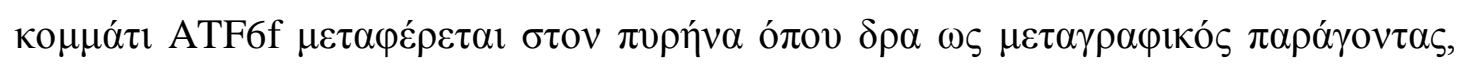

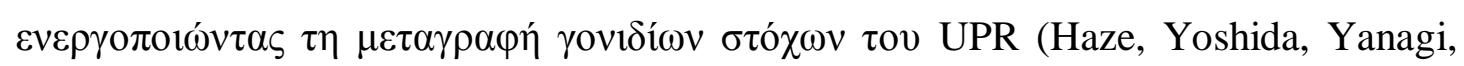
Yura, \& Mori, 1999) (Eıkóva 1.30). 


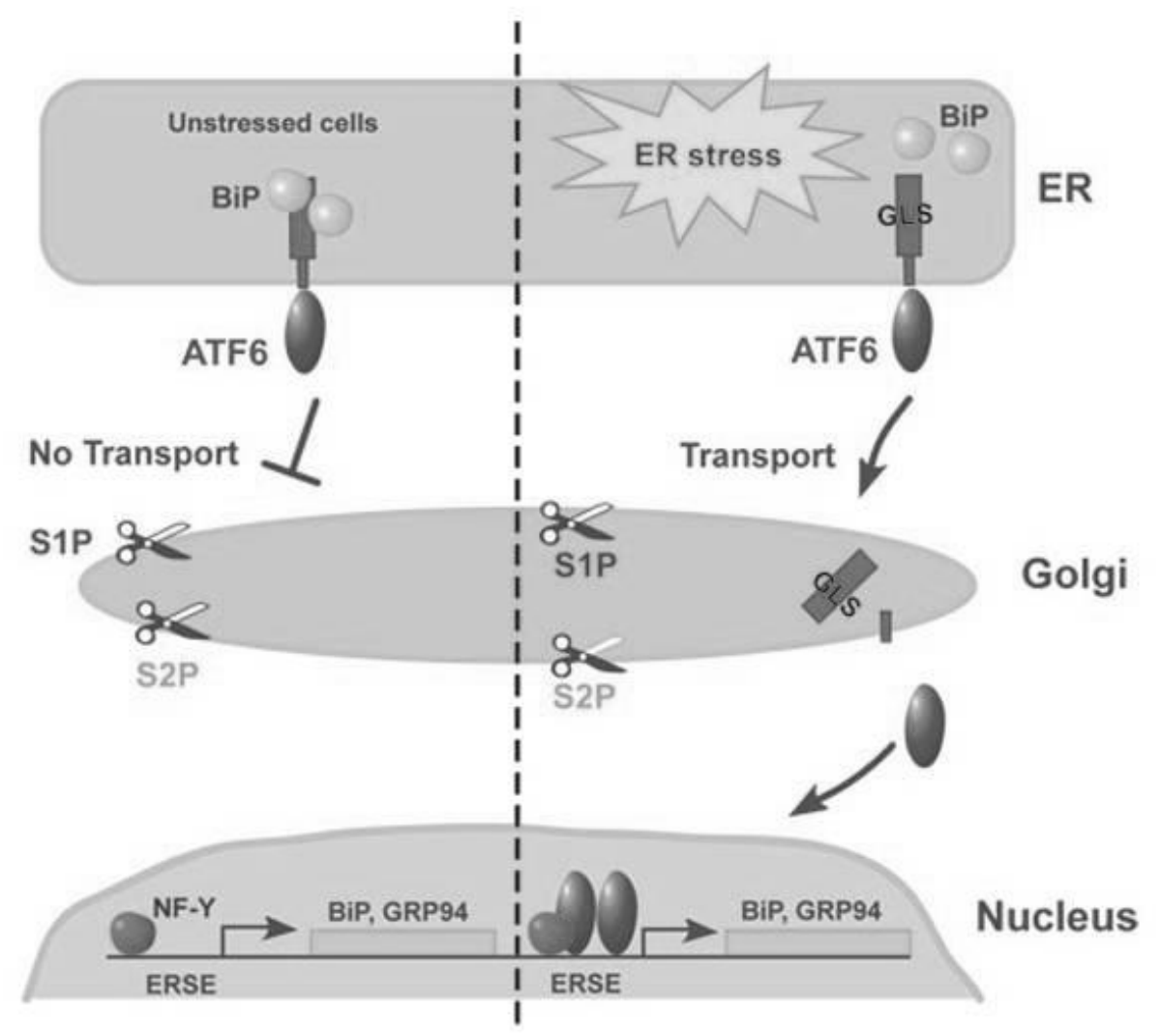

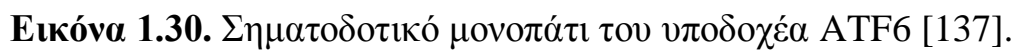

\section{PERK}

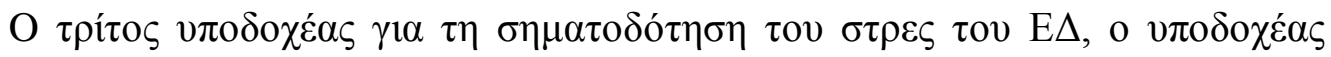

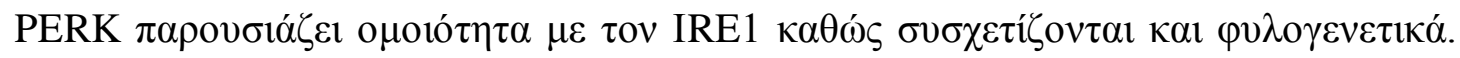

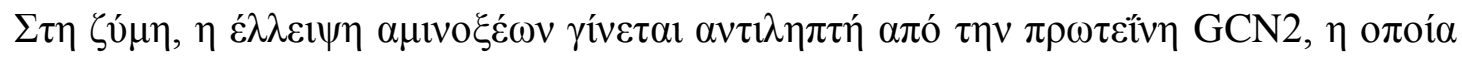

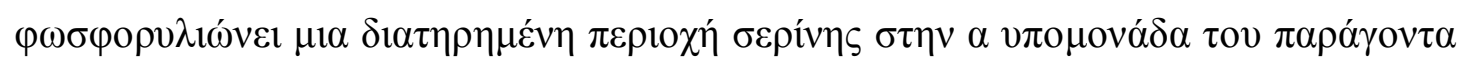

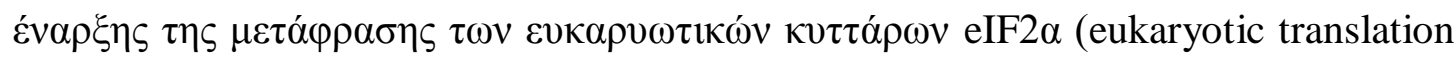

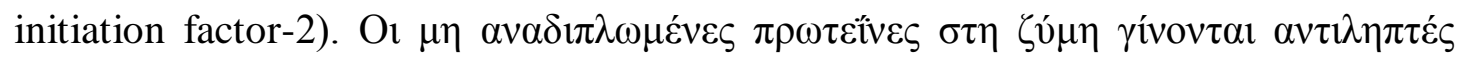

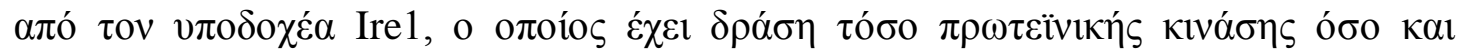

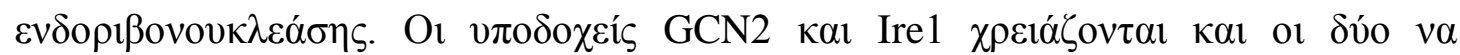

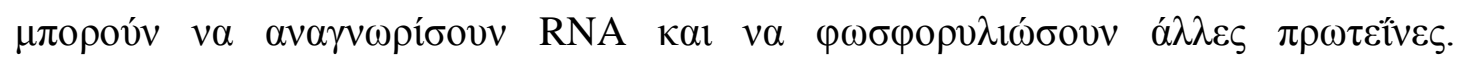

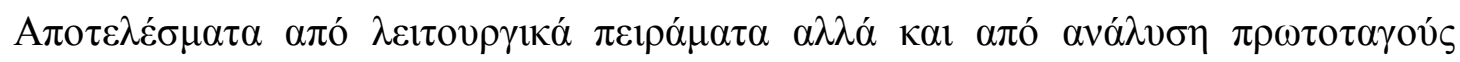

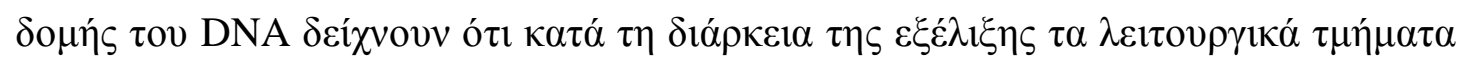

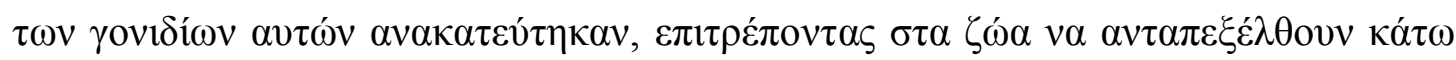

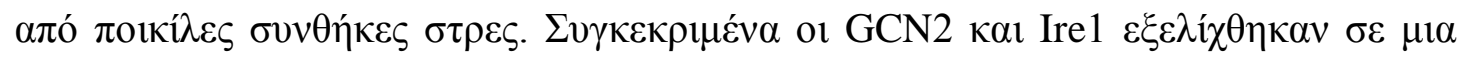




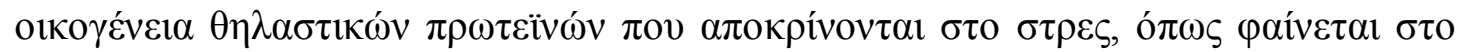
(Eıкóva 1.31)..

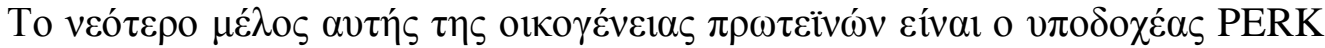

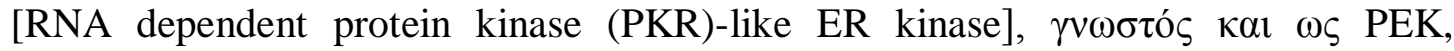

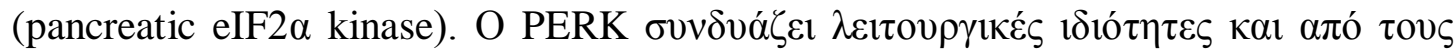

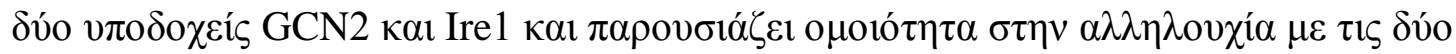

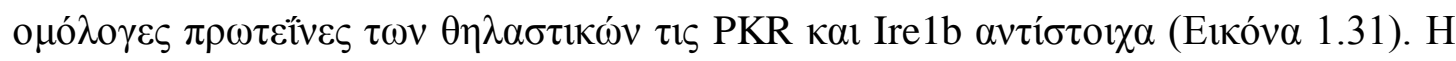

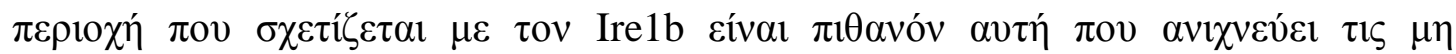

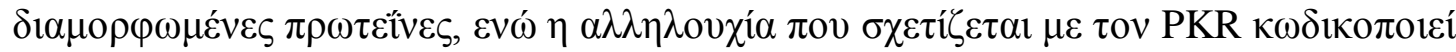

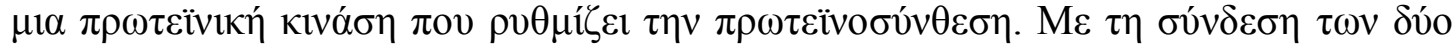

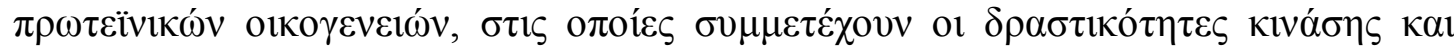

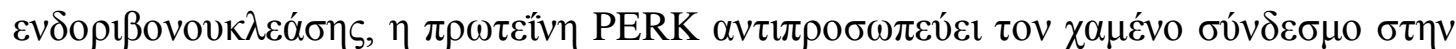

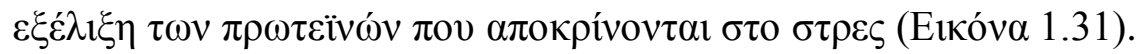

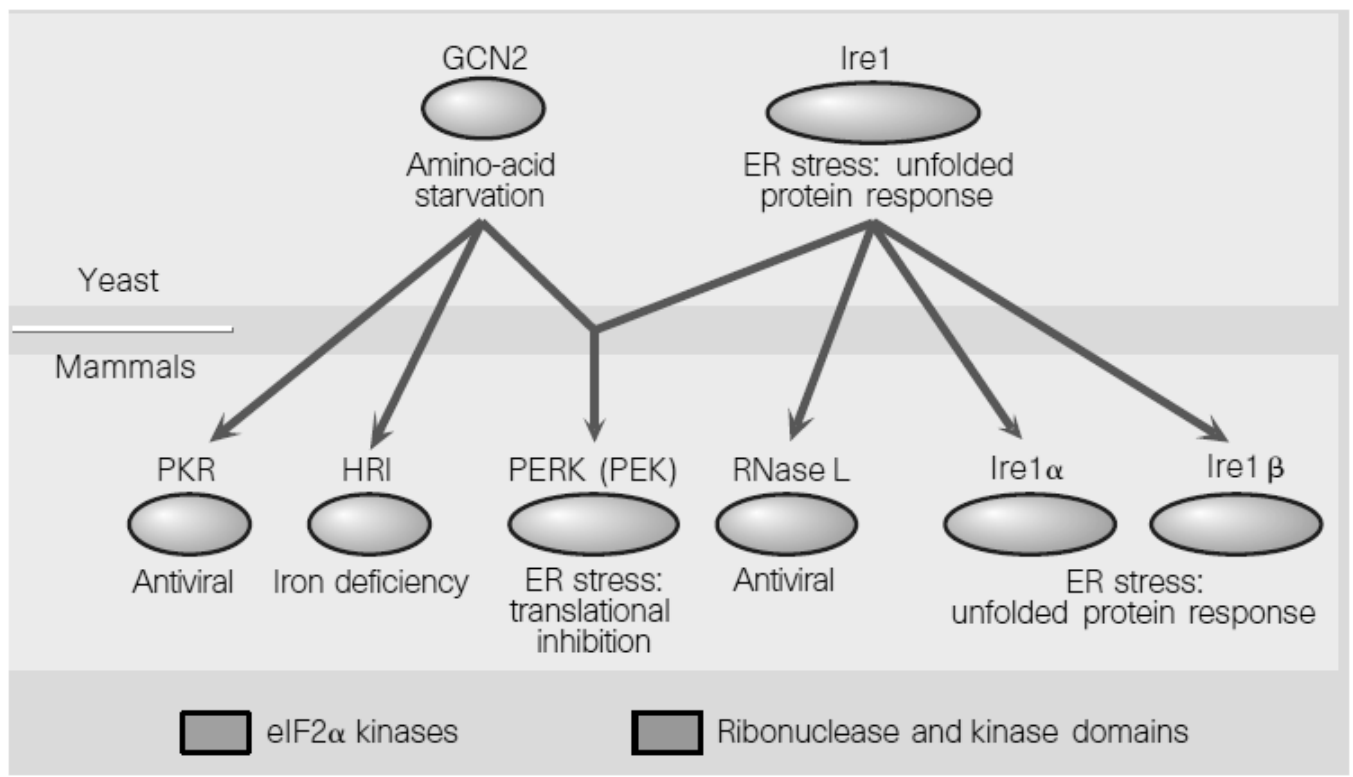

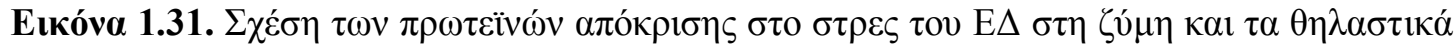
[139].

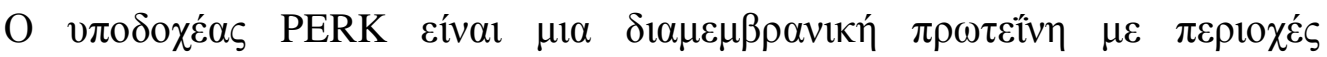

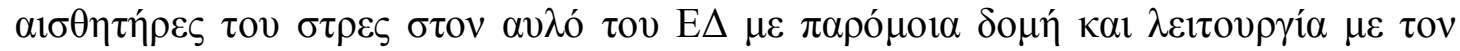

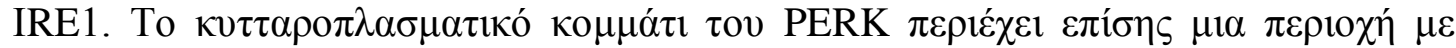

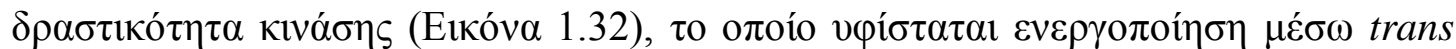




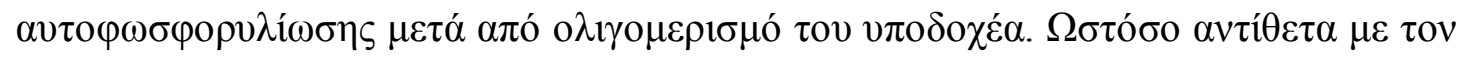

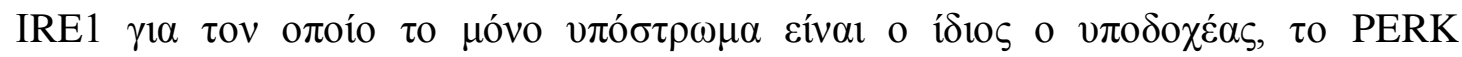

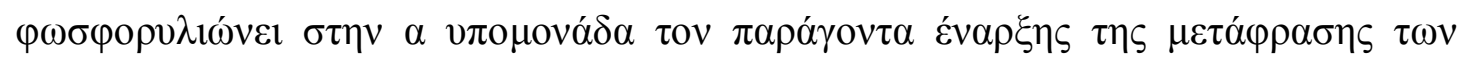

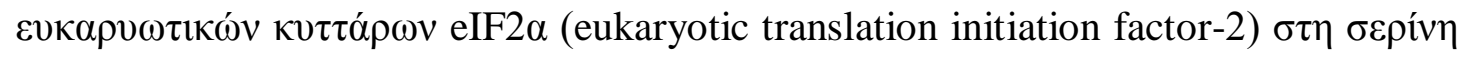

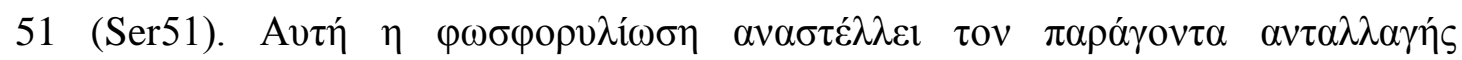

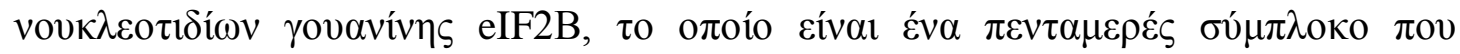

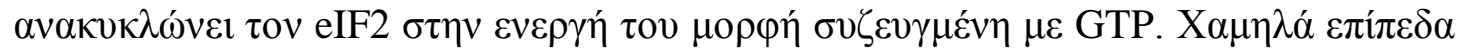

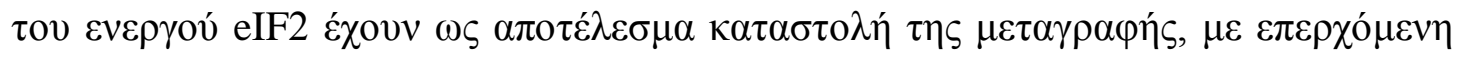

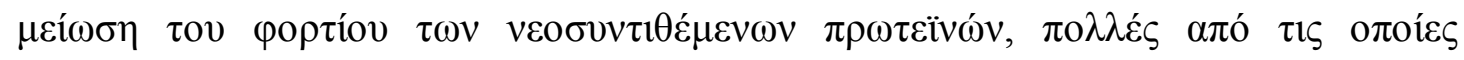

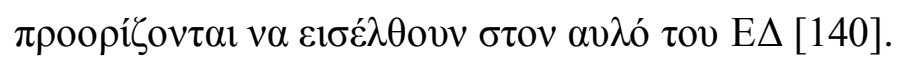

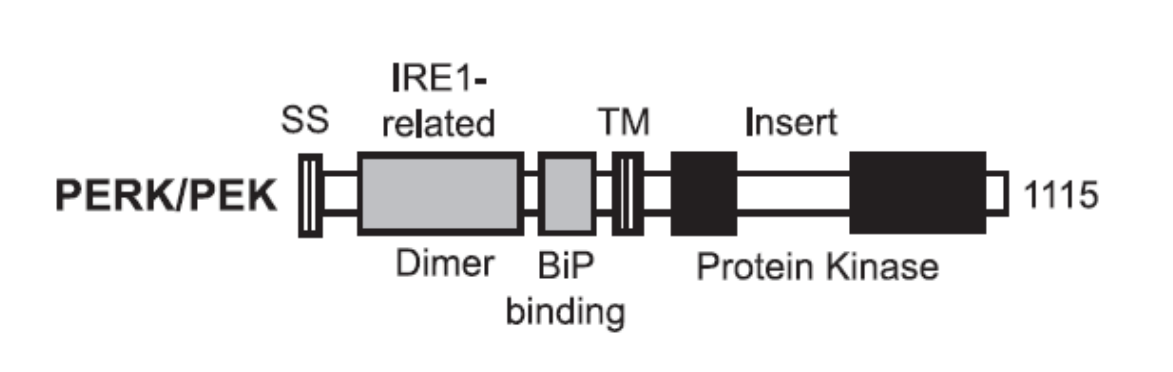

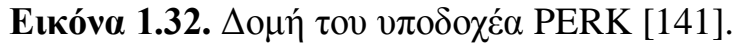

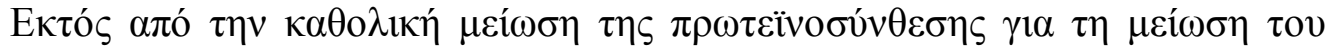

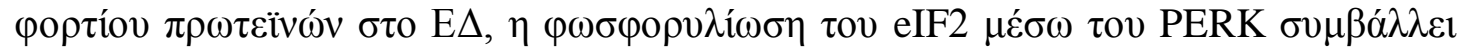

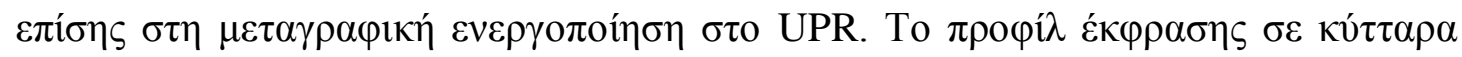

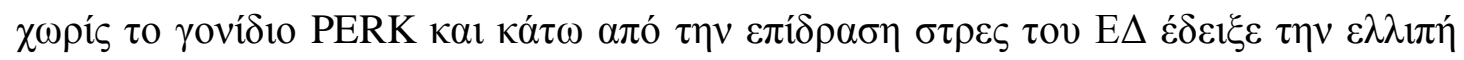

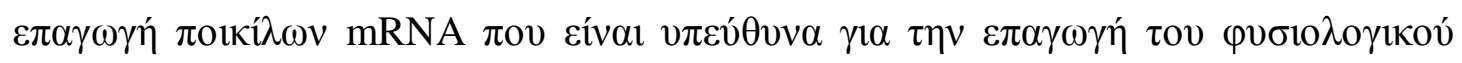

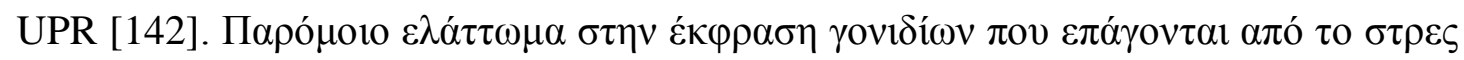

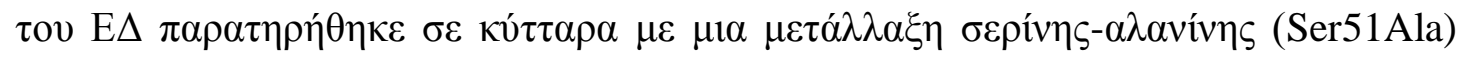

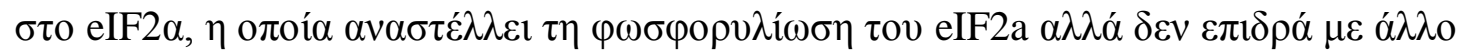

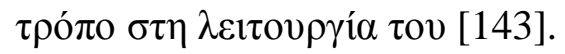

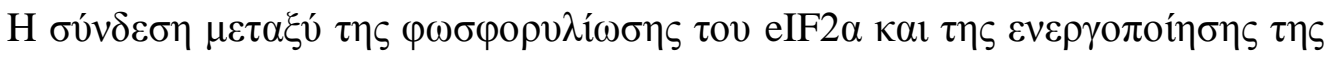

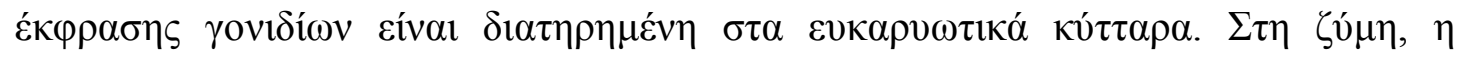

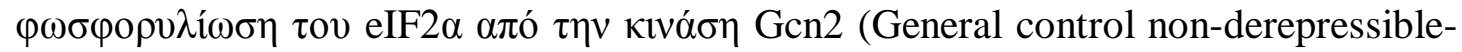

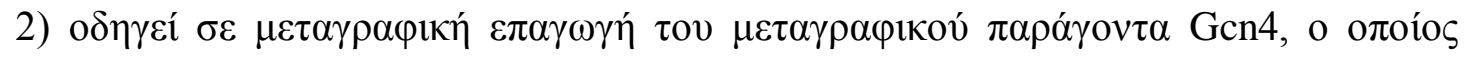

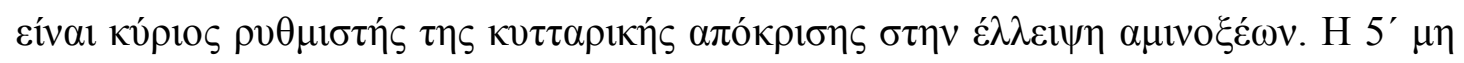

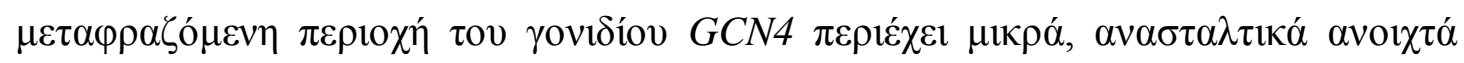




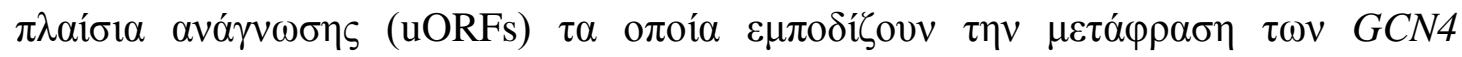

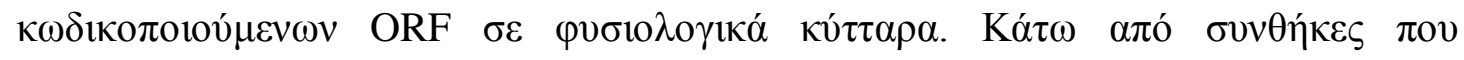

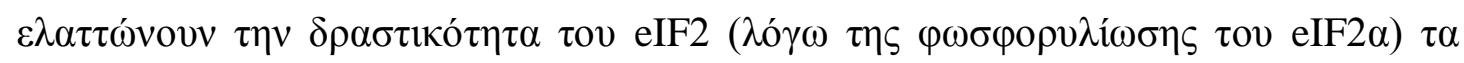

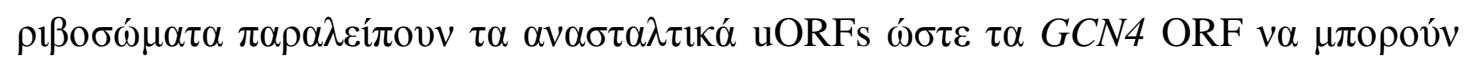

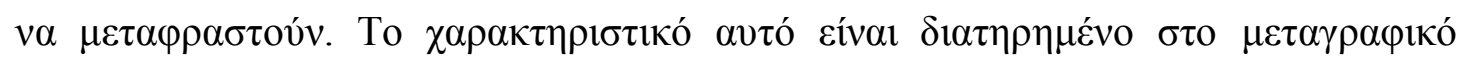

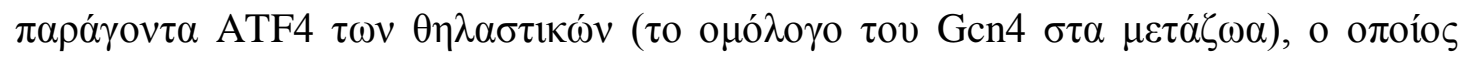

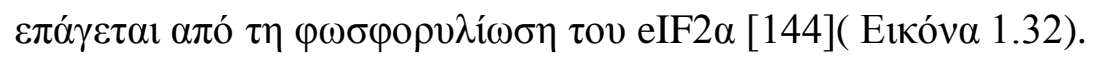

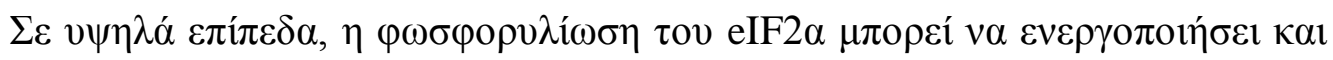

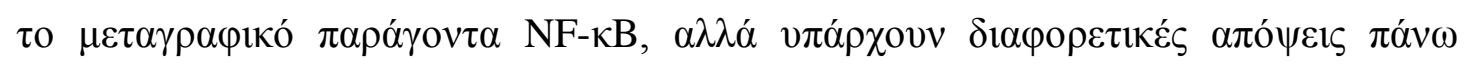

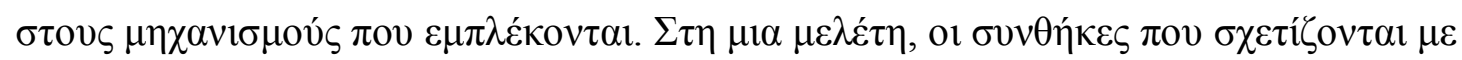

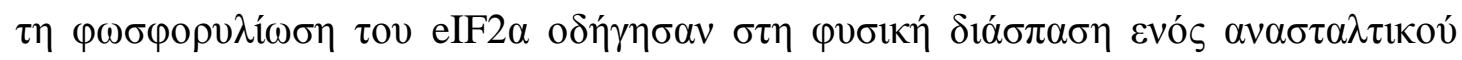

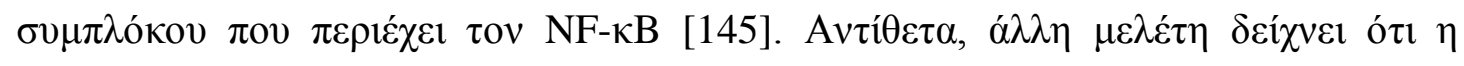

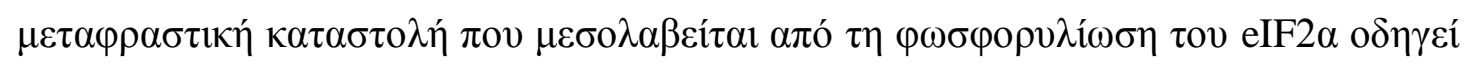

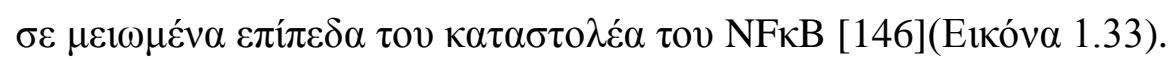

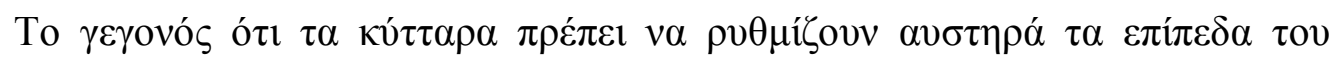

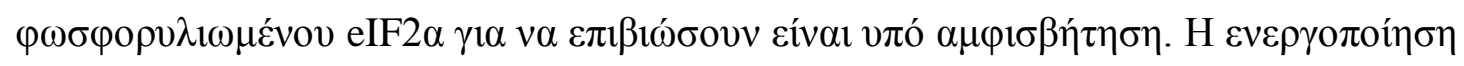

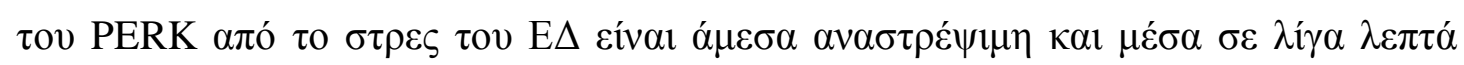

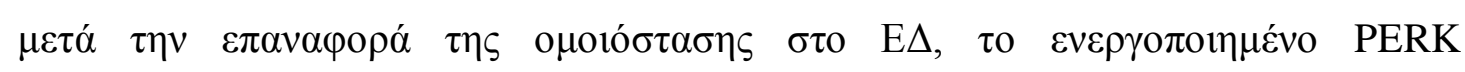

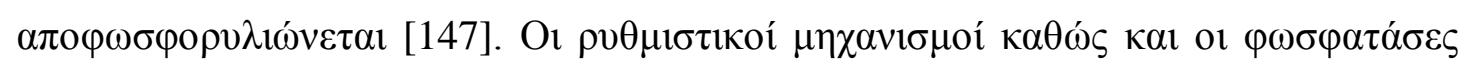

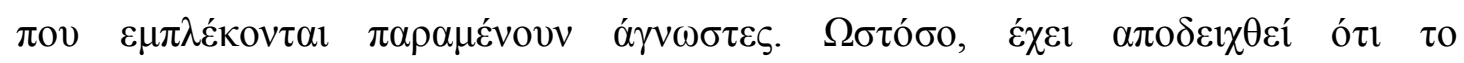

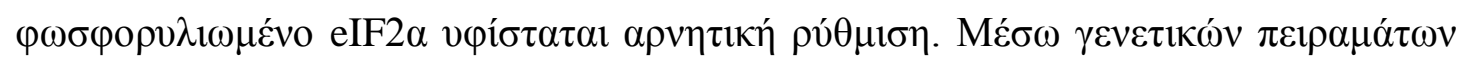

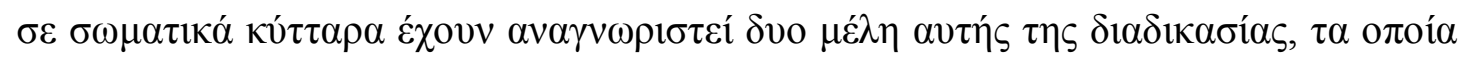

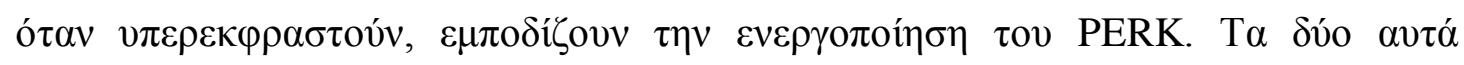

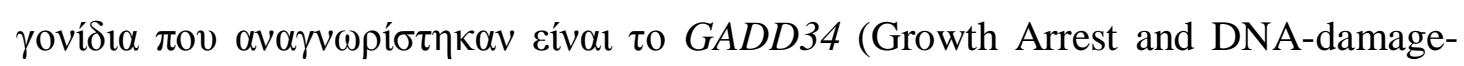

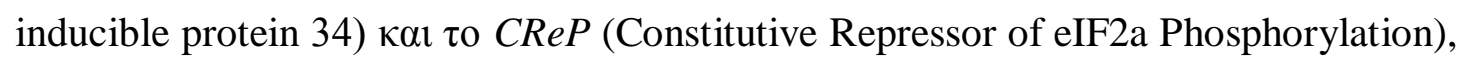

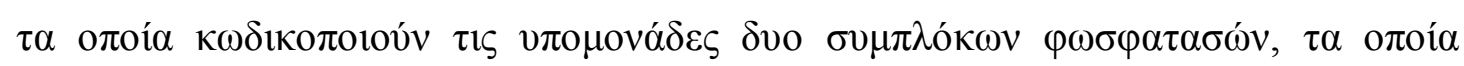

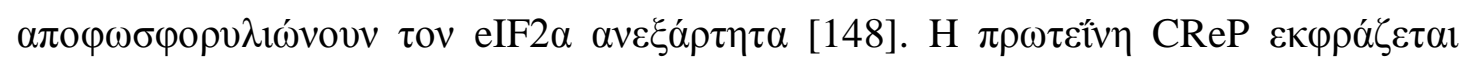

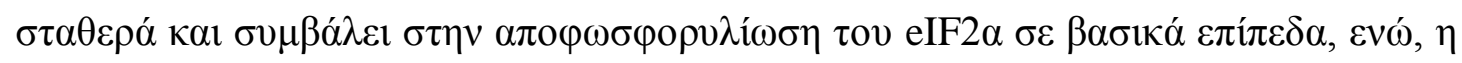

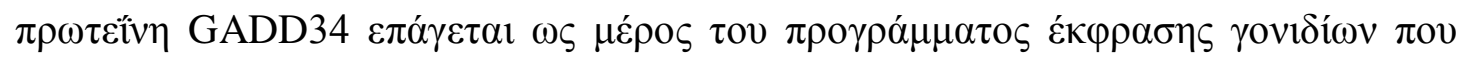

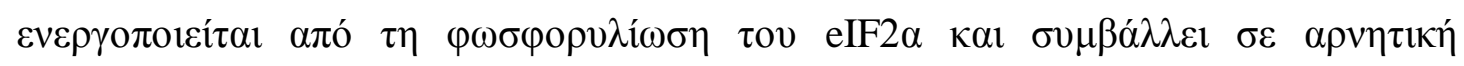

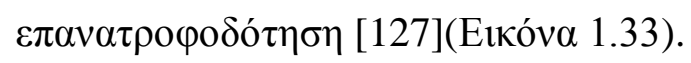




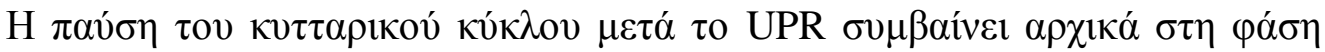

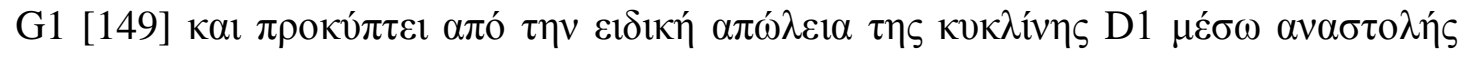

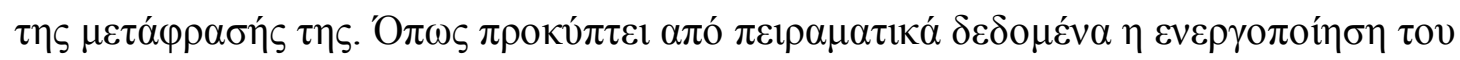

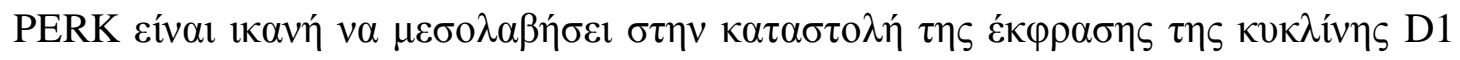

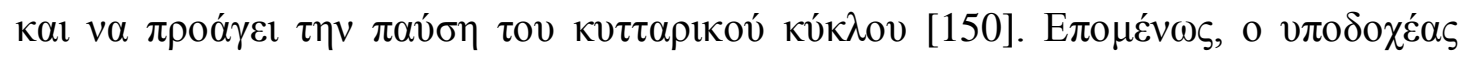

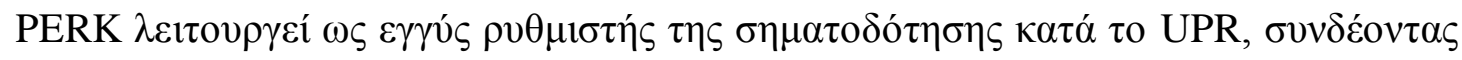

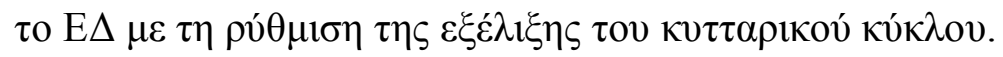

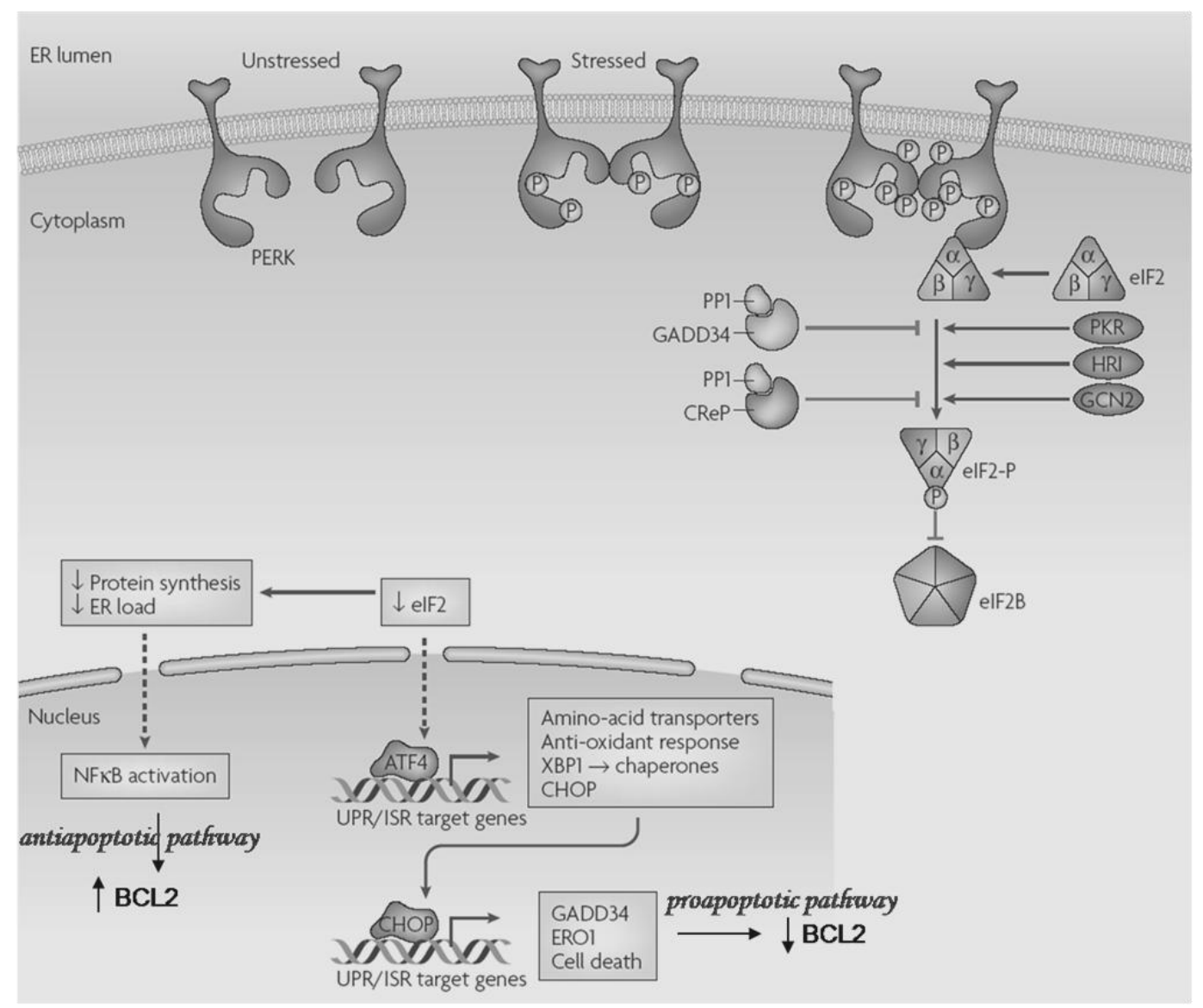

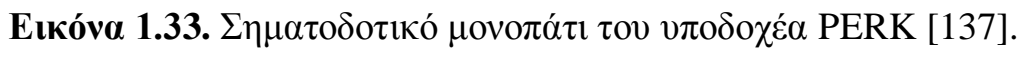

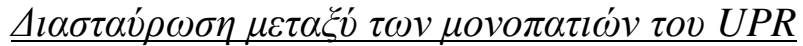

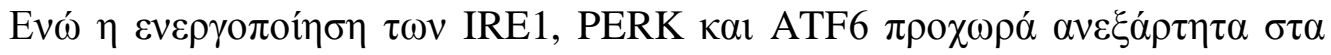

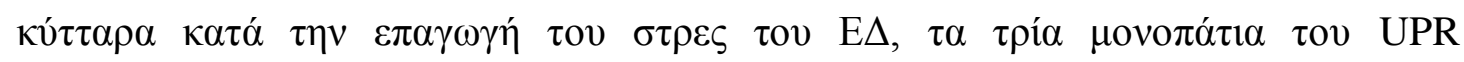

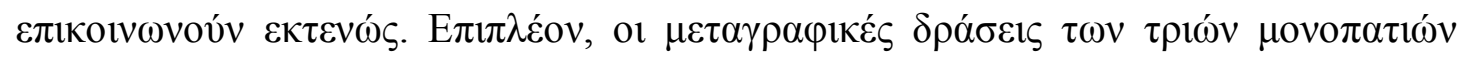

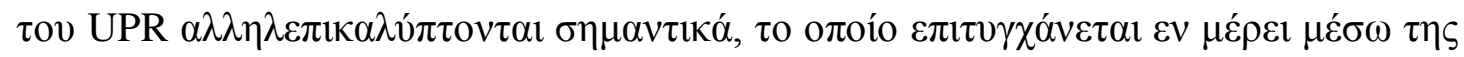

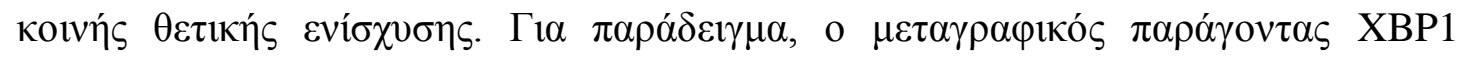

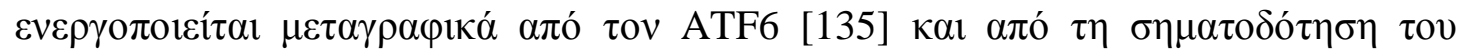




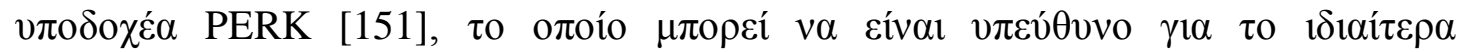

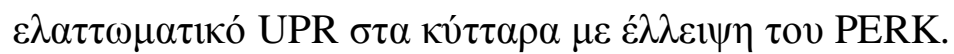

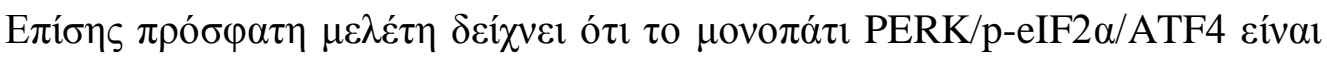

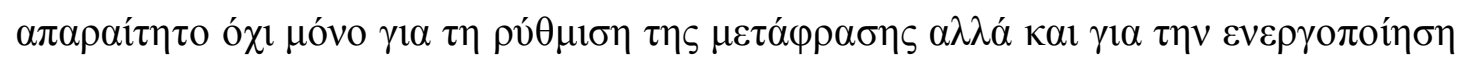

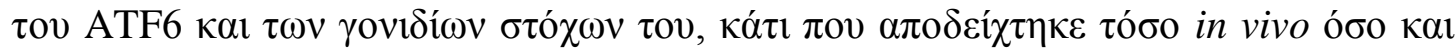

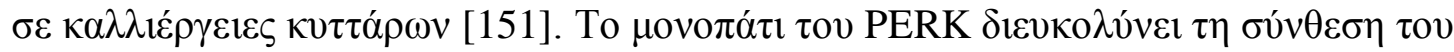

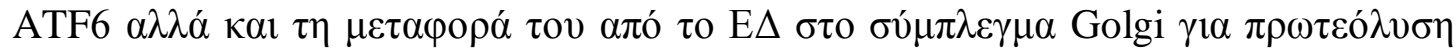

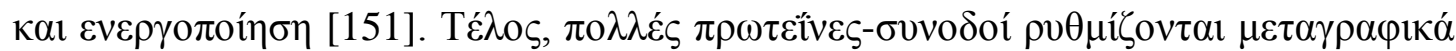

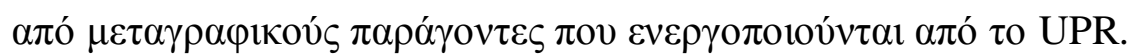

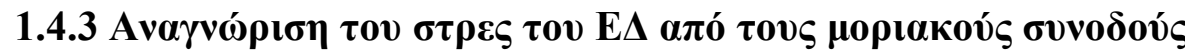

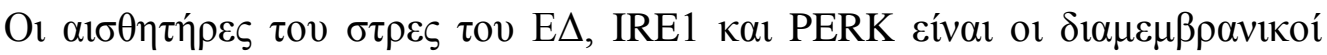

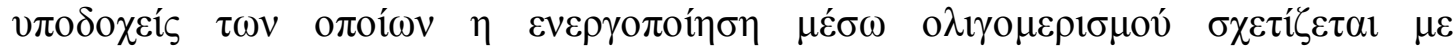

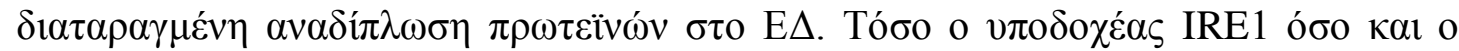

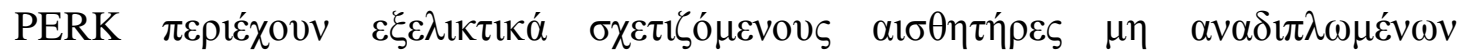

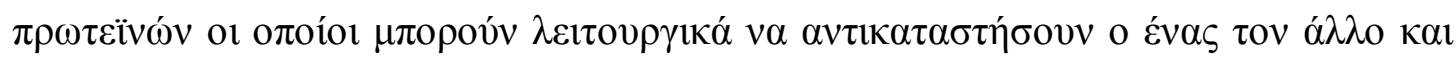

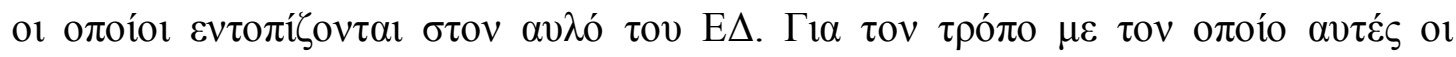

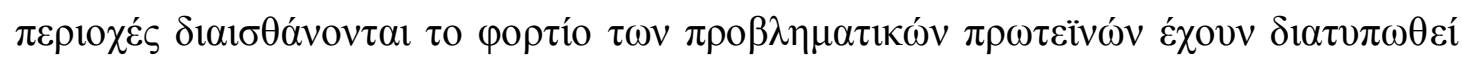

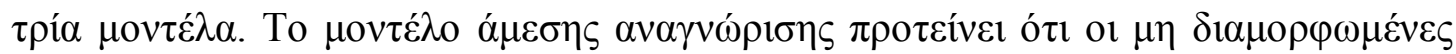

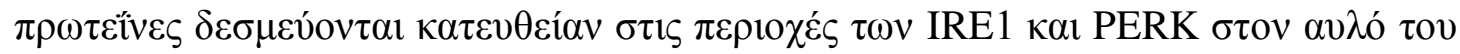

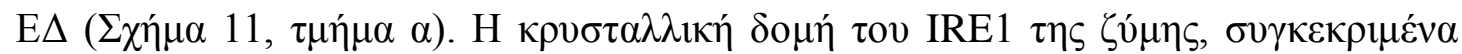

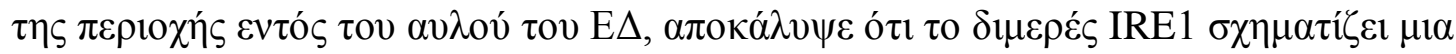

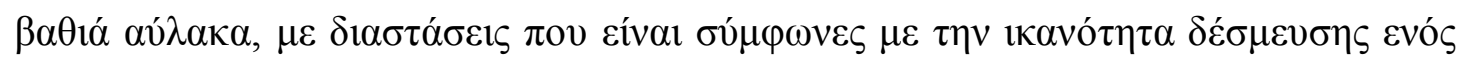

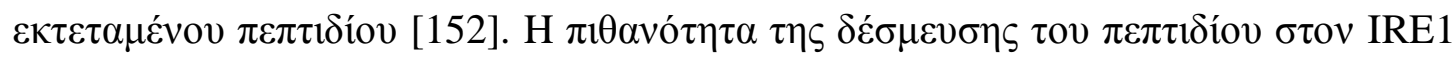

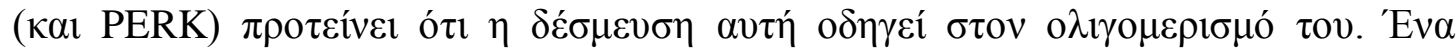

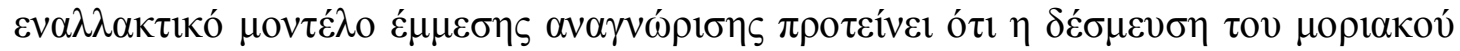

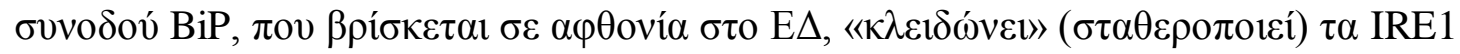

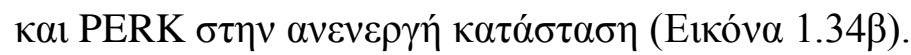

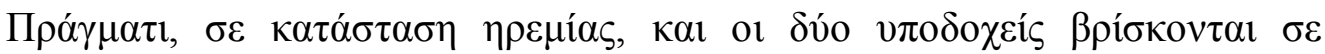

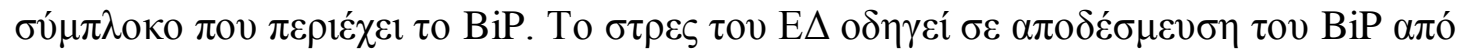

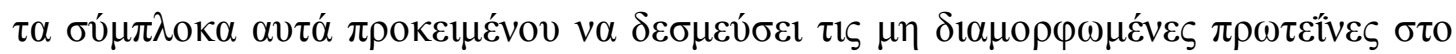




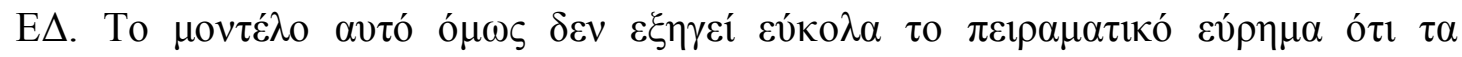

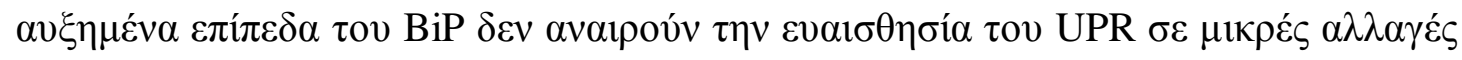

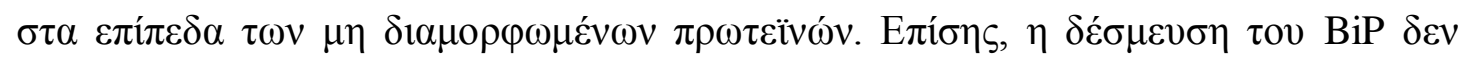

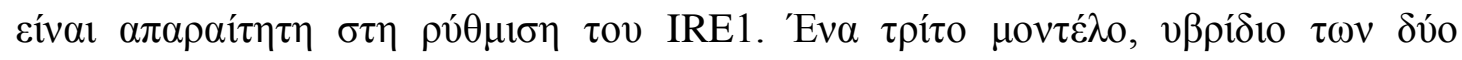

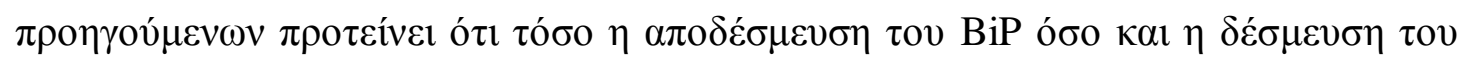

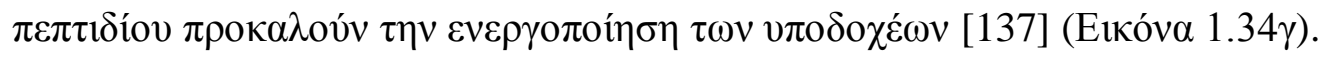

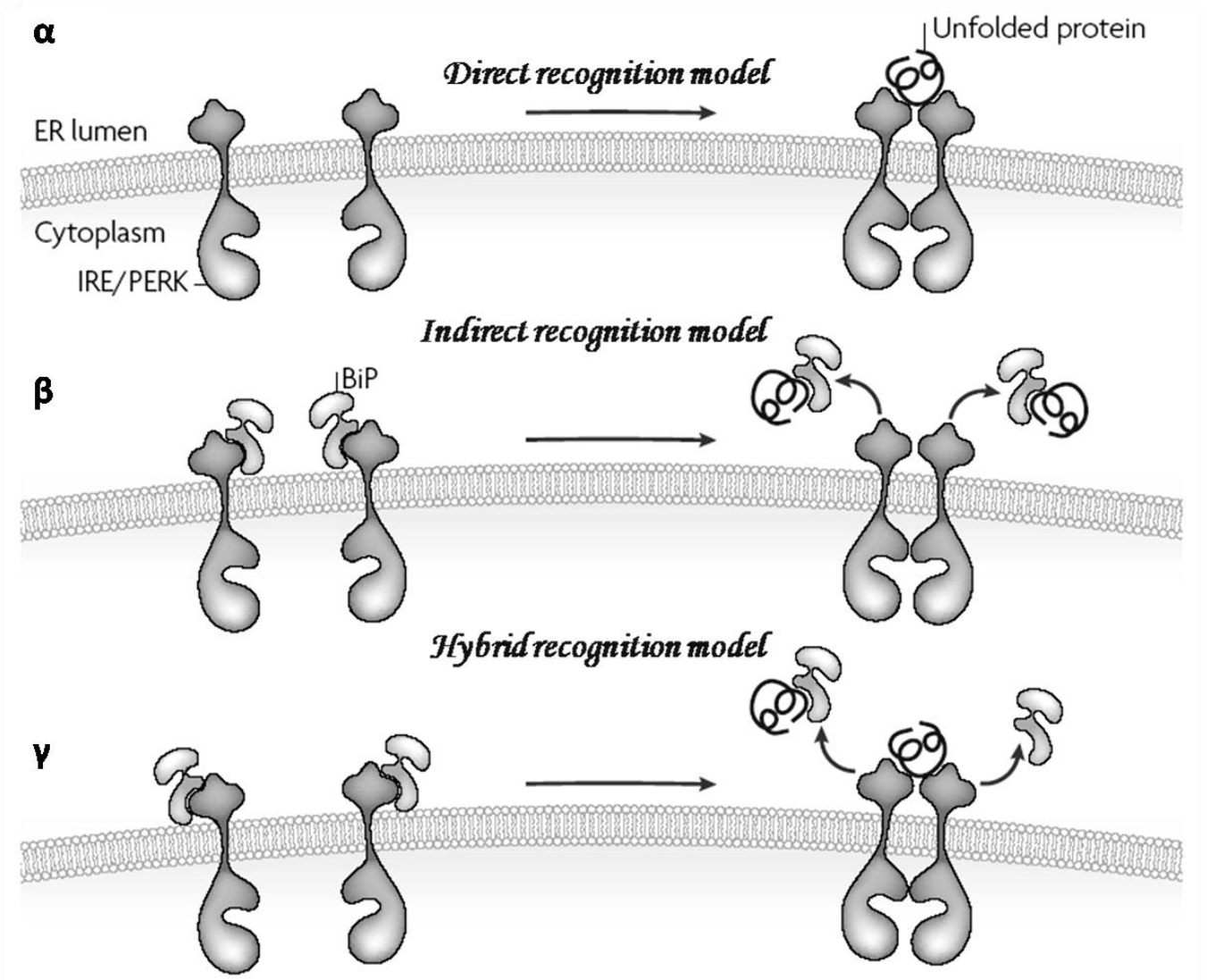

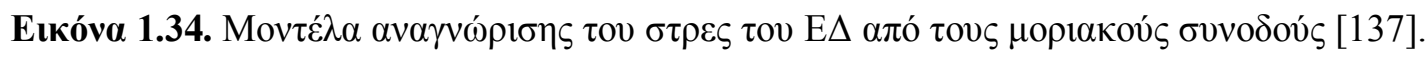

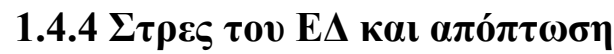

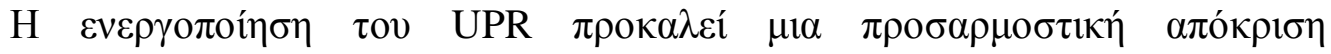

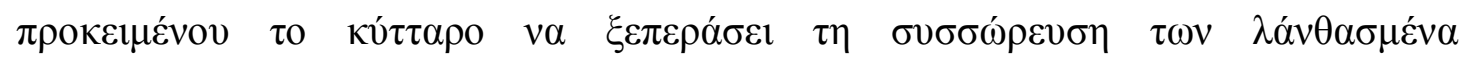

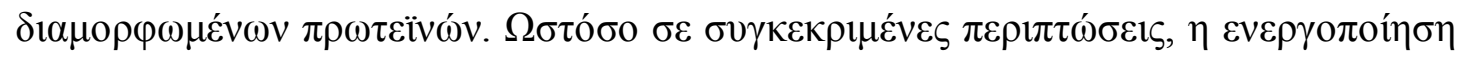

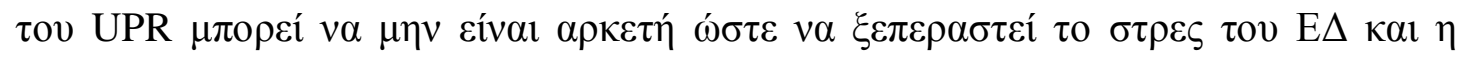

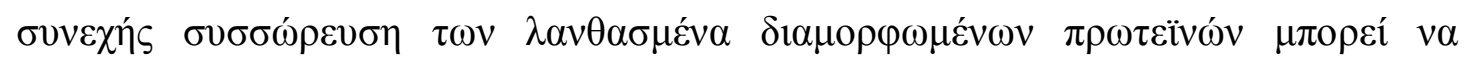

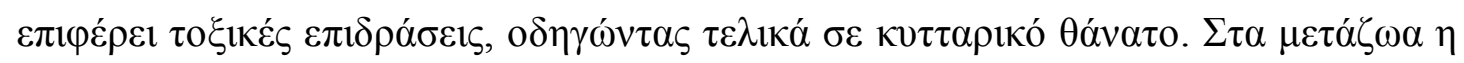




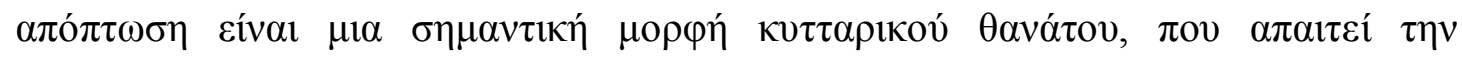

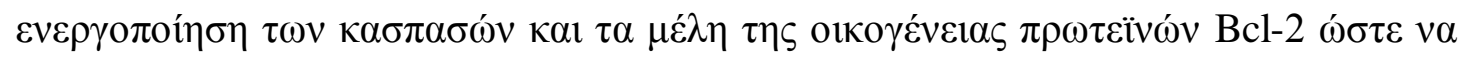

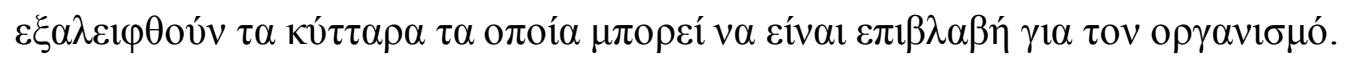

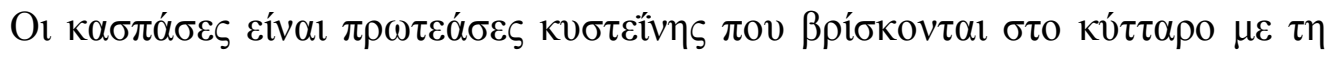

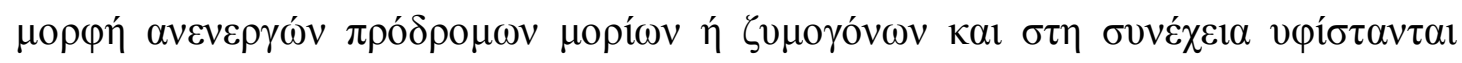

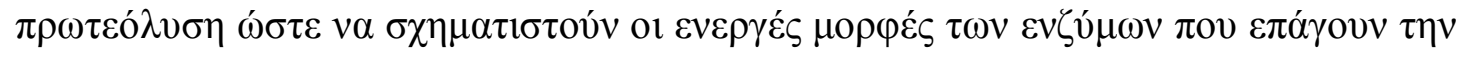

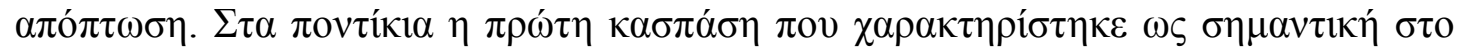

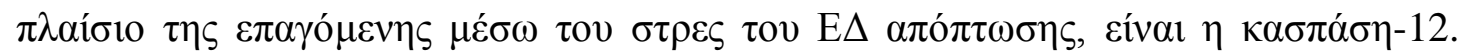

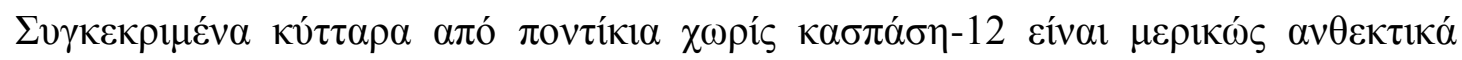

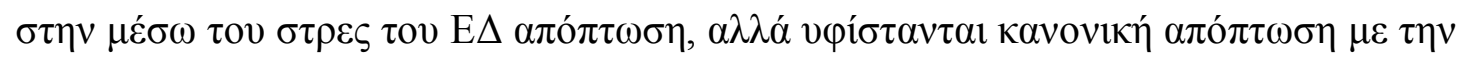

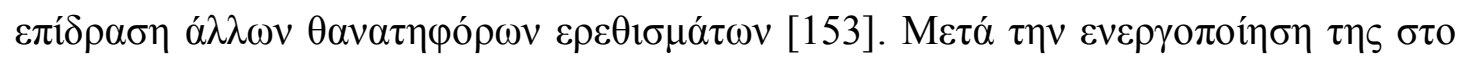

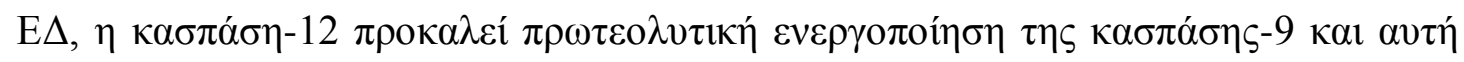

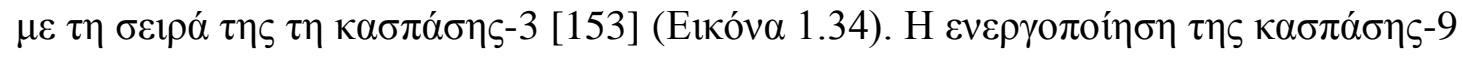

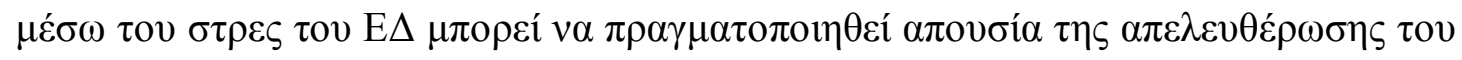

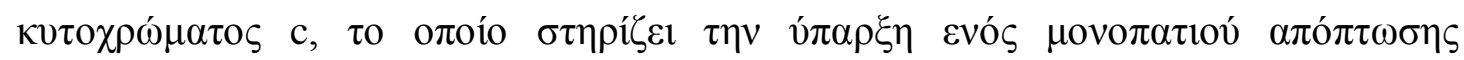

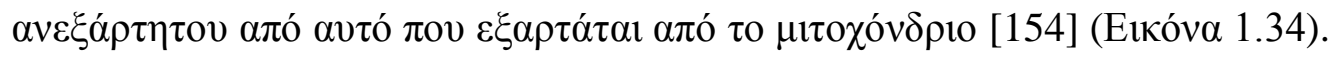

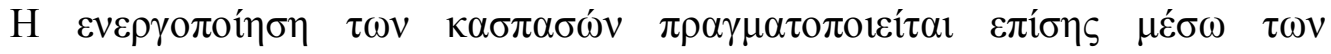

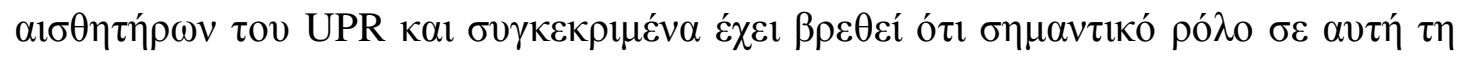

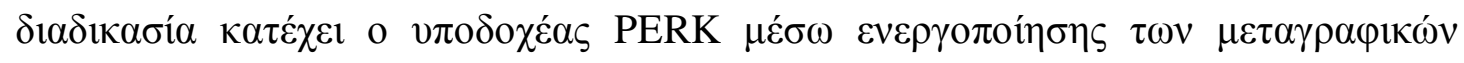

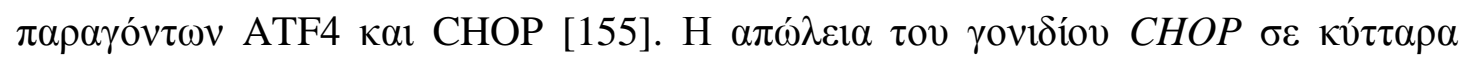

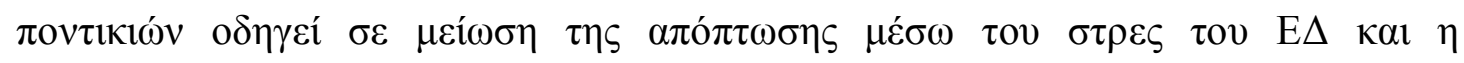

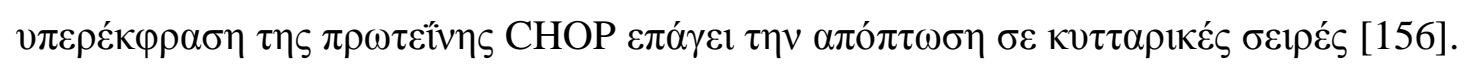

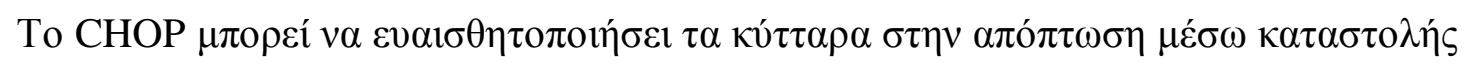

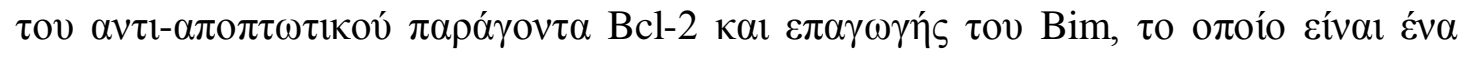

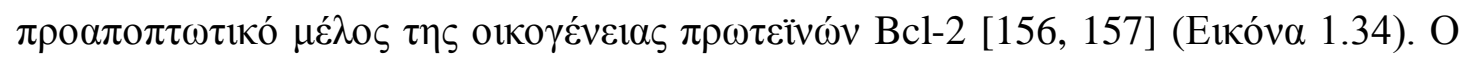

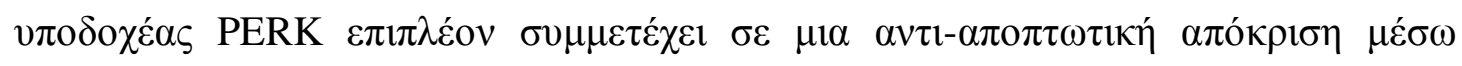

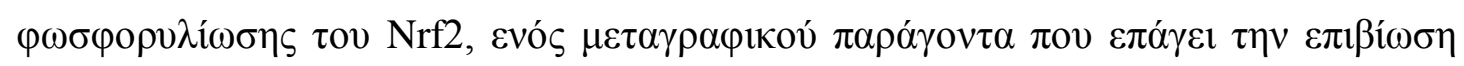

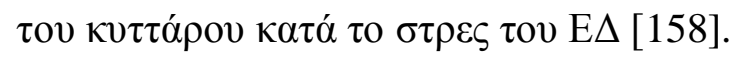

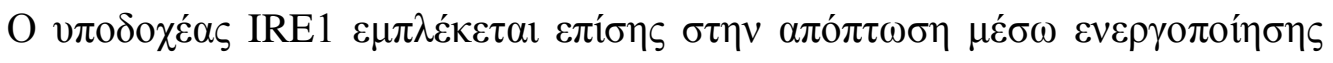

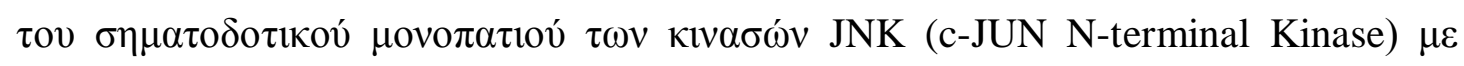

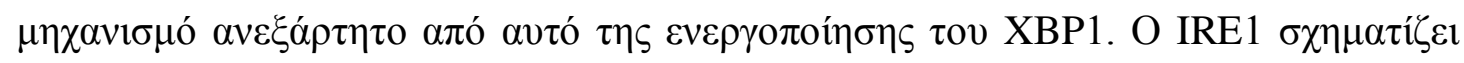

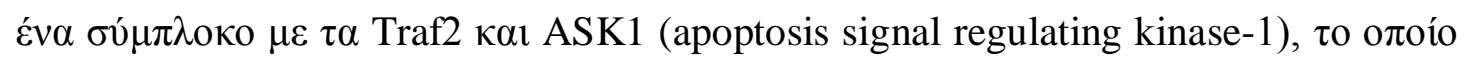




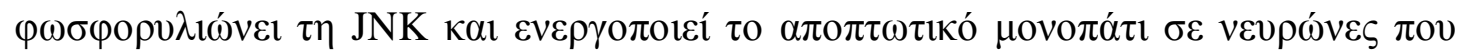

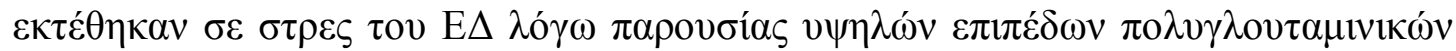

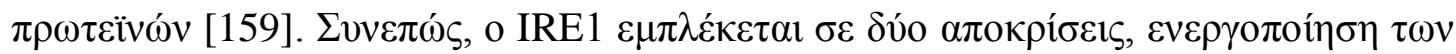

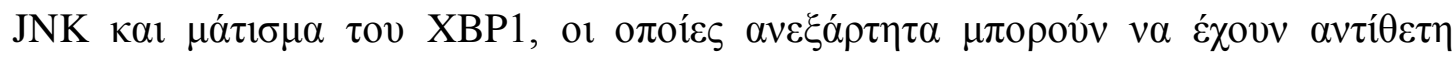

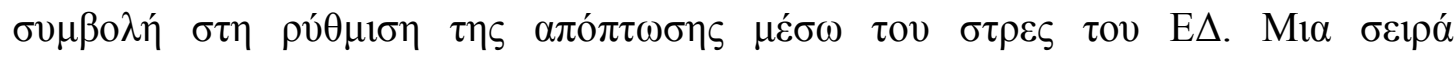

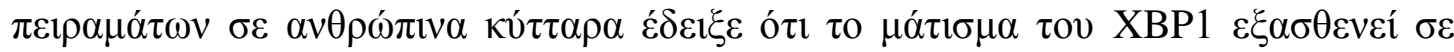

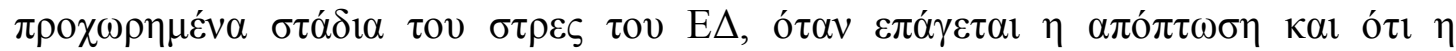

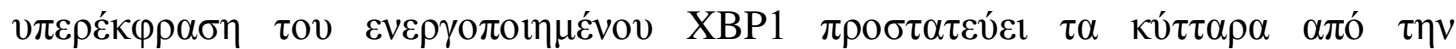
$\alpha \pi \pi_{\pi \tau \omega \sigma \eta}[160]$.

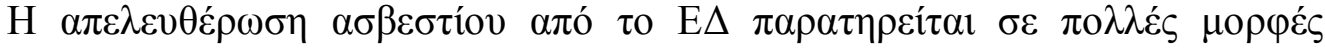

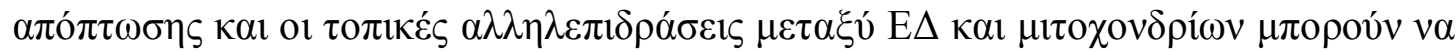

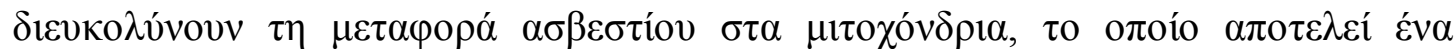

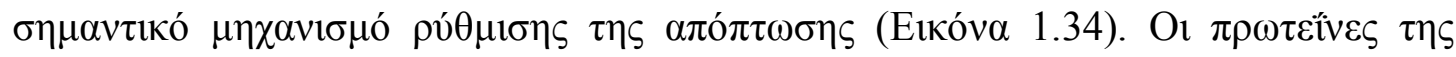

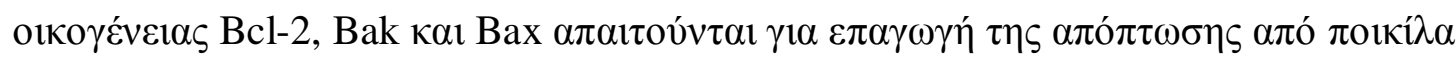

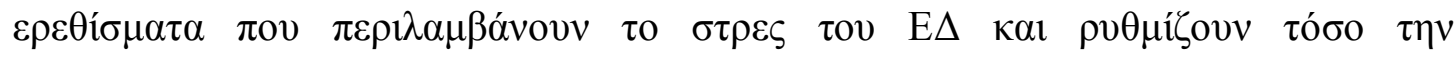

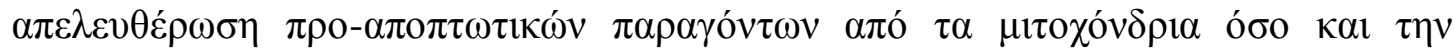

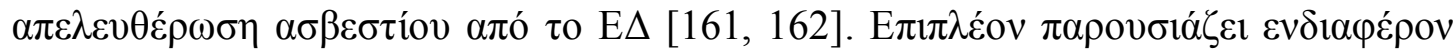

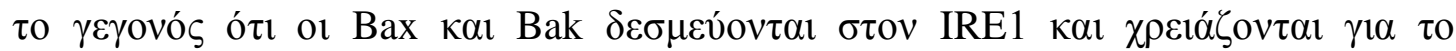

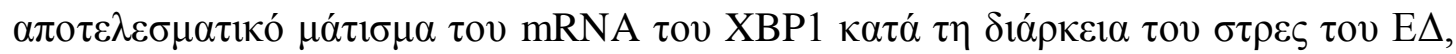

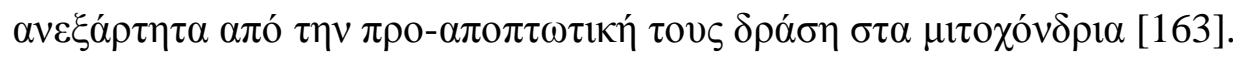

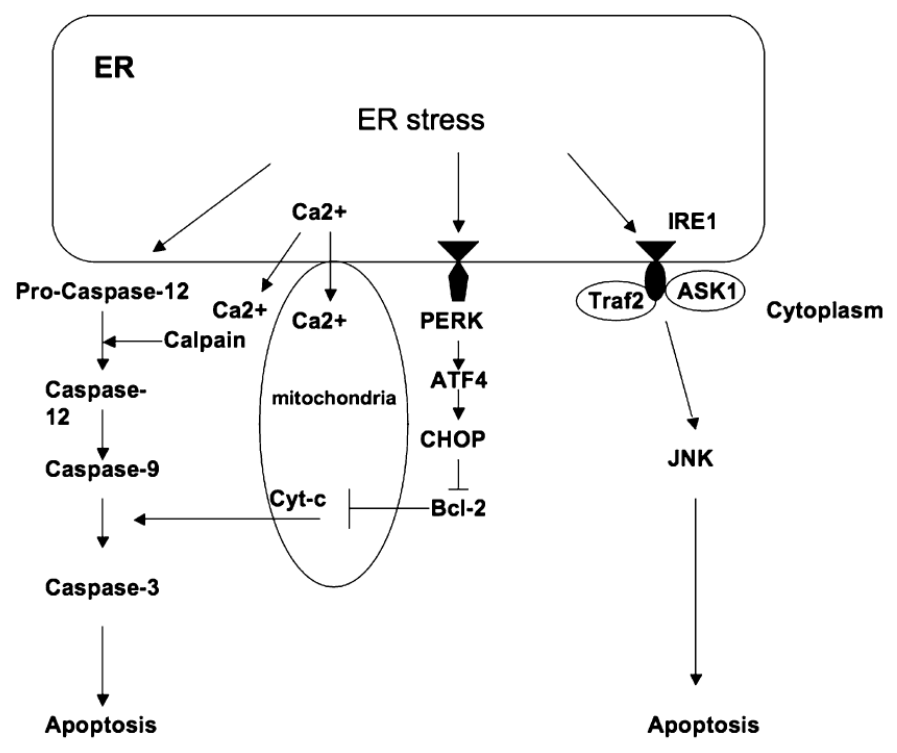

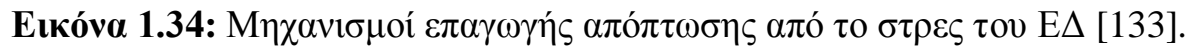




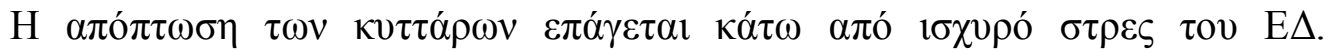

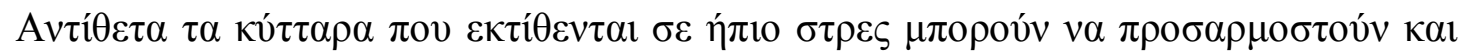

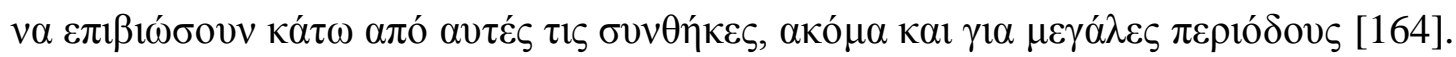

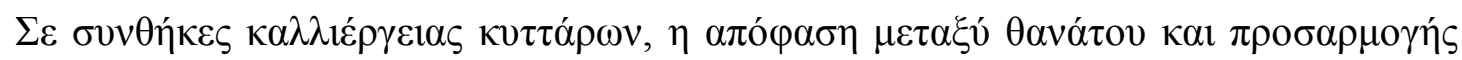

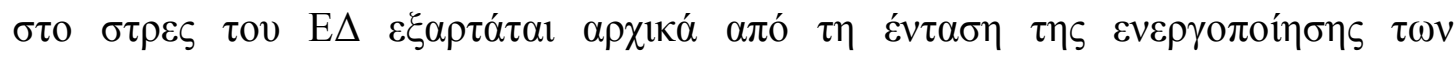

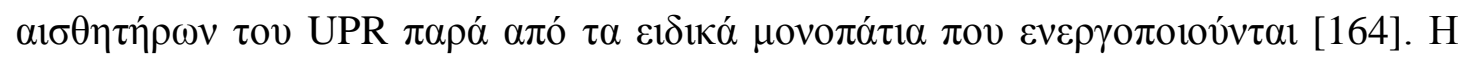

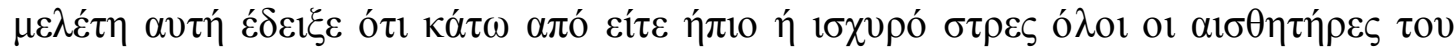

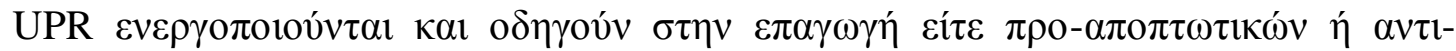

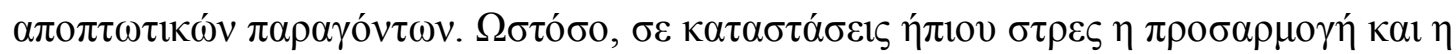

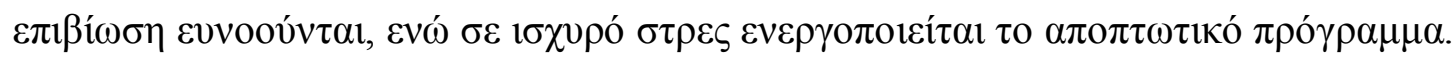

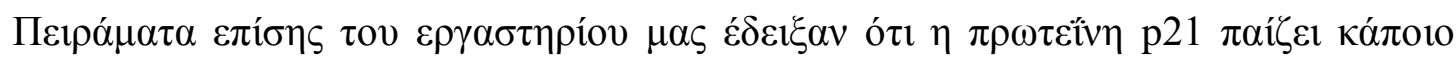

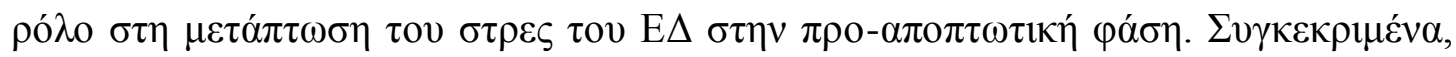

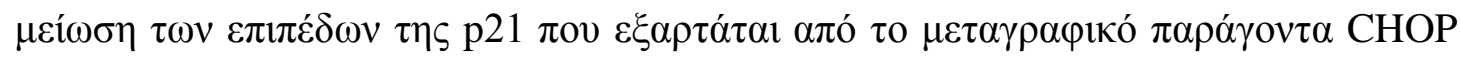

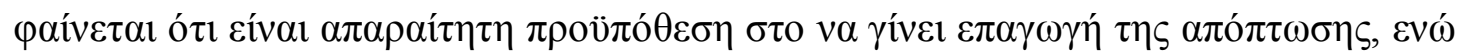

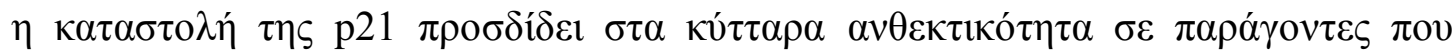

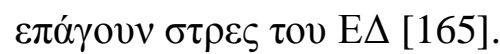




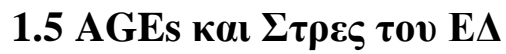

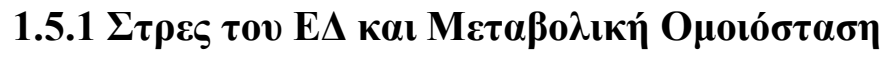

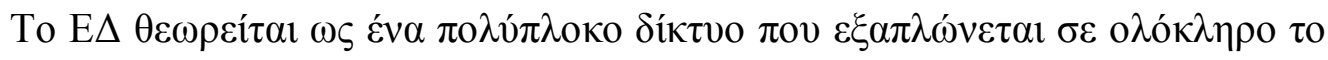

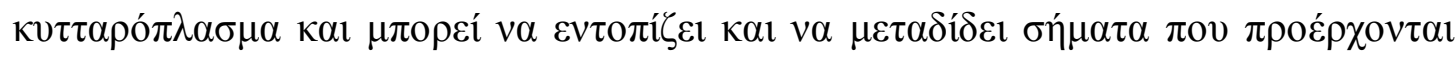

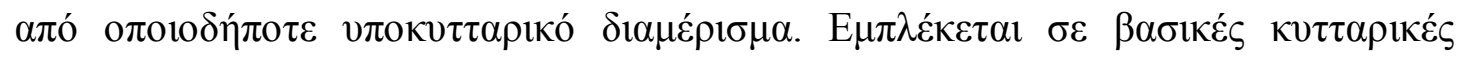

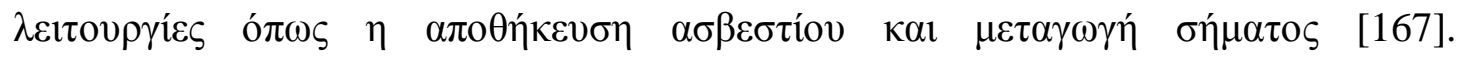

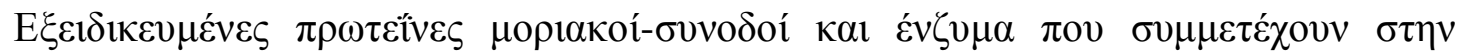

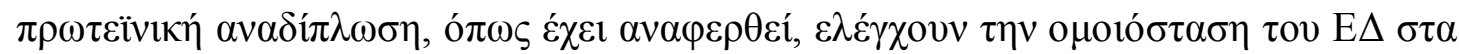

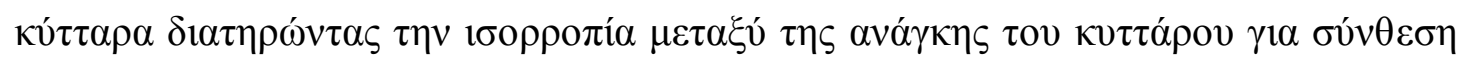

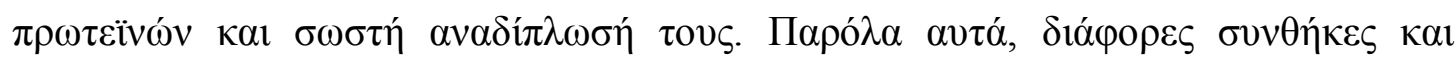

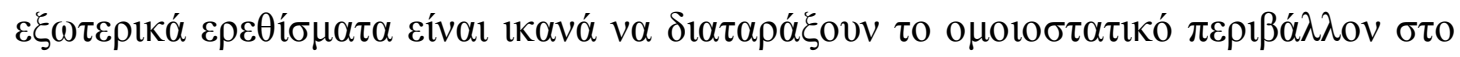

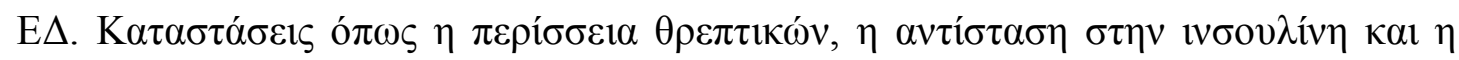

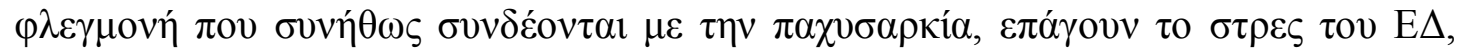

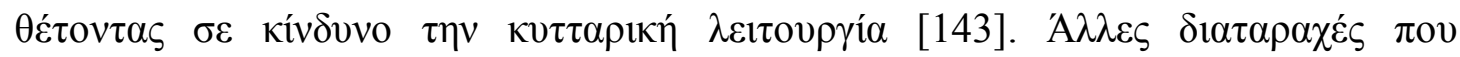

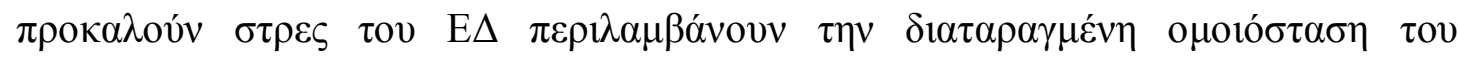

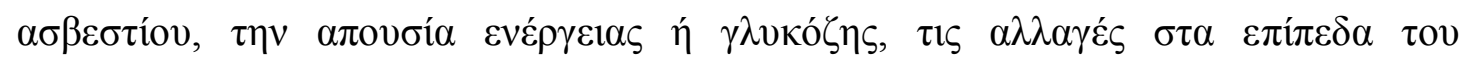

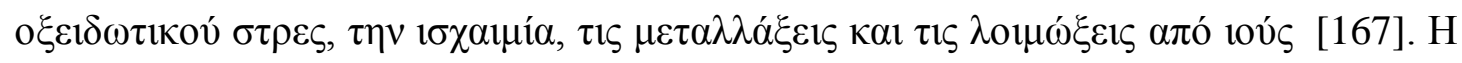

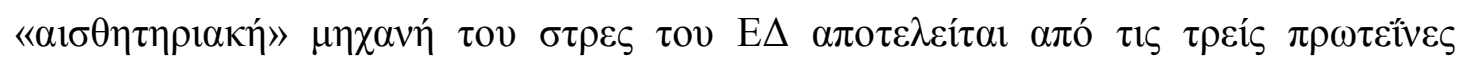

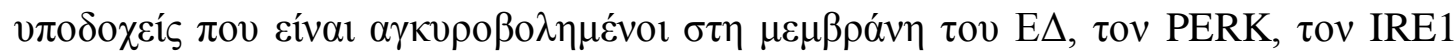

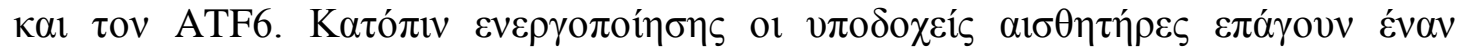

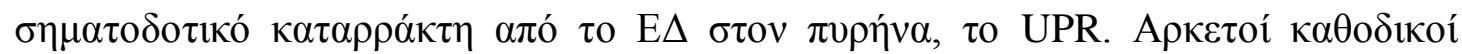

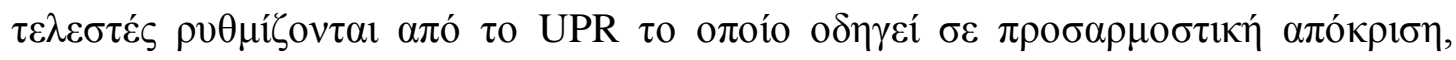

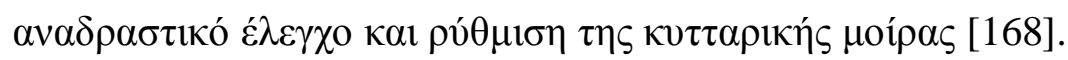

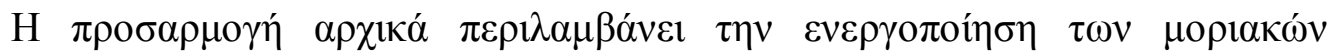

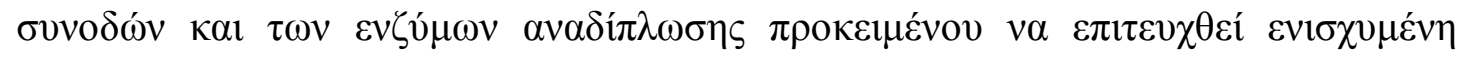

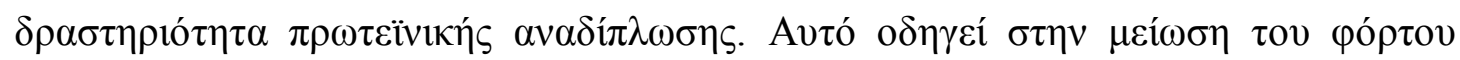

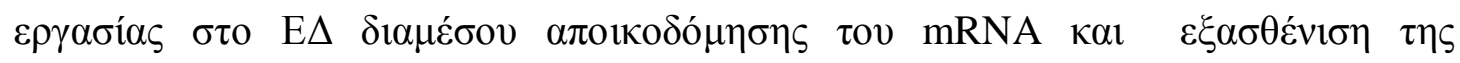

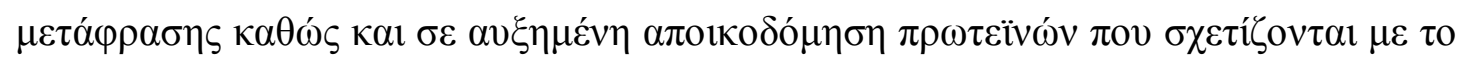

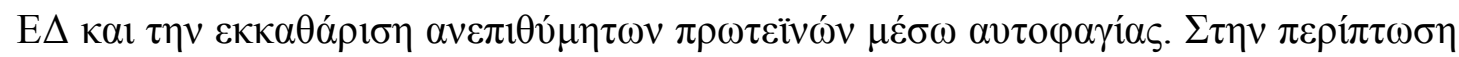

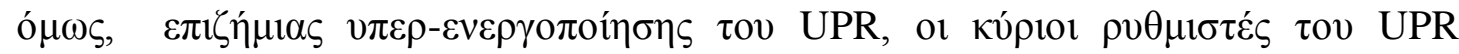

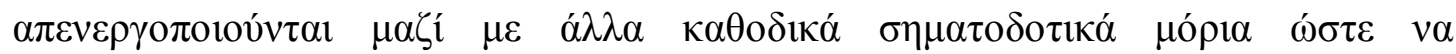




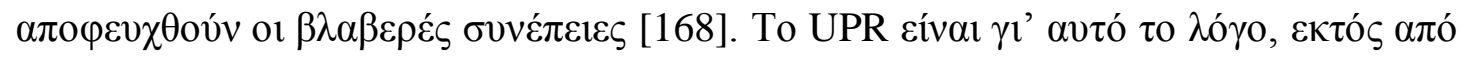

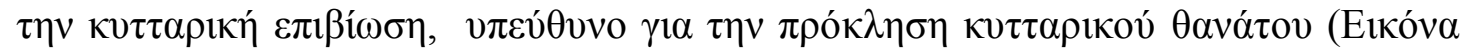
1.35) [166].

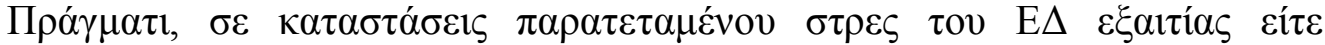

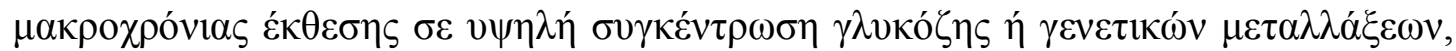

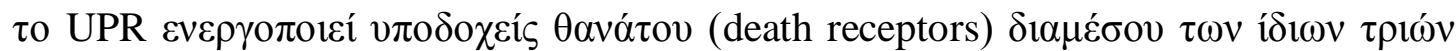

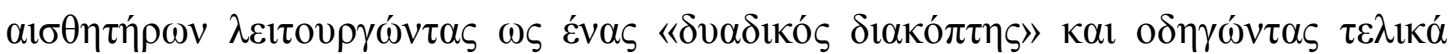
$\sigma \tau \eta v \alpha \pi{ }_{\pi} \tau \omega \sigma \eta[168]$.

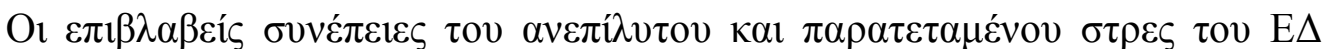

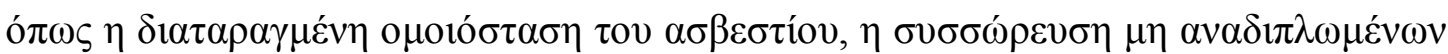

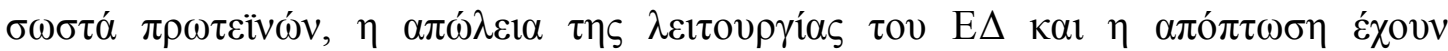

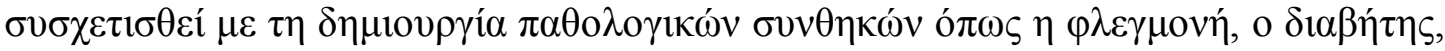

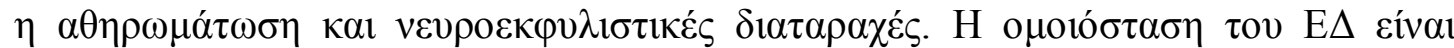

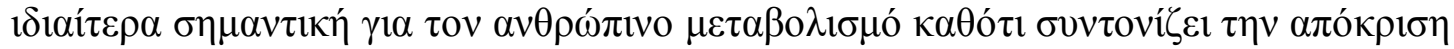

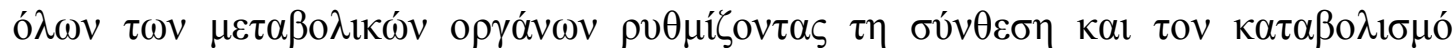

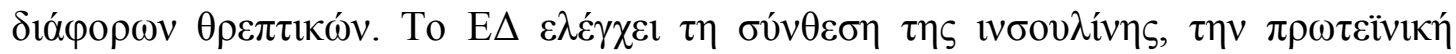

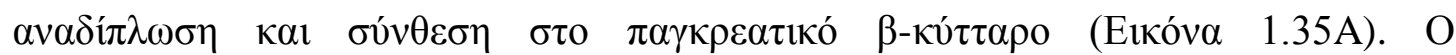

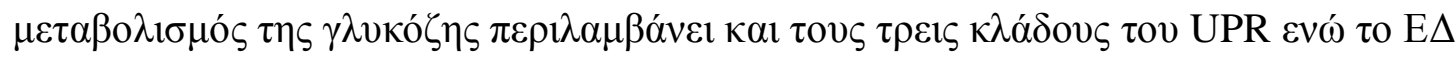

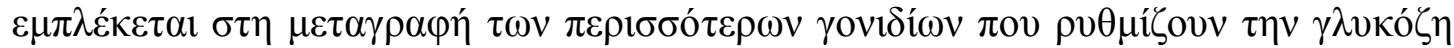
[169].

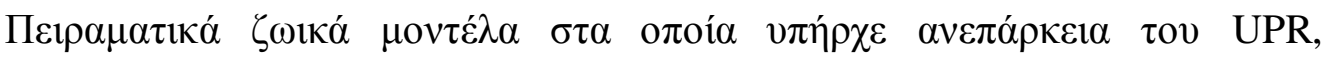

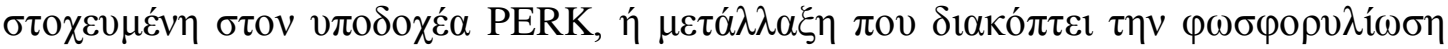

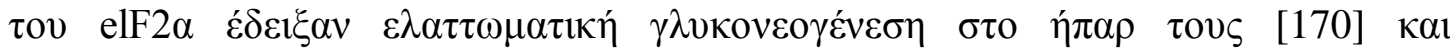

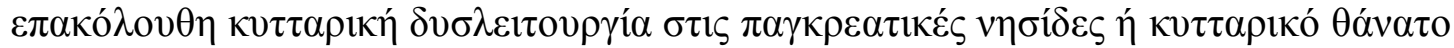

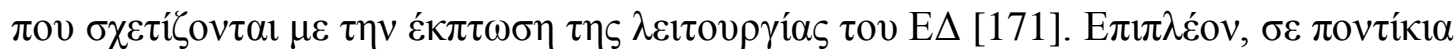

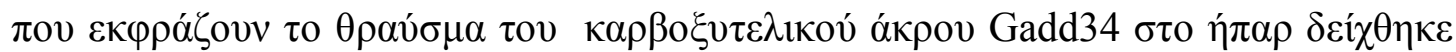

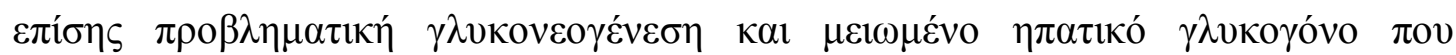

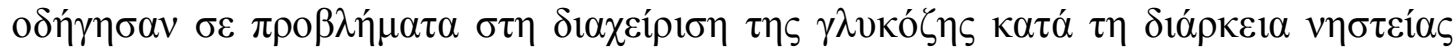

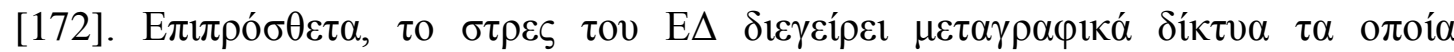

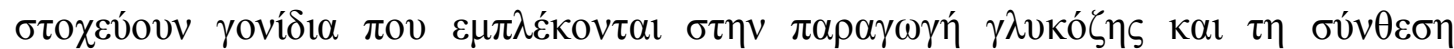

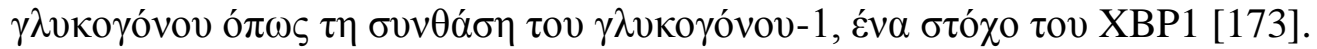




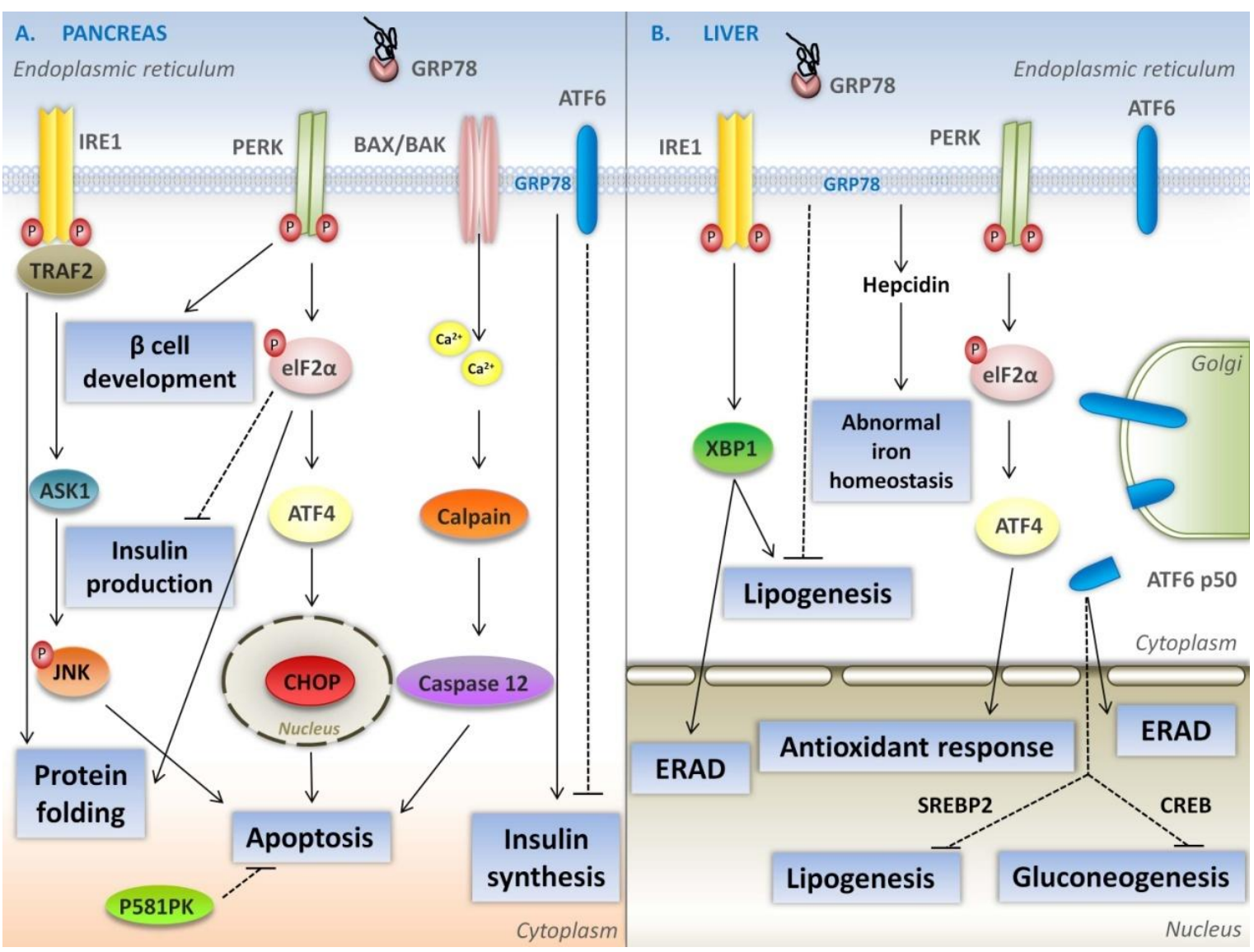

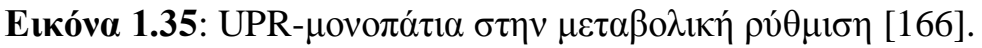

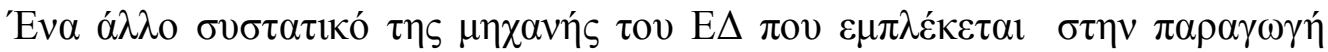

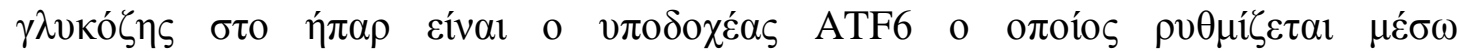

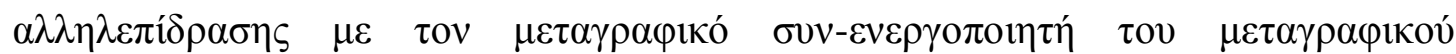
$\pi \alpha \rho \alpha ́ \gamma o v \tau \alpha$ CREB (cAMP response element binding), CRTC2/TORC2 (CREB-

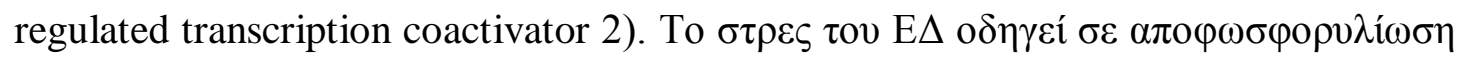

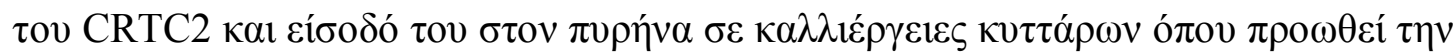

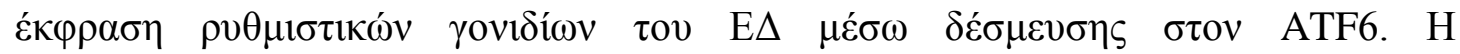

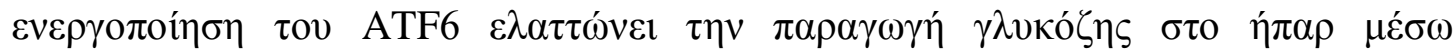

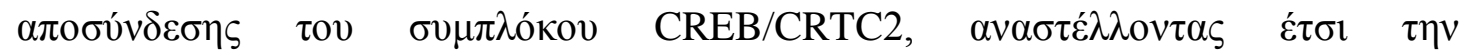

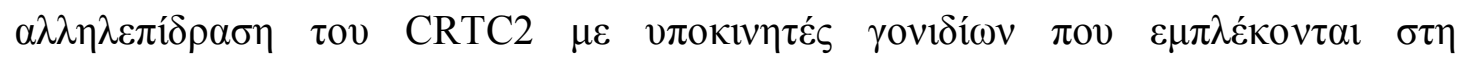

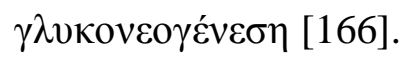

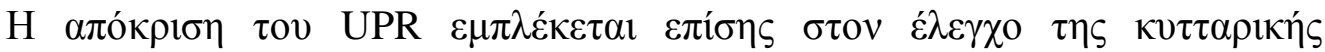

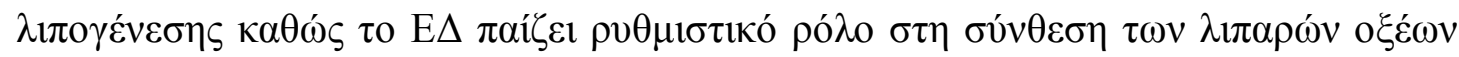




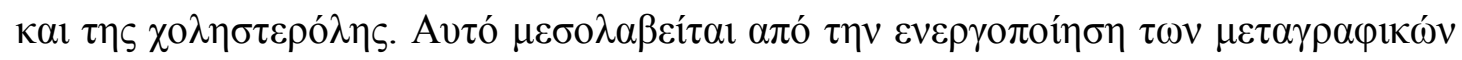
$\pi \alpha \rho \alpha \gamma o ́ v \tau \omega v$ SREBPs (sterol regulatory element-binding proteins), ol о $\pi$ oíor

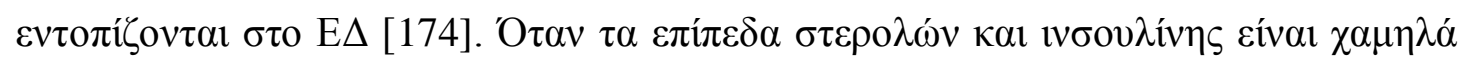

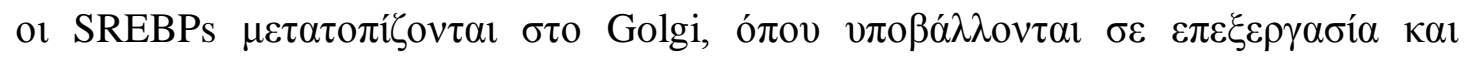

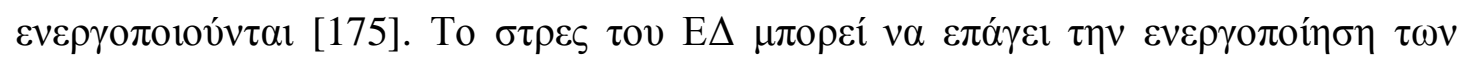

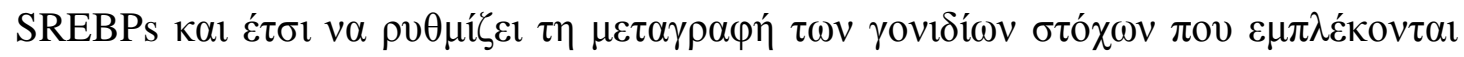

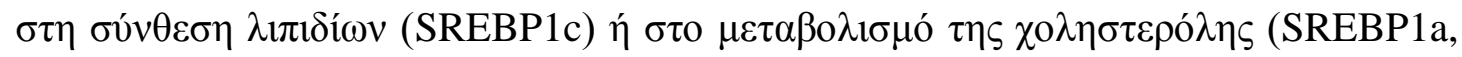

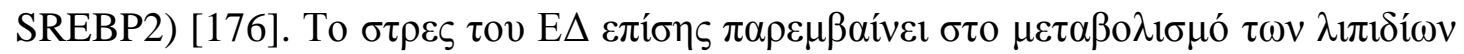

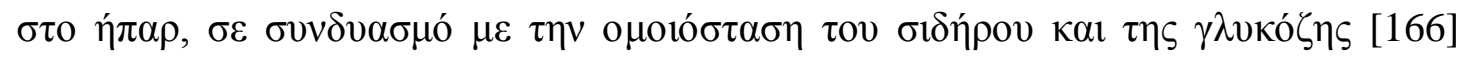
(Eıкóva 1.35B).

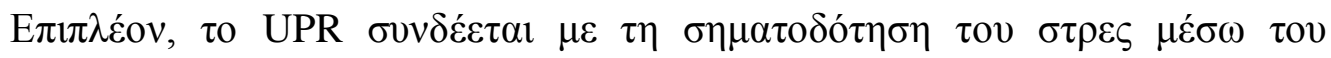

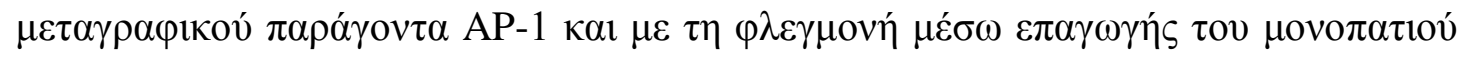

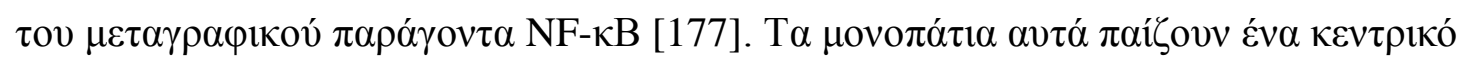

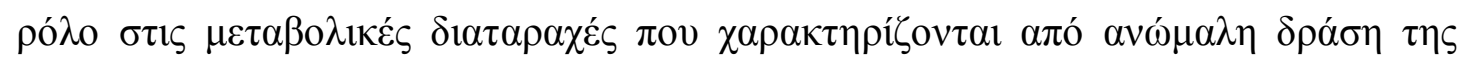

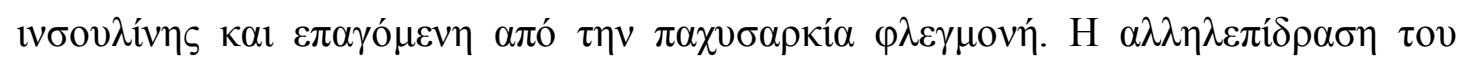

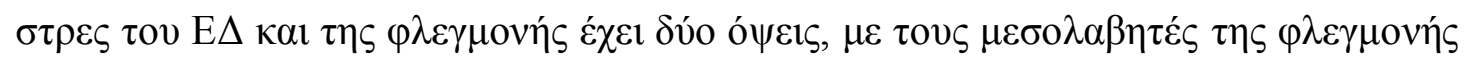

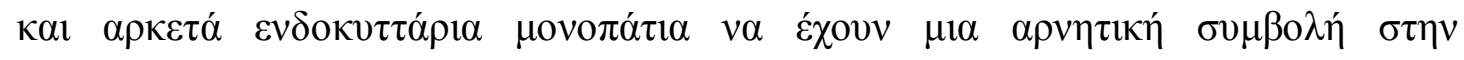

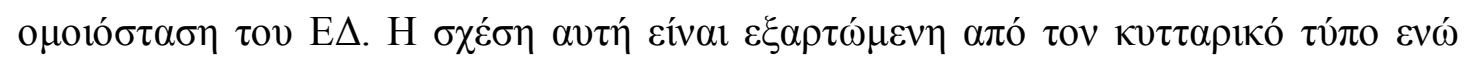

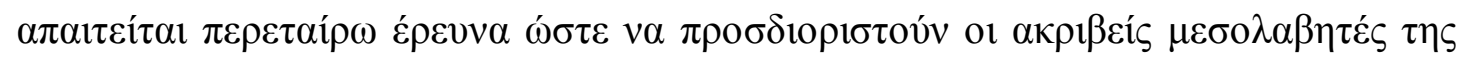

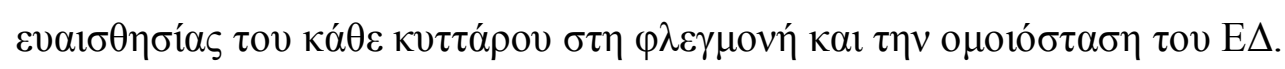

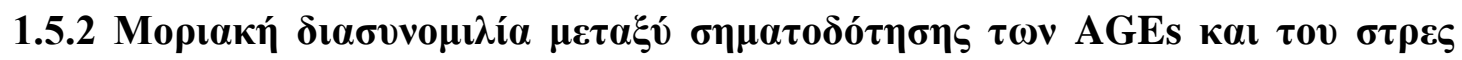 $\tau \mathbf{\tau o v} \mathbf{E} \Delta$}

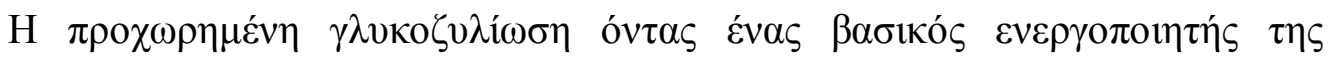

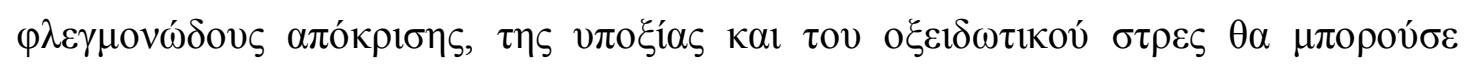

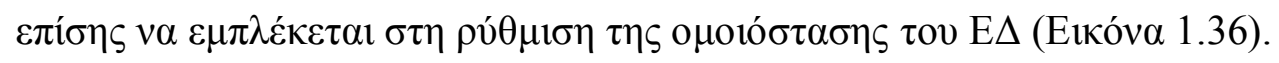




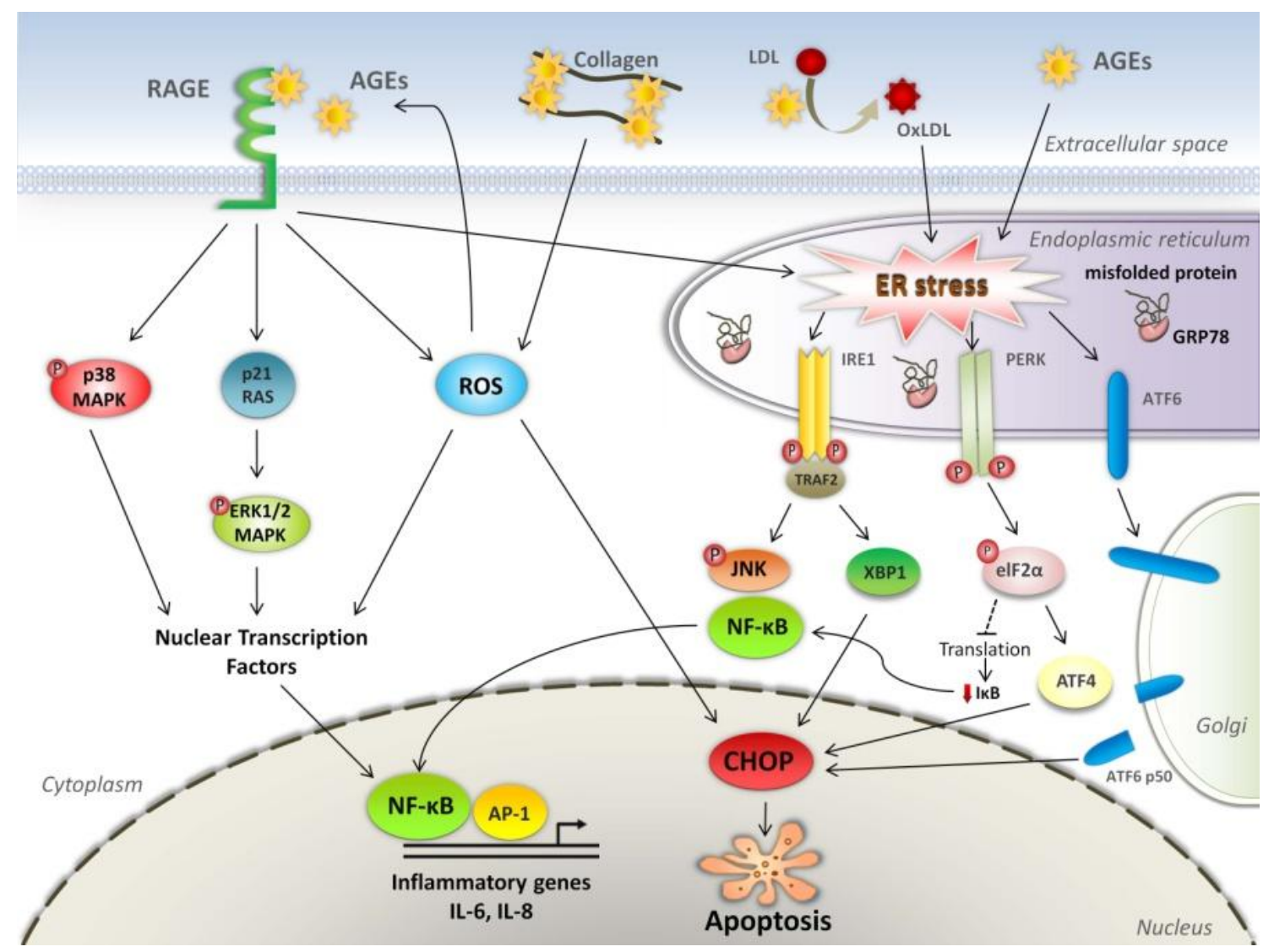

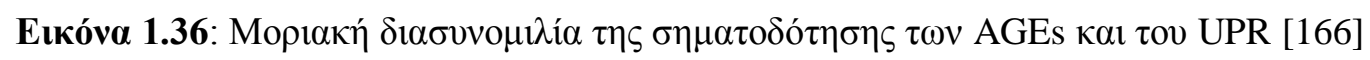

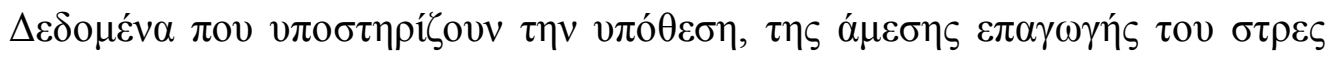

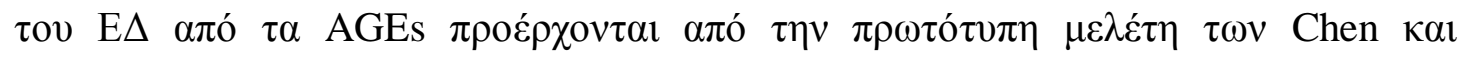

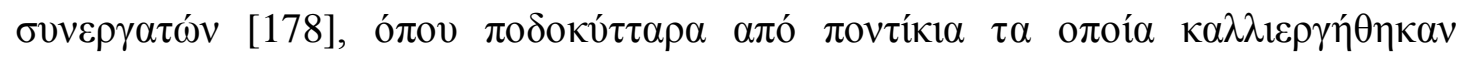

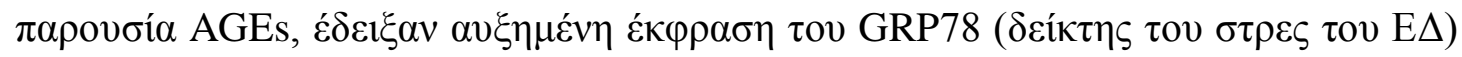

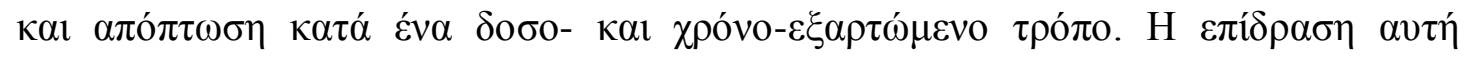

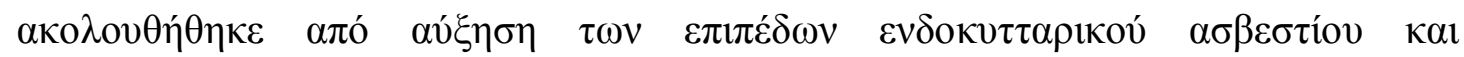

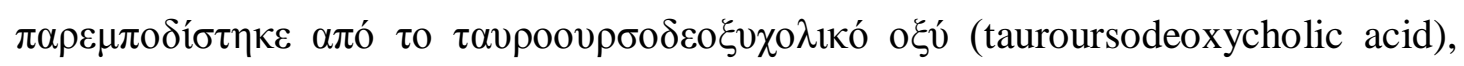

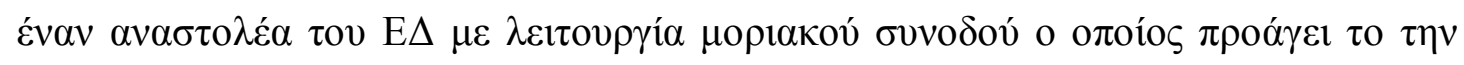

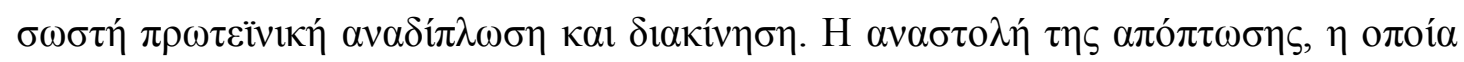

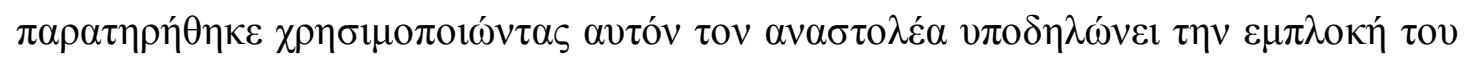

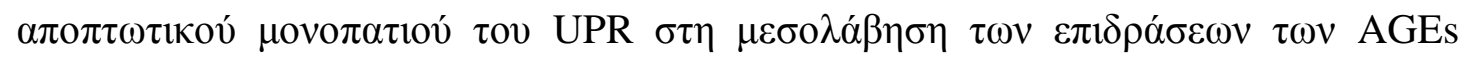

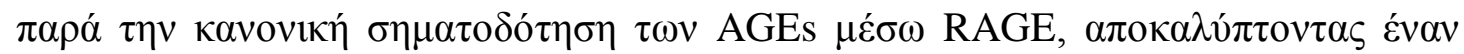

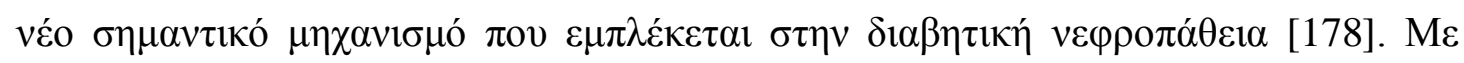

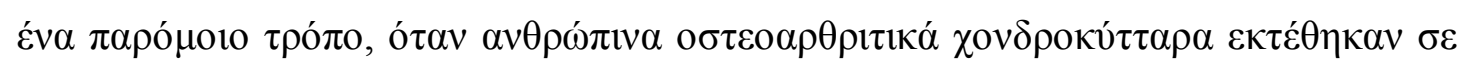

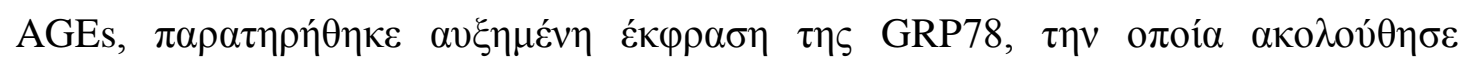




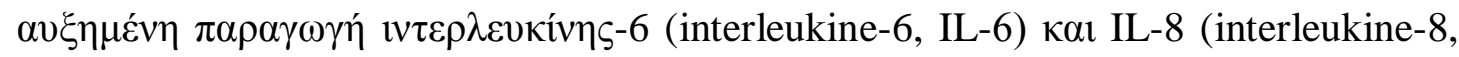

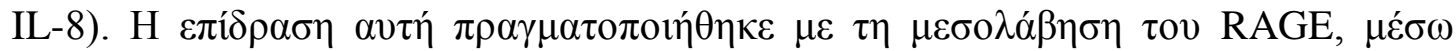

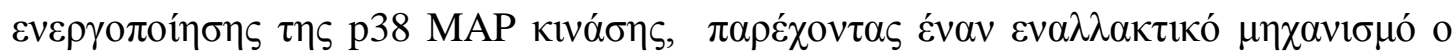

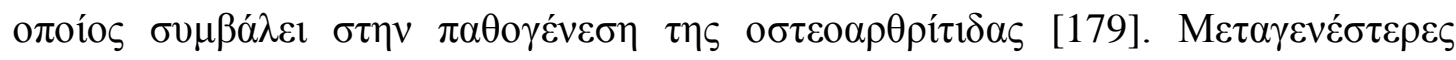
$\mu \varepsilon \lambda \varepsilon \dot{\tau \varepsilon \varsigma} \mu \varepsilon \alpha v \theta \rho \omega ́ \pi ı v o v \varsigma \delta \varepsilon \rho \mu \alpha \tau$ เ

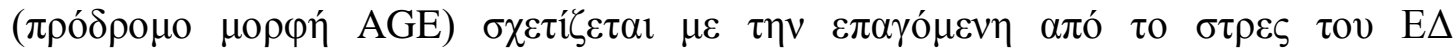

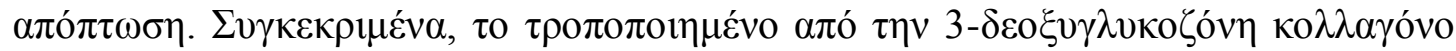

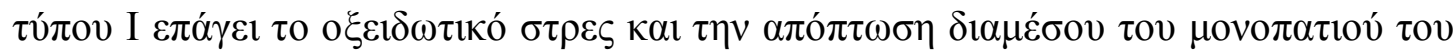

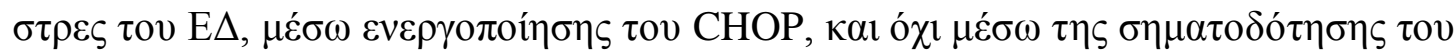

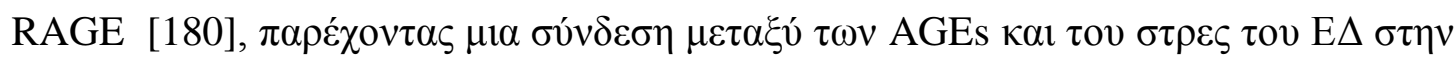

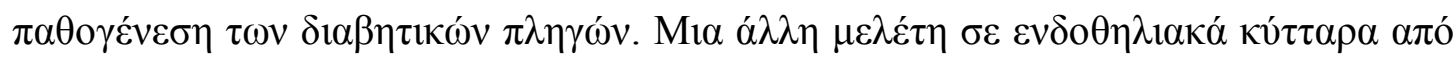

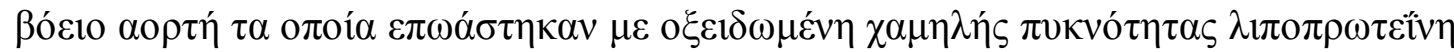

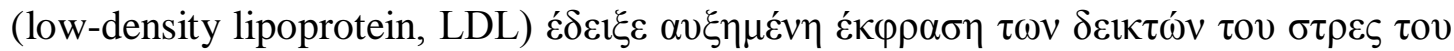

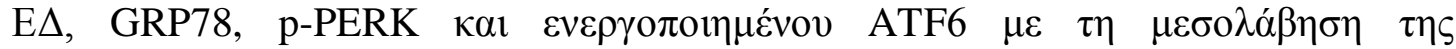

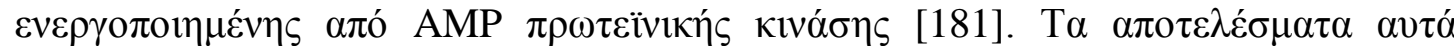

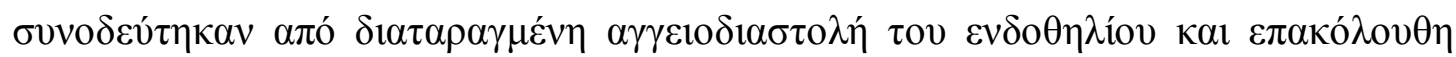

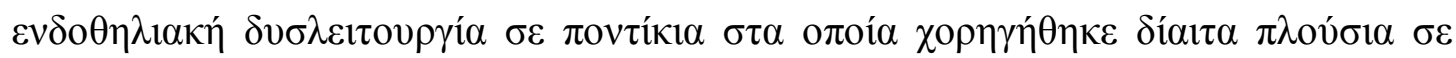
$\lambda \imath \pi \alpha \rho \alpha$. 


\section{2. $\Sigma$ КОПО $\Sigma$}

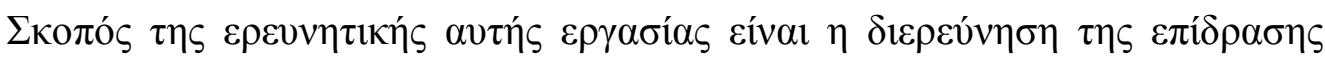

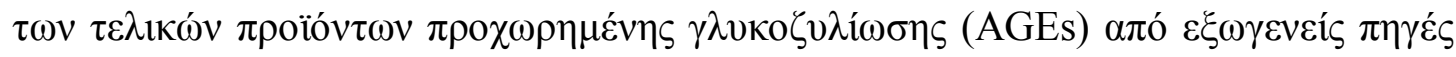

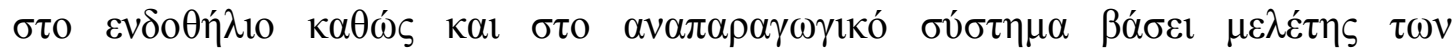

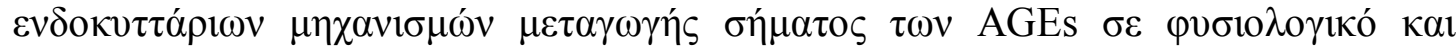

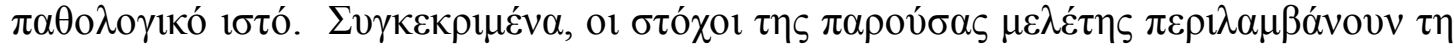

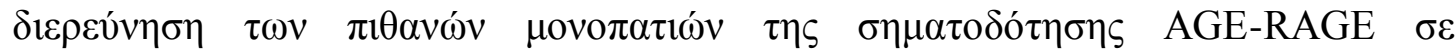

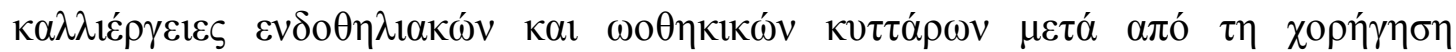

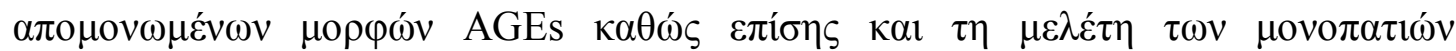

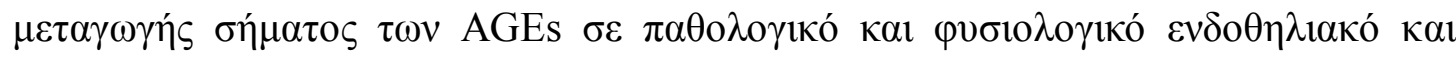

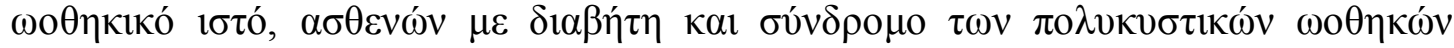

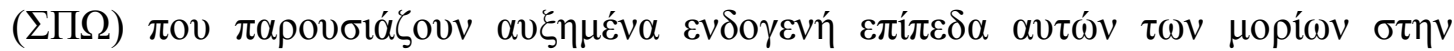

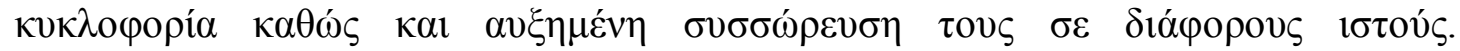

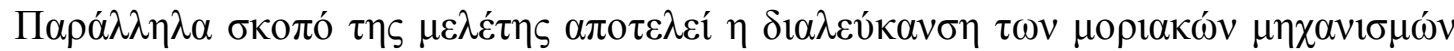

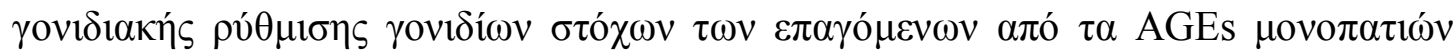

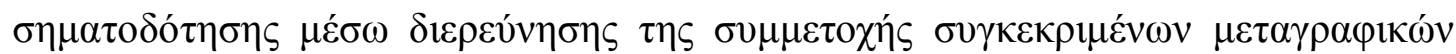
$\pi \alpha \rho \alpha \gamma o ́ v \tau \omega v$. 


\section{Y}

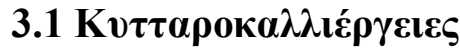

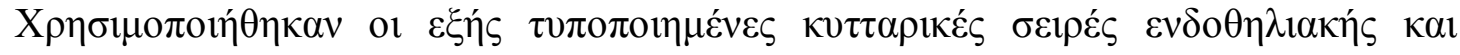

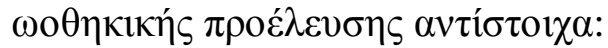

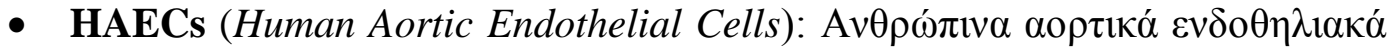
$\kappa v ́ \tau \tau \alpha \rho \alpha$

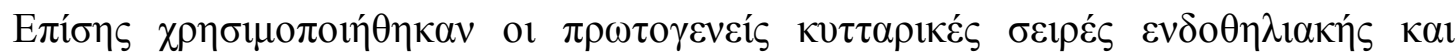

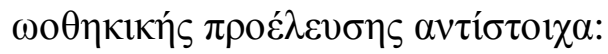

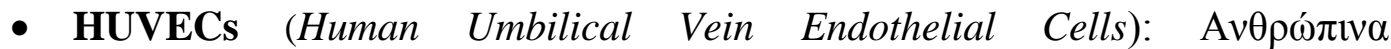

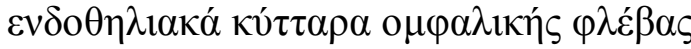

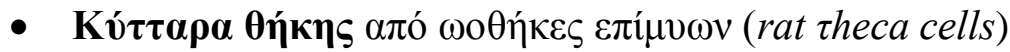

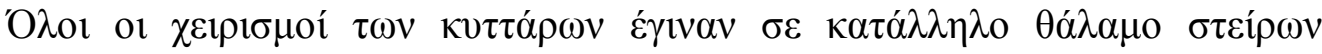

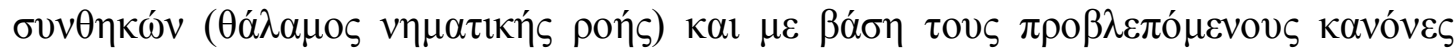

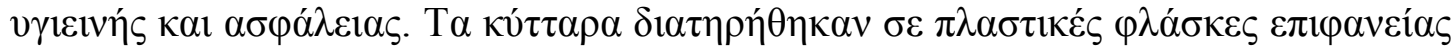

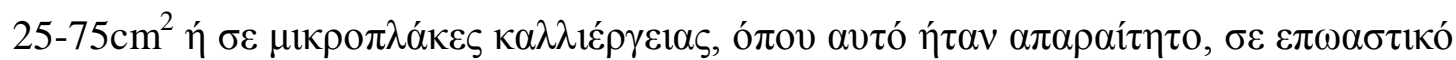

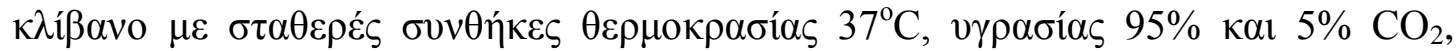

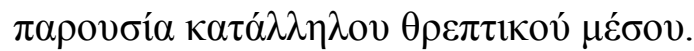

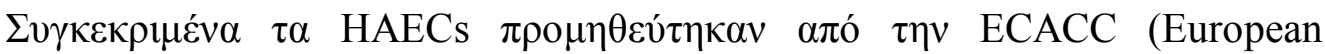

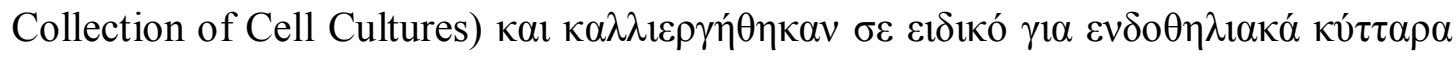

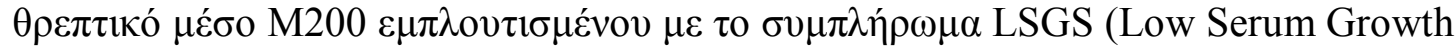

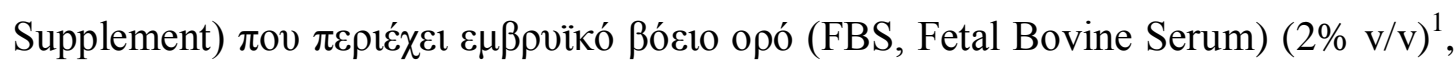

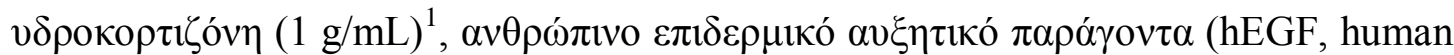

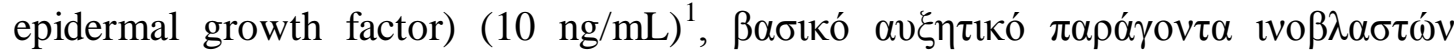
(bFGF, basic fibroblast growth factor) $(3 \mathrm{ng} / \mathrm{mL})^{1}, \eta \pi \alpha \rho i ́ v \eta(10 \mathrm{~g} / \mathrm{mL})^{1}, \gamma \varepsilon v \tau \alpha \mu v \kappa i ́ v \eta$ $(50 \mu \mathrm{g} / \mathrm{mL})^{1} \kappa \alpha \imath \alpha \mu \varphi о \tau \varepsilon \rho 1 \kappa i ́ v \eta ~(50 \mathrm{ng} / \mathrm{mL})^{1}$.

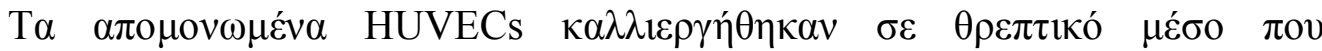

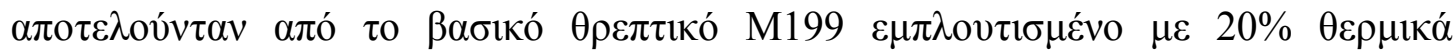

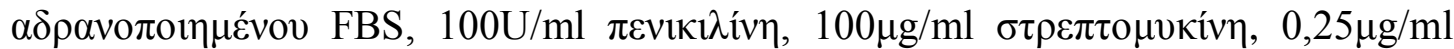

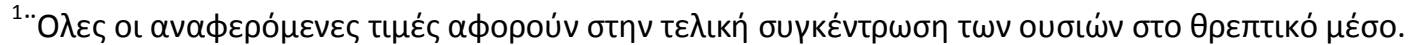




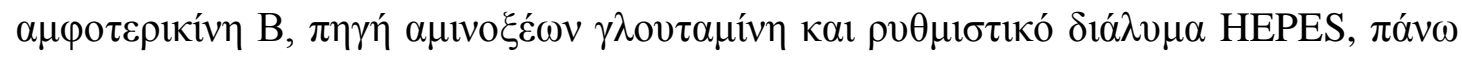

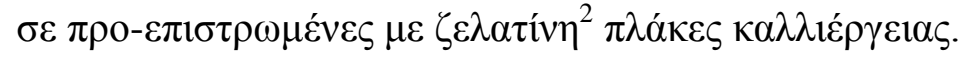

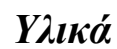

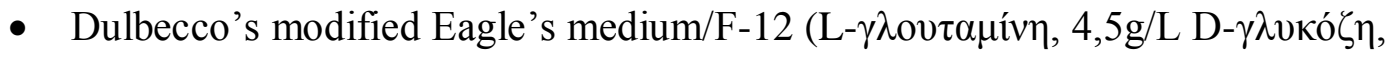

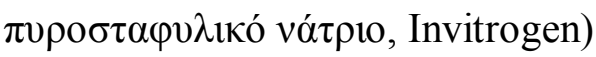

- Medium M200 (Invitrogen)

- Low Serum Growth Supplement (Invitrogen)

- Medium McCoy's 5A (Sigma-Aldrich St. Louis, MO)

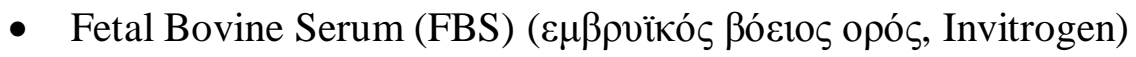

- Bovine Serum Albumin (BSA)

- Insulin (100ng/ml) (Lilly, Indianapolis, Indiana, USA)

- Antibiotic/Antimycotic (100X) (10000U/ml $\pi \varepsilon v \imath \kappa ı \lambda i ́ v \eta, 10000 \mu \mathrm{g} / \mathrm{ml}$

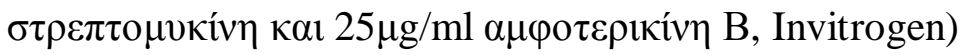

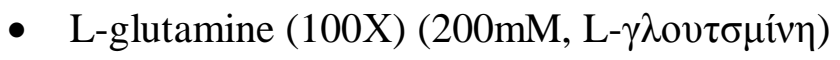

- Trypsin-EDTA (0,5\%w/v $\theta \rho v \psi i ́ v \eta ~ \kappa \alpha ~ 0,53 \mathrm{mM}$ EDTA, Invitrogen)

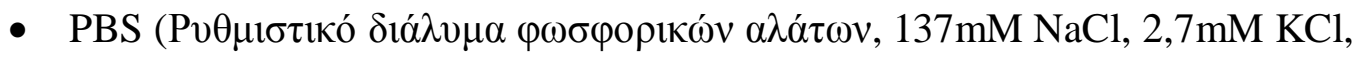
$8 \mathrm{mM} \mathrm{Na}_{2} \mathrm{HPO}_{4} \times 7 \mathrm{H}_{2} \mathrm{O}$, Invitrogen)

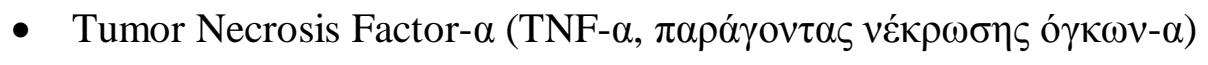

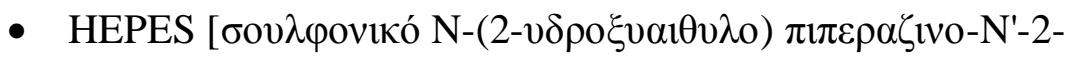
$\alpha \imath \theta \alpha ́ v i o]($ Invitrogen)

- Human fibronectin (Sigma-Aldrich St. Louis, MO-Aldrich)

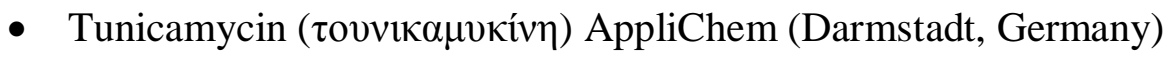

- Human gelatin (Sigma-Aldrich St. Louis, MO-Aldrich

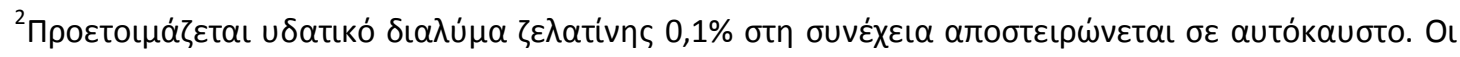

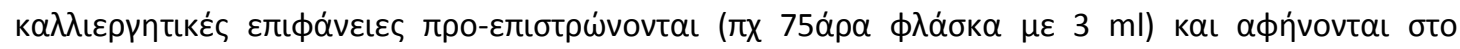

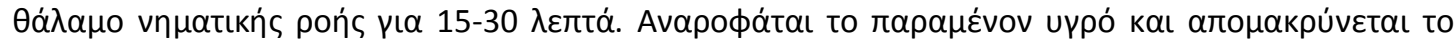

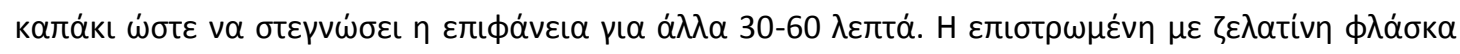

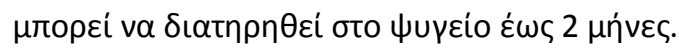




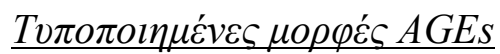

- $\Gamma \lambda v o \xi \alpha ́ \lambda \eta(G:$ Glyoxal)(Sigma-Aldrich St. Louis, MO)

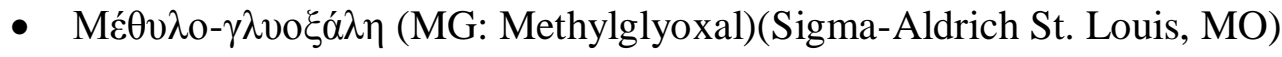

- $\operatorname{K} \alpha \rho \beta 0 \xi v-\mu \varepsilon \theta v \lambda-\lambda v \sigma i ́ v \eta(\mathrm{CML})$

- AGE-bovine serum albumin (AGE/BSA) (Abcam, Cambridge, UK)

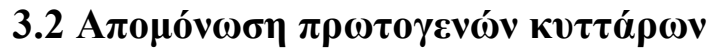

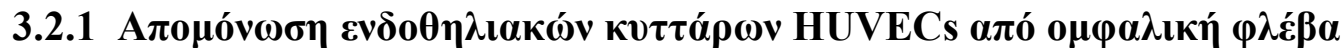

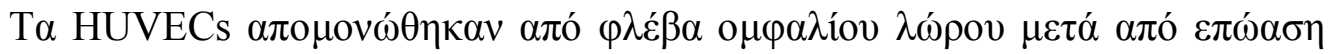

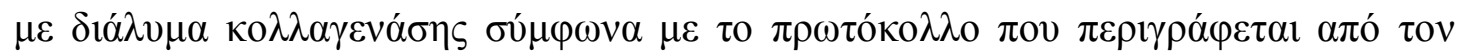

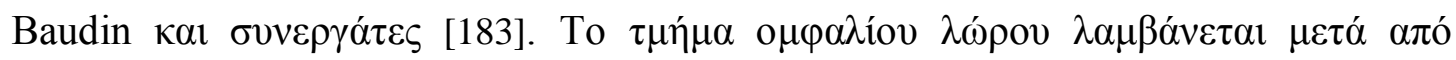

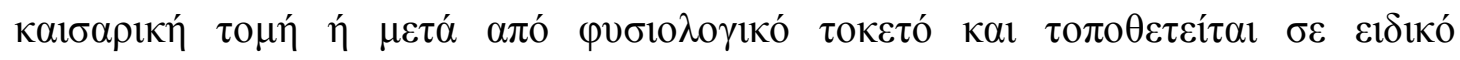

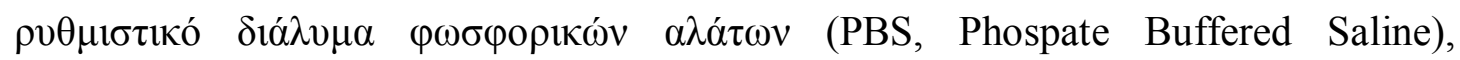

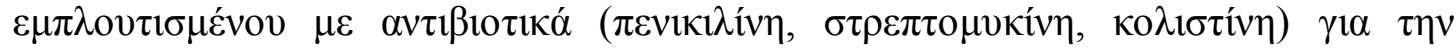

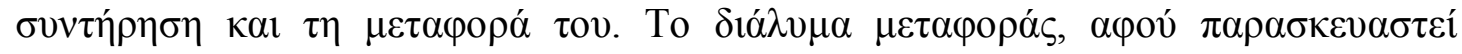

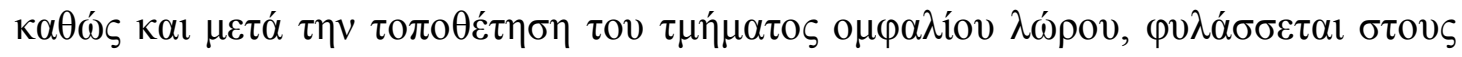

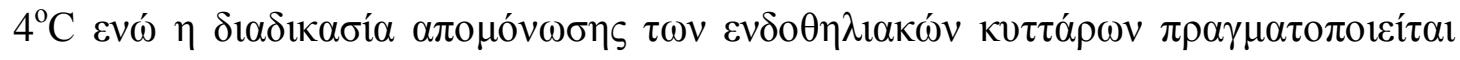

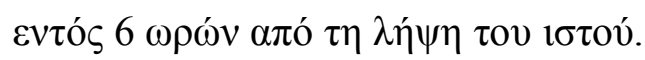

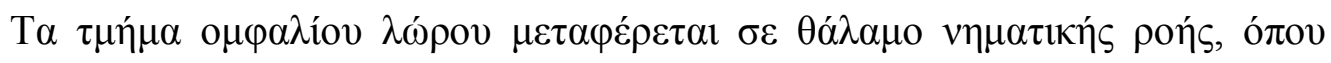

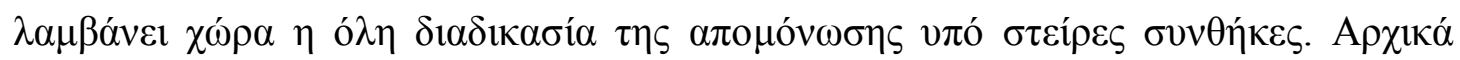

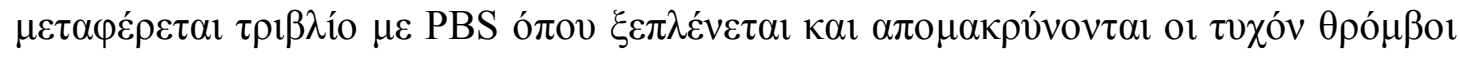

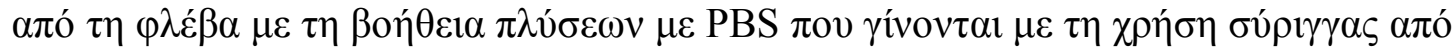

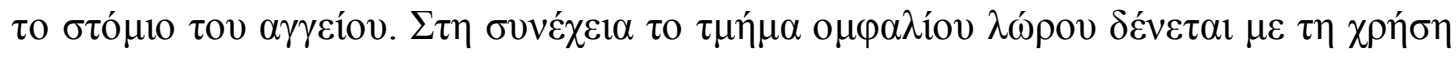

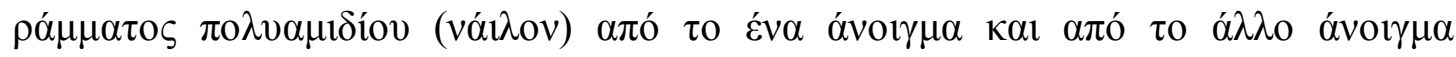

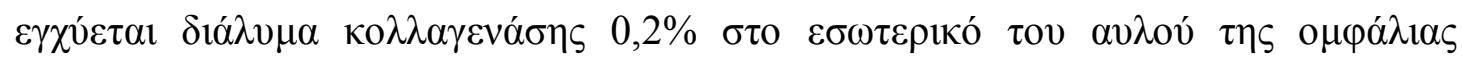

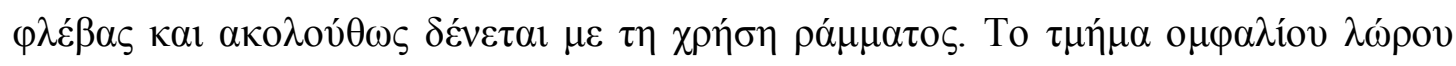

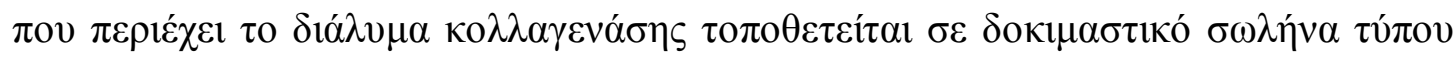

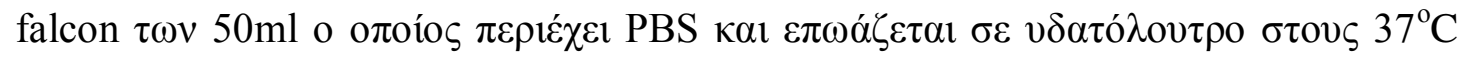
$\gamma 1 \alpha 10 \lambda \varepsilon \pi \tau \alpha$.

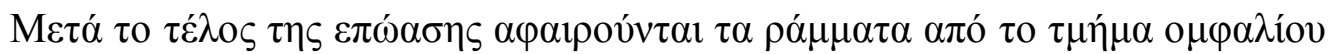

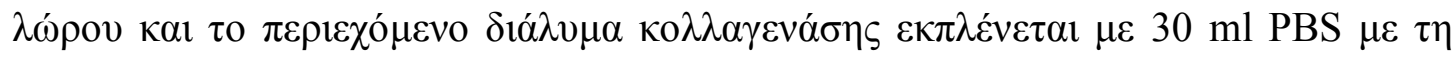




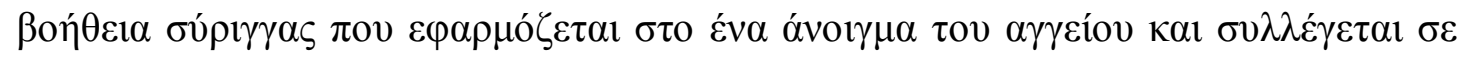

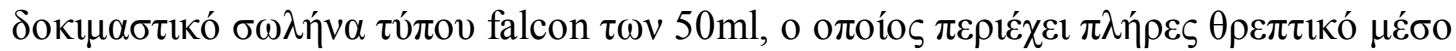

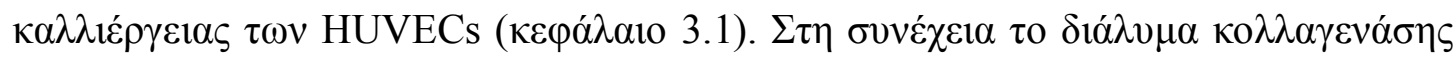

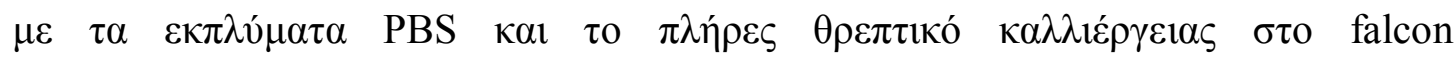

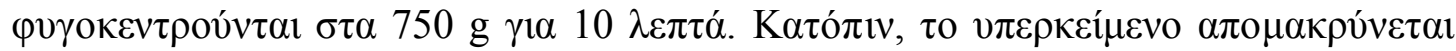

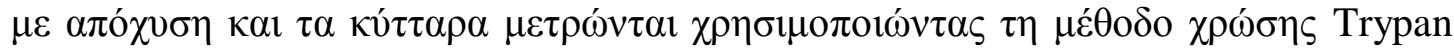

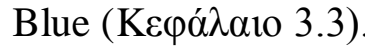

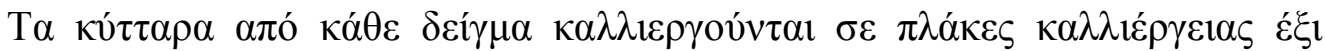

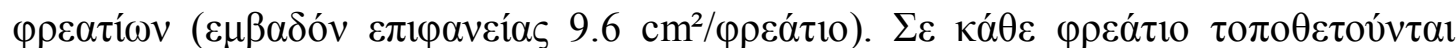

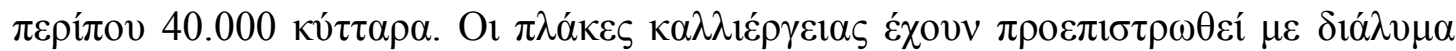

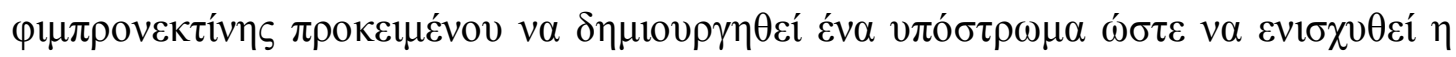

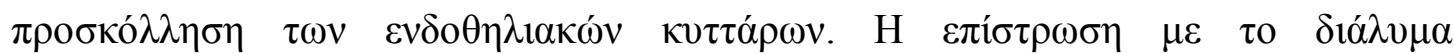

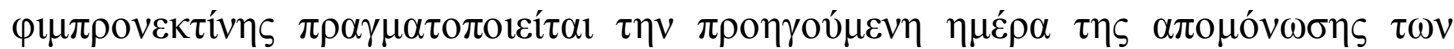

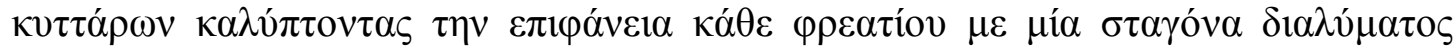

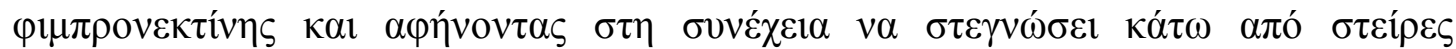

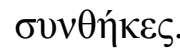

\section{$\sum v \lambda \lambda \sigma \gamma \eta \dot{~} \delta \varepsilon \imath \gamma \mu \alpha \dot{\tau} \omega \nu$}

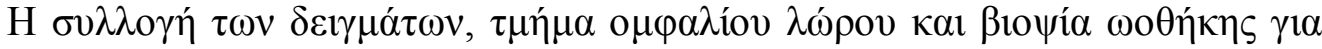

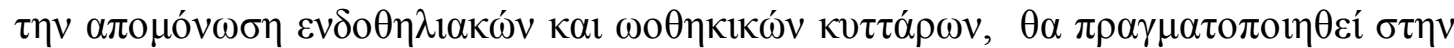

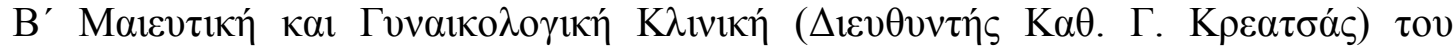

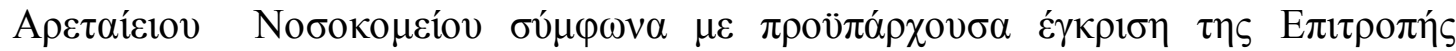

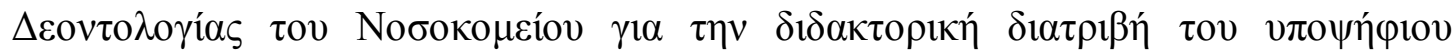

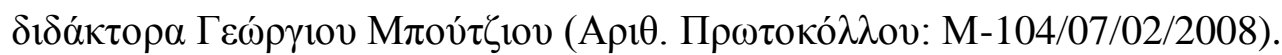




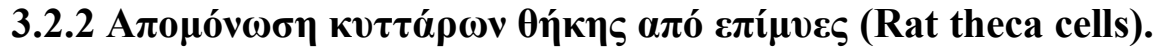

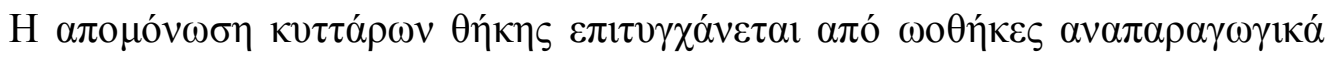

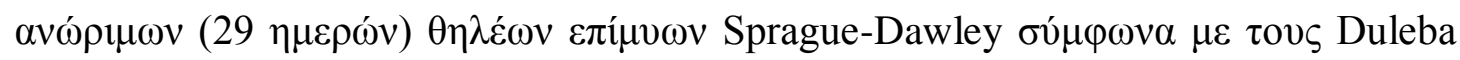

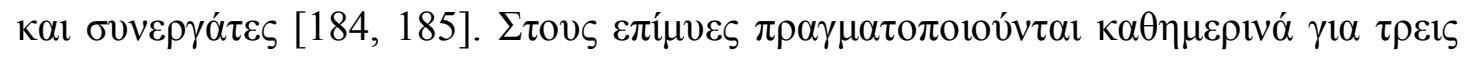

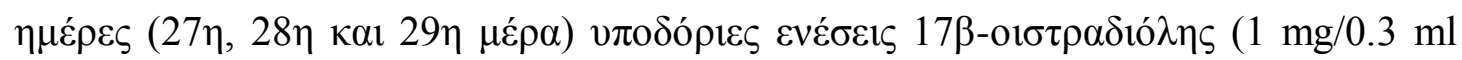

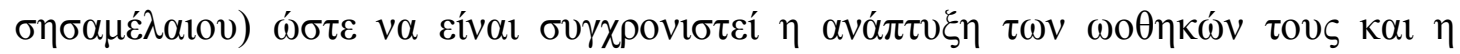

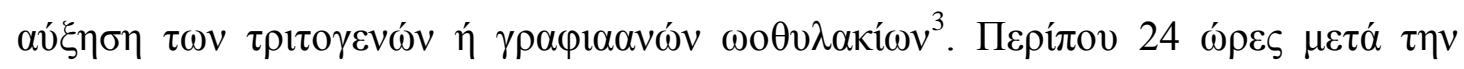

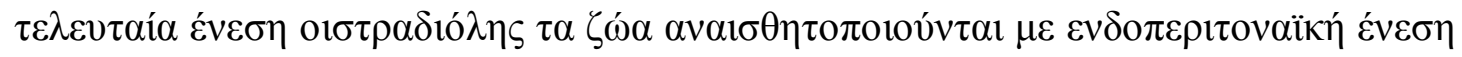

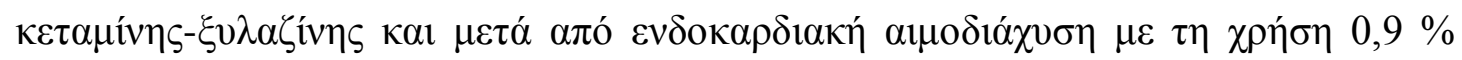

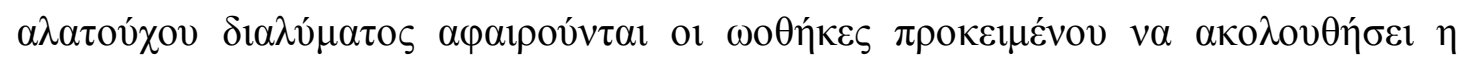

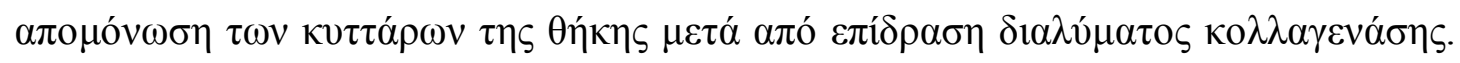

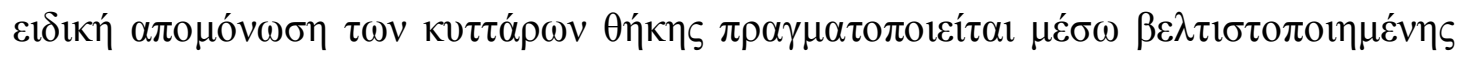

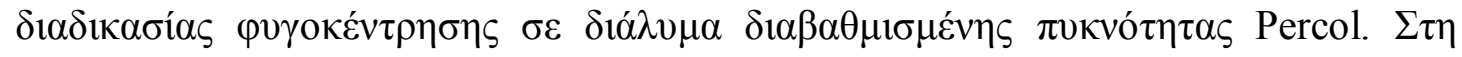

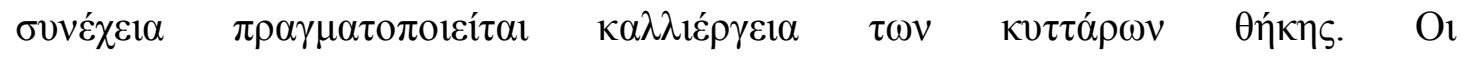

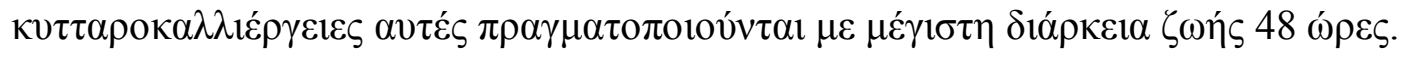

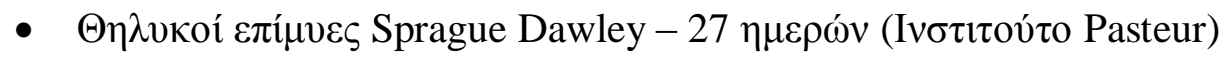

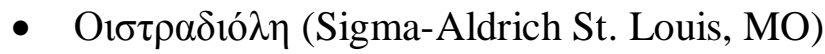

- Sesame oil (Sigma-Aldrich St. Louis, MO)

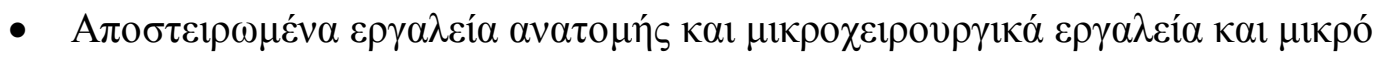

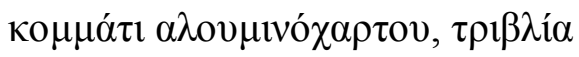

- M199 with Hank’s Salts (Sigma-Aldrich St. Louis, MO)

- McCoy5a (Sigma-Aldrich St. Louis, MO)

- L-Glutamine (Invitrogen)

- Antibiotics/Antimycotics (Invitrogen)

- BSA (Sigma-Aldrich St. Louis, MO)

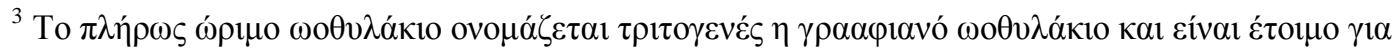

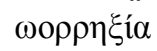




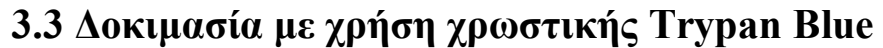

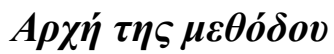

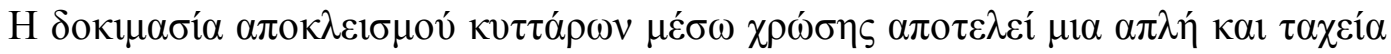

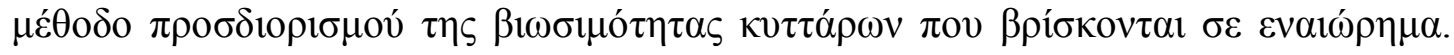

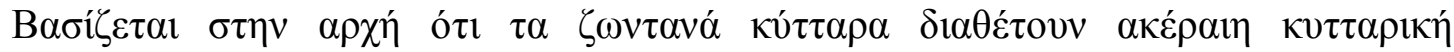

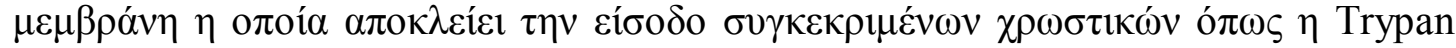

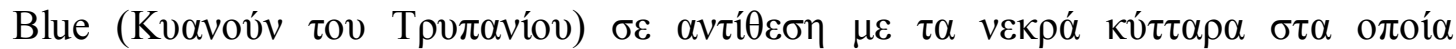

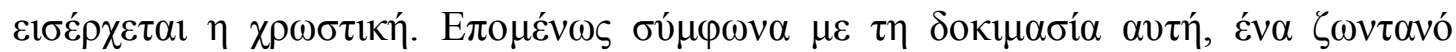

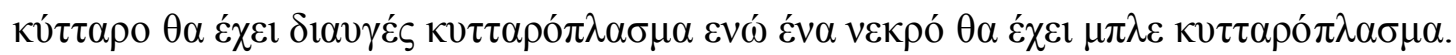

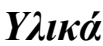

- Dulbecco's Modified Eagle Medium-DMEM (Invitrogen)

- Fetal Bovine Serum (FBS) (Invitrogen)

- Antibiotic/Antimycotic (100X) (Invitrogen)

- PBS (Invitrogen)

- Trypsin-EDTA (Invitrogen)

- Trypan Blue (Invitrogen)

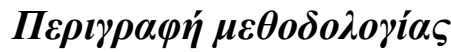

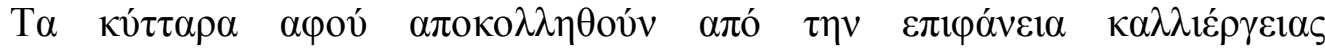

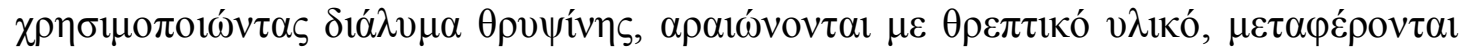

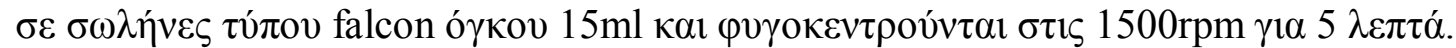

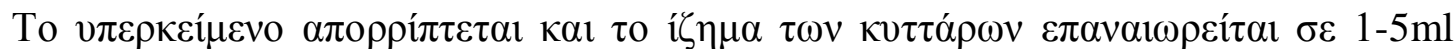

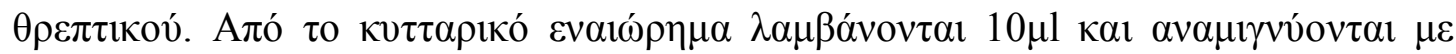

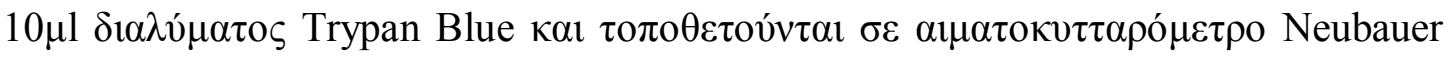

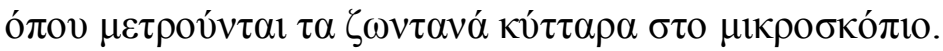




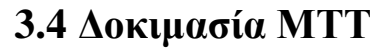

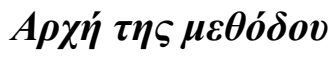

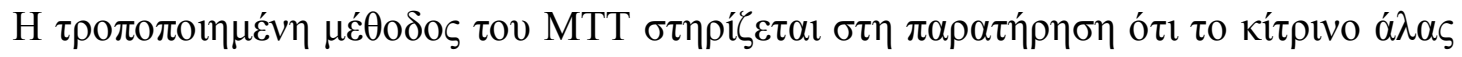

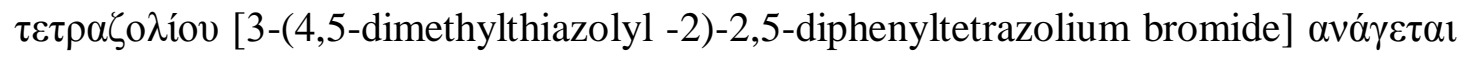

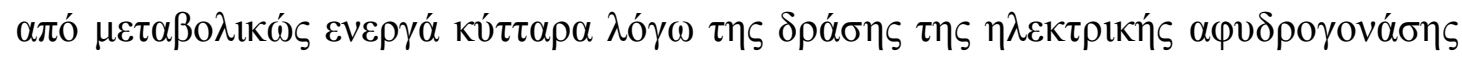

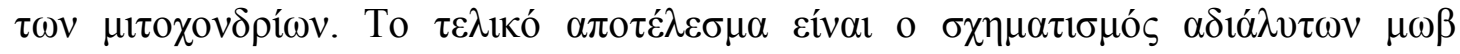

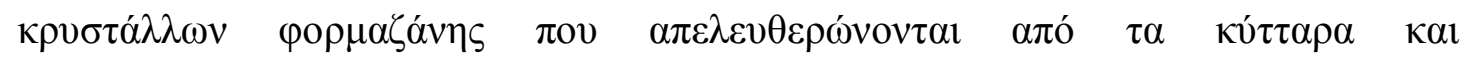

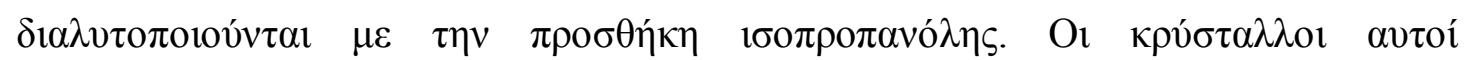

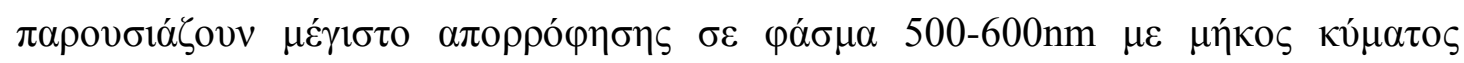

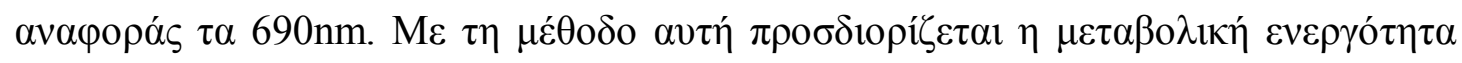

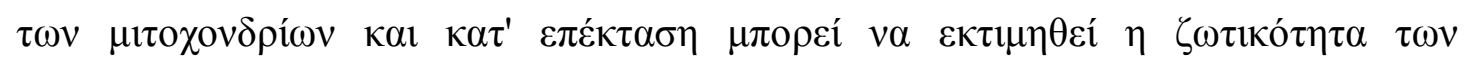

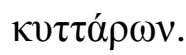

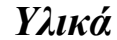

- Dulbecco's Modified Eagle Medium-DMEM (Invitrogen)

- Fetal Bovine Serum (FBS) (Invitrogen)

- Antibiotic/Antimycotic (100X) (Invitrogen)

- PBS 1X (Invitrogen)

- $\Delta \mathrm{lá} \lambda v \mu \alpha \varepsilon \rho \gamma \alpha \sigma i ́ \alpha \varsigma$

250ml DMEM phenol red free (Invitrogen)

$1 \mathrm{ml} \mathrm{NaHCO} 37,5 \% \mathrm{w} / \mathrm{v}$

2,5ml NEA (Invitrogen)

2,5ml HEPES 1M (Invitrogen)

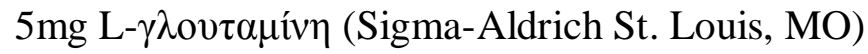

- $5 \mathrm{mg} / \mathrm{ml}$ Thiazolyl blue $\mathrm{C}_{18} \mathrm{H}_{16} \mathrm{~N}_{5} \mathrm{SBr}$ (MTT) (Sigma-Aldrich St. Louis, MO)

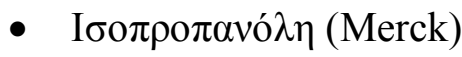

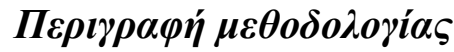

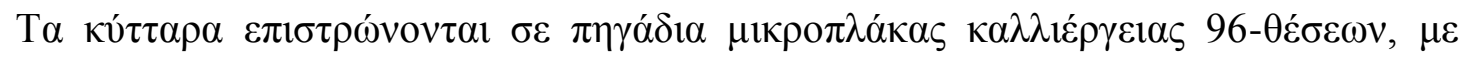

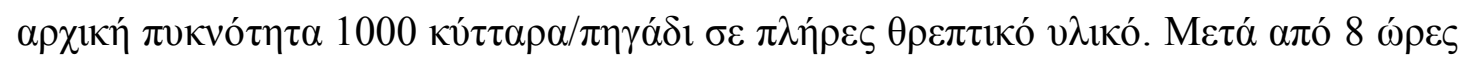

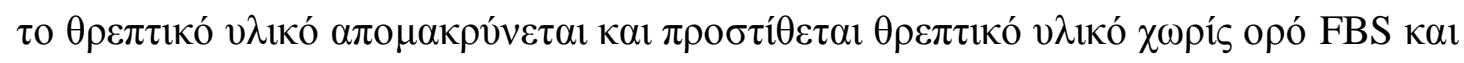

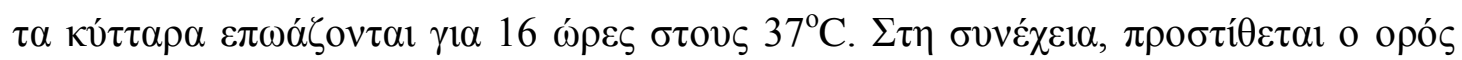




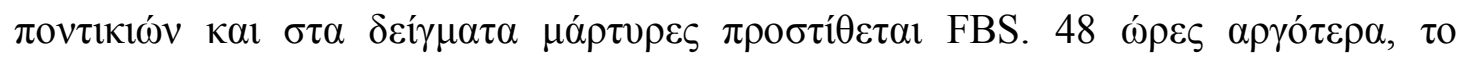

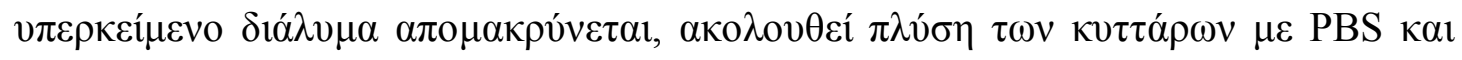

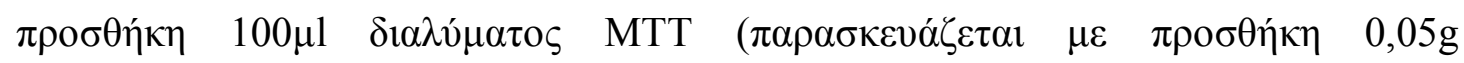

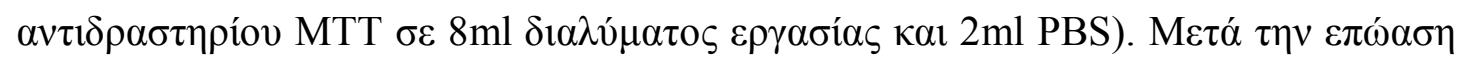

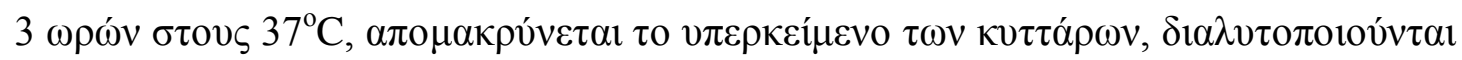

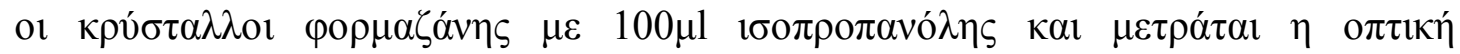

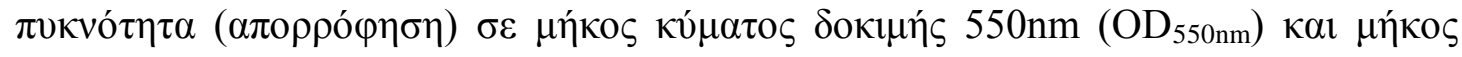

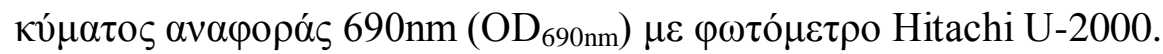

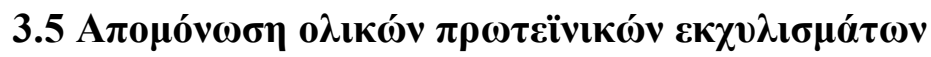

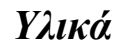

- Dulbecco's Modified Eagle Medium-DMEM (Invitrogen)

- Fetal Bovine Serum (FBS) (Invitrogen)

- Antibiotic/Antimycotic (100X) (Invitrogen)

- PBS (Invitrogen)

- Trypsin-EDTA (Invitrogen)

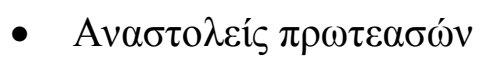

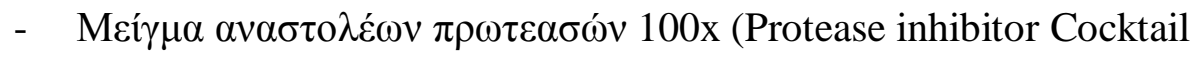
kit, Thermo Scientific)

- 1mM PMSF (phenylmethanesulfonylfluoride)

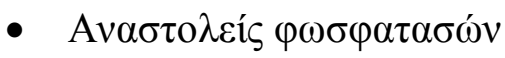

- 2,5mM sodium pyrophosphate,

- $50 \mathrm{mM} \mathrm{NaF}$ (sodium fluoride)

- $1 \mathrm{mM} \beta$-glycerophosphate,

- $1 \mathrm{mM} \mathrm{Na}_{3} \mathrm{VO}_{4}$ (sodium orthovanadate)

- $\quad \underline{\mathrm{t} \alpha} \lambda v \mu \alpha \operatorname{RIPA}$ (Thermo Scientific)

$25 \mathrm{mM}$ Tris- $\mathrm{HCl}(\mathrm{pH} 7,6)$

$150 \mathrm{mM} \mathrm{NaCl}$

$1 \%$ NP-40

$1 \% \Delta \varepsilon 0 \xi v \chi 0 \lambda$ 1кó vó

$0,1 \%$ SDS (Sodium Dodecyl Sulfate) 


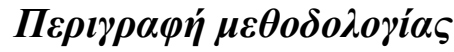

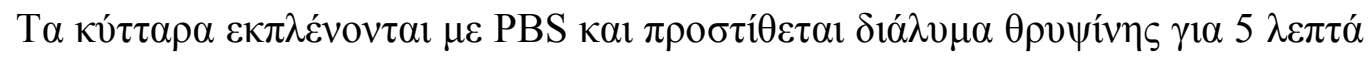

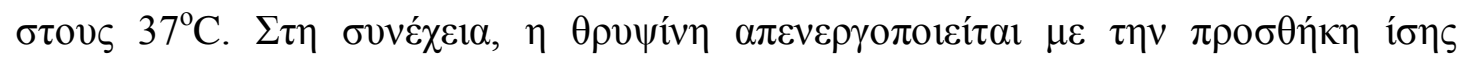

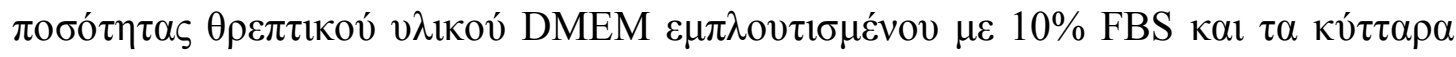

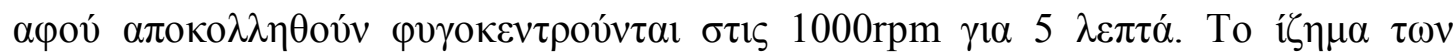

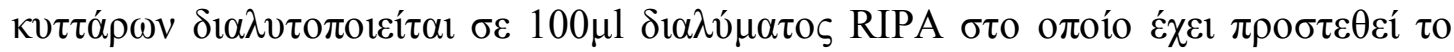

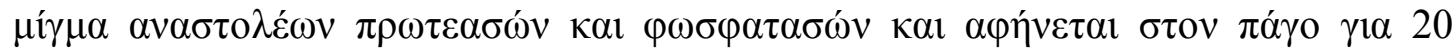

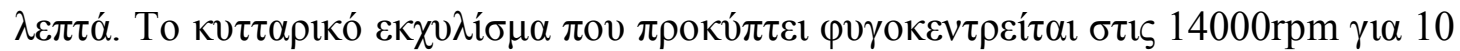

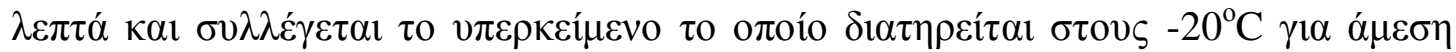

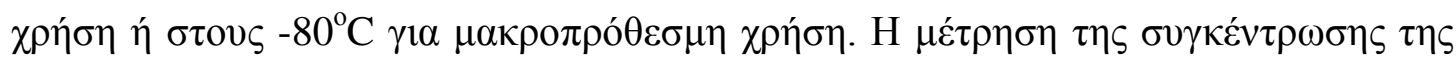

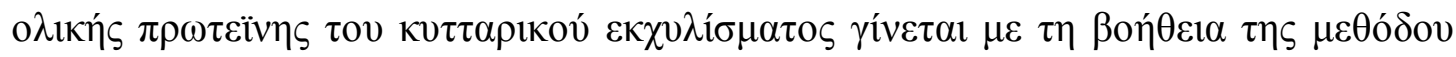

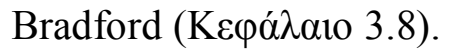

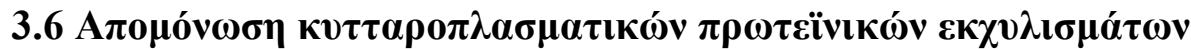

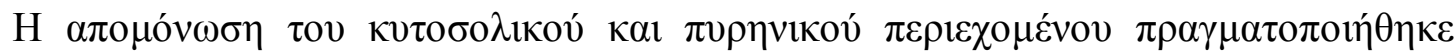

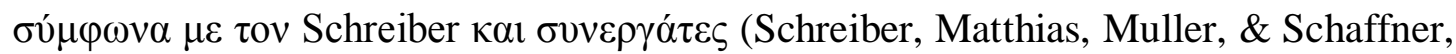

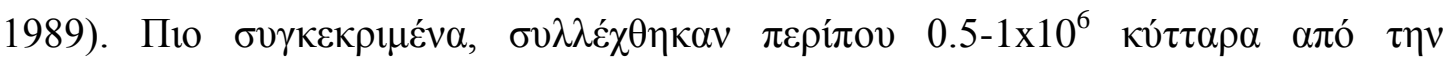

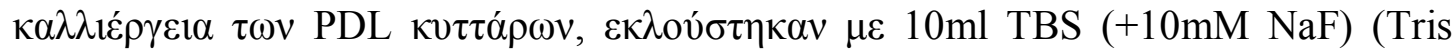

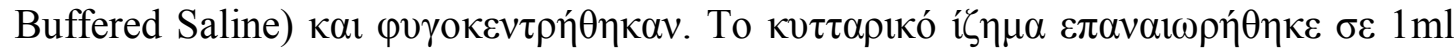

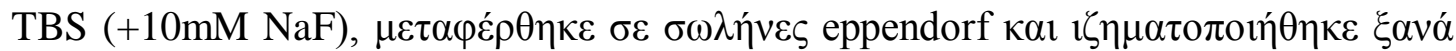

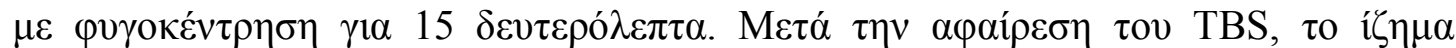

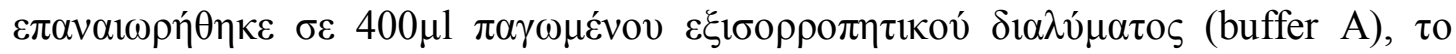

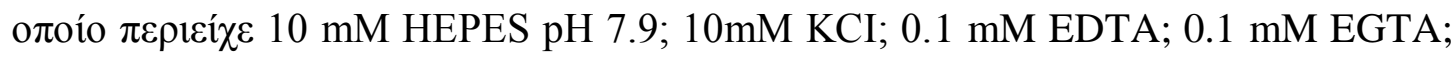

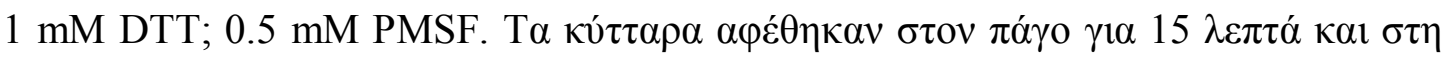

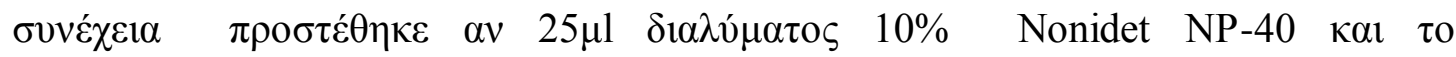

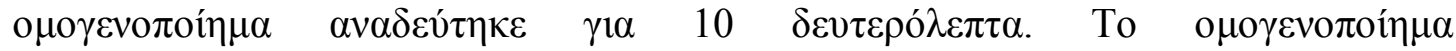

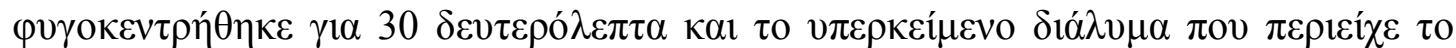

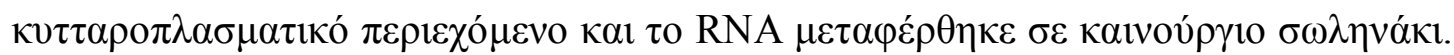

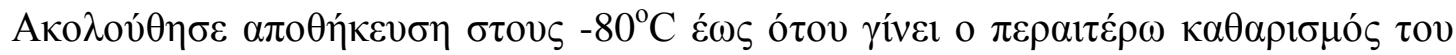
RNA. 


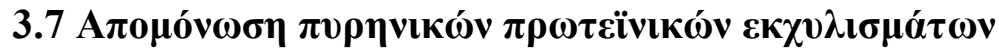

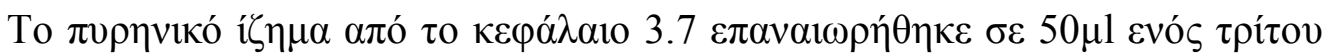
$\pi \alpha \gamma \omega \mu \varepsilon ́ v o v$ buffer C (20mM HEPES pH 7.5; $0.4 \mathrm{M} \mathrm{NaCl} ; 20 \%$ Glycerol; $2 \mathrm{mM}$ $\mathrm{MgCl} 2$; 30mM NaF; 0,1mM Na3VO4; 2 mM DTT; 2 mM PMSF; 5 $\mu \mathrm{g} / \mathrm{ml}$ aprotinin,

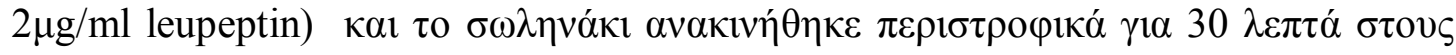

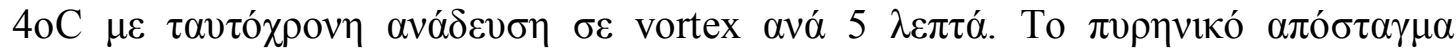

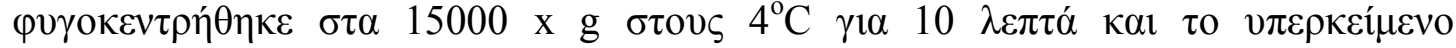
$\alpha \pi \circ \theta \eta \kappa \varepsilon v ́ \tau \eta \kappa \varepsilon \sigma \tau о \cup \varsigma-80^{\circ} \mathrm{C}$.

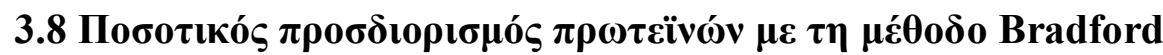

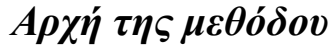

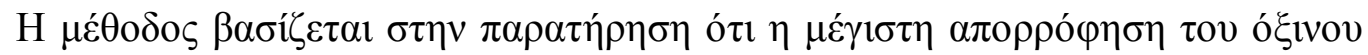

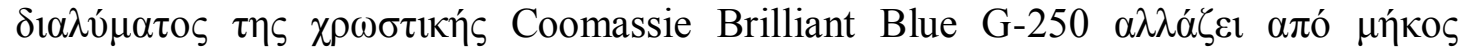

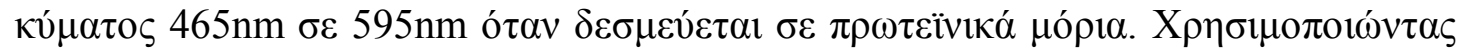

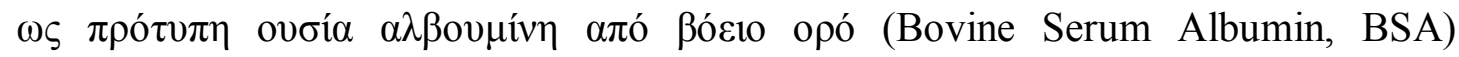

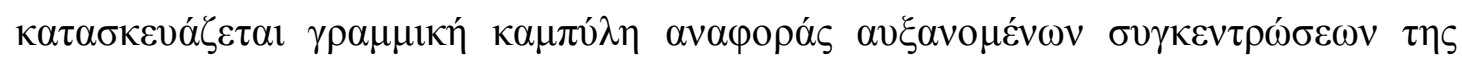

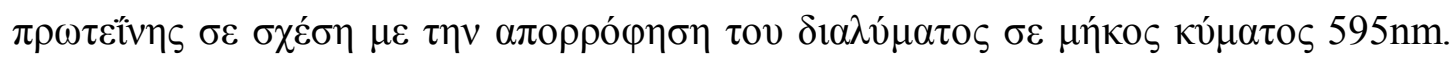

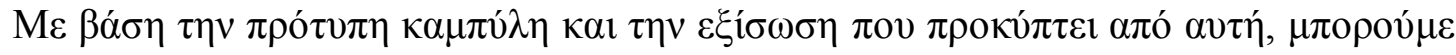

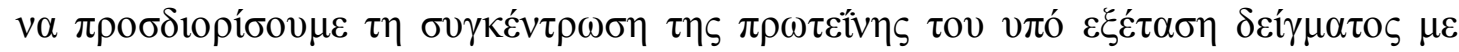

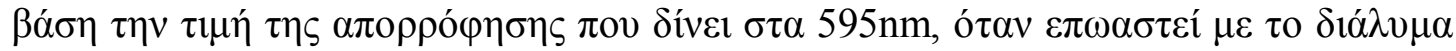

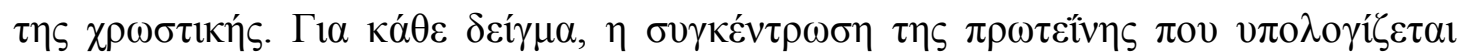

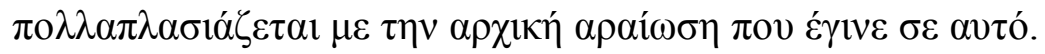

\section{Y}

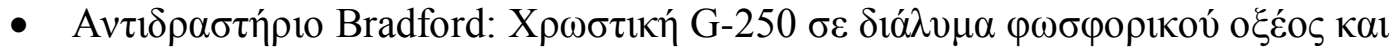
$\mu \varepsilon \theta \alpha v o ́ \lambda \eta \varsigma$ (Quick Start Bradford Dye Reagent 1x, Biorad)

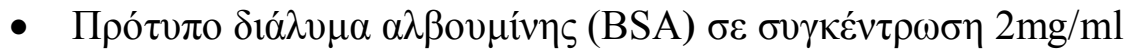

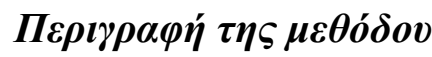

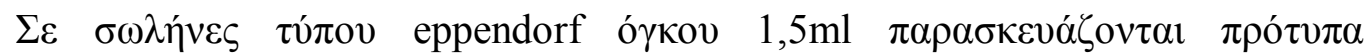

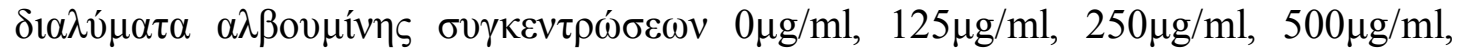

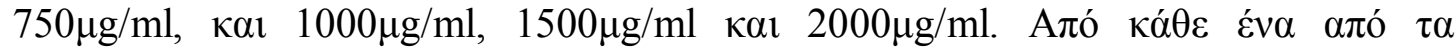




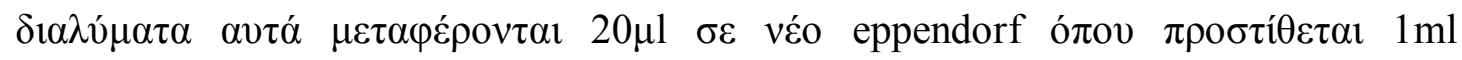

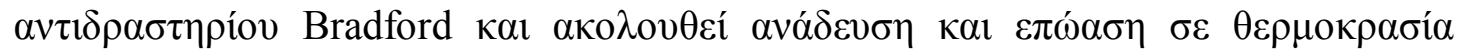

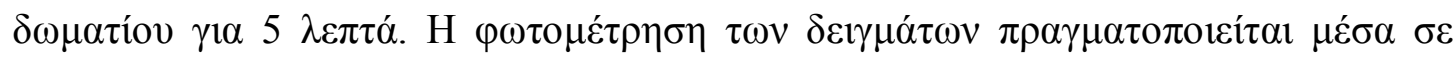

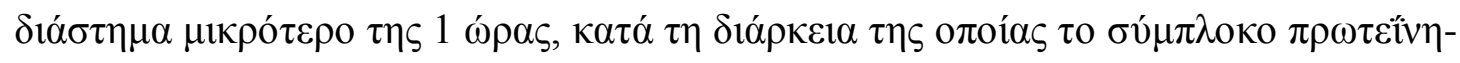

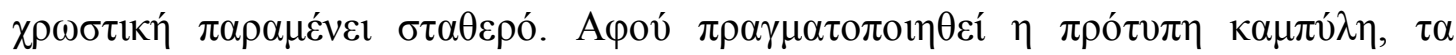

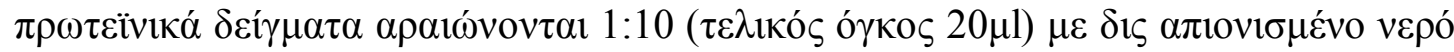

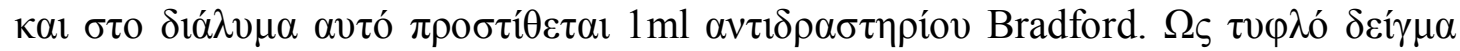

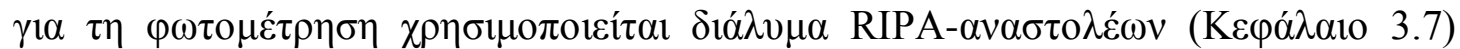

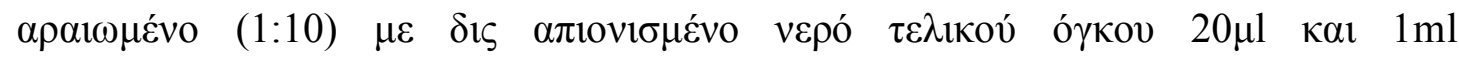
$\alpha v \tau 1 \delta \rho \alpha \sigma \tau \eta \rho i ́ o v ~ B r a d f o r d$.

\section{9 $\Delta \iota \alpha \chi \omega \rho \iota \sigma \mu o ́ \varsigma \pi \rho \omega \tau \varepsilon і ̈ v \omega ́ v ~ \mu \varepsilon$ SDS-PAGE $\eta \lambda \varepsilon \kappa \tau \rho о \varphi o ́ \rho \eta \sigma \eta$}

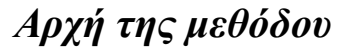

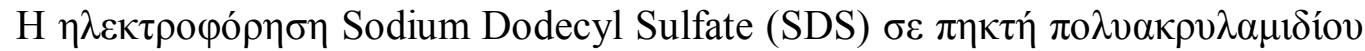

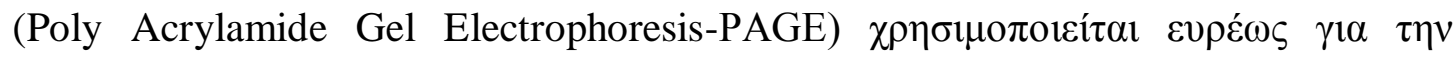

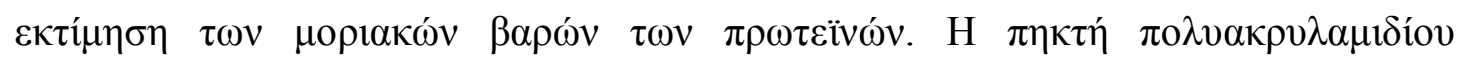

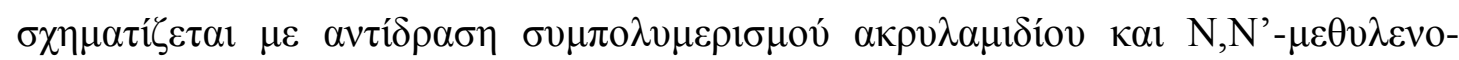

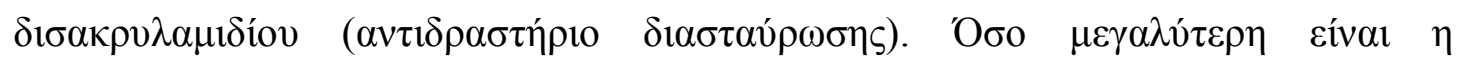

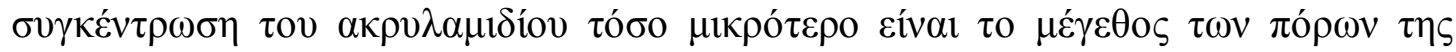

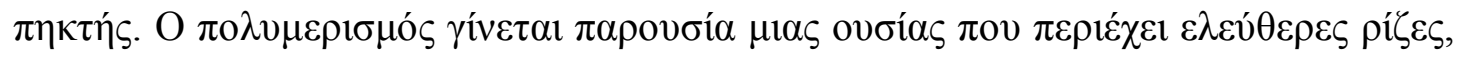

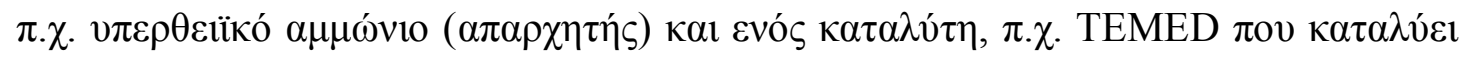

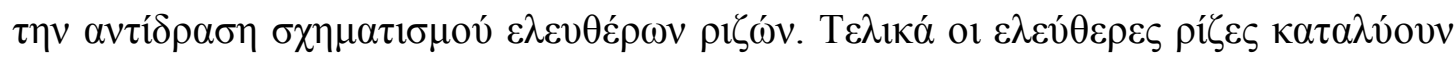

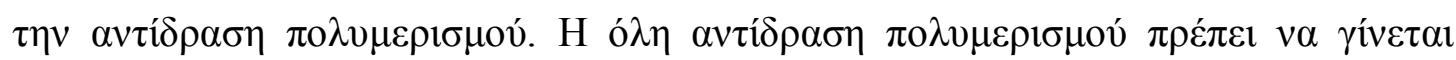

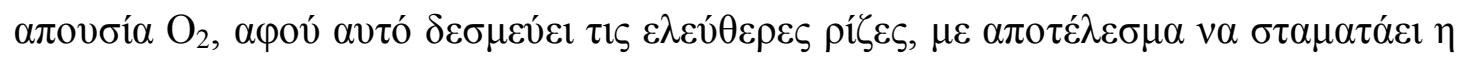

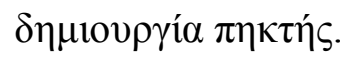

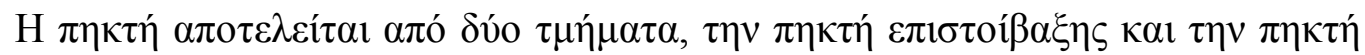

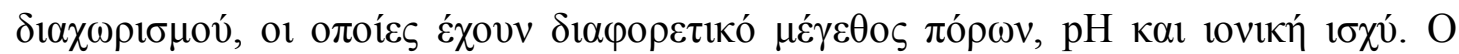

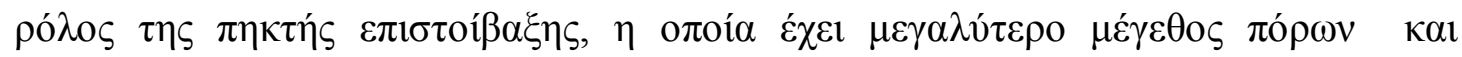

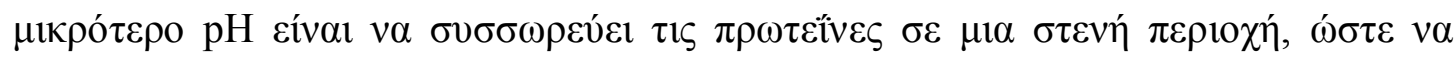

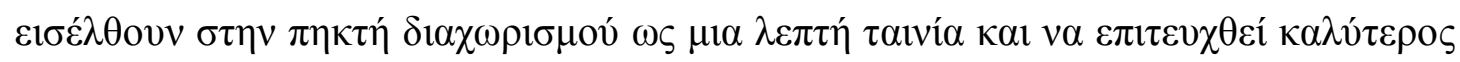

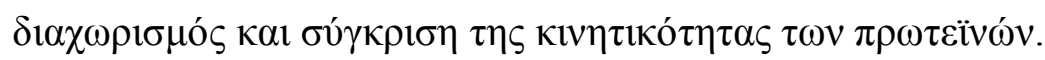




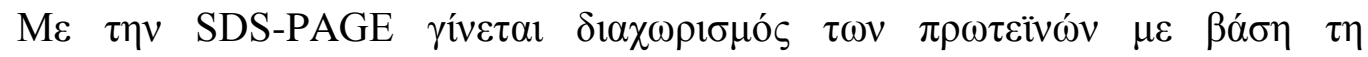

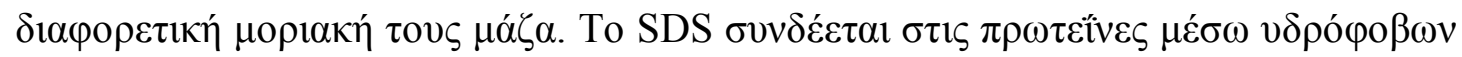

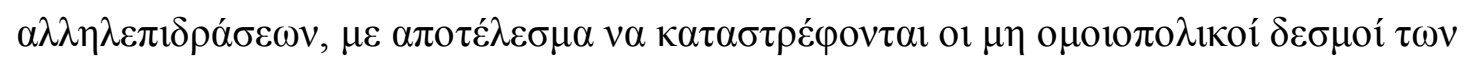

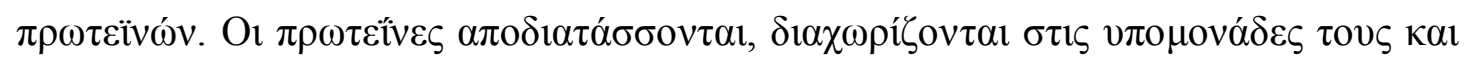

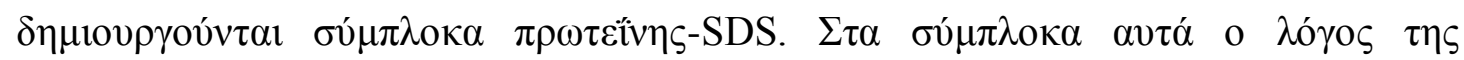

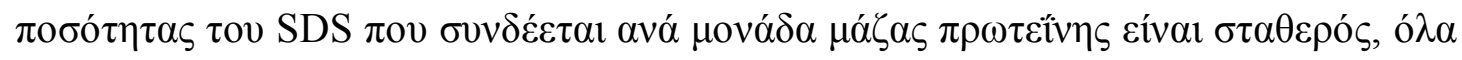

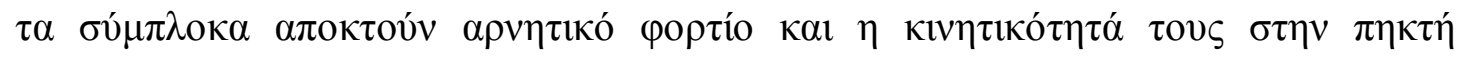

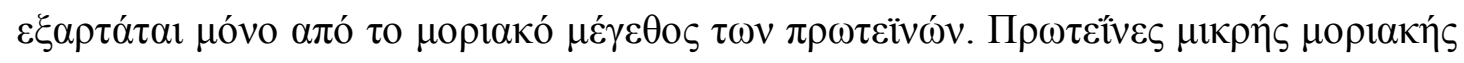

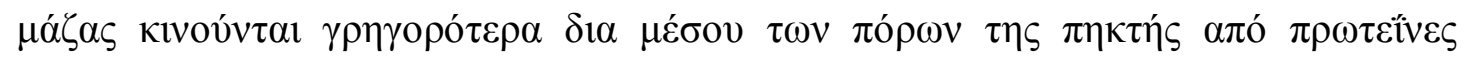

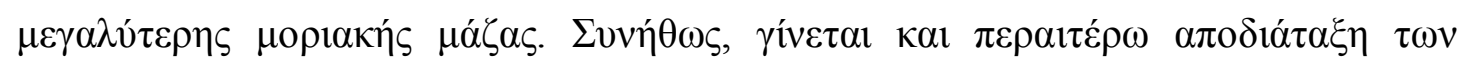

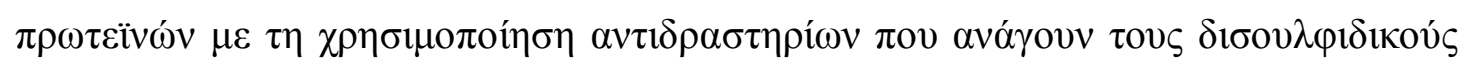

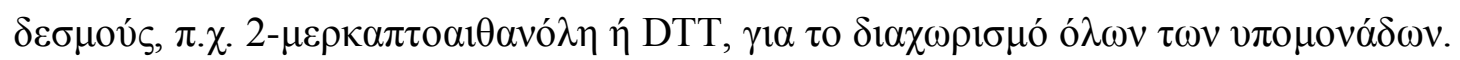

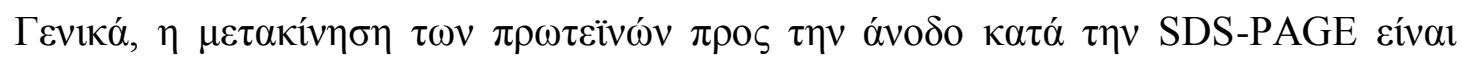

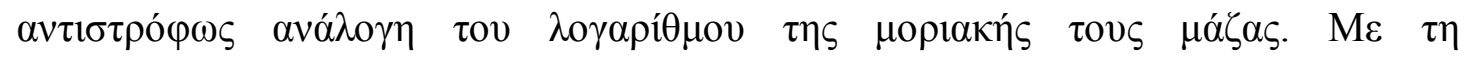

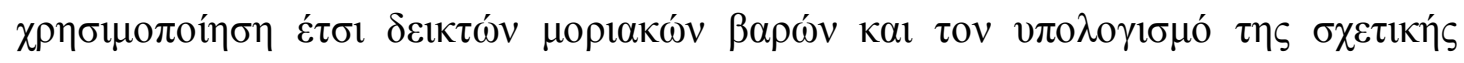

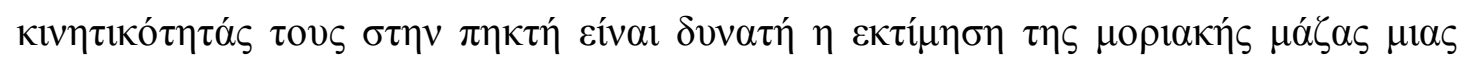

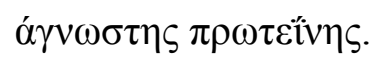

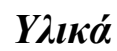

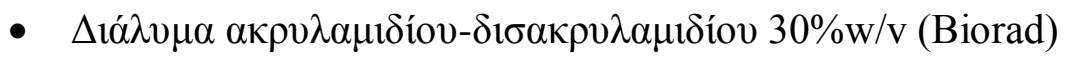

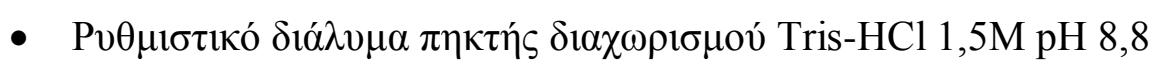

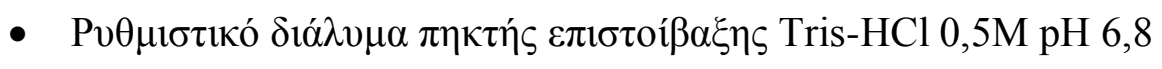

- $\mathrm{N}, \mathrm{N}, \mathrm{N}^{\prime}, \mathrm{N}^{\prime}-\tau \varepsilon \tau \rho \alpha \mu \varepsilon \theta v \lambda-\alpha \imath \theta \alpha v 0-1,2-\delta 1 \alpha \mu i ́ v \eta$ (TEMED) (Sigma-Aldrich St. Louis, MO)

- Y $\pi \varepsilon \rho \theta \varepsilon i \ddot{\kappa o ́ ~} \alpha \mu \mu \omega ́ v i o$ (AmmoniumPerSulfate-APS) 0,1g/ml

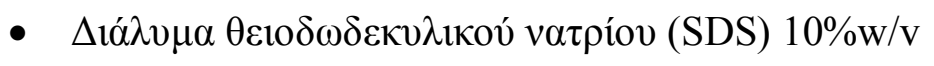

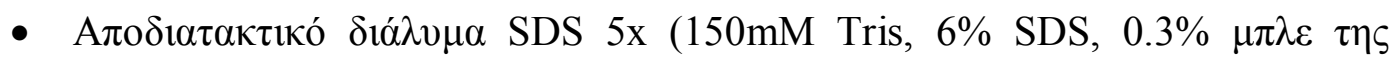

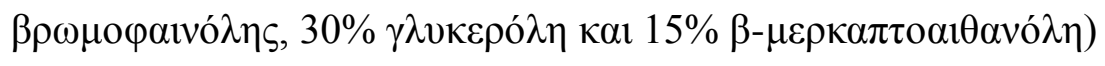

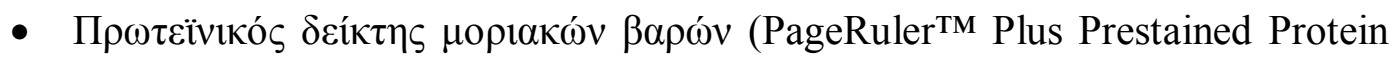
Ladder, Fermentas)

- $\Delta \mathrm{ló} \lambda v \mu \alpha \eta \eta \lambda \varepsilon \kappa \tau \rho \circ \varphi o ́ \rho \eta \sigma \eta s:$

25mM Tris (Sigma-Aldrich St. Louis, MO) 


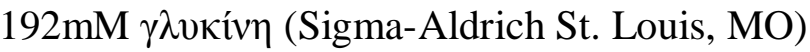

$1 \%$ SDS (Sigma-Aldrich St. Louis, MO)

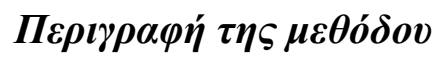

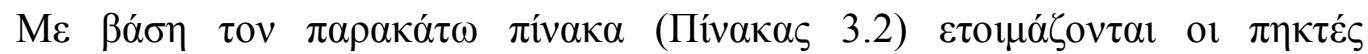

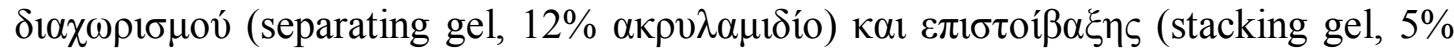

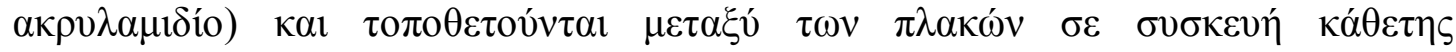

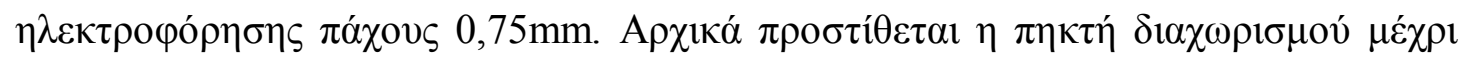

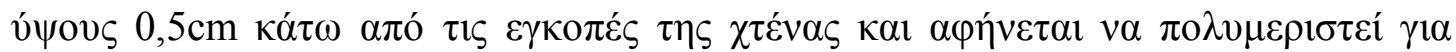

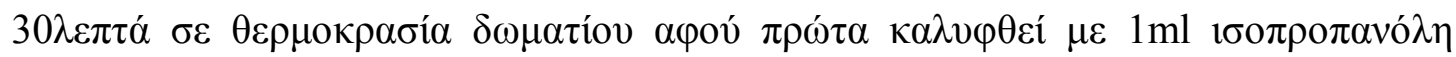

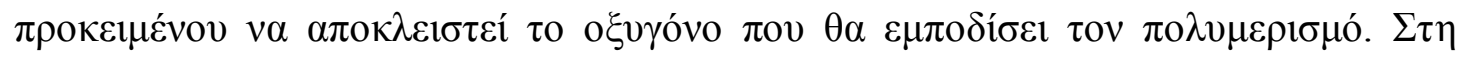

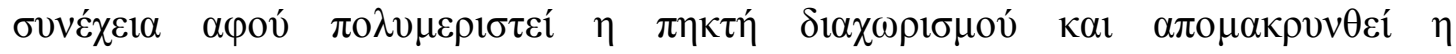

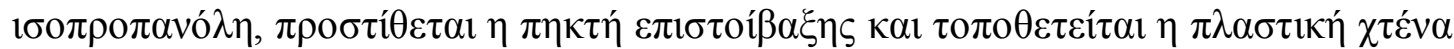

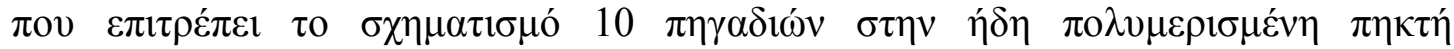

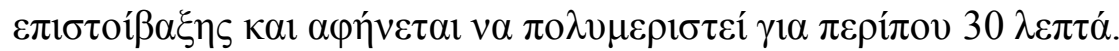

\begin{tabular}{|c|c|c|}
\hline & 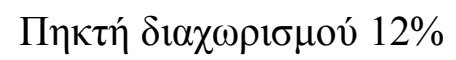 & 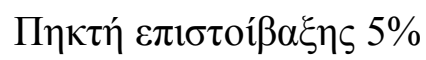 \\
\hline $\begin{array}{l}30 \% \alpha \kappa \rho v \lambda \alpha \mu 1 \delta i ́ o v- \\
\Delta 1 \sigma \alpha \kappa \rho v \lambda \alpha \mu \imath \delta \text { íov }\end{array}$ & $4 \mathrm{ml}$ & $0,83 \mathrm{ml}$ \\
\hline 1,5M Tris $\mathrm{pH} 8,8$ & $2,5 \mathrm{ml}$ & - \\
\hline $0,5 \mathrm{M}$ Tris $\mathrm{pH} 6,8$ & - & $2,5 \mathrm{ml}$ \\
\hline $\mathrm{ddH}_{2} \mathrm{O}$ & $3,3 \mathrm{ml}$ & $1,57 \mathrm{ml}$ \\
\hline $10 \% \mathrm{SDS}$ & $100 \mu 1$ & $50 \mu 1$ \\
\hline TEMED & $10 \mu 1$ & $5 \mu 1$ \\
\hline $\operatorname{APS}(0,1 \mathrm{~g} / \mathrm{ml})$ & $100 \mu 1$ & $50 \mu 1$ \\
\hline
\end{tabular}

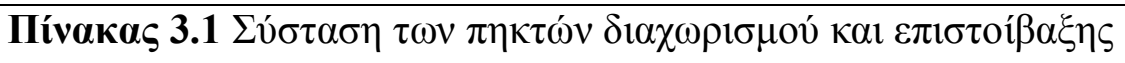

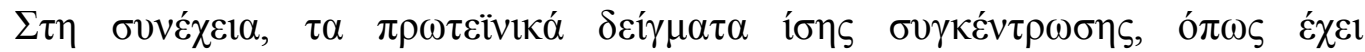

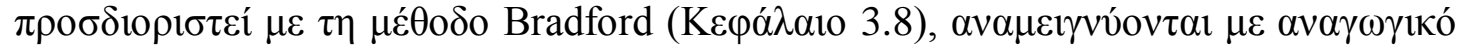

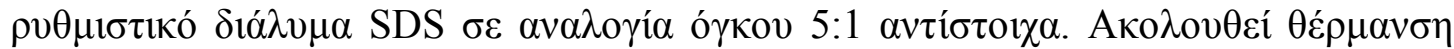




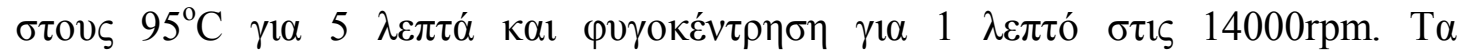

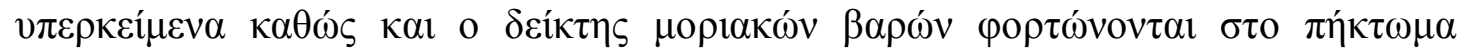

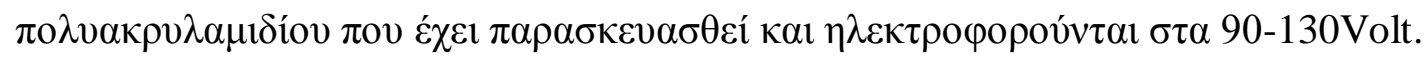

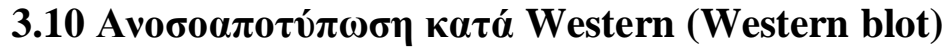

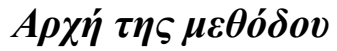

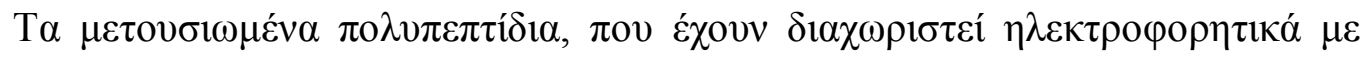

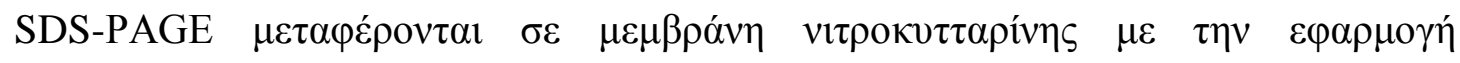

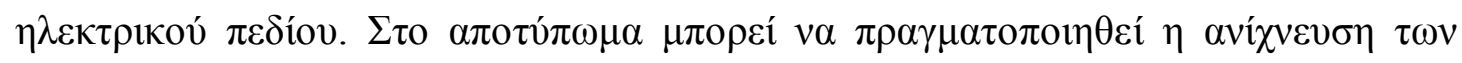

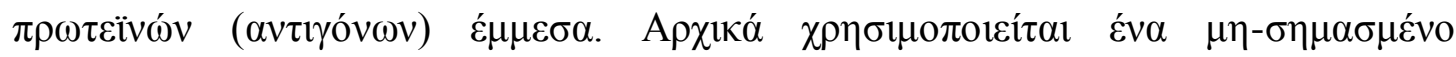

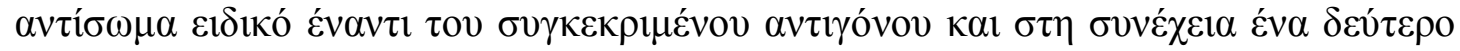

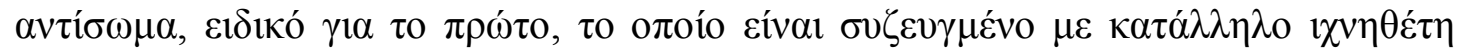

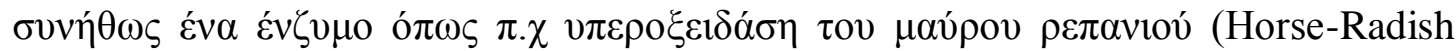

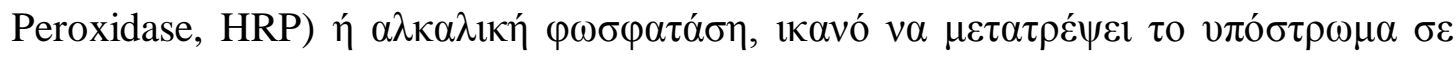

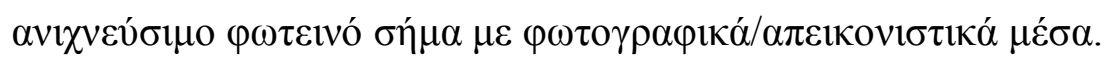

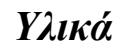

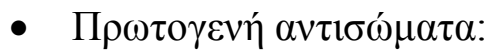

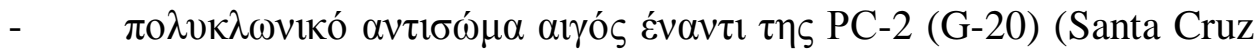
$\mathrm{Bt})$

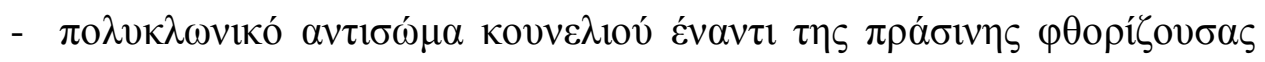

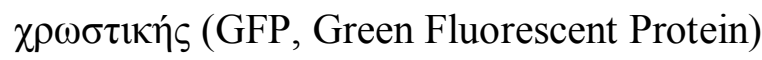

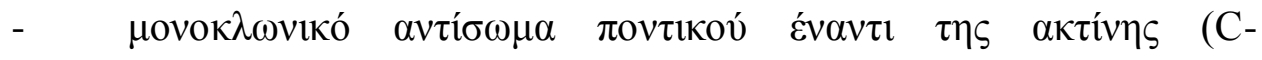
4)(Chemicon)

- $\Delta \varepsilon v \tau \varepsilon \rho о \gamma \varepsilon v \eta ́ ~ \alpha \nu \tau \imath \sigma \omega ́ \mu \alpha \tau \alpha:$

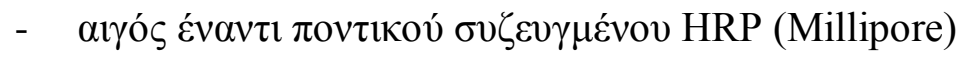

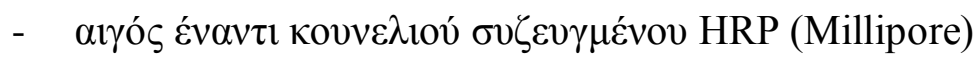

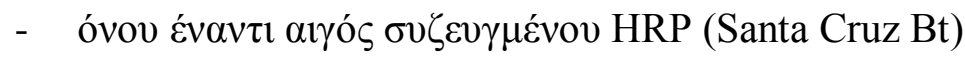

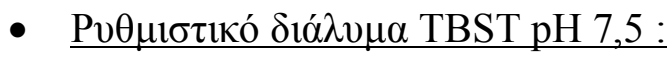

20mM Tris (Sigma-Aldrich St. Louis, MO)

$150 \mathrm{mM} \mathrm{NaCl}$ 
$0,05 \%$ Tween-20

- $\quad \Delta \mathrm{l} \alpha ́ \lambda v \mu \alpha \mu \varepsilon \tau \alpha \varphi \circ \rho \alpha ́ s:$

$25 \mathrm{mM}$ Tris (Sigma-Aldrich St. Louis, MO)

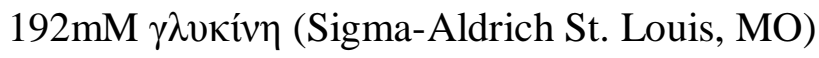

$20 \% \mu \varepsilon \theta \alpha v o ́ \lambda \eta$ (Sigma-Aldrich St. Louis, MO)

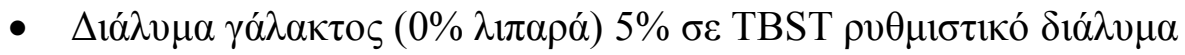

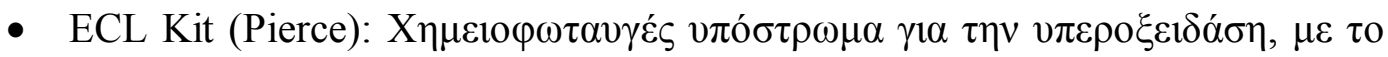

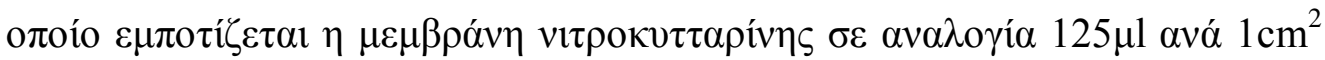

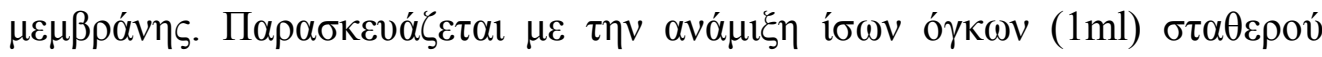

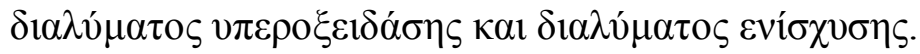

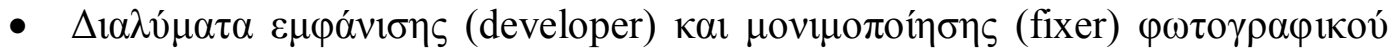
$\varphi \imath \lambda \mu$ (Sigma-Aldrich St. Louis, MO)

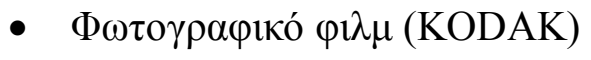

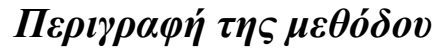

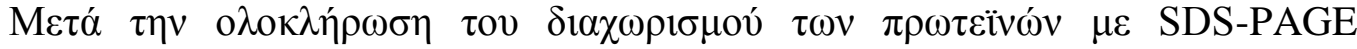

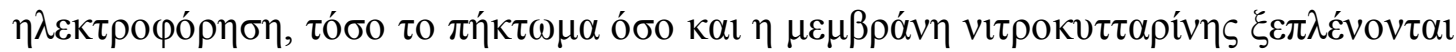

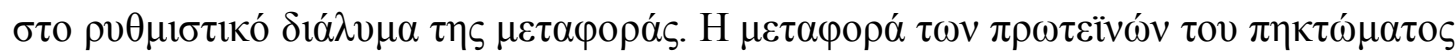

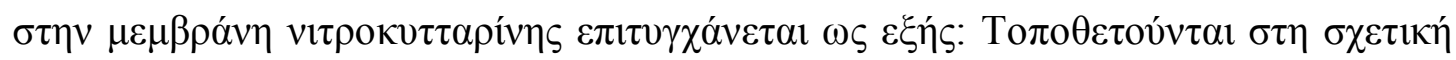

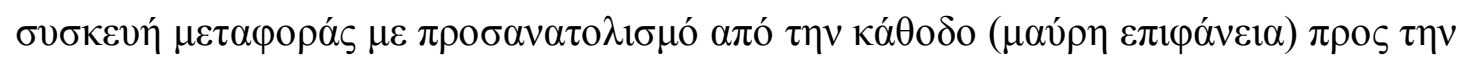

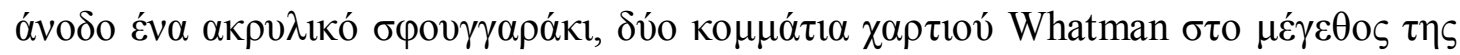

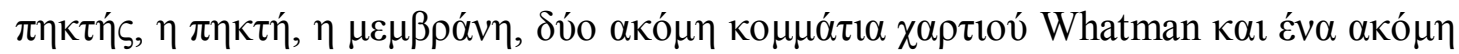

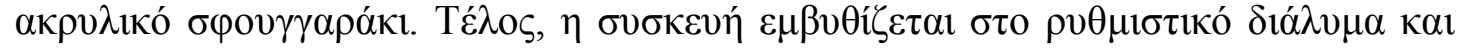

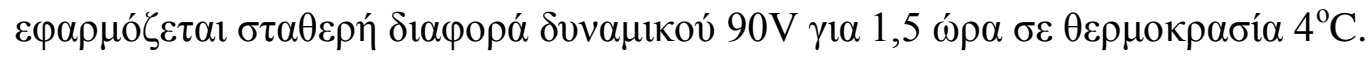

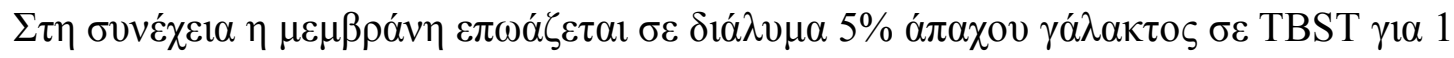

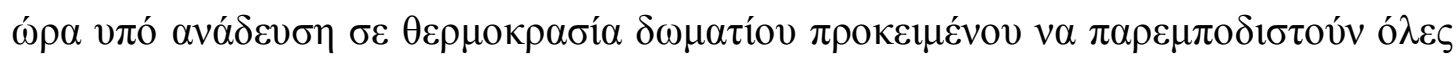

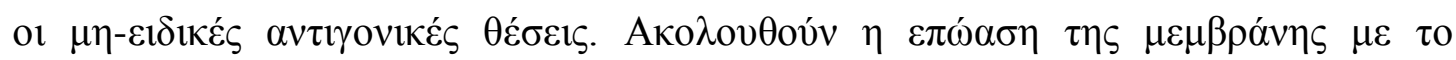

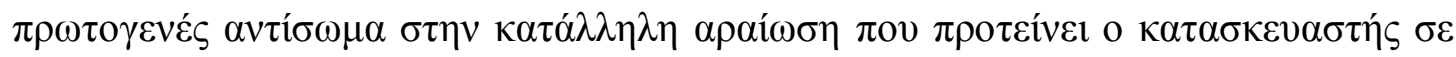

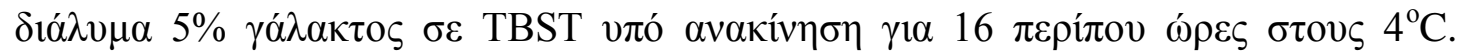

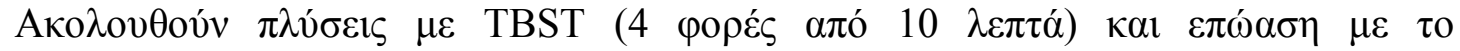

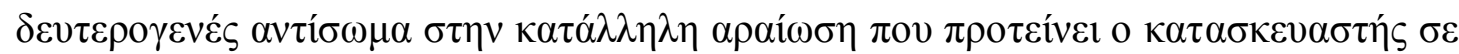

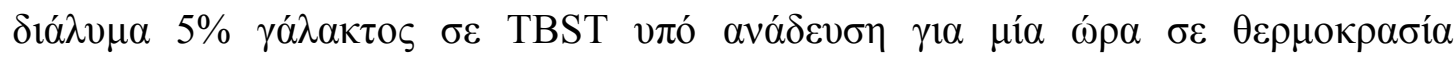
$\delta \omega \mu \alpha \tau i ́ o v$. 


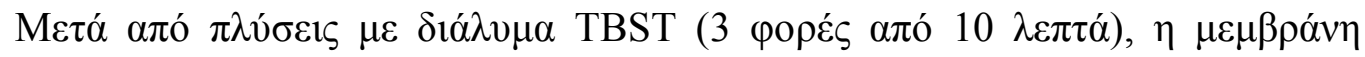

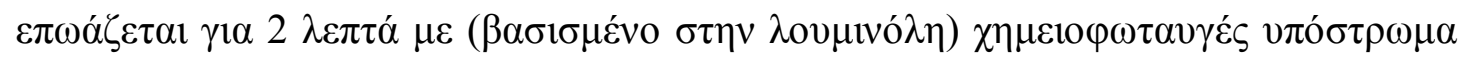

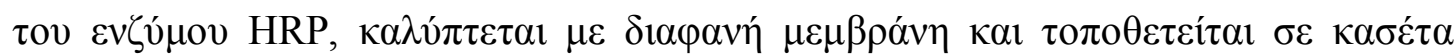

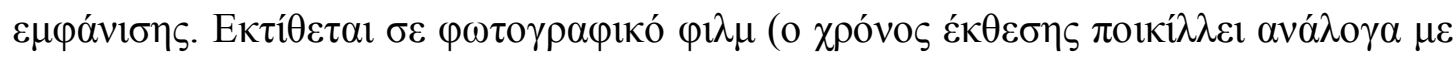

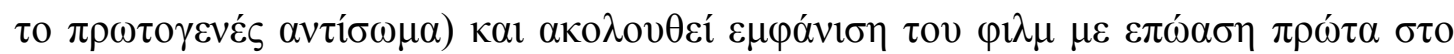

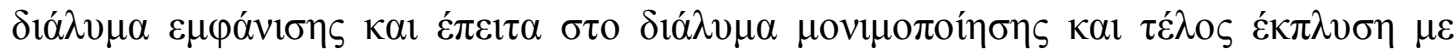

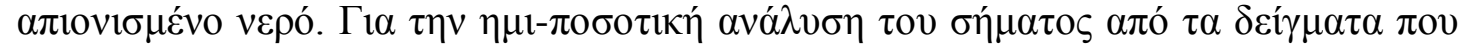

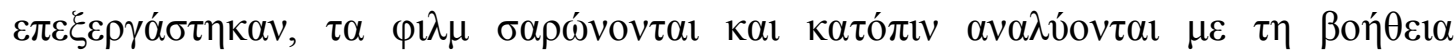

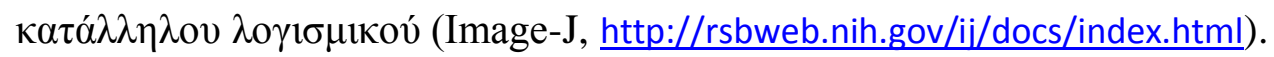

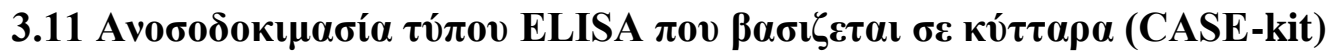

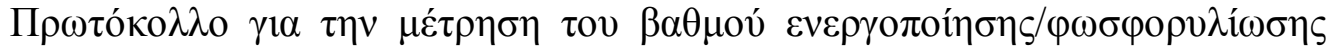

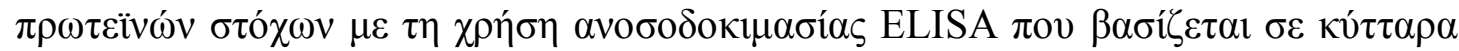

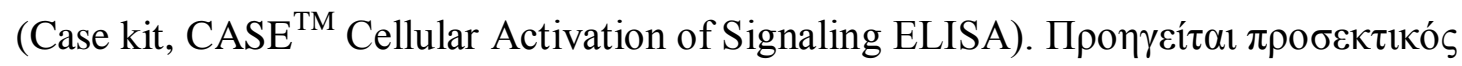

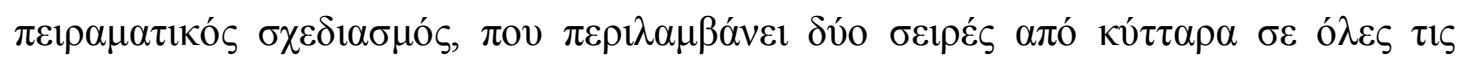

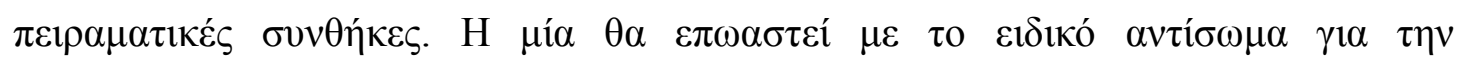

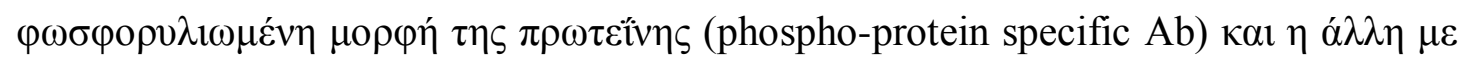

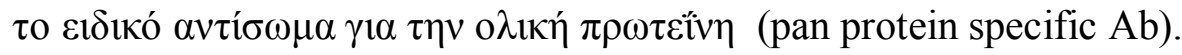

\section{CASE-kit: Cellular Activation of Signaling ELISA}

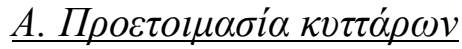

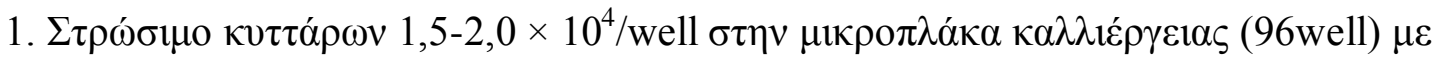

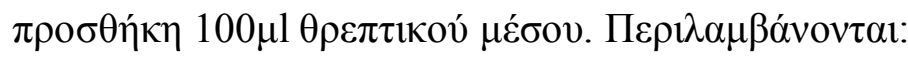

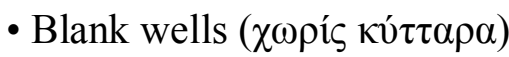

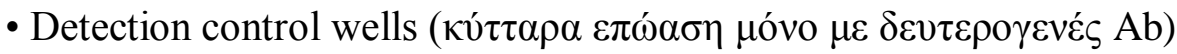

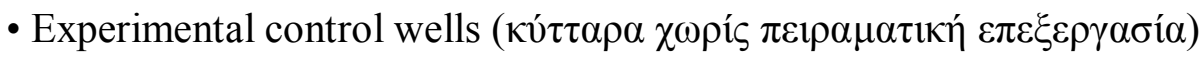

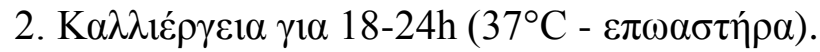

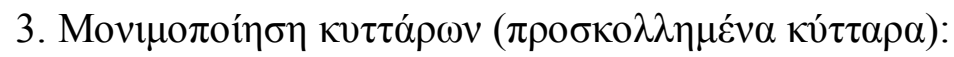

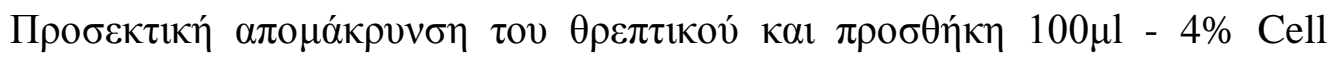

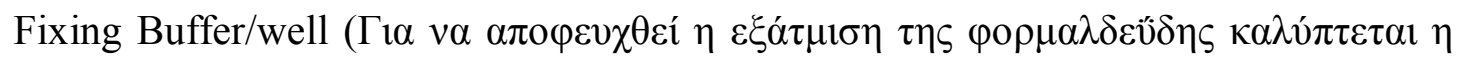

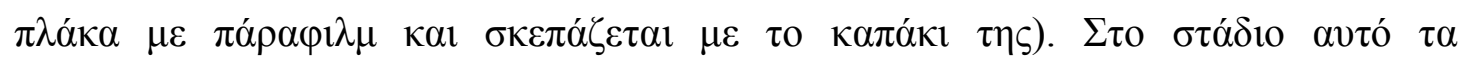




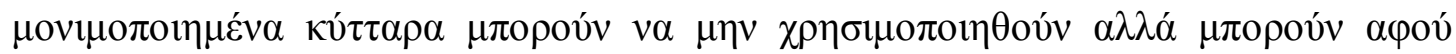

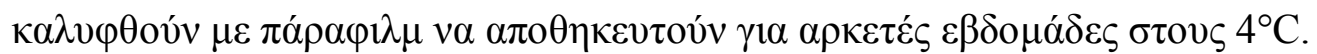

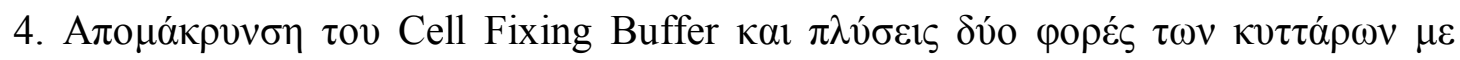

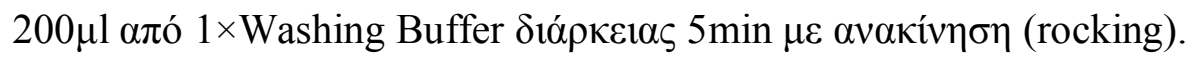

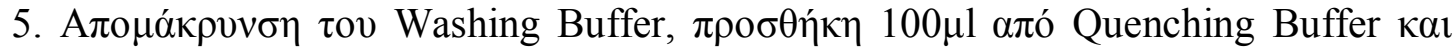

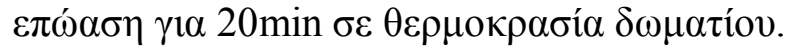

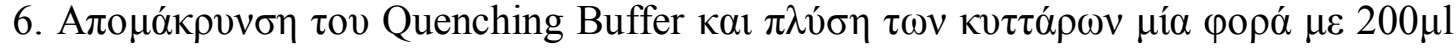

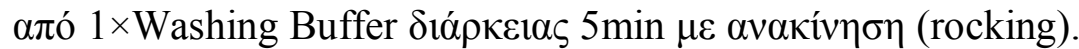

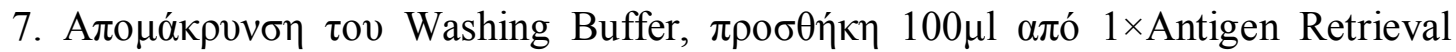

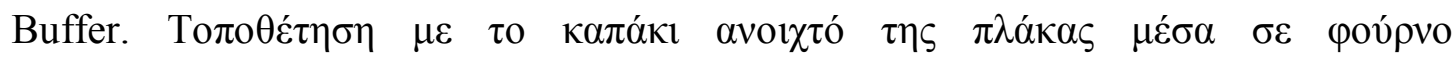

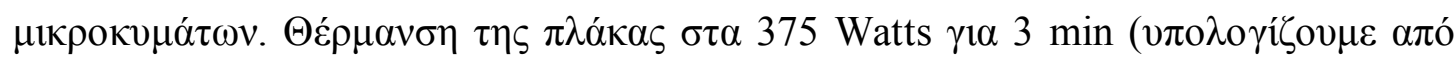

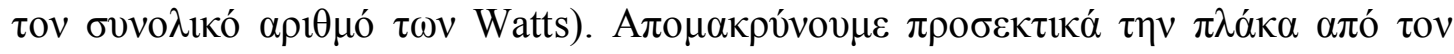

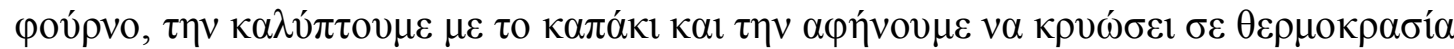
$\delta \omega \mu \alpha \tau i ́ o v$.

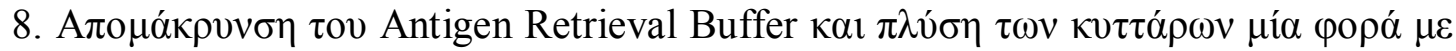

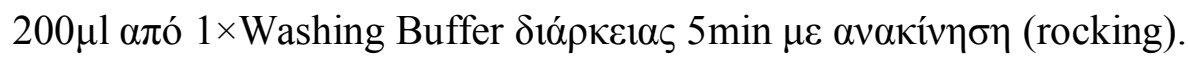

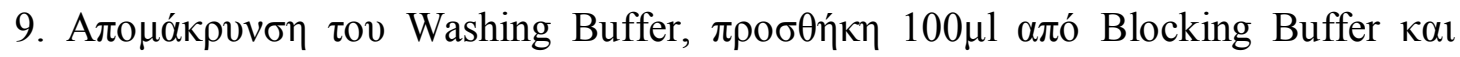

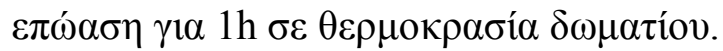

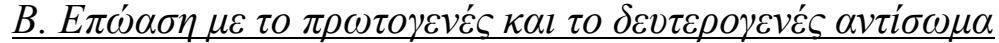

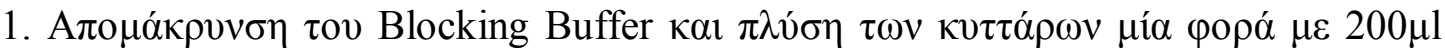

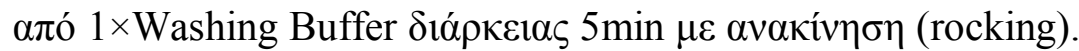

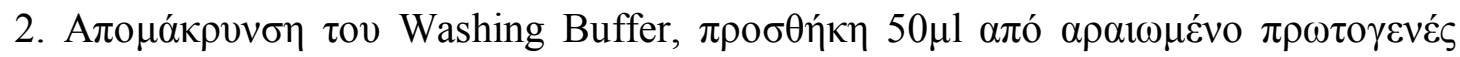

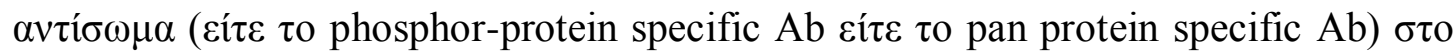

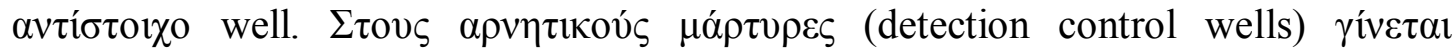

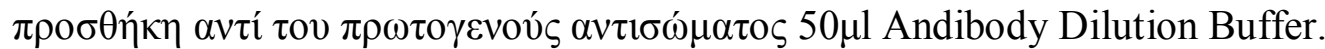

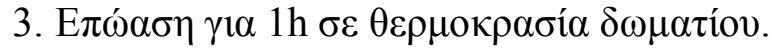

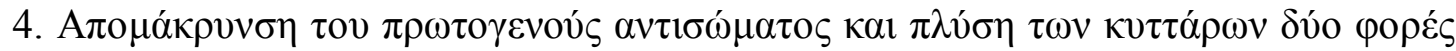

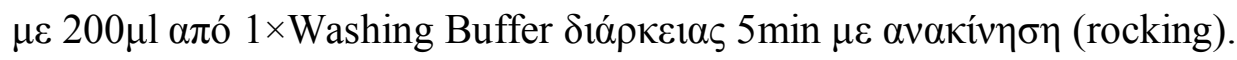

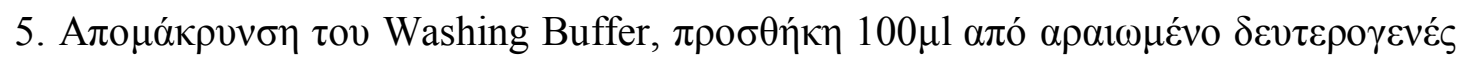

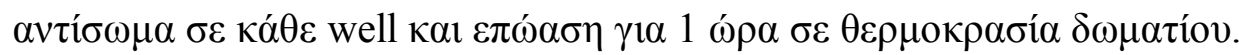




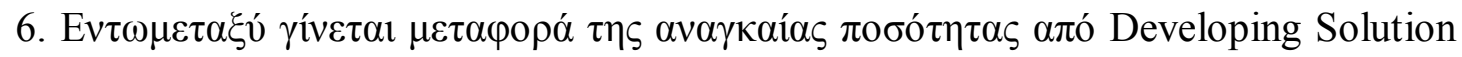

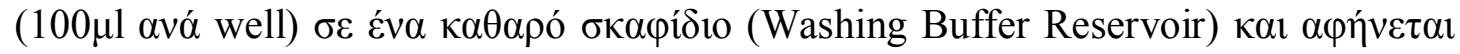

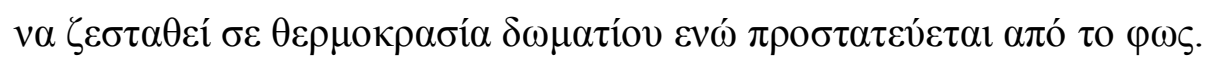

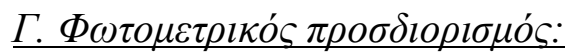

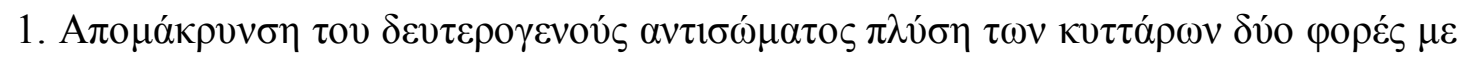

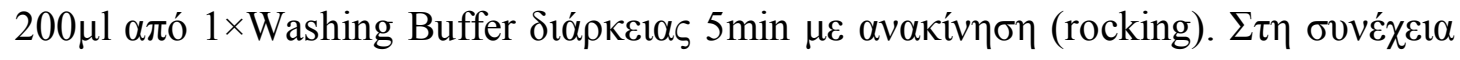

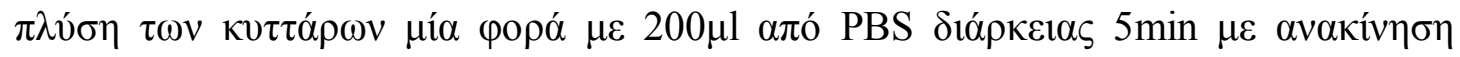
(rocking).

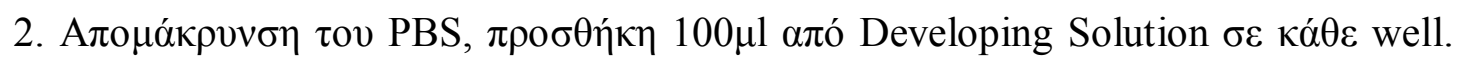

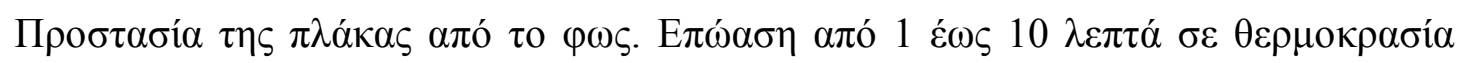
$\delta \omega \mu \alpha \tau i ́ o v$.

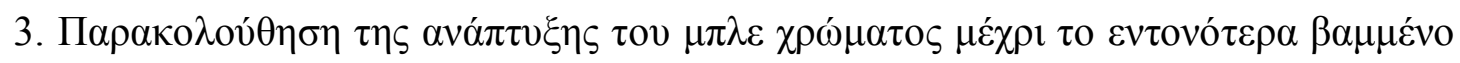

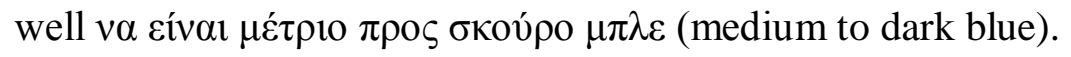

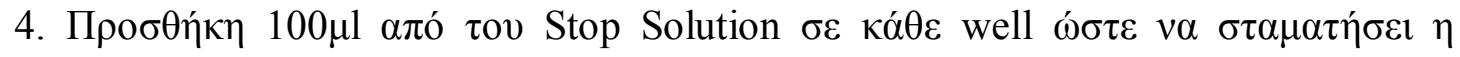

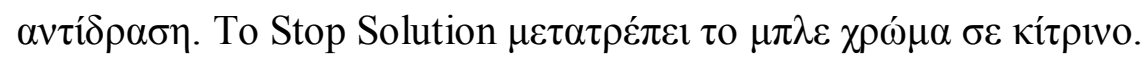

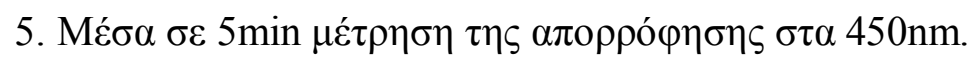

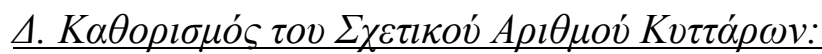

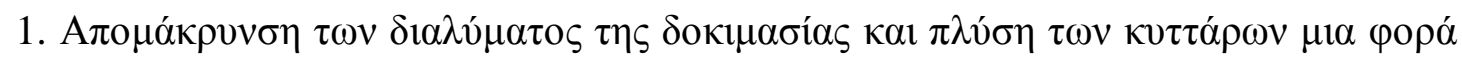

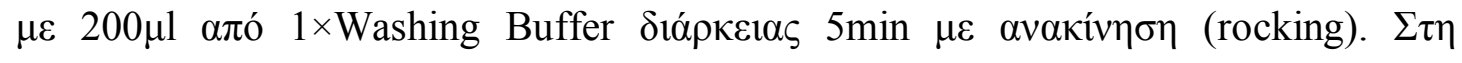

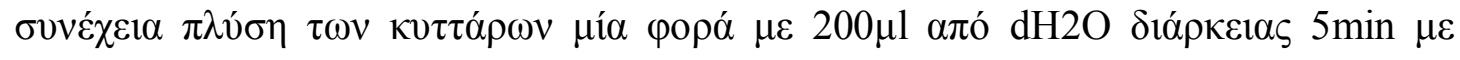

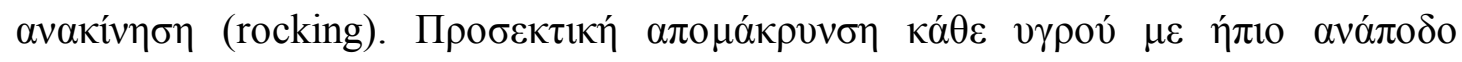

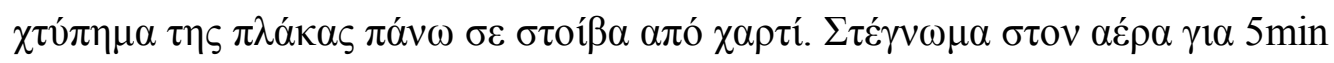

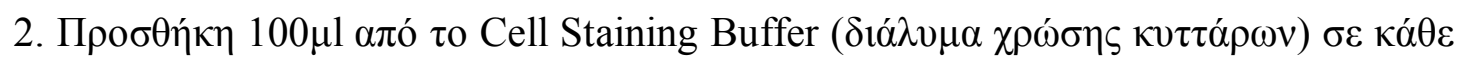

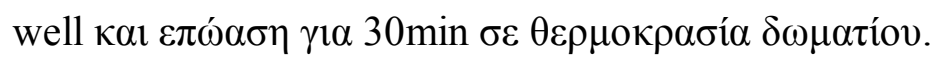

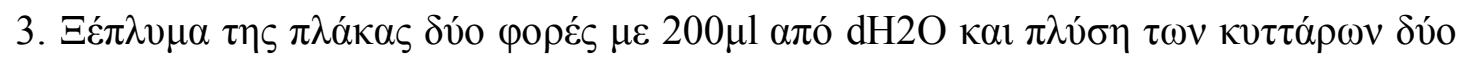

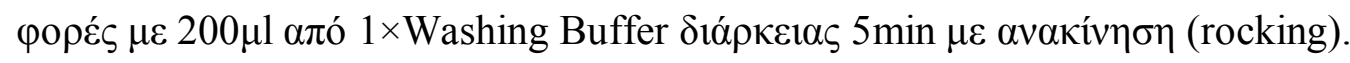

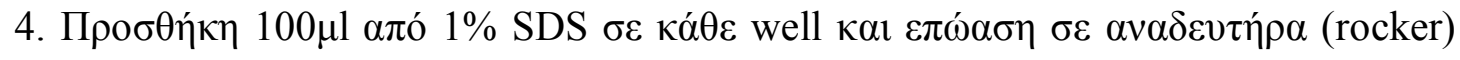

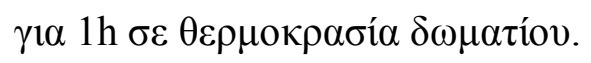

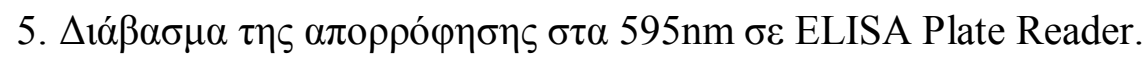

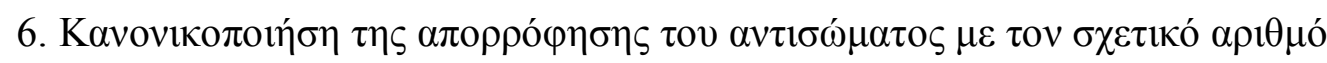

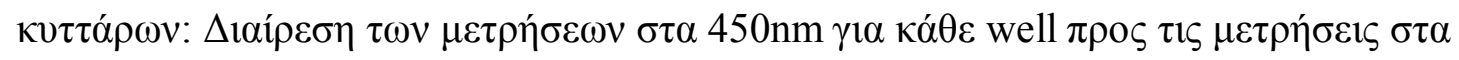




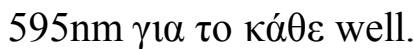

OD450 / OD595 $\gamma 1 \alpha \kappa \alpha ́ \theta \varepsilon$ well

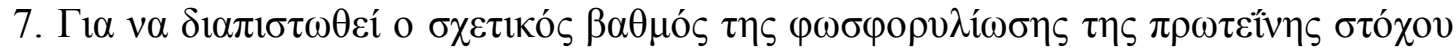

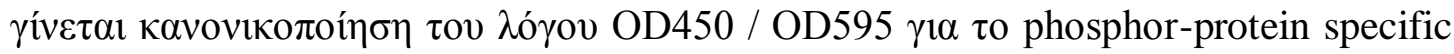

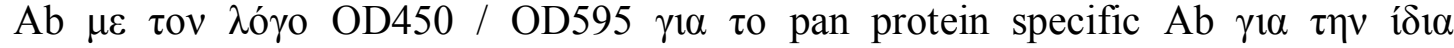

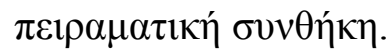

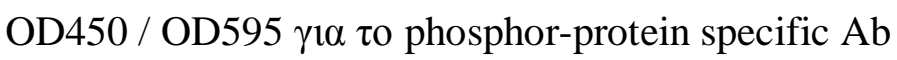

OD450 / OD595 $\gamma 1 \alpha$ to pan protein specific Ab

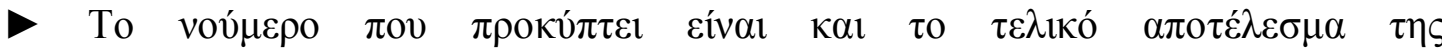

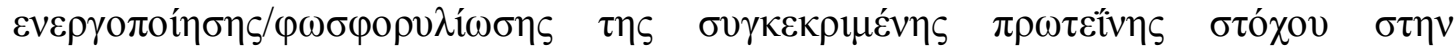

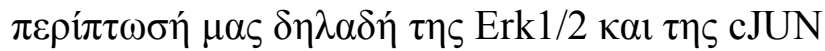

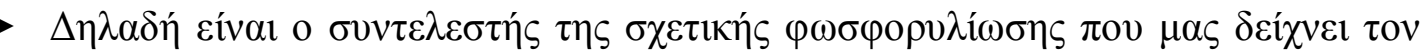

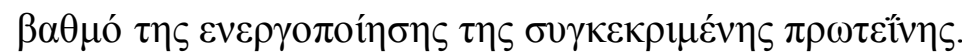

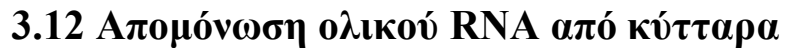

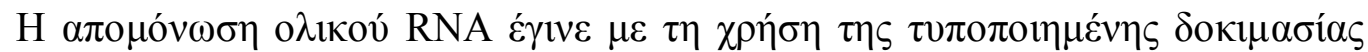

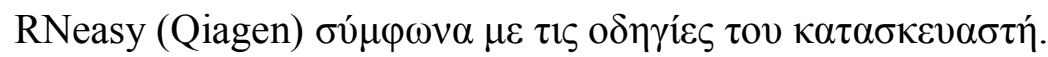

\subsection{RT-PCR (Reverse Transcription-PCR)}

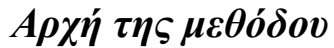

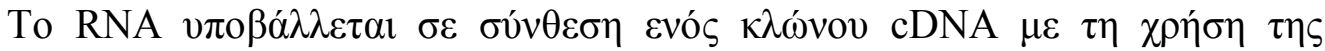

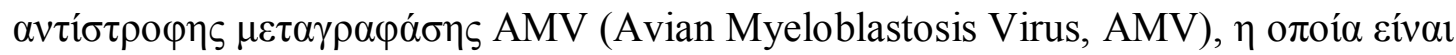

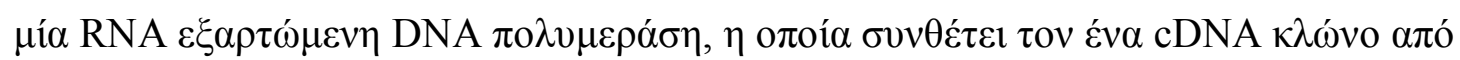

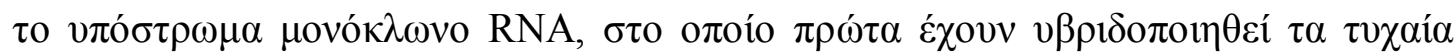




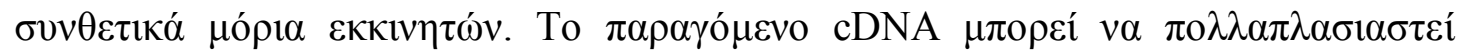

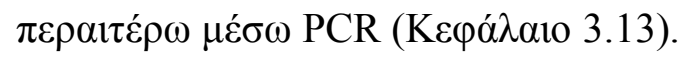

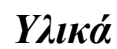

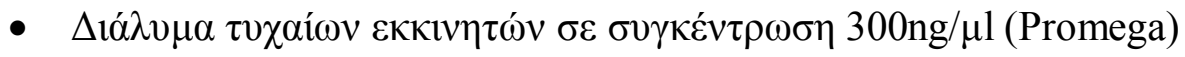

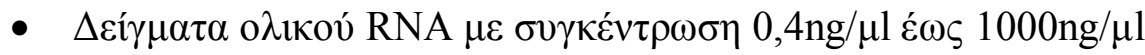

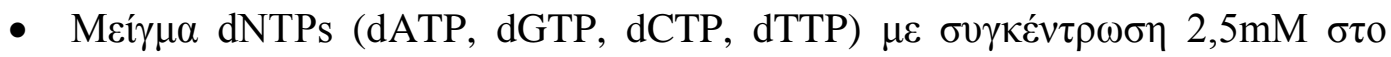
$\kappa \alpha \theta \varepsilon ́ v \alpha($ Promega)

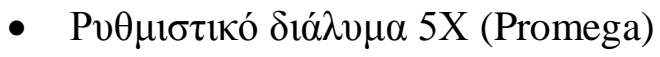

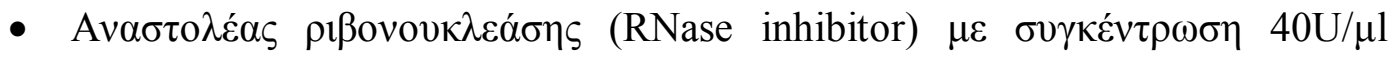
(Invitrogen)

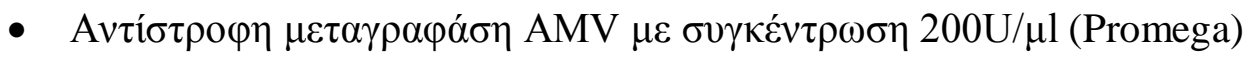

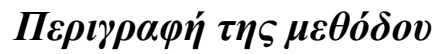

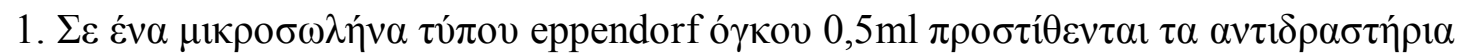

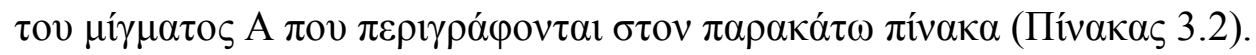

\begin{tabular}{|c|c|}
\hline 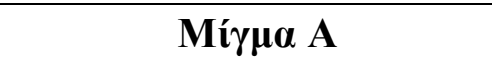 & 'Оүко૬ \\
\hline 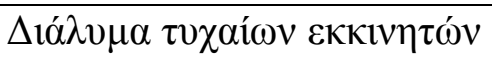 & $1 \mu \mathrm{l}$ \\
\hline$\Delta \varepsilon^{\prime} \gamma \mu \alpha$ одıкои́ RNA & $2 \mu l$ \\
\hline $\mathrm{ddH}_{2} \mathrm{O} \alpha \pi \sigma \sigma \tau \varepsilon \varphi \omega \mu \varepsilon v$ & $7 \mu 1$ \\
\hline
\end{tabular}

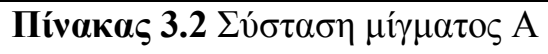

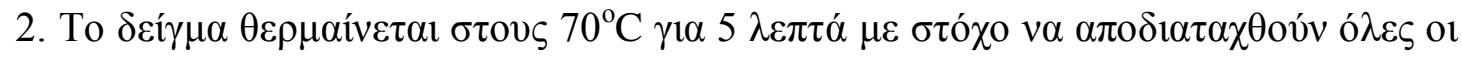

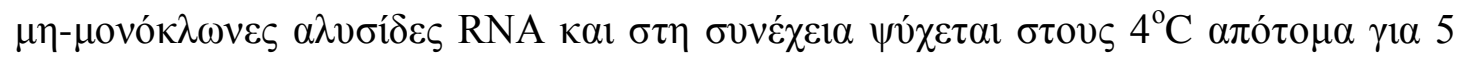
$\lambda \varepsilon \pi \tau \dot{\alpha}$.

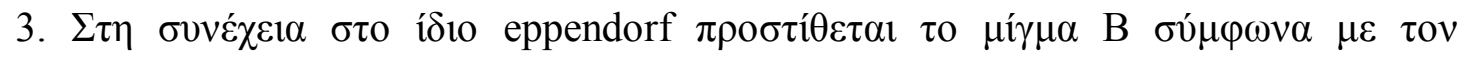

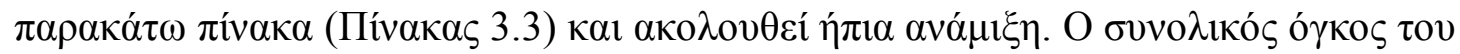

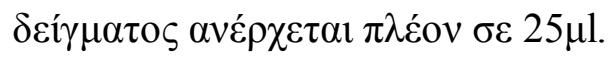




\begin{tabular}{|c|c|}
\hline Mí $\gamma \mu \alpha$ B & 'Оүко૬ \\
\hline 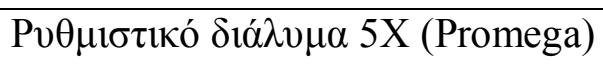 & $5 \mu 1$ \\
\hline dNTPs & $2,5 \mu 1$ \\
\hline 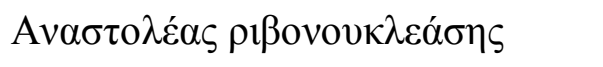 & $1 \mu 1$ \\
\hline AMV & $1 \mu 1$ \\
\hline $\mathrm{ddH}_{2} \mathrm{O} \alpha \pi \circ \sigma \tau \varepsilon 1 \rho \omega \mu \varepsilon \varepsilon^{\prime}{ }^{2}$ & $5,5 \mu 1$ \\
\hline
\end{tabular}

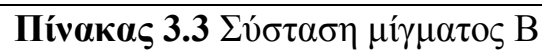

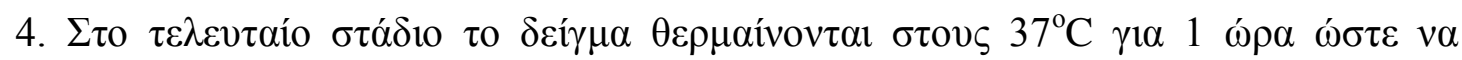

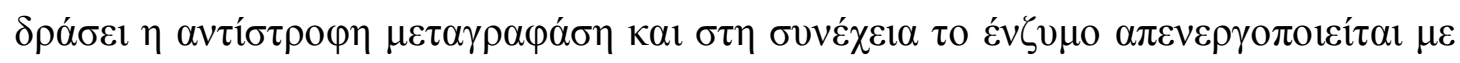

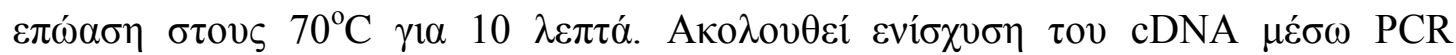

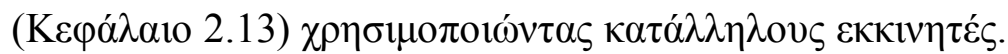

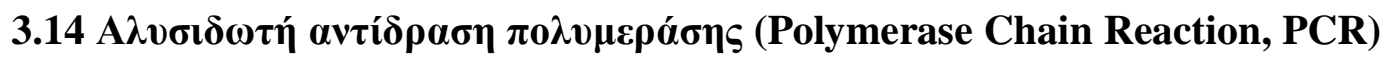

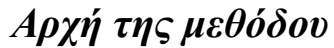

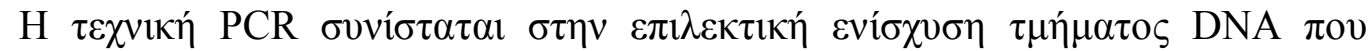

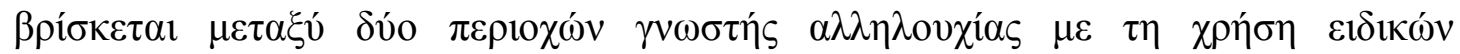

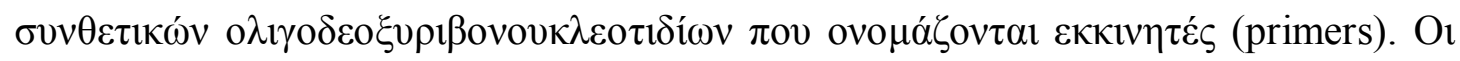

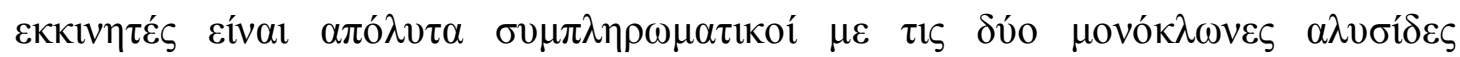

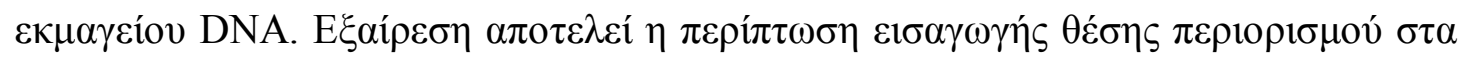

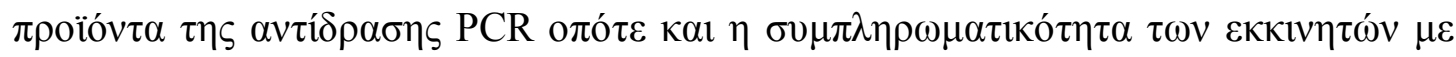

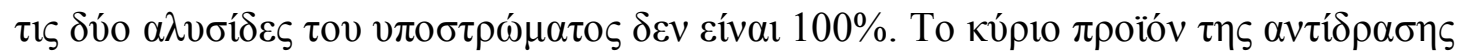

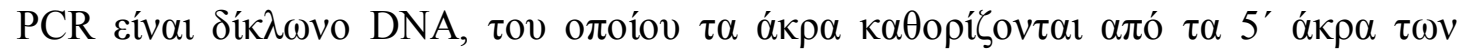

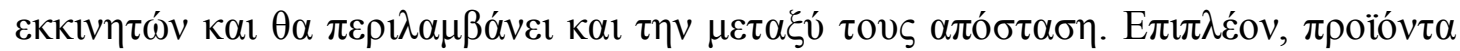

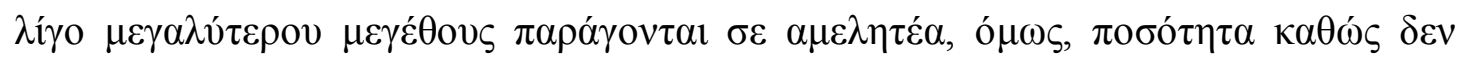

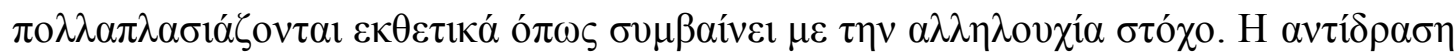

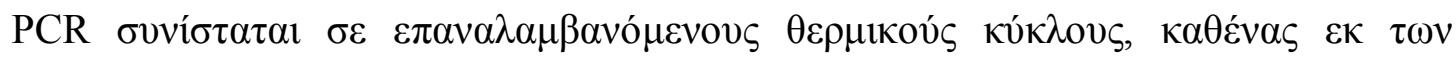

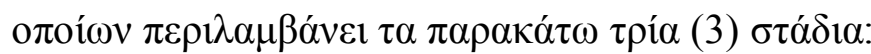

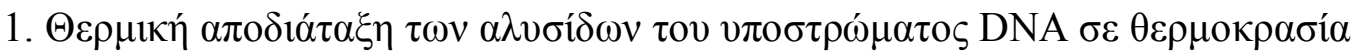

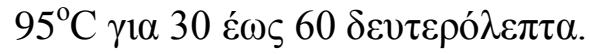




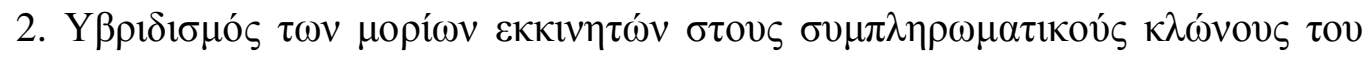

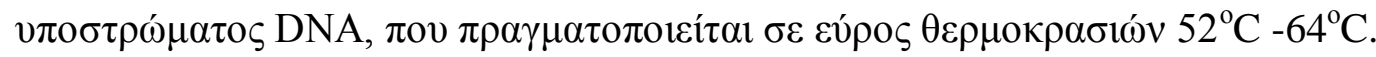

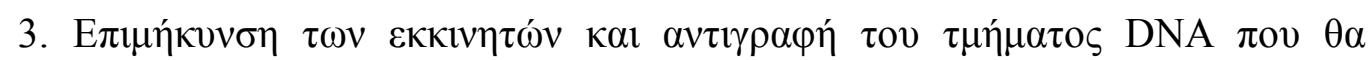

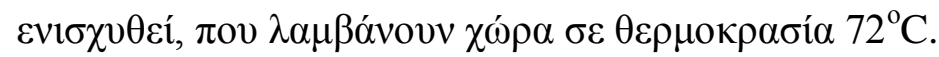

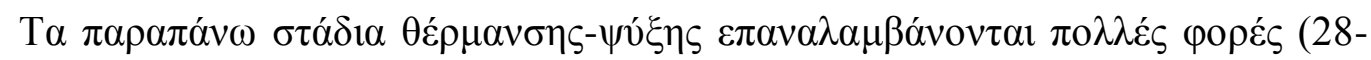

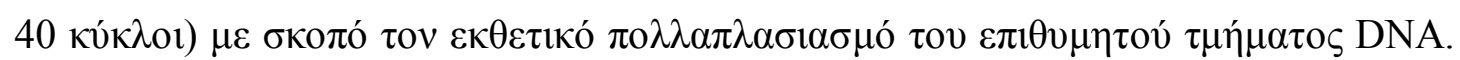

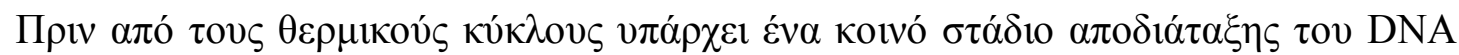

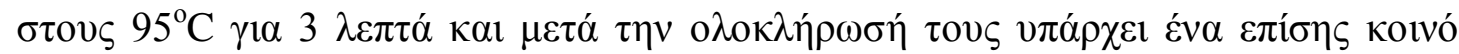

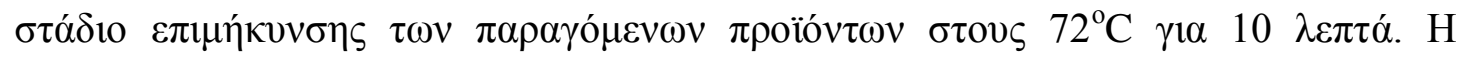

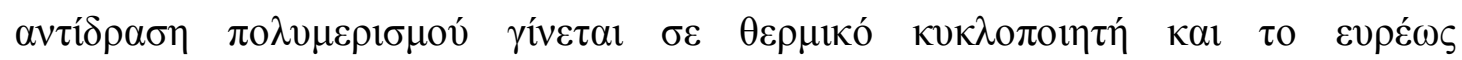

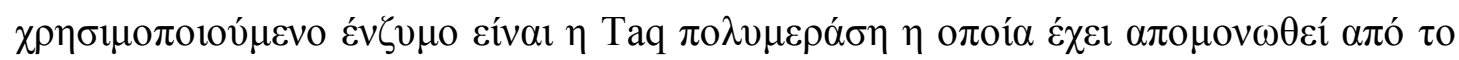

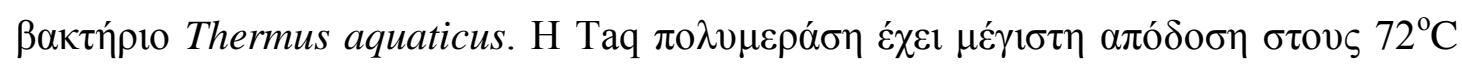

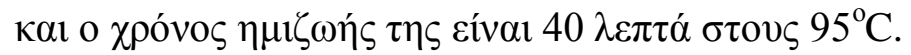

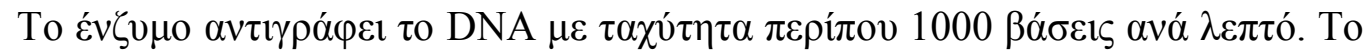

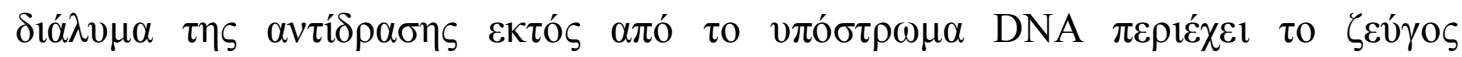

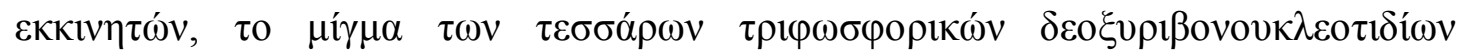

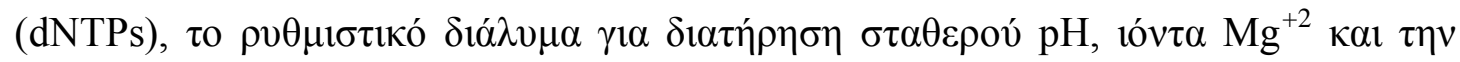

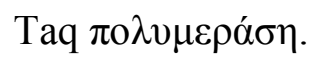

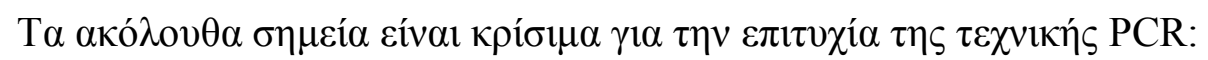

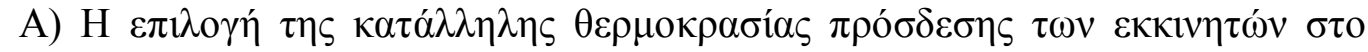

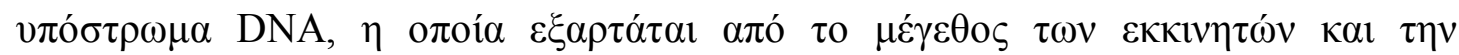

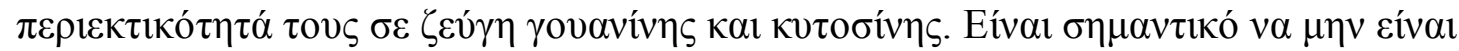

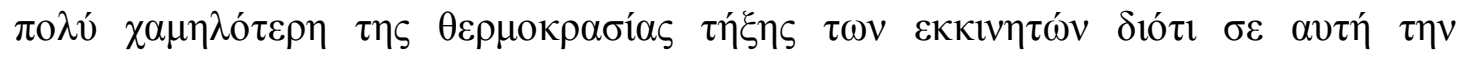

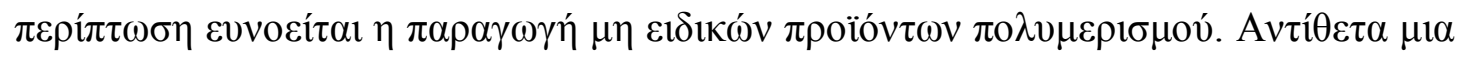

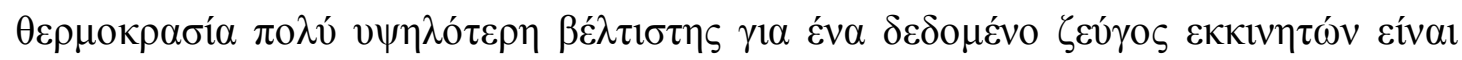

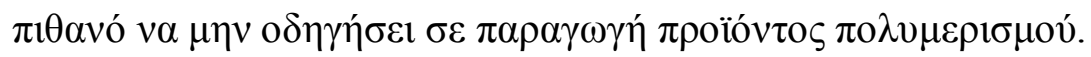

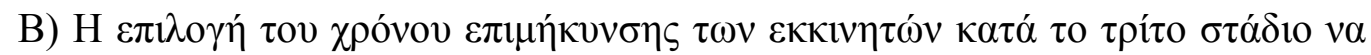

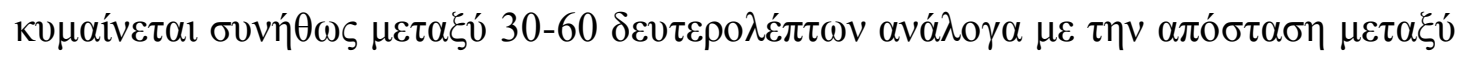

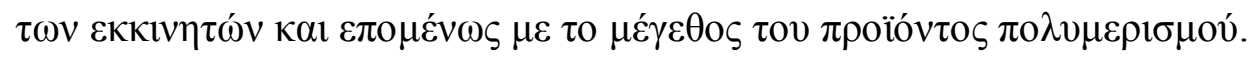

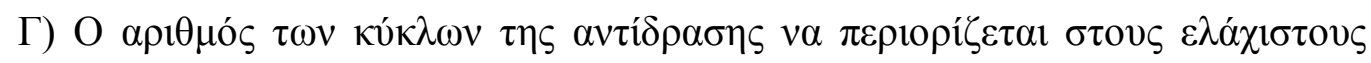

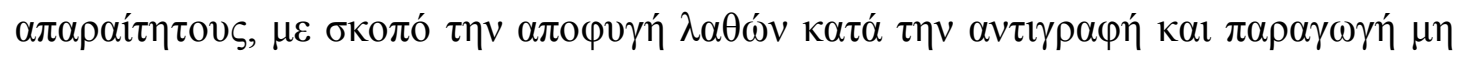
$\varepsilon 1 \delta 1 \kappa \omega ́ v \pi \rho 0 \ddot{o} v \tau \omega v$. 


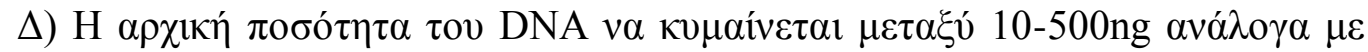

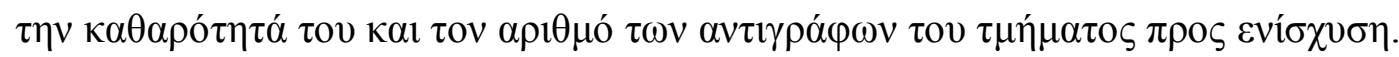

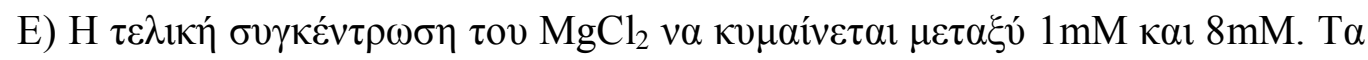

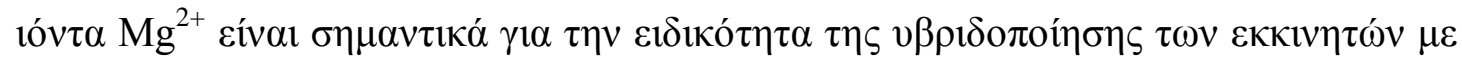

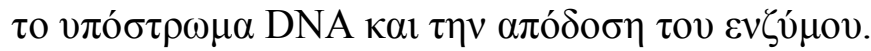

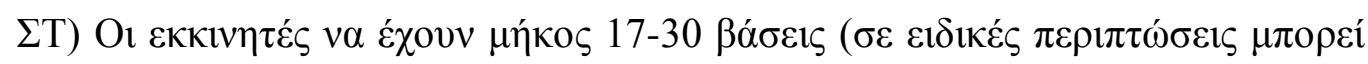

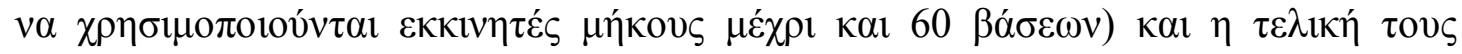

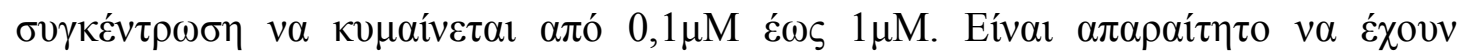

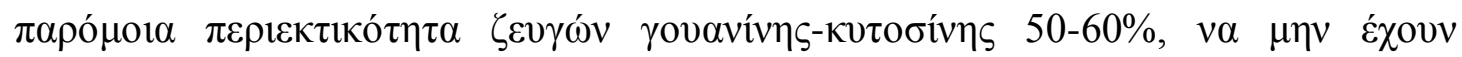

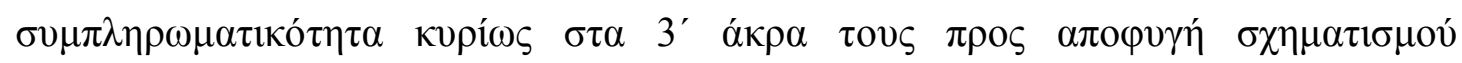

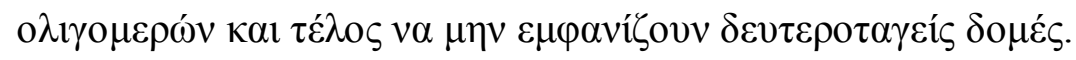

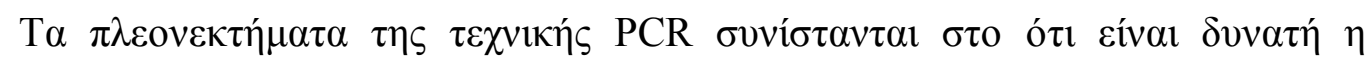

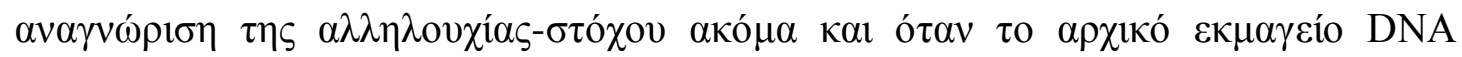

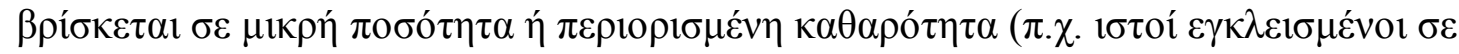

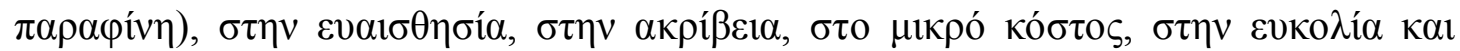

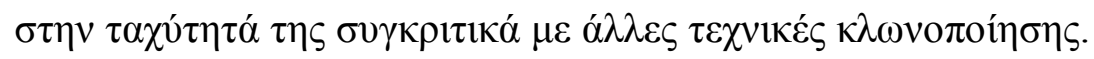

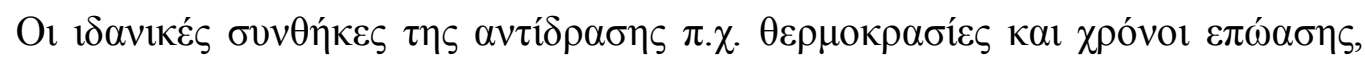

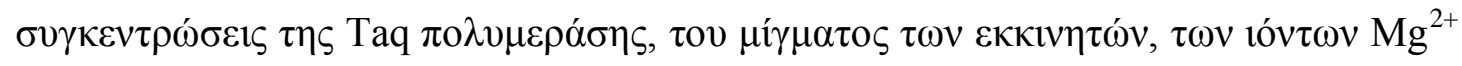

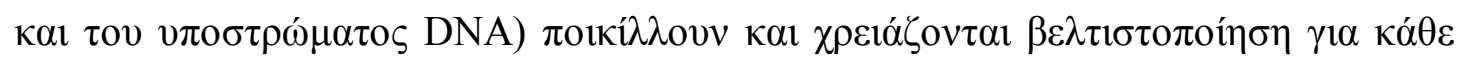
$\xi \varepsilon \chi \omega \rho 1 \sigma \tau \eta \dot{~} \alpha \nu \tau i ́ \delta \rho \alpha \sigma \eta$.

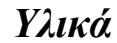

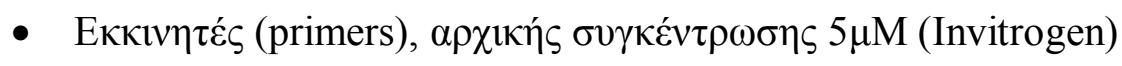

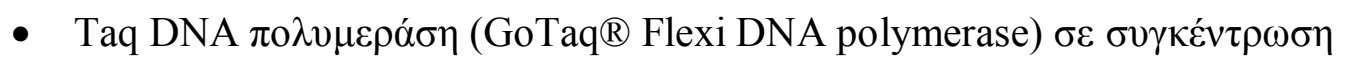

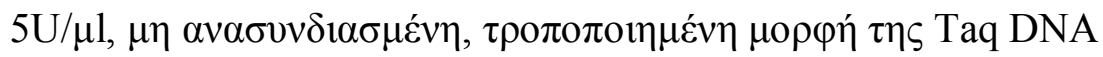

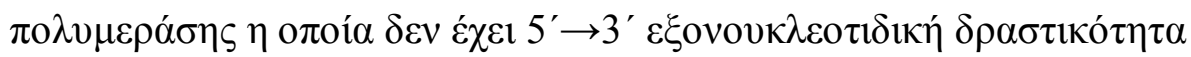
(Promega)

- $25 \mathrm{mM} \mathrm{MgCl}_{2}$ (Promega)

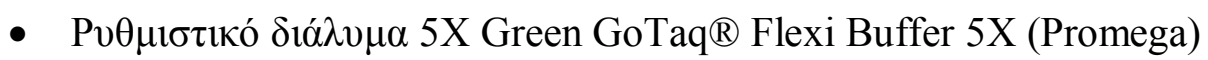

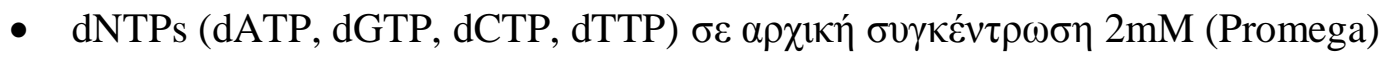




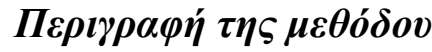

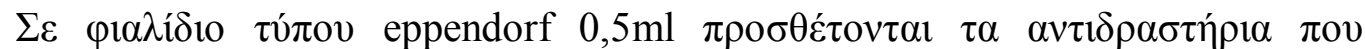

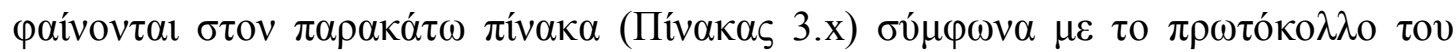
$\kappa \alpha \tau \alpha \sigma \kappa \varepsilon v \alpha \sigma \tau \eta ́$.

\begin{tabular}{lc}
\hline \multicolumn{2}{c}{ Mí $\gamma \boldsymbol{\mu \alpha} \boldsymbol{\alpha}$ PCR } \\
\hline $\mathrm{ddH}_{2} \mathrm{O}$ & $10,6 \mu \mathrm{l}$ \\
$\mathrm{MgCl}_{2}(25 \mathrm{mM})$ & $1,2 \mu \mathrm{l}$ \\
$\operatorname{Buffer}(5 \mathrm{x})$ & $4 \mu \mathrm{l}$ \\
$\mathrm{dNTPs}(2 \mathrm{mM})$ & $2 \mu \mathrm{l}$ \\
Primers $(5 \mu \mathrm{M})$ & $1 \mu \mathrm{l}$ \\
Taq polymerase $(5 \mathrm{U} / \mu \mathrm{l})$ & $0,2 \mu \mathrm{l}$ \\
DNA & $1 \mu \mathrm{l}$ \\
\hline$\Sigma v ́ v o \lambda o$ & $20 \mu \mathrm{l}$
\end{tabular}

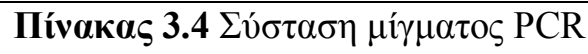

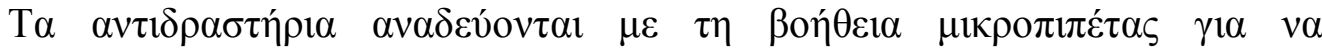

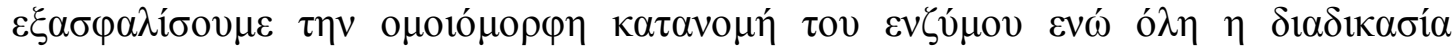

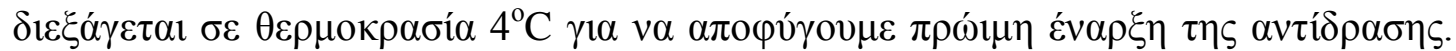

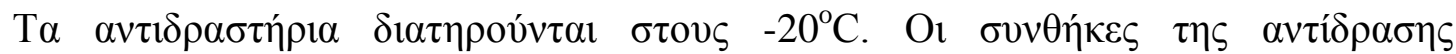

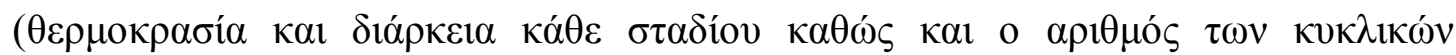

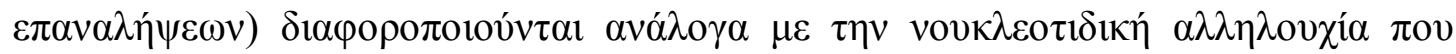

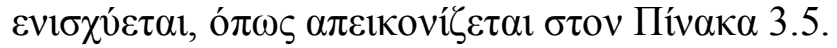

\begin{tabular}{|c|c|c|c|c|c|}
\hline 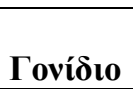 & 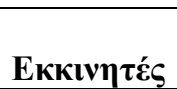 & $A \lambda \lambda \eta \lambda \mathbf{0 v} \chi \mathbf{i} \alpha$ & 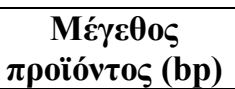 & 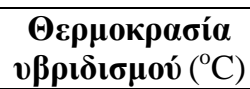 & 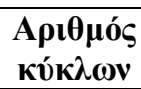 \\
\hline \multirow[t]{2}{*}{$\mathrm{BiP}$} & BIP-S & TAGCGTATGGTGCTGCTGTC & 241 & 60 & 25 \\
\hline & BIP-AS & TTTGTCAGGGGTCTTTCACC & & & \\
\hline \multirow[t]{2}{*}{ CHOP } & CHOP-S & AGTGCCACGGAGAAAGCTAA & 209 & 55 & 36 \\
\hline & CHOP-AS & CCATACAGCAGCCTGAGTGA & & & \\
\hline \multirow[t]{2}{*}{ ATF4 } & ATF4-S & AACCGACAAAGACACCTTCG & 674 & 60 & 25 \\
\hline & ATF4-AS & AGGGATCATGGCAACGTAAG & & & \\
\hline \multirow[t]{2}{*}{ GRP94 } & GRP94-S & TGGGAAGAGGTTCCAGAATG & 220 & 60 & 25 \\
\hline & GRP94-AS & GTTGCCAGACCATCCGTACT & & & \\
\hline
\end{tabular}

(S: sense, AS: antisense)

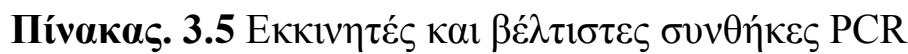




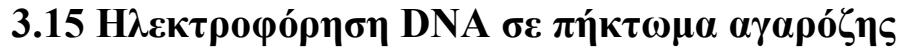

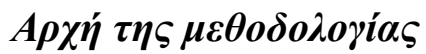

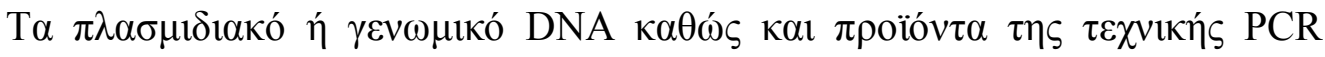

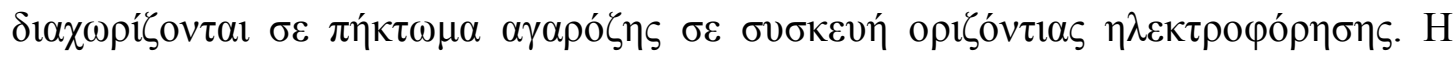

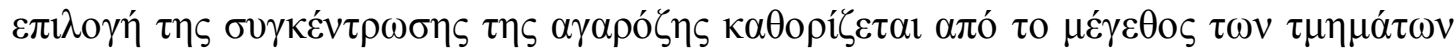

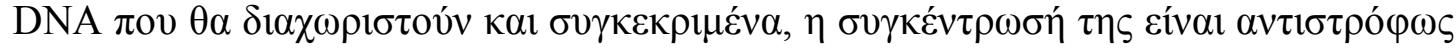

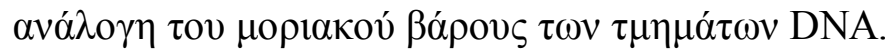

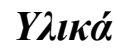

- A $\gamma \alpha \rho o ́ \zeta \eta ~(I n v i t r o g e n)$

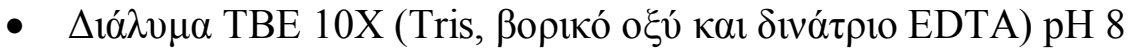

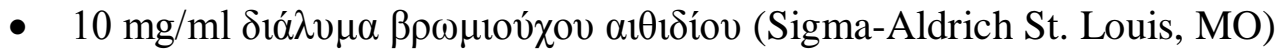

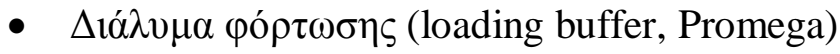

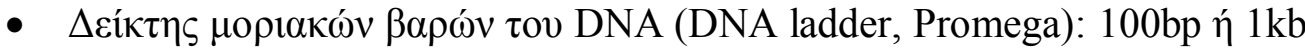

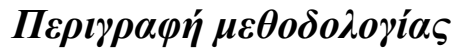

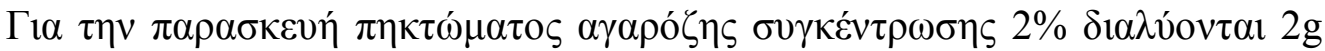

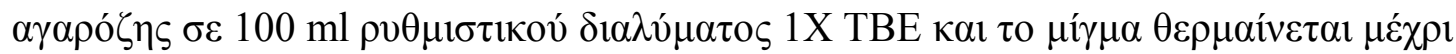

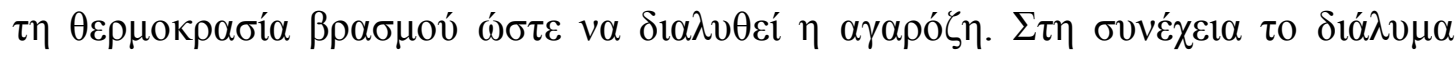

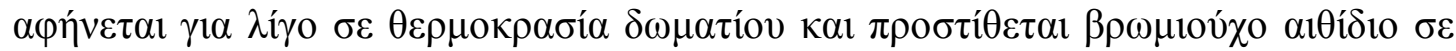

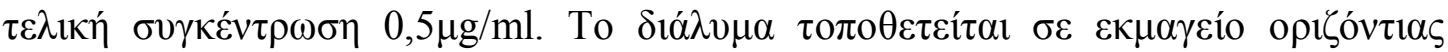

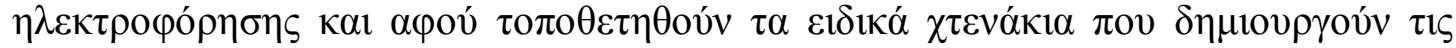

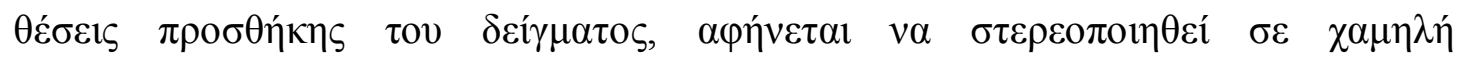

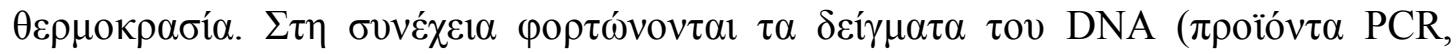

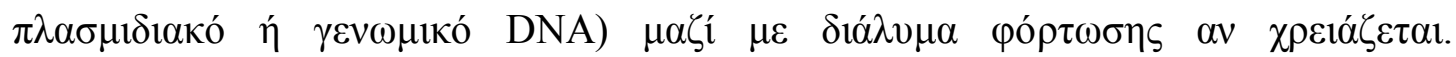

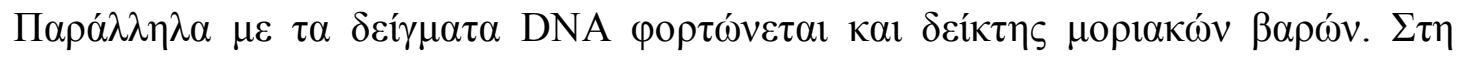

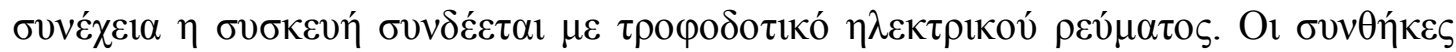

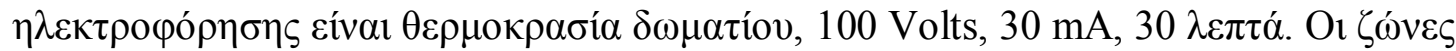

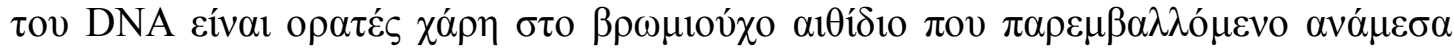

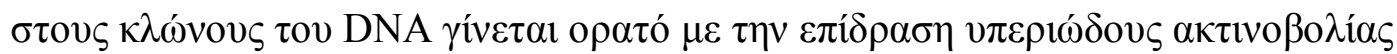




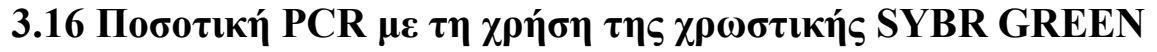

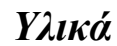

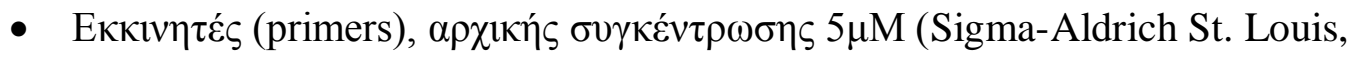

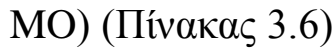

- 2x SYBR ${ }^{\circledR}$ Green PCR Master Mix

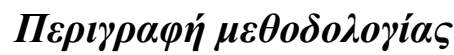

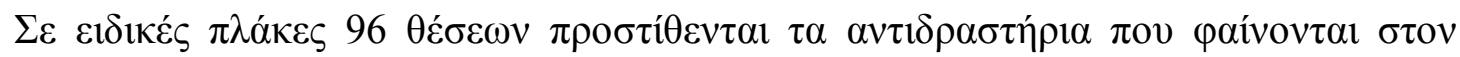

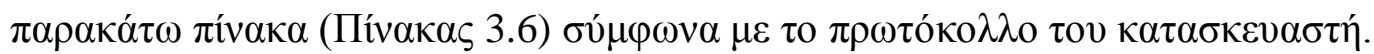

\begin{tabular}{|c|c|}
\hline \multicolumn{2}{|c|}{ 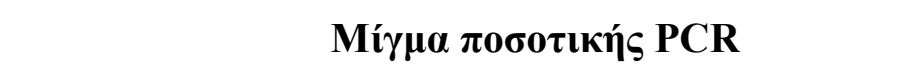 } \\
\hline 2x SYBR $®$ Green PCR Master Mix & $12,5 \mu$ \\
\hline Primers $(5 \mu \mathrm{M})$ & $1,5 \mu 1$ \\
\hline cDNA (500ng) & $10 \mu 1$ \\
\hline $\mathrm{ddH}_{2} \mathrm{O}$ & 1 \\
\hline$\Sigma u ́ v o \lambda o$ & $25 \mu 1$ \\
\hline
\end{tabular}

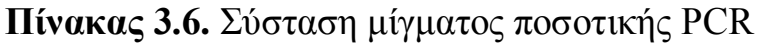

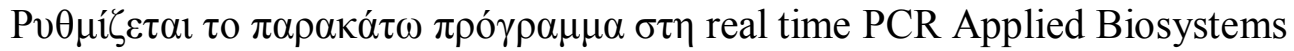

1. $10 \mathrm{~min} / 95^{\circ} \mathrm{C}$

2. $15 \mathrm{sec} / 95^{\circ} \mathrm{C}$

3. $1 \mathrm{~min} / 60^{\circ} \mathrm{C}$

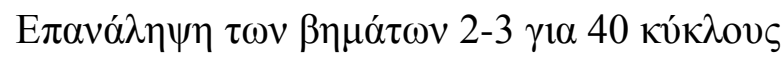

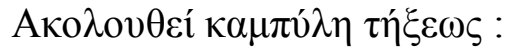

1. $15 \mathrm{sec} / 95^{\circ} \mathrm{C}$

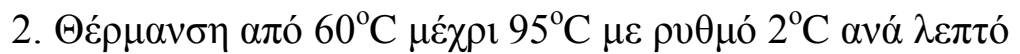




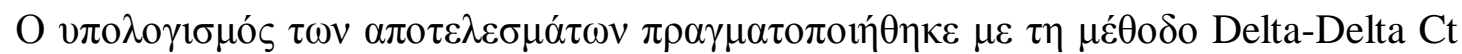

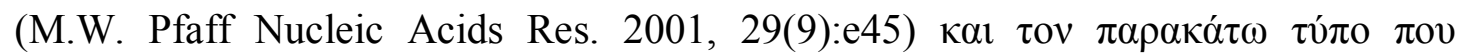

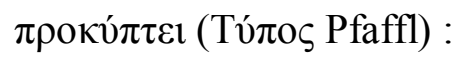

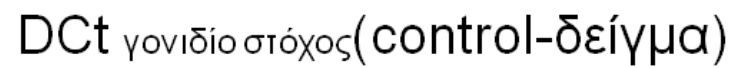

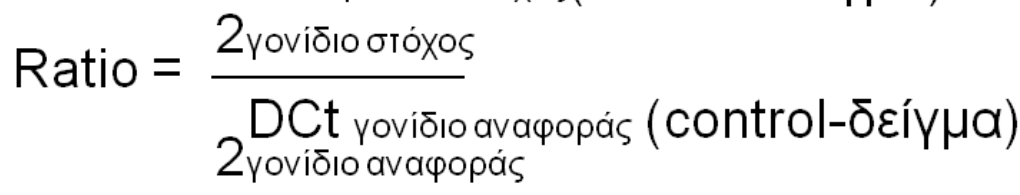

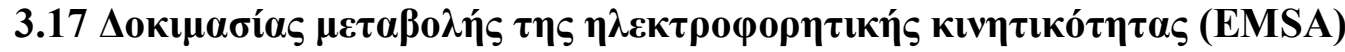

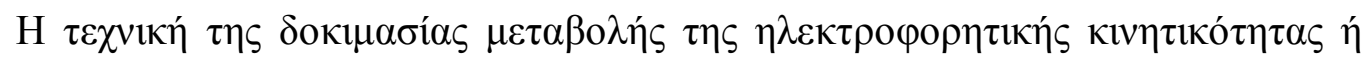

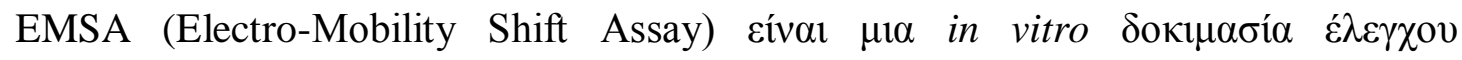

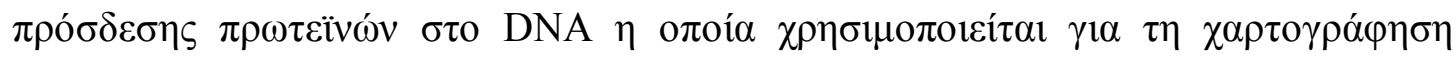

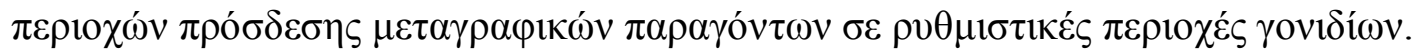

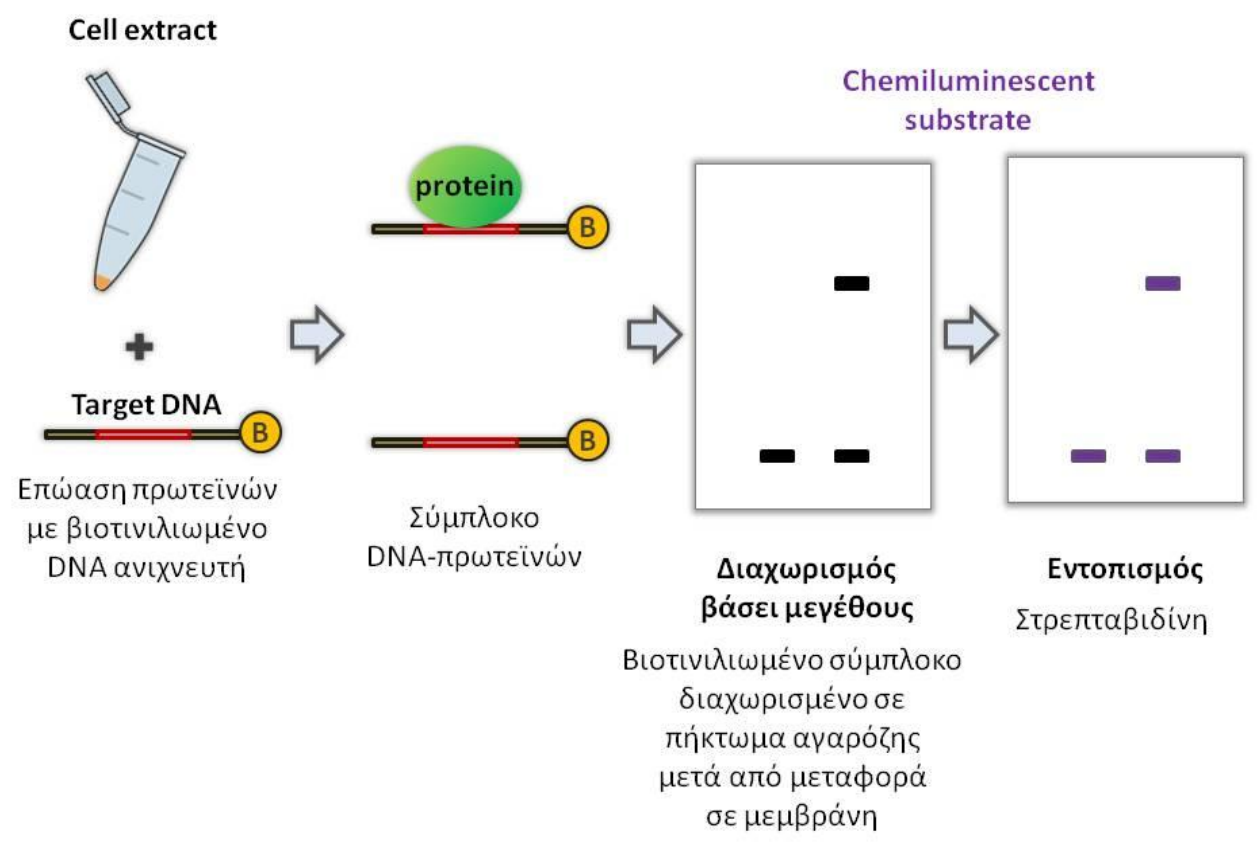

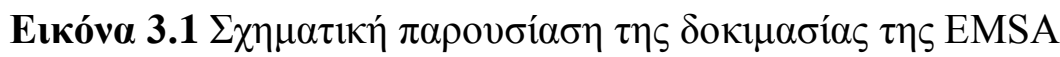




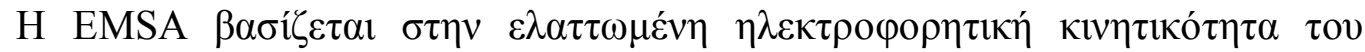

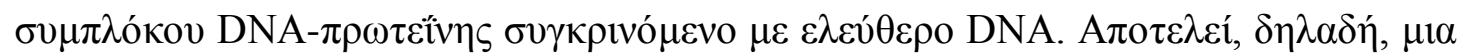

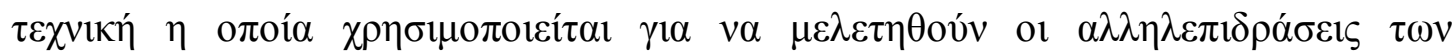

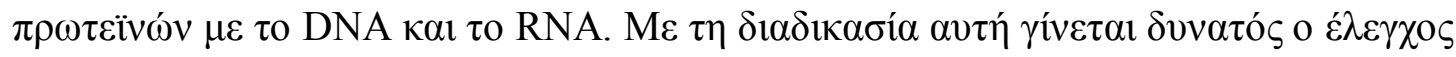

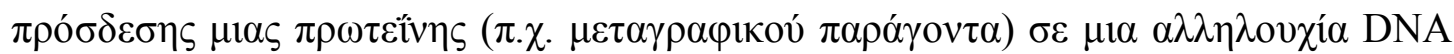
ท́ RNA (Eıкóva 3.1).

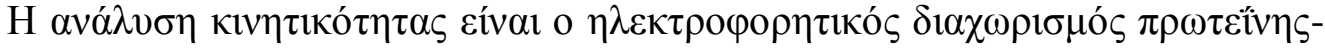

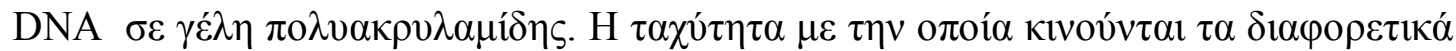

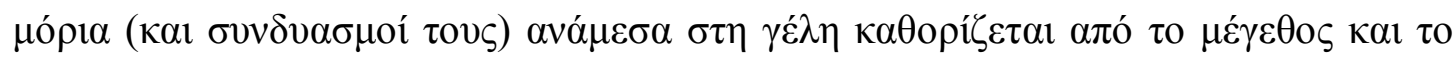

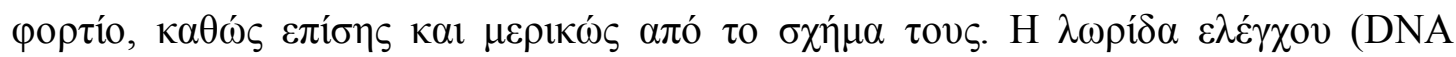

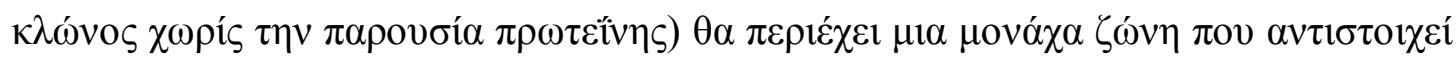

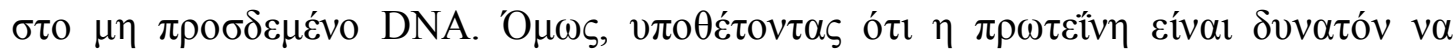

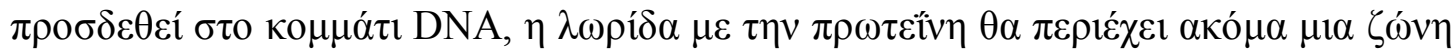

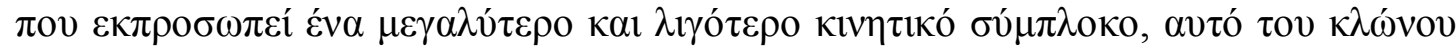

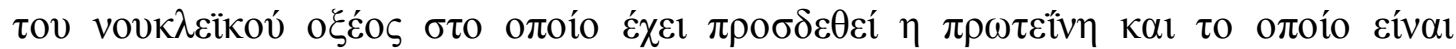

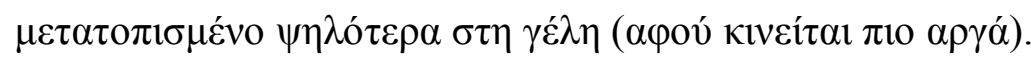

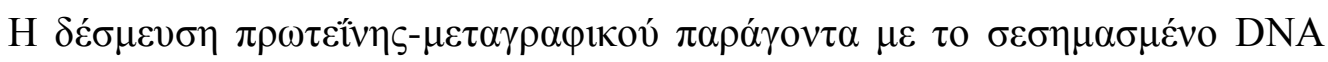

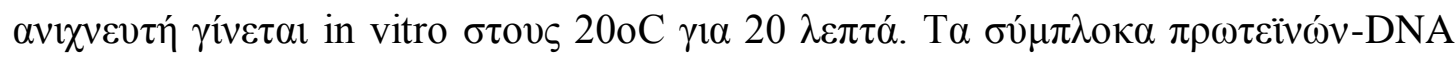

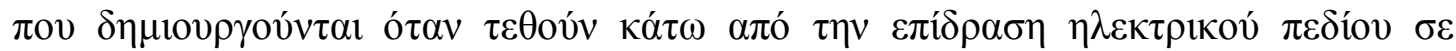

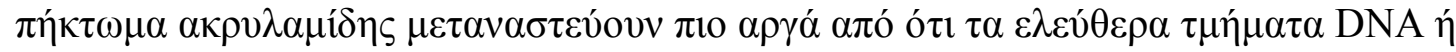

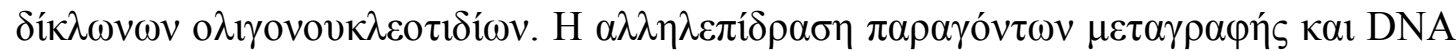

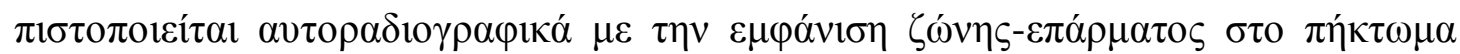

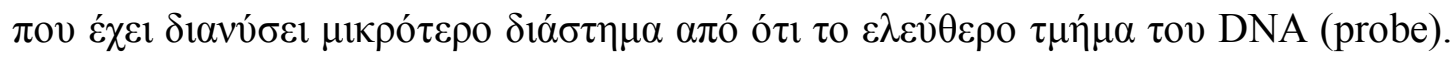

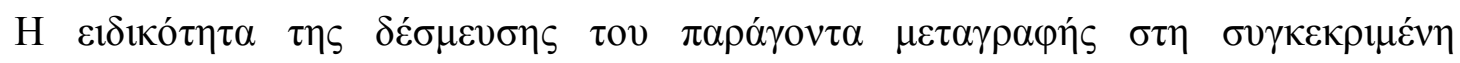

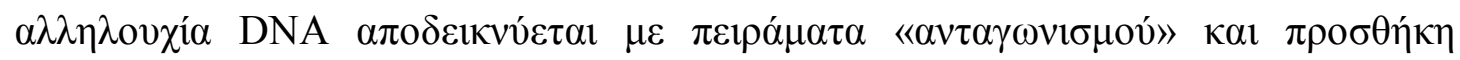

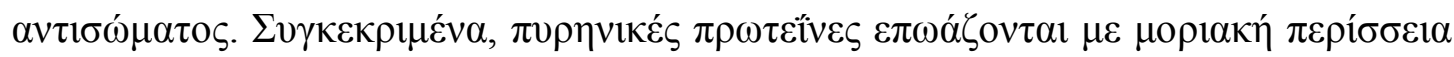

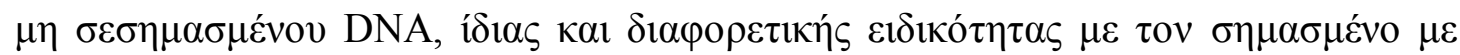

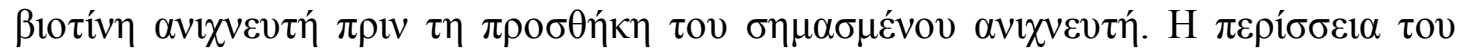

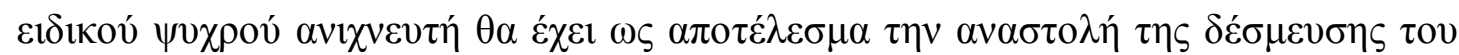

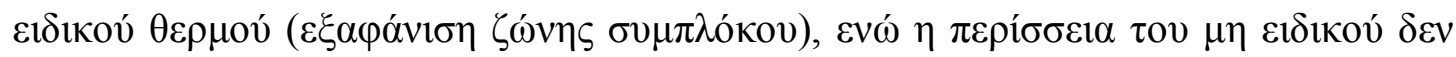

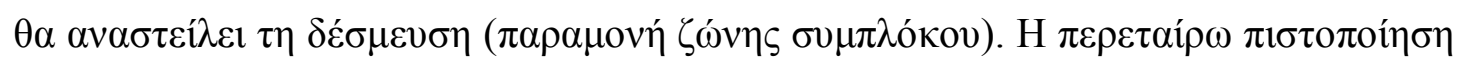

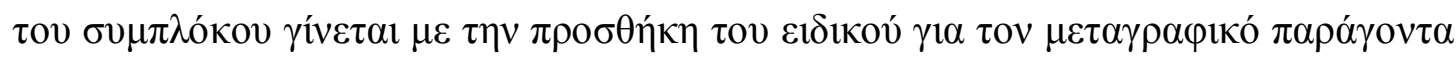

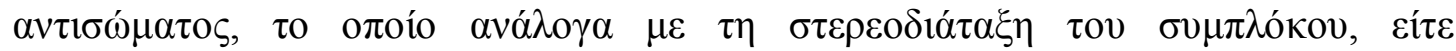




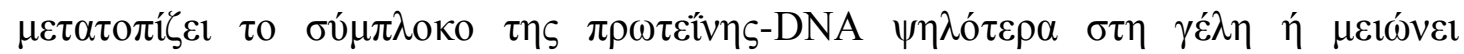

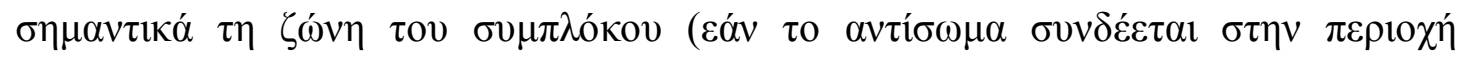

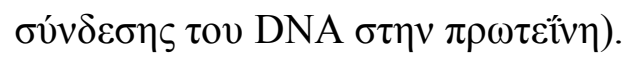

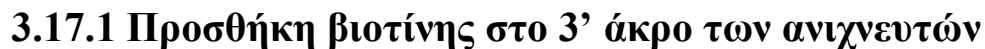

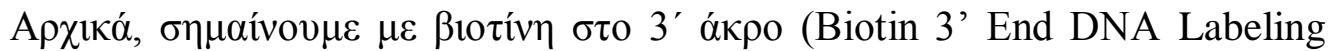

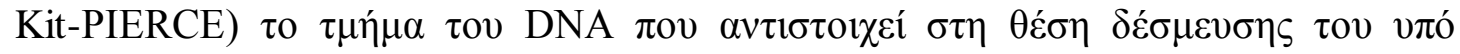

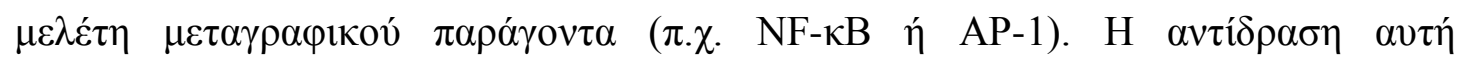

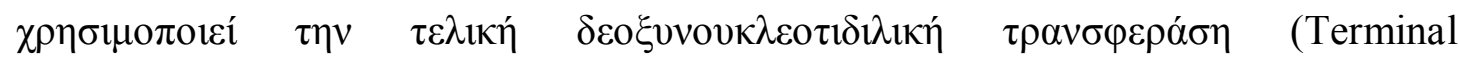

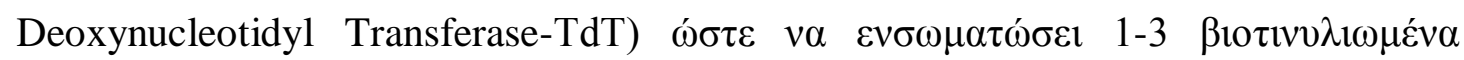

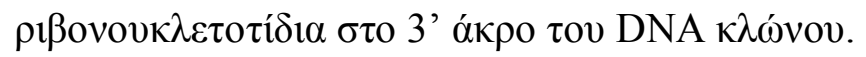

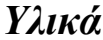

- $\quad 5 x$ TdT Reaction buffer

- $\mathrm{TdT}$

- Biotin-11-dUTP

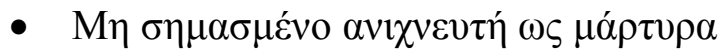

- $\Sigma \eta \mu \alpha \sigma \mu \varepsilon \dot{v o} \alpha v \imath \chi v \varepsilon v \tau \eta ́ ~ \omega \varsigma \mu \alpha ́ \rho \tau v \rho \alpha$

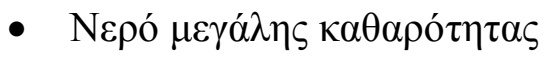

- $1 \mu \mathrm{M} \alpha v \imath \chi v \varepsilon v \tau \eta ́ n \pi \rho \circ \sigma \eta ́ \mu \alpha v \sigma \eta$

- $\quad 0.2 \mathrm{M}$ EDTA, $\mathrm{pH} 8.0$

- Х Х

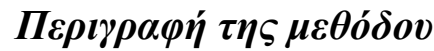

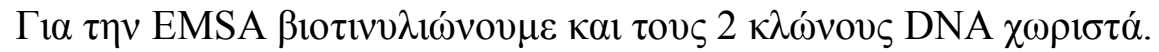

$\Sigma v \gamma \kappa \varepsilon \kappa \rho \mu \varepsilon ́ v \alpha:$

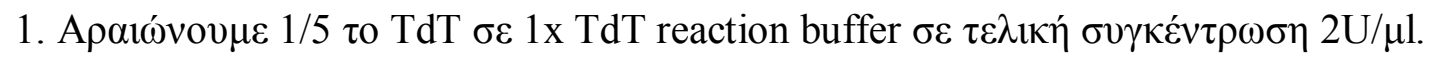

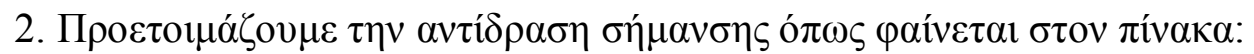




\begin{tabular}{|c|c|c|}
\hline$\Sigma v \sigma \tau \alpha \tau$ ¿ & 'О $\gamma \kappa о \varsigma$ & 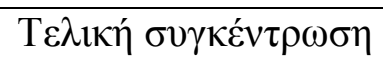 \\
\hline Nepó ultrapure & $25 \mu 1$ & --- \\
\hline 5x TdT Reaction Buffer & $10 \mu 1$ & $1 \mathrm{X}$ \\
\hline DNA $\alpha v i \chi v \varepsilon v \tau \eta \dot{ }{ }^{\prime}$ & $5 \mu 1$ & $5 \mathrm{pmol}$ \\
\hline Biotin-11-dUTP $(5 \mu \mathrm{M})$ & $5 \mu 1$ & $0.5 \mu \mathrm{M}$ \\
\hline 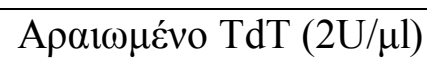 & $5 \mu 1$ & $0.2 \mathrm{U} / \mu \mathrm{l}$ \\
\hline$\Sigma v v o \lambda \imath \kappa o ́ \varsigma$ ó $\gamma \kappa о \varsigma$ & $50 \mu 1$ & $\overline{---}$ \\
\hline
\end{tabular}

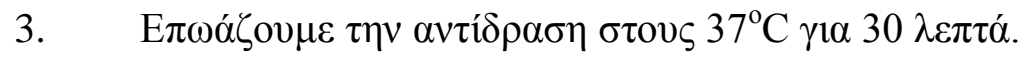

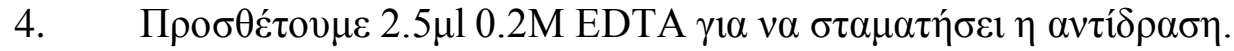

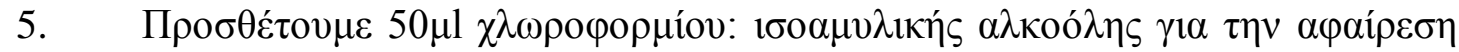

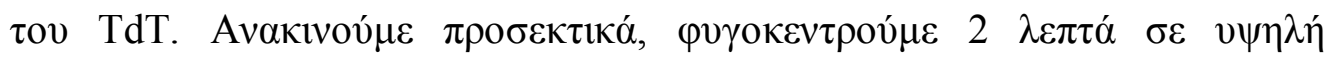

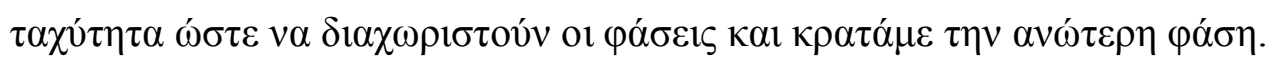

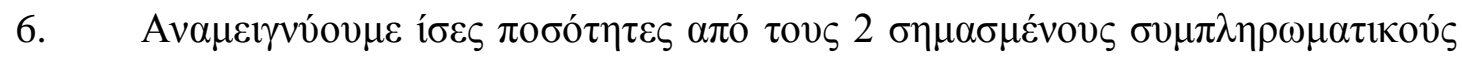

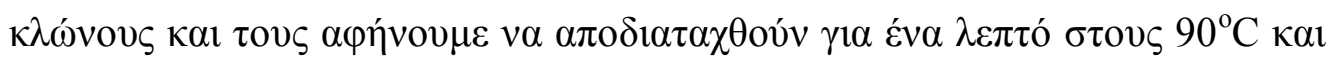

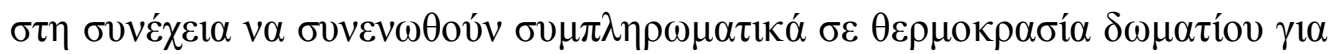

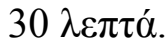

\subsubsection{Electrophoretic Mobility Shift Assay (EMSA)}

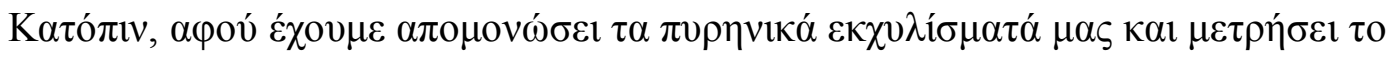

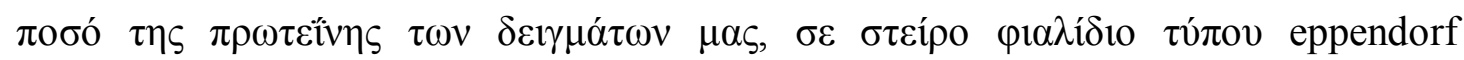

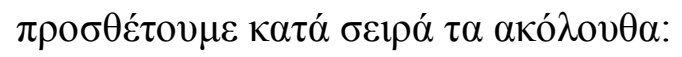

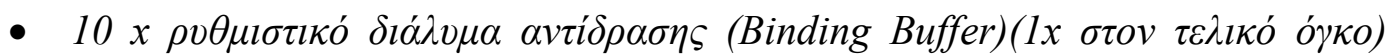
(PIERCE)

- $100 n g / \mu l$ poly dIdC (SIGMA-ALDRICH ST. LOUIS, MO)

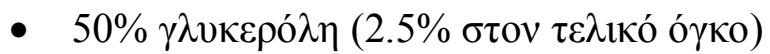

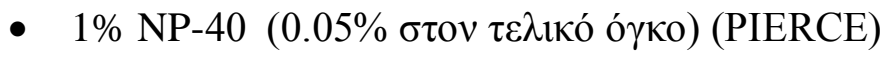

- 200mM EDTA (PIERCE)

- $10 \mu g \pi v \rho \eta v \imath \kappa \eta ́ \varsigma \pi \rho \omega \tau \varepsilon i ̈ v \eta \varsigma(\delta \varepsilon i ́ \gamma \mu \alpha)$

- Inhibitor mix $\left(\mathrm{NaF}+\mathrm{DTT}+\mathrm{PIC}+\mathrm{NA}_{3} \mathrm{VO}_{4}+\mathrm{PMSF}\right)$ 


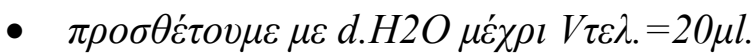

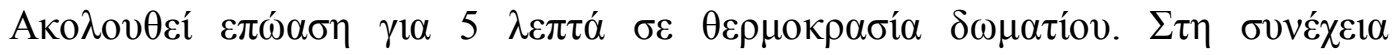

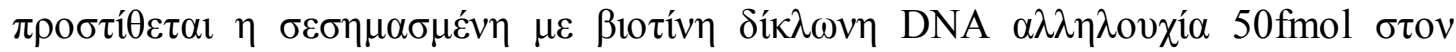

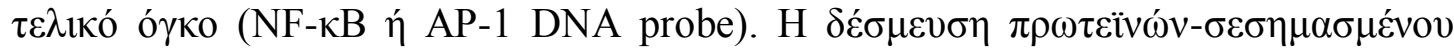

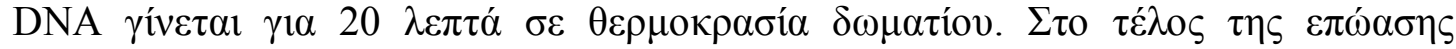

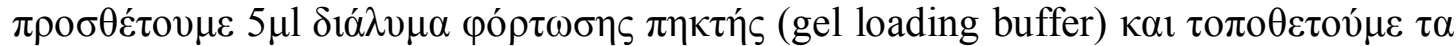

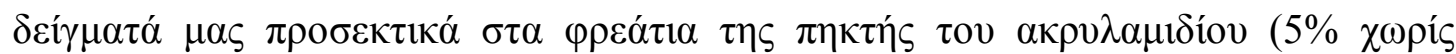
$\alpha \pi \circ \delta 1 \alpha \tau \alpha \kappa \tau 1 \kappa o v ́ \varsigma \pi \alpha \rho \alpha ́ \gamma о v \tau \varepsilon \varsigma)$.

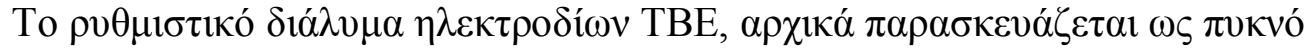

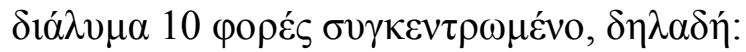

- 10.8 gr Tris-Base

- $5.5 \mathrm{gr}$ Boric acid

- $\quad 0.83 g r$ EDTA

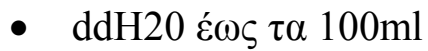

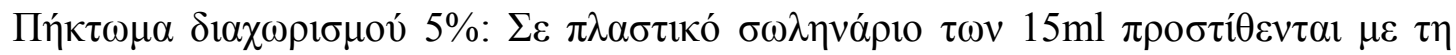

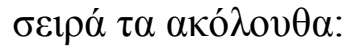

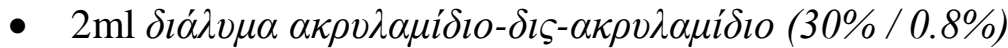

- $1.2 \mathrm{ml}$ TBEx10

- $150 \mu \mathrm{l}$ APS $10 \%$

- $\quad 20 \mu 1$ TEMED

- $\quad 8.8 \mathrm{ml}$ ddH20

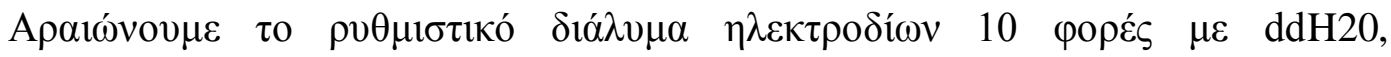

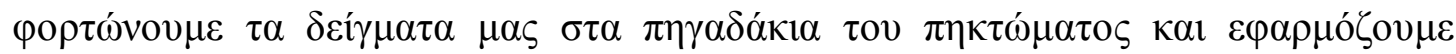

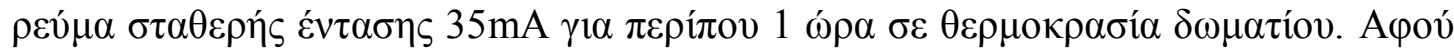

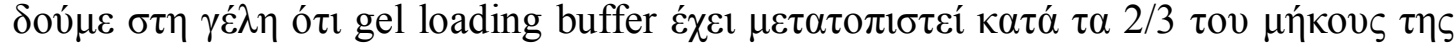

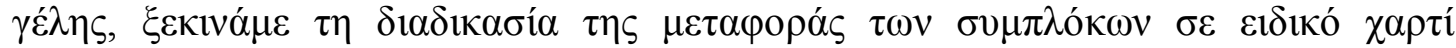

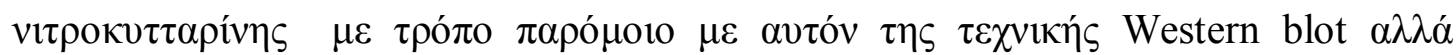

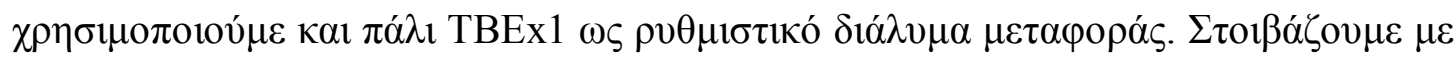

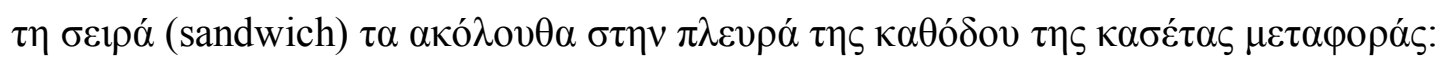




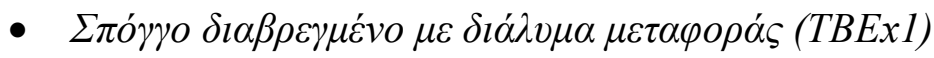

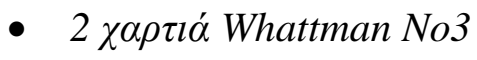

- $\Pi \dot{\eta} \kappa \tau \omega \mu \alpha(\mathrm{gel})$

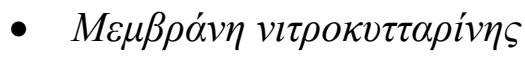

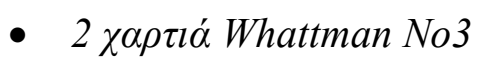

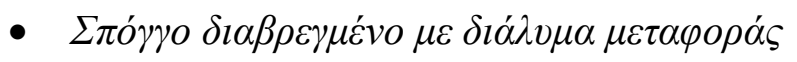

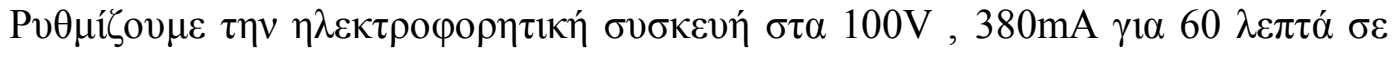

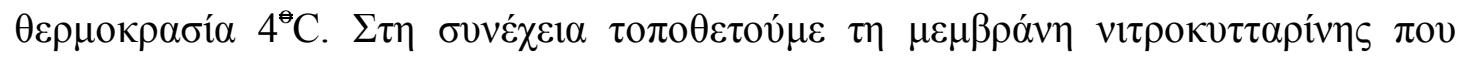

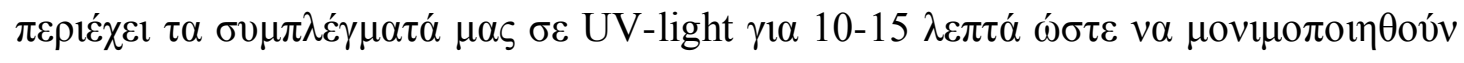

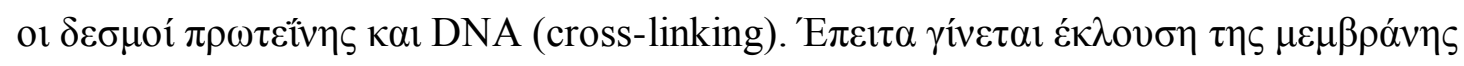

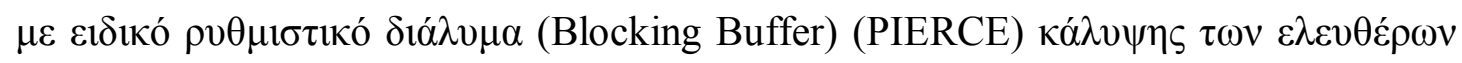

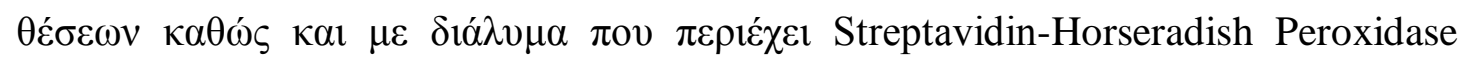

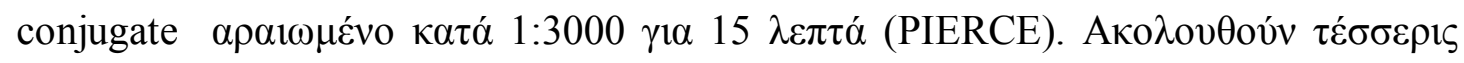

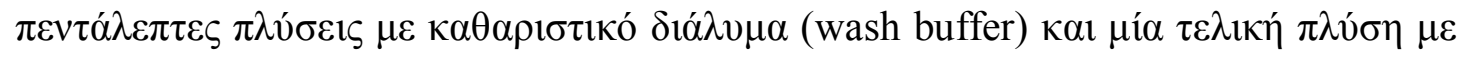
Substrate Equilibration Buffer $\gamma 1 \alpha 5 \lambda \varepsilon \pi \tau \alpha$ (PIERCE).

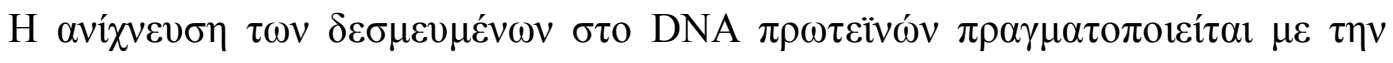

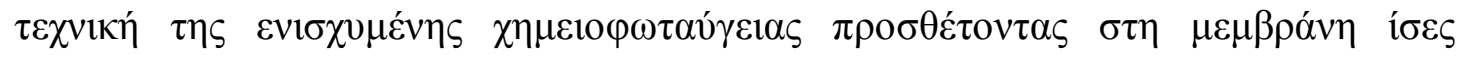

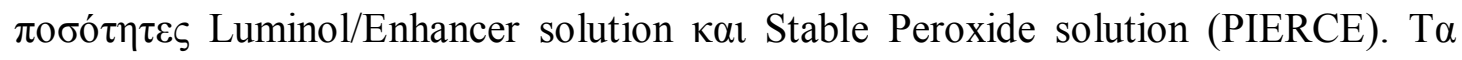

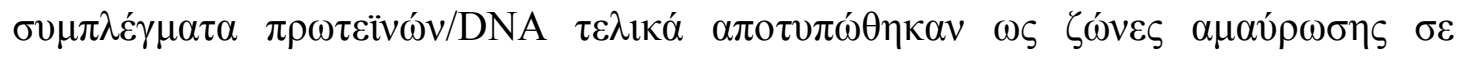

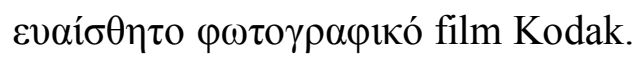

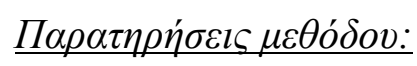

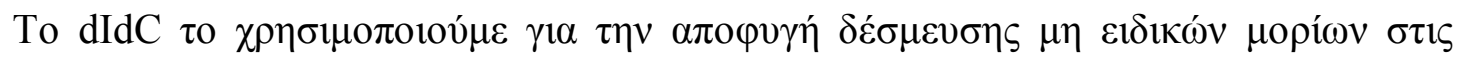

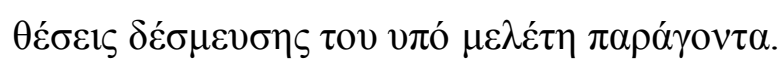

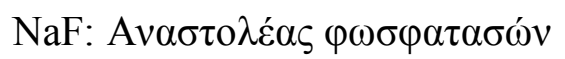

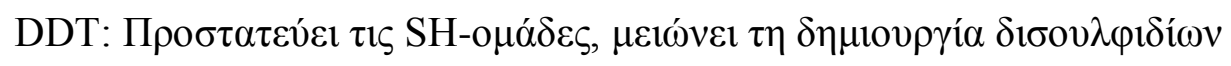

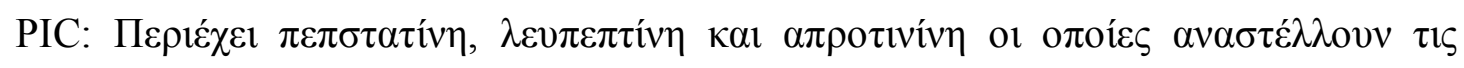

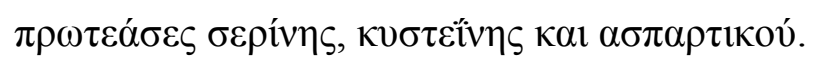

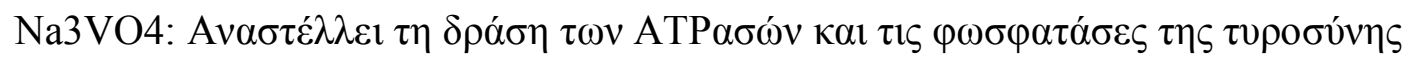

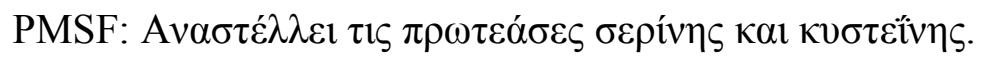




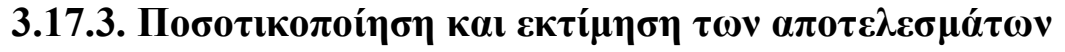

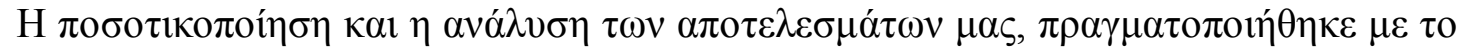

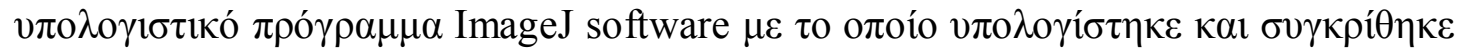

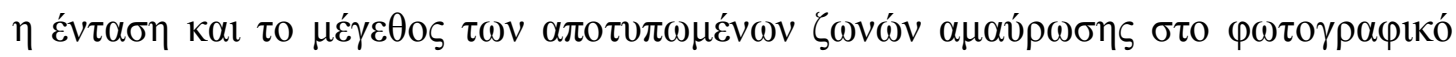
$\varphi \imath \lambda \mu$.

\subsection{Avoбoï $\tau \tau \chi \chi \mu \varepsilon i ́ \alpha$}

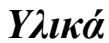

- $\Xi v \lambda o ́ \lambda \eta, \alpha \imath \theta \alpha v o ́ \lambda \eta$ (Sigma-Aldrich St. Louis, MO)

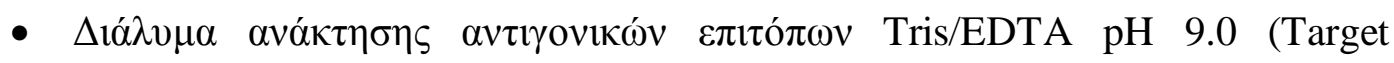
Retrieval Solution 3-in-1, Dako)

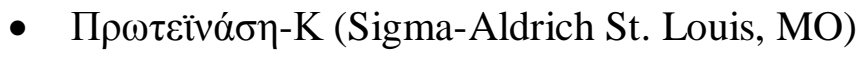

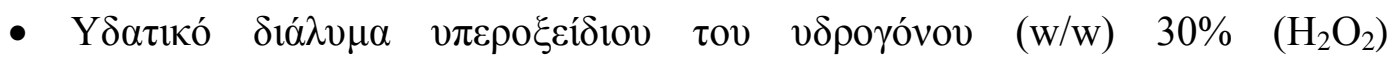
(Applichem)

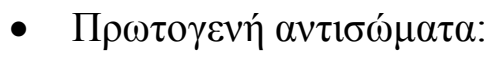

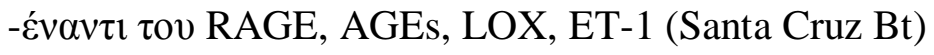

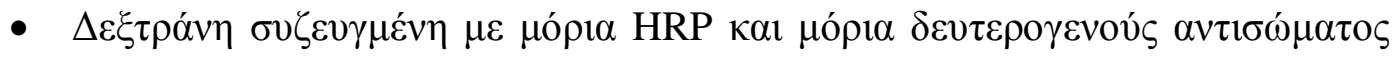

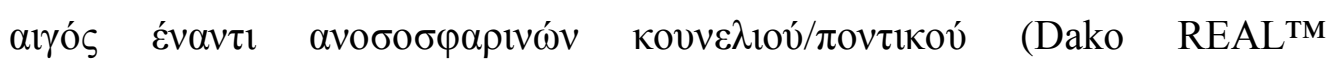
EnVision ${ }^{\mathrm{TM}} / \mathrm{HRP}$, Rabbit/Mouse) (Dako)

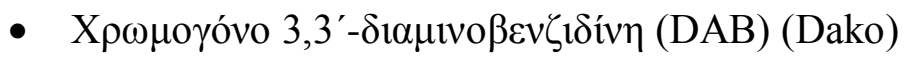

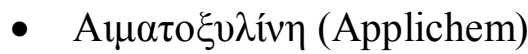

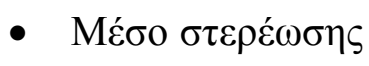

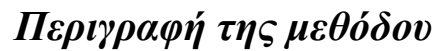

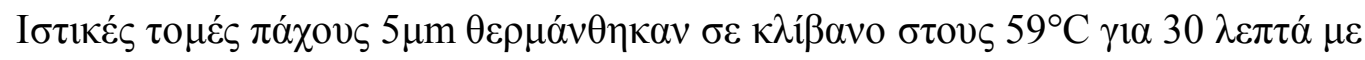

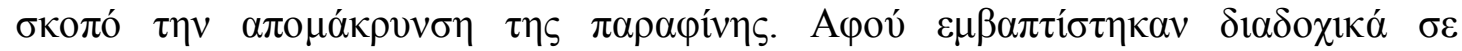

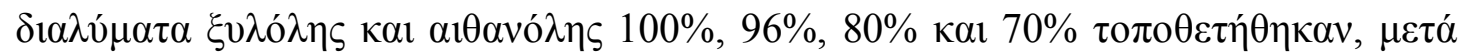

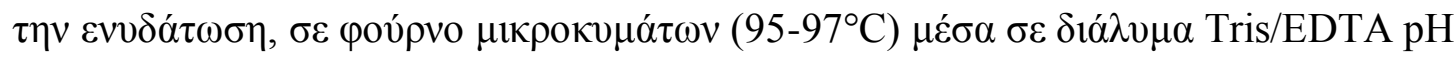

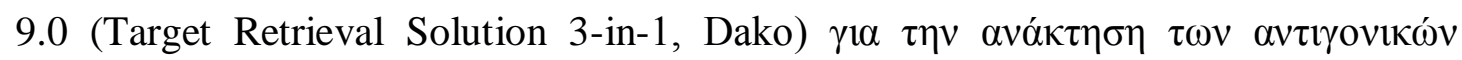

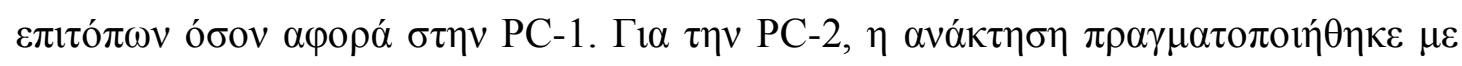




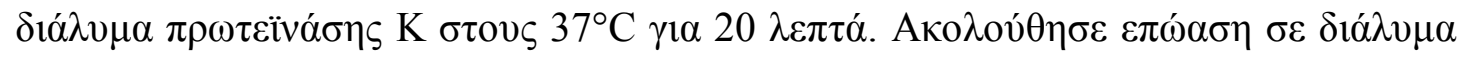

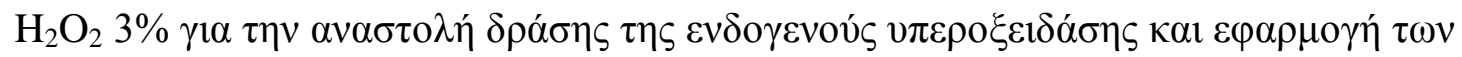

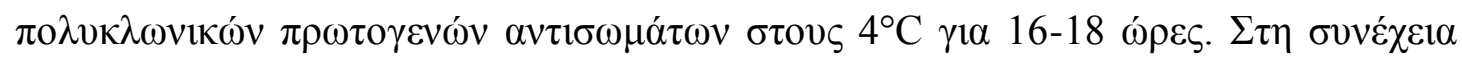

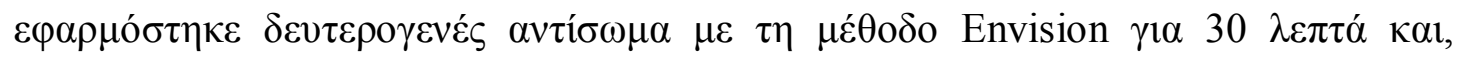

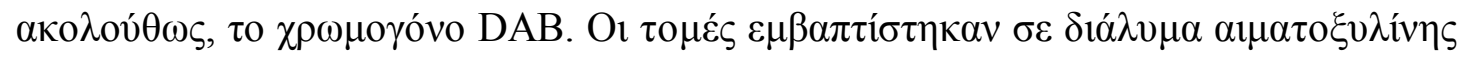

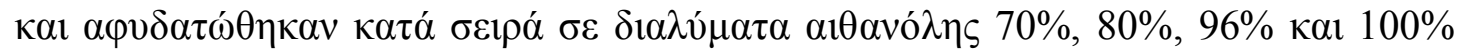

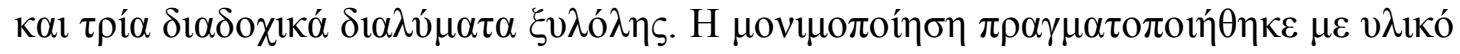

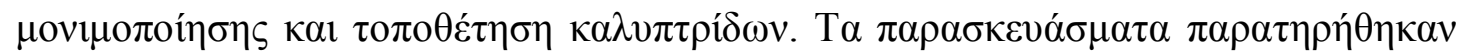

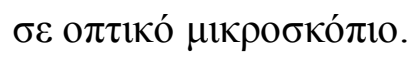

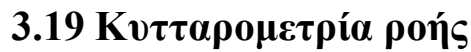

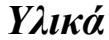

- $\Delta 1 \alpha \dot{\lambda} v \mu \mu \alpha \operatorname{PBS}$

- $\Delta \mathrm{l} \alpha \dot{\lambda} v \mu \alpha 1 \%$ (w/v) $\pi \alpha \rho \alpha \varphi \rho \rho \mu \alpha \lambda \delta \varepsilon v ́ \delta \eta \varsigma \sigma \varepsilon$ PBS

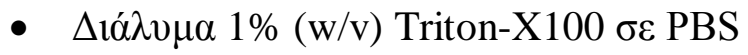

- $\Delta \mathrm{i} \alpha \dot{\lambda} v \mu \alpha 5 \%(\mathrm{v} / \mathrm{v})$ FBS $\sigma \varepsilon$ PBS

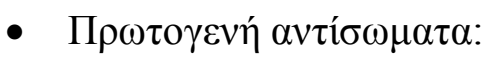

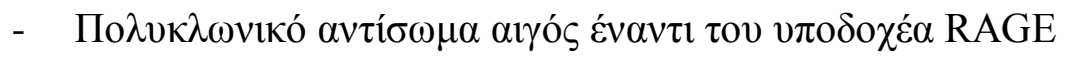
(Fitzerald)

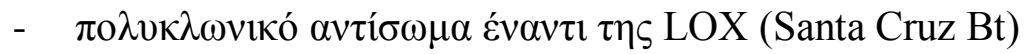

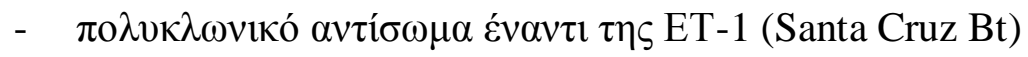

- $\Delta \varepsilon v ́ \tau \varepsilon \rho о \gamma \varepsilon v \eta \alpha \nu \tau i ́ \sigma \omega \mu \alpha \tau \alpha$ :

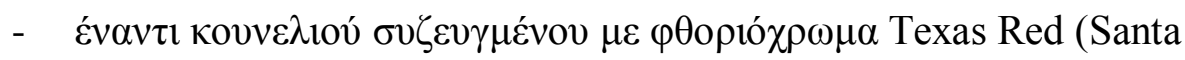
Cruz Bt)

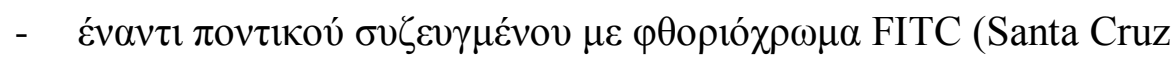

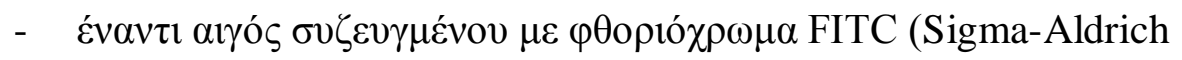
St. Louis, MO)

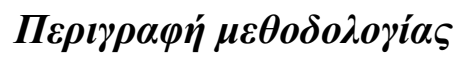

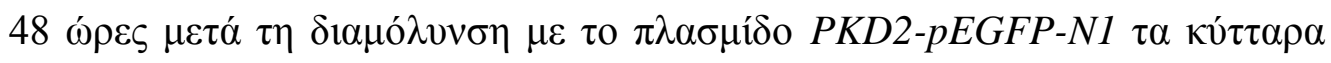

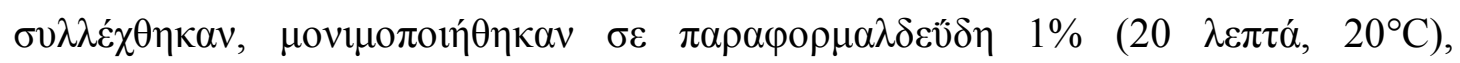




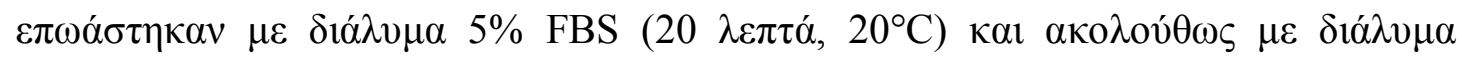

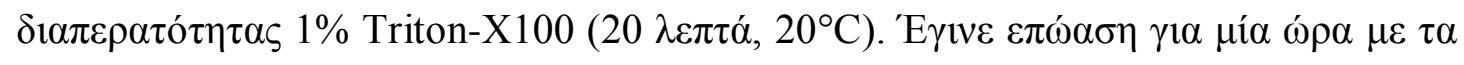

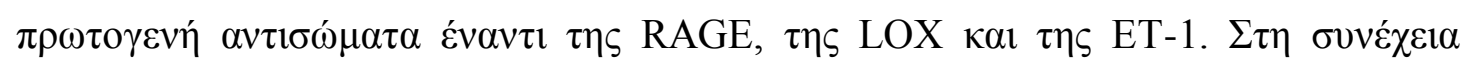

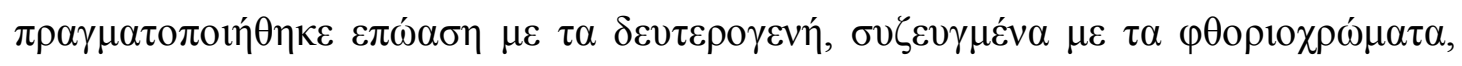

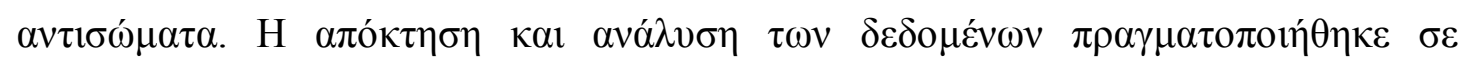

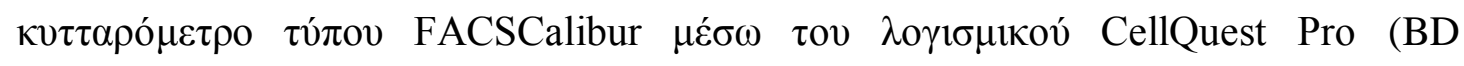
Biosciences).

\section{$3.20 \Sigma \tau \alpha \tau \iota \sigma \tau \iota \kappa \eta ́ ~ \alpha v \alpha ́ \lambda v \sigma \eta$}

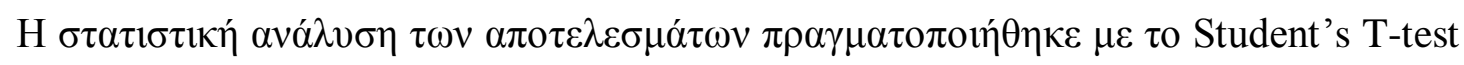

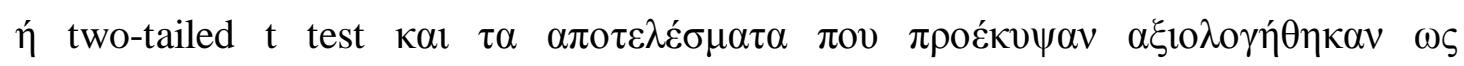

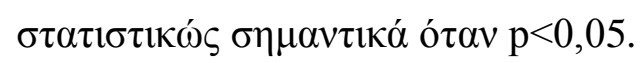




\section{AПOTE}

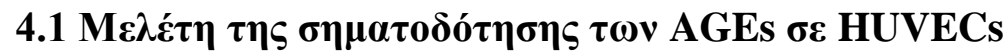

\section{$\underline{E \kappa \varphi \rho \alpha \sigma \eta ~ \tau o v ~ R A G E ~ \sigma \tau \eta v ~ \varepsilon \pi \iota \varphi \alpha ́ v \varepsilon l \alpha ~ \tau \omega v ~ H U V E C s ~}$}

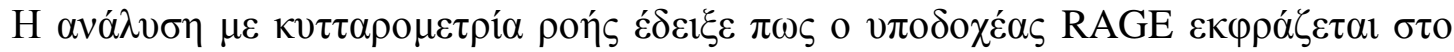

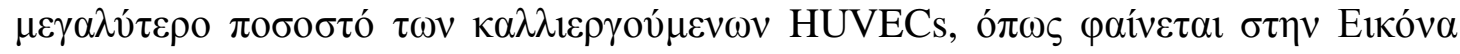
4.1B.

A

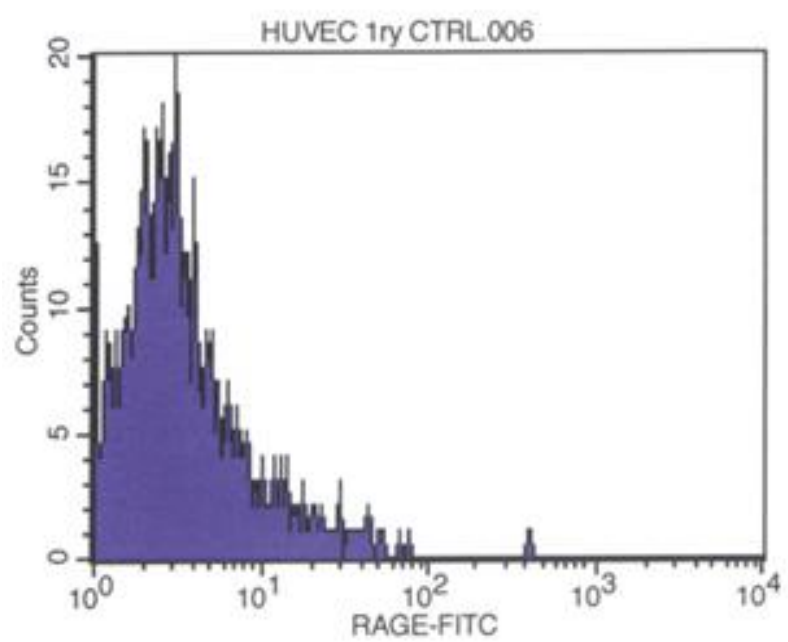

B

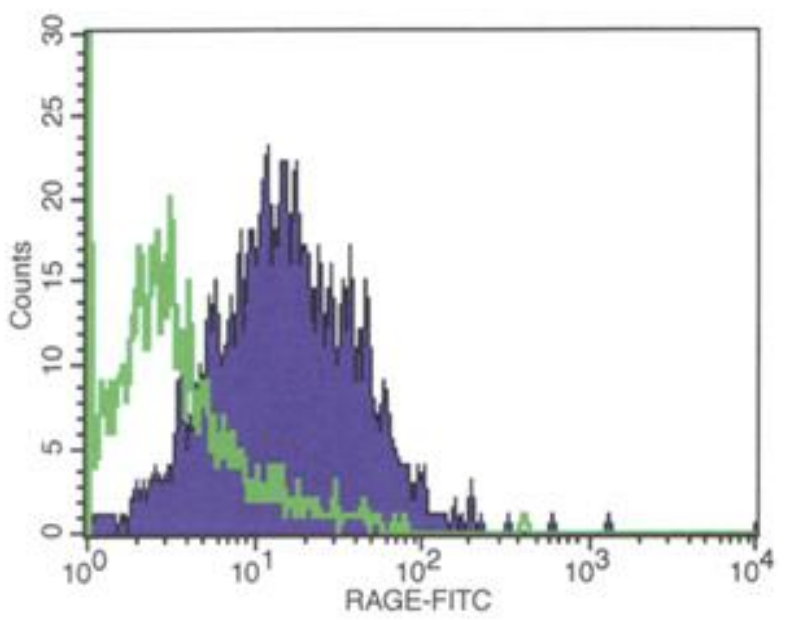

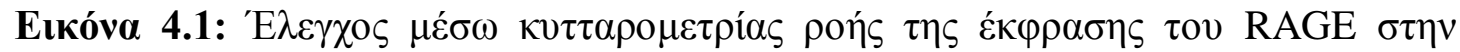

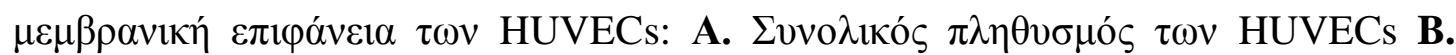

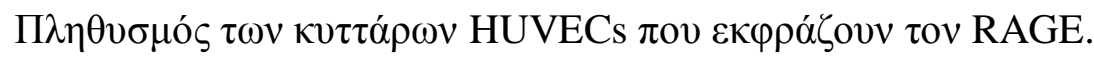

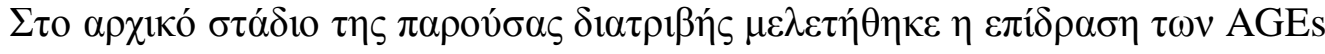

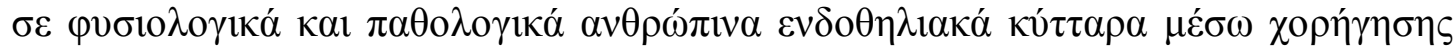

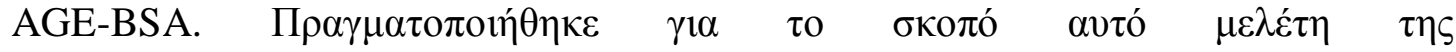

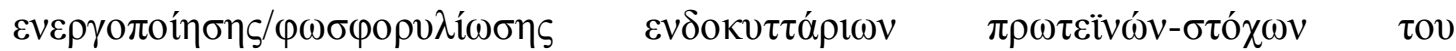

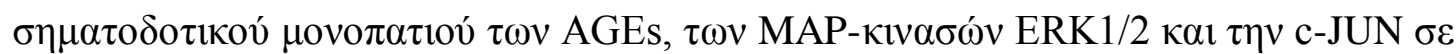

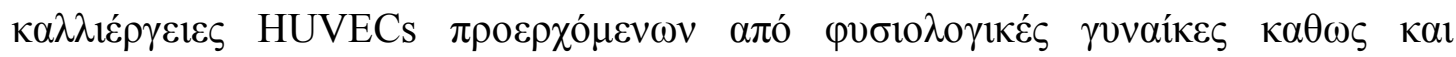

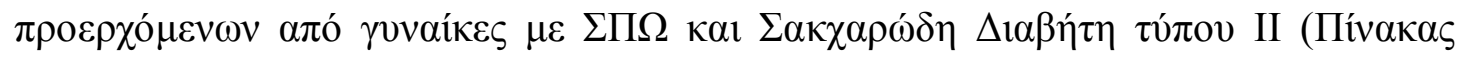

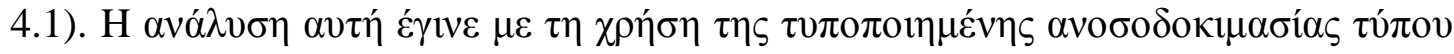

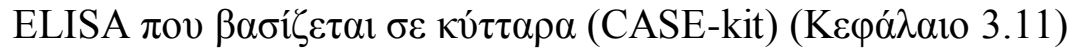




\begin{tabular}{|c|c|c|}
\hline 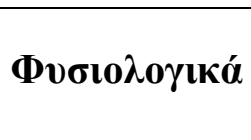 & $\Sigma \Pi \Omega$ & 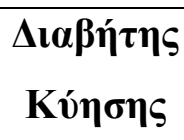 \\
\hline 15 & 15 & 24 \\
\hline
\end{tabular}

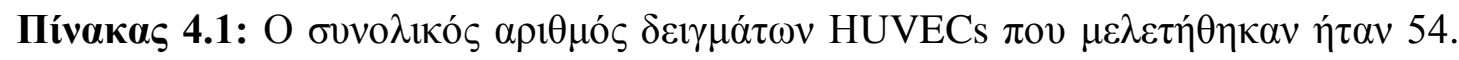

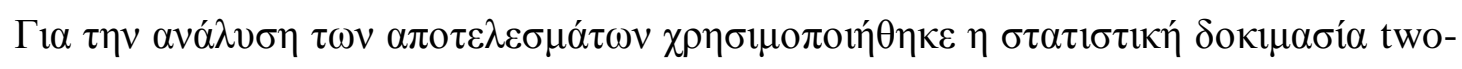
tailed $\mathrm{t}$ test.

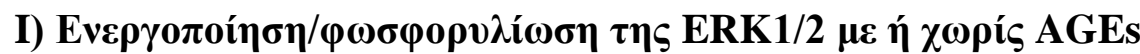

A

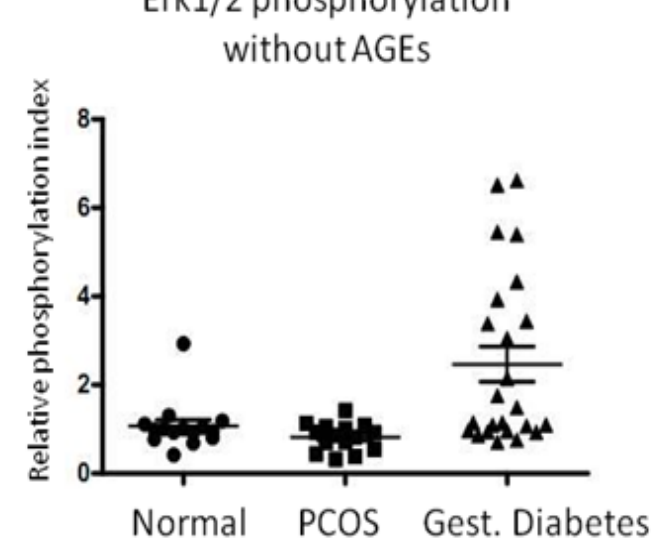

B

Erk1/2 phosphorylation with AGEs

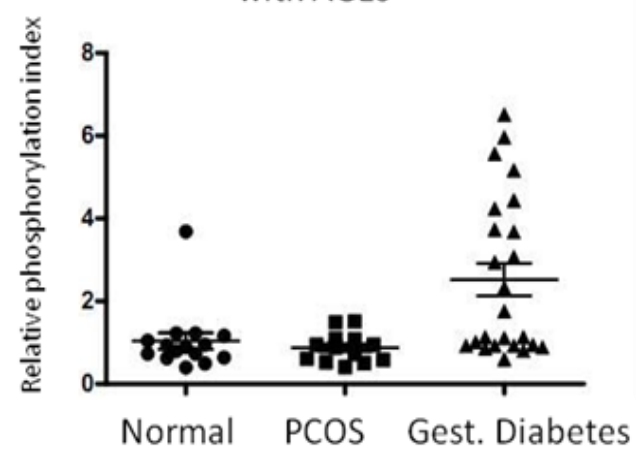

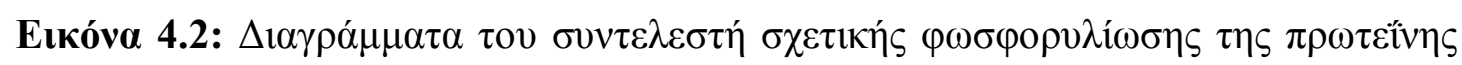

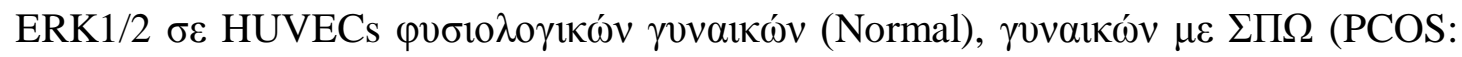

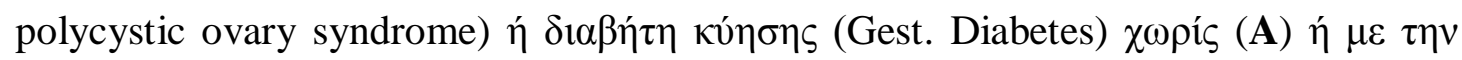
$\pi \alpha \rho o v \sigma i ́ \alpha$ AGE-BSA (B).

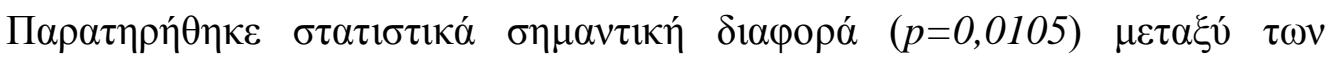

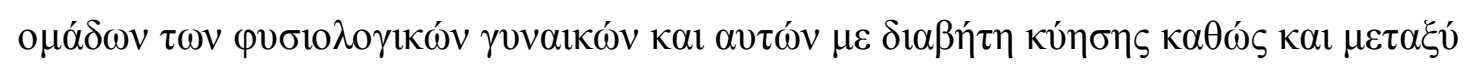

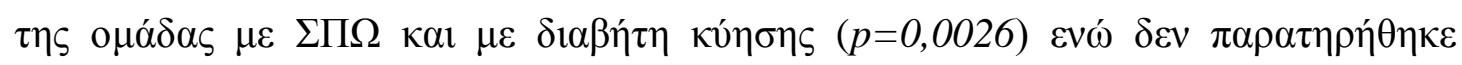

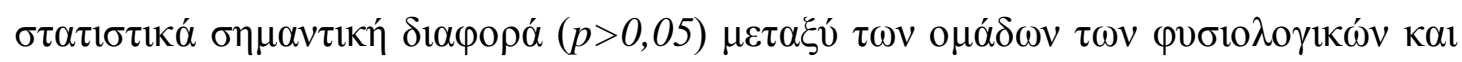

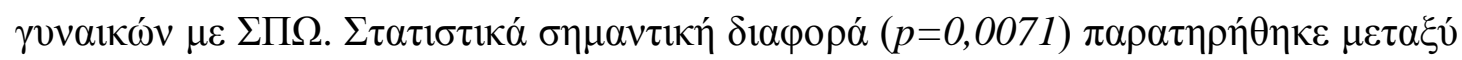

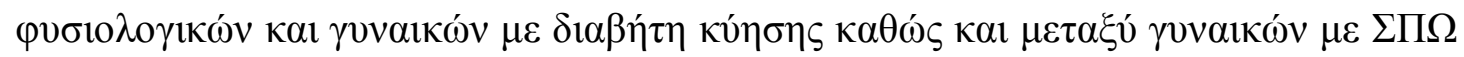

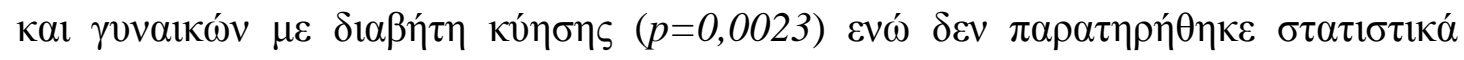




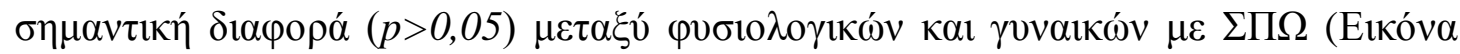
4.2).

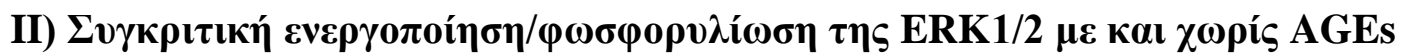

A

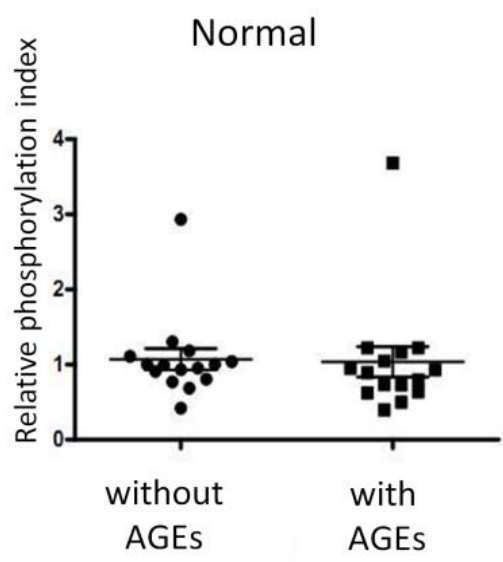

B

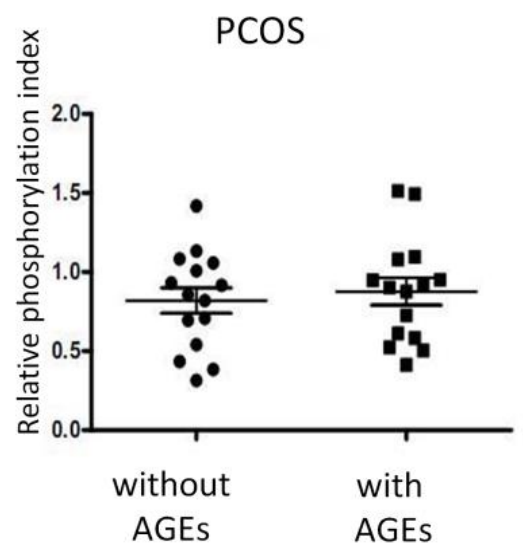

$\Gamma$

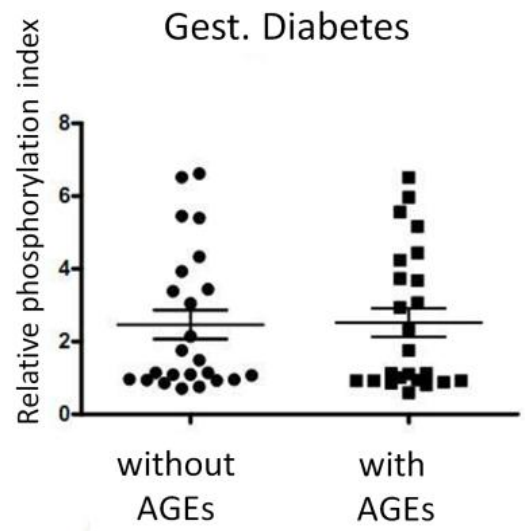

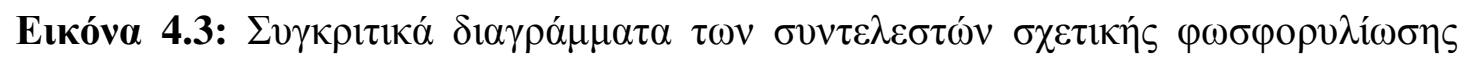

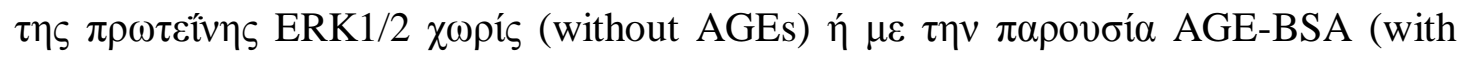

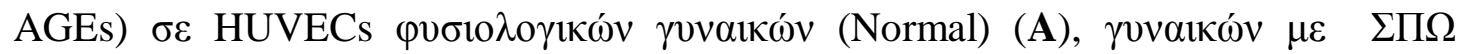

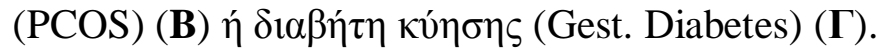

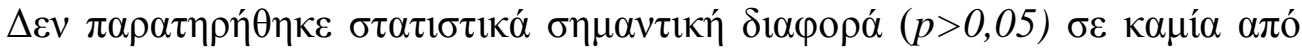

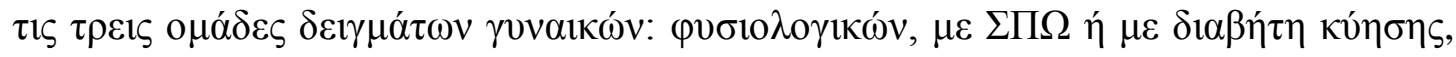

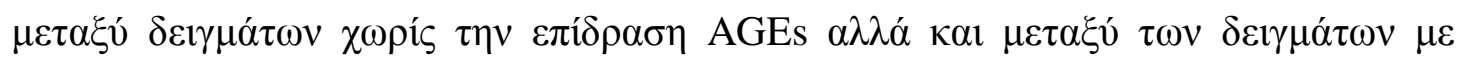

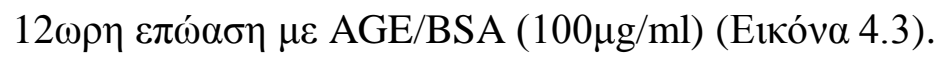

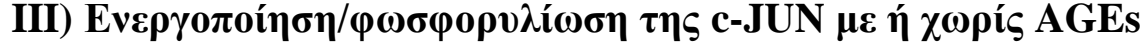

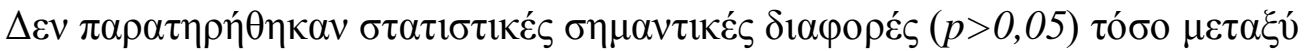

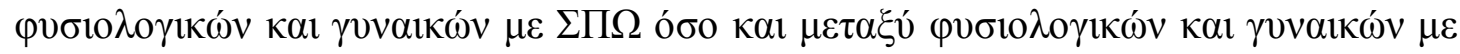

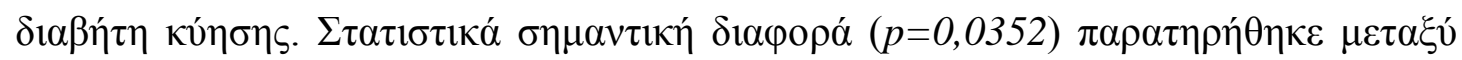

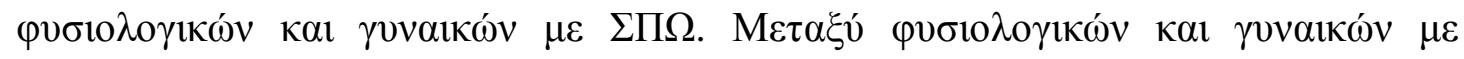

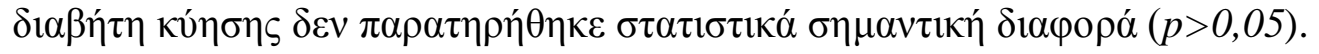


A

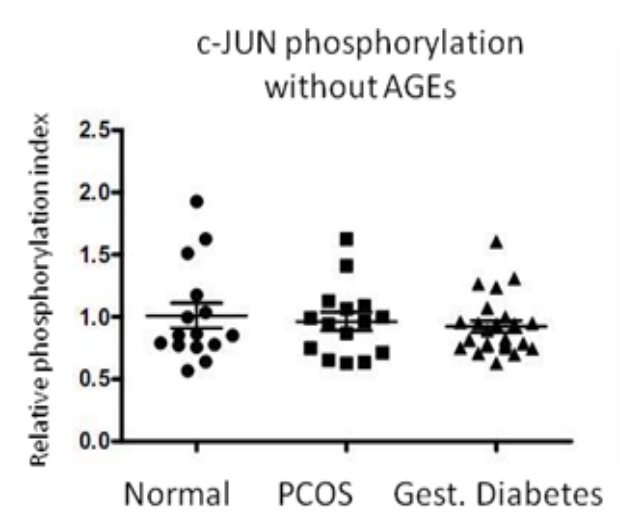

B

C-JUN phosphorylation

with AGEs

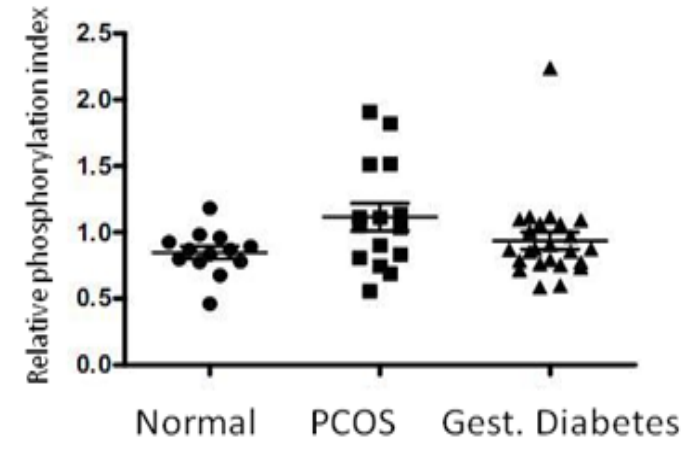

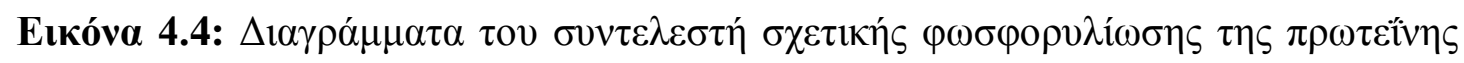

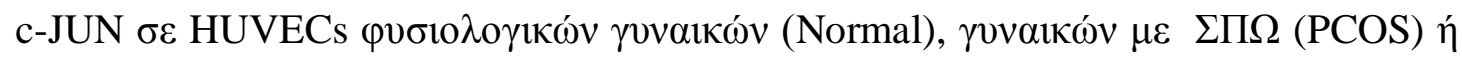

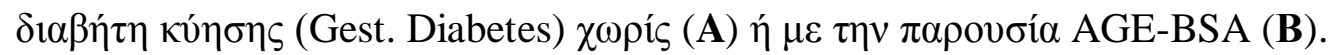

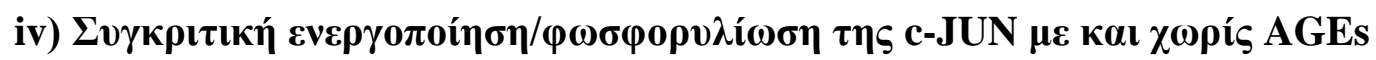

A

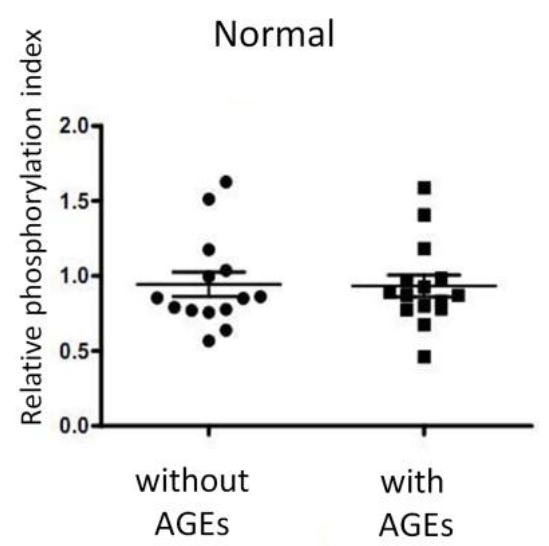

B

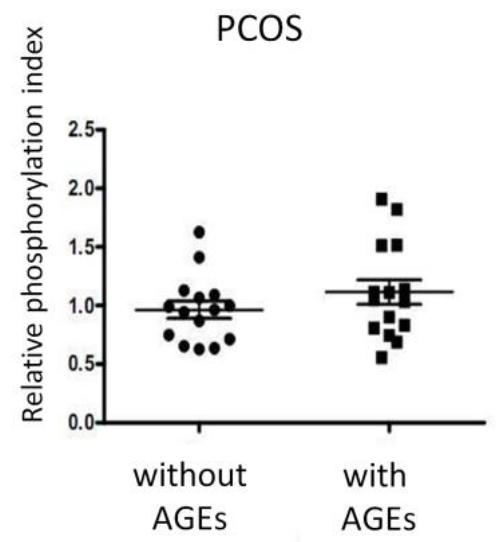

$\Gamma$

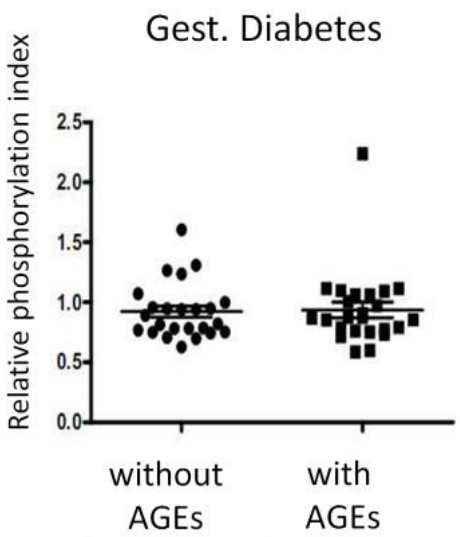

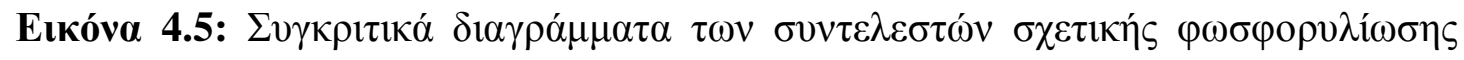

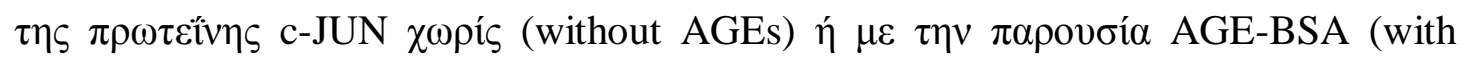

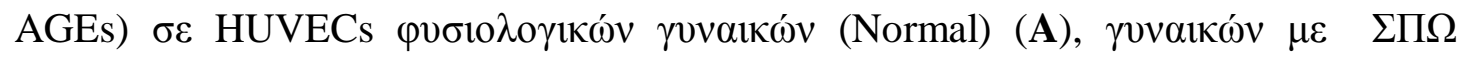

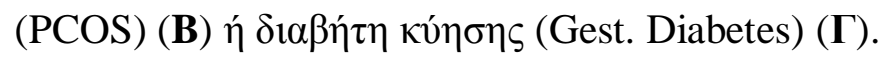

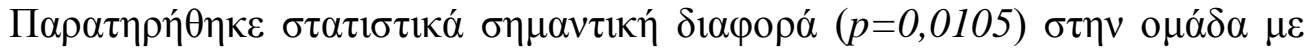

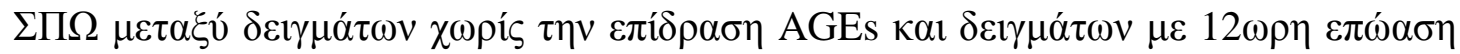

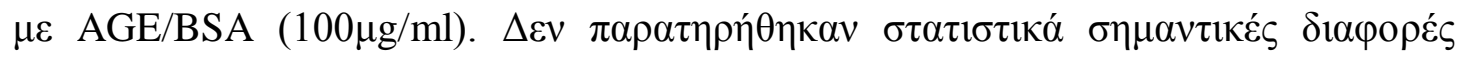

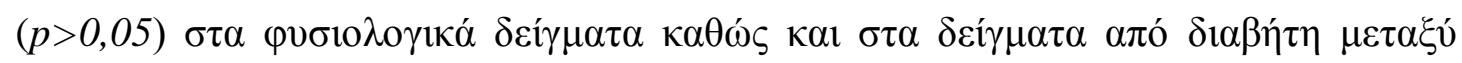




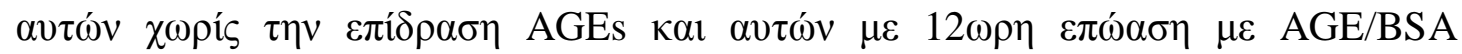
$(100 \mu \mathrm{g} / \mathrm{ml})$.

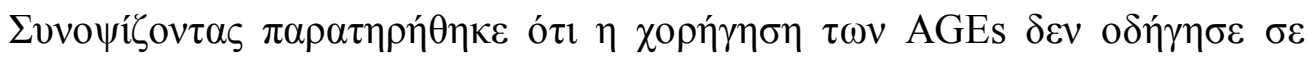

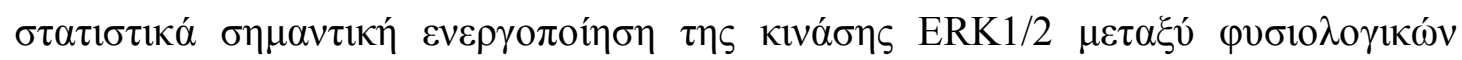

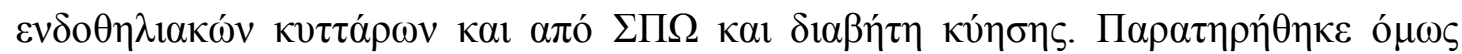

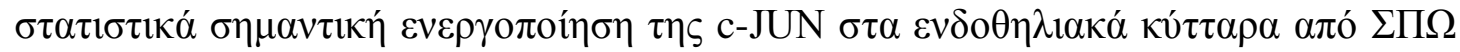

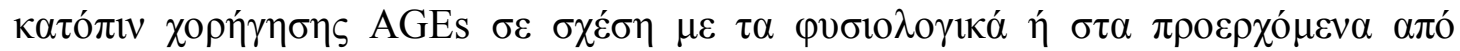

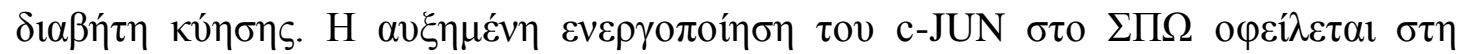

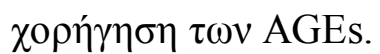




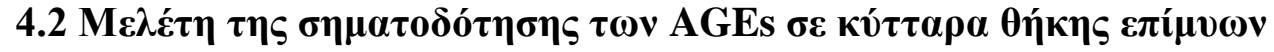

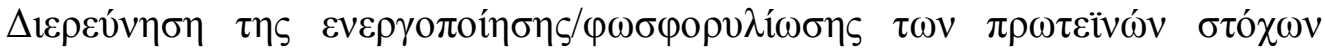

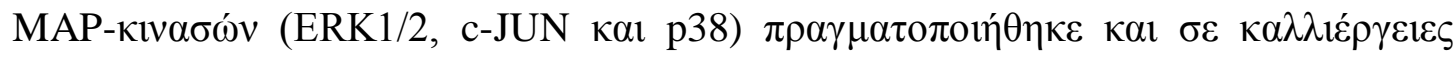

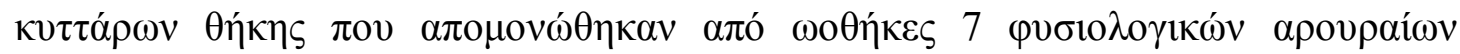

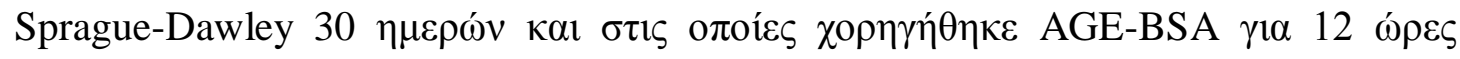

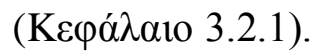

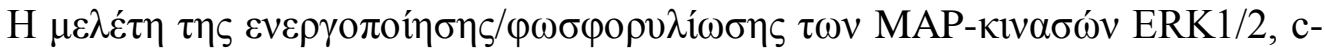

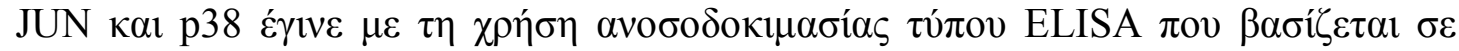

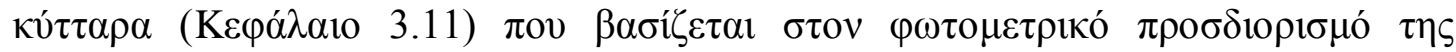

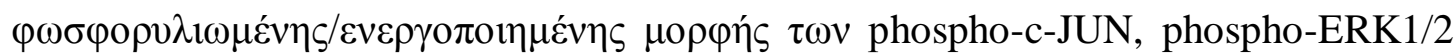

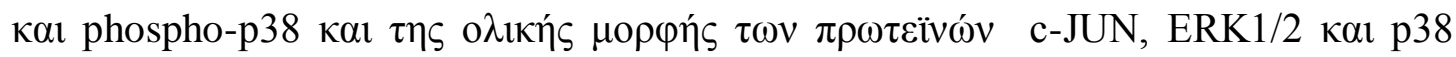

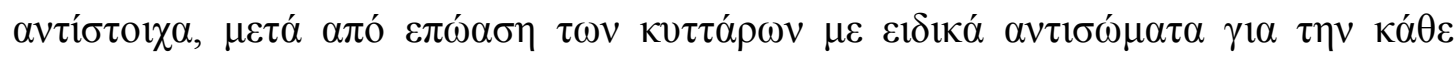

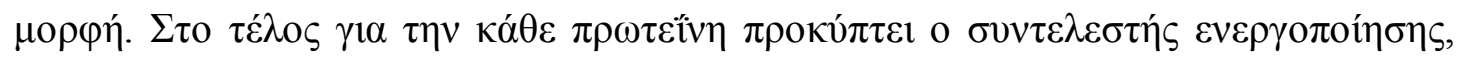

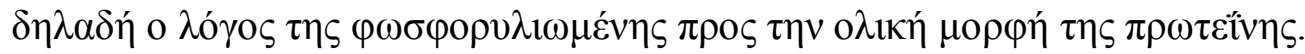

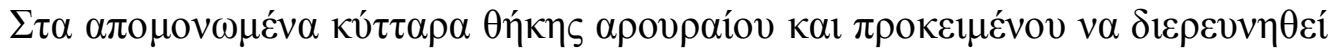

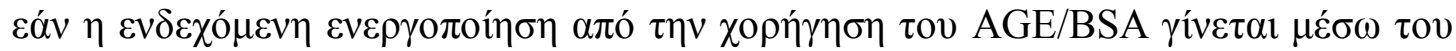

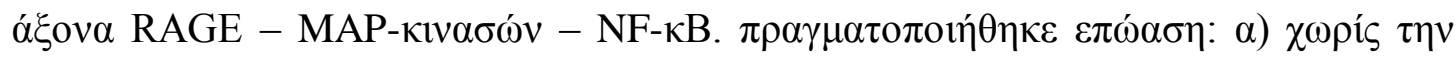

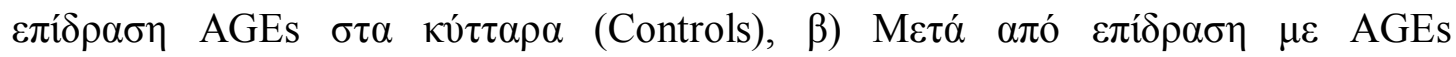

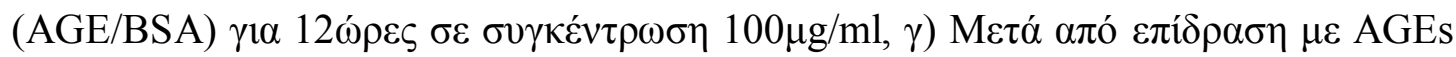

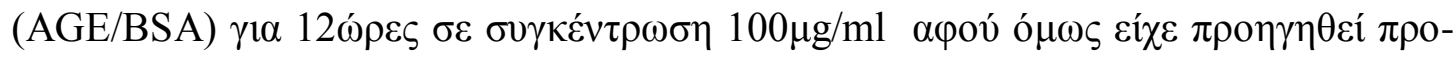

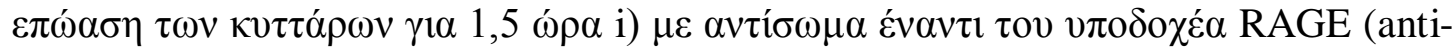

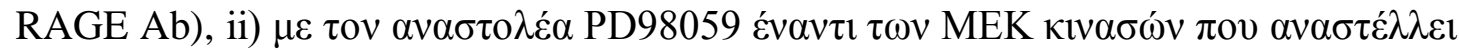

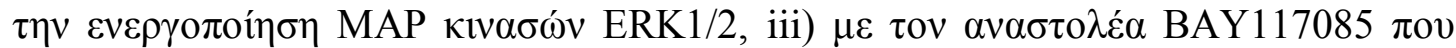

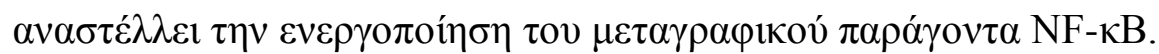

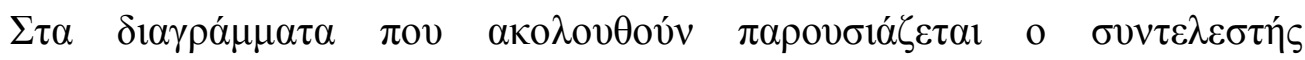

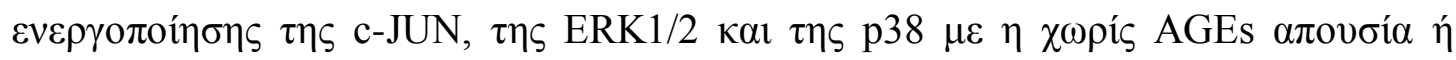
$\pi \alpha \rho o v \sigma i ́ \alpha \tau \omega \nu \alpha v \alpha \sigma \tau o \lambda \varepsilon ́ \omega v$ (anti-RAGE Ab, PD98059, BAY117085). 


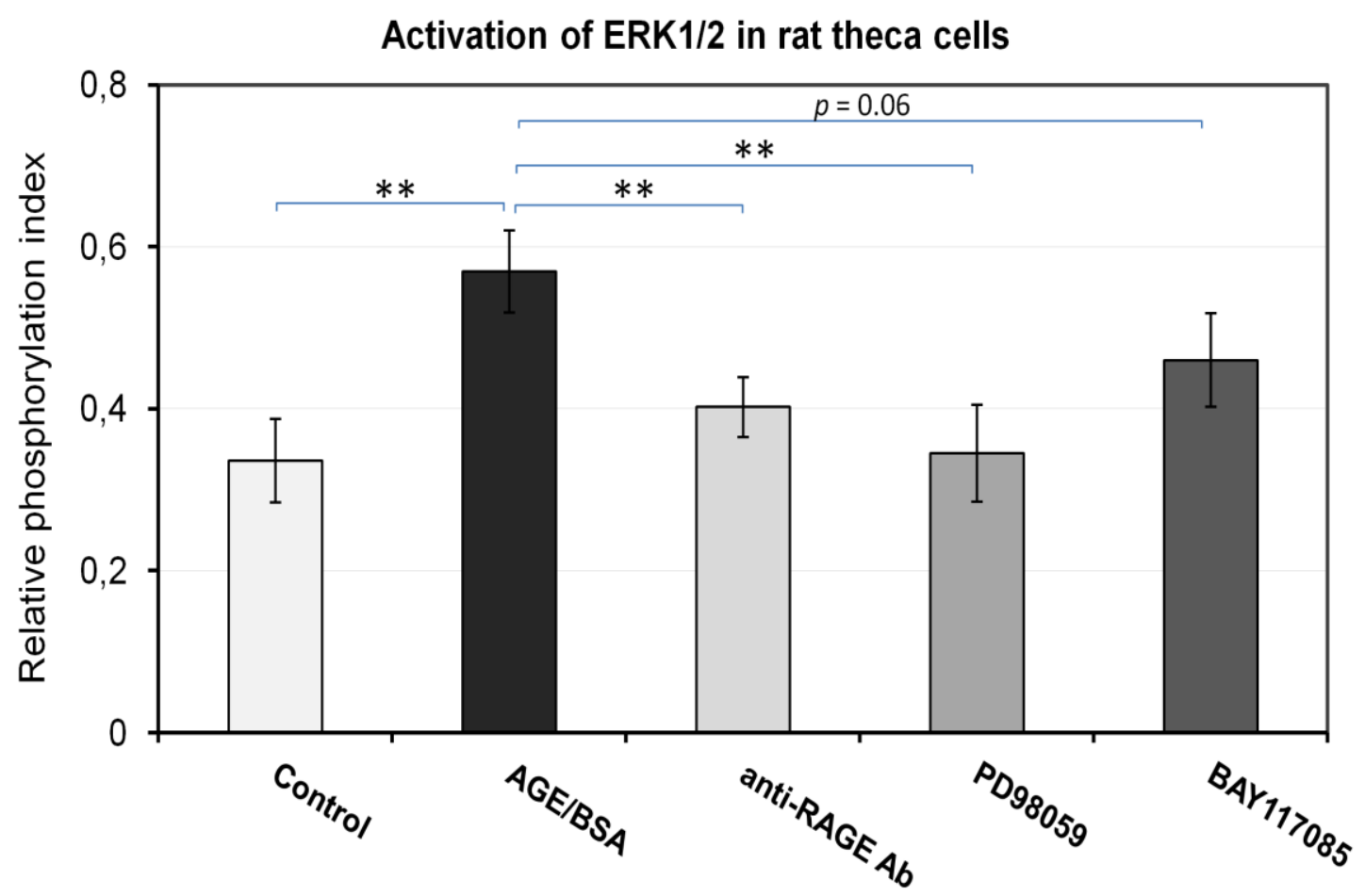

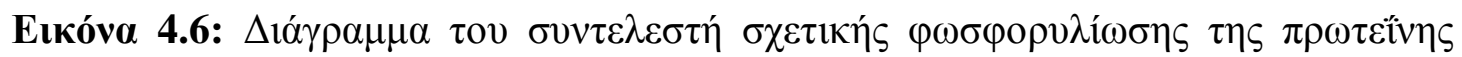

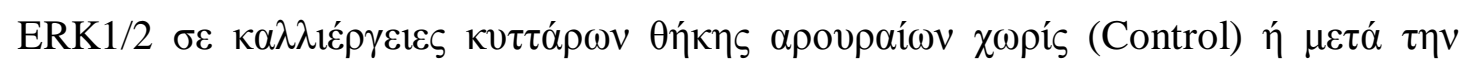

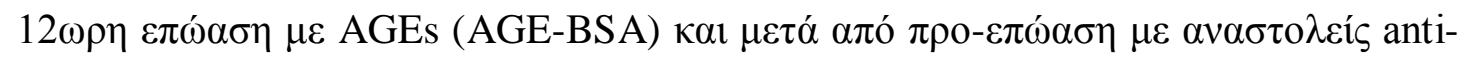
RAGE Ab, PD98059 ка1 BAY117085.

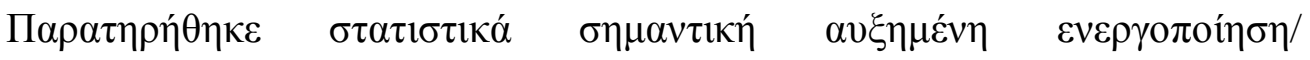

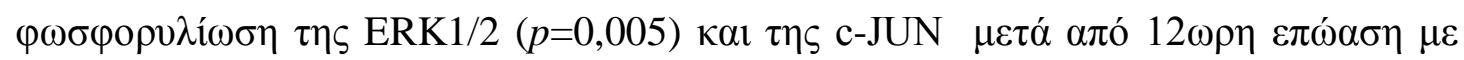

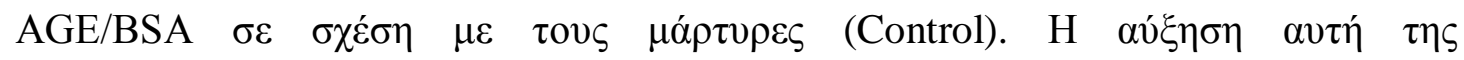

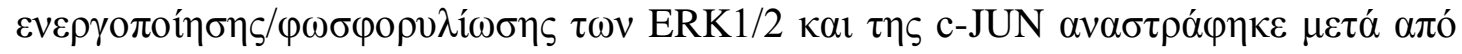

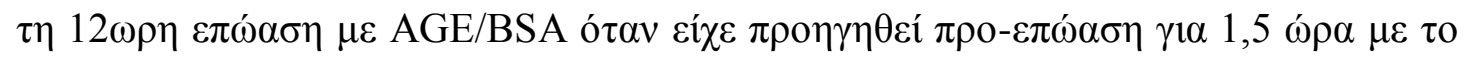

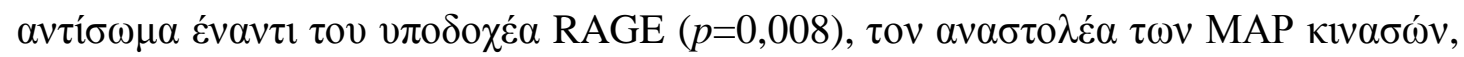

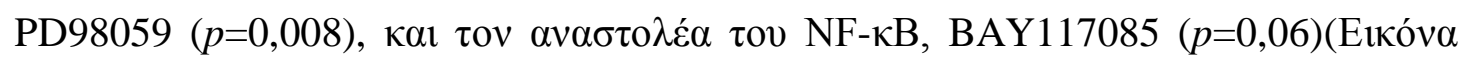
$4.6 \kappa \alpha 14.7)$. 


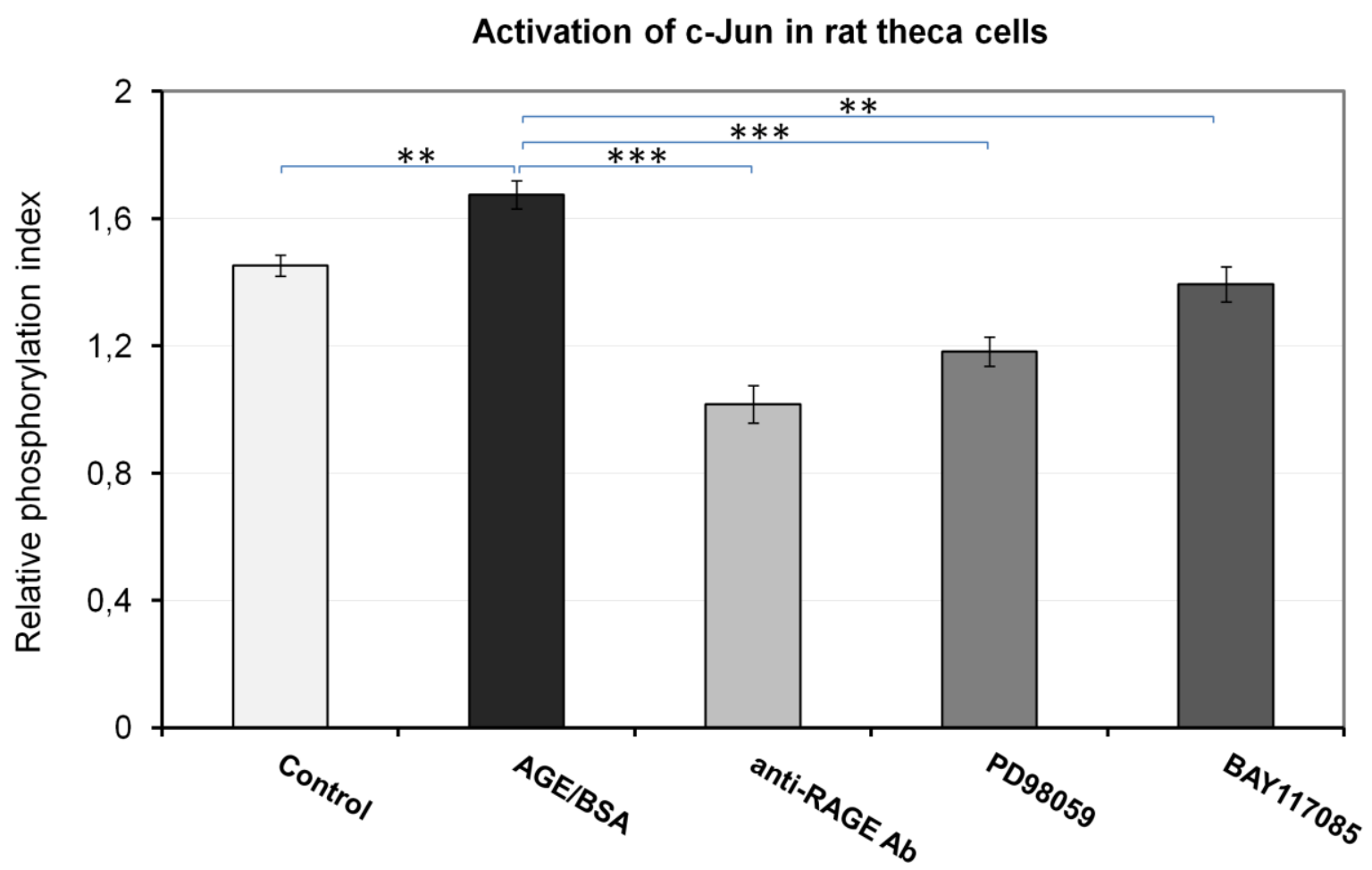

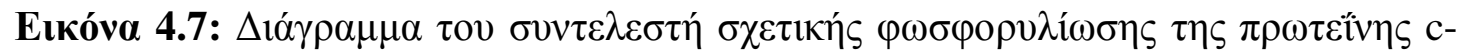

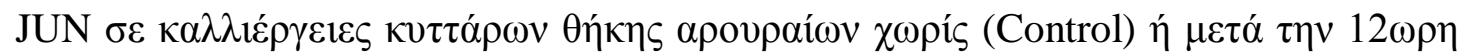

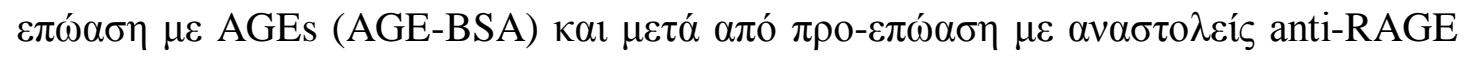
Ab, PD98059 каı BAY117085.

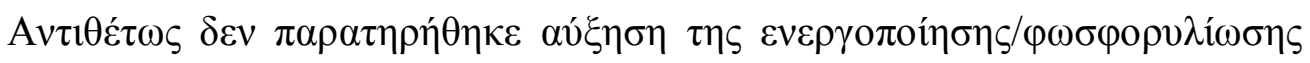

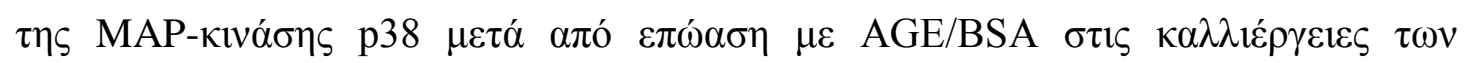

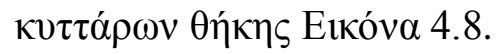




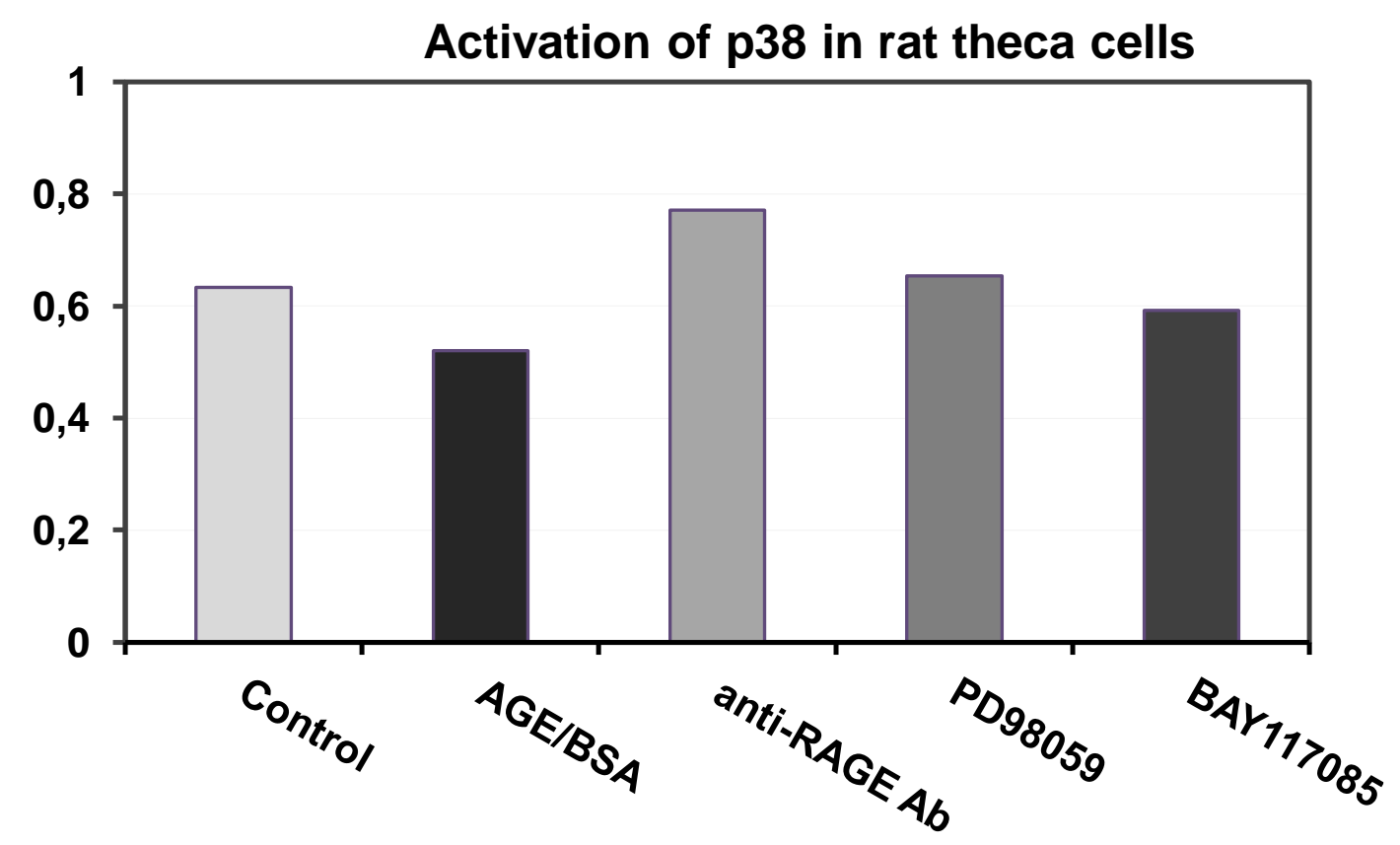

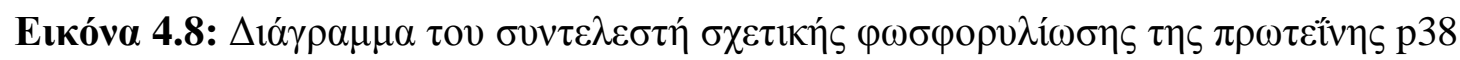

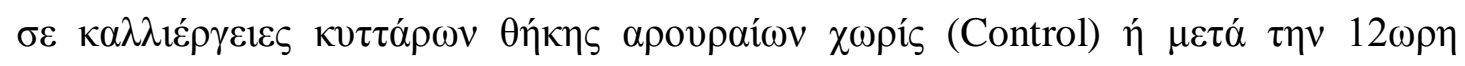

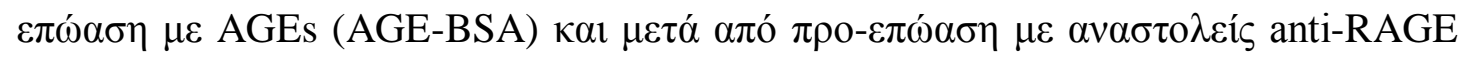
Ab, PD98059 каı BAY117085. 


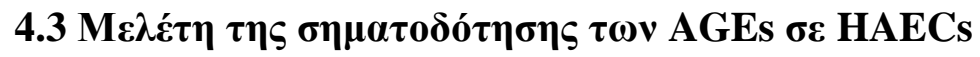

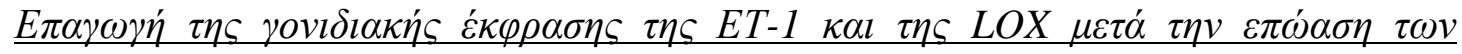
HAECs $\mu \varepsilon A G E s$

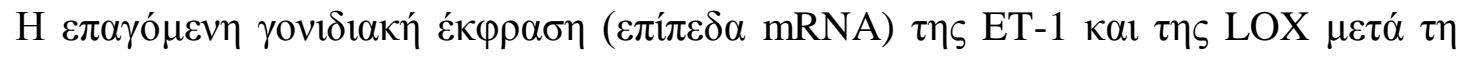

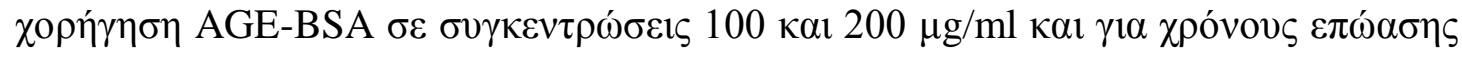

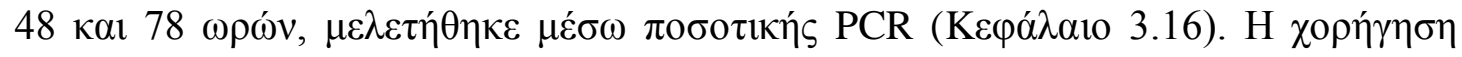

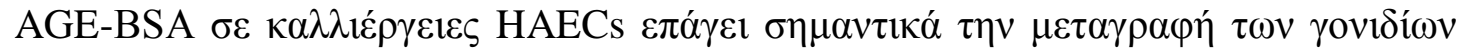

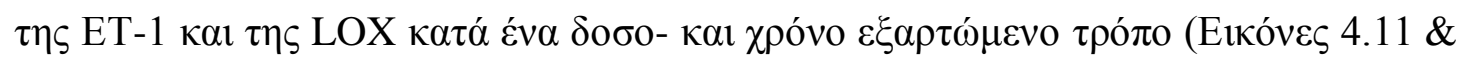

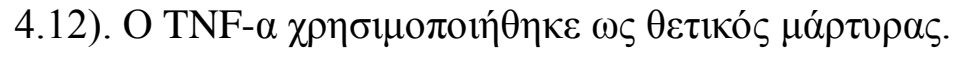

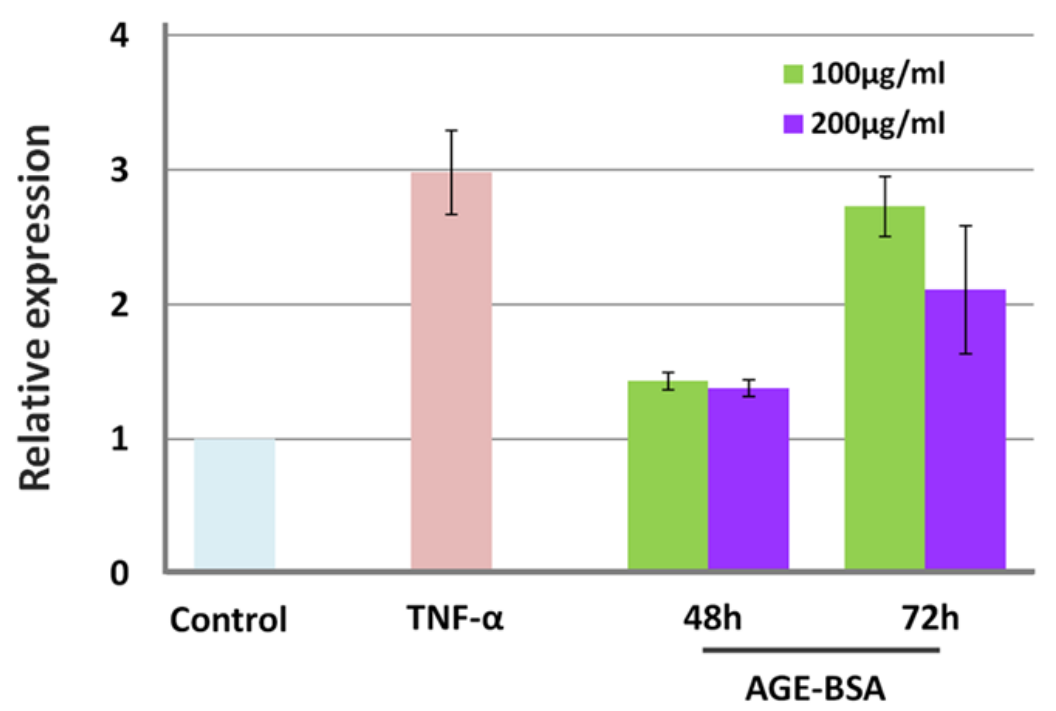

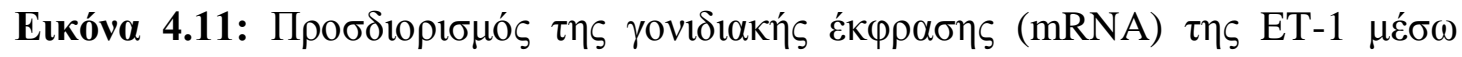

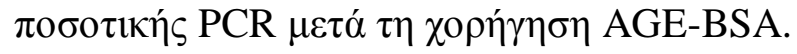

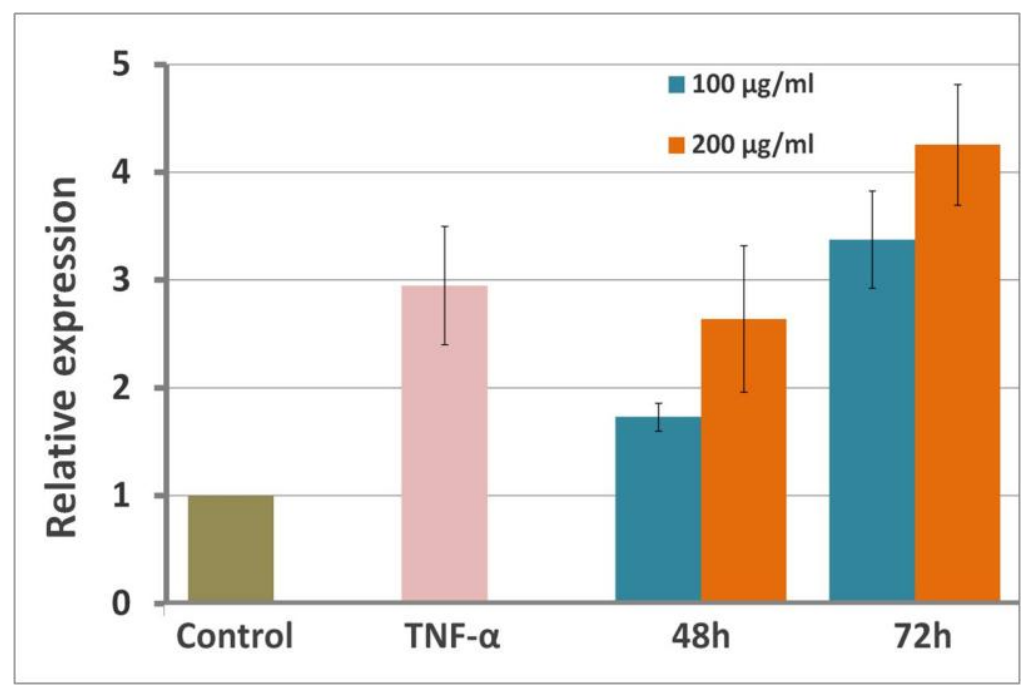

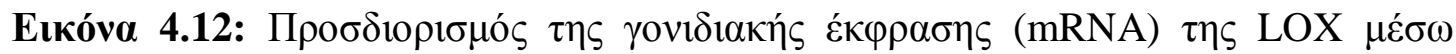

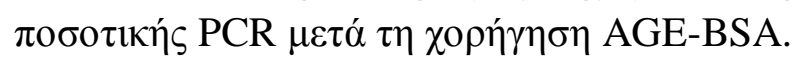




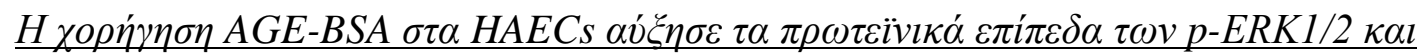

$$
p-c-J U N
$$
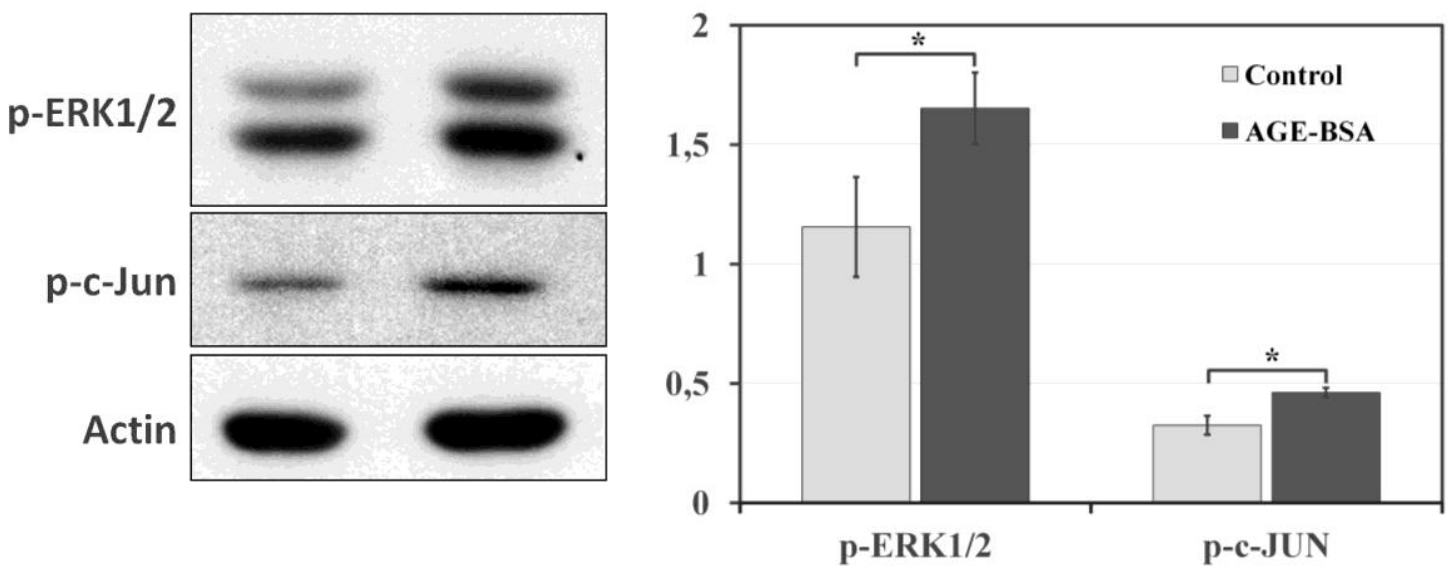

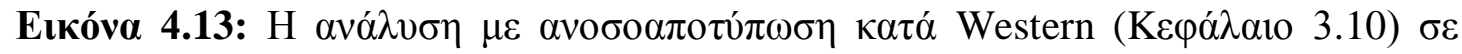

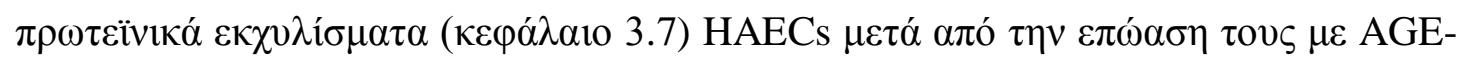

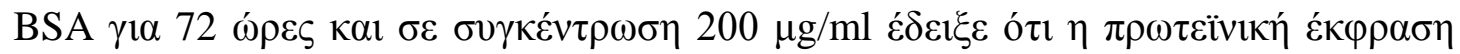

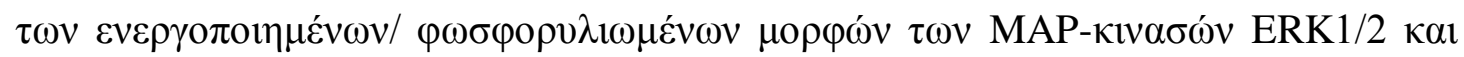

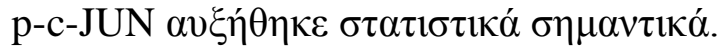




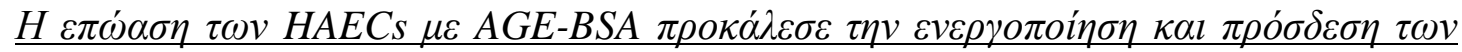

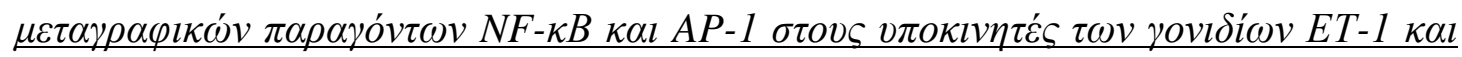
$\underline{\operatorname{LOX}}$

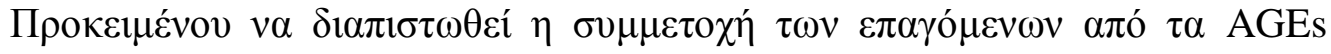

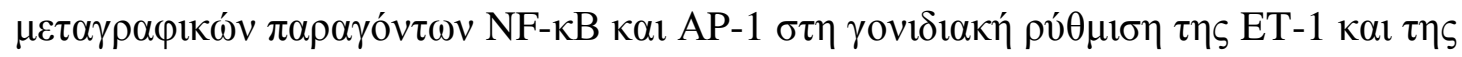

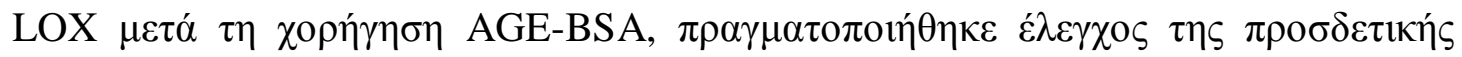

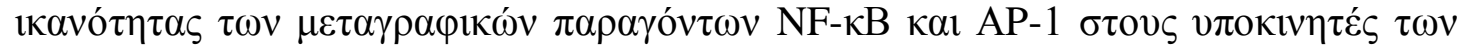

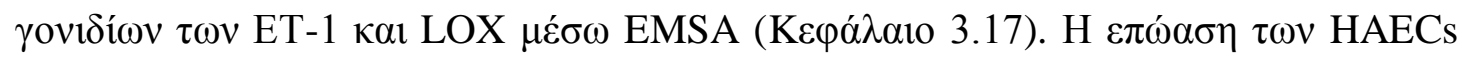

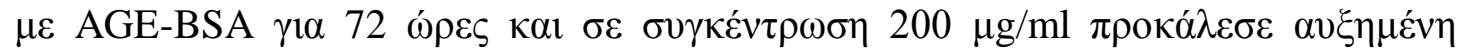

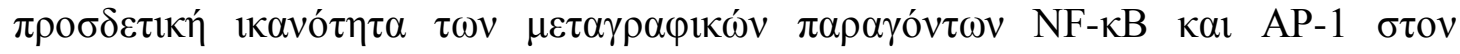

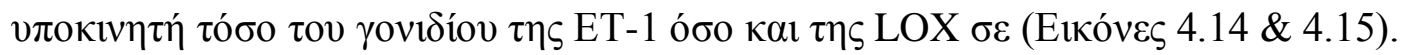

A

\begin{tabular}{r|cccccccccccc|} 
HAECS & - & + & - & - & - & - & - & + & - & - & - & - \\
HAECS +AGE-BSA & - & - & + & + & + & - & - & - & + & + & + & - \\
NF-kB p65 Ab & - & - & - & + & - & - & - & - & - & - & + & - \\
p-C-JUn Ab & - & - & - & - & + & - & - & - & - & + & - & - \\
\cline { 2 - 7 } & & & & & & & & & & & &
\end{tabular}

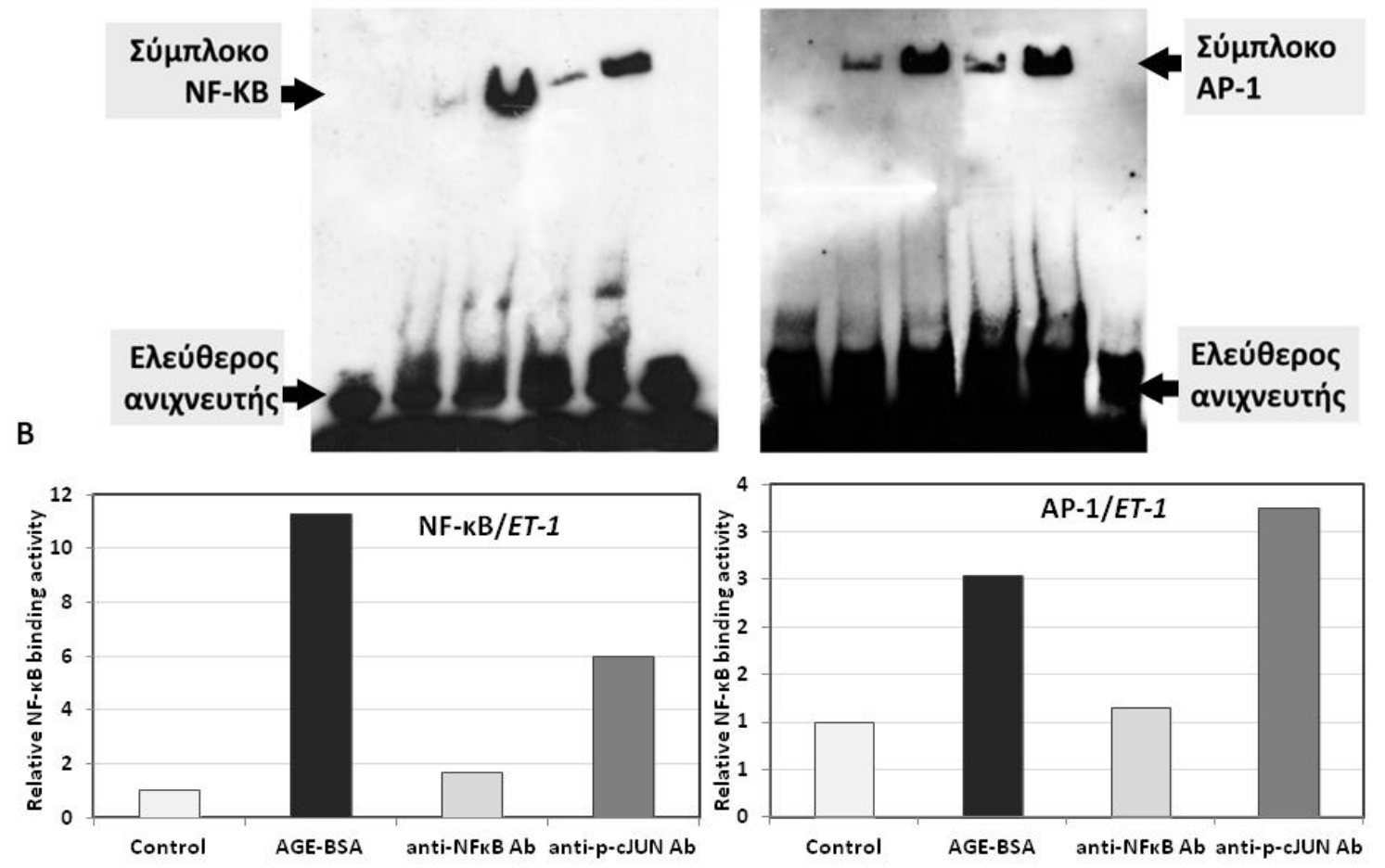

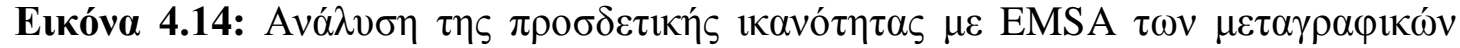

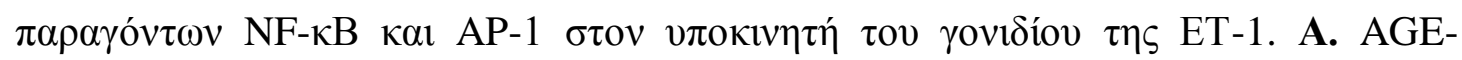

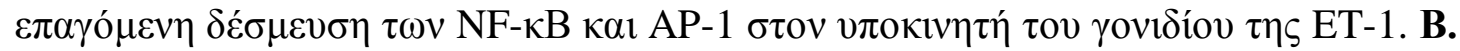

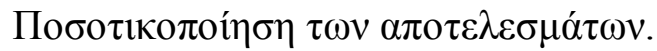



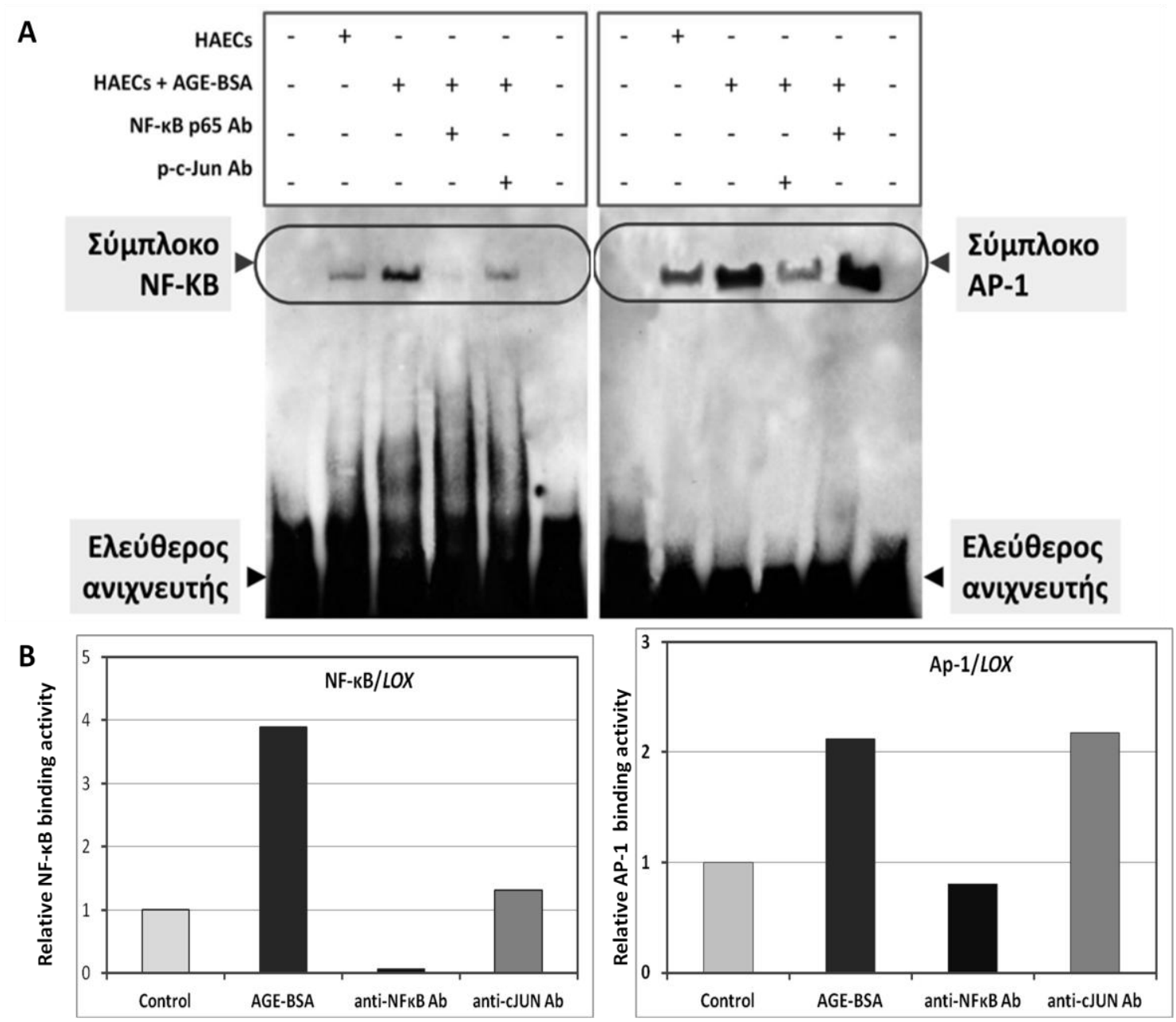

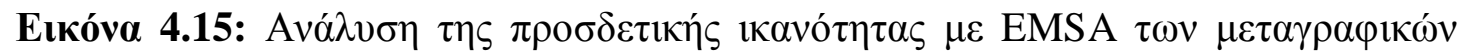

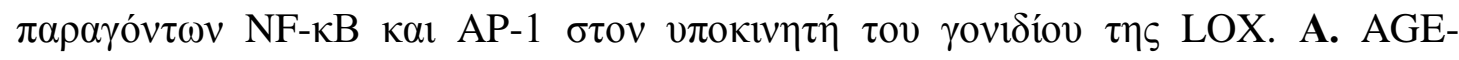

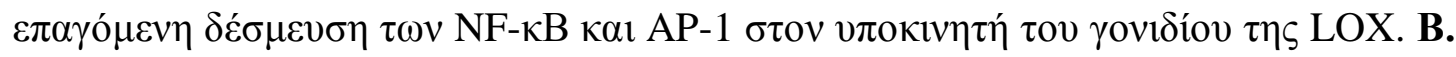

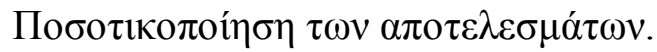




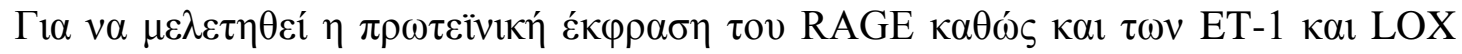

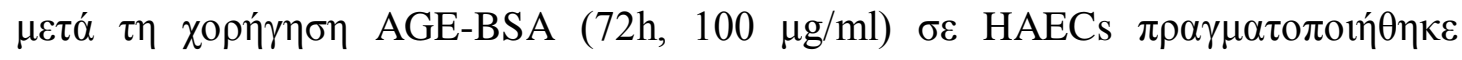

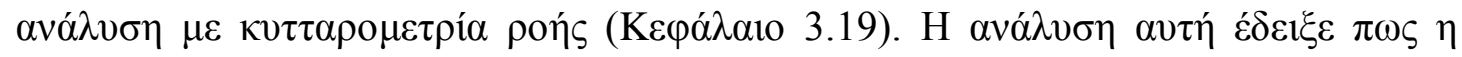

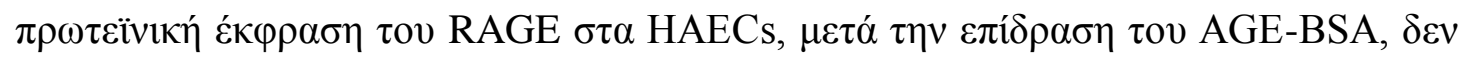

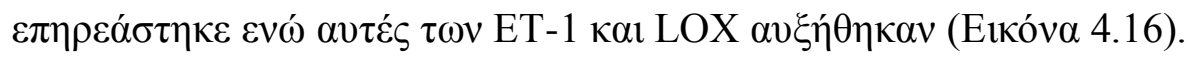
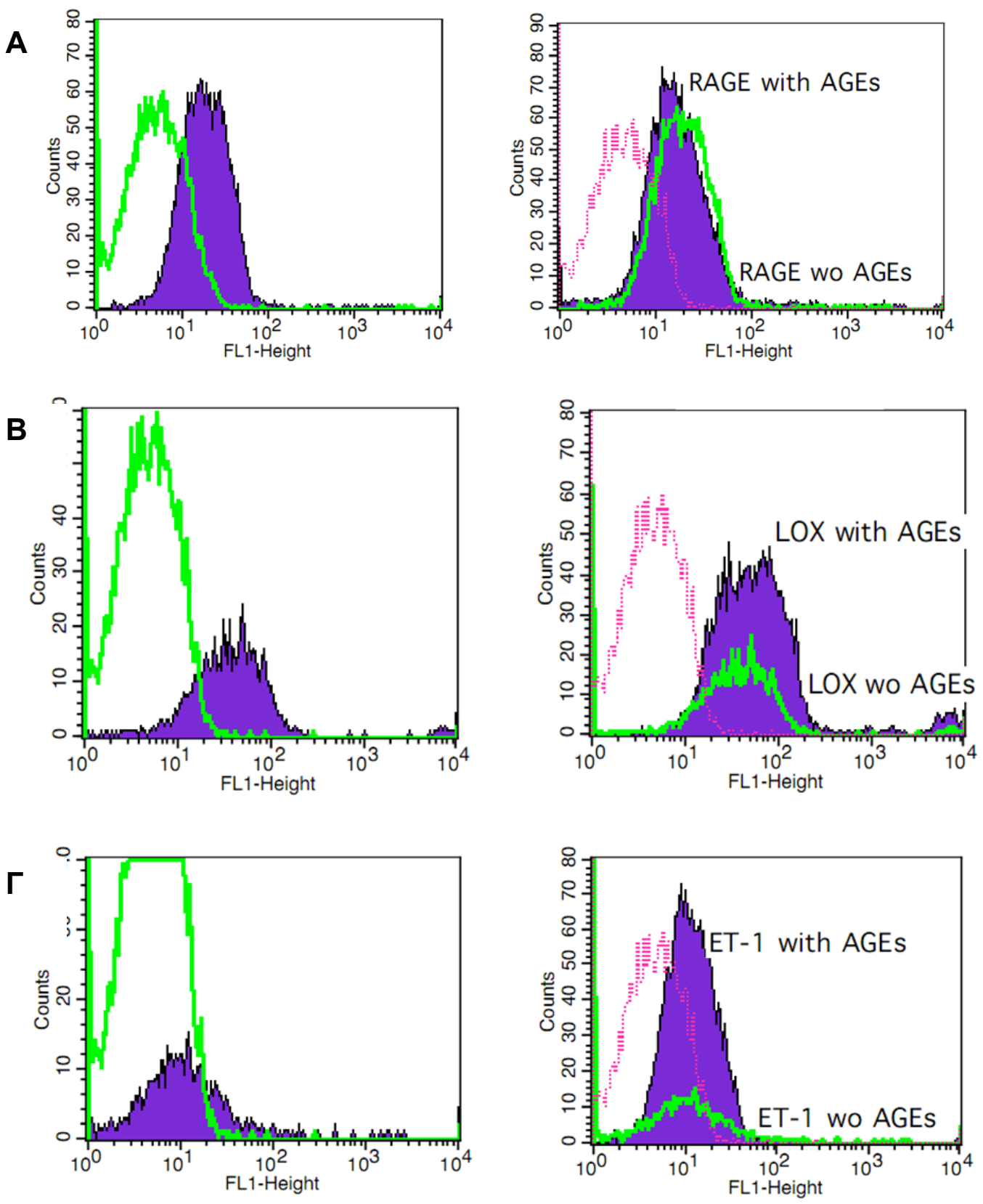

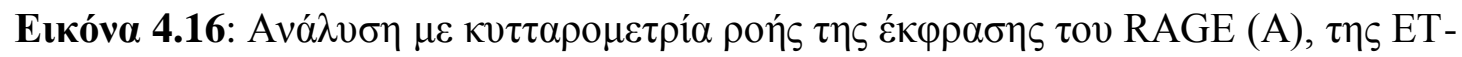
1 (B) $\kappa \alpha \imath \tau \eta \varsigma \operatorname{LOX}(\Gamma)$. 


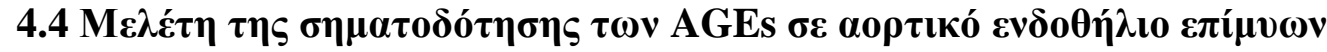

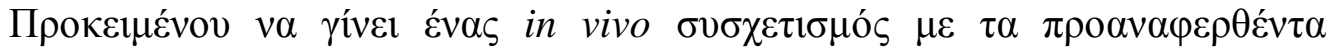

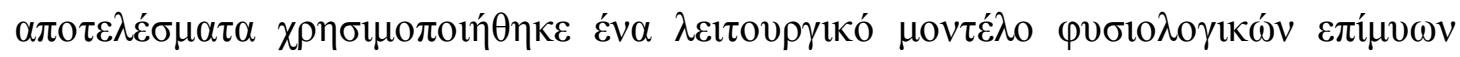

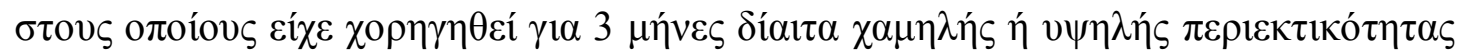
$\sigma \varepsilon$ AGEs (LOW-AGEs $\kappa \alpha \imath$ HIGH-AGEs $\alpha v \tau i ́ \sigma \tau o \imath \chi \alpha)(K \varepsilon \varphi \alpha ́ \lambda \alpha 10$ 3.18). H

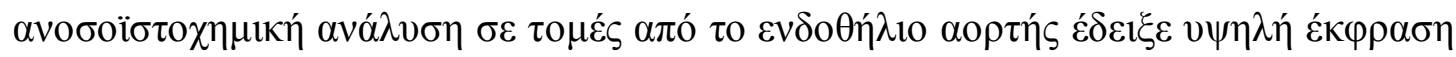

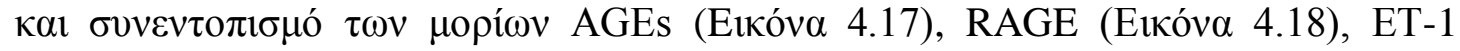

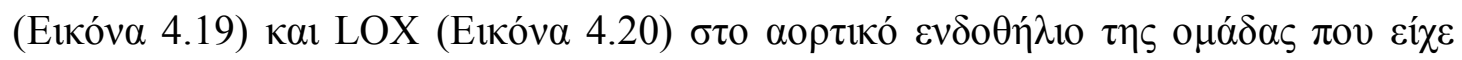

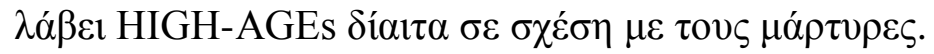

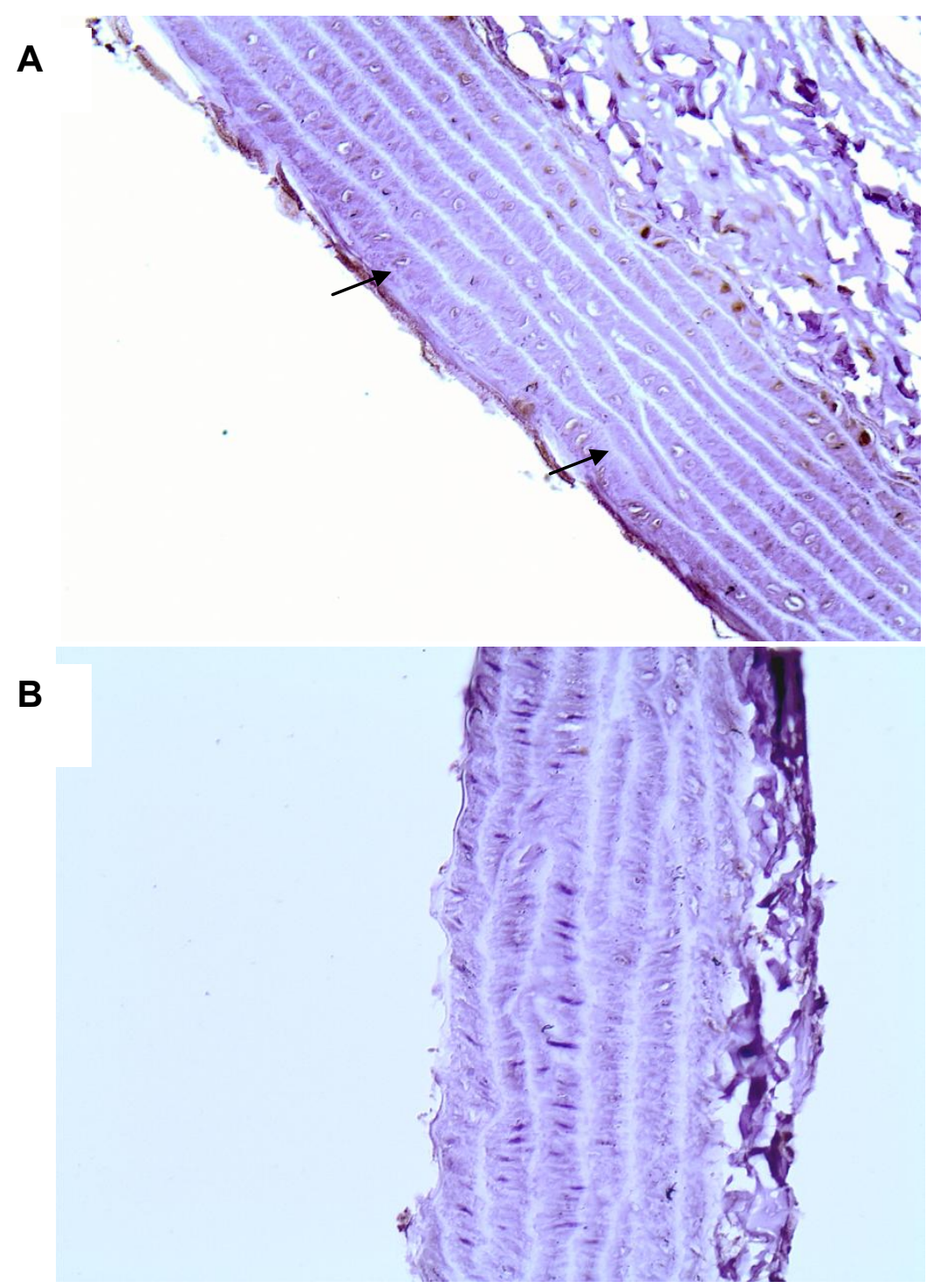

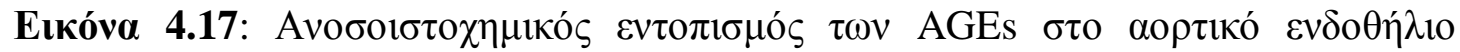

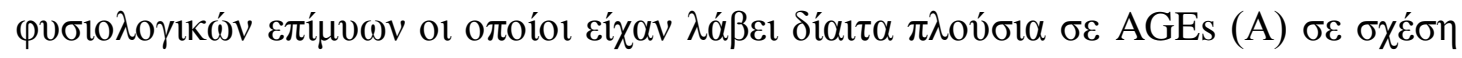

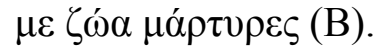




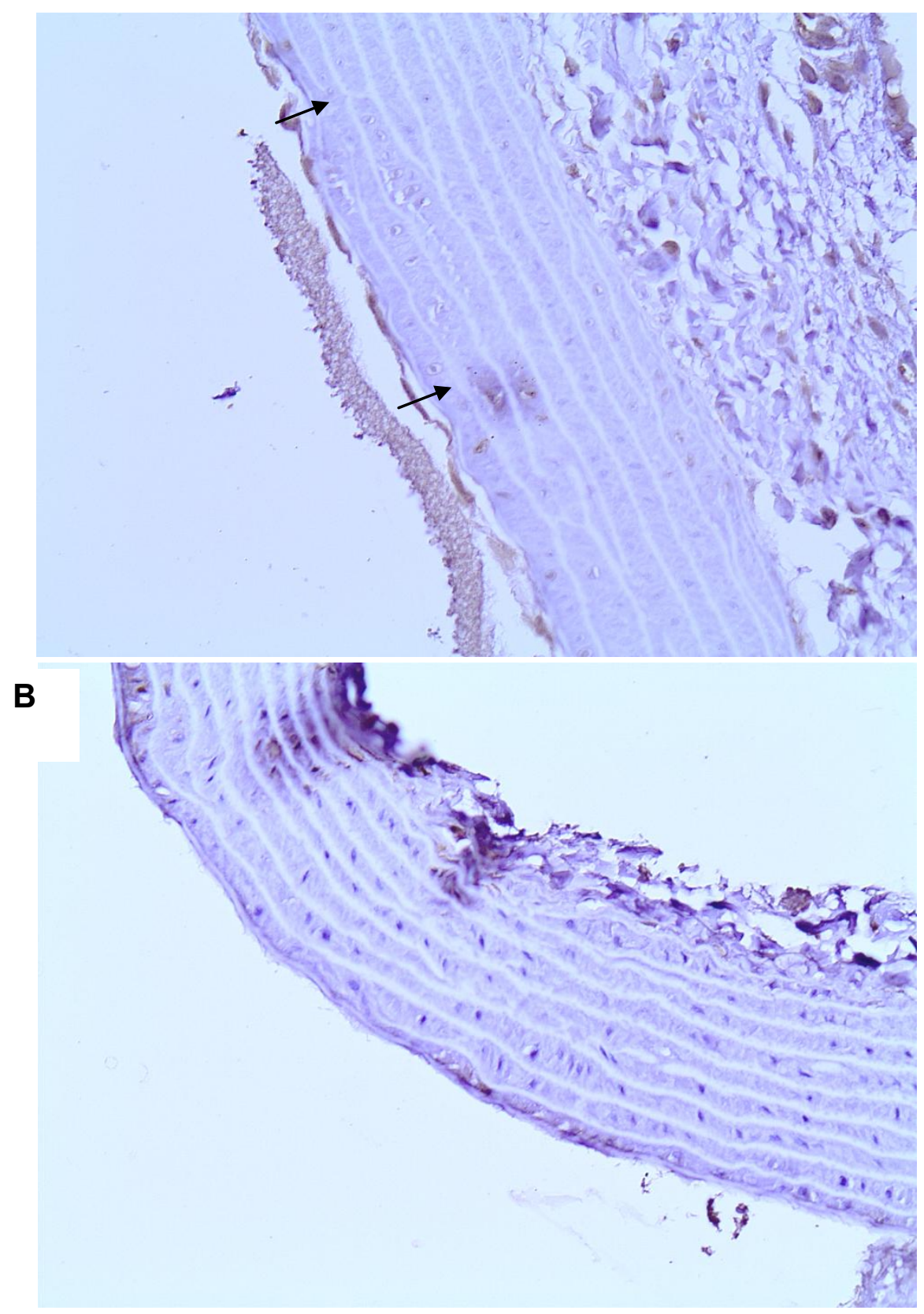

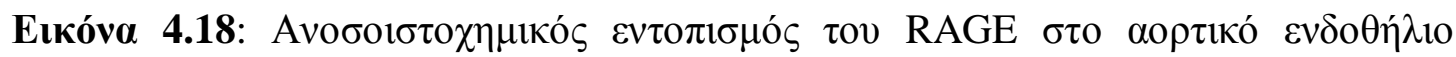

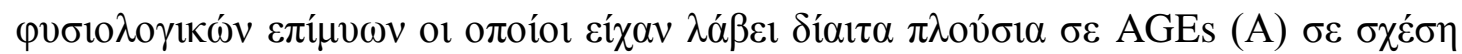
$\mu \varepsilon \zeta \omega ́ \alpha \mu \alpha ́ \rho \tau v \rho \varepsilon \varsigma(B)$. 

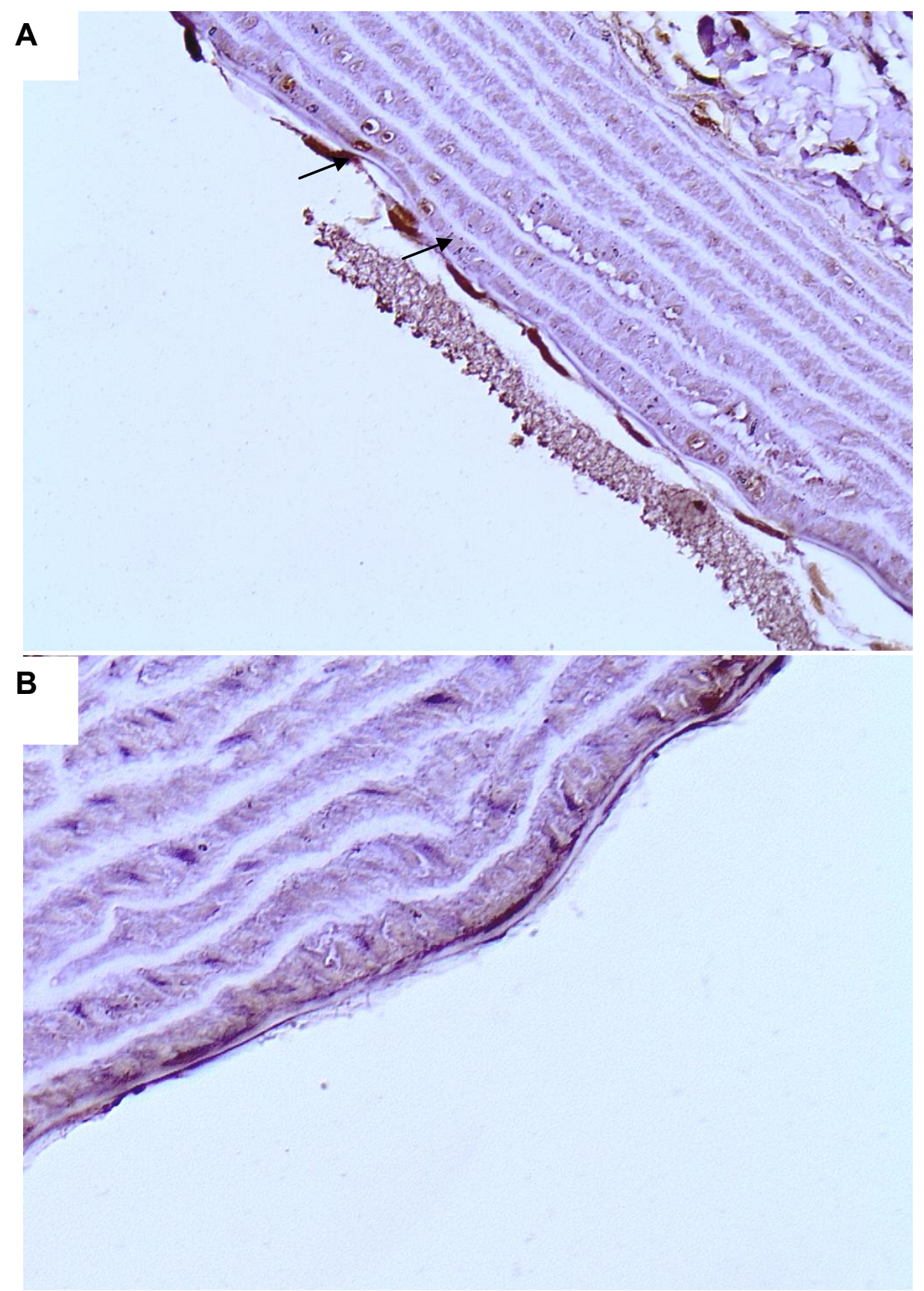

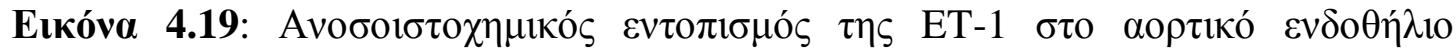

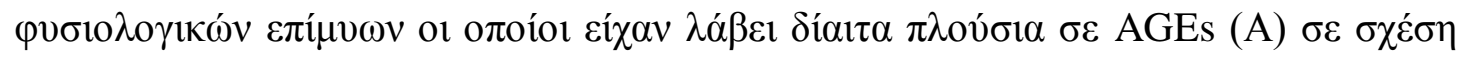

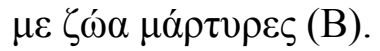




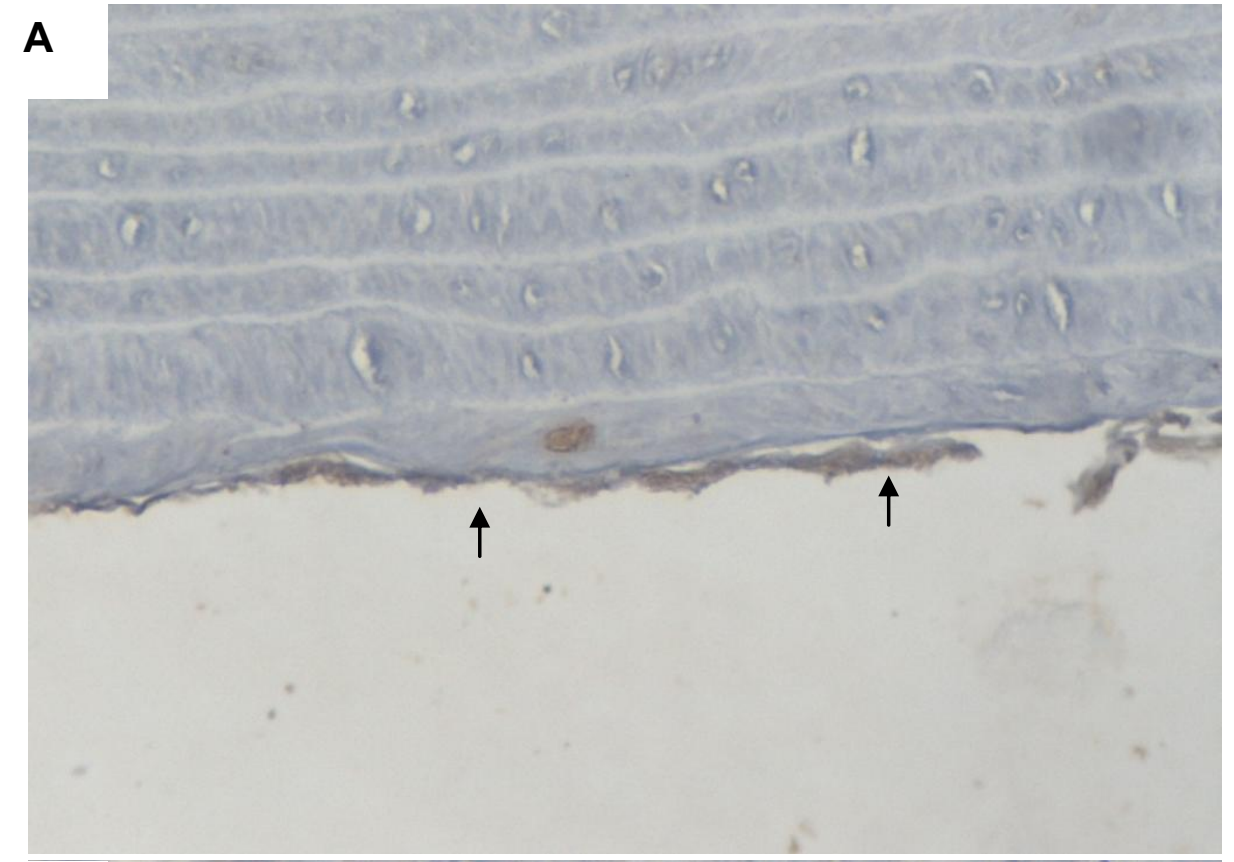

B

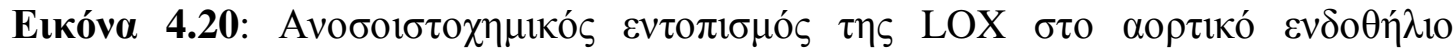

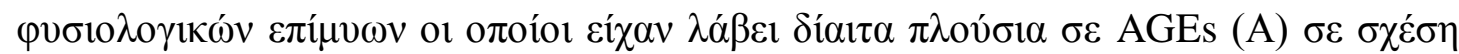
$\mu \varepsilon \zeta \dot{\omega} \alpha \mu \alpha \dot{\alpha} \rho \tau \rho \rho \varepsilon(B)$. 


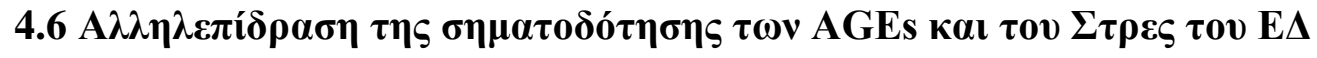

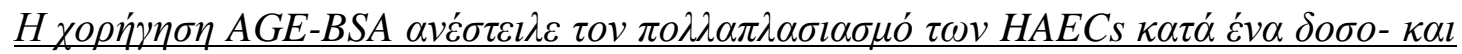

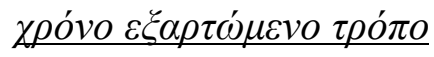

$\Gamma 1 \alpha v \alpha \delta 1 \alpha \lambda \varepsilon v \kappa \alpha v \theta \varepsilon i ́ \eta \eta$

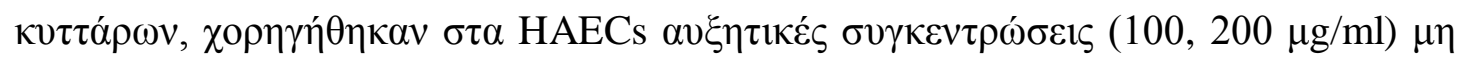

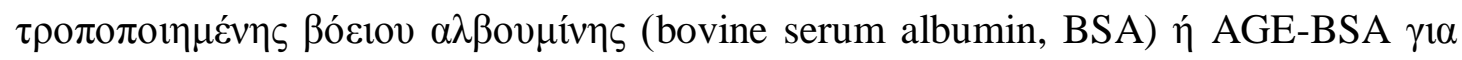

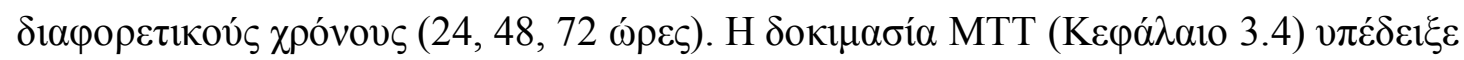

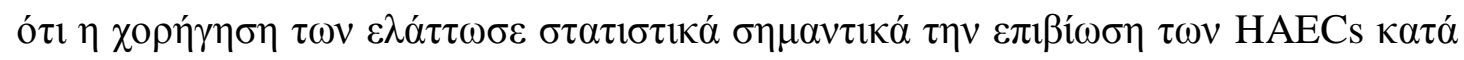

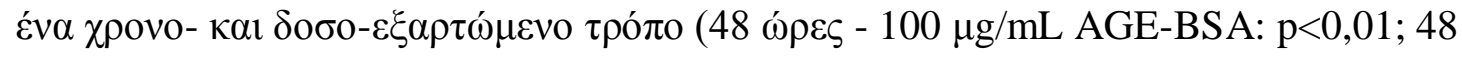
$\omega ́ \rho \varepsilon \varsigma$ - $200 \mu \mathrm{g} / \mathrm{mL}$ AGE-BSA: $\mathrm{p}<0,05 ; 72 \omega ́ \rho \varepsilon \varsigma$ - $100 \mu \mathrm{g} / \mathrm{mL}$ AGE-BSA: p<0,05; 72h

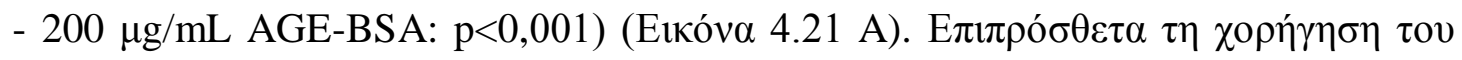

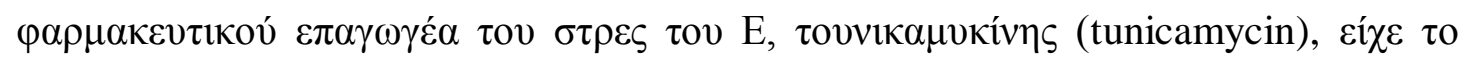

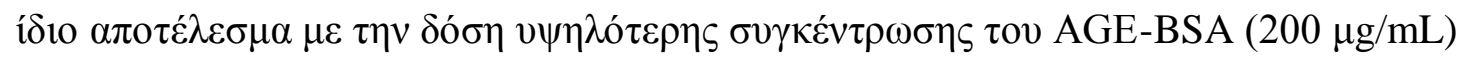

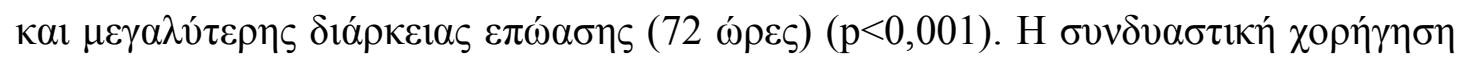

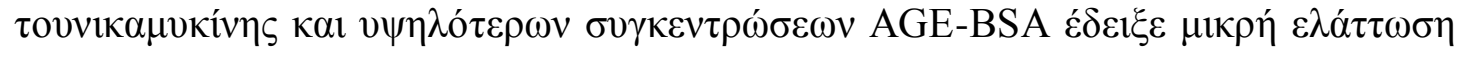

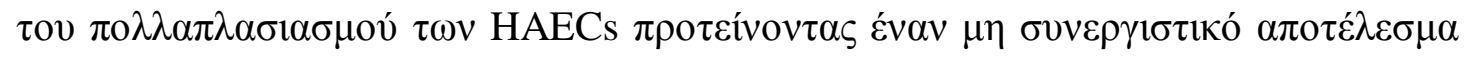

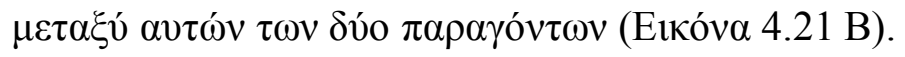




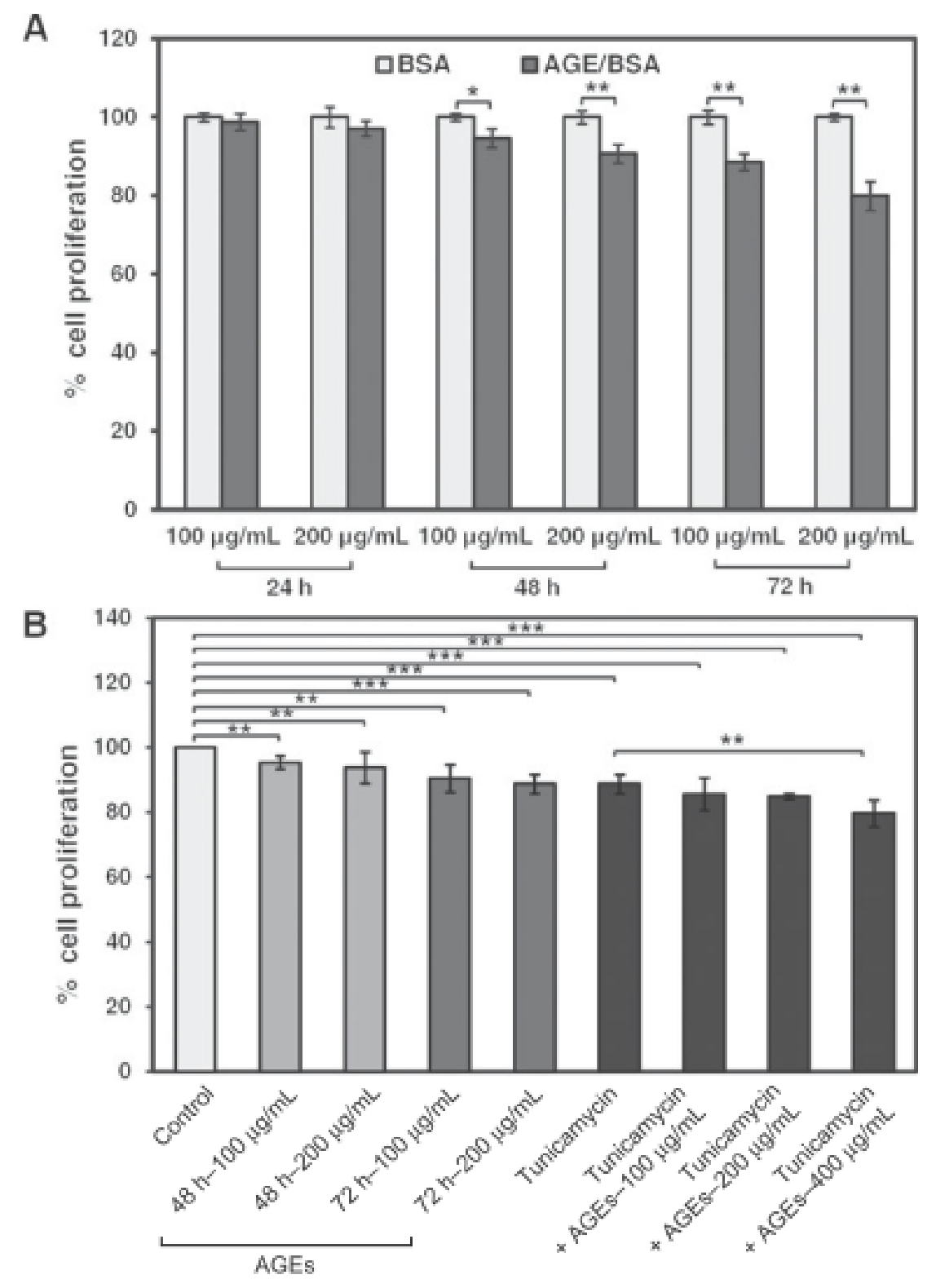

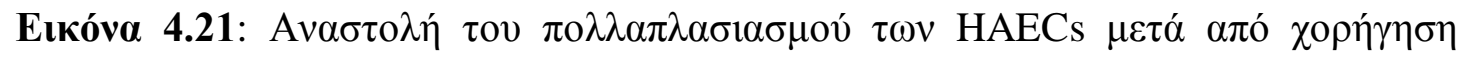

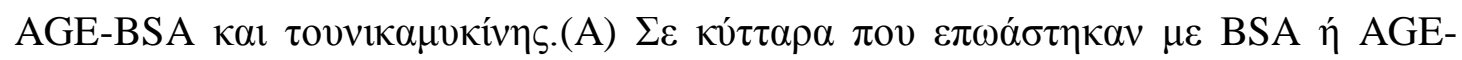

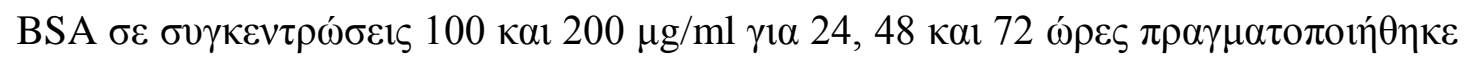

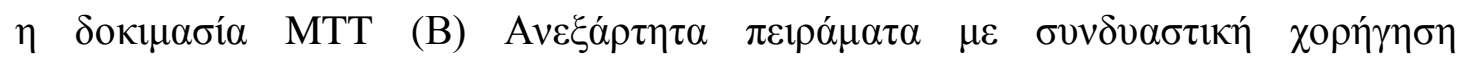

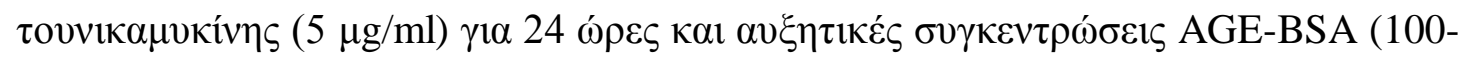

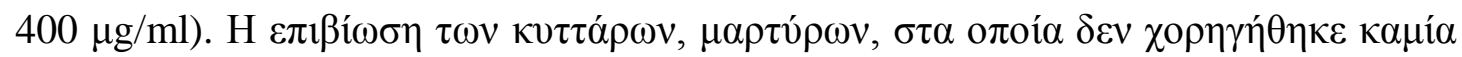
ovбía (control) $\theta \varepsilon \omega \rho \eta \dot{\theta \eta \kappa \varepsilon ~ 100 \% . ~ * p ~}<0.05$, **p $<0.01, * * * p<0.001$. 


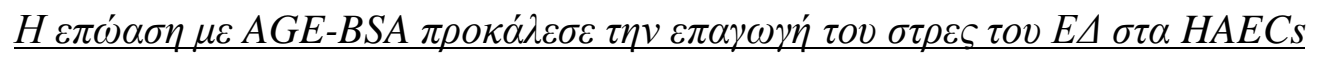

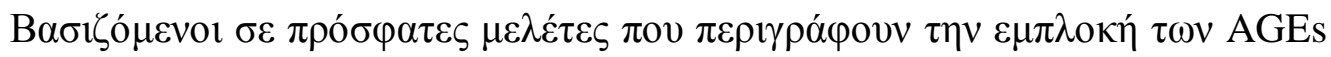

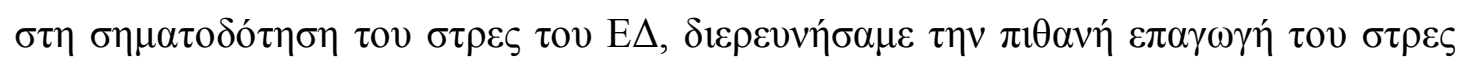

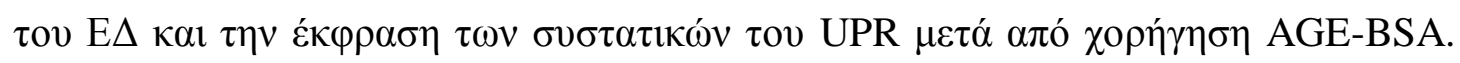

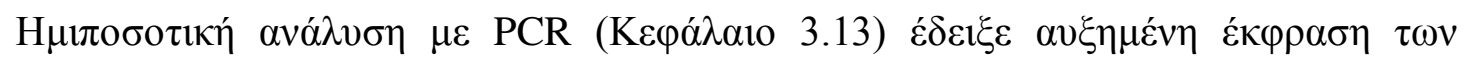

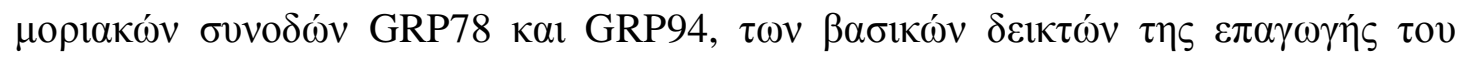

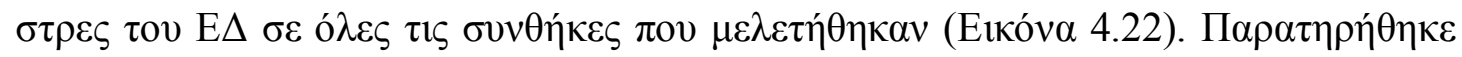

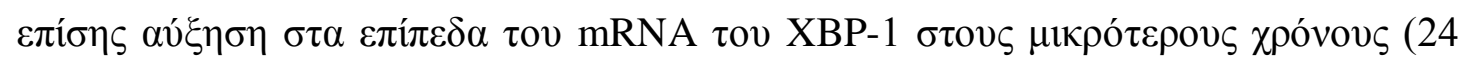

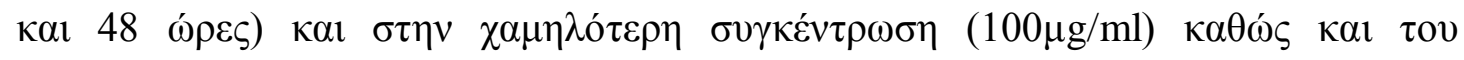

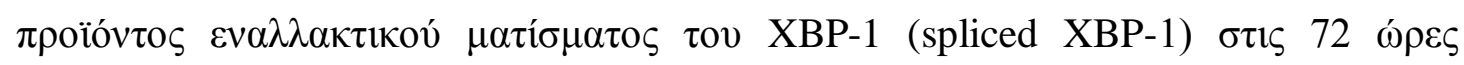

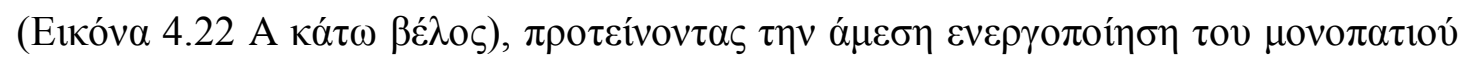

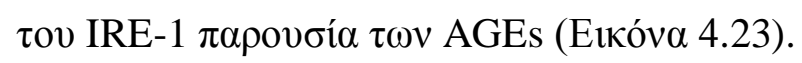

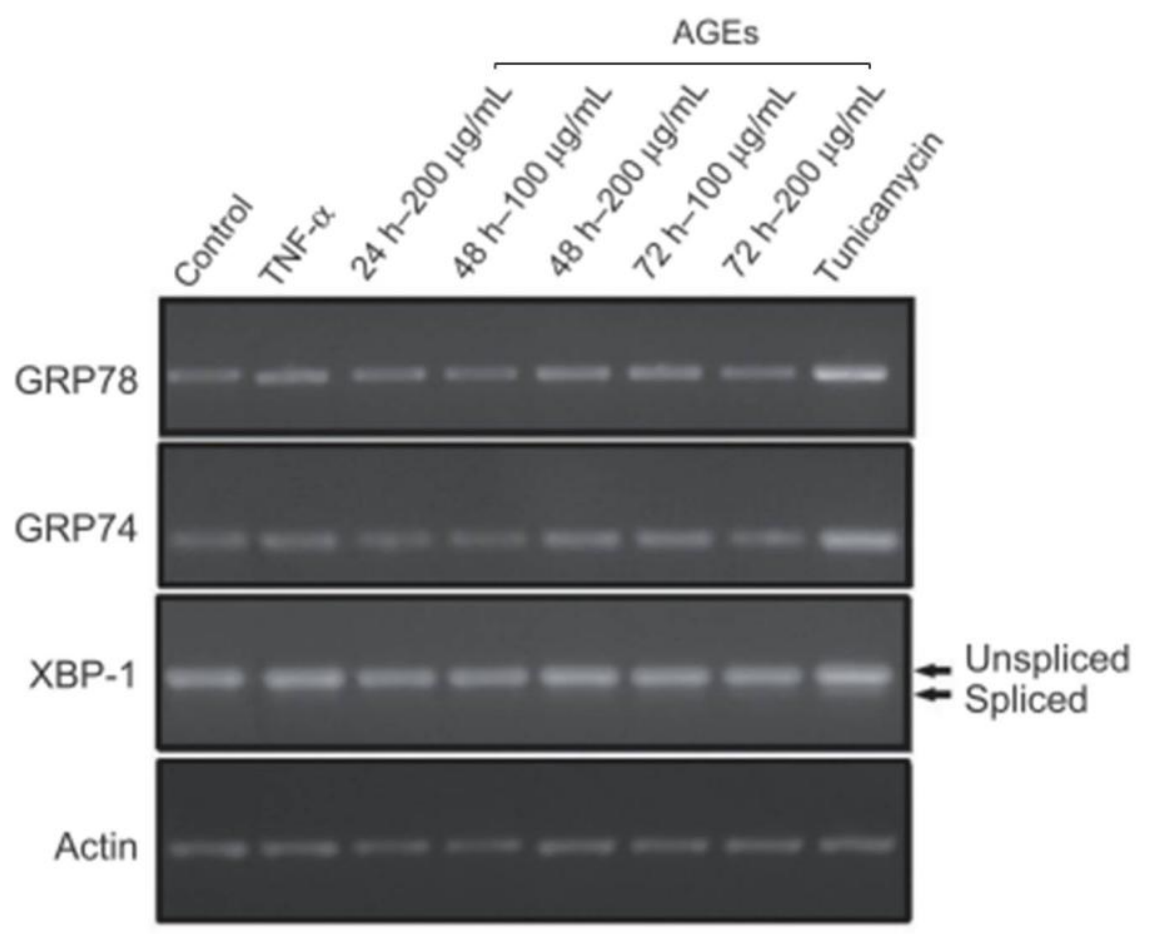

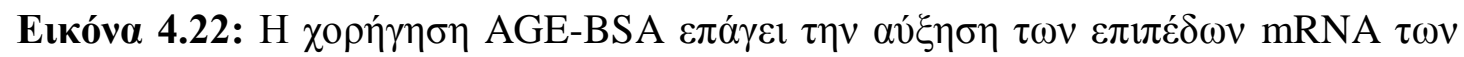

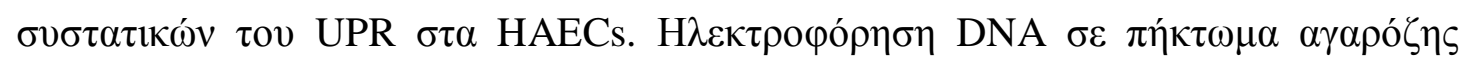

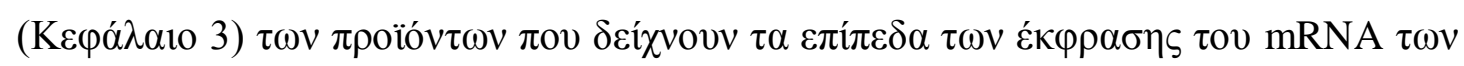
GRP78, GRP74, XBP-1 $\mu \varepsilon \tau \alpha ́ \alpha ~ \alpha \pi o ́ ~ \alpha v \alpha ́ \lambda v \sigma \eta ~ \mu \varepsilon$ RT-PCR. 


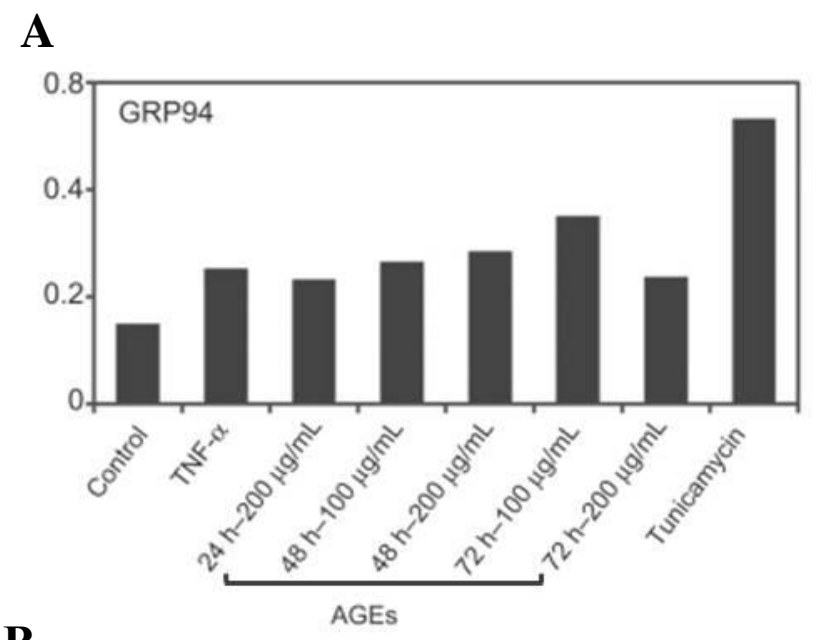

B

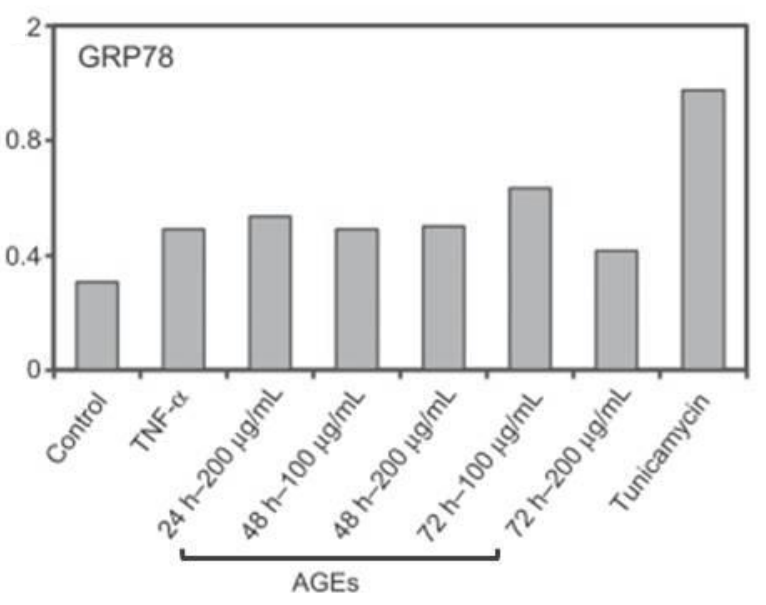

$\Gamma$

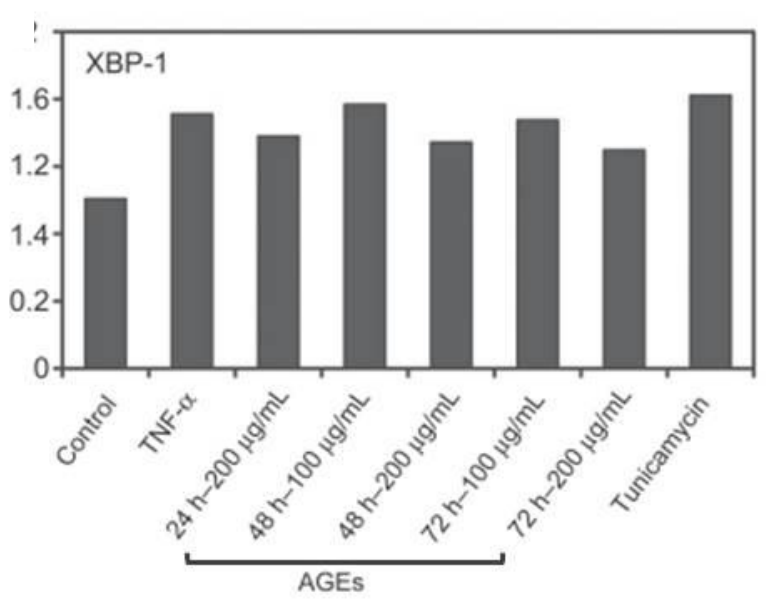

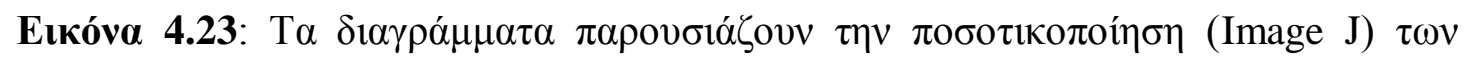

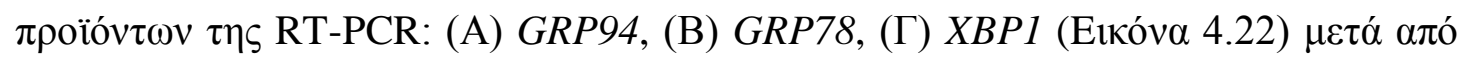

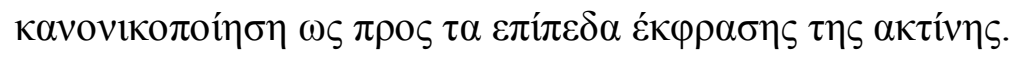

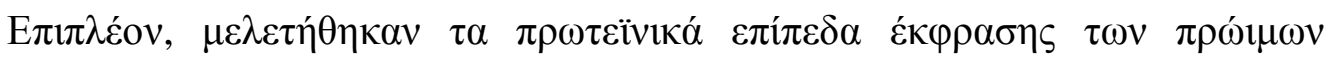

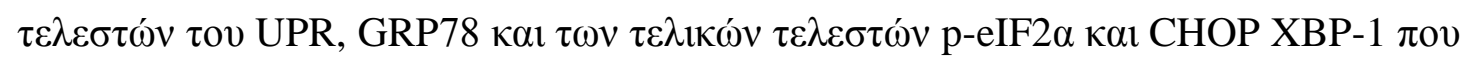
$\alpha \kappa \circ \lambda \circ v \theta o v ́ v \tau \eta v \varepsilon \pi \omega ́ \alpha \sigma \eta \mu$ AGE-BSA. 

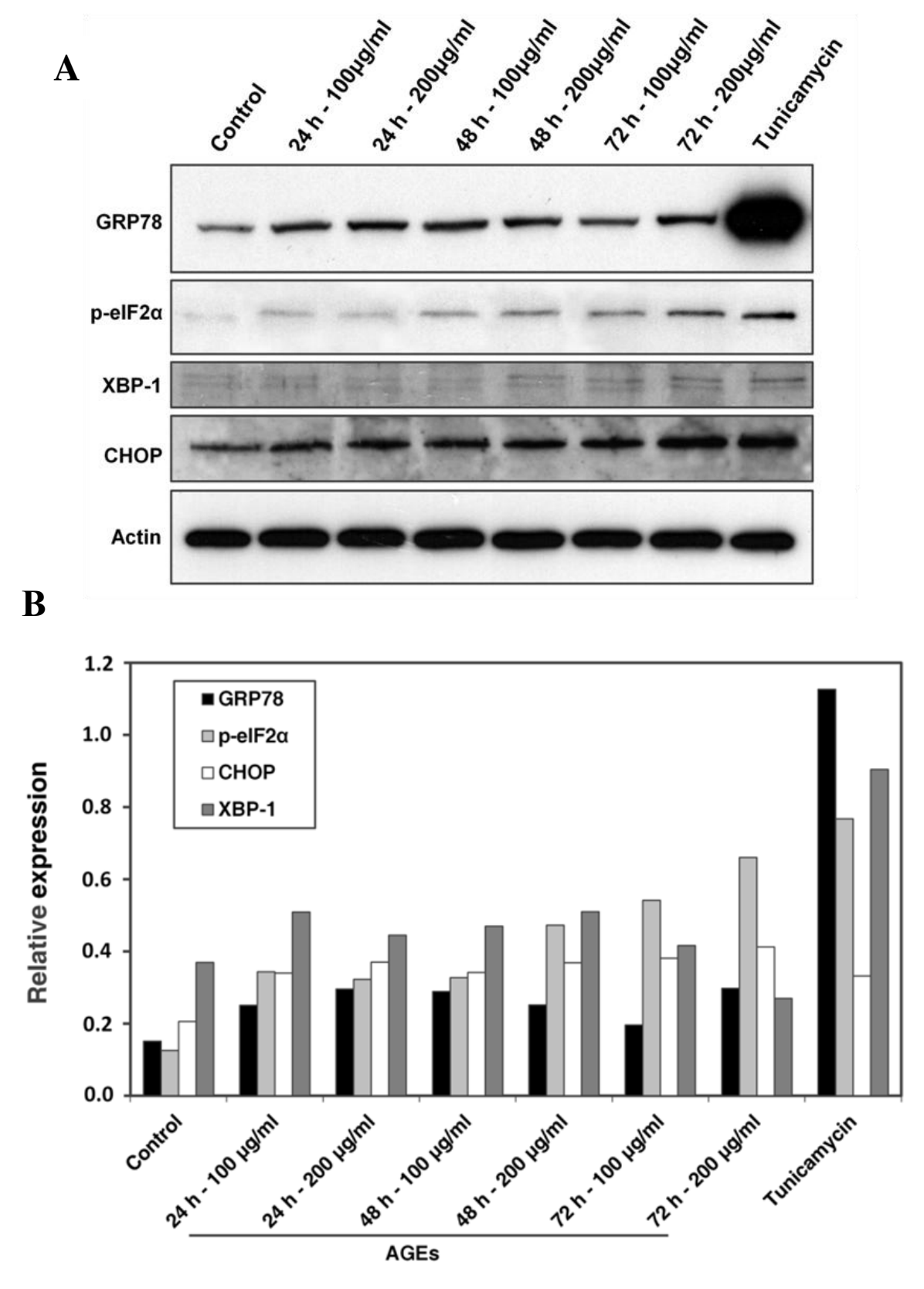

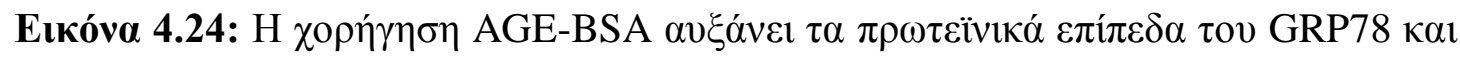

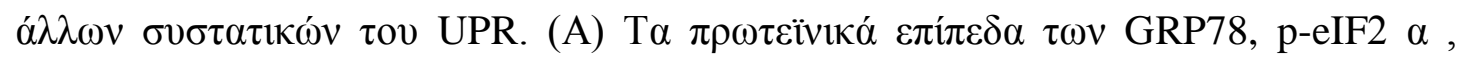

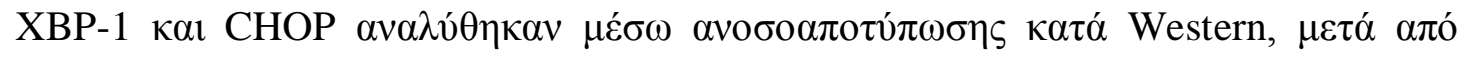

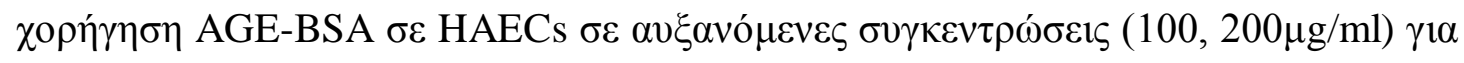

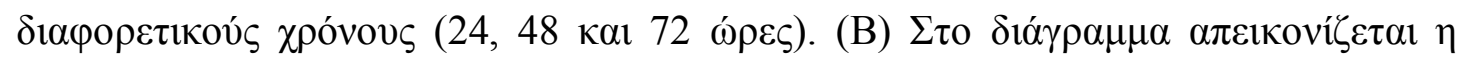

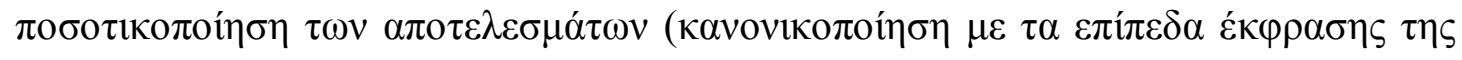

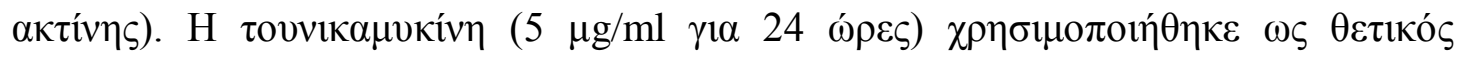

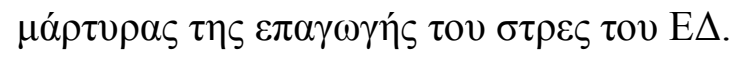

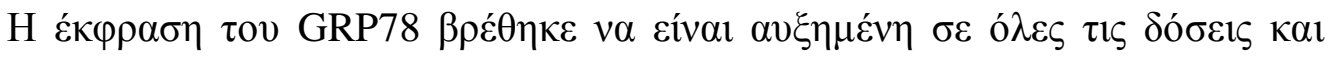

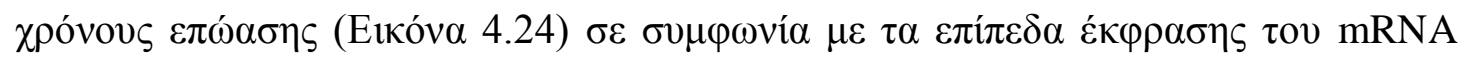




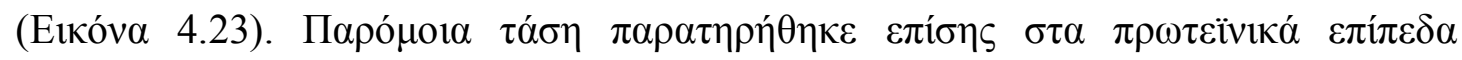

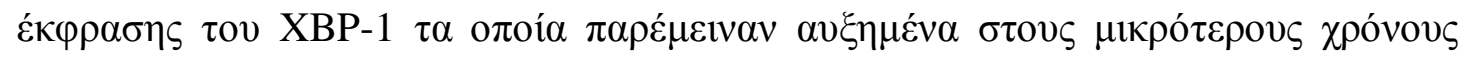

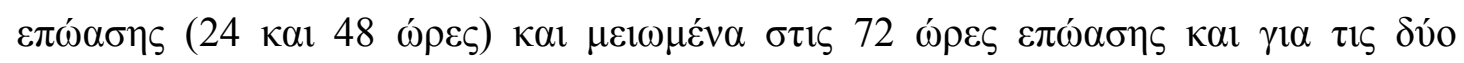

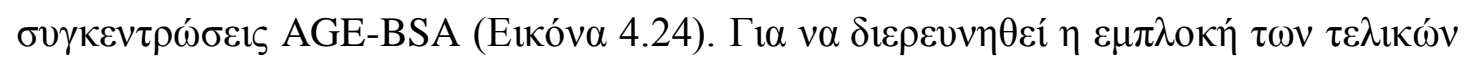

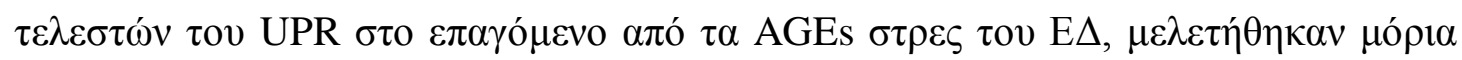

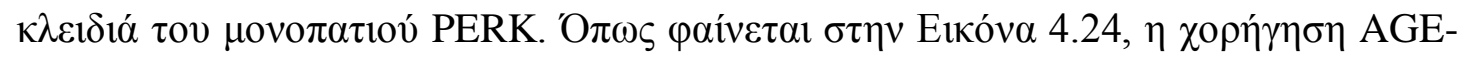

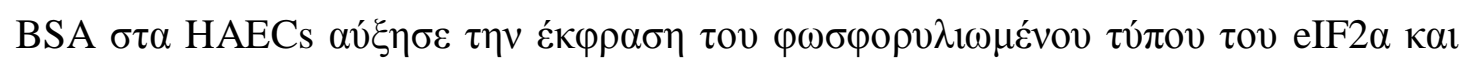

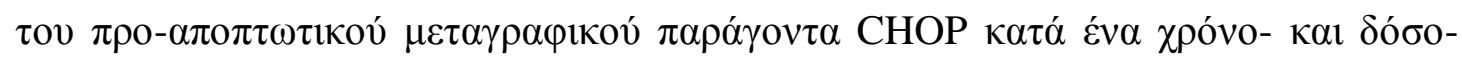

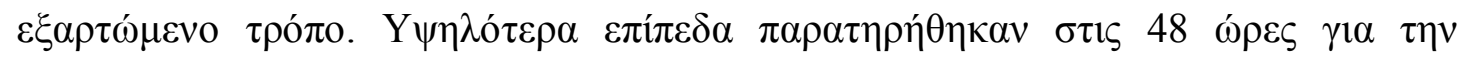

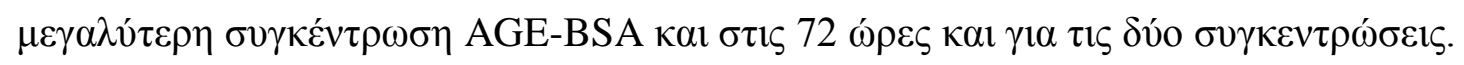

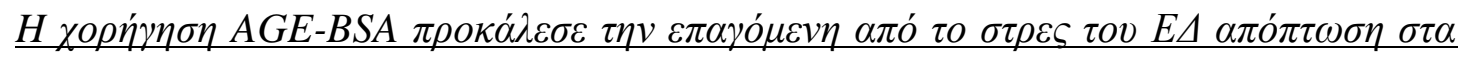
$\underline{H A E C S}$

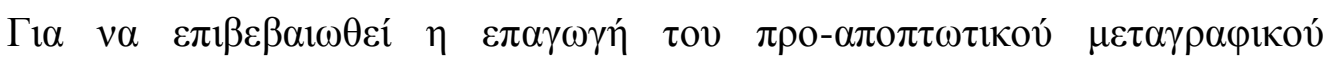

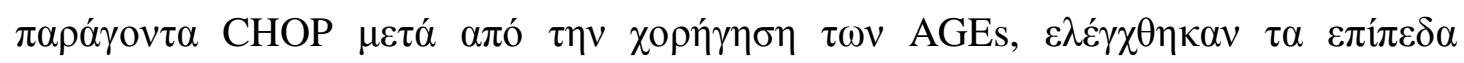

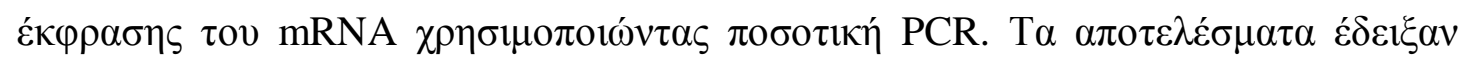

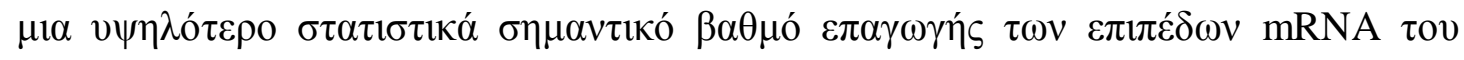

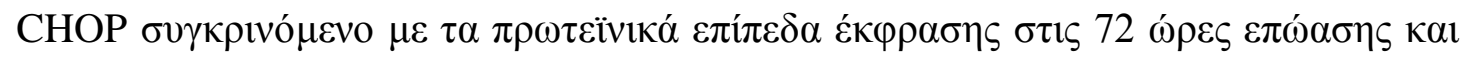

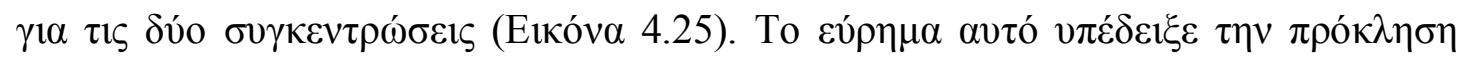

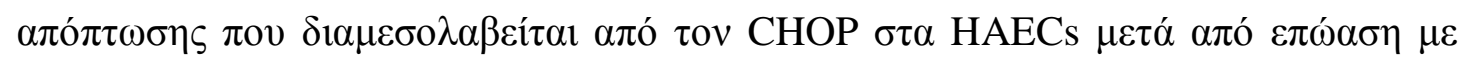

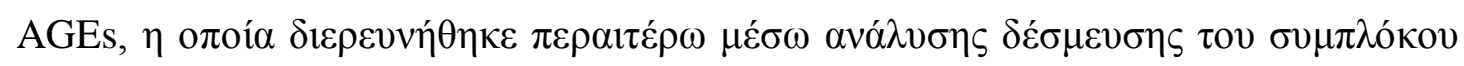

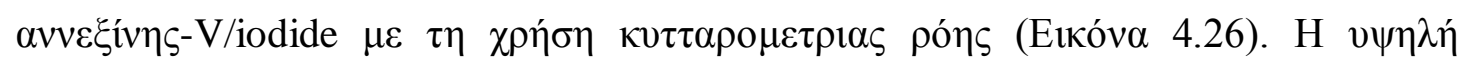

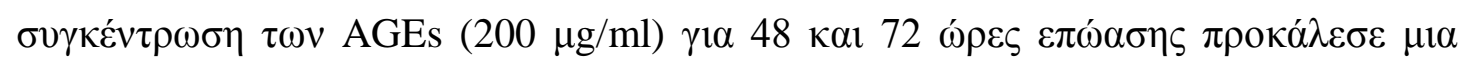

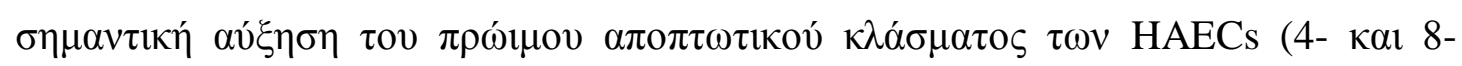

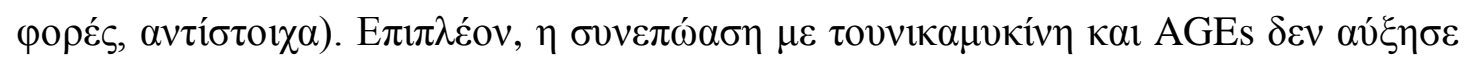

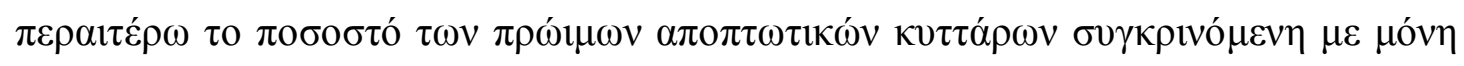

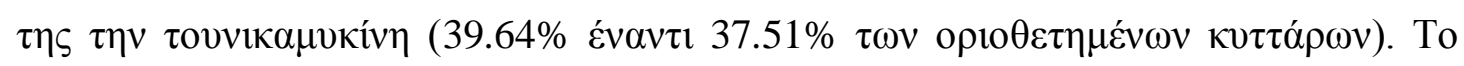

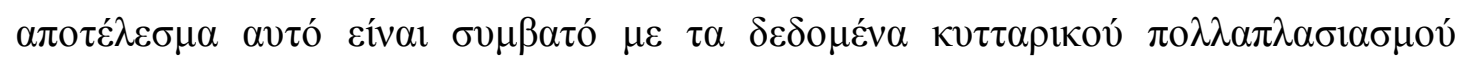

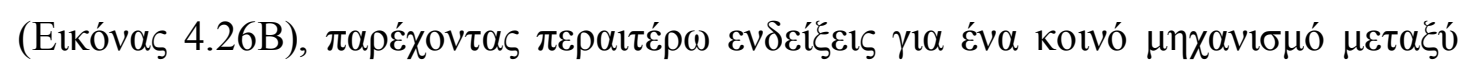

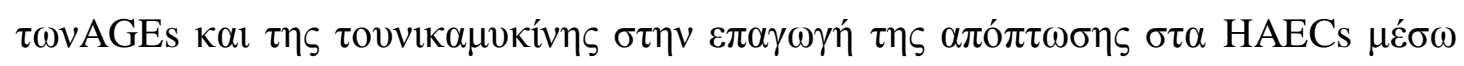

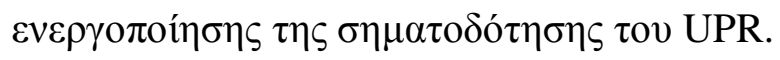




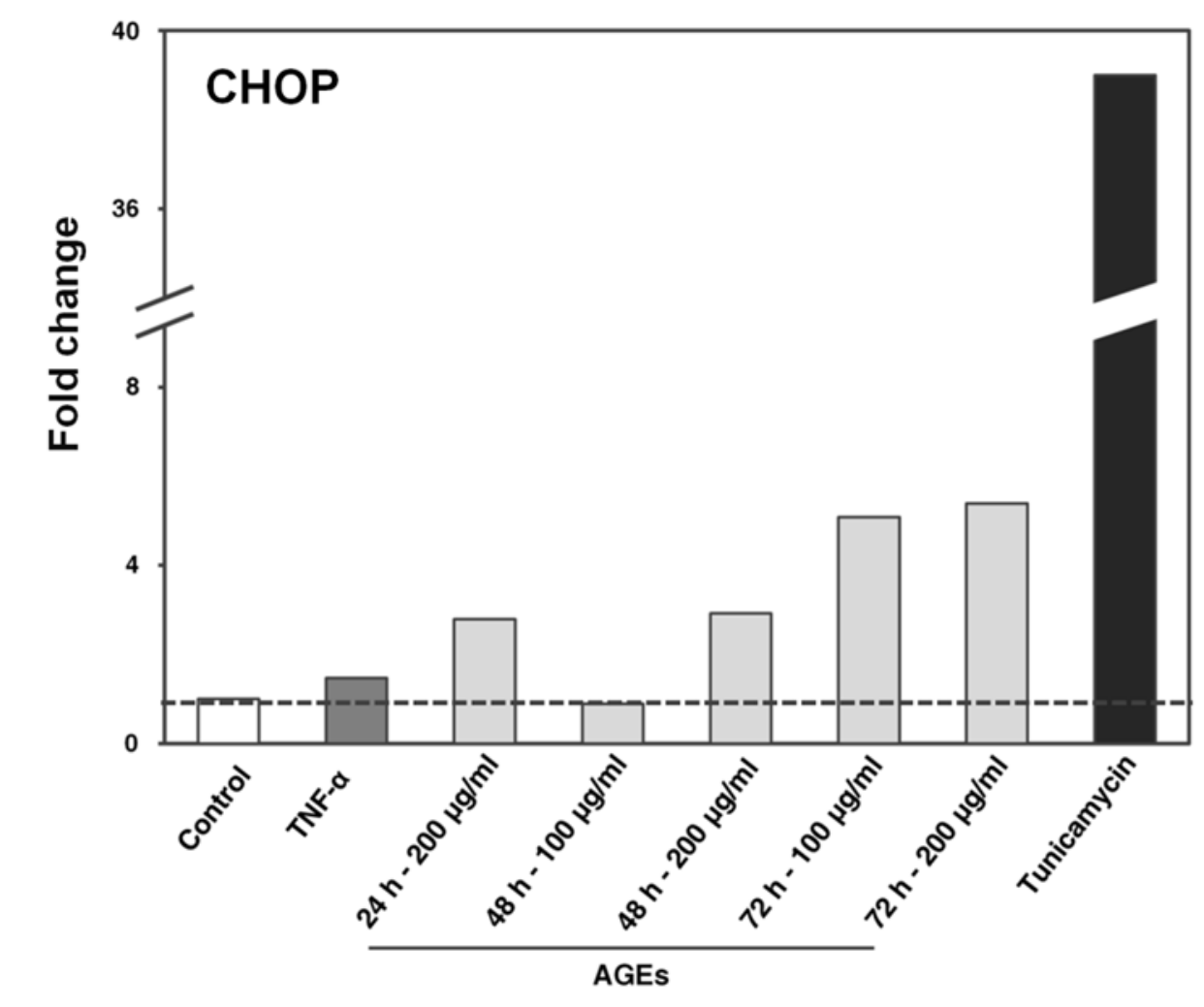

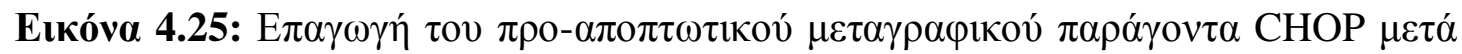

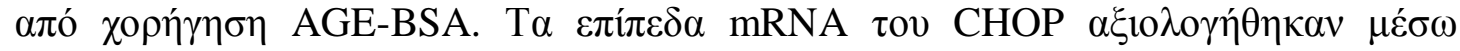

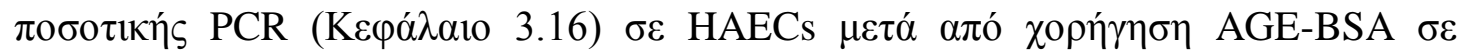

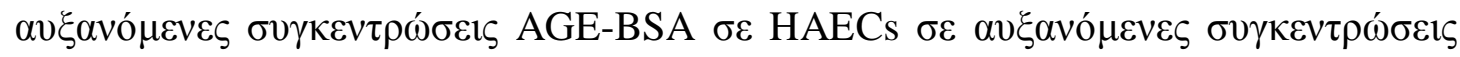

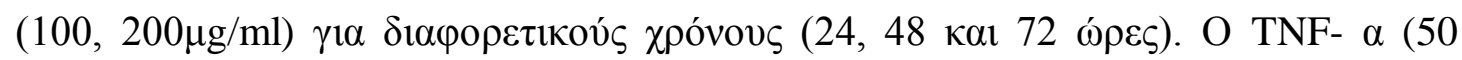

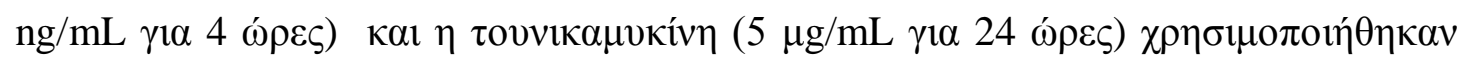

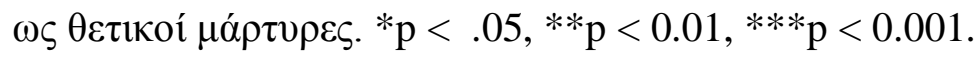



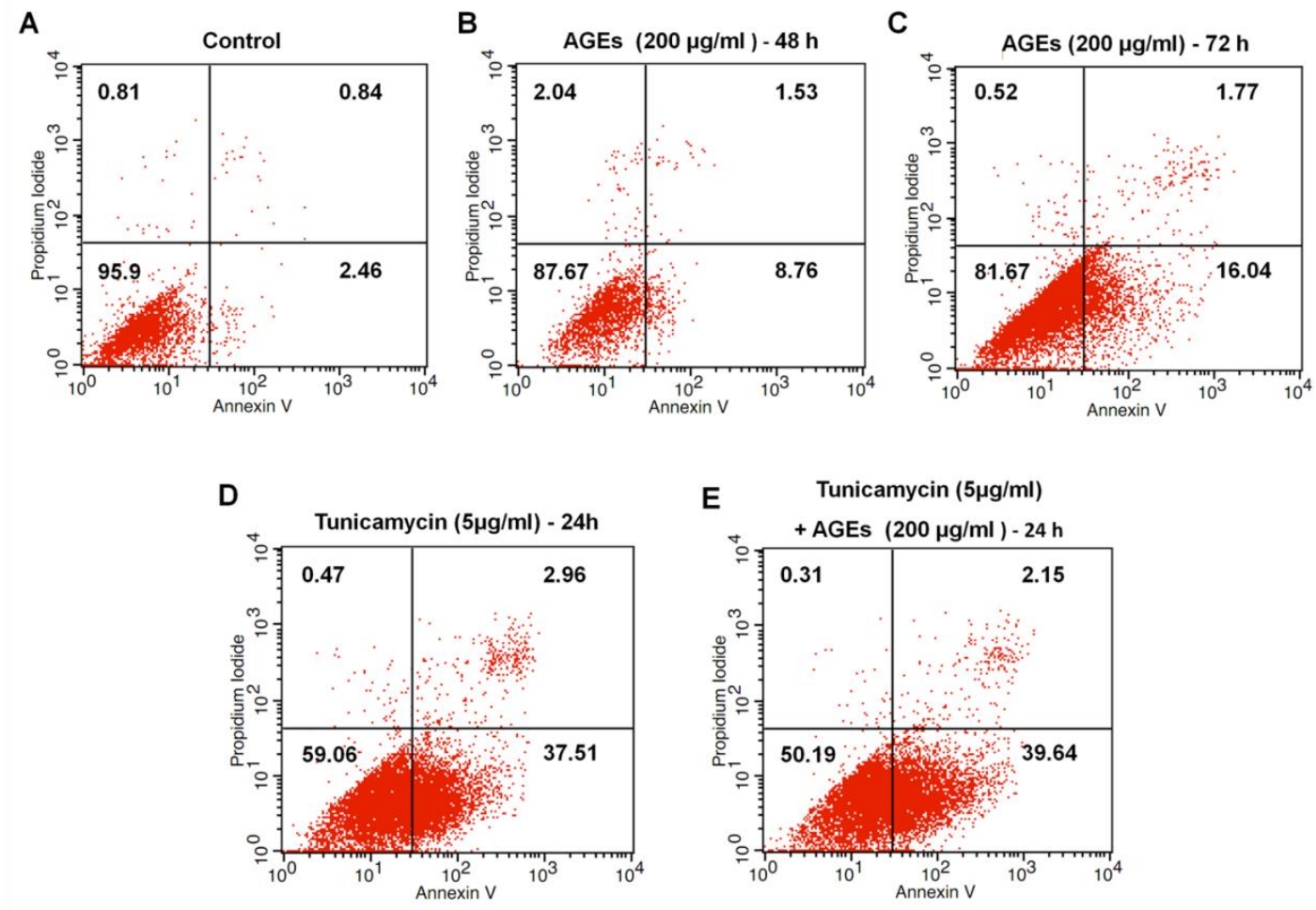

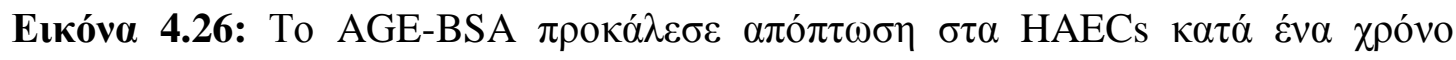

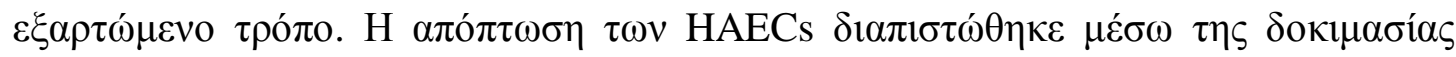

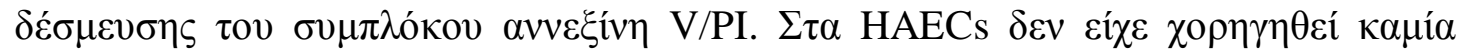

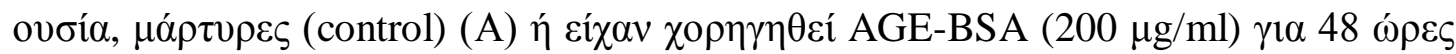

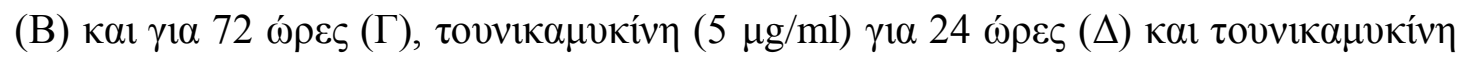

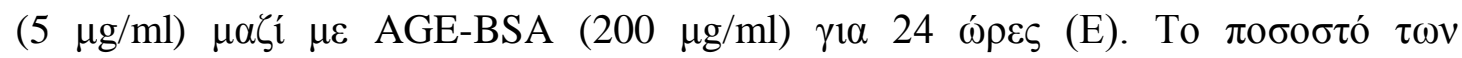
o

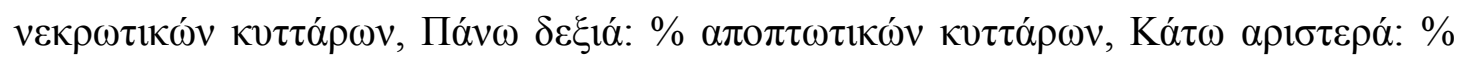

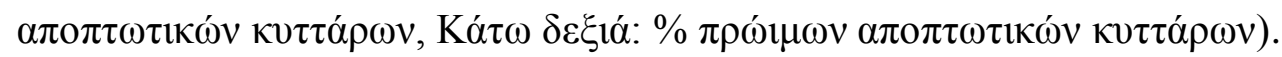




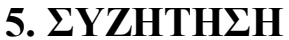

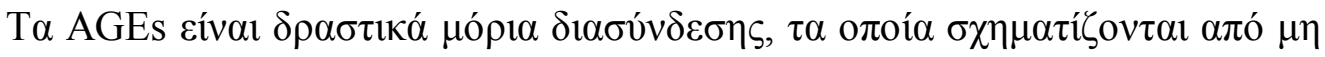

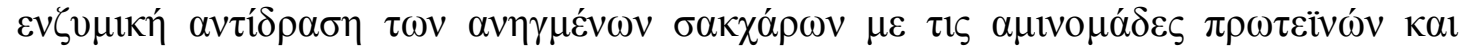

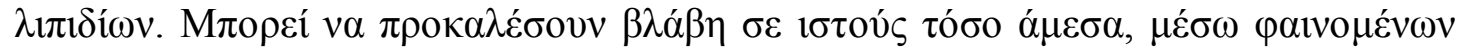

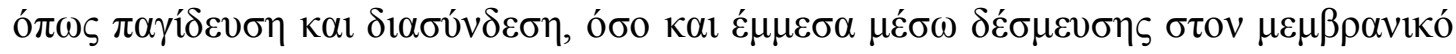

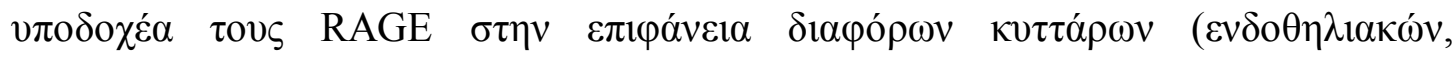

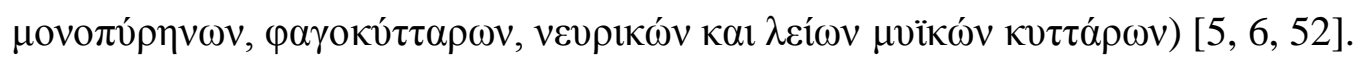

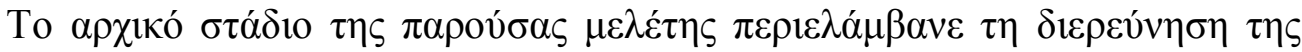

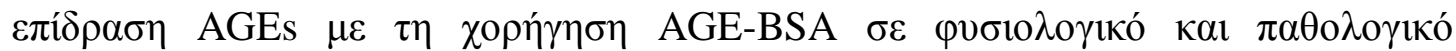

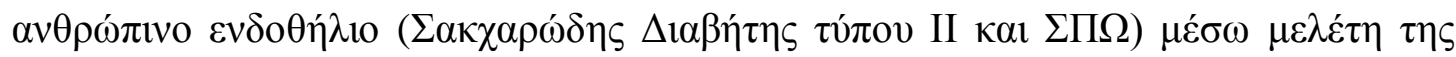

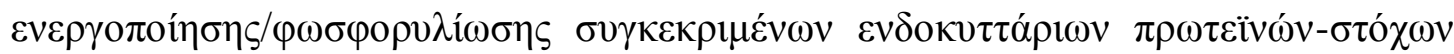

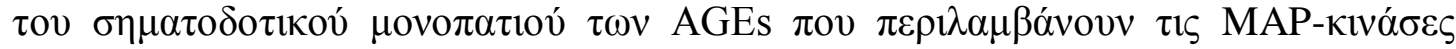

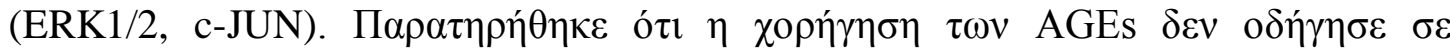

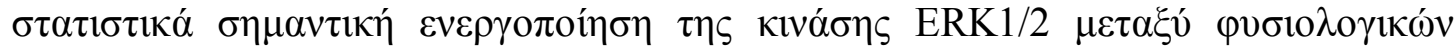

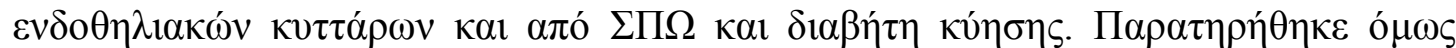

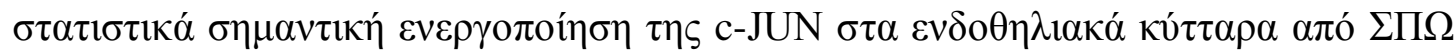

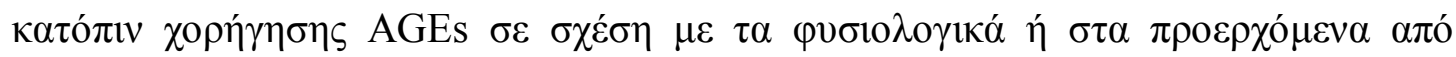

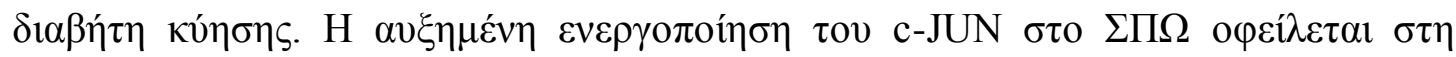

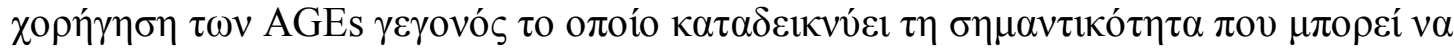

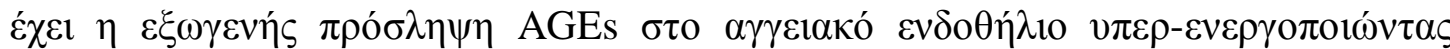

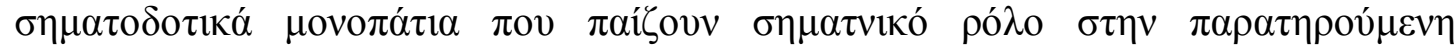

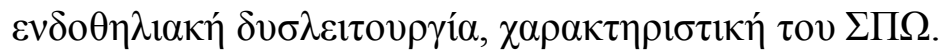

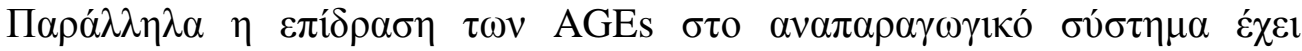

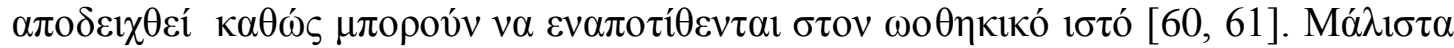

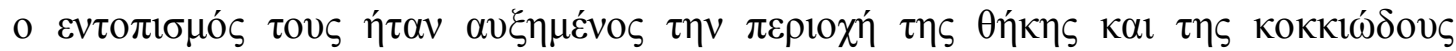

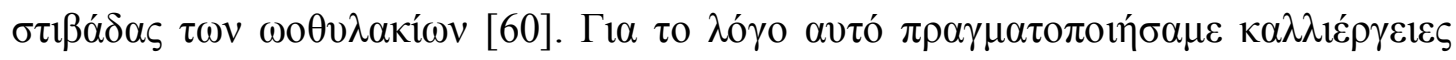

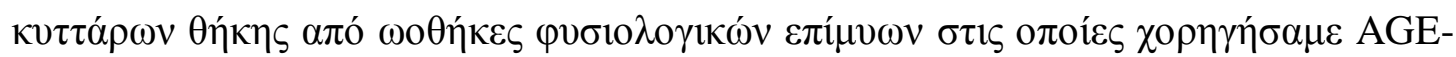

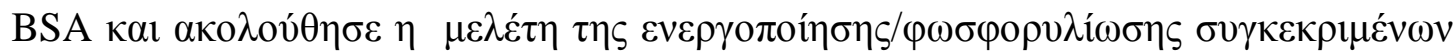

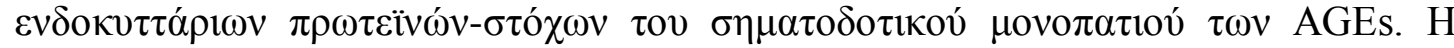

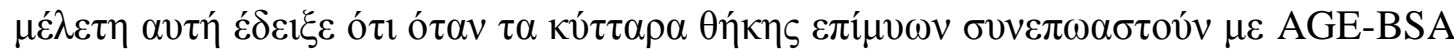

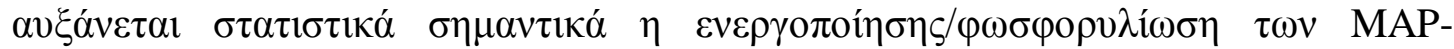




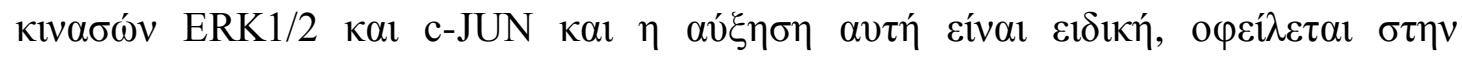

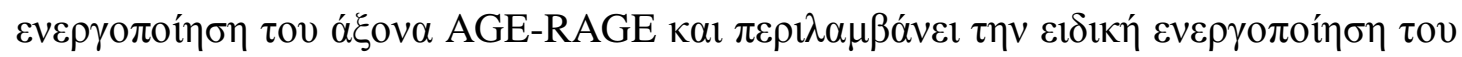

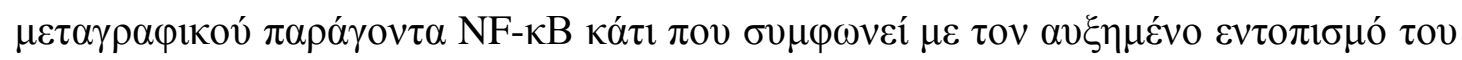

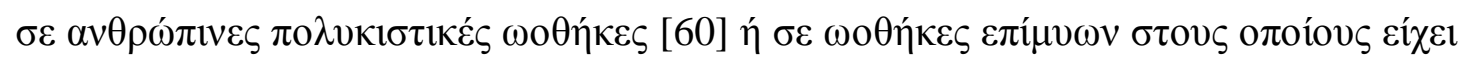

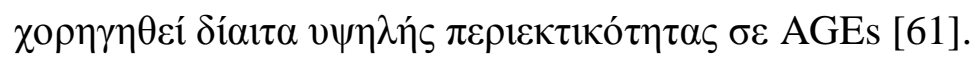

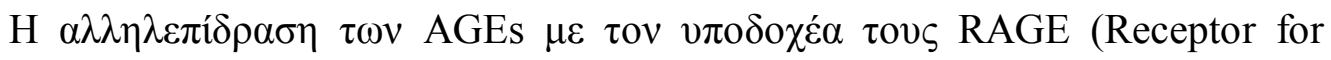

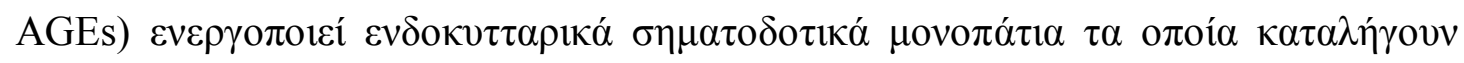

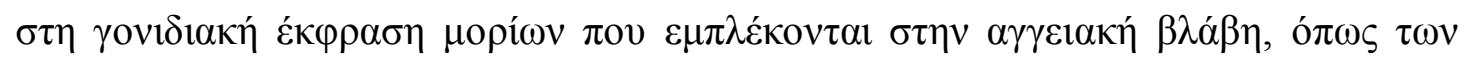

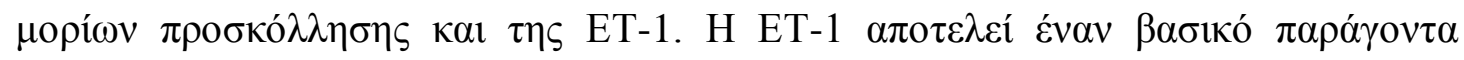

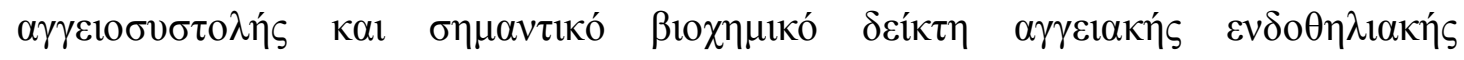
$\delta v \sigma \lambda \varepsilon ı \tau o v \rho \gamma i ́ \alpha$ [89].

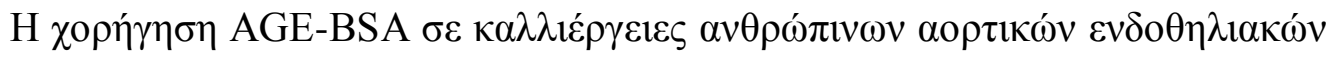

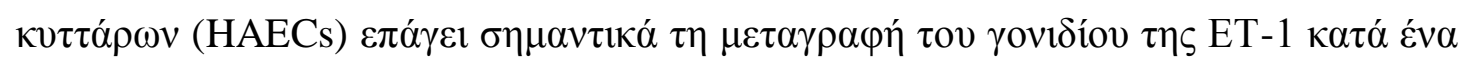

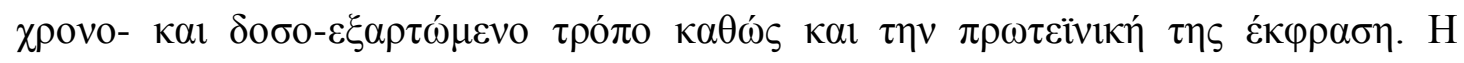

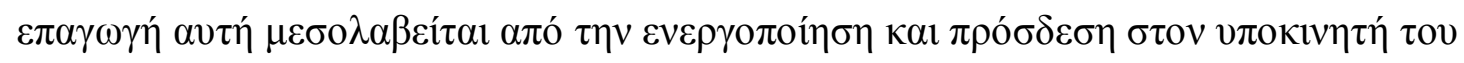

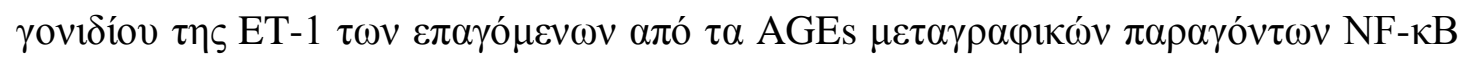

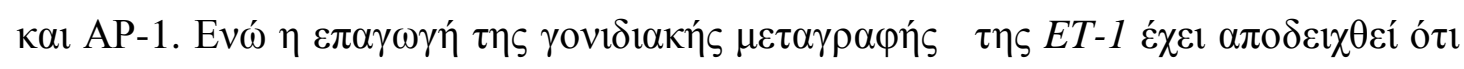

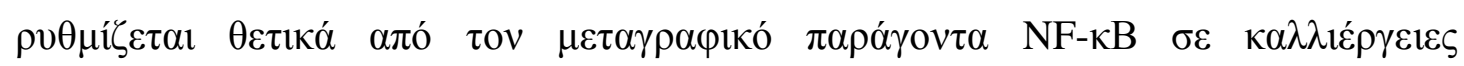

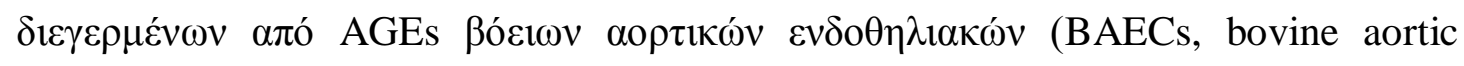

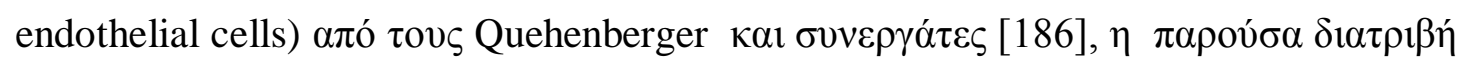

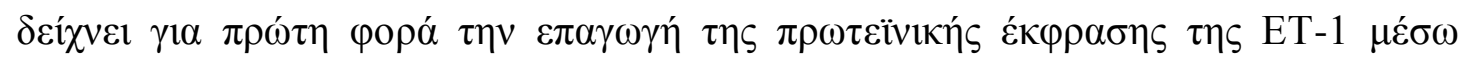

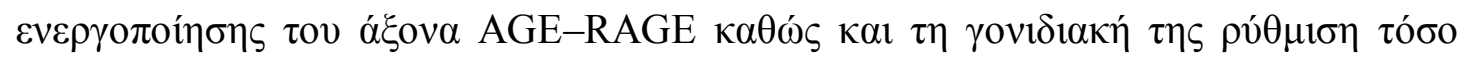

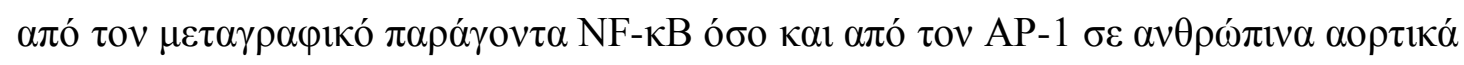

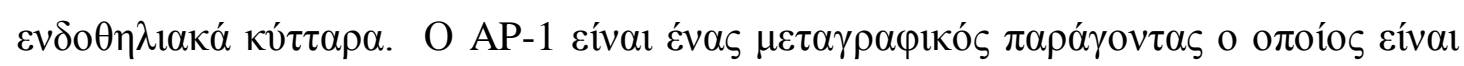

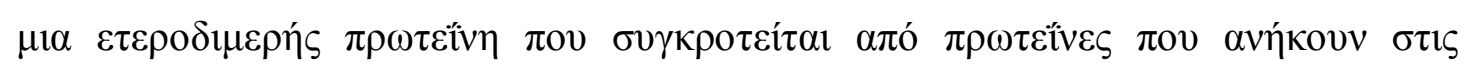

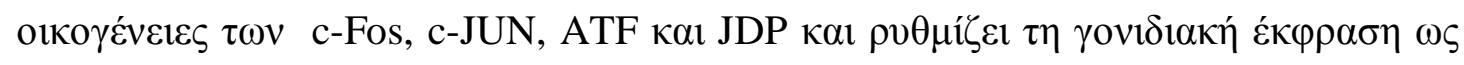

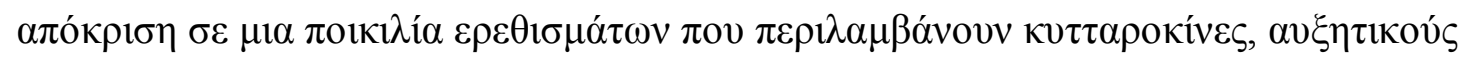

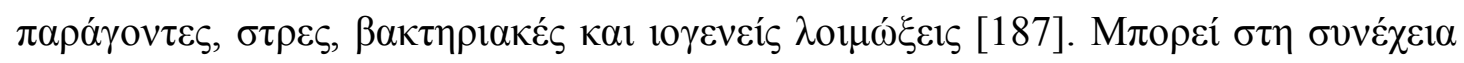

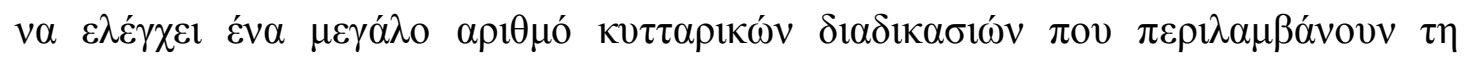

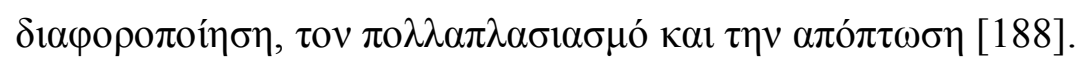




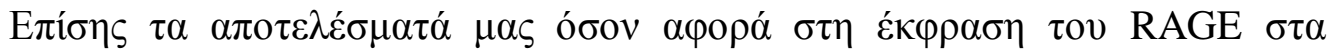

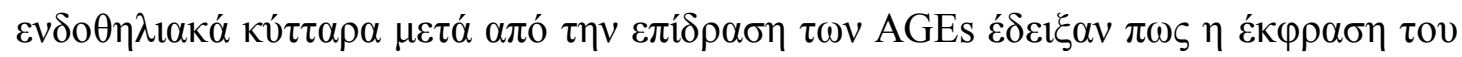

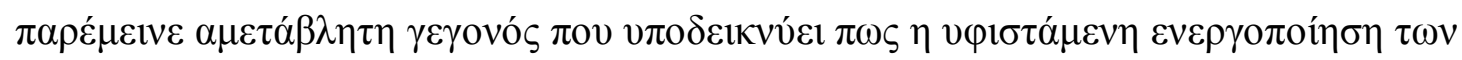

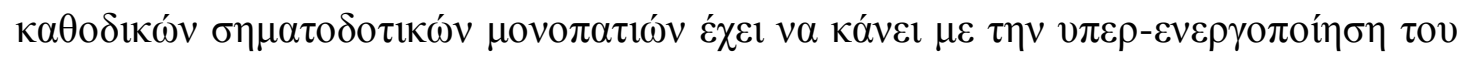

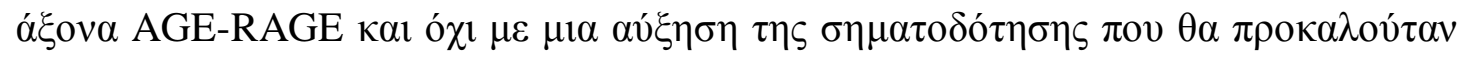

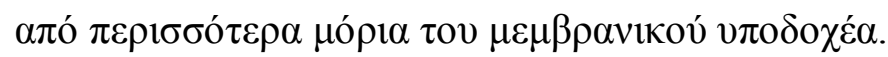

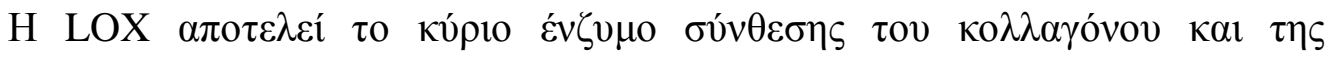

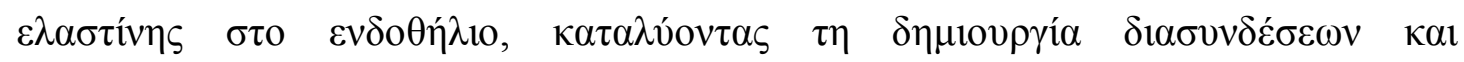

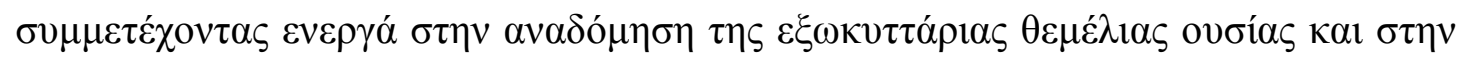

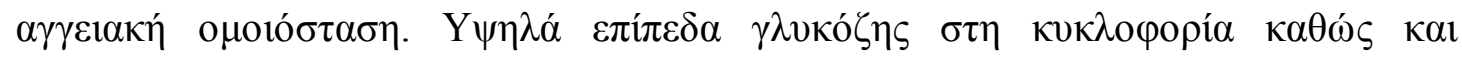

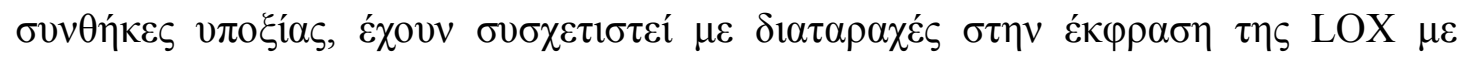

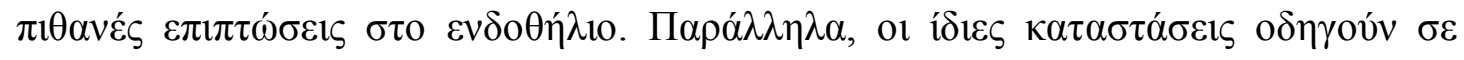

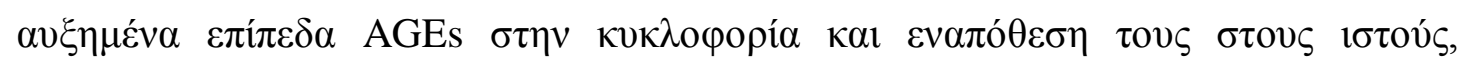

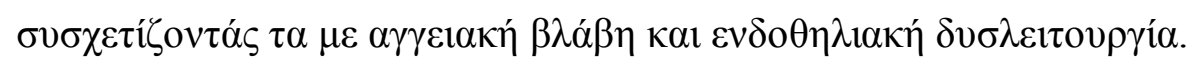

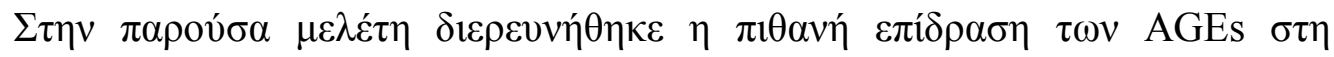

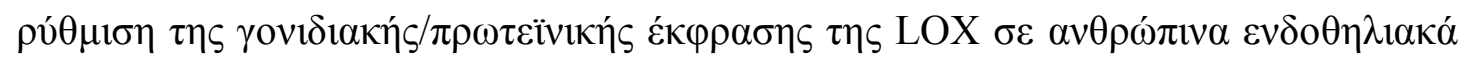

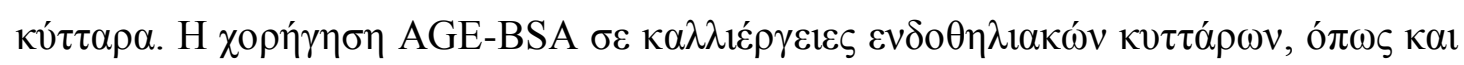

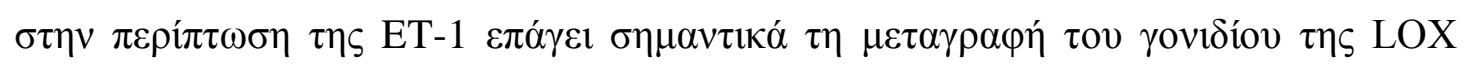

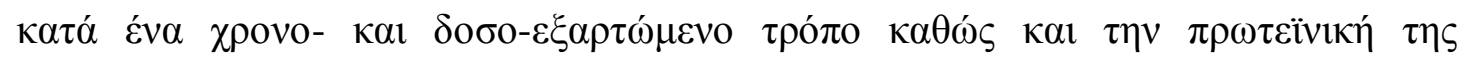

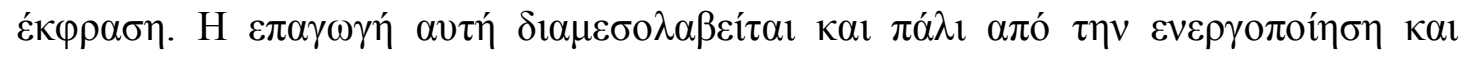

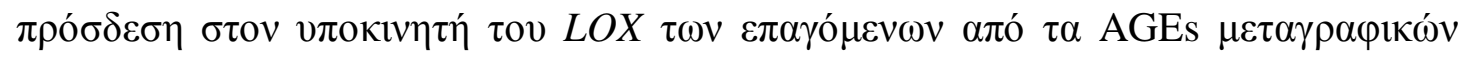

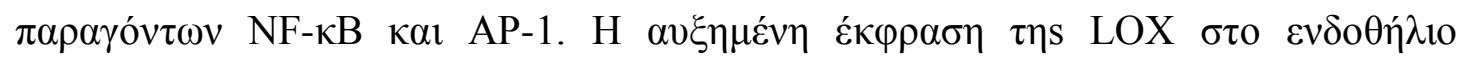

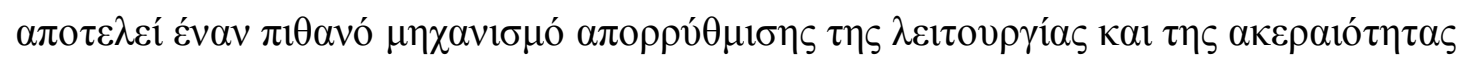

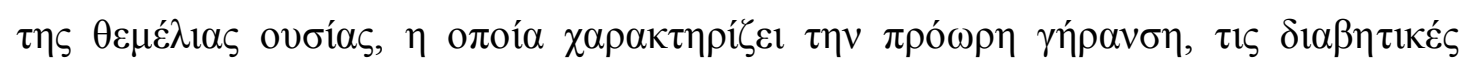

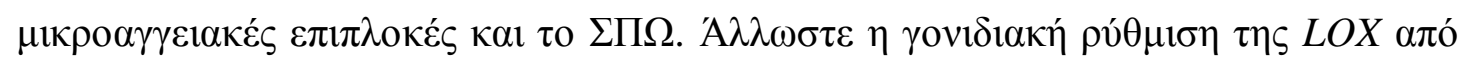

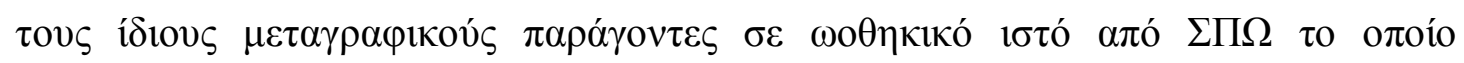

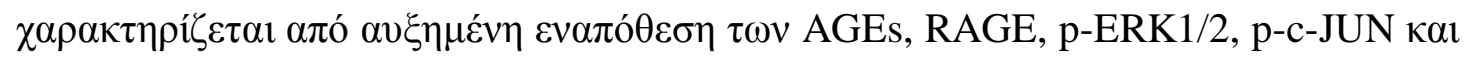

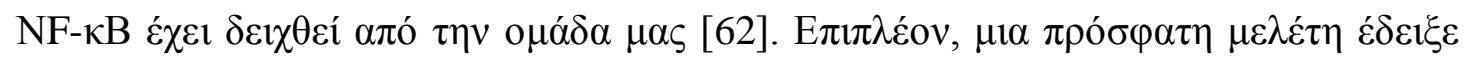

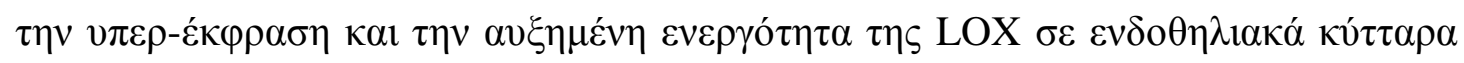

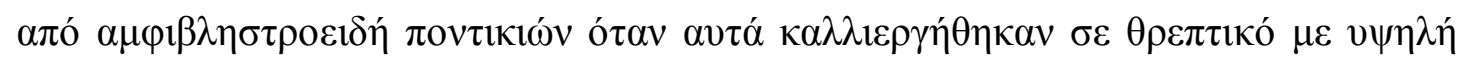

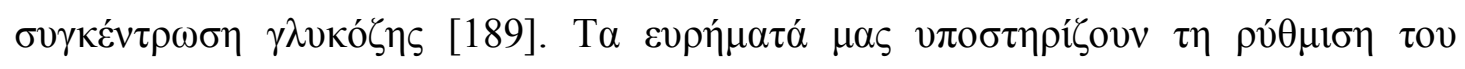

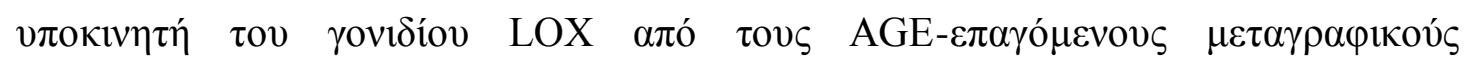




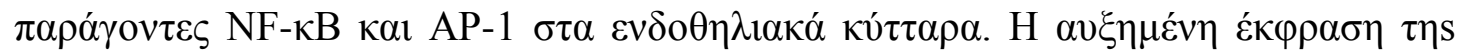

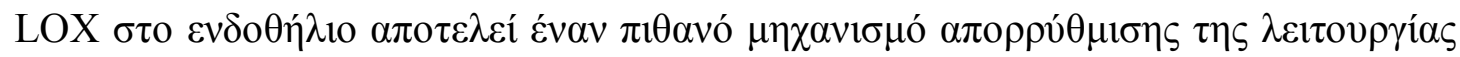

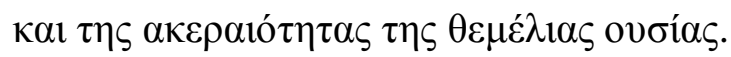

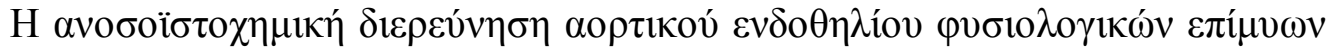

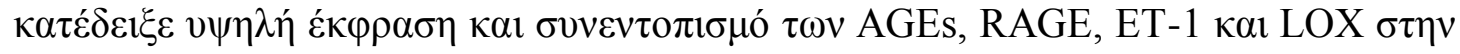

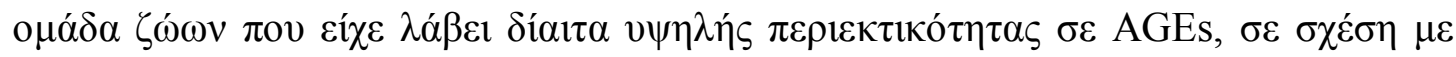

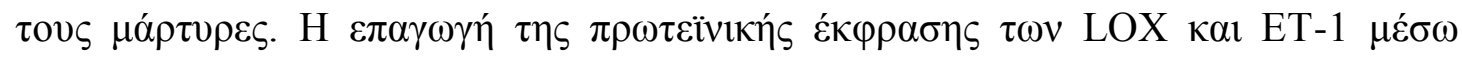

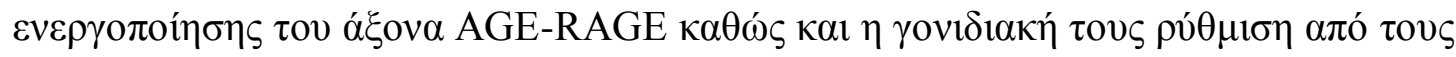

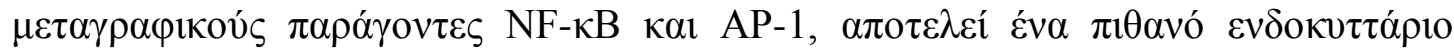

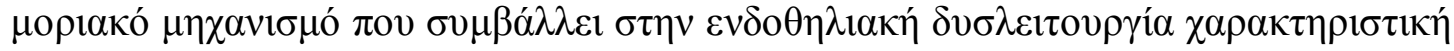

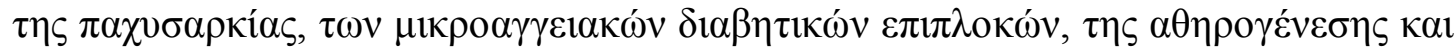
$\tau$ cov $\Sigma \Pi \Omega$.

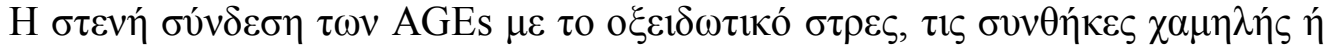

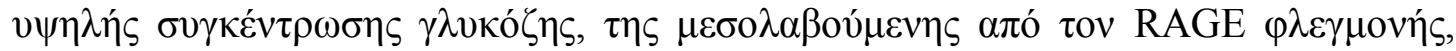

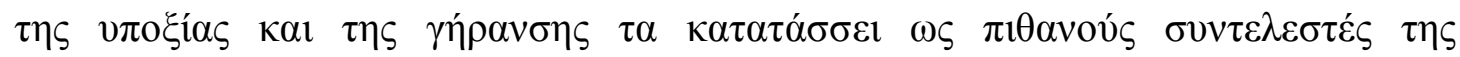

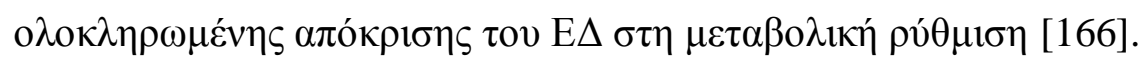

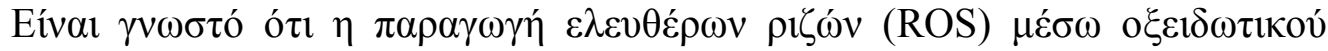

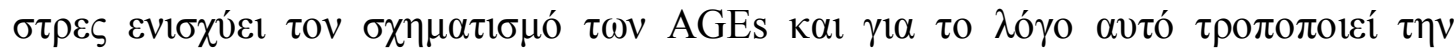

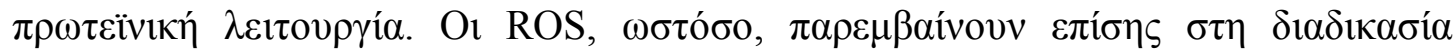

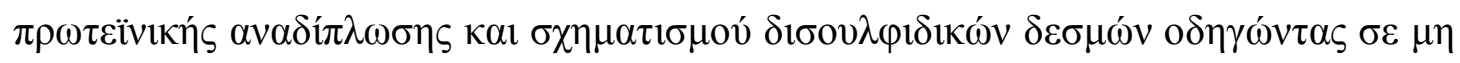

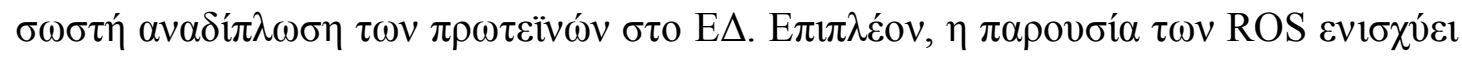

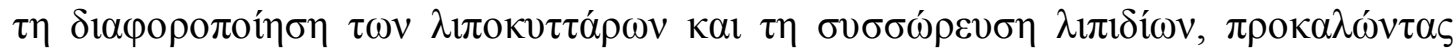

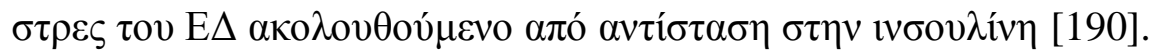

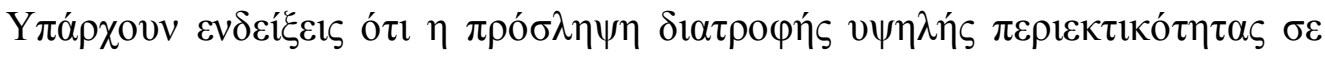

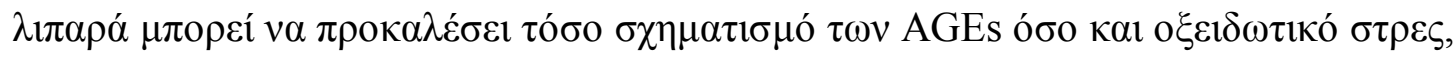

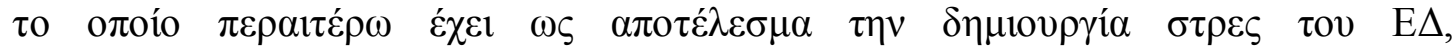

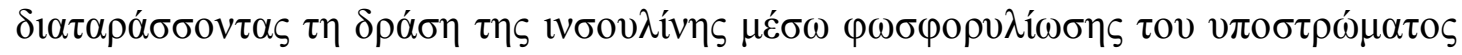

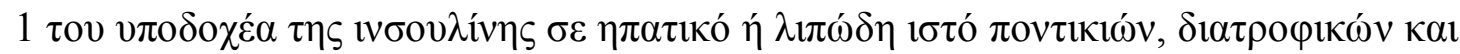

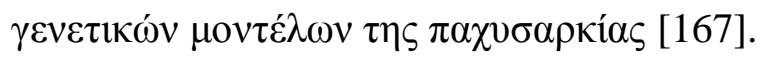




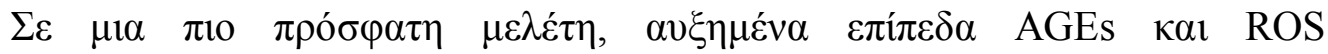

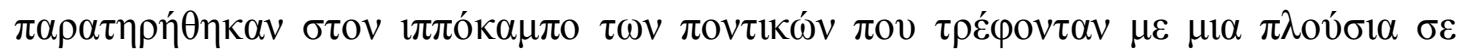

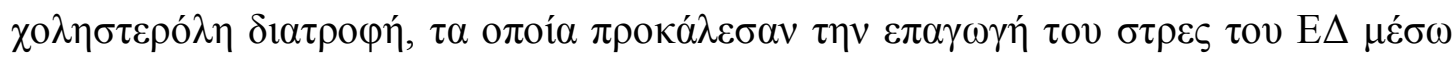

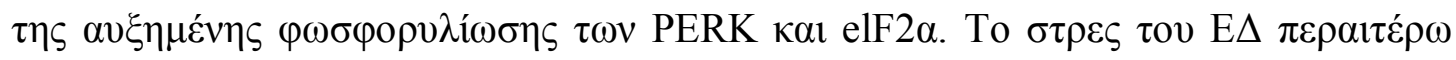

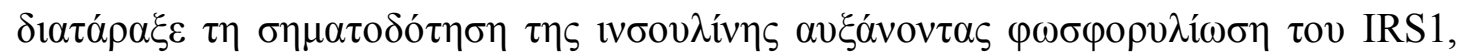

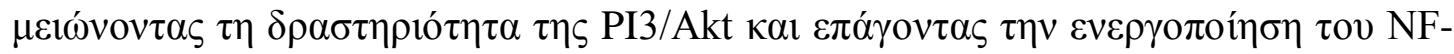
$\kappa \mathrm{B}[191]$.

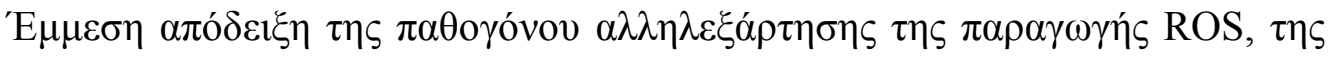

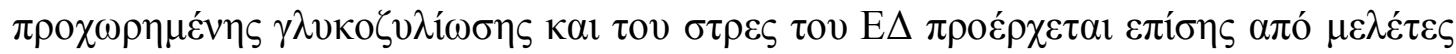

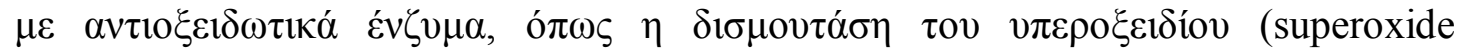

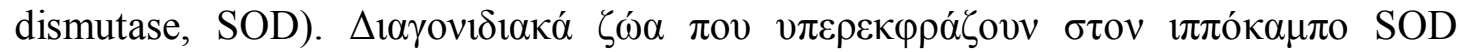

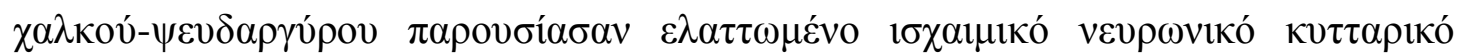

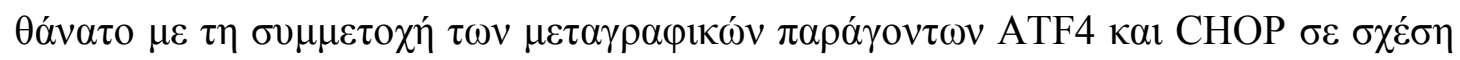

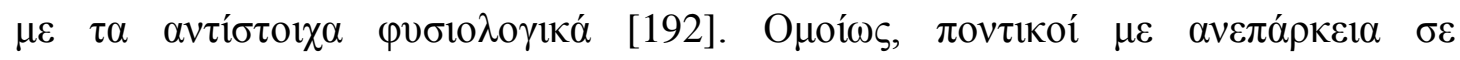

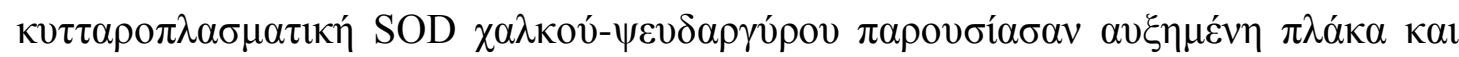

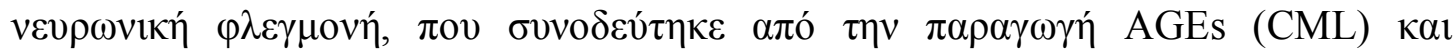

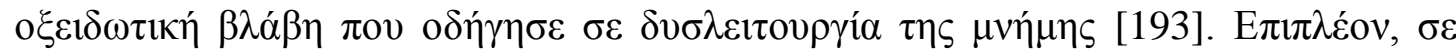

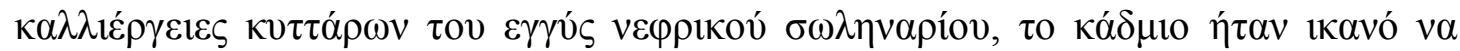

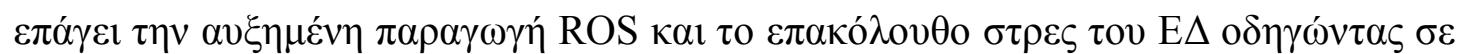

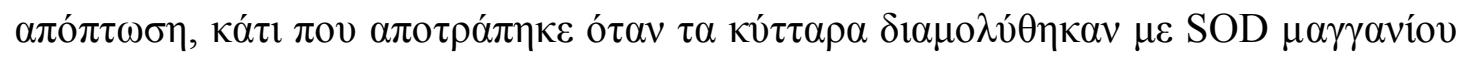

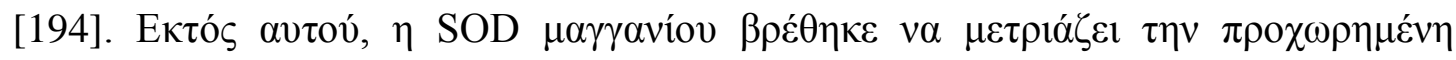

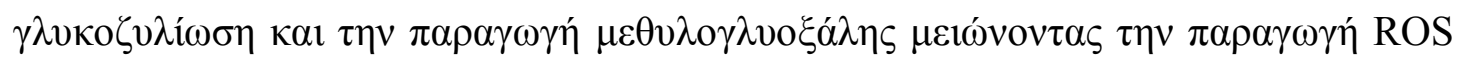

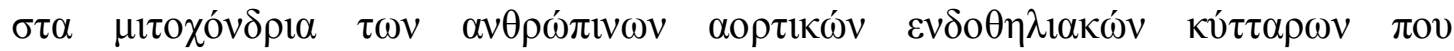

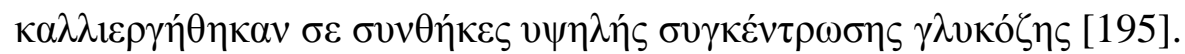

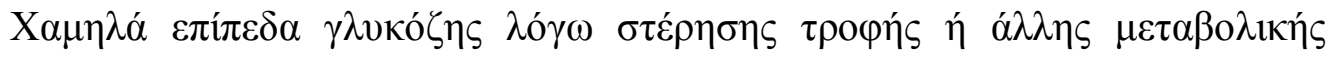

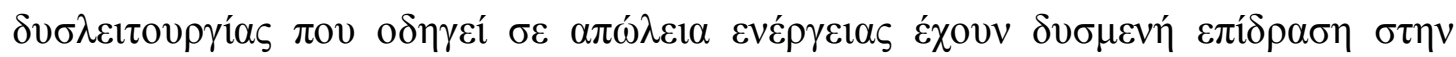

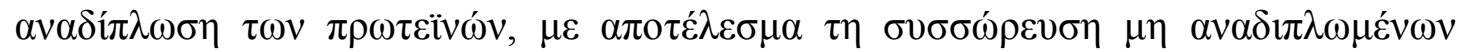

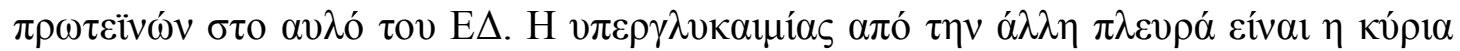

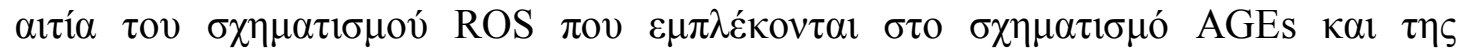

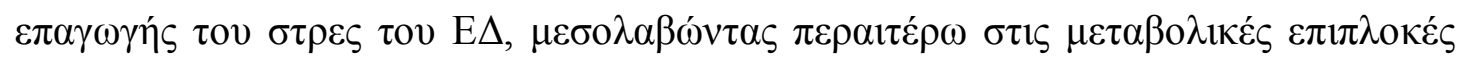

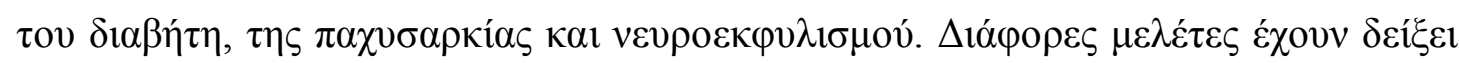

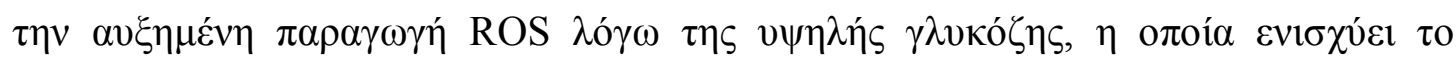




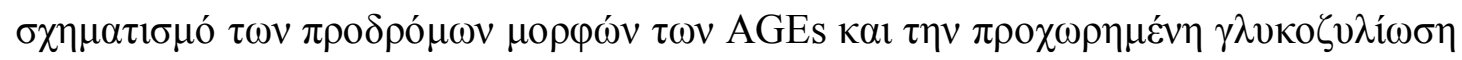

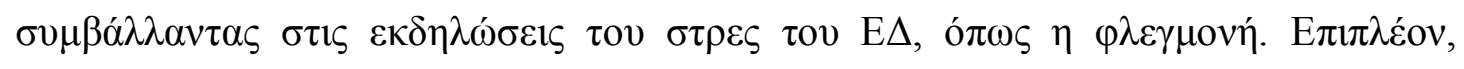

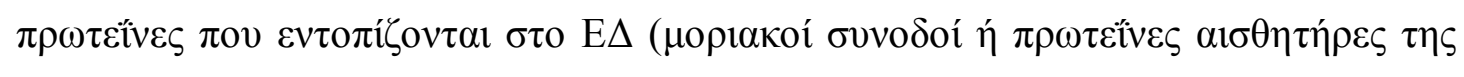

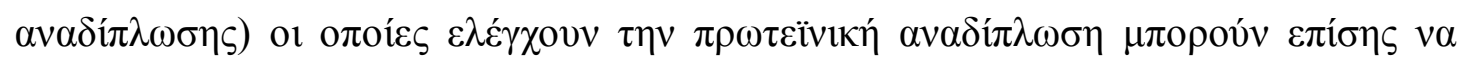

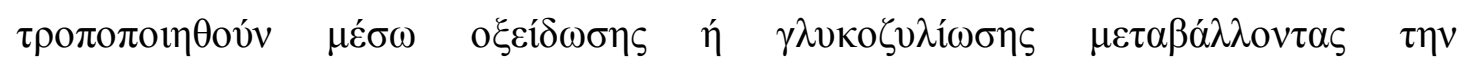

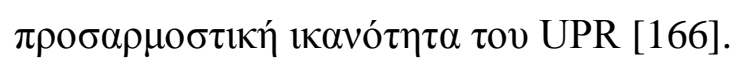

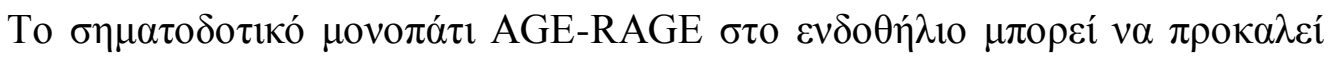

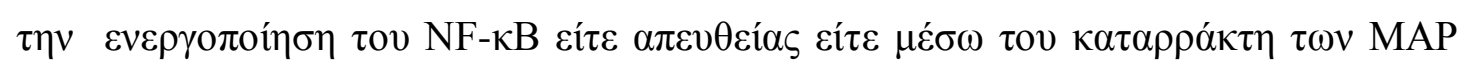

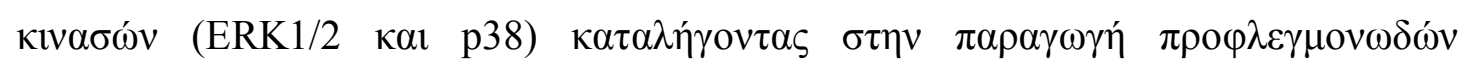

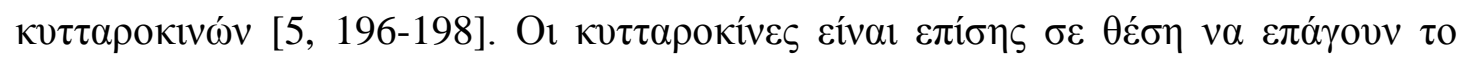

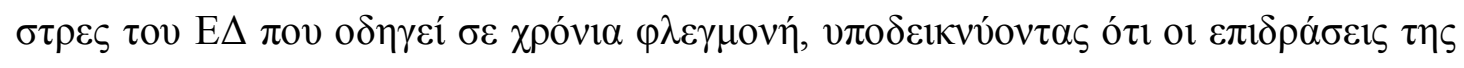

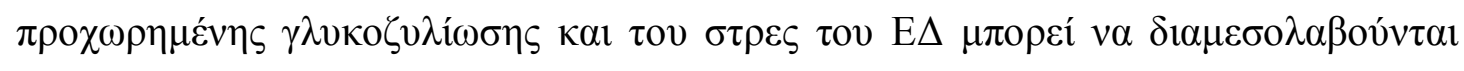

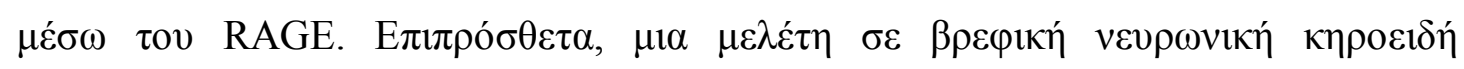

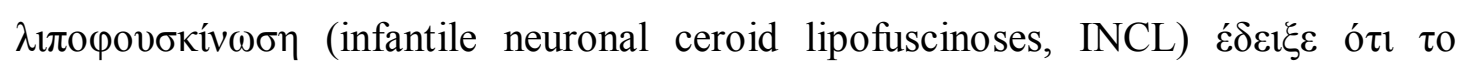

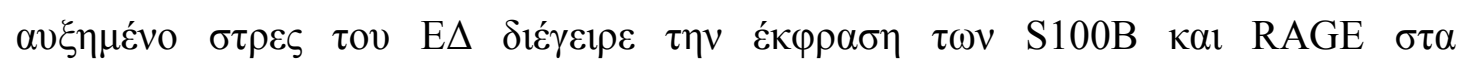

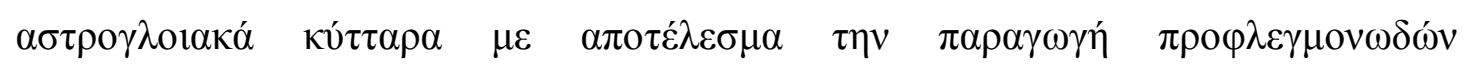

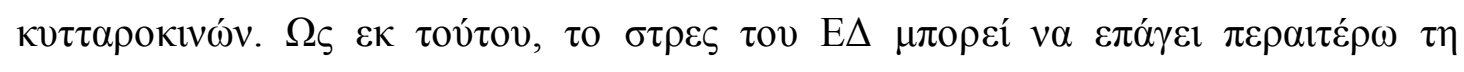

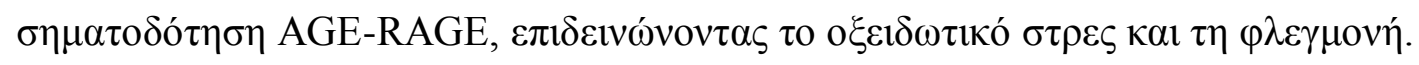

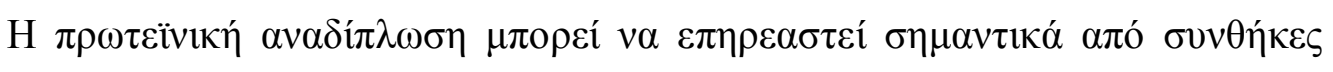

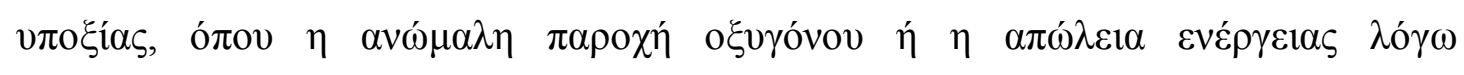

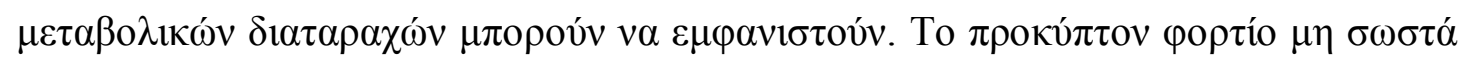

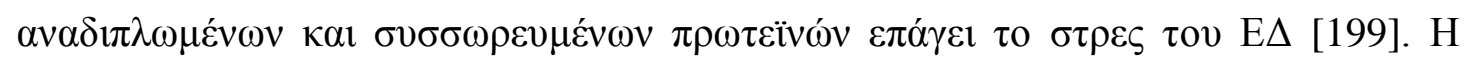

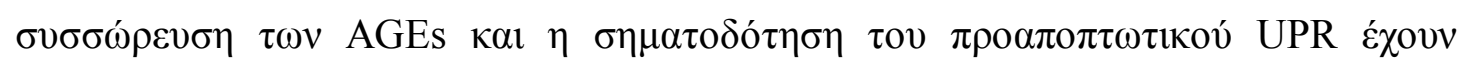

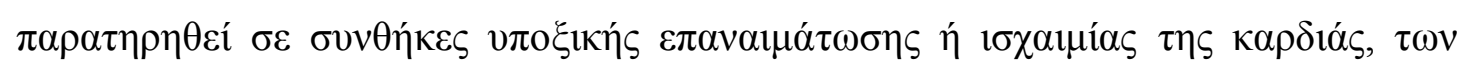

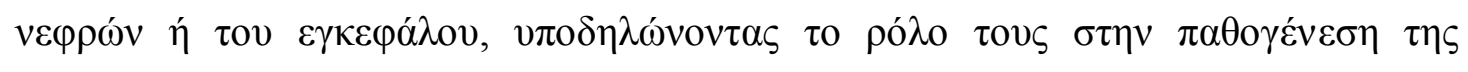

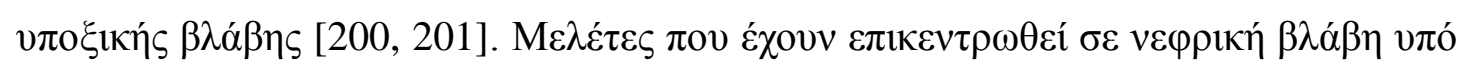

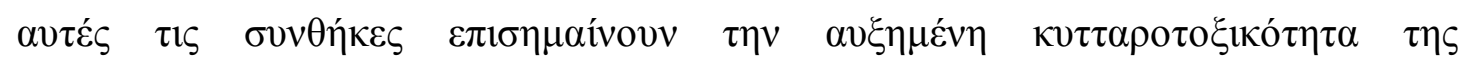

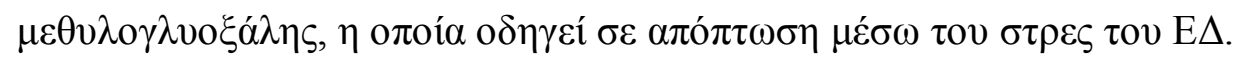

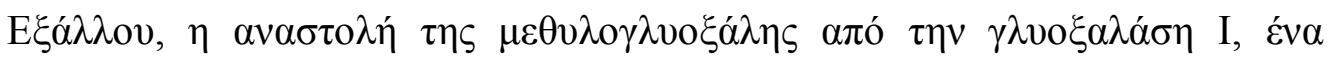

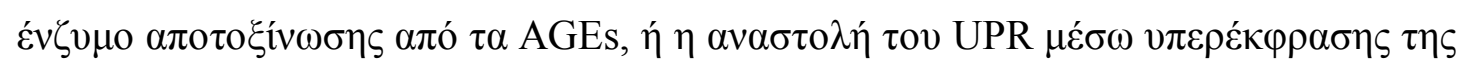

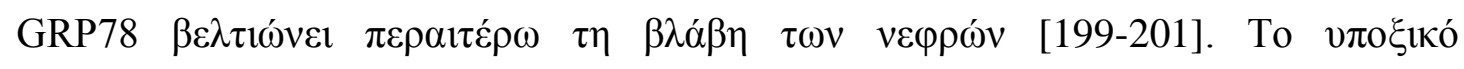




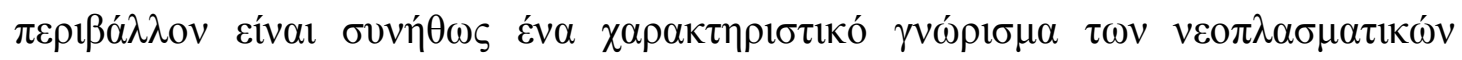

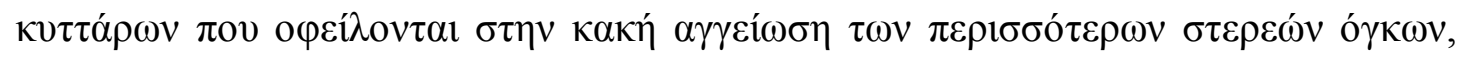

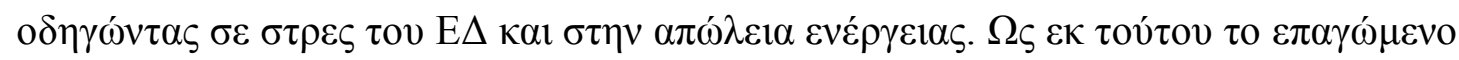

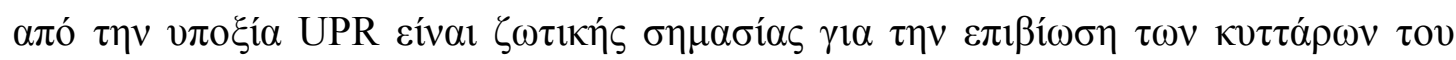

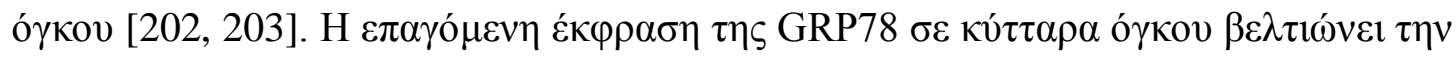

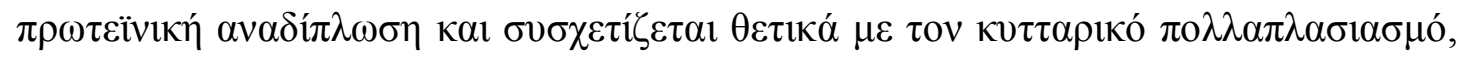

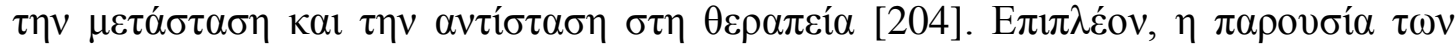

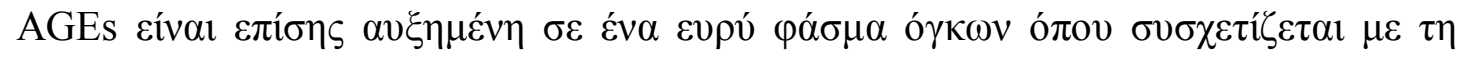

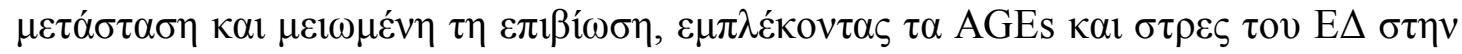

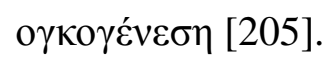

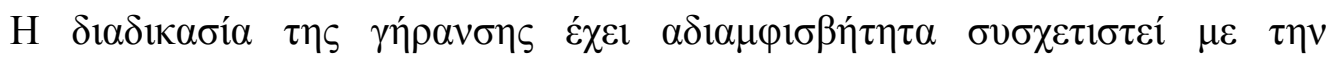

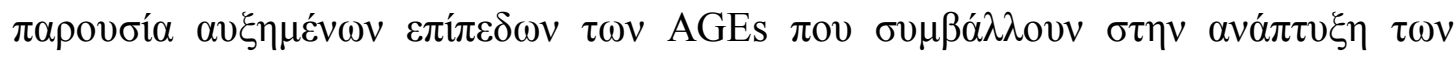

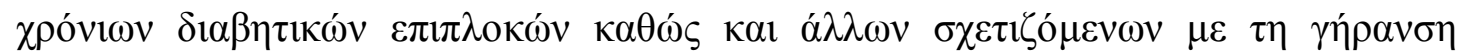

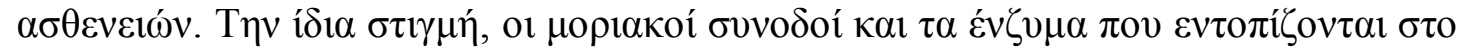

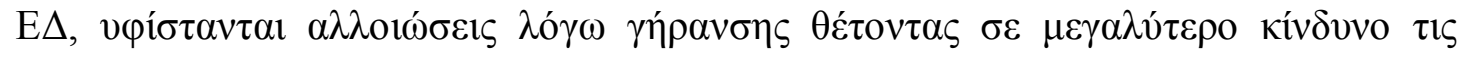

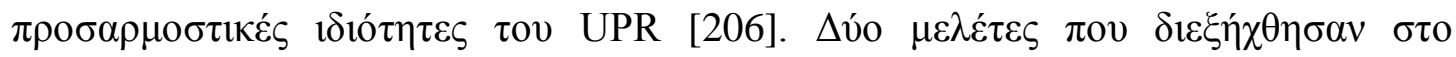

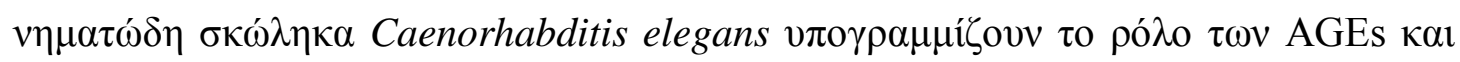

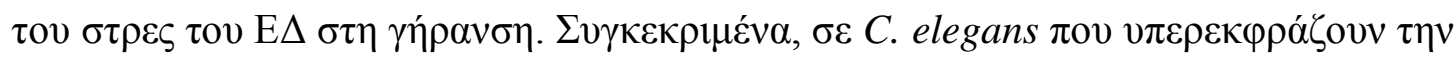

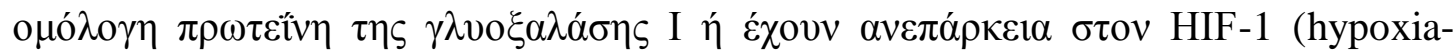

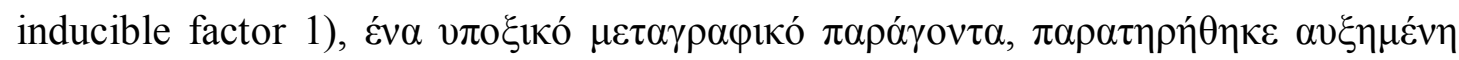

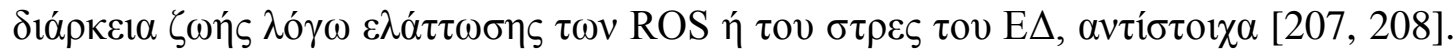

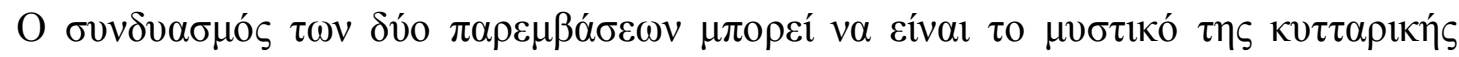

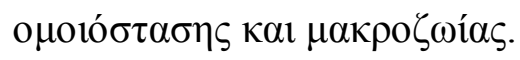

Н

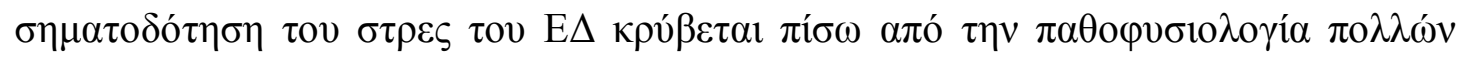

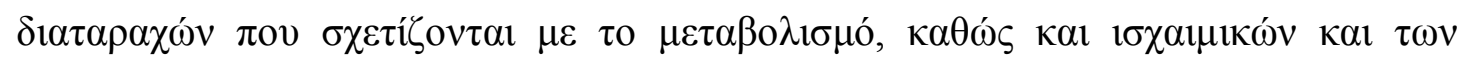

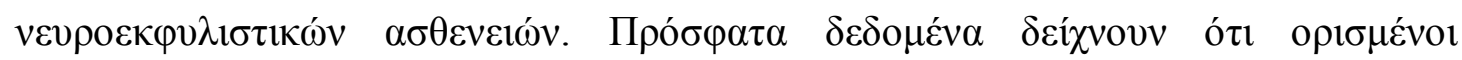

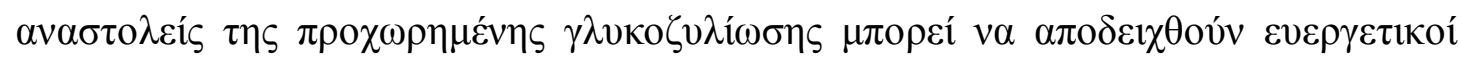
$\gamma 1 \alpha \tau \eta \delta 1 \alpha \tau \eta ́ p \eta \sigma \eta \tau \eta \varsigma$ o UPR [166].

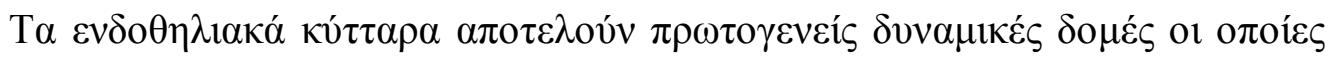

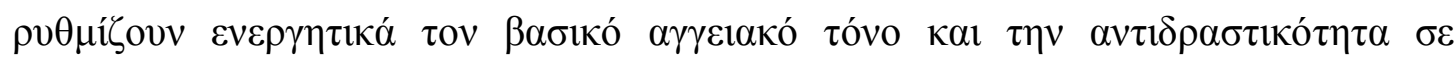




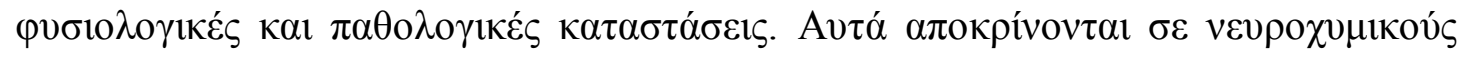

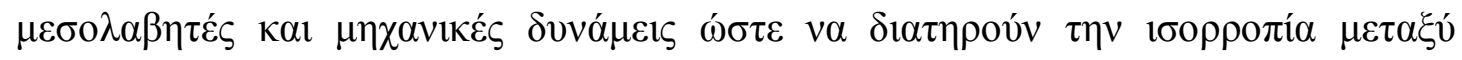

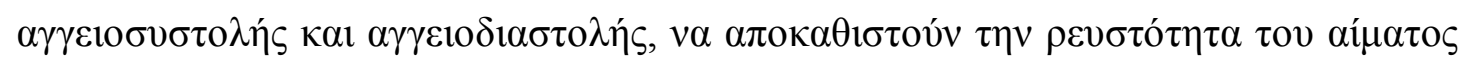

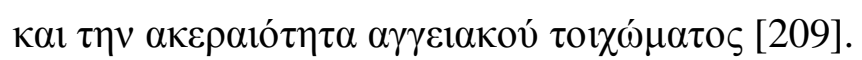

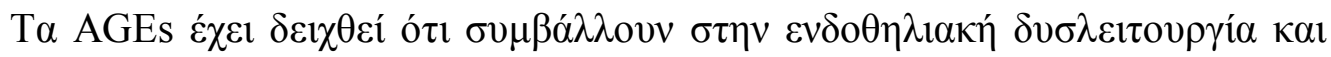

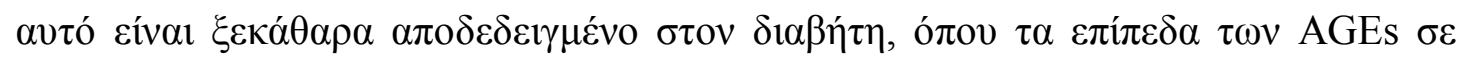

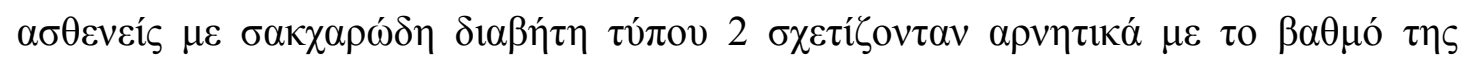

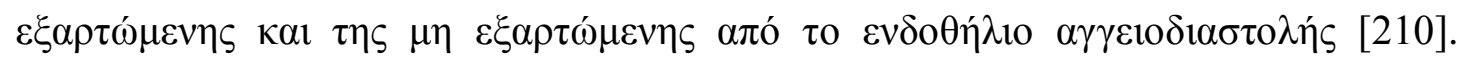

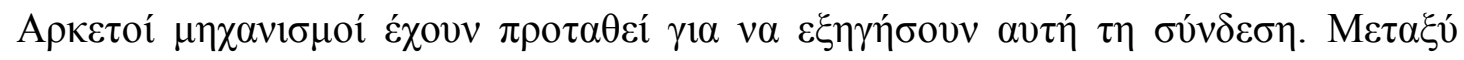

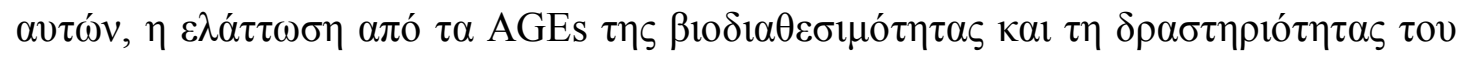
$\pi \rho \circ \varepsilon \rho \chi o ́ \mu \varepsilon v o v$ a

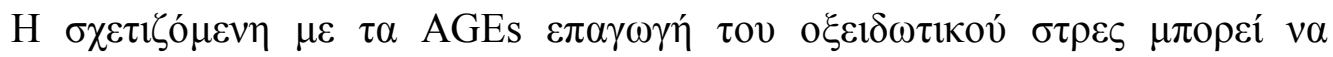

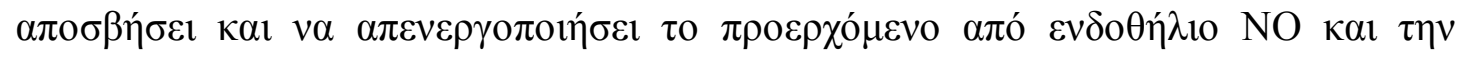

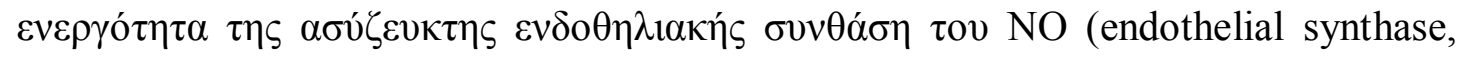

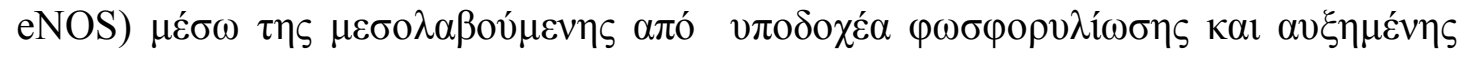

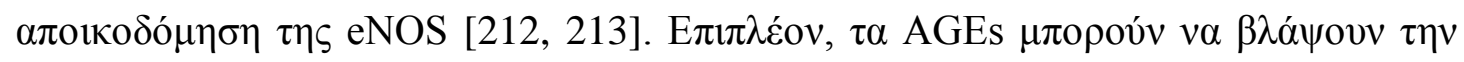

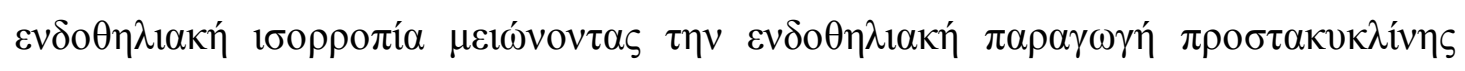

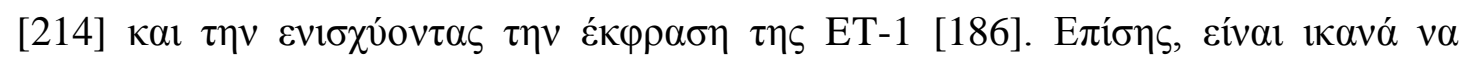

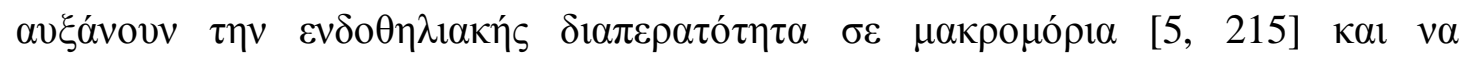

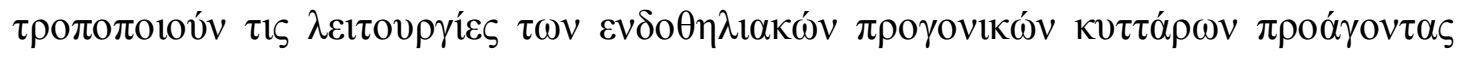
$\tau \eta \nu$ a

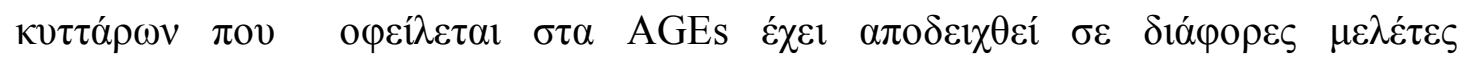

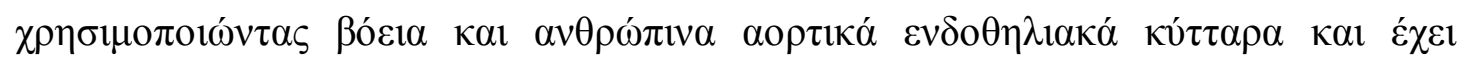

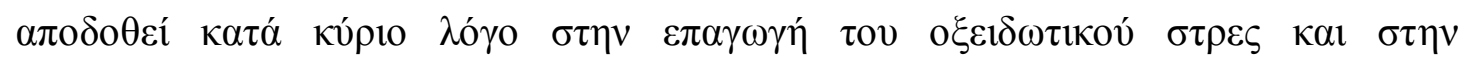

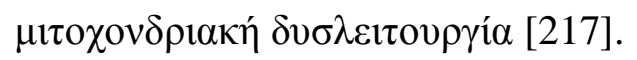

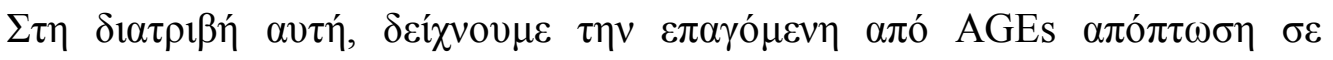

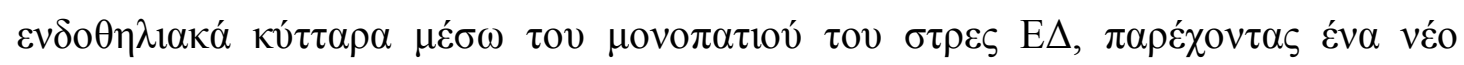

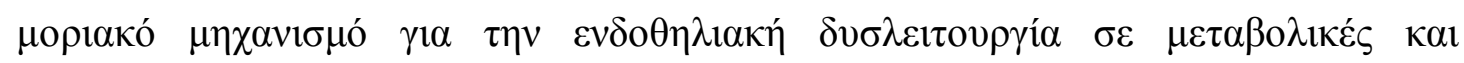
$\kappa \alpha \rho \delta 1 \alpha \gamma \gamma \varepsilon 1 \alpha \kappa \varepsilon \dot{\zeta} \delta 1 \alpha \tau \alpha \rho \alpha \chi \varepsilon \dot{\varepsilon}$.

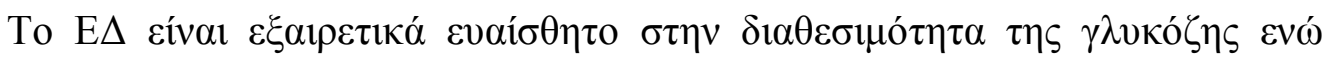

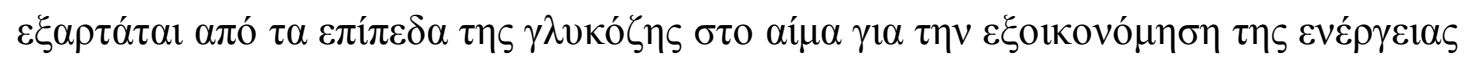




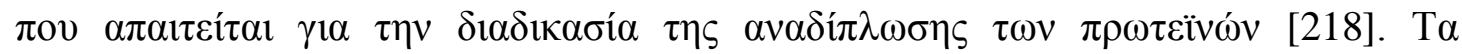

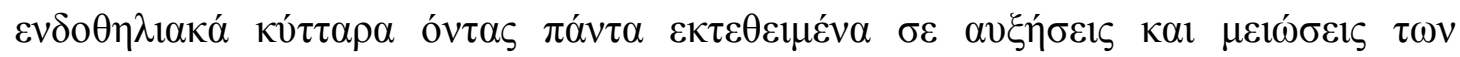

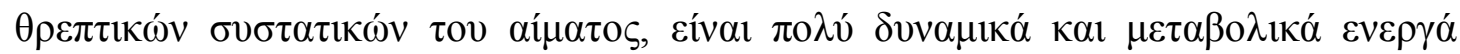

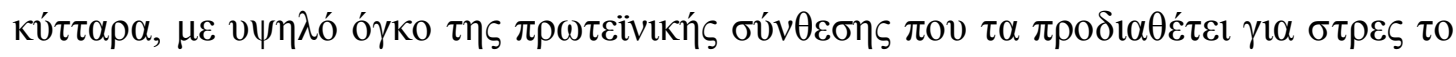

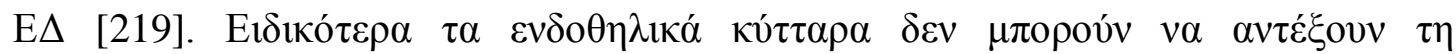

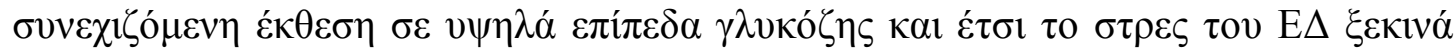

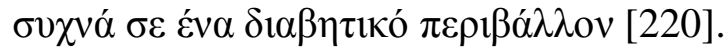

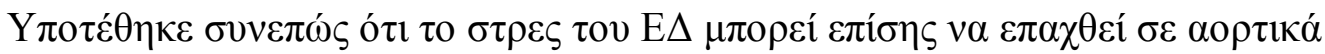

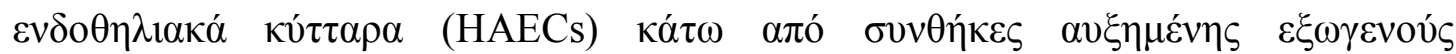

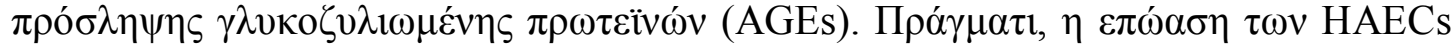

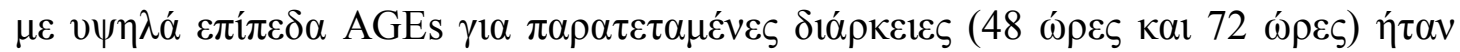

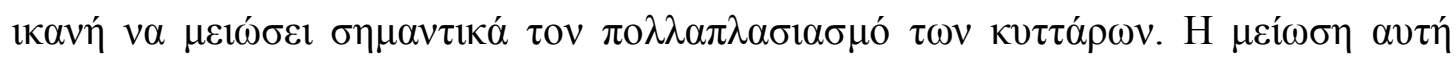

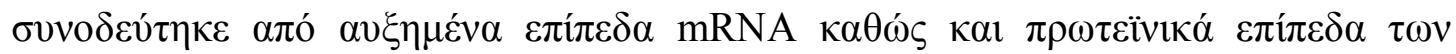

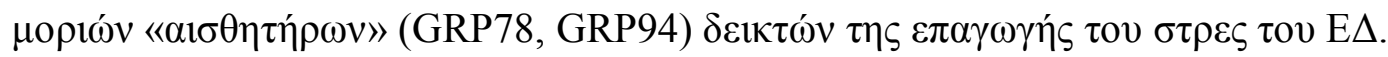

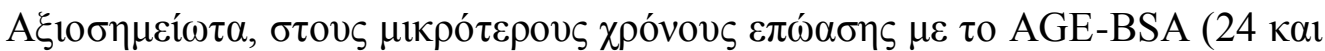

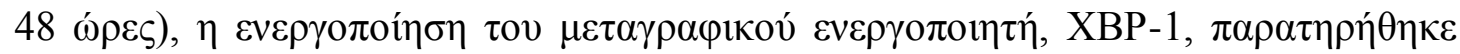

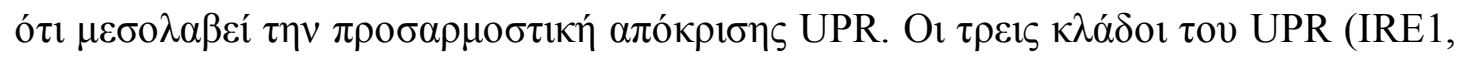

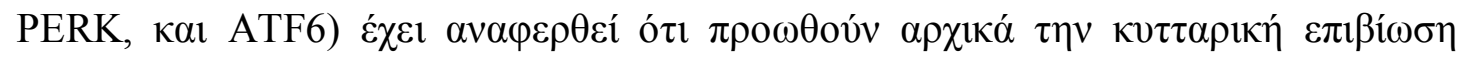

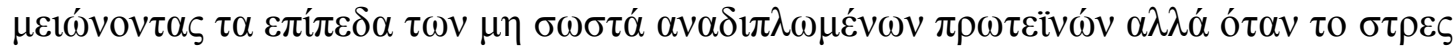

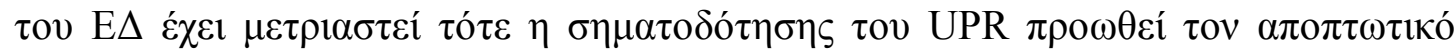

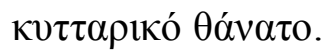

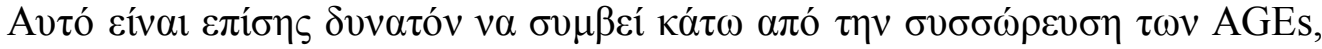

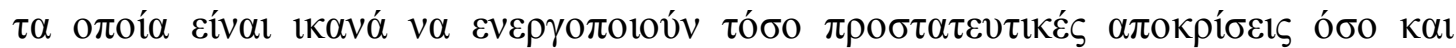

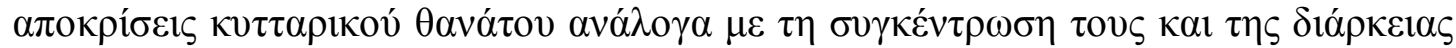

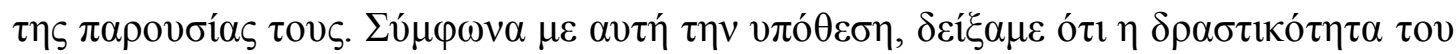

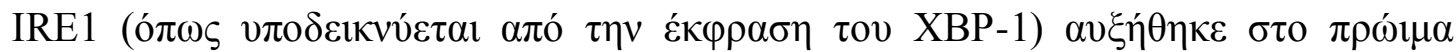

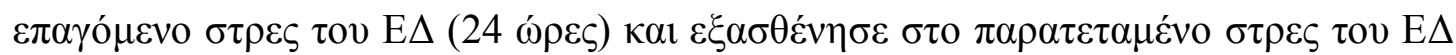

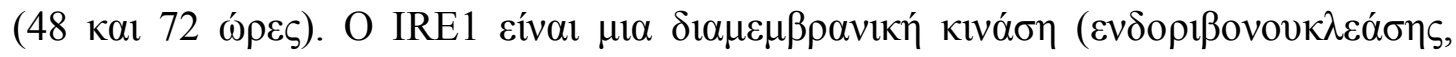

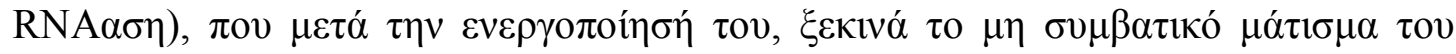

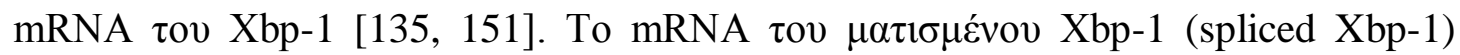

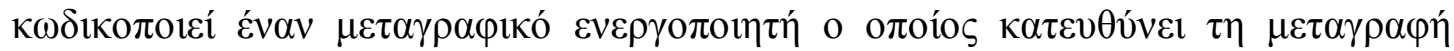




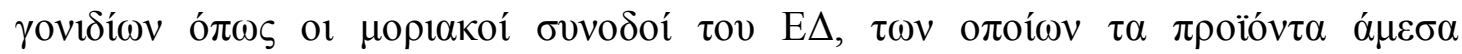

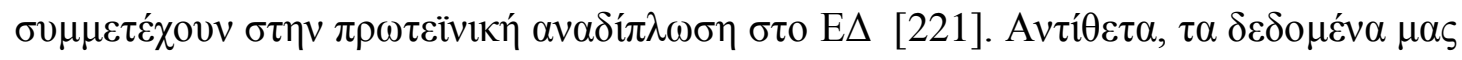

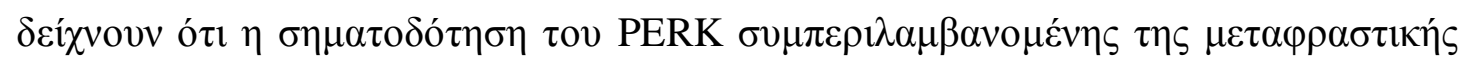

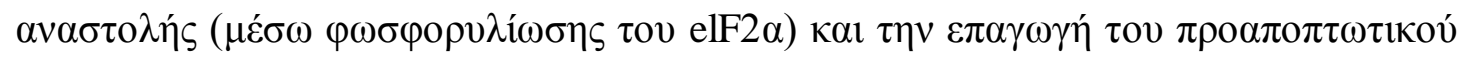

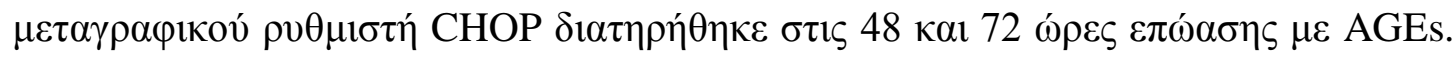

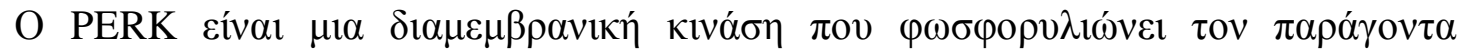

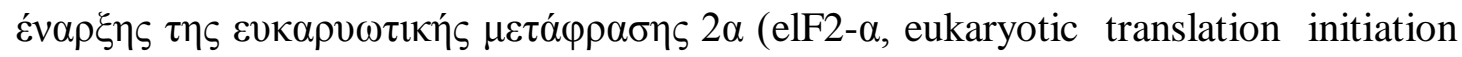

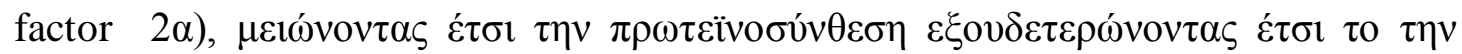

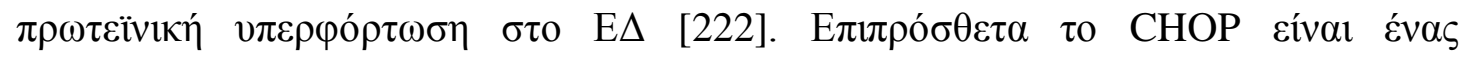

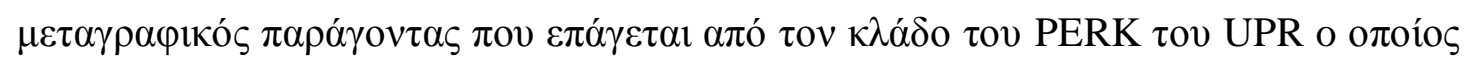

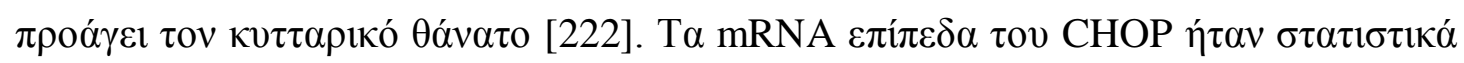

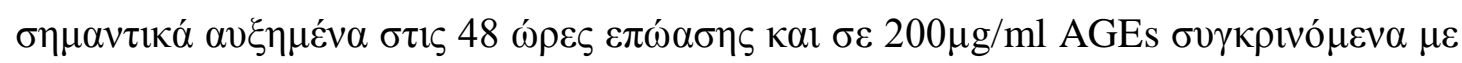

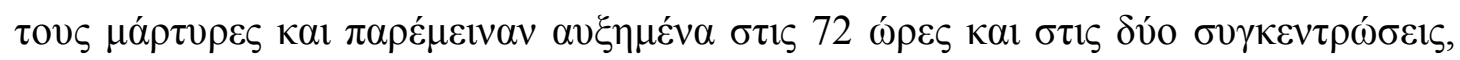

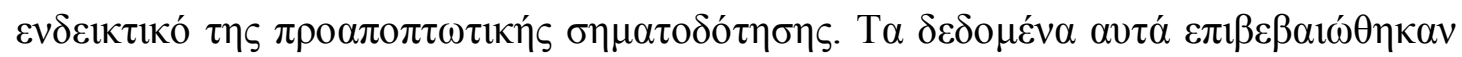

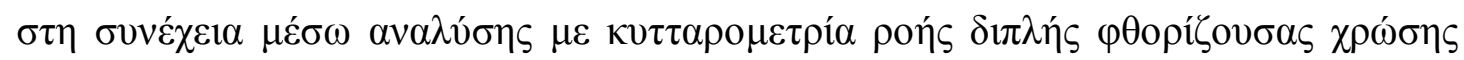

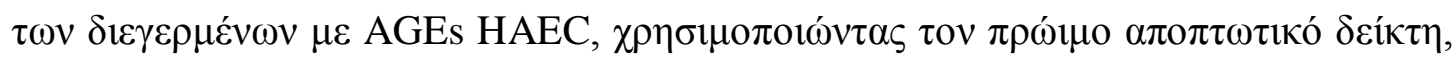

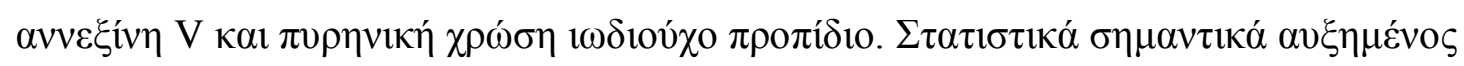

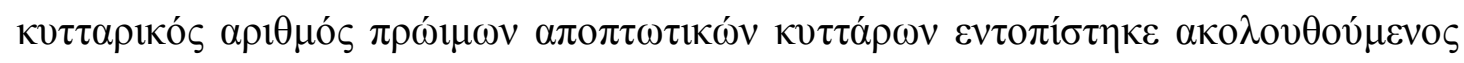

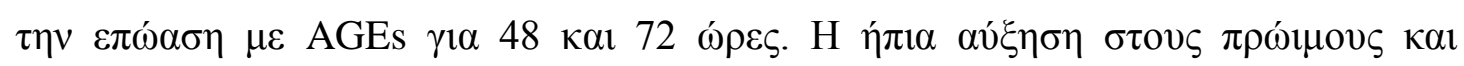

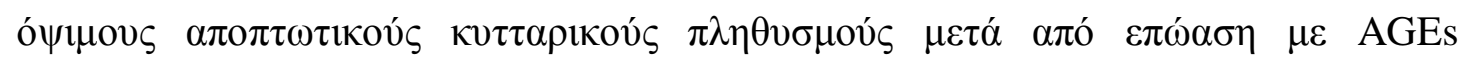

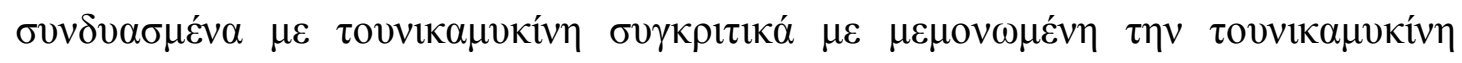

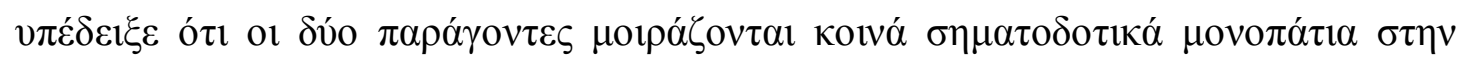

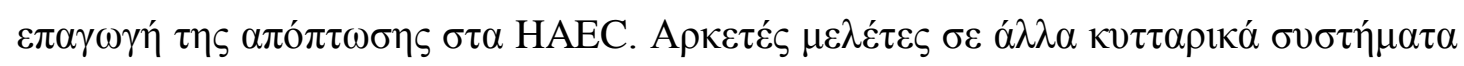

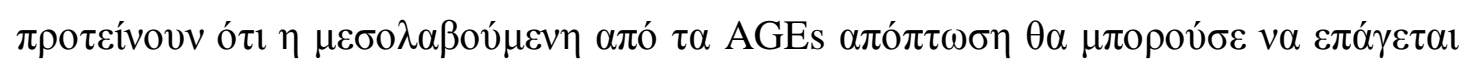

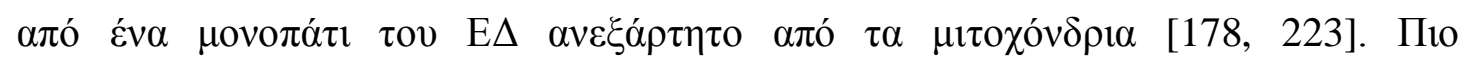

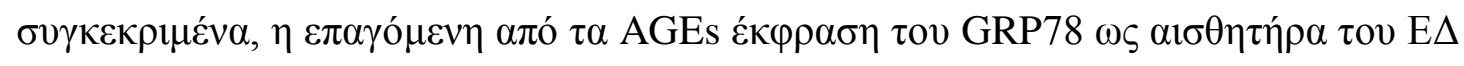

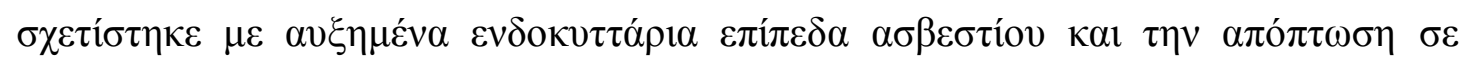

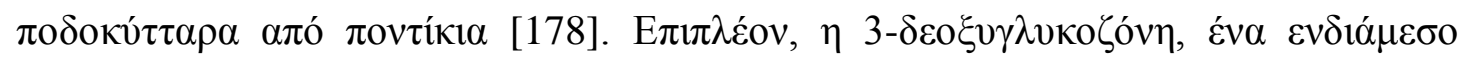

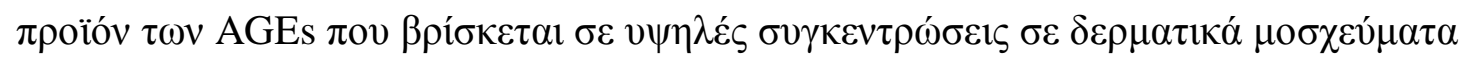

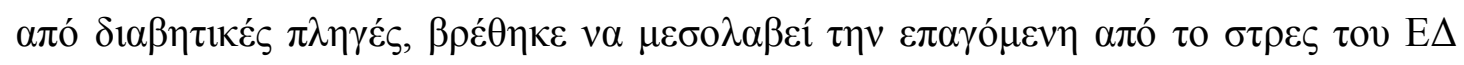

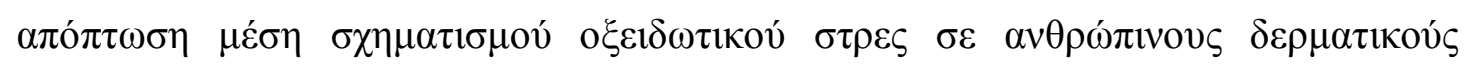

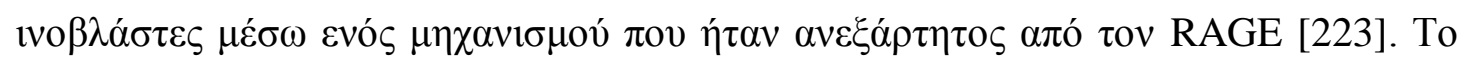

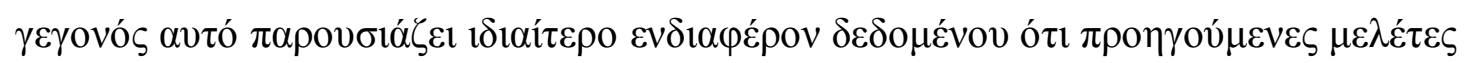




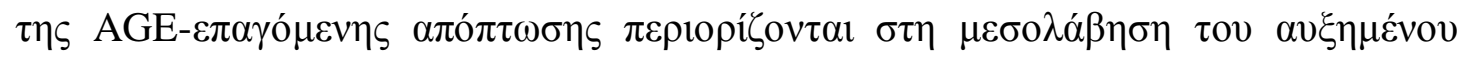

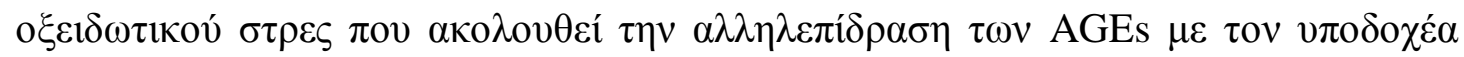

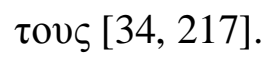

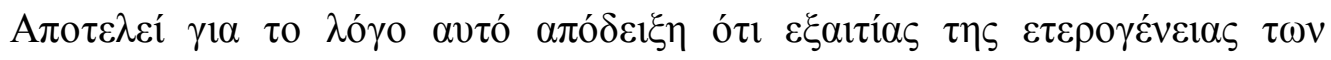

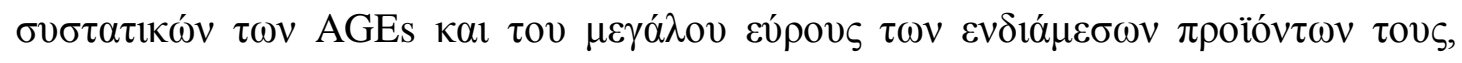

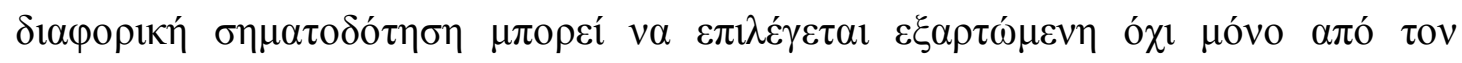

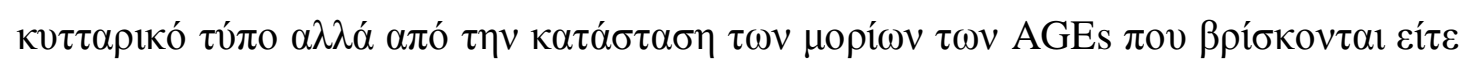

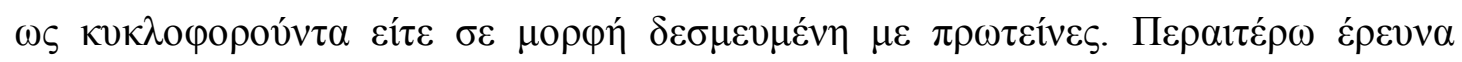

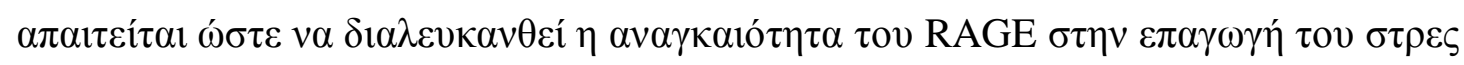

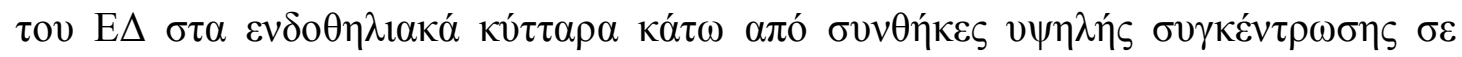
AGEs.

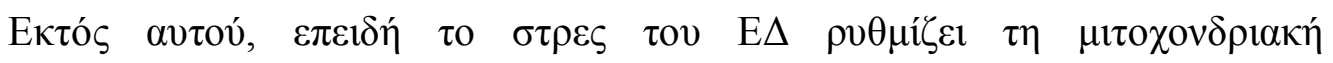

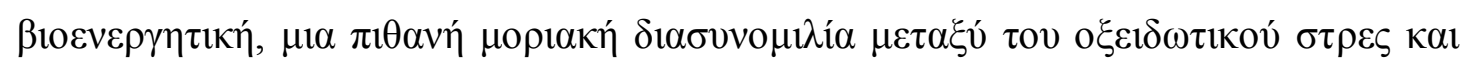

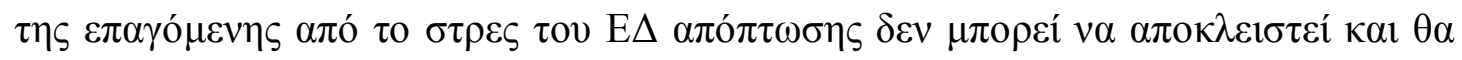

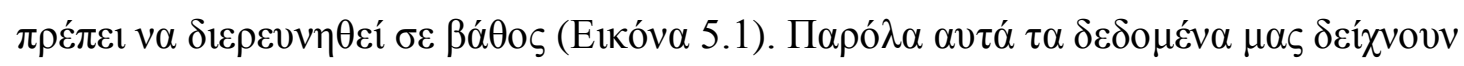

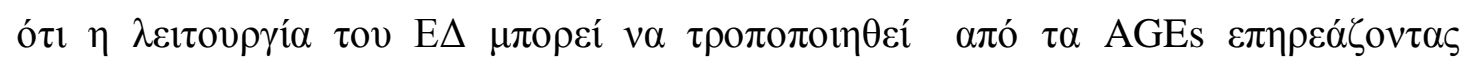

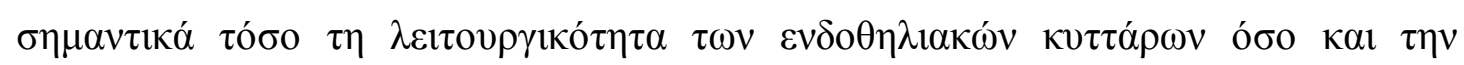

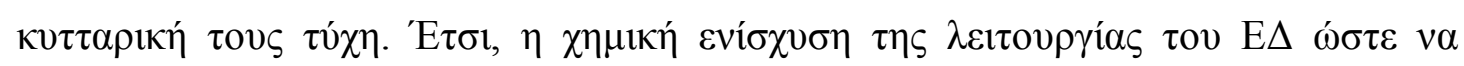

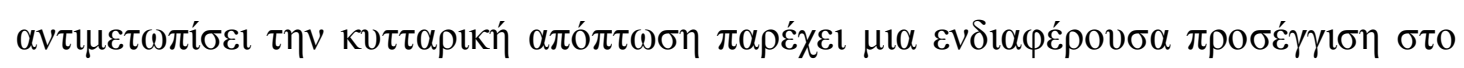

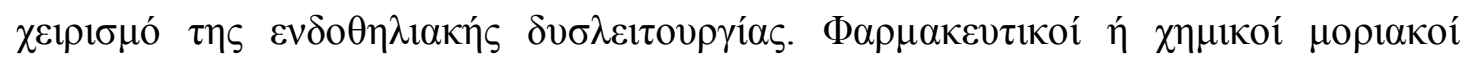

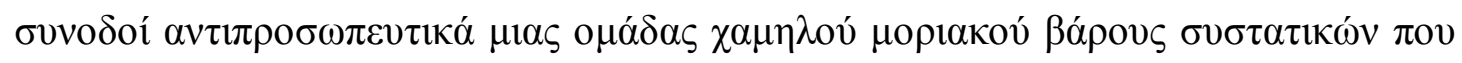

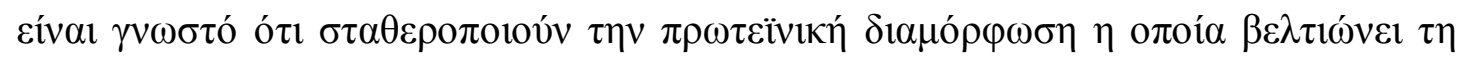

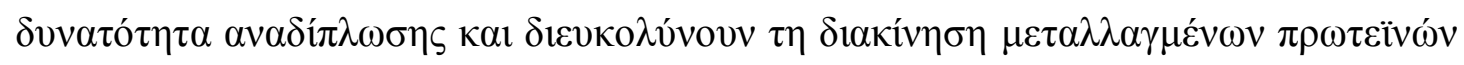
[166, 199]. H $\alpha \pi$

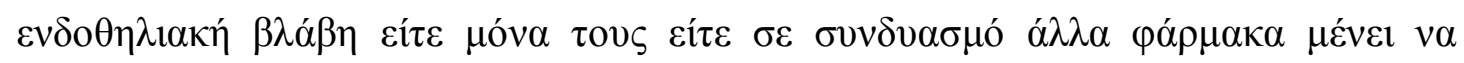
$\delta 1 \varepsilon \rho \varepsilon v \vee \eta \theta \varepsilon i ́$.

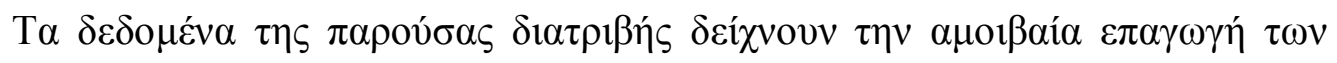

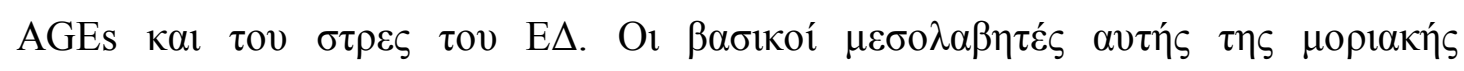

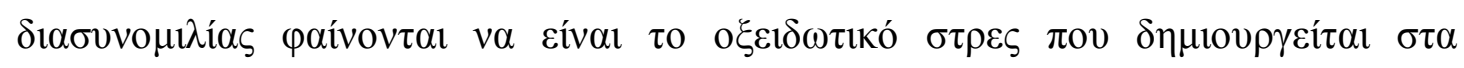

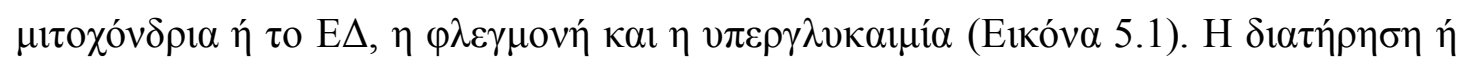

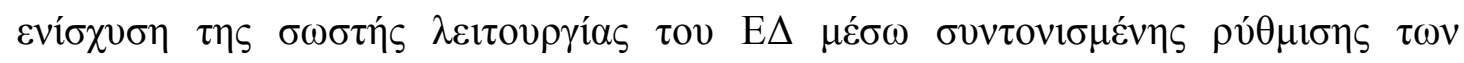




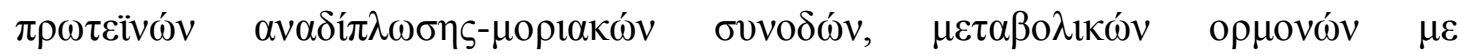

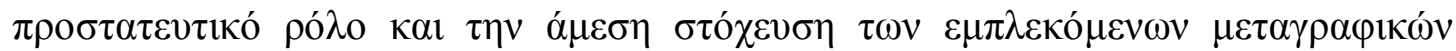

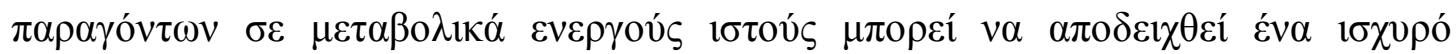

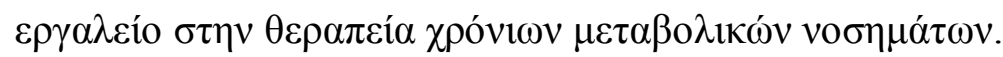

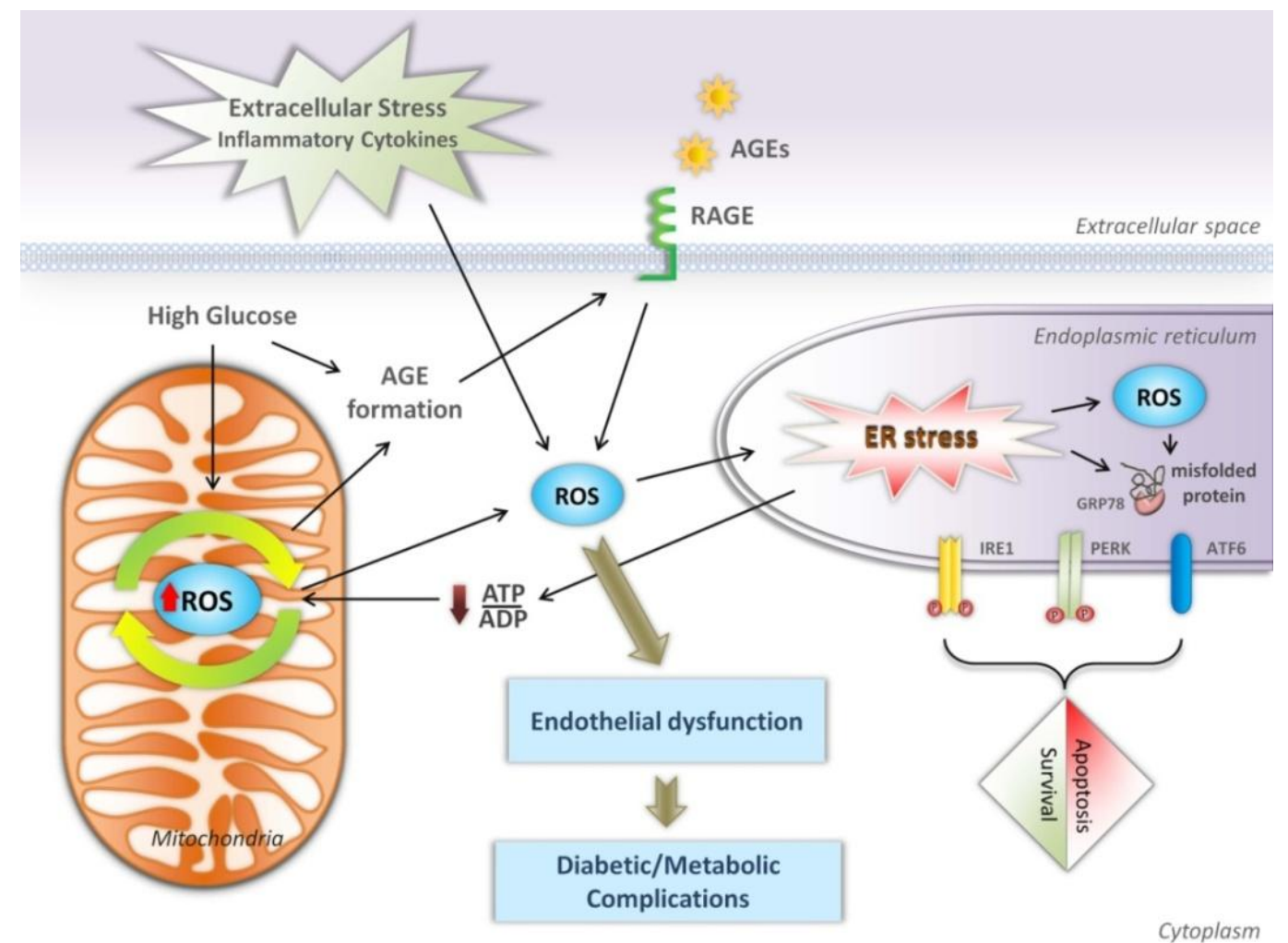

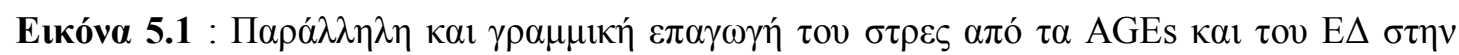

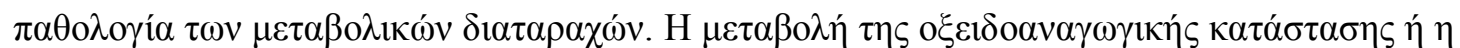

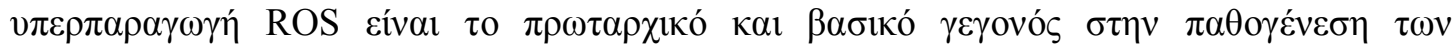

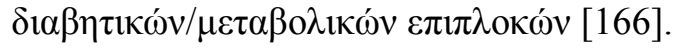




\section{6. ВIВАІОГРАФІА}

[1] Monnier, V. M. (2003) Intervention against the Maillard reaction in vivo. Arch Biochem Biophys, 419, 1-15.

[2] Ahmed, N. (2005) Advanced glycation endproducts--role in pathology of diabetic complications. Diabetes Res Clin Pract, 67, 3-21.

[3] Rahbar, S., Blumenfeld, O., \& Ranney, H. M. (1969) Studies of an unusual hemoglobin in patients with diabetes mellitus. Biochem Biophys Res Commun, 36, 838-843.

[4] Peyroux, J., \& Sternberg, M. (2006) Advanced glycation endproducts (AGEs): Pharmacological inhibition in diabetes. Pathol Biol (Paris), 54, 405-419.

[5] Basta, G., Schmidt, A. M., \& De Caterina, R. (2004) Advanced glycation end products and vascular inflammation: implications for accelerated atherosclerosis in diabetes. Cardiovasc Res, 63, 582-592.

[6] Huebschmann, A. G., Regensteiner, J. G., Vlassara, H., \& Reusch, J. E. (2006) Diabetes and advanced glycoxidation end products. Diabetes Care, 29, 1420-1432.

[7] Ramasamy R, Yan S. F., \& Schmidt A. M. (2005) The RAGE axis and endothelial dysfunction: maladaptive roles in the diabetic vasculature and beyond. Trends Cardiovasc Med, 15, 237-243.

[8] Januszewski A.S., Alderson N.L., Metz T.O., Thorpe S.R., Baynes J.W. (2003) Role of lipids in chemical modification of proteins and development of complications in diabetes Biochem Soc Trans, 31,1413-1416.

[9] Anderson MM, Requena JR, Crowley JR, Thorpe SR, Heinecke JW. (1999), The myeloperoxidase system of human phagocytes generates Nepsilon-(carboxymethyl)lysine on proteins: a mechanism for producing advanced glycation end products at sites of inflammation J Clin Invest, 104, 103-113.

[10] Anderson M.M., Heinecke J.W. (2003) Production of N(epsilon)-(carboxymethyl)lysine is impaired in mice deficient in NADPH oxidase: a role for phagocyte-derived oxidants in the formation of advanced glycation end products during inflammation Diabetes, 52,2137-2143.

[11] Sebekova K., Somoza V. (2007) Dietary advanced glycation endproducts (AGEs) and their health effects, PRO Mol Nutr Food Res, 51, 1079-1084.

[12] Meerwaldt, R., Links, T., Zeebregts, C., Tio, R., Hillebrands, J. L., \& Smit, A. (2008) The clinical relevance of assessing advanced glycation endproducts accumulation in diabetes. Cardiovasc Diabetol, 7, 29.

[13] Yan, S. F., Ramasamy, R., \& Schmidt, A. M. (2010) Soluble RAGE: therapy and biomarker in unraveling the RAGE axis in chronic disease and aging. Biochem Pharmacol, 79, 1379-1386.

[14] Kalea, A. Z., Schmidt, A. M., \& Hudson, B. I. (2009) RAGE: a novel biological and genetic marker for vascular disease. Clin Sci (Lond), 116, 621-637.

[15] Malherbe, P., Richards, J. G., Gaillard, H., Thompson, A., Diener, C., Schuler, A., et al. (1999) cDNA cloning of a novel secreted isoform of the human receptor for advanced glycation end products and characterization of cells co-expressing cell-surface scavenger receptors and Swedish mutant amyloid precursor protein. Brain Res Mol Brain Res, 71, 159170. 
[16] Yonekura, H., Yamamoto, Y., Sakurai, S., Petrova, R. G., Abedin, M. J., Li, H., et al. (2003) Novel splice variants of the receptor for advanced glycation end-products expressed in human vascular endothelial cells and pericytes, and their putative roles in diabetes-induced vascular injury. Biochem J, 370, 1097-1109.

[17] Schlueter, C., Hauke, S., Flohr, A. M., Rogalla, P., \& Bullerdiek, J. (2003) Tissuespecific expression patterns of the RAGE receptor and its soluble forms--a result of regulated alternative splicing? Biochim Biophys Acta, 1630, 1-6.

[18] Park, I. H., Yeon, S. I., Youn, J. H., Choi, J. E., Sasaki, N., Choi, I. H., et al. (2004) Expression of a novel secreted splice variant of the receptor for advanced glycation end products (RAGE) in human brain astrocytes and peripheral blood mononuclear cells. Mol Immunol, 40, 1203-1211.

[19] Bierhaus, A., Humpert, P. M., Morcos, M., Wendt, T., Chavakis, T., Arnold, B., et al. (2005) Understanding RAGE, the receptor for advanced glycation end products. J Mol Med (Berl), 83, 876-886.

[20] Ishihara, K., Tsutsumi, K., Kawane, S., Nakajima, M., \& Kasaoka, T. (2003) The receptor for advanced glycation end-products (RAGE) directly binds to ERK by a D-domainlike docking site. FEBS Lett, 550, 107-113.

[21] Hudson, B. I., Kalea, A. Z., Del Mar Arriero, M., Harja, E., Boulanger, E., D'Agati, V., et al. (2008) Interaction of the RAGE cytoplasmic domain with diaphanous-1 is required for ligand-stimulated cellular migration through activation of Rac1 and Cdc42. J Biol Chem, 283, 34457-34468.

[22] Dattilo, B. M., Fritz, G., Leclerc, E., Kooi, C. W., Heizmann, C. W., \& Chazin, W. J. (2007) The extracellular region of the receptor for advanced glycation end products is composed of two independent structural units. Biochemistry, 46, 6957-6970.

[23] Ostendorp, T., Leclerc, E., Galichet, A., Koch, M., Demling, N., Weigle, B., et al. (2007) Structural and functional insights into RAGE activation by multimeric S100B. EMBO J, 26, 3868-3878.

[24] Xie, J., Reverdatto, S., Frolov, A., Hoffmann, R., Burz, D. S., \& Shekhtman, A. (2008) Structural basis for pattern recognition by the receptor for advanced glycation end products (RAGE). J Biol Chem, 283, 27255-27269.

[25] Chaney, M. O., Stine, W. B., Kokjohn, T. A., Kuo, Y. M., Esh, C., Rahman, A., et al. (2005) RAGE and amyloid beta interactions: atomic force microscopy and molecular modeling. Biochim Biophys Acta, 1741, 199-205.

[26] Rojas A., Delgado-López F., González I., Pérez-Castro R., Romero J., Rojas I. (2013) The receptor for advanced glycation end-products: a complex signaling scenario for a promiscuous receptor, Cell Signal, 25, 609-614

[27] Mahajan N, Dhawan V. (2013) Receptor for advanced glycation end products (RAGE) in vascular and inflammatory diseases, Int J Cardiol, May 27. doi:pii: S0167-5273(13)00922-4.

[28] Schmidt A.M., Vianna M., Gerlach M., Brett J., Ryan J., Kao J., et al. (1992) Isolation and characterization of two binding proteins for advanced glycosylation end products from bovine lung which are present on th endothelial cell surface. J Biol Chem, 14987-14997.

[29] Riehl A, Németh J, Angel P, Hess J. (2009) The receptor RAGE: Bridging inflammation and cancer. Cell Commun Signal, 7,12. 
[30] Li J., Schmidt A.M. (1997) Characterization and functional analysis of the promoter of RAGE, the receptor for advanced glycation end products. J Biol Chem, 272,16498-16506.

[31] Brett J, Schmidt AM, Yan SD, Zou YS, Weidman E, Pinsky D, et al. (1993) Survey of the distribution of a newly characterized receptor for advanced glycation end products in tissues. Am J Pathol, 143, 1699-1712.

[32] Sakaguchi M, Murata H, Yamamoto K, Ono T, Sakaguchi Y, Motoyama A, et al. (2011) TIRAP, an adaptor protein for TLR2/4, transduces a signal from RAGE phosphorylated upon ligand binding. PLoS One, 6:e23132.

[33] Hu P, Lai D, Lu P, Gao J, He H. (2012) ERK and Akt signaling pathways are involved in advanced glycation end product-induced autophagy in rat vascular smooth muscle cells. Int $\mathbf{J}$ Mol Med, 29,613-618.

[34] Xiang Y, Li Q, Li M, Wang W, Cui C, Zhang J. (2011) Ghrelin inhibits AGEs-induced apoptosis in human endothelial cells involving ERK1/2 and PI3K/Akt pathways. Cell Biochem Funct, 29, 149-155.

[35] Liu Y, Liang C, Liu X, Liao B, Pan X, Ren Y, et al.(2010) AGEs increased migration and inflammatory responses of adventitial fibroblasts via RAGE, MAPK and NF-kappaB pathways. Atherosclerosis, 208, 34-42.

[36] Alikhani M, Maclellan CM, Raptis M, Vora S, Trackman PC, Graves DT. (2007) Advanced glycation end products induce apoptosis in fibroblasts through activation of ROS, MAP kinases, and the FOXO1 transcription factor. Am J Physiol Cell Physiol, 292, C850C856.

[37] Yamagishi S, Inagaki $\mathrm{Y}$, Okamoto $\mathrm{T}$, Amano S, Koga K, Takeuchi M, et al. (2002) Advanced glycation end products induced apoptosis and overexpression of vascular endothelial growth factor in bovine retinal pericytes. Biochem Biophys Res Commun ;290:973-978.

[38] Fukami K, Ueda S, Yamagishi S, Kato S, Inagaki Y, Takeuchi M, et al. (2004) AGEs activate mesangial TGF-beta-Smad signaling via an angiotensin II type I receptor interaction. Kidney Int.;66:2137-2147.

[39] Cai W, Ramdas M, Zhu L, Chen X, Striker GE, Vlassara H. (2012) Oral advanced glycation end products (AGEs) promote insulin resistance and diabetes by depleting the antioxidant defenses AGE receptor-1 and sirtuin 1. Proc Natl Acad Sci USA;109:15888-15893.

[40] Molinari J, Ruszova E, Velebny V, Robert L. (2008) Effect of advanced glycation endproducts on gene expression profiles of human dermal fibroblasts. Biogerontology;9:177-182.

[41] Bansal S, Siddarth M, Chawla D, Banerjee BD, Madhu SV, Tripathi AK. (2012) Advanced glycation end products enhance reactive oxygen and nitrogen species generation in neutrophils in vitro. Mol Cell Biochem;361:289-296.

[42] Nedić O, Rattan SI, Grune T, Trougakos IP. (2013) Molecular effects of advanced glycation end products on cell signalling pathways, ageing and pathophysiology.Free Radic Res. Aug;47 Suppl 1:28-38. doi: 10.3109/10715762.2013.806798. 
[43] Wautier MP, Chappey O, Corda S, Stern DM, Schmidt AM, Wautier JL. (2001).Activation of NADPH oxidase by AGE links oxidant stress to altered gene expression via RAGE. Am J Physiol Endocrinol Metab;280:E685-E694.

[44] Coughlan MT, Thorburn DR, Penfold SA, Laskowski A, Harcourt BE, Sourris $\mathrm{KC}$, et al. (2009) RAGE-induced cytosolic ROS promote mitochondrial superoxide generation in diabetes. J Am Soc Nephrol;20:7425-752.

[45] Cai W, He JC, Zhu L, Lu C, Vlassara H. (2006) Advanced glycation end product (AGE) receptor 1 suppresses cell oxidant stress and activation signaling via EGF receptor. Proc Natl Acad Sci USA;103:13801-13806.

[46] Cai W, He JC, Zhu L, Chen X, Striker GE, Vlassara H. (2008) AGE-receptor-1 counteracts cellular oxidant stress induced by AGEs via negative regulation of p66 shc -dependent FKHRL1 phosphorylation. Am J Physiol Cell Physiol;294:C145-C152.

[47] Nagai R, Mera K, Nakajou K, Fujiwara Y, Iwao Y, Imai H, et al. (2007) The ligand activity of AGE-proteins to scavenger receptors is dependent on their rate of modification by AGEs. Biochim Biophys Acta;1772:1192-1198.

[48] Pluddemann A, Neyen C, Gordon S. (2007) Macrophage scavenger receptors and host-derived ligands. Methods;43:207-217.

[49] Saito A, Takeda T, Hama H, Oyama Y, Hosaka H, Tanuma A, et al. (2005) Role of megalin, a proximal tubular endocytic receptor, in the pathogenesis of diabetic and metabolic syndrome-related nephopathies: Protein metabolic overload hypothesis. Nephrology 10:S26-S31.

[50] Rojas A, Delgado-Lopez F, Gonzalez I, Perez-Castro R, Romero J, Rojas (2013) The receptor for advanced glycation end-products: A complex signaling scenario for a promiscuous receptor. Cell Signall, 25, 609-614.

[51] Basta G, Schmidt AM, De Caterina R. (2004) Advanced glycation end products and vascular inflammation: implications for accelerated atherosclerosis in diabetes. Cardiovasc Res. Sep 1;63(4):582-92.

[52] Schmidt AM, Yan SD, Wautier JL, Stern DM. (1999) Activation of receptorfor advanced glycation end products - a mechanism for chronic vas-cular dysfunction in diabetic vasculopathy and atherosclerosis. Circ Res, 84:489-97.

[53] Junttila MR, Li SP, Westermarck J (2008) Phosphatase-mediated crosstalk between MAPK signaling pathways in the regulation of cell survival. FASEB J. 22(4), 954-65.

[54] Murphy LO, Blenis J (2006) MAPK signal specificity: the right place at the right time. Trends Biochem. Sci. 31(5), 268-75.

[55] Pimienta G, Pascual J (2007) Canonical and alternative MAPK signaling. Cell Cycle 6(21), 2628-32.

[56] Raman M, Chen W, Cobb MH (2007) Differential regulation and properties of MAPKs. Oncogene 26(22), 3100-12.

[57] Turjanski AG, Vaqué JP, Gutkind JS (2007) MAP kinases and the control of nuclear events. Oncogene 26(22), 3240-53.

[58] Zhang Y, Dong C (2007) Regulatory mechanisms of mitogen-activated kinase signaling. Cell. Mol. Life Sci. 64(21), 2771-89. 
[59] Kate L. Jeffrey, Montserrat Camps, Christian Rommel \& Charles R. Mackay (2007) Targeting dual-specificity phosphatases: manipulating MAP kinase signalling and immune responsesNature Reviews Drug Discovery 6, 391-403

[60] Diamanti-Kandarakis E, Piperi C, Patsouris E, Korkolopoulou P, Panidis D, Pawelczyk L, Papavassiliou AG, Duleba AJ. (2007) Immunohistochemical localization of advanced glycation end-products (AGEs) and their receptor (RAGE) in polycystic and normal ovaries. Histochem Cell Biol,. 127(6): p. 581-9.

[61] Diamanti-Kandarakis, E., Piperi C, Korkolopoulou P, Kandaraki E, Levidou G, Papalois A, Patsouris E, Papavassiliou AG., (2007). Accumulation of dietary glycotoxins in the reproductive system of normal female rats. J Mol Med (Berl), 85, p. 1413-20.

[62] Papachroni KK, Piperi C, Levidou G, Korkolopoulou P, Pawelczyk L, DiamantiKandarakis E, Papavassiliou AG. (2010) Lysyl oxidase interacts with AGE signalling to modulate collagen synthesis in polycystic ovarian tissue.J Cell Mol Med. 14:2460-9.

[63] Diamanti-Kandarakis E, Piperi C, Creatsas G, Kalofoutis A (2005) Increased levels of Advanced glycation end products in women with polycystic ovary syndrome Clin Endocrinol $62,37-43$

[64] Diamanti-Kandarakis, E. and A. Dunaif, (1996) New perspectives in polycystic ovary syndrome. Trends Endocrinol Metab,. 7, 267-71.

[65] Diamanti-Kandarakis, E., et al., (1999) A survey of the polycystic ovary syndrome in the Greek island of Lesbos: hormonal and metabolic profile. J Clin Endocrinol Metab,. 84, 40064011.

[66] Clement F, Monniaux D, Thalabard JC, Claude D. (2002) Contribution of a mathematical modelling approach to the understanding of the ovarian function. C R Biol.; 325: 473-85

[67] Aronson D. (2003) Cross-linking of glycated collagen in the pathogenesis of arterial and myocardial stiffening of aging and diabetes. J Hyperten.; 21: 3-12

[68] Facchiano F, Lentini A, Fogliano V, et al. (2002) Sugar-induced modification of fibroblast growth factor 2 reduces its angiogenic activity in vivo. Am J Pathol.; 161: 531-41

[69] Verzijl N, Degroot J, Ben ZC, et al. (2002) Crosslinking by advanced glycation end products increases the stiffness of the collagen network in human articular cartilage: a possible mechanism through which age is a risk factor for osteoarthritis. Arthr Rheumatism.; 46: $114-23$

[70] Abe H, Matsubara T, Iehara N, et al. Type IV collagen is transcriptionally regulated by Smad1 under advanced glycation end product (AGE) stimulation. J Biol Chem. 2004; 279: 14201-6

[71] Auersperg N, Maines-bandiera SL, Dyck HG, Kruk PA. Characterization of cultured human ovarian surface epithelial cells: phenotypic plasticity and premalignant changes. Lab Invest. 1994; 71: 510-8

[72] Kruk PA, Auersperg N. (1994) A line of rat ovarian surface epithelium provides a continuous source of complex extracellular matrix. In Vitro Cell Dev Biol Anim. 30A: 217-25

[73] Woessner JF, JR. Catabolism of collagen and non-collagen protein in the rat uterus during post-partum involution. Biochem J. 1962; 83: 304-14 
[74] Royce PM, Camakaris J, Danks DM. (1980) Reduced lysyl oxidase activity in skin fibroblasts from patients with Menkes' syndrome. Biochem J.; 192: 579-86

[75] Kuivaniemi H, Peltonen L, Kivirikko KI. (1985) Type IX Ehlers-Danlos syndrome and Menkes syndrome: the decrease in lysyl oxidase activity is associated with a corresponding deficiency in the enzyme protein. Am J Hum Gen.; 37, 798-808

[76] Murawaki Y, Kusakabe Y, Hirayama C. (1991) Serum lysyl oxidase activity in chronic liver disease in comparison with serum levels of prolyl hydroxylase and laminin. Hepatology.; 14: 1167-73

[77] Riley DJ, Kerr JS, Berg RA, et al. (1982) Beta-Aminopropionitrile prevents bleomycininduced pulmonary fibrosis in the hamster. Am Rev Resp Dis.; 125: 67-73

[78] Di donato A, Ghiggeri GM, Di duca M, et al. (1997) Lysyl oxidase expression and collagen cross-linking during chronic adriamycin nephropathy. Nephron.; 76: 192-200

[79] Trivedy C, WArnakulasuriya KA, Hazarey VK, et al. (1999) The upregulation of lysyl oxidase in oral submucous fibrosis and squamous cell carcinoma. J Oral Pathol Med.; 28: 246-51

[80] Sommer P, Gleyzal C, Raccurt M, et al. (1993) Transient expression of lysyl oxidase by liver myofibroblasts in murine schistosomiasis. Lab Invest.; 69: 460-70

[81] Jourdan-Le Saux C, Gleyzal C, Garnier JM, et al. (1994) Lysyl oxidase cDNA of myofibroblast from mouse fibrotic liver. Biochem Biophys Res Commun.; 199: 587-92

[82] Decitre M, Gleyzal C, Raccurt M, et al. (1998) Lysyl oxidase-like protein localizes to sites of de novo fibrinogenesis in fibrosis and in the early stromal reaction of ductal breast carcinomas. Lab Invest.; 78: 143-51

[83] Chanoki M, Ishii M, Kobayashi H, et al. (1995) Increased expression of lysyl oxidase in skin with scleroderma. British J Dermatol.; 133: 710-5

[84] Himeno N. (1986) Effect of prostaglandins on collagen synthesis in rabbit ovarian follicles during the ovulatory process. Nippon Naibunpi Gakkai zasshi.; 62: 1181-1193

[85] Slee RB, Hillier SG, Largue P, et al. (2001) Differentiation-dependent expression of connective tissue growth factor and lysyl oxidase messenger ribonucleic acids in rat granulosa cells. Endocrinology.; 142: 1082-9

[86] Kendall NR, Marsters P, Scaramuzzi RJ, Campbell BK (2003). Expression of lysyl oxidase and effect of copper chloride and ammonium tetrathiomolybdate on bovine ovarian follicle granulosa cells cultured in serum-free media. Reproduction.; 125: 657-65

[87] Harlow CR, Rae M, Davidson L, et al. (2003) Lysyl oxidase gene expression and enzyme activity in the rat ovary: regulation by follicle-stimulating hormone, androgen, and transforming growth factor-beta superfamily members in vitro. Endocrinology.; 144: 154-62

[88] Henmi H, Endo T, Nagasawa K, et al. (2001) Lysyl Oxidase and MMP-2 expression in Dehydroepiandrosterone-induced Polycystic Ovary in rats. Biol Reproduct.; 64: 157-62

[89] Christakou, C., Christakou C, Economou F, Livadas S, Piperi C, Adamopoulos C, Marinakis E, Jdiamanti-Kandarakis E.., Strong and positive association of endothelin-1 with AGEs in PCOS: a causal relationship or a bystander? Hormones (Athens). 10(4): p. 292-7. 
[90] Kaya, C., Kaya C, Erkan AF, Cengiz SD, Dünder I, Demirel OE, Bilgihan A. (2009) Advanced oxidation protein products are increased in women with polycystic ovary syndrome: relationship with traditional and nontraditional cardiovascular risk factors in patients with polycystic ovary syndrome. Fertil Steril,. 92(4): p. 1372-7.

[91] Tatone, C. and F. Amicarelli, (2013) The aging ovary-the poor granulosa cells. Fertil Steril. 99(1): p. 12-7.

[92] Wautier JL, Wautier MP, Schmidt AM, et al. (1994) Advanced glycation end products (AGEs) on the surface of diabetic erythrocytes bind to the vessel wall via a specific receptor inducing oxidant stress in the vasculature: a link between surface-associated AGEs and diabetic complications. Proc Natl Acad Sci USA.;91:7742-6.

[93] Schmidt AM, Hori O, Chen JX, et al. (1995) Advanced glycation end products interacting with their endothelial receptor induce expression of vascular cell adhesion molecule-1 (VCAM-1) in cultured human endothelial cells and in mice. A potential mechanism for the accelerated vasculopathy of diabetes. J Clin Invest.;96:1395-403.

[94] Onat D, Brillon D, Colombo PC, Schmidt AM. (2011) Human vascular endothelial cells: a model system for studying vascular inflammation in diabetes and atherosclerosis. Curr Diab Rep. Jun;11(3):193-202.

[95] Zhou, Z., Wang, K., Penn, M. S., Marso, S. P., Lauer, M. A., Forudi, F., et al. (2003) Receptor for AGE (RAGE) mediates neointimal formation in response to arterial injury. Circulation, 107, 2238-2243.

[96] Morigi M, Angioletti S, Imberti B, Donadelli R, Micheletti G, Figliuzzi M, Remuzzi A, Zoja C, Remuzzi G. (1998) Leukocyte-endothelial interaction is augmented by high glucose concentrations and hyperglycemia in a NF-kB-dependent fashion. J Clin Invest.; 101: 19051915. Medline

[97] Edelstein D, Brownlee M. (1992) Mechanistic studies of advanced glycosylation end product inhibition by aminoguanidine. Diabetes.; 41: 26-29.

[98] Bucala, R., Tracey, K. J., \& Cerami, A. (1991) Advanced glycosylation products quench nitric oxide and mediate defective endothelium-dependent vasodilatation in experimental diabetes. J Clin Invest, 87, 432-438.

[99] Aronson, D., \& Rayfield, E. J. (2002) How hyperglycemia promotes atherosclerosis: molecular mechanisms. Cardiovasc Diabetol, 1,1 .

[100] Zhang, J., Ren, S., Sun, D., \& Shen, G. X. (1998) Influence of glycation on LDLinduced generation of fibrinolytic regulators in vascular endothelial cells. Arterioscler Thromb Vasc Biol, 18, 1140-1148.

[101] Hedrick, C. C., Thorpe, S. R., Fu, M. X., Harper, C. M., Yoo, J., Kim, S. M., et al. (2000) Glycation impairs high-density lipoprotein function. Diabetologia, 43, 312-320.

[102] Kume, S., Takeya, M., Mori, T., Araki, N., Suzuki, H., Horiuchi, S., et al. (1995) Immunohistochemical and ultrastructural detection of advanced glycation end products in atherosclerotic lesions of human aorta with a novel specific monoclonal antibody. Am J Pathol, 147, 654-667.

[103] Schleicher, E. D., Wagner, E., \& Nerlich, A. G. (1997) Increased accumulation of the glycoxidation product $\mathrm{N}$ (epsilon)-(carboxymethyl)lysine in human tissues in diabetes and aging. J Clin Invest, 99, 457-468. 
[104] Tan, K. C., Chow, W. S., Ai, V. H., Metz, C., Bucala, R., \& Lam, K. S. (2002) Advanced glycation end products and endothelial dysfunction in type 2 diabetes. Diabetes Care, 25, 1055-1059.

[105] Zieman, S. J., Melenovsky, V., \& Kass D. A. (2005) Mechanisms, pathophysiology, and therapy of arterial stiffness. Arterioscler Thromb Vasc Biol, 25, 932-943.

[106] Yanagisawa, M., Kurihara, H., Kimura, S., Tomobe, Y., Kobayashi, M., Mitsui, Y., et al. (1988) A novel potent vasoconstrictor peptide produced by vascular endothelial cells. Nature, 332, 411-415.

[107] Stow, L. R., Jacobs, M. E., Wingo, C. S., \& Cain, B. D. (2011) Endothelin-1 gene regulation. FASEB J, 25, 16-28.

[108] Abman, S. H. (2009) Role of endothelin receptor antagonists in the treatment of pulmonary arterial hypertension. Annu Rev Med, 60, 13-23.

[109] Barton, M. (2008) Reversal of proteinuric renal disease and the emerging role of endothelin. Nat Clin Pract Nephrol, 4, 490-501.

[110] Yanagisawa, M., \& Masaki, T. (1989) Molecular biology and biochemistry of the endothelins. Trends Pharmacol Sci, 10, 374-378.

[111] Davenport, A. P. (2002) International Union of Pharmacology. XXIX. Update on endothelin receptor nomenclature. Pharmacol Rev, 54, 219-226.

[112] Fabbrini, M. S., Valsasina, B., Nitti, G., Benatti, L., \& Vitale, A. (1991) The signal peptide of human preproendothelin-1. FEBS Lett, 286, 91-94.

[113] Harrison, V. J., Barnes, K., Turner, A. J., Wood, E., Corder, R., \& Vane, J. R. (1995) Identification of endothelin 1 and big endothelin 1 in secretory vesicles isolated from bovine aortic endothelial cells. Proc Natl Acad Sci U S A, 92, 6344-6348.

[114] Doi, Y., Kudo, H., Nishino, T., Yamamoto, O., Nagata, T., Nara, S., et al. (2002) Enhanced expression of endothelin-1 and endothelin-converting enzyme-1 in acute hypoxic rat aorta. Histol Histopathol, 17, 97-105.

[115] Lowenstein, C. J., Morrell, C. N., \& Yamakuchi, M. (2005) Regulation of WeibelPalade body exocytosis. Trends Cardiovasc Med, 15, 302-308.

[116] Doi, Y., Kudo, H., Nishino, T., Nagata, T., \& Fujimoto, S. (2004) The enhancement of preproendothelin-1 synthesis and the acceleration of endothelin-1 processing in the acute hypoxic rat aorta. J Cardiovasc Pharmacol, 44 Suppl 1, S207-210.

[117] Popowski, K., Sperker, B., Kroemer, H. K., John, U., Laule, M., Stangl, K., et al. (2003) Functional significance of a hereditary adenine insertion variant in the 5'-UTR of the endothelin-1 gene. Pharmacogenetics, 13, 445-451.

[118] Winker, R., Garland, E. M., Rudiger, H. W., Diedrich, A., Biaggioni, I., Ponocny, I., et al. (2005) Influence of an insertion variant in the 5'UTR of the endothelin-1 gene on orthostatic intolerance. Am J Med Sci, 330, 166-171.

[119] Delerive, P., Martin-Nizard, F., Chinetti, G., Trottein, F., Fruchart, J. C., Najib, J., et al. (1999) Peroxisome proliferator-activated receptor activators inhibit thrombin-induced endothelin-1 production in human vascular endothelial cells by inhibiting the activator protein-1 signaling pathway. Circ Res, 85, 394-402. 
[120] Geese, W. J., Achanzar, W., Rubin, C., Hariharan, N., Cheng, P., Tomlinson, L., et al. (2008) Genetic and gene expression studies implicate renin and endothelin-1 in edema caused by peroxisome proliferator-activated receptor gamma agonists. Pharmacogenet Genomics, 18, 903-910.

[121] Benatti, L., Bonecchi, L., Cozzi, L., \& Sarmientos, P. (1993) Two preproendothelin 1 mRNAs transcribed by alternative promoters. J Clin Invest, 91, 1149-1156.

[122] Lee, M. E., Bloch, K. D., Clifford, J. A., \& Quertermous, T. (1990) Functional analysis of the endothelin-1 gene promoter. Evidence for an endothelial cell-specific cis-acting sequence. J Biol Chem, 265, 10446-10450.

[123] Mantovani, R. (1998) A survey of 178 NF-Y binding CCAAT boxes. Nucleic Acids Res, 26, 1135-1143.

[124] Yamashita, K., Discher, D. J., Hu, J., Bishopric, N. H., \& Webster, K. A. (2001) Molecular regulation of the endothelin-1 gene by hypoxia. Contributions of hypoxia-inducible factor-1, activator protein-1, GATA-2, AND p300/CBP. J Biol Chem, 276, 12645-12653.

[125] Ellgaard, L., \& Helenius, A. (2003) Quality control in the endoplasmic reticulum. Nat Rev Mol Cell Biol, 4, 181-191.

[126] Hammond, C., \& Helenius, A. (1994) Quality control in the secretory pathway: retention of a misfolded viral membrane glycoprotein involves cycling between the ER, intermediate compartment, and Golgi apparatus. J Cell Biol, 126, 41-52

[127] Ma, Y., \& Hendershot, L. M. (2004) ER chaperone functions during normal and stress conditions. J Chem Neuroanat, 28, 51-65.

[128] Parodi, A. J. (2000) Protein glucosylation and its role in protein folding. Annu Rev Biochem, 69, 69-93.

[129] Hendershot, L. M. (2004) The ER function BiP is a master regulator of ER function. Mt Sinai J Med, 71, 289-297.

[130] Meunier, L., Usherwood, Y. K., Chung, K. T., \& Hendershot, L. M. (2002) A subset of chaperones and folding enzymes form multiprotein complexes in endoplasmic reticulum to bind nascent proteins. Mol Biol Cell, 13, 4456-4469.

[131] Yang, Y., \& Li, Z. (2005) Roles of heat shock protein gp96 in the ER quality control: redundant or unique function? Mol Cells, 20, 173-182.

[132] Hampton, R. Y. (2002) ER-associated degradation in protein quality control and cellular regulation. Curr Opin Cell Biol, 14, 476-482.

[133] Rasheva, V. I., \& Domingos, P. M. (2009) Cellular responses to endoplasmic reticulum stress and apoptosis. Apoptosis, 14, 996-1007.

[134] Cox, J. S., Shamu, C. E., \& Walter, P. (1993) Transcriptional induction of genes encoding endoplasmic reticulum resident proteins requires a transmembrane protein kinase. Cell, 73, 1197-1206.

[135] Yoshida, H., Matsui, T., Yamamoto, A., Okada, T., \& Mori, K. (2001) XBP1 mRNA is induced by ATF6 and spliced by IRE1 in response to ER stress to produce a highly active transcription factor. Cell, 107, 881-891. 
[136] Yoshida, H., Oku, M., Suzuki, M., \& Mori, K. (2006) pXBP1(U) encoded in XBP1 premRNA negatively regulates unfolded protein response activator pXBP1(S) in mammalian ER stress response. J Cell Biol, 172, 565-575.

[137] Ron, D., \& Walter, P. (2007) Signal integration in the endoplasmic reticulum unfolded protein response. Nat Rev Mol Cell Biol, 8, 519-529.

[138] Haze, K., Yoshida, H., Yanagi, H., Yura, T., \& Mori, K. (1999) Mammalian transcription factor ATF6 is synthesized as a transmembrane protein and activated by proteolysis in response to endoplasmic reticulum stress. Mol Biol Cell, 10, 3787-3799.

[139] Silverman, R. H., \& Williams, B. R. (1999) Translational control perks up. Nature, 397, 208-209, 211.

[140] Harding, H. P., Zhang, Y., \& Ron, D. (1999) Protein translation and folding are coupled by an endoplasmic-reticulum-resident kinase. Nature, 397, 271-274.

[141] Wek, R. C., \& Cavener, D. R. (2007) Translational control and the unfolded protein response. Antioxid Redox Signal, 9, 2357-2371.

[142] Harding, H. P., Zhang, Y., Zeng, H., Novoa, I., Lu, P. D., Calfon, M., et al. (2003) An integrated stress response regulates amino acid metabolism and resistance to oxidative stress. Mol Cell, 11, 619-633.

[143] Scheuner, D., Song, B., McEwen, E., Liu, C., Laybutt, R., Gillespie, P., et al. (2001) Translational control is required for the unfolded protein response and in vivo glucose homeostasis. Mol Cell, 7, 1165-1176.

[144] Vattem, K. M., \& Wek, R. C. (2004) Reinitiation involving upstream ORFs regulates ATF4 mRNA translation in mammalian cells. Proc Natl Acad Sci U S A, 101, 11269-11274.

[145] Jiang, H. Y., Wek, S. A., McGrath, B. C., Scheuner, D., Kaufman, R. J., Cavener, D. R., et al. (2003) Phosphorylation of the alpha subunit of eukaryotic initiation factor 2 is required for activation of NF-kappaB in response to diverse cellular stresses. Mol Cell Biol, $23,5651-5663$.

[146] Deng, J., Lu, P. D., Zhang, Y., Scheuner, D., Kaufman, R. J., Sonenberg, N., et al. (2004) Translational repression mediates activation of nuclear factor kappa B by phosphorylated translation initiation factor 2. Mol Cell Biol, 24, 10161-10168.

[147] Bertolotti, A., Zhang, Y., Hendershot, L. M., Harding, H. P., \& Ron, D. (2000) Dynamic interaction of BiP and ER stress transducers in the unfolded-protein response. Nat Cell Biol, 2, 326-332.

[148] Jousse, C., Oyadomari, S., Novoa, I., Lu, P., Zhang, Y., Harding, H. P., et al. (2003) Inhibition of a constitutive translation initiation factor 2alpha phosphatase, CReP, promotes survival of stressed cells. J Cell Biol, 163, 767-775.

[149] Brewer, J. W., Hendershot, L. M., Sherr, C. J., \& Diehl, J. A. (1999) Mammalian unfolded protein response inhibits cyclin D1 translation and cell-cycle progression. Proc Natl Acad Sci U S A, 96, 8505-8510.

[150] Brewer, J. W., \& Diehl, J. A. (2000) PERK mediates cell-cycle exit during the mammalian unfolded protein response. Proc Natl Acad Sci U S A, 97, 12625-12630. 
[151] Calfon, M., Zeng, H., Urano, F., Till, J. H., Hubbard, S. R., Harding, H. P., et al. (2002) IRE1 couples endoplasmic reticulum load to secretory capacity by processing the XBP-1 mRNA. Nature, 415, 92-96.

[151] Teske, B. F., Wek, S. A., Bunpo, P., Cundiff, J. K., McClintick, J. N., Anthony, T. G., et al. The eIF2 kinase PERK and the integrated stress response facilitate activation of ATF6 during endoplasmic reticulum stress. Mol Biol Cell.

[152] Credle, J. J., Finer-Moore, J. S., Papa, F. R., Stroud, R. M., \& Walter, P. (2005) On the mechanism of sensing unfolded protein in the endoplasmic reticulum. Proc Natl Acad Sci U S A, 102, 18773-18784.

[153] Nakagawa, T., Zhu, H., Morishima, N., Li, E., Xu, J., Yankner, B. A., et al. (2000) Caspase-12 mediates endoplasmic-reticulum-specific apoptosis and cytotoxicity by amyloidbeta. Nature, 403, 98-103.

[154] Rao, R. V., Castro-Obregon, S., Frankowski, H., Schuler, M., Stoka, V., del Rio, G., et al. (2002) Coupling endoplasmic reticulum stress to the cell death program. An Apaf-1independent intrinsic pathway. J Biol Chem, 277, 21836-21842.

[155] Lin, J. H., Li, H., Zhang, Y., Ron, D., \& Walter, P. (2009) Divergent effects of PERK and IRE1 signaling on cell viability. PLoS One, 4, e4170.

[156] McCullough, K. D., Martindale, J. L., Klotz, L. O., Aw, T. Y., \& Holbrook, N. J. (2001) Gadd153 sensitizes cells to endoplasmic reticulum stress by down-regulating Bcl2 and perturbing the cellular redox state. Mol Cell Biol, 21, 1249-1259.

[157] Puthalakath, H., O'Reilly, L. A., Gunn, P., Lee, L., Kelly, P. N., Huntington, N. D., et al. (2007) ER stress triggers apoptosis by activating BH3-only protein Bim. Cell, 129, 13371349.

[158] Cullinan, S. B., Zhang, D., Hannink, M., Arvisais, E., Kaufman, R. J., \& Diehl, J. A. (2003) Nrf2 is a direct PERK substrate and effector of PERK-dependent cell survival. Mol Cell Biol, 23, 7198-7209.

[159] Nishitoh, H., Matsuzawa, A., Tobiume, K., Saegusa, K., Takeda, K., Inoue, K., et al. (2002) ASK1 is essential for endoplasmic reticulum stress-induced neuronal cell death triggered by expanded polyglutamine repeats. Genes Dev, 16, 1345-1355.

[160] Lin, J. H., Li, H., Yasumura, D., Cohen, H. R., Zhang, C., Panning, B., et al. (2007) IRE1 signaling affects cell fate during the unfolded protein response. Science, 318, 944-949.

[161] Scorrano, L., Oakes, S. A., Opferman, J. T., Cheng, E. H., Sorcinelli, M. D., Pozzan, T., et al. (2003) BAX and BAK regulation of endoplasmic reticulum $\mathrm{Ca} 2+$ : a control point for apoptosis. Science, 300, 135-139.

[162] Wei, M. C., Zong, W. X., Cheng, E. H., Lindsten, T., Panoutsakopoulou, V., Ross, A. J., et al. (2001) Proapoptotic BAX and BAK: a requisite gateway to mitochondrial dysfunction and death. Science, 292, 727-730.

[163] Hetz, C., Bernasconi, P., Fisher, J., Lee, A. H., Bassik, M. C., Antonsson, B., et al. (2006) Proapoptotic BAX and BAK modulate the unfolded protein response by a direct interaction with IRE1alpha. Science, 312, 572-576. 
[164] Rutkowski, D. T., Arnold, S. M., Miller, C. N., Wu, J., Li, J., Gunnison, K. M., et al. (2006) Adaptation to ER stress is mediated by differential stabilities of pro-survival and proapoptotic mRNAs and proteins. PLoS Biol, 4, e374.

[165] Mihailidou, C., Papazian, I., Papavassiliou, A. G., \& Kiaris, H. (2010) CHOPdependent regulation of p21/waf1 during ER stress. Cell Physiol Biochem, 25, 761-766.

[166] Piperi C, Adamopoulos C, Dalagiorgou G, Diamanti-Kandarakis E, Papavassiliou AG. (2012) Crosstalk between advanced glycation and endoplasmic reticulum stress: emerging therapeutic targeting for metabolic diseases. J Clin Endocrinol Metab. Jul;97(7):2231-42. doi: 10.1210/jc.2011-3408. Epub 2012 Apr 16. Review.

[167] Kaufman RJ, Scheuner D, Schröder M, Shen X, Lee K, Liu CY, Arnold SM (2002) The unfolded protein response in nutrient sensing and differentiation. Nat Rev Mol Cell Biol $3: 411-421$

[168] Oslowski CM, Urano F (2010) The binary switch between life and death of endoplasmic reticulum-stressed $\beta$ cells. Curr Opin Endocrinol Diabetes Obes 17:107-112

[169] Wang Y, Vera L, Fischer WH, Montminy M (2009) The CREB coactivator CRTC2 links hepatic ER stress and fasting gluconeogenesis. Nature 460:534-537

[170] Harding HP, Novoa I, Zhang Y, Zeng H, Wek R, Schapira M, Ron D (2000) Regulated translation initiation controls stress-induced gene expression in mammalian cells. Mol Cell 6:1099-1108

[171] Back SH, Scheuner D, Han J, Song B, Ribick M, Wang J, Gildersleeve RD, Pennathur S, Kaufman RJ (2009) Translation attenuation through eIF2 $\alpha$ phosphorylation prevents oxidative stress and maintains the differentiated state in $\beta$ cells. Cell Metab 10:13-26

[172] Oyadomari S, Harding HP, Zhang Y, Oyadomari M, Ron D (2008) Dephosphorylation of translation initiation factor $2 \alpha$ enhances glucose tolerance and attenuates hepatosteatosis in mice. Cell Metab 7:520-532

[173] Lee AH, Scapa EF, Cohen DE, Glimcher LH (2008) Regulation of hepatic lipogenesis by the transcription factor XBP1. Science 320:1492-1496

[174] Gregor MF, Hotamisligil GS (2007) Adipocyte stress: the endoplasmic reticulum and metabolic disease. J Lipid Res 48:1905-1914 Abstract/FREE Full Text

[175] Brown MS, Ye J, Rawson RB, Goldstein JL (2000) Regulated intramembrane proteolysis: a control mechanism conserved from bacteria to humans. Cell 100:391-398

[176] Gregor MF, Yang L, Fabbrini E, Mohammed BS, Eagon JC, Hotamisligil GS, Klein S 2009 Endoplasmic reticulum stress is reduced in tissues of obese subjects after weight loss. Diabetes 58:693-700

[177] Hotamisligil GS (2010) Endoplasmic reticulum stress and the inflammatory basis of metabolic disease. Cell 140:900-917

[178] Chen Y, Liu CP, Xu KF, Mao XD, Lu YB, Fang L, Yang JW, Liu C (2008) Effect of taurine-conjugated ursodeoxycholic acid on endoplasmic reticulum stress and apoptosis induced by advanced glycation end products in cultured mouse podocytes. Am J Nephrol 28:1014-1022

[179] Rasheed Z, Haqqi TM (2012) Endoplasmic reticulum stress induces the expression of COX-2 through activation of eIF2 $\alpha, \mathrm{p} 38$-MAPK and NF- $\mathrm{KB}$ in advanced glycation end 
products stimulated human chondrocytes. Biochim Biophys Acta. Dec;1823(12):2179-89. doi: 10.1016/j.bbamcr.2012.08.021. Epub 2012 Sep 6.

[180] Loughlin DT, Artlett CM (2010) Precursor of advanced glycation end products mediates ER-stress-induced caspase-3 activation of human dermal fibroblasts through NAD(P)H oxidase 4. PLoS One 5:e11093

[181] Dong Y, Zhang M, Wang S, Liang B, Zhao Z, Liu C, Wu M, Choi HC, Lyons TJ, Zou MH (2010) Activation of AMP-activated protein kinase inhibits oxidized LDL-triggered endoplasmic reticulum stress in vivo. Diabetes 59:1386-1396

[182] Nishi Y, Yanase T, Mu Y, Oba K, Ichino I, Saito M, Nomura M, Mukasa C, Okabe T, Goto K, Takayanagi R, Kashimura Y, Haji M, Nawata H. (2001) Establishment and characterization of a steroidogenic human granulosa-like tumor cell line, KGN, that expresses functional follicle-stimulating hormone receptor. Endocrinology. Jan;142(1):437-45.

[183] Baudin B, Bruneel A, Bosselut N, Vaubourdolle M. (2007) A protocol for isolation and culture of human umbilical vein endothelial cells. Nat Protoc.;2(3):481-5.

[184] Duleba AJ, Spaczynski RZ, Olive DL, Behrman HR. (1997) Effects of insulin and insulin-like growth factors on proliferation of rat ovarian theca-interstitial cells. Biol Reprod. Apr;56(4):891-7.

[185] Duleba AJ, Spaczynski RZ, Arici A, Carbone R, Behrman HR (1999) Proliferation and differentiation of rat theca-interstitial cells: comparison of effects induced by platelet-derived growth factor and insulin-like growth factor-I. Biol Reprod. Mar;60(3):546-50.

[186] Quehenberger P, Bierhaus A, Fasching P, Muellner C, Klevesath M, Hong M, Stier G, Sattler M, Schleicher E, Speiser W, Nawroth PP. (2000) Endothelin 1 transcription is controlled by nuclear factor-kappaB in AGE-stimulated cultured endothelial cells. Diabetes. Sep;49, 1561-70.

[187] Hess J, Angel P, Schorpp-Kistner M (2004). AP-1 subunits: quarrel and harmony among siblings. J. Cell. Sci. 117: 5965-5973.

[188] Ameyar M, Wisniewska M, Weitzman JB (2003). A role for AP-1 in apoptosis: the case for and against. Biochimie 85, 747-752.

[189] Chronopoulos A, Tang A, Beglova E, Trackman PC, Roy S. (2010) High glucose increases lysyl oxidase expression and activity in retinal endothelial cells: mechanism for compromised extracellular matrix barrier function. Diabetes, 59, 3159-66.

[190] Sekiya M, Hiraishi A, Touyama M, Sakamoto K.Biochem Biophys Res Commun. (2008) Oxidative stress induced lipid accumulation via SREBP1c activation in HepG2 cells., $375,602-607$.

[191] Lu J, Wu DM, Zheng ZH, Zheng YL, Hu B, Zhang ZF (2011) Troxerutin protects against high cholesterol-induced cognitive deficits in mice. Brain 134:783-797

[192] Hayashi T, Saito A, Okuno S, Ferrand-Drake M, Dodd RL, Chan PH (2005) Damage to the endoplasmic reticulum and activation of apoptotic machinery by oxidative stress in ischemic neurons. J Cereb Blood Flow Metab 25:41-53

[193] Murakami K, Murata N, Noda Y, Tahara S, Kaneko T, Kinoshita N, Hatsuta H, Murayama S, Barnham KJ, Irie K, Shirasawa T, Shimizu T (2011) SOD1 (copper/zinc 
superoxide dismutase) deficiency drives amyloid $\beta$ protein oligomerization and memory loss in mouse model of Alzheimer disease. J Biol Chem, 286, 44557-44568.

[194] Yokouchi M, Hiramatsu N, Hayakawa K, Okamura M, Du S, Kasai A, Takano Y, Shitamura A, Shimada T, Yao J, Kitamura M (2008) Involvement of selective reactive oxygen species upstream of proapoptotic branches of unfolded protein response. J Biol Chem 283:4252-4260

[195] Yao D, Brownlee M (2010) Hyperglycemia-induced reactive oxygen species increase expression of the receptor for advanced glycation end products (RAGE) and RAGE ligands. Diabetes 59:249-255

[196] Vlassara H, Cai W, Crandall J, Goldberg T, Oberstein R, Dardaine V, Peppa M, Rayfield EJ (2002) Inflammatory mediators are induced by dietary glycotoxins, a major risk factor for diabetic angiopathy. Proc Natl Acad Sci USA 99:15596-15601

[197] Logsdon CD, Fuentes MK, Huang EH, Arumugam T (2007) RAGE and RAGE ligands in cancer. Curr Mol Med 7:777-789

[198] Morcos M, Du X, Pfisterer F, Hutter H, et al (2008) Glyoxalase-1 prevents mitochondrial protein modification and enhances lifespan in Caenorhabditis elegans. Aging Cell 7:260-269

[199] Inagi R (2011) Inhibitors of advanced glycation and endoplasmic reticulum stress. Methods Enzymol 491:361-380

[200] Hu C, Cong XD, Dai DZ, Zhang Y, Zhang GL, Dai Y (2011) Argirein alleviates diabetic nephropathy through attenuating NADPH oxidase, $\mathrm{Cx} 43$, and PERK in renal tissue. Naunyn Schmiedebergs Arch Pharmacol 383:309-319

[201] Battah S, Ahmed N, Thornalley JP (2002) Kinetics and mechanism of the reaction of metformin with methylglyoxal. Int Congr Ser 1245, 355-356

[202] Diamanti-Kandarakis E, Alexandraki K, Piperi C, Aessopos A, Paterakis T, Katsikis I, Panidis D (2007) Effect of metformin administration on plasma advanced glycation end product levels in women with polycystic ovary syndrome. Metabolism 56:129-134

[203] Kim DS, Jeong SK, Kim HR, Kim DS, Chae SW, Chae HJ (2010) Metformin regulates palmitate-induced apoptosis and ER stress response in HepG2 liver cells. Immunopharmacol Immunotoxicol 32:251-7

[204] Jung TW, Lee MW, Lee YJ, Kim SM, Jung TW (2011) Metformin prevents thapsigargin-induced apoptosis via inhibition of c-Jun NH(2) terminal kinase in NIT-1 cells. Biochem Biophys Res Commun Nov 23[Epub ahead of print]

[205] Miyata T, Dan T (2008) Inhibition of advanced glycation end products (AGEs): an implicit goal in clinical medicine for the treatment of diabetic nephropathy? Diabetes Res Clin Pract 82 Suppl 1:S25-29

[206] Lee AS (2007) GRP78 induction in cancer: Therapeutic and prognostic implications. Cancer Res 67:3496-3499

[207] Yoshiuchi K, Kaneto H, Matsuoka TA, Kasami R, Kohno K, Iwawaki T, Nakatani Y, Yamasaki Y, Shimomura I, Matsuhisa M (2009) Pioglitazone reduces ER stress in the liver: direct monitoring of in vivo ER stress using ER stress-activated indicator transgenic mice. Endocr J 56:1103-1111 
[208] Akiyama M, Hatanaka M, Ohta Y, Ueda K, Yanai A, Uehara Y, Tanabe K, Tsuru M, Miyazaki M, Saeki S, Saito T, Shinoda K, Oka Y, Tanizawa Y (2009) Increased insulin demand promotes while pioglitazone prevents pancreatic beta cell apoptosis in Wfs1 knockout mice. Diabetologia 52:653-663

[209] Basha B, Samuel SM, Triggle CR, Ding H. (2012) Endothelial dysfunction in diabetes mellitus: possible involvement of endoplasmic reticulum stress? Exp Diabetes Res; 2012:481840.

[210] Tan KC, Chow WS, Ai VH, Metz C, Bucala R, Lam KS. (2002) Advanced glycation end products and endothelial dysfunction in type 2 diabetes. Diabetes Care 25:1055-9.

[211] Bucala R, Tracey KJ, Cerami A. (1991) Advanced glycosylation products quench nitric oxide and mediate defective endothelium-dependent vasodilatation in experimental diabetes. $\mathrm{J}$ Clin Invest;87:432-8.

[212] Xu B, Chibber R, Ruggiero D, Kohner E, Ritter J, Ferro A. (2003) Impairment of vascular endothelial nitric oxide synthase activity by advanced glycation end products. FASEB J;17:1289-91.

[213] Rojas A, Romay S, Gonzalez D, Herrera B, Delgado R, Otero K. (2000) Regulation of endothelial nitric oxide synthase expression by albumin-derived advanced glycosylation end products. Circ Res;86:E50-4.

[214] Yamagishi S, Fujimori H, Yonekura H, Yamamoto Y, Yamamoto H. (1998) Advanced glycation endproducts inhibit prostacyclin production and induce plasminogen activator inhibitor-1 in human microvascular endothelial cells. Diabetologia;41:1435-41.

[215] Bierhaus A, Illmer T, Kasper M, Luther T, Quehenberger P, Tritschler H, et al. (1997) Advanced glycation end product (AGE)-mediated induction of tissue factor in cultured endothelial cells is dependent on RAGE. Circulation;96:2262-71.

[216] Sun C, Liang C, Ren Y, Zhen Y, He Z, Wang H, et al. (2009) Advanced glycation end products depress function of endothelial progenitor cells via p38 and ERK $1 / 2$ mitogenactivated protein kinase pathways. Basic Res Cardiol;104:42-9.

[217] Oba T, Tatsunami R, Sato K, Takahashi K, Hao Z, Tampo Y. (2012) Methylglyoxal has deleterious effects on thioredoxin in human aortic endothelial cells. Environ Toxicol Pharmacol;34:117-26.

[218] Zhang K. (2010) Integration of ER stress, oxidative stress and the inflammatory response in health and disease. Int J Clin Exp Med;3:33-40.

[219] Witte I, Horke S. (2011) Assessment of endoplasmic reticulum stress and the unfolded protein response in endothelial cells. Methods Enzymol;489:127-46.

[220] Sheikh-Ali M, Sultan S, Alamir AR, Haas MJ, Mooradian AD (2010) Hyperglycemiainduced endoplasmic reticulum stress in endothelial cells. Nutrition;26:1146-50.

[221] Lee AH, Iwakoshi NN, Glimcher LH. (2003) XBP-1 regulates a subset of endoplasmic reticulum resident chaperone genes in the unfolded protein response. Mol Cell Biol;23:744859.

[222] Zinszner H, Kuroda M, Wang X, Batchvarova N, Lightfoot RT, Remotti H, et al. (1998) CHOP is implicated in programmed cell death in response to impaired function of the endoplasmic reticulum. Genes Dev;12:982-95. 
[223] Loughlin DT, Artlett CM. (2010) Precursor of advanced glycation end products mediates ER-stress-induced caspase-3 activation of human dermal fibroblasts through NAD(P)H oxidase 4. PLoS One;5:e11093. 


\section{Christos Adamopoulos, Elena Farmaki, Eliana Spilioti, Hippokratis Kiaris, Christina Piperi*} and Athanasios G. Papavassiliou

\section{Advanced glycation end-products induce endoplasmic reticulum stress in human aortic endothelial cells}

\begin{abstract}
Background: Advanced glycation end products (AGEs), the final products of the Maillard reaction, have been shown to impair endothelial proliferation and function, thus contributing to endothelial cell injury present in diabetes, inflammatory and cardiovascular diseases. Endoplasmic reticulum (ER) stress triggered under hyperglycemic, hypoxic and oxidative conditions has been implicated in endothelial dysfunction through activation of the unfolded protein response (UPR). The present study investigates the role of AGEs in ER stress induction in human aortic endothelial cells exposed to variable AGE treatments.
\end{abstract}

Methods: Human aortic endothelial cells (HAEC) were treated with increasing concentrations $(100,200 \mu \mathrm{g} / \mathrm{mL})$ of AGE-bovine serum albumin (AGE-BSA) at different time-points $(24,48,72 \mathrm{~h})$. The induction of ER stress and the involved UPR components were investigated on mRNA and protein levels. Apoptosis was quantitatively determined by flow cytometry detecting propidium iodide expression and annexin $\mathrm{V}$ binding simultaneously.

Results: AGEs administration significantly reduced HAEC proliferation in a time- and dose-dependent manner. An immediate induction of the ER chaperones GRP78, GRP94 and the transcriptional activator, XBP-1 was observed at $24 \mathrm{~h}$ and $48 \mathrm{~h}$. A later induction of the phospho-elF2 $\alpha$ and proapoptotic transcription factor $\mathrm{CHOP}$ was observed at $48 \mathrm{~h}$ and $72 \mathrm{~h}$, being correlated with elevated early apoptotic cell numbers at the same time-points.

Conclusions: The present study demonstrates that AGEs directly induce ER stress in human aortic endothelial cells, playing an important role in endothelial cell apoptosis. Targeting AGEs signaling pathways in order to alleviate ER stress may prove of therapeutic potential to endothelial dysfunction-related disorders.

Keywords: advanced glycation end-products (AGEs); apoptosis; endoplasmic reticulum (ER) stress; human aortic endothelial cells; unfolded protein response (UPR).
*Corresponding author: Assistant Professor Christina Piperi, PhD, Department of Biological Chemistry, Medical School, University of Athens, 75, M. Asias Street, 11527 Athens, Greece, Phone: +30 210 7462610, Fax: +30 2108037372 , E-mail: cpiperi@med.uoa.gr Christos Adamopoulos, Elena Farmaki, Eliana Spilioti, Hippokratis Kiaris and Athanasios G. Papavassiliou: Department of Biological Chemistry, Medical School, University of Athens, Athens, Greece

\section{Introduction}

Advanced glycation end products (AGEs) are highly reactive molecules resulting from the irreversible post-translational modification reactions of glucose with the amino groups on proteins, lipids or nucleic acids [1, 2]. Increased circulating AGE levels are a common finding and a pathogenic mediator of hyperglycemic, oxidative and inflammatory conditions including diabetes, polycystic ovarian syndrome, neurodegenerative and cardiovascular diseases [3-6]. However, exogenous intake of AGEs from high fat and/or protein diets, beverages or cigarette smoke may also contribute to increased AGEs tissue deposition under normal physiology predisposing individuals to metabolic, cardiovascular disorders and progressive aging $[7,8]$. AGEs have been shown to accumulate in almost all major organs including vascular tissues mediating both extracellular and intracellular functions [9].

Endothelial cells (ECs) have been proposed as primary targets of AGEs, affecting their proliferation, migration and adhesion [10]. Extracellular effects of AGEs in endothelium involve irreversible crosslinking formation with stable, long-lived extracellular matrix proteins such as collagen, elastin or laminin [11]. At the same time they can induce cell activation, proinflammatory cytokine release and oxidative stress through interaction with their receptor, RAGE, leading to endothelial injury and dysfunction $[12,13]$. The role of AGEs in the induction of aortic endothelial apoptosis has not been adequately addressed. It has been suggested that AGEs trigger oxidative stress and thus 
accelerate apoptosis of bovine aortic ECs contributing to vascular complications associated with diabetes mellitus [14]. This effect has been attributed to AGE-RAGE interaction leading to increased intracellular accumulation of reactive oxygen species and elevation of intracellular $\mathrm{Ca}^{2+}$ concentration. Additionally, AGE-RAGE dependent activation promotes apoptosis of rat endothelial progenitor cells and inhibits their migration [15]. Recently methyglyoxal, the main AGE precursor has also been shown to induce apoptosis of human aortic endothelial cells (HAEC), through oxidative stress and by triggering the mitochondrial route of apoptotic machinery [16].

Relevant studies challenge the central role of mitochondria in apoptosis and suggest that some apoptotic signals may bypass mitochondria to directly activate caspases [17]. Endoplasmic reticulum (ER) stress presents such an alternative mechanism triggering apoptosis without the involvement of mitochondria [18]. ER is the major organelle involved in the synthesis and folding of secreted and membrane bound proteins, calcium homeostasis and lipid biosynthesis [19]. The lumen of ER is characterized by high $\mathrm{Ca}^{2+}$ concentration and a very oxidizing environment [19]. Stressors that interfere with the ER functions, such as oxidant or reducing agents, result in the disruption of $\mathrm{Ca}^{2+}$ homeostasis or glucose deprivation leading to the accumulation of unfolded and/or misfolded proteins in the ER lumen, known as ER stress [20].

The mammalian ER stress response known as unfolded protein response (UPR) can be separated into two phases, adaptation and apoptosis. The cells initially adapt to the accumulation of unfolded proteins by increasing the concentration of chaperones in the ER lumen, namely the glucose-regulated protein 78 (GRP78) and glucose-regulated protein 94 (GRP94). The UPR response is triggered by the activation of three main sensors of ER stress: protein kinase-like ER kinase (PERK), inositol requiring kinase 1 (IRE1) and the activating transcription factor 6 (ATF6) [20]. All these proteins are maintained in the inactive form by binding the N-terminus of GRP78 that serves both as chaperone and sensor of ER stress. When misfolded proteins accumulate, GRP78 is required for its chaperone function and releases these sensors to initiate the UPR [21]. PERK, upon activation, phosphorylates the eukaryotic translation initiation factor $2 \alpha$ (eIF $2 \alpha)$, decreases mRNA translation and induces UPR-related genes [20]. Similarly, activation of IRE1 $\alpha$ and ATF6 also leads to regulation of various UPR-related genes, such as protein chaperones and calnexin [22]. However, if this adaptation does not prove sufficient, the apoptotic response is initiated.

Previous studies have shown that ER stress is implicated in endothelial dysfunction associated with diabetes mellitus and cardiovascular diseases indicating that ER stress-induced apoptosis can be an important pathophysiological factor [23, 24]. Furthermore, AGEs have been shown to induce ER stress-mediated apoptosis in mouse podocytes [25], human neuroblastoma cells and cortical neurons [26]. However, the role of AGEs in ER stressinduced apoptosis in human aortic ECs remains to be elucidated. The present study aims to delineate ER stress induction in human ECs exposed to different AGE concentrations at variable time-points, monitoring the series of events leading to apoptosis.

\section{Materials and methods}

\section{Reagents}

All reagents were purchased from Life Technologies (Carlsbad, CA, USA) unless otherwise stated. AGE-bovine serum albumin (AGE-BSA) was obtained from Abcam plc (ab51995; Cambridge, UK). Tunicamycin was purchased from AppliChem (Darmstadt, Germany). BSA, TNF- $\alpha$ and 3-(4,5-dimethylthiazol-2-yl)-2,5-diphenyltetrazolium bromide (MTT) were obtained from Sigma Aldrich (St Louis, MO, USA).

\section{AGE-BSA characteristics}

According to manufacturer's instructions, glycated BSA has been produced by reacting BSA with glycoaldehyde under sterile conditions followed by extensive dialysis and purification steps. Fluorescence of AGEs was confirmed by fluorescence spectrophotometry with Ex./Em.=370/440 nm. Glycated BSA showed 7000\% increase in fluorescence when compared to control BSA. The purity of the final stock is $>95 \%$ as analyzed by SDS-PAGE and filter sterilized using $0.22 \mu \mathrm{m}$ filter.

\section{Culture of endothelial cells}

HAEC were obtained as cryopreserved cells from European Collection of Cell Cultures (ECACC) and were cultured in endothelial cell growth medium M200 supplemented with Low Serum Growth Supplement containing Fetal Bovine Serum ( $2 \% \mathrm{v} / \mathrm{v})$, hydrocortisone, $(1 \mathrm{~g} / \mathrm{mL})$, human epidermal growth factor $(10 \mathrm{ng} / \mathrm{mL})$, basic fibroblast growth factor, $(3 \mathrm{ng} / \mathrm{mL})$, heparin $(10 \mathrm{~g} / \mathrm{mL})$, gentamicin $(50 \mu \mathrm{g} / \mathrm{mL})$ and amphotericin B $(50 \mathrm{ng} / \mathrm{mL})$. Cells were cultured at $37^{\circ} \mathrm{C}$ in a humidified $95 \%$ air $-5 \% \mathrm{CO}_{2}$ atmosphere and were split according to standard procedures. HAEC were used in all assays between passage four and eight.

\section{Cell proliferation assay}

For the proliferation assay, HAEC were plated on 96-well microplates at a density of approximately $1 \times 10^{4}$ cells per well, and then treated 
with either unmodified BSA or AGE-BSA at concentrations of 100 and $200 \mu \mathrm{g} / \mathrm{mL}$ for 24,48 and $72 \mathrm{~h}$ or with tunicamycin at concentration of $5 \mu \mathrm{g} / \mathrm{mL}$ for $24 \mathrm{~h}$. Co-treatment with both tunicamycin at the same concentration and AGE-BSA at concentrations of 100, 200 and $400 \mu \mathrm{g} / \mathrm{mL}$ was also performed. Cell viability was subsequently determined by the MTT assay as described previously [27]. Each experiment was conducted in triplicate.

\section{RT-PCR analysis}

Total RNA was isolated from cultured cells using RNeasy Mini Kit (Qiagen, Hilden, Germany), according to the manufacturer's instructions. cDNA was synthesized in a two-step reaction using iScript cDNA synthesis kit (Biorad, Hercules, CA, USA).

For semi-quantitative PCR, cDNA was amplified using gene specific primer pairs in 25 cycles. Detailed PCR conditions have been described previously [28]. PCR fragments were resolved on EtBr agarose gel. Densitometric analysis of PCR fragments was performed using image analysis software Image J (La Jolla, CA, USA) after normalization to actin levels. All experiments have been performed at least three times. Data and densitometric analysis from one representative experiment are presented.

\section{Real-time quantitative PCR}

Real-time quantitative PCR was performed using an iCycler real time instrument (Biorad). RT-PCR product was amplified using the iQ SYBR Green Supermix (Biorad) in a total reaction volume of $20 \mu \mathrm{L}$. Primer efficiencies were calculated from a standard curve of serially diluted cDNA. Product identity was confirmed by a single pick in the melt curve. Relative expression values were calculated using the $2^{-(\Delta \Delta C t)}$ formula. The data are presented as fold change in gene expression normalized to GAPDH and relative to the untreated control. The primers used for the amplification of $\mathrm{CHOP}$ were forward $5^{\prime}$-agtgccacggagaaagctaa-3', and reverse primer, $5^{\prime}$-ccatacagcagcctgagtga- $3{ }^{\prime}$, and for GAPDH were forward primer $5^{\prime}$-gggtgtgaaccatgagaagt $-3^{\prime}$, and reverse primer $5^{\prime}$-catgccagtgagcttcccgttc-3'.

\section{Western blot analysis}

For immunoblot analysis cells were solubilized with ice-cold RIPA buffer (Thermo Scientific, Rockford, IL, USA) supplemented with protease inhibitor cocktail (Thermo Scientific). The protein concentration in the lysates was determined by using Bradford assay (Biorad). Equal amounts of total protein were resolved by SDS-PAGE and immunoblotted with anti-GRP78 (\#3177S; Cell Signaling, Beverly, MA, USA), anti-CHOP (sc-71817; Santa Cruz Biotechnology, Santa Cruz, CA, USA), anti-p-eIF2 $\alpha$ (\#33985; Cell Signaling), anti-XBP-1 (sc-7160; Santa Cruz) and anti-Actin (MAB150; Millipore, Bedford, MA, USA). Relative protein amounts were evaluated by a densitometric analysis using Image J software and normalized to the corresponding actin levels. All experiments have been performed at least three times and representative results and the corresponding quantification data of one experiment are shown.

\section{Assessment of apoptosis by flow cytometry}

For the apoptosis assay, HAEC were seeded in six-well plates at a density of approximately $2 \times 10^{5}$ cells per well and then treated with BSA or AGE-BSA at a concentration of $200 \mu \mathrm{g} / \mathrm{mL}$ for $48 \mathrm{~h}$ and $72 \mathrm{~h}$, with tunicamycin at a concentration of $5 \mu \mathrm{g} / \mathrm{mL}$ for $24 \mathrm{~h}$ and with both AGE-BSA and tunicamycin at the same concentrations for $24 \mathrm{~h}$. Cells were then harvested, rinsed twice with PBS, collected by centrifugation at $2000 \mathrm{rpm}$ for $5 \mathrm{~min}$ and annexin V-FITC apoptosis detection kit (Trevigen, Gaithersburg, MD, Germany) was used according to the manufacturer's protocol. Samples were scanned with a fluorescenceactivated cell sorter (FACSCalibur, Becton Dickinson, USA), and the data were analyzed with CellQuest software (Becton Dickinson). A total of 10,000 events were measured per sample.

Plots in the annexin V-positive/propidium iodide-negative quadrant were counted as apoptotic cells. Each experiment was performed in triplicate.

\section{Statistics}

The data are presented as mean $\pm \mathrm{SE}$ for the number of experiments indicated. Statistical analysis of the results was performed using Student's t-test. A p-value of $<0.05$ was considered to indicate statistical significance.

\section{Results}

\section{AGE-BSA administration inhibited HAEC proliferation in a time- and dose-dependent manner}

In order to elucidate the impact of AGEs in endothelial cell proliferation, HAEC were treated with increasing concentrations $(100,200 \mu \mathrm{g} / \mathrm{mL})$ of unmodified bovine serum albumin (BSA) or AGE-BSA for different periods of time $(24,48,72 \mathrm{~h})$. MTT proliferation assay indicated that AGEs administration significantly reduced HAEC viability in a time- and dose-dependent manner $(48 \mathrm{~h}-100 \mu \mathrm{g} / \mathrm{mL}$ AGE-BSA: $\mathrm{p}<0.01 ; 48 \mathrm{~h}-200 \mu \mathrm{g} / \mathrm{mL}$ AGE-BSA: $\mathrm{p}<0.05 ; 72 \mathrm{~h}-100 \mu \mathrm{g} / \mathrm{mL}$ AGE-BSA: $\mathrm{p}<0.05$; $72 \mathrm{~h}-200 \mu \mathrm{g} / \mathrm{mL}$ AGE-BSA: $\mathrm{p}<0.001$ ) (Figure $1 \mathrm{~A}$ ). In addition the administration of the ER stress-inducer tunicamycin had the same effect with the higher concentration dose of AGE-BSA $(200 \mu \mathrm{g} / \mathrm{mL})$ and longer incubation time $(72 \mathrm{~h})(\mathrm{p}<0.001)$. The combined administration of tunicamycin and increasing concentrations of AGE-BSA indicated minor reduction of HAEC proliferation suggesting a non-synergistic effect between these two agents (Figure 1B). 
A
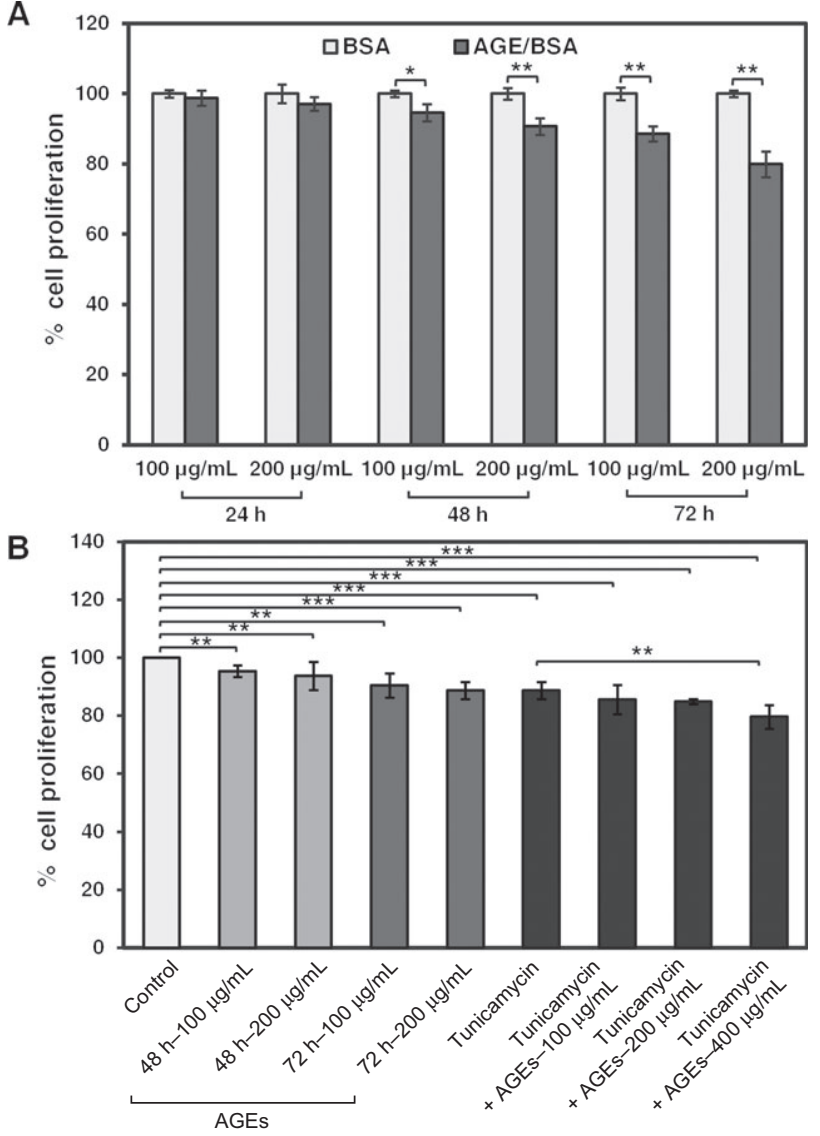

Figure 1 Inhibition of HAEC proliferation by AGE-BSA and tunicamycin treatment.

(A) Cells treated with BSA or AGE-BSA at concentrations of 100 and $200 \mu \mathrm{g} / \mathrm{mL}$ for $24 \mathrm{~h}, 48 \mathrm{~h}$ and $72 \mathrm{~h}$ were subjected to the MTT cell proliferation assay. (B) Independent experiments with combined administration of tunicamycin $(5 \mu \mathrm{g} / \mathrm{mL})$ for $24 \mathrm{~h}$ and increasing concentration of AGE-BSA $(100-400 \mu \mathrm{g} / \mathrm{mL})$ were performed. The viability of untreated cells (control) was considered $100 \%$. ${ }^{*} p<0.05$, ${ }^{\star *} \mathrm{p}<0.01,{ }^{* \star *} \mathrm{p}<0.001$

\section{AGE-BSA treatment-induced ER stress in HAEC}

Based on recent studies that describe the involvement of AGEs in ER stress signaling, we investigated the possible induction of ER stress and the expression of UPR components upon AGE-BSA administration. Semi quantitative PCR analysis demonstrated the up-regulation of chaperones GRP78 and GRP94, the major indicators of ER stress induction in all conditions under study (Figure 2). We also observed an increase in XBP-1 mRNA levels at early time points $(24 \mathrm{~h}$, $48 \mathrm{~h}$ ) and lower concentrations $-100 \mu \mathrm{g} / \mathrm{mL}$ ) and of the spliced form of $X B P-1$ at $72 \mathrm{~h}$, suggesting the early activation of IRE-1 pathway upon AGEs presence (Figure 2B-D).

Furthermore, we assessed the protein levels of the early GRP78, XBP-1 and late UPR effectors, p-eIF2 $\alpha$ and
CHOP following AGE-BSA incubation. GRP78 expression was found to be up-regulated in all dose- and time-points (Figure 3) being in accordance with the elevated mRNA levels (Figure 2). Similar trend was also observed in XBP-1 protein expression levels that remained elevated at early time points ( $24 \mathrm{~h}, 48 \mathrm{~h}$ ) and decreased at $72 \mathrm{~h}$ at both AGE-BSA concentrations (Figure 3). In order to investigate the involvement of late UPR effectors in AGEs-induced ER stress, key molecules of the PERK pathway were studied. As shown in Figure 3, AGE-BSA administration in HAEC increased the phosphorylated form of eIF2 $\alpha$ and of the pro-apoptotic transcription factor $\mathrm{CHOP}$ expression in a time- and dose- dependent manner. Higher levels were observed in $48 \mathrm{~h}(200 \mu \mathrm{g} / \mathrm{mL}$ AGE-BSA) and in $72 \mathrm{~h}$ (at both concentrations).

\section{AGE-BSA administration-induced ER stress-mediated apoptosis in HAEC}

In order to confirm the proapoptotic transcription factor CHOP induction upon AGEs treatment, we monitored CHOP mRNA expression levels using quantitative real time PCR analysis. Our results demonstrated an even higher statistically significant $\mathrm{CHOP}$ mRNA fold induction compared with protein expression levels in $72 \mathrm{~h}$ treatment at both concentrations (Figure 4).

This finding indicated the development of CHOPmediated apoptosis in HAEC upon AGEs treatment that was further investigated by annexin $\mathrm{V} /$ propidium iodide flow cytometric analysis (Figure 5). High concentration of AGEs $(200 \mu \mathrm{g} / \mathrm{mL})$ for $48 \mathrm{~h}$ and $72 \mathrm{~h}$ incubation resulted in a significant increase of the early apoptotic fraction of HAEC (four- and eight-fold, respectively). In addition tunicamycin and AGE-BSA co-treatment did not further increase the early apoptotic cells compared to tunicamycin alone (39.64\% vs. $37.51 \%$ of gated cells). This result is consistent with the proliferation data (Figure 1B), providing further evidence for a common mechanism between AGEs and tunicamycin in inducing HAEC apoptosis via activation of UPR signaling.

\section{Discussion}

Endothelial cells present primary dynamic structures that actively regulate basal vascular tone and reactivity in physiological and pathological conditions. They respond to neurohumoral mediators and mechanical forces as to maintain the balance between vasodilatation and 

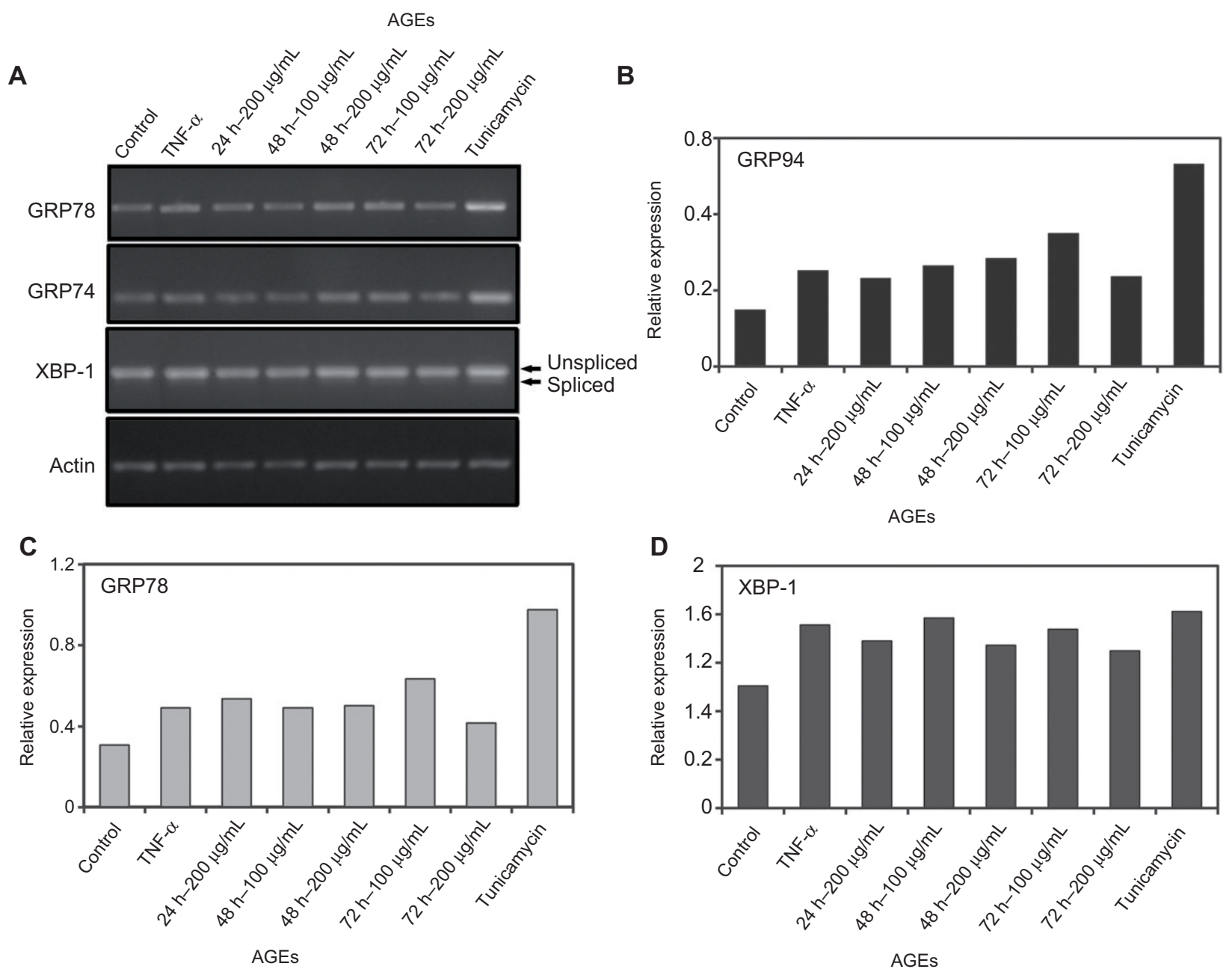

Figure 2 AGE-BSA administration induces mRNA levels of UPR components in HAEC.

(A) ER stress induction in HAEC following AGE-BSA treatment. RT-PCR analysis of GRP78, GRP94 and XBP-1 mRNA expression after AGE-BSA administration at increasing concentrations $(100,200 \mu \mathrm{g} / \mathrm{mL})$ for different periods of time $(24 \mathrm{~h}, 48 \mathrm{~h}, 72 \mathrm{~h})$. The lower band represents the spliced form of activated XBP-1(arrow). (B) The panel represents results of densitometric analysis of GRP78, GRP94 and XBP-1 PCR fragments after normalization to actin levels. TNF- $\alpha(50 \mathrm{ng} / \mathrm{mL}$ for $4 \mathrm{~h})$ and tunicamycin $(5 \mu \mathrm{g} / \mathrm{mL}$ for $24 \mathrm{~h})$ treatments were used as positive ER stress induction controls. All experiments have been performed at least three times and representative results as well as corresponding quantification data of one experiment are shown (B-D).

vasoconstriction, restore blood fluidity and vessel wall integrity [29]. Loss of endothelial function may be a critical and initiating factor for several conditions including inflammation, diabetic micro- and macrovascular complications as well as cardiovascular diseases [29].

AGEs have been shown to contribute to endothelial dysfunction and this has been clearly demonstrated in diabetes, where levels of serum AGEs in patients with type 2 diabetes were negatively associated with the degree of endothelium-dependent and endothelium independent vasodilation [30]. Several mechanisms have been suggested to explain the association. Among them, bioavailability and activity of the endothelium-derived NO has been demonstrated to be reduced by AGEs [31]. AGE associated induction of oxidative stress may quench and inactivate endothelium-derived NO and uncouple endothelial NO synthase (eNOS) activity through receptor-mediated phosphorylation and increased eNOS degradation [32, 33]. In addition, AGEs may impair endothelial balance by reducing the endothelial production of prostacyclin [34] and enhancing the expression of endothelin-1 [35]. They are also capable of increasing endothelial permeability to macromolecules [36, 37] and modify the functions of endothelial progenitor cells by promoting their apoptosis and migration [38]. Endothelial cell apoptosis due to AGEs has been demonstrated in several studies using bovine and human aortic ECs and has been mainly attributed to the induction of oxidative stress and mitochondrial dysfunction $[14,16]$. 


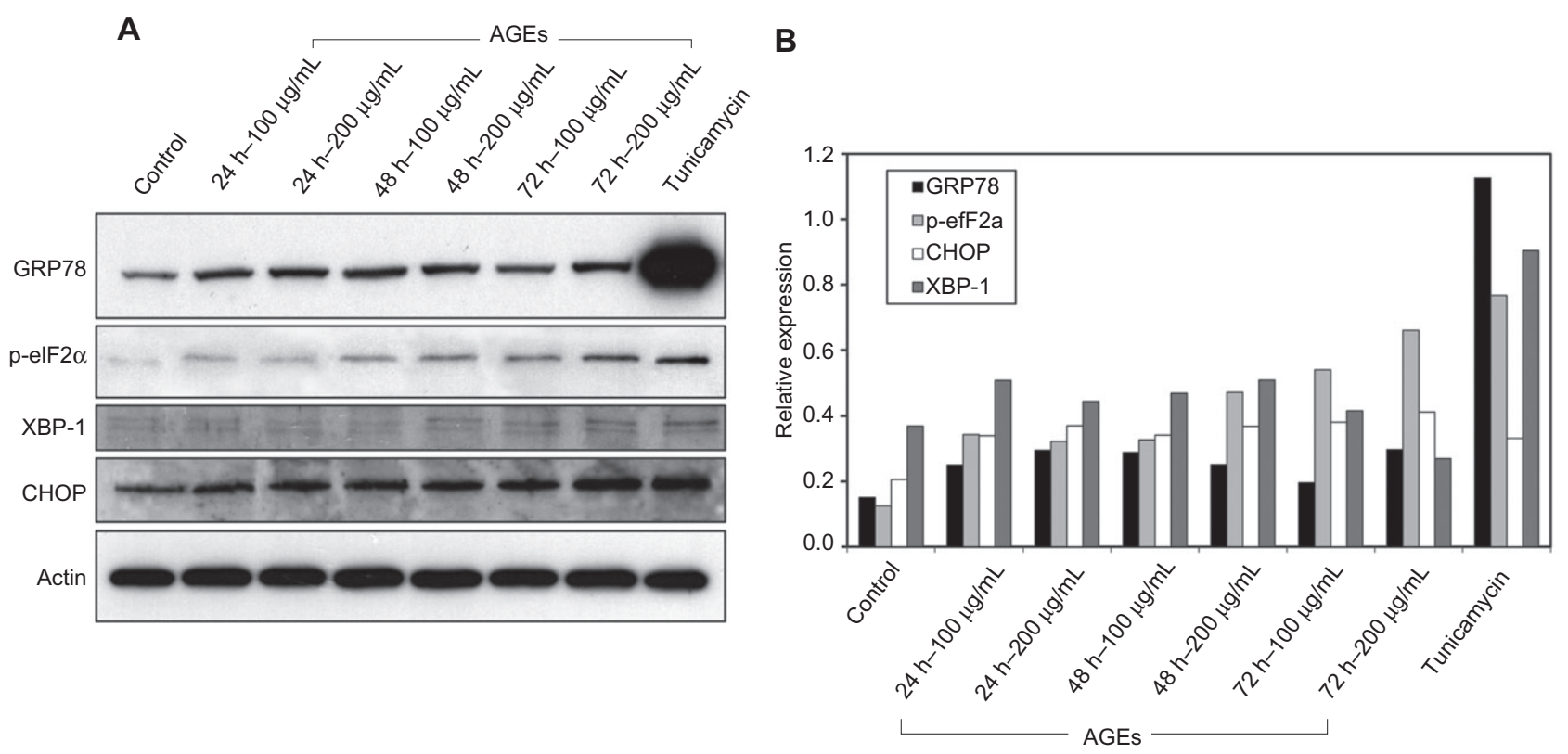

Figure 3 AGE-BSA administration increases GRP78 and other UPR component protein levels.

GRP78, p-elF2 $\alpha$, XBP-1 and CHOP protein levels were assessed by Western blot analysis after AGE-BSA treatment of HAEC at increasing concentrations $(100,200 \mu \mathrm{g} / \mathrm{mL})$ for various time points $(24 \mathrm{~h}, 48 \mathrm{~h}, 72 \mathrm{~h})(\mathrm{A})$. The densitometric quantification of these results (normalized to the actin levels) is shown in the graph (B). Tunicamycin $(5 \mu \mathrm{g} / \mathrm{mL}$ for $24 \mathrm{~h})$ treatment was used as positive ER stress induction control. All experiments have been performed at least three times and representative results as well as corresponding quantification data of one experiment are shown.

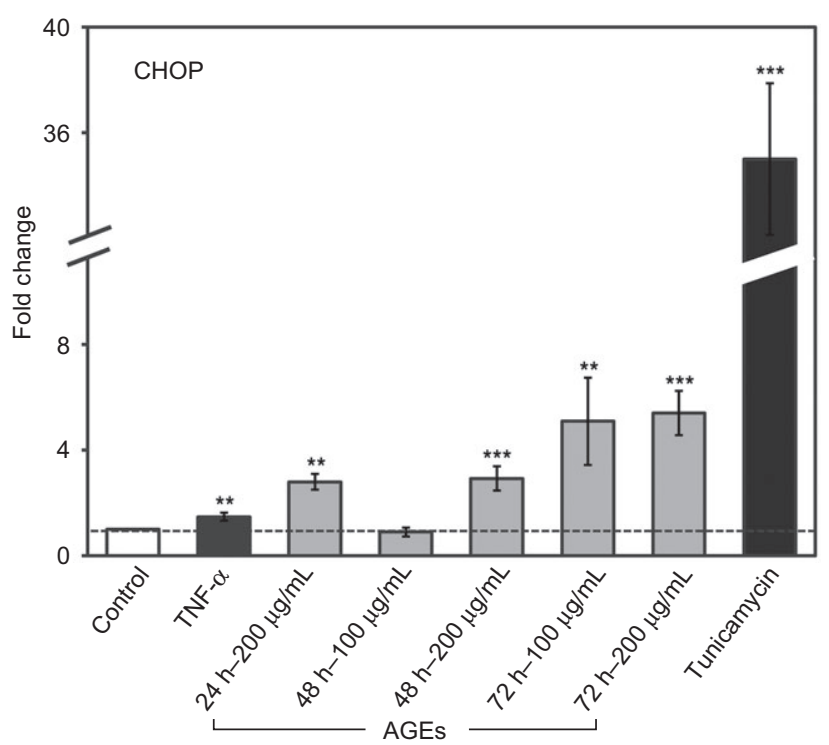

Figure 4 Induction of proapoptotic transcription factor CHOP upon AGE-BSA treatment.

CHOP mRNA levels were monitored by quantitative real time PCR analysis in HAEC following treatment with AGE-BSA at increasing concentrations $(100,200 \mu \mathrm{g} / \mathrm{mL})$ for different time points. TNF- $\alpha$ $(50 \mathrm{ng} / \mathrm{mL}$ for $4 \mathrm{~h})$ and tunicamycin $(5 \mu \mathrm{g} / \mathrm{mL}$ for $24 \mathrm{~h})$ treatments were used as positive ER stress induction controls. ${ }^{*} p<0.05$, ${ }^{\star *} p<0.01,{ }^{* * *} p<0.001$.
Here, we show AGEs-induced apoptosis in ECs through ER stress pathway, providing a novel molecular mechanism for endothelial dysfunction in metabolic and cardiovascular disorders. ER is exquisitely sensitive to glucose availability and depends on blood glucose levels for the energy supply required for the protein folding process [39]. ECs always exposed to elevations and reductions of blood nutrients, are very dynamic, metabolically active cells, with a high volume of protein synthesis which predispose them to ER stress [40]. In particular ECs cannot tolerate the continued high glucose exposure, and thus ER stress is commonly initiated in a diabetic milieu [41]. It was thus hypothesized that ER stress may also be induced in HAECs under conditions of increased exogenous glycated proteins (AGEs) uptake. Indeed, incubation of HAEC with high AGE levels for prolonged durations ( $48 \mathrm{~h}$ and $72 \mathrm{~h}$ ) was capable to significantly reduce cell proliferation. This reduction was accompanied by increased mRNA and protein levels of ER stress sensors (GRP78, GRP94) indicative of ER stress induction. Recent studies have also shown the direct induction of ER stress by AGEs in murine podocytes, human neuroblastoma cell lines and human chondrocytes where they are implicated in inflammatory and oxidative processes as well as cell death pathways [25, 26, 42]. 
A

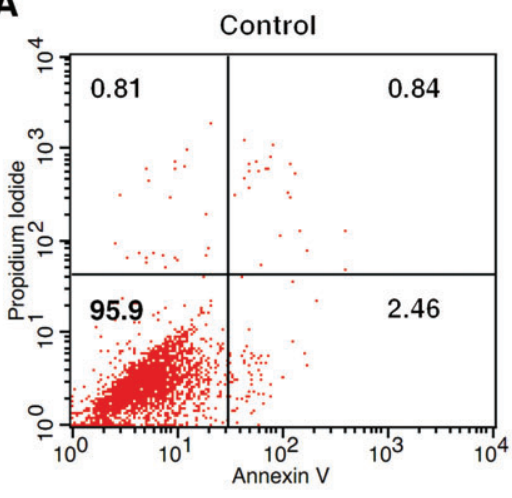

B

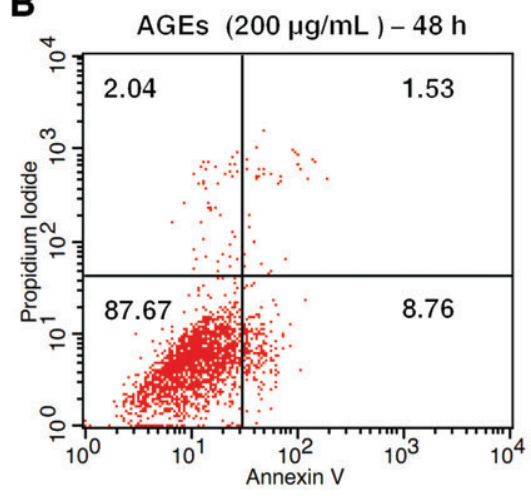

C

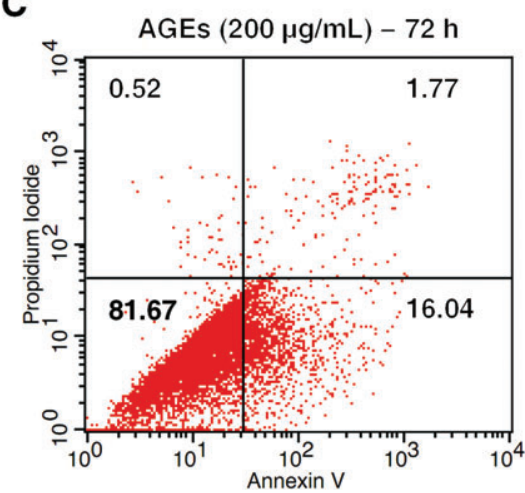

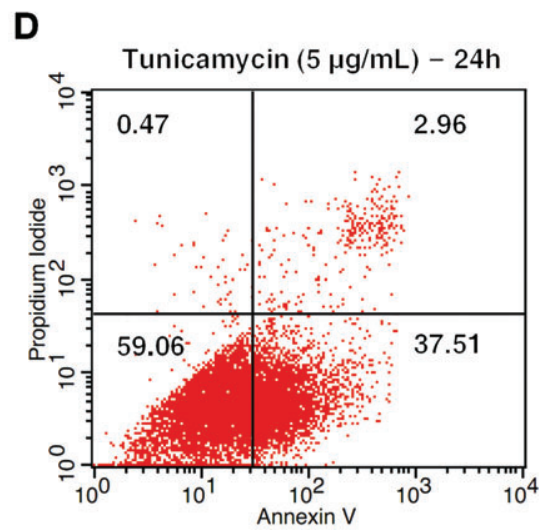

E Tunicamycin $(5 \mu \mathrm{g} / \mathrm{mL})$

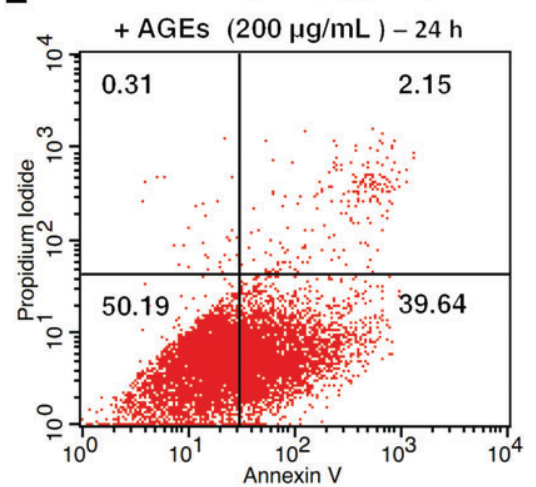

Figure 5 AGE-BSA induced apoptosis in HAEC in a time-dependent manner.

Apoptosis of HAEC was determined by annexin V/PI binding. HAEC were untreated (A), treated with $A G E-B S A(200 \mu g / m L)$ for $48 \mathrm{~h}(B)$ and $72 \mathrm{~h}(\mathrm{C})$, with tunicamycin $(5 \mu \mathrm{g} / \mathrm{mL})$ for $24 \mathrm{~h}$ (D) and with both tunicamycin $(5 \mu \mathrm{g} / \mathrm{mL})$ and AGE-BSA (200 $\mu \mathrm{g} / \mathrm{mL})$ for $24 \mathrm{~h}(\mathrm{E})$. Percentage of gated cells is shown in each quadrant (UL: \% of necrotic cells, UR: \% of apoptotic cells, LL: \% of viable cells, LR: \% of early apoptotic cells).

Notably, at early time points ( 24 h, 48 h) of AGE-BSA administration, activation of the transcriptional activator, XBP-1 was observed that is a mediator of the adaptive UPR response. The three UPR branches (IRE1, PERK, and ATF6) have been reported to initially promote cell survival by reducing misfolded protein levels but when ER stress is not alleviated UPR signaling promotes apoptotic cell death. This is also possible to occur under AGEs accumulation, being capable of triggering both protective and cell death responses depending on their concentration and the duration of their presence. In accordance with this hypothesis, we found that IRE1 activity (as demonstrated by XBP-1 expression) was increased at early AGE-induced ER stress $(24 \mathrm{~h})$ and attenuated by persistent ER stress (48 h, $72 \mathrm{~h}$ ). IRE1 is a transmembrane kinase (endoribonuclease, RNAse) that upon activation, initiates the nonconventional splicing of Xbp-1 mRNA [43, 44]. Spliced $X b p-1$ mRNA encodes a transcription activator that drives transcription of genes such as ER chaperones, whose products directly participate in ER protein folding [45]. By contrast, our data show that PERK signaling including translational inhibition (by phosphorylation of elF $2 \alpha$ ) and proapoptotic transcription regulator $\mathrm{CHOP}$ induction was maintained at $48 \mathrm{~h}$ and $72 \mathrm{~h}$ incubation with AGEs. PERK is a transmembrane kinase that phosphorylates the eukaryotic translation initiation factor $2 \alpha(\mathrm{elF} 2 \alpha)$, thus reducing protein synthesis and counteracting ER protein overload [46]. Additionally, CHOP is a B-ZIP transcription factor induced by the PERK branch of the UPR that promotes cell death [47]. Chop mRNA levels were significantly increased at $48 \mathrm{~h}$ incubation with $200 \mu \mathrm{g} / \mathrm{mL}$ AGEs compared to untreated control and remained elevated at $72 \mathrm{~h}$ at both concentrations, indicative of proapoptotic signaling. These data were further confirmed by double staining flow cytometric analysis of AGE-stimulated HAEC using the early apoptotic marker, annexin $\mathrm{V}$ and nucleic acid stain, propidium iodide. Significantly increased cell numbers of early apoptotic cells were detected following AGEs treatment for $48 \mathrm{~h}$ and $72 \mathrm{~h}$. The modest increase in early and late apoptotic cell population after treatment with AGEs combined with tunicamycin compared to tunicamycin alone indicated that both agents share common 
UPR signaling pathways in the induction of apoptosis in HAEC.

Several studies in other cell systems suggest that apoptosis mediated by AGEs could be induced by an ER pathway independent from mitochondria [25, 48]. More specifically, AGE-induced GRP78 expression as an ER stress sensor was associated with elevated intracellular $\mathrm{Ca}^{2+}$ and apoptosis in murine podocytes [25]. Additionally, 3-deoxyglucosone, a precursor for AGEs, that is highly upregulated in skin explants of diabetic cutaneous wounds, was found to mediate ER stress-induced apoptosis through ROS formation in human dermal fibroblasts via a RAGE-independent mechanism [48]. This is particularly interesting since previous studies limited AGEinduced apoptosis in being mediated through increased oxidative stress following interaction of AGEs with their receptor RAGE [14-16].

It is therefore evident that due to the heterogeneity of AGE compounds and the wide range of their precursor molecules; different signaling may be selected depending not only upon the cell type but the state of AGE molecules being either in circulating or protein-bound form. Further research is required in elucidating the necessity of RAGE in endothelial ER stress induction under high AGE environments. Additionally, since ER stress regulates mitochondrial bioenergetics, a potential crosstalk between oxidative stress in ER stress-induced apoptosis cannot be excluded and should be explored in detail.

However, our data show that ER function can be modulated by AGEs affecting critically both ECs functionality and cell fate. Thus, chemical enhancement of ER function to cope with cell apoptosis provides an interesting approach to managing endothelial dysfunction. Pharmaceutical or chemical chaperones such as trimethylamine $\mathrm{N}$-oxide dehydrate, 4-phenyl butyric acid and taurine-conjugated ursodeoxycholic acid are representatives of a group of low-molecular weight compounds known to stabilize protein conformation that improve ER folding capacity and facilitate trafficking of mutant proteins $[49,50]$. The effectiveness of these molecules in alleviating endothelial injury either alone or in conjunction with standard medications remains to be investigated.

\section{Conflict of interest statement}

Authors' conflict of interest disclosure: The authors stated that there are no conflicts of interest regarding the publication of this article. Research support played no role in the study design; in the collection, analysis, and interpretation of data; in the writing of the report; or in the decision to submit the report for publication.

Research funding: This research has been co-financed by the European Union (European Social Fund - ESF) and Greek national funds through the Operational Program 'Education and Lifelong Learning' of the National Strategic Reference Framework (NSRF) - Research Funding Program: Heracleitus II. Investing in knowledge society through the European Social Fund.

Employment or leadership: None declared.

Honorarium: None declared.

Received November 29, 2012; accepted February 7, 2013

\section{References}

1. Brownlee M. Advanced protein glycosylation in diabetes and aging. Annu Rev Med 1995;46:223-34.

2. Degenhardt TP, Thorpe SR, Baynes JW. Chemical modification of proteins by methylglyoxal. Cell Mol Biol (Noisy-le-grand) 1998;44:1139-45.

3. Bierhaus A, Hofmann MA, Ziegler R, Nawroth PP. AGEs and their interaction with AGE-receptors in vascular disease and diabetes mellitus. I. The AGE concept. Cardiovasc Res 1998;37:586-600.

4. Diamanti-Kandarakis E, Piperi C, Kalofoutis A, Creatsas G. Increased levels of serum advanced glycation end-products in women with polycystic ovary syndrome. Clin Endocrinol (0xf) 2005;62:37-43.

5. Li J, Liu D, Sun L, Lu Y, Zhang Z. Advanced glycation end products and neurodegenerative diseases: mechanisms and perspective. J Neurol Sci 2012;317:1-5.

6. Barlovic DP, Thomas MC, Jandeleit-Dahm K. Cardiovascular disease: what's all the AGE/RAGE about? Cardiovasc Hematol Disord Drug Targets 2010;10:7-15.
7. Uribarri J, Cai W, Peppa M, Goodman S, Ferrucci L, Striker G, et al. Circulating glycotoxins and dietary advanced glycation endproducts: two links to inflammatory response, oxidative stress, and aging. J Gerontol A Biol Sci Med Sci 2007;62:427-33.

8. Gursinsky T, Ruhs S, Friess U, Diabate S, Krug HF, Silber RE, et al. Air pollution-associated fly ash particles induce fibrotic mechanisms in primary fibroblasts. Biol Chem 2006;387: 1411-20.

9. Schalkwijk CG, Miyata T. Early- and advanced non-enzymatic glycation in diabetic vascular complications: the search for therapeutics. Amino Acids 2012;42:1193-204.

10. Li H, Zhang X, Guan X, Cui X, Wang Y, Chu H, et al. Advanced glycation end products impair the migration, adhesion and secretion potentials of late endothelial progenitor cells. Cardiovasc Diabetol 2012;11:46.

11. Goldin A, Beckman JA, Schmidt AM, Creager MA. Advanced glycation end products: sparking the development of diabetic vascular injury. Circulation 2006;114:597-605. 
12. Madonna R, De Caterina R. Cellular and molecular mechanisms of vascular injury in diabetes - part II: cellular mechanisms and therapeutic targets. Vascul Pharmacol 2011;54:75-9.

13. Zhou YJ, Yang HW, Wang XG, Zhang H. Hepatocyte growth factor prevents advanced glycation end products-induced injury and oxidative stress through a PI3K/Akt-dependent pathway in human endothelial cells. Life Sci 2009;85: 670-7.

14. Xiang M, Yang M, Zhou C, Liu J, Li W, Qian Z. Crocetin prevents AGEs-induced vascular endothelial cell apoptosis. Pharmacol Res 2006;54:268-74.

15. Chen J, Song M, Yu S, Gao P, Yu Y, Wang H, et al. Advanced glycation endproducts alter functions and promote apoptosis in endothelial progenitor cells through receptor for advanced glycation endproducts mediate overpression of cell oxidant stress. Mol Cell Biochem 2010;335:137-46.

16. Oba T, Tatsunami R, Sato K, Takahashi K, Hao Z, Tampo Y. Methylglyoxal has deleterious effects on thioredoxin in human aortic endothelial cells. Environ Toxicol Pharmacol 2012;34: 117-26.

17. Finkel E. The mitochondrion: is it central to apoptosis? Science 2001;292:624-6.

18. Nakagawa T, Zhu H, Morishima N, Li E, Xu J, Yankner BA, et al. Caspase-12 mediates endoplasmic-reticulum-specific apoptosis and cytotoxicity by amyloid-beta. Nature 2000;403:98-103.

19. Gorlach A, Klappa P, Kietzmann T. The endoplasmic reticulum: folding, calcium homeostasis, signaling, and redox control. Antioxid Redox Signal 2006;8:1391-418.

20. Ron D, Walter P. Signal integration in the endoplasmic reticulum unfolded protein response. Nat Rev Mol Cell Biol 2007;8: 519-29.

21. Bertolotti A, Zhang Y, Hendershot LM, Harding HP, Ron D. Dynamic interaction of BiP and ER stress transducers in the unfolded-protein response. Nat Cell Biol 2000;2:326-32.

22. Hetz C, Glimcher LH. Fine-tuning of the unfolded protein response: assembling the IRE1alpha interactome. Mol Cell 2009;35:551-61.

23. Chaube R, Kallakunta VM, Espey MG, McLarty R, Faccenda A, Ananvoranich S, et al. Endoplasmic reticulum stress-mediated inhibition of NSMase 2 elevates plasma membrane cholesterol and attenuates NO production in endothelial cells. Biochim Biophys Acta 2012;1821:313-23.

24. Tabas I. The role of endoplasmic reticulum stress in the progression of atherosclerosis. Circ Res 2010;107:839-50.

25. Chen Y, Liu CP, Xu KF, Mao XD, Lu YB, Fang L, et al. Effect of taurine-conjugated ursodeoxycholic acid on endoplasmic reticulum stress and apoptosis induced by advanced glycation end products in cultured mouse podocytes. Am J Nephrol 2008;28:1014-22.

26. Yin QQ, Dong CF, Dong SQ, Dong XL, Hong Y, Hou XY, et al. AGES induce cell death via oxidative and endoplasmic reticulum stresses in both human SH-SY5Y neuroblastoma cells and rat cortical neurons. Cell Mol Neurobiol 2012;32:1299-309.

27. Kassi E, Papoutsi Z, Pratsinis H, Aligiannis N, Manoussakis M, Moutsatsou P. Ursolic acid, a naturally occurring triterpenoid, demonstrates anticancer activity on human prostate cancer cells. J Cancer Res Clin Oncol 2007;133:493-500.

28. Dioufa N, Kassi E, Papavassiliou AG, Kiaris H. Atypical induction of the unfolded protein response by mifepristone. Endocrine 2010;38:167-73.
29. Basha B, Samuel SM, Triggle CR, Ding H. Endothelial dysfunction in diabetes mellitus: possible involvement of endoplasmic reticulum stress? Exp Diabetes Res 2012;2012:481840.

30. Tan KC, Chow WS, Ai VH, Metz C, Bucala R, Lam KS. Advanced glycation end products and endothelial dysfunction in type 2 diabetes. Diabetes Care 2002;25:1055-9.

31. Bucala R, Tracey KJ, Cerami A. Advanced glycosylation products quench nitric oxide and mediate defective endotheliumdependent vasodilatation in experimental diabetes. J Clin Invest 1991;87:432-8.

32. Xu B, Chibber R, Ruggiero D, Kohner E, Ritter J, Ferro A. Impairment of vascular endothelial nitric oxide synthase activity by advanced glycation end products. FASEB J 2003;17: 1289-91.

33. Rojas A, Romay S, Gonzalez D, Herrera B, Delgado R, Otero K. Regulation of endothelial nitric oxide synthase expression by albumin-derived advanced glycosylation end products. Circ Res 2000;86:E50-4.

34. Yamagishi S, Fujimori H, Yonekura H, Yamamoto Y, Yamamoto H. Advanced glycation endproducts inhibit prostacyclin production and induce plasminogen activator inhibitor-1 in human microvascular endothelial cells. Diabetologia 1998;41:1435-41.

35. Quehenberger P, Bierhaus A, Fasching P, Muellner C, Klevesath $M$, Hong $M$, et al. Endothelin 1 transcription is controlled by nuclear factor-kappaB in AGE-stimulated cultured endothelial cells. Diabetes 2000;49:1561-70.

36. Bierhaus A, Illmer T, Kasper M, Luther T, Quehenberger P, Tritschler $\mathrm{H}$, et al. Advanced glycation end product (AGE)-mediated induction of tissue factor in cultured endothelial cells is dependent on RAGE. Circulation 1997;96:2262-71.

37. Basta G, Schmidt AM, De Caterina R. Advanced glycation end products and vascular inflammation: implications for accelerated atherosclerosis in diabetes. Cardiovasc Res 2004;63:582-92.

38. Sun C, Liang C, Ren Y, Zhen Y, He Z, Wang H, et al. Advanced glycation end products depress function of endothelial progenitor cells via p38 and ERK $1 / 2$ mitogen-activated protein kinase pathways. Basic Res Cardiol 2009;104:42-9.

39. Zhang K. Integration of ER stress, oxidative stress and the inflammatory response in health and disease. Int J Clin Exp Med 2010;3:33-40.

40. Witte I, Horke S. Assessment of endoplasmic reticulum stress and the unfolded protein response in endothelial cells. Methods Enzymol 2011;489:127-46.

41. Sheikh-Ali M, Sultan S, Alamir AR, Haas MJ, Mooradian AD. Hyperglycemia-induced endoplasmic reticulum stress in endothelial cells. Nutrition 2010;26:1146-50.

42. Rasheed Z, Haqqi TM. Endoplasmic reticulum stress induces the expression of COX-2 through activation of elF2alpha, p38-MAPK and NF-kappaB in advanced glycation end products stimulated human chondrocytes. Biochim Biophys Acta 2012;1823: 2179-89.

43. Calfon M, Zeng H, Urano F, Till JH, Hubbard SR, Harding HP, et al. IRE1 couples endoplasmic reticulum load to secretory capacity by processing the XBP-1 mRNA. Nature 2002;415:92-6.

44. Yoshida H, Matsui T, Yamamoto A, Okada T, Mori K. XBP1 mRNA is induced by ATF6 and spliced by IRE1 in response to ER stress to produce a highly active transcription factor. Cell 2001;107:881-91. 
45. Lee AH, Iwakoshi NN, Glimcher LH. XBP-1 regulates a subset of endoplasmic reticulum resident chaperone genes in the unfolded protein response. Mol Cell Biol 2003;23:7448-59.

46. Harding HP, Zhang Y, Ron D. Protein translation and folding are coupled by an endoplasmic-reticulum-resident kinase. Nature 1999;397:271-4.

47. Zinszner $H$, Kuroda M, Wang X, Batchvarova N, Lightfoot RT, Remotti $\mathrm{H}$, et al. CHOP is implicated in programmed cell death in response to impaired function of the endoplasmic reticulum. Genes Dev 1998;12:982-95.
48. Loughlin DT, Artlett CM. Precursor of advanced glycation end products mediates ER-stress-induced caspase-3 activation of human dermal fibroblasts through NAD(P)H oxidase 4. PLoS One 2010;5:e11093.

49. Inagi R. Inhibitors of advanced glycation and endoplasmic reticulum stress. Methods Enzymol 2011;491:361-80.

50. Piperi C, Adamopoulos C, Dalagiorgou G, Diamanti-Kandarakis E, Papavassiliou AG. Crosstalk between advanced glycation and endoplasmic reticulum stress: emerging therapeutic targeting for metabolic diseases. J Clin Endocrinol Metab 2012;97:2231-42. 


\title{
Crosstalk between Advanced Glycation and Endoplasmic Reticulum Stress: Emerging Therapeutic Targeting for Metabolic Diseases
}

\author{
Christina Piperi, Christos Adamopoulos, Georgia Dalagiorgou, \\ Evanthia Diamanti-Kandarakis, and Athanasios G. Papavassiliou \\ Department of Biological Chemistry (C.P., C.A., G.D., A.G.P.), University of Athens Medical School, \\ 11527 Athens, Greece; and Third Department of Medicine (E.D.-K.), University of Athens Medical \\ School, 'Sotiria' Hospital, 11527 Athens, Greece
}

Context: Advanced glycation, the major posttranslational modification of proteins, DNA, and lipids, is accelerated under conditions of increased oxidative stress, hyperglycemia, and hypoxia contributing to a variety of metabolic diseases such as diabetes mellitus, obesity, inflammation, polycystic ovarian syndrome, ischemic cardiovascular disease, and neurodegenerative disorders. The potential role of advanced glycation in endoplasmic reticulum (ER) homeostasis is largely unknown.

Evidence Acquisition: Basic and clinical peer-reviewed articles on advanced glycation and ER stress related to metabolic regulation were searched in PubMed from 2000-2011. The resulting articles as well as relevant cited references were reviewed.

Evidence Synthesis: Recent evidence indicates that hyperglycemia, hypoxia, and oxidative stress, apart of triggering advanced glycation, can also adversely affect ER function, leading to pathogenic ER stress, followed by the unfolded protein response. The concomitant presence of advanced glycation in the same conditions with ER stress suggests their crosstalk in the progression of diseases associated with hypoxic and oxidative stress.

Conclusion: Current data support the direct or indirect induction of ER stress response by advanced glycation end products or advanced glycation end product precursors in the pathogenesis of metabolic diseases. Inhibitors of advanced glycation acting as potent ER stress modulators with beneficial effects in restoring ER homeostasis and adjusting physiological unfolded protein response level present an emerging therapeutic approach with significant applications, especially in the context of metabolic dysfunction. (J Clin Endocrinol Metab 97: 2231-2242, 2012)

$l^{n}$ $\mathrm{n}$ the last century, an increased prevalence of chronic metabolic disorders including obesity, type 2 diabetes (T2D), and insulin resistance has been observed in developed countries, affecting adults as well as children $(1,2)$. Abnormal insulin action and increased adiposity are the hallmarks of numerous health problems associated with elevated incidence of T2D, hepatobilliary and gall bladder disorders, cardiovascular complications, neurodegenerative diseases, and tumors $(3,4)$. The underlying causative

ISSN Print 0021-972X ISSN Online 1945-7197

Printed in U.S.A.

Copyright (C) 2012 by The Endocrine Society

doi: 10.1210/jc.2011-3408 Received December 20, 2011. Accepted March 21, 2012.

First Published Online April 25, 2012 mechanisms usually involve dysregulation or lack of cooperation between homeostatic mechanisms implicated in nutrient and energy management.

Metabolic dysregulation as characterized by high energy and excess nutrient intake is often associated to unbalanced glucose homeostasis and subsequent insulin secretion affecting a variety of organs, including pancreatic $\beta$-cells, liver, fat, and muscle tissue as well as brain.

Abbreviations: AGE, Advanced glycation end product; ATF6, activating transcription factor 6; CHOP, C/EBP homologous protein; CML, N-carboxymethyl-lysine; CRCT2, CREB-regulated transcription activator 2; elF2 $\alpha$, E74-like factor $2 \alpha$; ER, endoplasmic reticulum; GRP, glucose regulated protein; IRE1, inositol requiring 1; LDL, low-density lipoprotein; NF- $\kappa B$, nuclear factor$\kappa B$; PCOS, polycystic ovarian syndrome; PERK, protein kinase-like ER kinase; RAGE, receptor for $A G E ; R O S$, reactive oxygen species; SOD, superoxide dismutase; SREBP, sterol regulatory element-binding protein; T2D, type 2 diabetes; UPR, unfolded protein response. 
Among the most important posttranslational modifications is the nonenzymatic modification of proteins, lipids, and nucleic acids by glucose, converting them to advanced glycation end products (AGE). Advanced glycation results in irreversible cross-linking of proteins, loss of protein structure and function, followed by apoptosis (5). Hyperglycemic conditions, oxidative stress, and hypoxia accelerate the generation of AGE precursors contributing to variable pathologies such as T2D, metabolic syndrome and obesity, polycystic ovarian syndrome (PCOS), cardiovascular disease, inflammation, and neurodegenerative disorders.

Endoplasmic reticulum (ER), being the primary regulatory organelle of protein synthesis, folding, maturation, and transport, presents the most important nutrient cell sensor and a critical coordinator of metabolic responses. Conditions that challenge ER function such as increased synthesis of misfolded proteins and their accumulation in ER lumen activate an elaborate adaptive response, the unfolded protein response (UPR) (6). Canonical UPR is mediated by three main branches that intersect with different inflammatory and signaling pathways, commonly implicated in metabolic dysfunction (4).

Recent experimental evidence indicates the concomitant presence of AGE and ER stress in the same pathological entities commonly associated with oxidative stress or hypoxia, suggesting a pathophysiological interplay.

\section{Advanced Glycation and Metabolic Implications}

Advanced glycation takes place in all cell types and refers to reaction between reducing sugars and amino residues present in DNA, proteins, and lipids, known as the Maillard reaction. This is followed by rearrangements and final cross-linking to generate AGE (7). AGE constitute a heterogeneous group of compounds of more than 20 members including the 1,2-dicarbonyl precursor compounds glyoxal, methylglyoxal, and the end products, N-carboxymethyl-lysine (CML), pentosidine, and hydroimidazolone being the best characterized, and serving as markers of AGE accumulation in various tissues $(8,9)$.

Although AGE are formed slowly under physiological conditions, their generation and subsequent accumulation are enhanced in hyperglycemic and insulin resistance states as well as during oxidative stress (5). In normoglycemia, however, advanced glycation can be induced by hypoxia and oxidative stress as characterized by increased production of reactive oxygen species (ROS). Under hypoxic conditions, it is triggered by mitochondrial dysfunction followed by ROS generation and altered adaptive hypoxic response. A vicious circle is therefore developed between conditions of hypoxia, oxidative stress, and enhanced advanced glycation (5).

Diet presents an alternative source of AGE in the human body along with consumption of beverages and cigarette smoke (10). Food processing such as prolonged heating, frying, or grilling accelerates the glycol oxidation and lipoxidation reactions, resulting in increased formation of AGE (10-13). Intake of high-AGE diets has been correlated with increased circulating AGE levels and insulin resistance as well as tissue deposition interfering with metabolic homeostasis $(13,14)$. Administration of the pancreatic lipase inhibitor orlistat in women with PCOS after a high-AGE diet intake was able to acutely reduce serum AGE levels and improve their metabolic profile (15-17).

AGE effects can be classified as receptor dependent or independent, and their mode of action is both intracellular and extracellular. Intracellular effects are mediated through binding of AGE to specific receptors (RAGE) present on cell surface, triggering the activation of signaling pathways such as protein kinase C, MAPK, ROS, and nitric oxide (5), with most of them converging in the activation of transcription factor nuclear factor $-\kappa \mathrm{B}(\mathrm{NF}-\kappa \mathrm{B})$ that regulates transcription of a number of proteins implicated in endothelial function (vascular cell adhesion protein-1, intercellular adhesion molecule-1, endothelin-1) and inflammation (proinflammatory cytokines, IL-1 $\beta$, IL-6) (18-20). Extracellular effects of AGE include modification of structural proteins, usually connective tissue components such as collagen type IV and laminin, leading to alteration of structure and function, increased stiffness, and resistance to proteolytic digestion (21-23).

Every tissue in the human body can be affected by AGE, including kidney, liver, arteries, reproductive, muscle, skeletal tissue, and brain, whereas there is increasing evidence that sustained exposure to AGE is related to the risk of metabolic disorders and adverse aging-related outcomes (24-26). Apart from diabetes and its complications, AGE are associated with certain liver diseases such as nonalcoholic steatohepatitis and cirrhosis due to their clearance through liver as well as to end-stage renal disease due to their metabolism by the kidney (27). Deposition of AGE in arterial walls, especially in the elastic membrane and intimal extracellular matrix, allows formation of cross-links with matrix proteins in the wall of blood vessels, reducing elasticity and promoting vessel rigidity, therefore implicating AGE in atherosclerosis (28). Recently, increased localization of AGE has been observed in the female reproductive system of experimental animals, after intake of a high-AGE diet (29), and in the ovarian 


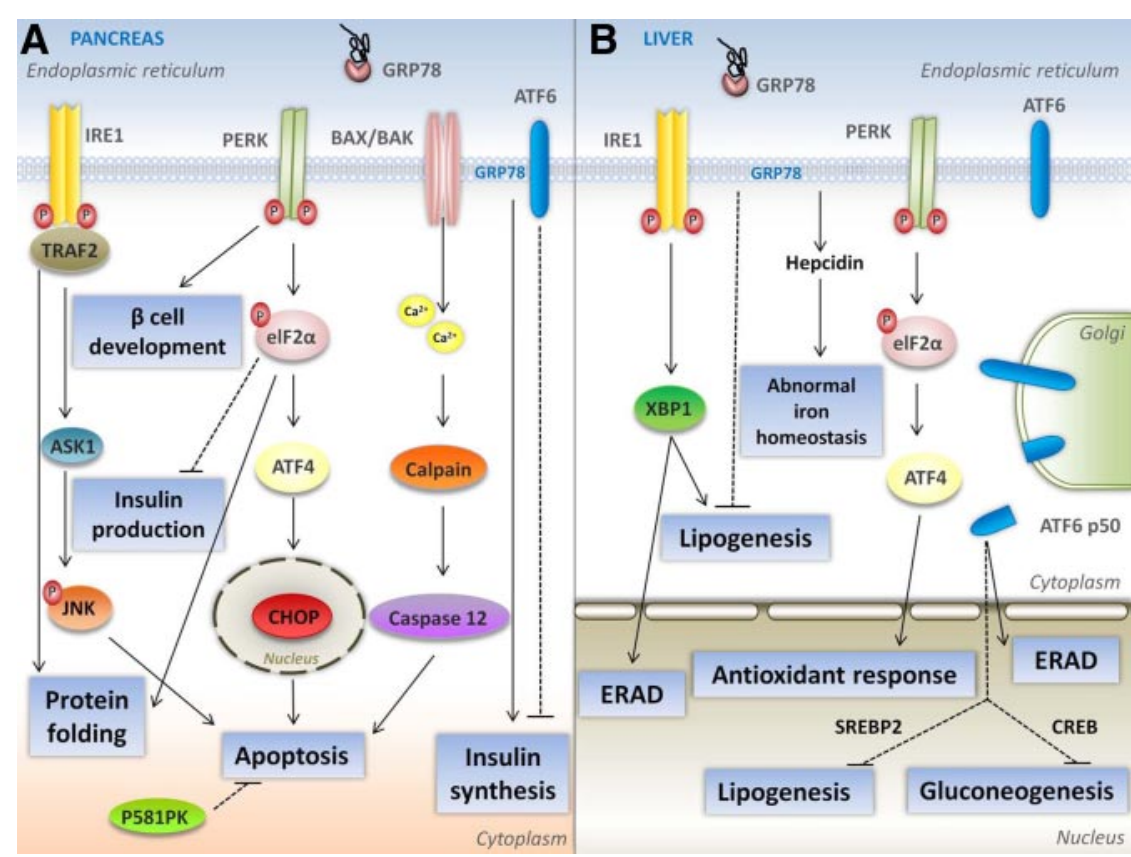

FIG. 1. UPR pathways in metabolic regulation. Under ER stress, the canonical UPR pathways are implicated in the homeostasis of glucose, insulin synthesis, and lipid metabolism. A, The pancreatic $\beta$-cell controls the synthesis, folding, and production of insulin through the ER. PERK expression is a requirement for $\beta$-cell formation and survival during early stages of development, along with another chaperone, p581PK, that is induced in ER stress (51, 52, $110,111)$. Insulin production requires IRE 1 , and elF $2 \alpha$ phosphorylation is necessary to halt insulin synthesis until folding needs are met (47). The loss of gene WFS1 that encodes the protein wolframin present in ER membrane leads to ER stress and cellular death (112). Prolonged ER stress in $\beta$-cells results in apoptosis through c-Jun terminal kinase phosphorylation mediated via IRE-1 activation as well as through CHOP, a downstream mediator of UPR (113). Down-regulation of CHOP in conditions of high insulin requirements leads in $\beta$-cell survival and amelioration of glucose homeostasis (114). B, In the liver, UPR is implicated in lipogenesis and gluconeogenesis. The activity of XBP1 in liver cells, which is downstream of the UPR sensor IRE1a, regulates transcription of many genes implicated in fatty acid synthesis (115). In addition, overexpression of Grp78 inhibits the activation of SREBP1 and SREBP2, the major transcriptional regulators of lipogenic gene expression, and reduces hepatic steatosis in obese mice (116). In hepatocytes, ER stress also induces the production of hepcidin, an iron-regulating peptide hormone, leading to abnormal iron metabolism (117). [Adapted and reprinted in modified form from G. S. Hotamisligil: Endoplasmic reticulum stress and the inflammatory basis of metabolic disease. Cell 140:900917, 2010 (4), with permission. (C) Elsevier.]

tissue of women with PCOS, correlating positively with an altered metabolic (insulin resistance) and hormonal (hyperandrogenemia) profile (30-32). Furthermore, muscle tissue can also be affected from AGE deposition due to collagen cross-linking in muscle tendons and cartilage that leads to muscle stiffness and reduced muscle function (33, 34). Emerging evidence suggests AGE involvement in bone remodeling and in reduced bone quality due to altered mechanical properties and increased stiffness of collagen matrix $(35,36)$. At last, neurofibrillary tangles and senile plaques can also be sites of AGE accumulation in aging human brain as well as in Alzheimer's disease, where they are implicated in cognitive deficits $(37,38)$.

Adverse AGE-mediated signaling from all major metabolic tissues is then directed to ER lumen where cell fate decisions are made through a specialized protein network.

\section{ER Response and Metabolic Homeostasis}

ER is envisioned as a complex network spread throughout the cytoplasm that can detect and transmit signals originating in any compartment of the cell. It is implicated in essential cellular activities including $\mathrm{Ca}^{2+}$ storage and signal transduction $(39,40)$. Specialized chaperones and folding enzymes control ER homeostasis in the cells by keeping the balance between the cellular need for protein synthesis and proper protein folding. However, various conditions and external inputs are capable to disrupt ER homeostatic environment. Situations of excess nutrients, insulin resistance, and inflammation commonly associated with obesity induce ER stress, compromising cellular function $(41,42)$. Other disturbances that provoke ER stress include disrupted calcium homeostasis, lack of energy or glucose, changes in ROS level, ischemia, mutations, and viral infections (43).

The sensory machinery of ER stress is composed of three proteins bound to ER membrane, namely double-stranded RNA-dependent protein kinase-like ER kinase (PERK), inositol requiring 1 (IRE1), and activating transcription factor 6 (ATF6). Upon activation, these sensor molecules transduce a signaling cascade from ER to nucleus, the UPR. Several downstream effectors are regulated by UPR that lead to adaptive response, feedback control, and regulation of cell fate (44). Adaptation initially involves activation of molecular chaperones and folding enzymes to achieve enhanced protein-folding activity. This leads to reduced ER workload through mRNA degradation and attenuation of translation as well as elevated ER-associated protein degradation and clearance of unwanted proteins through autophagy. In the case of harmful UPR hyperactivation, however, UPR master regulators are switched off along with downstream signaling molecules to resolve the unwanted effects (44). The UPR is therefore responsible, apart from cell survival, to also promote cell death (Fig. 1). Indeed, in situations of prolonged ER stress due to either chronic exposure to high glucose or genetic mutations, UPR activates death receptors through the same three transducers, acting as a 
"binary switch" and leading to apoptosis (44). The deleterious effects of unresolved and sustained ER stress such as perturbed calcium homeostasis, accumulation of unfolded proteins, loss of ER function, and apoptosis have been associated with the development of pathological conditions such as inflammation, diabetes, atherosclerosis, and neurodegenerative disorders.

ER homeostasis is particularly important for human metabolism because it coordinates the response of all metabolic organs by controlling the synthesis and catabolism of various nutrients. ER controls insulin synthesis, folding, and production in the pancreatic $\beta$-cell (Fig. 1A). Glucose metabolism involves all the three branches of UPR, and ER is implicated in the transcription of most glucose-regulating genes (45). Experimental animal models harboring UPR deficiency (PERK-targeted) or mutation disrupting elF2 $\alpha$ phosphorylation show defective gluconeogenesis in their livers $(42,46)$ and subsequent pancreatic islet cell dysfunction or death linked to ER failure (47). Additionally, mice expressing the C-terminal fragment of Gadd34 in liver demonstrate defective gluconeogenesis and reduced hepatic glycogen that lead to problems in blood glucose handling during fasting (48). Furthermore, ER stress triggers transcriptional networks that target genes implicated in glucose output and glycogen synthesis such as glycogen synthase 1 , an XBP1 target (45, 49). Another component of ER machinery that is implicated in glucose production in the liver is ATF6, which is regulated through the interaction with the cAMP response element binding-regulated transcription activator 2 (CRTC2/ TORC2) (45). ER stress leads to dephosphorylation of CRTC2 and entry in the nucleus of cultured cells where it promotes expression of ER control genes through binding to ATF6. ATF6 activation reduces glucose output in the liver through dissociation of the cAMP response element bindingCRTC2 complex, thus inhibiting CRTC2 interaction with gene promoters implicated in gluconeogenesis (45).

UPR response is also involved in the control of cellular lipogenesis because ER plays a regulatory role in the synthesis of fatty acids and cholesterol. This is mediated through activation of the transcription factors, sterol regulatory element-binding protein (SREBP) located in the ER (50). When sterol or insulin levels are low, SREBP are translocated to the Golgi, where they are processed and activated (51). ER stress may also induce SREBP activation and thus regulate the transcription of target genes involved in lipid synthesis (SREBP1c) or cholesterol metabolism (SREBP1a, SREBP2) (52). ER stress also interferes with lipid metabolism in the liver, along with iron and glucose homeostasis (Fig. 1B).

Furthermore, UPR pathways are linked to stress signaling through activator protein-1 and to inflammation through induction of NF- $\kappa$ B pathway, nitric oxide, and
ROS production (4). These pathways play a central role in metabolic abnormalities characterized by abnormal insulin action and obesity-induced inflammation. The interplay of ER stress and inflammation is two-sided, with inflammatory mediators and several cellular pathways having a negative input on ER homeostasis. This relationship is cell type-dependent, and further research is required to establish the exact mediators of each cell's susceptibility to inflammation and ER homeostasis. Advanced glycation being a major trigger of inflammatory response, hypoxia and oxidative stress could also be involved in modulation of ER homeostasis (Fig. 2).

\section{Crosstalk between AGE and ER Stress Signaling}

Evidence of the direct induction of ER stress by AGE comes from the original study of Chen et al. (53) where murine podocytes exposed to AGE-BSA showed increased expression of glucose regulated protein (GRP)78 (an ER stress marker) and apoptosis in a dose- and time-dependent manner. This effect was accompanied by increased intracellular $\mathrm{Ca}^{2+}$ levels and inhibited by tauroursodeoxycholic acid, an ER stress inhibitor with chaperone function that promotes proper folding and trafficking. The reduction of apoptosis that was observed by using this inhibitor indicates the involvement of UPR apoptotic pathway in mediating AGE effects rather than canonical RAGE signaling, providing an important molecular mechanism implicated in diabetic nephropathy (53).

In a similar way, when human osteoarthritic chondrocytes were exposed to AGE-BSA, an increased expression of GRP78 was observed, followed by elevated expression of IL-6 and IL-8. This effect was mediated through RAGE via p-38 MAPK activation, providing an alternative mechanism that contributes to the pathogenesis of osteoarthritis (54).

Subsequent studies with human dermal fibroblasts showed that deoxyglucosone, an AGE-precursor, is associated with ER stress-induced apoptosis. Collagen type I modified by 3-deoxyglucosone induces ROS and apoptosis mediated through ER stress pathway [via C/EBP homologous protein (CHOP) activation] and not through the RAGE signaling (55), providing a link between AGE and ER stress in the pathogenesis of diabetic wounds. These data are in accordance with preliminary findings of our group where glycated albumin induced direct activation of ER stress through GRP78 in endothelial cells (human aortic endothelial cells) that was followed by CHOP induction and apoptosis in a concentration-dependent manner.

Another study of endothelial cells from bovine aortas incubated with oxidized low-density lipoprotein (LDL) 


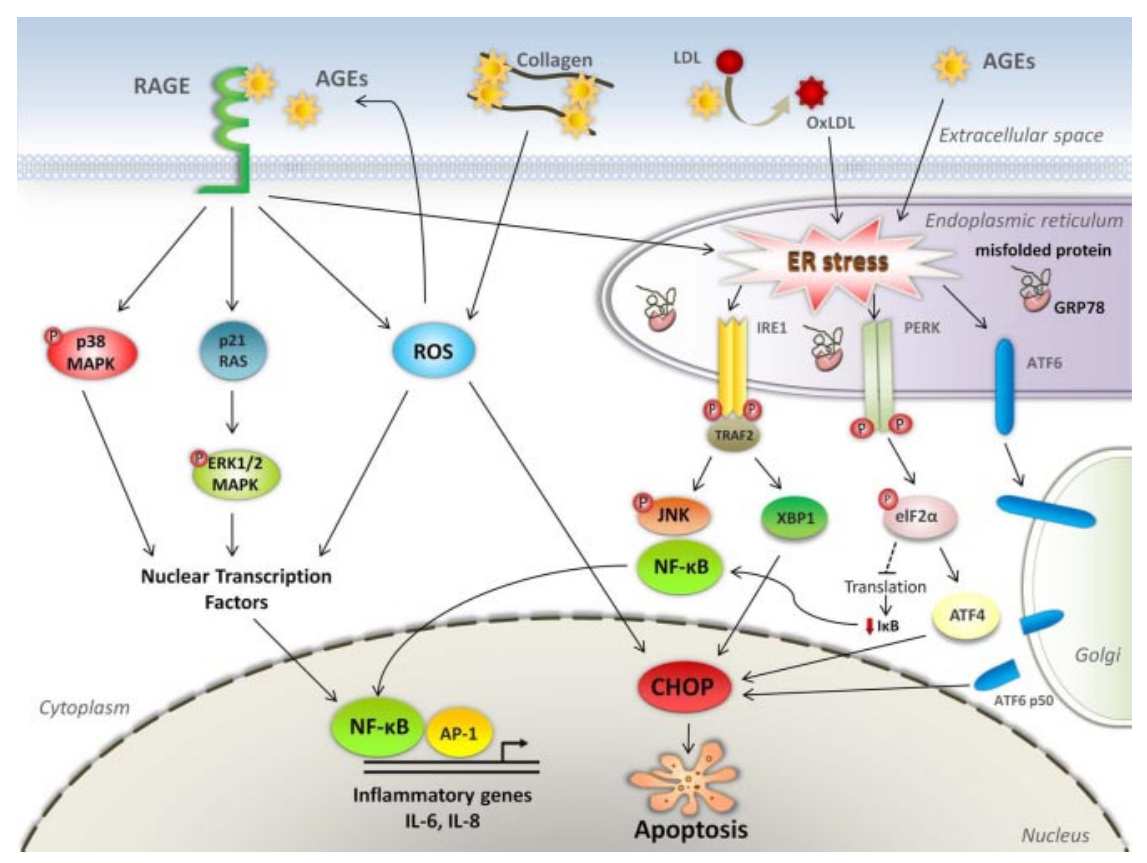

FIG. 2. Crosstalk of AGE signaling and UPR. AGE and oxidized-LDL can directly or indirectly (through receptor for AGE) induce ER stress. AGE-RAGE signaling and AGE-modified longlived proteins (such as collagen) lead to increased ROS production and UPR, via CHOP activation, resulting in apoptosis. AGE and UPR pathways are linked to stress signaling through activator protein-1 and to inflammation through induction of NF- $\kappa$ B pathway and ROS production.

showed increased ER stress markers expression, GRP78, p-PERK and activated ATF6 mediated through AMP-activated protein kinase activation (56). These effects were accompanied by impaired vasorelaxation of the endothelium and subsequent endothelial dysfunction in mice fed with a rich-in-fat diet.

\section{Indirect Mechanisms Contributing to ER Stress Induction by AGE}

The close link of AGE with oxidative stress, low or high glucose conditions, RAGE-mediated inflammation, hypoxia, and aging allocates them as likely contributors of the integrated ER response in metabolic regulation (Fig. 2).

\section{Oxidative Stress}

It is known that production of ROS by oxidative stress enhances AGE formation, therefore altering protein function. ROS, however, also interfere with the protein-folding process and disulfide bonds leading to misfolding of proteins in the ER. Furthermore, ROS presence enhances adipocyte differentiation and lipid accumulation, leading to ER stress followed by insulin resistance $(57,58)$.

There is evidence that intake of a high-fat diet can induce both AGE formation and oxidative stress, which further re- sult in ER stress, impairing insulin action through insulin receptor substrate 1 phosphorylation in liver or adipose tissue of dietary and genetic mouse models of obesity $(53,59-62)$. In a more recent study, increased AGE and ROS were observed in the hippocampus of mice fed with a rich-in-cholesterol diet and promoted ER stress induction through increased phosphorylation of PERK and elF2 $\alpha$. ER stress further impaired insulin signaling by increasing IRS1 phosphorylation, reducing phosphoinositide kinase-3/Akt activity and inducing NF- $\kappa \mathrm{B}$ activation (63).

Indirect evidence of the pathogenic interrelation of ROS generation, advanced glycation, and ER stress comes also from studies with antioxidant enzymes such as superoxide dismutase (SOD). Transgenic animals overexpressing copper-zinc SOD showed reduced ischemic neuronal cell death mediated through ATF4 and CHOP than in wild-type hippocampus (64). Similarly, mice deficient in cytoplasmic copper-zinc SOD exhibited increased plaque and neuronal inflammation, accompanied by AGE production (CML) and oxidative damage leading to memory impairment (65). Furthermore, in cultures of renal proximal tubular cells, cadmium was able to induce increased ROS production and subsequent ER stress leading to apoptosis that was prevented when cells were transfected with manganese SOD (66). Moreover, manganese SOD was found to attenuate advanced glycation and methylglyoxal production by decreasing ROS production in mitochondria of human aortic endothelial cells cultured in high glucose (67).

\section{Glucose Levels}

Low glucose levels due to starvation or other metabolic dysregulation leading to energy loss have a detrimental effect in protein folding, resulting in unfolded protein accumulation in the ER lumen (5). Hyperglycemia on the other hand is the main trigger of ROS formation involved in AGE formation and ER stress induction, further mediating the metabolic complications of diabetes, obesity, and neurodegeneration $(19,20,64,67)$. Various studies have shown the increased ROS generation due to high glucose that enhances AGE precursor formation and advanced glycation contributing to ER stress manifestations such as 
inflammation. In addition, resident proteins of the ER (chaperones or folding sensor proteins) that control protein folding can also be modified by oxidation or glycation altering the UPR adaptive ability $(68,69)$.

\section{Inflammation}

AGE-RAGE signaling pathway in endothelium may result in NF- $\kappa \mathrm{B}$ activation either directly or through MAPK cascades (ERK1/2 and p38) resulting in proinflammatory cytokine production $(13,28,70,71)$. Cytokines are also able to induce ER stress leading to chronic inflammation, indicating that the effects of advanced glycation and ER stress can be mediated through RAGE $(3,4)$. Additionally, a study of infantile neuronal ceroid lipofuscinosis showed that elevated ER stress stimulated S100B and RAGE expression in astroglial cells resulting in proinflammatory cytokine production (72). Therefore, ER stress can further stimulate AGE-RAGE signaling, exacerbating oxidative stress and inflammation.

\section{Hypoxia}

Protein folding can be severely affected by hypoxic conditions where aberrant oxygen tension or loss of energy due to metabolic disturbances can occur (5). The resulted unfolded protein load and protein aggregates in the ER induce ER stress (5). Accumulation of AGE and proapoptotic UPR signaling has been observed in conditions of hypoxic reperfusion or ischemia of the heart, kidney, or brain, indicating their role in the pathogenesis of hypoxic injury $(73,74)$. Studies focused on kidney damage by these conditions point out the increased cytotoxicity of methylglyoxal, which leads to apoptosis through ER stress. Inhibition of methylglyoxal by an AGE detoxifying enzyme, glyoxalase I, or inhibition of UPR by GRP78 overexpression further improves kidney damage $(5,73,74)$.

Hypoxic environment is commonly a hallmark of malignant cells due to poor vascularization of most solid tumors leading to ER stress and energy loss. The hypoxiainduced UPR is thus crucial for tumor cell survival ( 75 , 76). Induced GRP78 expression in tumor cells improves protein folding and correlates positively with tumor proliferation, metastasis, and drug resistance (77). Furthermore, the presence of AGE is also increased in a range of tumors correlating with metastasis and reduced survival interrelating AGE and ER stress in tumorigenesis (78).

\section{Aging}

The aging process has been ultimately associated with elevated AGE presence contributing to chronic diabetic complications, senescence, and age-related diseases. At the same time, molecular chaperones and ER-resident enzymes are altered by aging, further compromising UPR adaptive properties (69). Two studies performed on Caenorhabditis elegans underline the role of AGE and ER stress in aging. C. elegans either overexpressing glyoxalase I homolog or being deficient in HIF-1, a hypoxic transcription factor, showed increased life span due to ROS or ER stress reduction, respectively $(79,80)$. The combination of both interventions may be the secret of cellular homeostasis and longevity.

\section{Targeting Advanced Glycation Improves ER Homeostasis}

The crosstalk of advanced glycation with ER stress signaling underlies the pathophysiology of several disorders related to metabolism as well as ischemic and neurodegenerative diseases. Current evidence indicates that some inhibitors of advanced glycation may prove beneficial in maintaining ER homeostasis through modulation of UPR response (Table 1).

\section{AGE Formation Inhibitors}

Aminoguanidine, a well-established AGE inhibitor that has the ability to sequester the toxic 1,2-dicarbonyl compounds and render them into nontoxic triazines has been currently tested in a rat model with diabetic nephropathy (81). It was proven effective in up-regulating PERK, therefore alleviating associated ER stress and reducing inflammatory cytokine production (81).

\section{Hypoglycemic Agents}

The bisguanidine metformin is a glucose-lowering agent with antioxidant properties and a common antidiabetic drug. Previous studies along with our data have shown that metformin acts as a moderate AGE inhibitor through its reaction with methylglyoxal to form dihydroimidazolone, a less potent derivative $(82,83)$. Recently, metformin was found to reduce ER stress induction in hepatocytes (HepG2) through blockage of GRP78, CHOP, and XBP-1, suggesting its potential use in nonalcoholic fatty liver disease (84). Furthermore, metformin has a protective role against dysfunction and ER stress-induced death in pancreatic $\beta$-cells (NIT-1) (85). Another hypoglycemic agent, pioglitazone, known to decrease AGE formation (86), is also able to reduce ER stress in liver and reduce 
TABLE 1. AGE inhibitors targeting ER stress

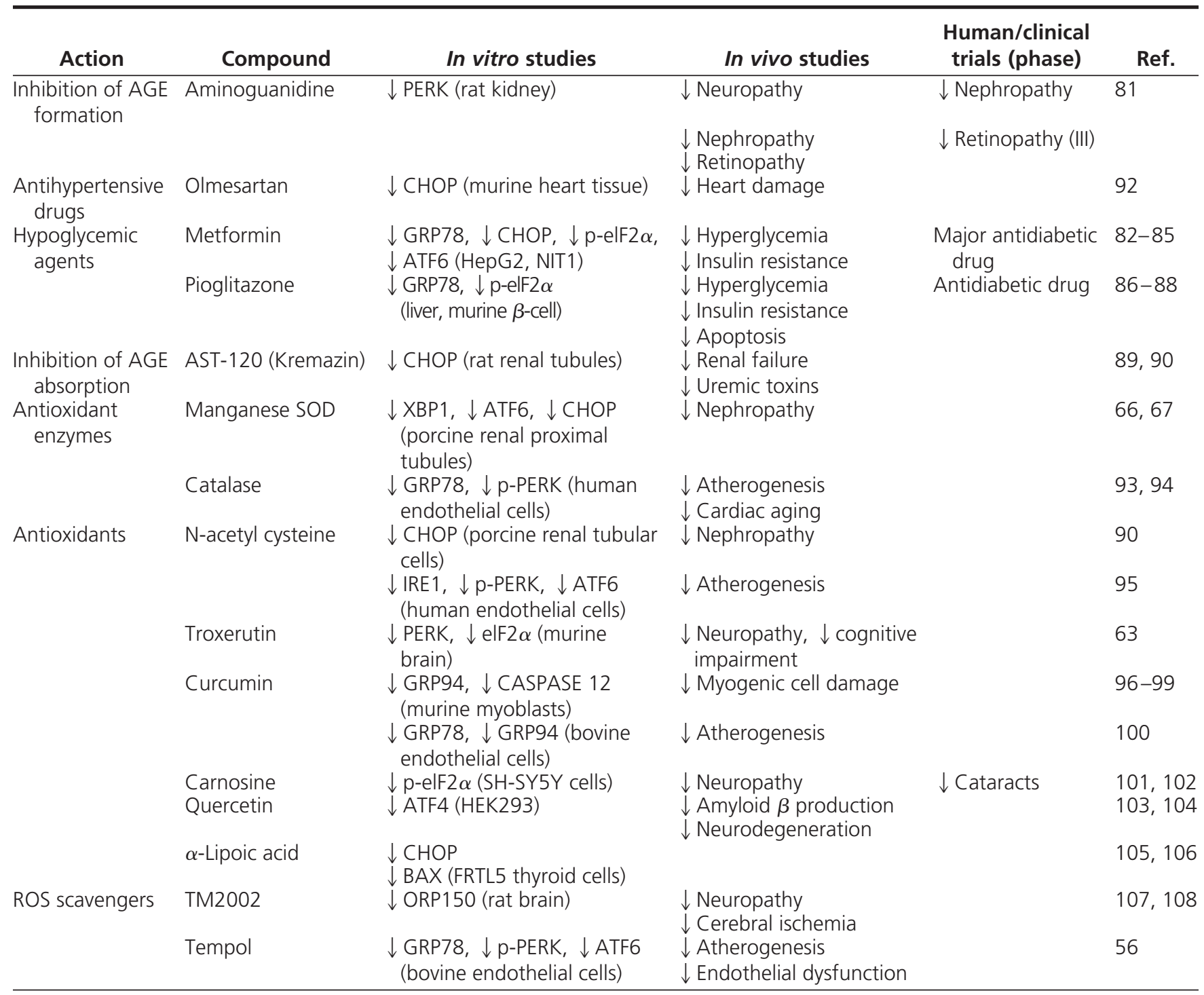

insulin resistance in diabetic mice (87) as well as in pancreatic $\beta$-cells of Wfs1-knockout mice (88).

\section{AGE Absorption Inhibitors}

A novel approach to reduce the deleterious effects of AGE is to block their absorption with oral absorbents. AST-120 is able to bind CML and decrease AGE levels in diabetic subjects with chronic renal failure (89). The same agent when administered in a nephrectomized rat model with chronic kidney disease was able to decrease tubular expression of CHOP, thus suppressing disease progression (90).

\section{Antihypertensive Drugs}

Antihypertensive drugs (angiotensin type 1 receptor blockers and inhibitors of angiotensin-converting en- zyme) acting on the renin-angiotensin system reduce oxidative stress through ROS scavenging and at the same time inhibit advanced glycation (91). In parallel, ER stress is induced by angiotensin II, suggesting an interaction between these pathways. In the study by Okada et al. (92), apoptosis of cardiac myocytes during hypertrophy and heart failure of mice subjected to transverse aortic constriction may be mediated through prolonged ER stress. The angiotensin type 1 receptor blocker olmesartan prevented ER stress induction and proapoptotic cascade in this model, improving heart damage (92).

\section{Antioxidants}

Various antioxidant compounds have been studied relative to their action over reduction of advanced glycation. Their potent reducing effect is attributed to reduction of 
ROS formation mainly through scavenging of free radicals and inhibition of glycoxidation. The scavenging activity of these agents is also capable of modulating UPR. In addition to SOD, catalase, an antioxidant enzyme involved in $\mathrm{H}_{2} \mathrm{O}_{2}$ detoxification, has been found to reduce AGE levels in cardiomyocytes during cardiac aging of transgenic mice overexpressing the cardiac isoform of the enzyme (93). In parallel, in human endothelial cells, catalase was effective in blocking UPR activation induced by tunicamycin and HIV-1 Tat (ER stressors) (94).

The precursor of the anti-oxidant glutathione, $\mathrm{N}$-acetyl cysteine inhibits ER stress-mediated apoptosis through inhibition of CHOP activation in tubular cells of patients with end-stage renal failure, therefore stabilizing ER homeostasis (90). In addition, $\mathrm{N}$-acetyl cysteine inhibits lipoxidation- and oxidized LDL-induced ER stress in human endothelial cells implicated in atherogenesis (95).

Troxerutin, a natural flavonoid, is able to decrease AGE levels and concomitantly reduce oxidative and ER stress in cholesterol-induced cognitive impairment in mice (63). It reduced PERK and elF $2 \alpha$ as well as NF- $\kappa$ B activation due to ER stress in the hippocampus and enhanced insulin signaling, thus preventing obesity and restoring blood glucose levels. The antioxidant and antiinflammatory effects of troxerutin make it a possible candidate for prevention of cognitive deficits in T2D and Alzheimer's disease.

Curcumin, a natural polyphenol with antioxidant capacity, acts through scavenging of superoxide anions and

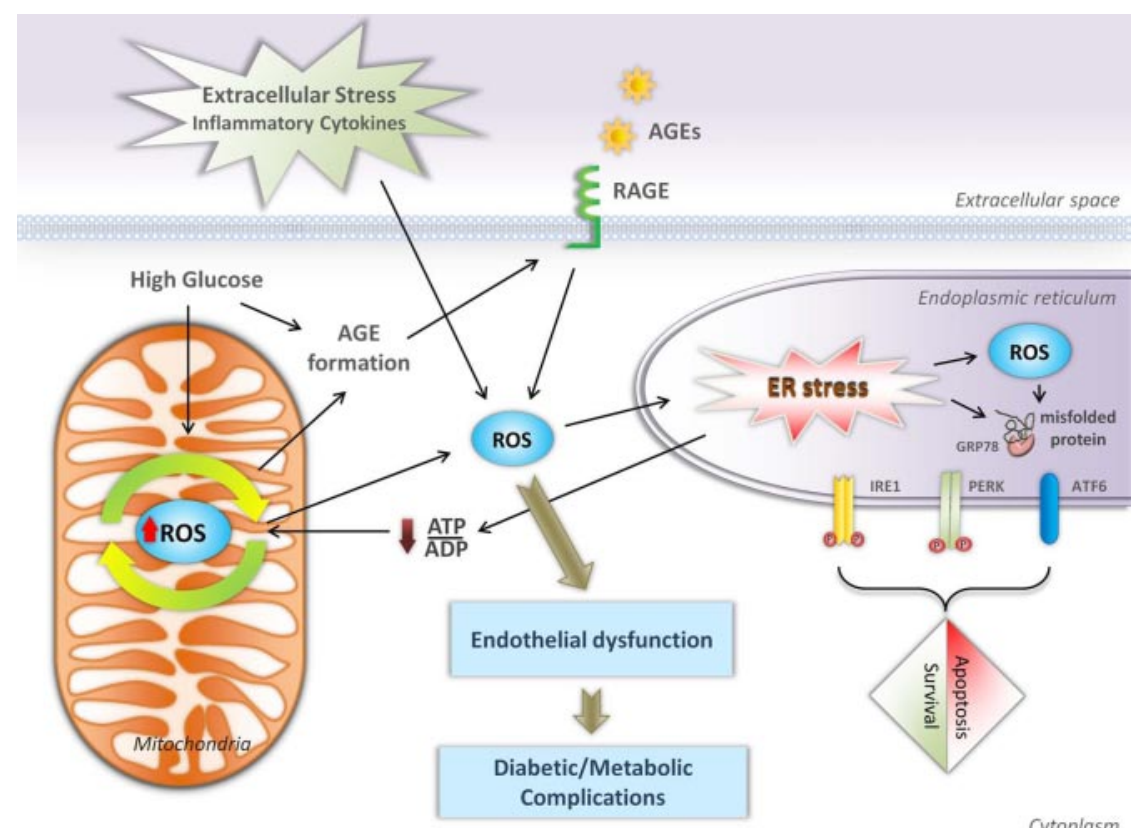

FIG. 3. Parallel and linear induction of AGE and ER stress in the pathology of metabolic diseases. Extracellular stress, AGE-RAGE signaling, intracellular hyperglycemia, or mitochondrial respiration by-products induce ROS generation that directly or indirectly affect ER homeostasis and protein folding. Alteration in redox status or overproduction of ROS is the primary and central event in the pathogenesis of diabetic/metabolic complications. hydroxyl radicals, being effective in reducing AGE levels and collagen cross-linking as well as decreasing oxidative stress and inflammation in diabetic rats $(96,97)$ and humans (98). Recently, curcumin has been associated with induction of the ER stress chaperone, GRP94, in myogenic cell damage where it restores calcium levels and protects from apoptosis (99). Lead-induced ER stress in endothelial cells of bovine aortas is inhibited by pretreatment with curcumin that specifically increases GRP78 and GRP94 expression (100).

Other antioxidant agents such as carnosine $(101,102)$, quercetin $(103,104)$, and $\alpha$-lipoic acid $(105,106)$ have been found effective in reducing advanced glycation in vivo as well as inhibiting ER stress response in independent studies. However, their concomitant action in these processes and estimation of physiological relevant doses to achieve antioxidant capacity seek further evaluation.

\section{ROS Scavengers}

Newly synthesized ROS scavenging compounds, such as TM2002 and tempol, were the first to be tested in experimental models. TM2002, being a derivative of edaravone, is a nontoxic scavenger of low molecular weight that inhibits AGE production by reducing oxidative stress. TM2002 has been tested in rat models of cerebral ischemia with neuroprotective properties that were attributed to its ability to reduce advanced glycation, oxidative stress, and ER stress (107). Although the clinical use of this agent is prohibited due to severe side effects, several new derivatives have been synthesized and are under testing (108).

Another scavenger of free radicals, namely tempol, mimics the effects of SOD and presents antioxidant properties in vitro and in vivo (56). Tempol is efficient in inhibiting LDL glycation and blocks associated ER stress response that leads to endothelial deregulation.

\section{Chemical Chaperones}

Several chemical chaperones that alleviate ER stress with protective role toward ER stress in liver have been found useful in protecting animals from obesity-induced ER stress (109). Administration of the chaperones in obese mice 
established normoglycemia and thus reduced advanced glycation, increased insulin sensitivity, and reduced fatty liver disease. The treatment suppressed inflammatory signaling along with ER stress and accelerated signal transduction through insulin receptor in liver and adipose tissue (109).

\section{Conclusions}

Current evidence indicates the mutual induction of AGE and ER stress in the pathology of various metabolic diseases. Key mediators of this crosstalk seem to be oxidative stress generated in mitochondria or ER, inflammation, and hyperglycemia (Fig. 3). Maintenance or enhancement of ER proper function through concerted up-regulation of protein-folding chaperones, metabolic hormones with protective effects, and direct targeting of the implicated transcription factors in metabolic active tissues may prove powerful in treating chronic metabolic diseases.

\section{Acknowledgments}

Address all correspondence and requests for reprints to: Professor Athanasios G. Papavassiliou, Department of Biological Chemistry, Medical School, University of Athens, 75, M. Asias Street, 11527 Athens, Greece. E-mail: papavas@med.uoa.gr.

Disclosure Summary: The authors have nothing to disclose.

\section{References}

1. Hossain P, Kawar B, El Nahas M 2007 Obesity and diabetes in the developing world-a growing challenge. N Engl J Med 356:213215

2. Ogden CL, Carroll MD, Curtin LR, Lamb MM, Flegal KM 2010 Prevalence of high body mass index in US children and adolescents. JAMA 303:242-249

3. Hotamisligil GS 2006 Inflammation and metabolic disorders. Nature 444:860-867

4. Hotamisligil GS 2010 Endoplasmic reticulum stress and the inflammatory basis of metabolic disease. Cell 140:900-917

5. Inagi R 2011 Inhibitors of advanced glycation and endoplasmic reticulum stress. Methods Enzymol 491:361-380

6. Ron D, Walter P 2007 Signal integration in the endoplasmic reticulum unfolded protein response. Nat Rev Mol Cell Biol 8:519-529

7. Cho SJ, Roman G, Yeboah F, Konishi Y 2007 The road to advanced glycation end products: a mechanistic perspective. Curr Med Chem 14:1653-1671

8. Brownlee M 2001 Biochemistry and molecular cell biology of diabetic complications. Nature 414:813-820

9. Thornalley JP 1996 Advanced glycation and the development of diabetic complications: unifying the involvement of glucose, methylglyoxal and oxidative stress. Endocrinol Metab 3:149-166

10. Cerami C, Founds H, Nicholl I, Mitsuhashi T, Giordano D, Vanpatten S, Lee A, Al-Abed Y, Vlassara H, Bucala R, Cerami A 1997 Tobacco smoke is a source of toxic reactive glycation products. Proc Natl Acad Sci USA 94:13915-13920
11. Koschinsky T, He CJ, Mitsuhashi T, Bucala R, Liu C, Buenting C, Heitmann K, Vlassara H 1997 Orally absorbed reactive glycation products (glycotoxins): an environmental risk factor in diabetic nephropathy. Proc Natl Acad Sci USA 94:6474-6479

12. Nicholl ID, Stitt AW, Moore JE, Ritchie AJ, Archer DB, Bucala R 1998 Increased levels of advanced glycation endproducts in the lenses and blood vessels of cigarette smokers. Mol Med 4:594-601

13. Vlassara H, Cai W, Crandall J, Goldberg T, Oberstein R, Dardaine V, Peppa M, Rayfield EJ 2002 Inflammatory mediators are induced by dietary glycotoxins, a major risk factor for diabetic angiopathy. Proc Natl Acad Sci USA 99:15596-15601

14. Zheng F, He C, Cai W, Hattori M, Steffes M, Vlassara H 2002 Prevention of diabetic nephropathy in mice by a diet low in glycoxidation products. Diabetes Metab Res Rev 18:224-237

15. Diamanti-Kandarakis E, Piperi C, Kalofoutis A, Creatsas G 2005 Increased levels of serum advanced glycation end-products in women with polycystic ovary syndrome. Clin Endocrinol (Oxf) 62:37-43

16. Diamanti-Kandarakis E, Katsikis I, Piperi C, Alexandraki K, Panidis D 2007 Effect of long-term orlistat treatment on serum levels of advanced glycation end-products in women with polycystic ovary syndrome. Clin Endocrinol (Oxf) 66:103-109

17. Diamanti-Kandarakis E, Piperi C, Alexandraki K, Katsilambros N, Kouroupi E, Papailiou J, Lazaridis S, Koulouri E, Kandarakis HA, Douzinas EE, Creatsas G, Kalofoutis A 2006 Short-term effect of orlistat on dietary glycotoxins in healthy women and women with polycystic ovary syndrome. Metabolism 55:494-500

18. Goldin A, Beckman JA, Schmidt AM, Creager MA 2006 Advanced glycation end products: sparking the development of diabetic vascular injury. Circulation 114:597-605

19. Schiekofer S, Andrassy M, Chen J, Rudofsky G, Schneider J, Wendt T, Stefan N, Humpert P, Fritsche A, Stumvoll M, Schleicher E, Häring HU, Nawroth PP, Bierhaus A 2003 Acute hyperglycemia causes intracellular formation of CML and activation of ras, p42/44 MAPK, and nuclear factor- $\kappa$ B in PBMCs. Diabetes 52:621633

20. Yan SD, Schmidt AM, Anderson GM, Zhang J, Brett J, Zou YS, Pinsky D, Stern D 1994 Enhanced cellular oxidant stress by the interaction of advanced glycation end products with their receptors/binding proteins. J Biol Chem 269:9889-9897

21. Vlassara H 1996 Advanced glycation end-products and atherosclerosis. Ann Med 28:419-426

22. Haitoglou CS, Tsilibary EC, Brownlee M, Charonis AS 1992 Altered cellular interactions between endothelial cells and non enzymatically glycosylated laminin/type IV collagen. J Biol Chem 267: 12404-12407

23. Zieman SJ, Melenovsky V, Kass DA 2005 Mechanisms, pathophysiology, and therapy of arterial stiffness. Arterioscler Thromb Vasc Biol 25:932-943

24. Hammes HP, Alt A, Niwa T, Clausen JT, Bretzel RG, Brownlee M, Schleicher ED 1999 Differential accumulation of advanced glycation end products in the course of diabetic retinopathy. Diabetologia 42:728-736

25. Bucala R, Vlassara H 1995 Advanced glycosylation end products in diabetic renal and vascular disease. Am J Kidney Dis 26:875888

26. Makita Z, Bucala R, Rayfield EJ, Friedman EA, Kaufman AM, Korbet SM, Barth RH, Winston JA, Fuh H, Manogue KR 1994 Reactive glycosylation endproducts in diabetic uraemia and treatment of renal failure. Lancet 343:1519-1522

27. Hyogo H, Yamagishi S 2008 Advance glycation end products (AGEs) and their involvement in liver disease. Curr Pharm Des 14:969-972

28. Basta G, Schmidt AM, De Caterina R 2004 Advanced glycation end products and vascular inflammation: implications for accelerated atherosclerosis in diabetes. Cardiovasc Res 63:582-592

29. Diamanti-Kandarakis E, Piperi C, Korkolopoulou P, Kandaraki E, Levidou G, Papalois A, Patsouris E, Papavassiliou AG 2007 Ac- 
cumulation of dietary glycotoxins in the reproductive system of normal female rats. J Mol Med Berl 85:1413-1420

30. Diamanti-Kandarakis E, Piperi C, Patsouris E, Korkolopoulou P, Panidis D, Pawelczyk L, Papavassiliou AG, Duleba AJ 2007 Immunohistochemical localization of advanced glycation end-products (AGEs) and their receptor (RAGE) in polycystic and normal ovaries. Histochem Cell Biol 127:581-589

31. Diamanti-Kandarakis E, Katsikis I, Piperi C, Kandaraki E, Piouka A, Papavassiliou AG, Panidis D 2008 Increased serum advanced glycation end-products is a distinct finding in lean women with polycystic ovary syndrome (PCOS). Clin Endocrinol (Oxf) 69: 634-641

32. Diamanti-Kandarakis E, Lambrinoudaki I, Economou F, Christou M, Piperi C, Papavassiliou AG, Creatsas G 2010 Androgens associated with advanced glycation end-products in postmenopausal women. Menopause 17:1182-1187

33. Haus JM, Carrithers JA, Trappe SW, Trappe TA 2007 Collagen, cross-linking and advanced glycation end-products in aging human skeletal muscle. J Appl Physiol 103:2068-2076

34. Snow LM, Fugere NA, Thompson LV 2007 Advanced glycation end-product accumulation and associated protein modification in type II skeletal muscle with aging. J Gerontol A Biol Sci Med Sci 62:1204-1210

35. Hein GE 2006 Glycation end products in osteoporosis - is there a pathophysiologic importance? Clin Chim Acta 371:32-36

36. Odetti P, Rossi S, Monacelli F, Poggi A, Cirnigliaro M, Federici M, Federici A 2005 Advanced glycation end-products and bone loss during aging. Ann NY Acad Sci 1043:710-717

37. Kimura T, Takamatsu J, Ikeda K, Kondo A, Miyakawa T, Horiuchi 1996 Accumulation of advanced glycation end products of the Maillard reaction with age in human hippocampal neurons. Neurosci Lett 208:53-56

38. Castellani RJ, Harris PL, Sayre LM, Fujii J, Taniguchi N, Vitek MP, Founds H, Atwood CS, Perry G, Smith MA 2001 Active glycation in neurofibrillary pathology of Alzheimer disease: Ne-(carboxymethyl) lysine and hexitol-lysine. Free Radic Biol Med 31:175-180

39. Melloul D, Marshak S, Cerasi E 2002 Regulation of insulin gene transcription. Diabetologia 45:309-326

40. Poitout V, Hagman D, Stein R, Artner I, Robertson RP, Harmon JS 2006 Regulation of the insulin gene by glucose and fatty acids. J Nutr 136:873-876

41. Goodge KA, Hutton JC 2000 Translational regulation of proinsulin biosynthesis and proinsulin conversion in the pancreatic $\beta$-cell. Semin Cell Dev Biol 11:235-242

42. Scheuner D, Vander Mierde D, Song B, Flamez D, Creemers JW, Tsukamoto K, Ribick M, Schuit FC, Kaufman RJ 2005 Control of mRNA translation preserves endoplasmic reticulum function in $\beta$ cells and maintains glucose homeostasis. Nat Med 11:757-764

43. Kaufman RJ, Scheuner D, Schröder M, Shen X, Lee K, Liu CY, Arnold SM 2002 The unfolded protein response in nutrient sensing and differentiation. Nat Rev Mol Cell Biol 3:411-421

44. Oslowski CM, Urano F 2010 The binary switch between life and death of endoplasmic reticulum-stressed $\beta$ cells. Curr Opin Endocrinol Diabetes Obes 17:107-112

45. Wang Y, Vera L, Fischer WH, Montminy M 2009 The CREB coactivator CRTC2 links hepatic ER stress and fasting gluconeogenesis. Nature 460:534-537

46. Harding HP, Novoa I, Zhang Y, Zeng H, Wek R, Schapira M, Ron D 2000 Regulated translation initiation controls stress-induced gene expression in mammalian cells. Mol Cell 6:1099-1108

47. Back SH, Scheuner D, Han J, Song B, Ribick M, Wang J, Gildersleeve RD, Pennathur S, Kaufman RJ 2009 Translation attenuation through eIF $2 \alpha$ phosphorylation prevents oxidative stress and maintains the differentiated state in $\beta$ cells. Cell Metab 10:13-26

48. Oyadomari S, Harding HP, Zhang Y, Oyadomari M, Ron D 2008 Dephosphorylation of translation initiation factor $2 \alpha$ enhances glucose tolerance and attenuates hepatosteatosis in mice. Cell Metab 7:520-532
49. Lee AH, Scapa EF, Cohen DE, Glimcher LH 2008 Regulation of hepatic lipogenesis by the transcription factor XBP1. Science 320: 1492-1496

50. Gregor MF, Hotamisligil GS 2007 Adipocyte stress: the endoplasmic reticulum and metabolic disease. J Lipid Res 48:1905-1914

51. Brown MS, Ye J, Rawson RB, Goldstein JL 2000 Regulated intramembrane proteolysis: a control mechanism conserved from bacteria to humans. Cell 100:391-398

52. Gregor MF, Yang L, Fabbrini E, Mohammed BS, Eagon JC, Hotamisligil GS, Klein S 2009 Endoplasmic reticulum stress is reduced in tissues of obese subjects after weight loss. Diabetes 58:693-700

53. Chen Y, Liu CP, Xu KF, Mao XD, Lu YB, Fang L, Yang JW, Liu C 2008 Effect of taurine-conjugated ursodeoxycholic acid on endoplasmic reticulum stress and apoptosis induced by advanced glycation end products in cultured mouse podocytes. Am J Nephrol 28:1014-1022

54. Rasheed Z, Akhtar N, Haqqi TM, Tariq M 2010 Advanced glycation end products (AGEs)-induced expression of IL- 6 and IL-8 in human osteoarthritis chondrocytes correlates with the induction of endoplasmic reticulum stress. Arthritis and Rheumatism, 62 Nov 2010, Abstract

55. Loughlin DT, Artlett CM 2010 Precursor of advanced glycation end products mediates ER-stress-induced caspase- 3 activation of human dermal fibroblasts through $\mathrm{NAD}(\mathrm{P}) \mathrm{H}$ oxidase 4 . PLoS One 5:e11093

56. Dong Y, Zhang M, Wang S, Liang B, Zhao Z, Liu C, Wu M, Choi HC, Lyons TJ, Zou MH 2010 Activation of AMP-activated protein kinase inhibits oxidized LDL-triggered endoplasmic reticulum stress in vivo. Diabetes 59:1386-1396

57. Lee H, Lee YJ, Choi H, Ko EH, Kim JW 2009 Reactive oxygen species facilitate adipocyte differentiation by accelerating mitotic clonal expansion. J Biol Chem 284:10601-10609

58. Sekiya M, Hiraishi A, Touyama M, Sakamoto K 2008 Oxidative stress induced lipid accumulation via SREBP1c activation in HepG2 cells. Biochem Biophys Res Commun 375:602-607

59. Sparks DL, Schreurs BG 2003 Trace amounts of copper in water induce b-amyloid plaques and learning deficits in a rabbit model of Alzheimer's disease. Proc Natl Acad Sci USA 100:11065-11069

60. Ozcan U, Cao Q, Yilmaz E, Lee AH, Iwakoshi NN, Ozdelen E, Tuncman G, Görgün C, Glimcher LH, Hotamisligil GS 2004 Endoplasmic reticulum stress links obesity, insulin action, and type 2diabetes. Science 306:457-461

61. Tokita Y, Hirayama Y, Sekikawa A, Kotake H, Toyota T, Miyazawa T, Sawai T, Oikawa S 2005 Fructose ingestion enhances atherosclerosis and deposition of advanced glycated end-products in cholesterol-fed rabbits. J Atheroscler Thromb 12:260-267

62. Sato M, Kawakami T, Kondoh M, Takiguchi M, Kadota Y, Himeno S, Suzuki S 2010 Development of high-fat-diet-induced obesity in female metallothionein-null mice. FASEB J 24:2375-2384

63. Lu J, Wu DM, Zheng ZH, Zheng YL, Hu B, Zhang ZF 2011 Troxerutin protects against high cholesterol-induced cognitive deficits in mice. Brain 134:783-797

64. Hayashi T, Saito A, Okuno S, Ferrand-Drake M, Dodd RL, Chan PH 2005 Damage to the endoplasmic reticulum and activation of apoptotic machinery by oxidative stress in ischemic neurons. J Cereb Blood Flow Metab 25:41-53

65. Murakami K, Murata N, Noda Y, Tahara S, Kaneko T, Kinoshita N, Hatsuta H, Murayama S, Barnham KJ, Irie K, Shirasawa T, Shimizu T 2011 SOD1 (copper/zinc superoxide dismutase) deficiency drives amyloid $\beta$ protein oligomerization and memory loss in mouse model of Alzheimer disease. J Biol Chem 286:4455744568

66. Yokouchi M, Hiramatsu N, Hayakawa K, Okamura M, Du S, Kasai A, Takano Y, Shitamura A, Shimada T, Yao J, Kitamura M 2008 Involvement of selective reactive oxygen species upstream of proapoptotic branches of unfolded protein response. J Biol Chem 283:4252-4260

67. Yao D, Brownlee M 2010 Hyperglycemia-induced reactive oxygen 
species increase expression of the receptor for advanced glycation end products (RAGE) and RAGE ligands. Diabetes 59:249-255

68. Hayashi T, Saito A, Okuno S, Ferrand-Drake M, Dodd RL, Nishi T, Maier CM, Kinouchi H, Chan PH 2003 Oxidative damage to the endoplasmic reticulum is implicated in ischemic neuronal cell death. J Cereb Blood Flow Metab 23:1117-1128

69. Naidoo N 2009 The endoplasmic reticulum stress response and aging. Rev Neurosci 20:23-37

70. Wautier JL, Schmidt AM 2004 Protein glycation: a firm link to endothelial cell dysfunction. Circ Res 95:233-238

71. Evans JL, Goldfine ID, Maddux BA, Grodsky GM 2002 Oxidative stress and stress-activated signaling pathways: a unifying hypothesis of type 2 diabetes. Endocr Rev 23:599-622

72. Saha A, Kim SJ, Zhang Z, Lee YC, Sarkar C, Tsai PC, Mukherjee AB 2008 RAGE signaling contributes to neuroinflammation in infantile neuronal ceroid lipofuscinosis. FEBS Lett 582:3823-3831

73. Kumagai T, Nangaku M, Kojima I, Nagai R, Ingelfinger JR, Miyata T, Fujita T, Inagi R 2009 Glyoxalase I overexpression ameliorates renal ischemia-reperfusion injury in rats. Am J Physiol Renal Physiol 296:F912-F921

74. Prachasilchai W, Sonoda H, Yokota-Ikeda N, Ito K, Kudo T, Imaizumi K, Ikeda M 2009 The protective effect of a newly developed molecular chaperone-inducer against mouse ischemic acute kidney injury. J Pharmacol Sci 109:311-314

75. Koumenis C 2006 ER stress, hypoxia tolerance and tumor progression. Curr Mol Med 6:55-69

76. Dioufa N, Kassi E, Papavassiliou AG, Kiaris H 2010 Atypical induction of the unfolded protein response by mifepristone. Endocrine 38:167-173

77. Lee AS 2007 GRP78 induction in cancer: therapeutic and prognostic implications. Cancer Res 67:3496-3499

78. Logsdon CD, Fuentes MK, Huang EH, Arumugam T 2007 RAGE and RAGE ligands in cancer. Curr Mol Med 7:777-789

79. Morcos M, Du X, Pfisterer F, Hutter H, Sayed AA, Thornalley P, Ahmed N, Baynes J, Thorpe S, Kukudov G, Schlotterer A, Bozorgmehr F, El Baki RA, Stern D, Moehrlen F, Ibrahim Y, Oikonomou D, Hamann A, Becker C, Zeier M, Schwenger V, Miftari N, Humpert P, Hammes HP, Buechler M, Bierhaus A, Brownlee M, Nawroth PP 2008 Glyoxalase-1 prevents mitochondrial protein modification and enhances lifespan in Caenorhabditis elegans. Aging Cell 7:260-269

80. Chen D, Thomas EL, Kapahi P 2009 HIF-1 modulates dietary restriction mediated lifespan extension via IRE-1 in Caenorhabditis elegans. PLoS Genet 5:e1000486

81. Hu C, Cong XD, Dai DZ, Zhang Y, Zhang GL, Dai Y 2011 Argirein alleviates diabetic nephropathy through attenuating NADPH oxidase, Cx43, and PERK in renal tissue. Naunyn Schmiedebergs Arch Pharmacol 383:309-319

82. Battah S, Ahmed N, Thornalley PJ 2002 Kinetics and mechanism of the reaction of metformin with methylglyoxal. Int Congr Ser 1245:355-356

83. Diamanti-Kandarakis E, Alexandraki K, Piperi C, Aessopos A, Paterakis T, Katsikis I, Panidis D 2007 Effect of metformin administration on plasma advanced glycation end product levels in women with polycystic ovary syndrome. Metabolism 56:129-134

84. Kim DS, Jeong SK, Kim HR, Kim DS, Chae SW, Chae HJ 2010 Metformin regulates palmitate-induced apoptosis and ER stress response in HepG2 liver cells. Immunopharmacol Immunotoxicol 32:251-257

85. Jung TW, Lee MW, Lee YJ, Kim SM, Jung TW 2012 Metformin prevents thapsigargin-induced apoptosis via inhibition of c-Jun $\mathrm{NH}(2)$ terminal kinase in NIT-1 cells. Biochem Biophys Res Commun 417:147-152

86. Miyata T, Dan T 2008 Inhibition of advanced glycation end products (AGEs): an implicit goal in clinical medicine for the treatment of diabetic nephropathy? Diabetes Res Clin Pract 82(Suppl 1):S25S29

87. Yoshiuchi K, Kaneto H, Matsuoka TA, Kasami R, Kohno K,
Iwawaki T, Nakatani Y, Yamasaki Y, Shimomura I, Matsuhisa M 2009 Pioglitazone reduces ER stress in the liver: direct monitoring of in vivo ER stress using ER stress-activated indicator transgenic mice. Endocr J 56:1103-1111

88. Akiyama M, Hatanaka M, Ohta Y, Ueda K, Yanai A, Uehara Y, Tanabe K, Tsuru M, Miyazaki M, Saeki S, Saito T, Shinoda K, Oka Y, Tanizawa Y 2009 Increased insulin demand promotes while pioglitazone prevents pancreatic $\beta$ cell apoptosis in Wfs1 knockout mice. Diabetologia 52:653-663

89. Yamagishi S, Nakamura K, Matsui T, Inoue H, Takeuchi M 2007 Oral administration of AST-120 (Kremezin) is a promising therapeutic strategy for advanced glycation end product (AGE)-related disorders. Med Hypotheses 69:666-668

90. Kawakami T, Inagi R, Wada T, Tanaka T, Fujita T, Nangaku M 2010 Indoxyl sulfate inhibits proliferation of human proximal tubular cells via endoplasmic reticulum stress. Am J Physiol Renal Physiol 299:F568-F576

91. Nangaku M, Miyata T, Sada T, Mizuno M, Inagi R, Ueda Y, Ishikawa N, Yuzawa H, Koike H, van Ypersele de Strihou C, Kurokawa K 2003 Anti-hypertensive agents inhibit in vivo the formation of advanced glycation end products and improve renal damage in a type 2 diabetic nephropathy rat model. J Am Soc Nephrol 14:1212-1222

92. Okada K, Minamino T, Tsukamoto Y, Liao Y, Tsukamoto O, Takashima S, Hirata A, Fujita M, Nagamachi Y, Nakatani T, Yutani C, Ozawa K, Ogawa S, Tomoike H, Hori M, Kitakaze M 2004 Prolonged endoplasmic reticulum stress in hypertrophic and failing heart after aortic constriction: possible contribution of endoplasmic reticulum stress to cardiac myocyte apoptosis. Circulation 110: 705-712

93. Ren J, Li Q, Wu S, Li SY, Babcock SA 2007 Cardiac overexpression of antioxidant catalase attenuates aging-induced cardiomyocyte relaxation dysfunction. Mech Ageing Dev 128:276-285

94. Wu RF, Ma Z, Liu Z, Terada LS 2010 Nox4-derived $\mathrm{H}_{2} \mathrm{O}_{2}$ mediates endoplasmic reticulum signaling through local Ras activation. Mol Cell Biol 30:3553-3568

95. Sanson M, Augé N, Vindis C, Muller C, Bando Y, Thiers JC, Marachet MA, Zarkovic K, Sawa Y, Salvayre R, Nègre-Salvayre A 2009 Oxidized low density lipoproteins trigger endoplasmic reticulum stress in vascular cells: prevention by oxygen-regulated protein 150 expression. Circ Res 104:328-336

96. Sajithlal GB, Chithra P, Chandrakasan G 1998 Effect of curcumin on the advanced glycation and cross-linking of collagen in diabetic rats. Biochem Pharmacol 56:1607-1614

97. Suryanarayana P, Krishnaswamy K, Reddy GB 2003 Effect of curcumin on galactose-induced cataractogenesis in rats. Mol Vis 9:223-230

98. Bengmark S, Mesa MD, Gil A 2009 Plant-derived health: the effects of turmeric and curcuminoids. Nutr Hosp 24:273-281

99. Pizzo P, Scapin C, Vitadello M, Florean C, Gorza L 2010 Grp94 acts as a mediator of curcumin-induced antioxidant defence in myogenic cells. J Cell Mol Med 14:970-981

100. Shinkai Y, Yamamoto C, Kaji T 2010 Lead induces the expression of endoplasmic reticulum chaperones GRP78 and GRP94 in vascular endothelial cells via the JNK-AP-1 pathway. Toxicol Sci 114: 378-386

101. Hipkiss AR, Michaelis J, Syrris P 1995 Non-enzymic glycosylation of the dipeptide L-carnosine, a potential anti-protein-crosslinking agent. FEBS Lett 371:81-85

102. Oh YM, Jang EH, Ko JH, Kang JH, Park CS, Han SB, Kim JS, Kim KH, Pie JE, Shin DW 2009 Inhibition of 6-hydroxydopamine-induced endoplasmic reticulum stress by l-carnosine in SH-SY5Y cells. Neurosci Lett 459:7-10

103. Sengupta B, Uematsu T, Jacobsson P, Swenson J 2006 Exploring the antioxidant property of bioflavonoid quercetin in preventing DNA glycation: a calorimetric and spectroscopic study. Biochem Biophys Res Commun 339:355-361

104. Ohta K, Mizuno A, Li S, Itoh M, Ueda M, Ohta E, Hida Y, Wang 
MX, Furoi M, Tsuzuki Y, Sobajima M, Bohmoto Y, Fukushima T, Kobori M, Inuzuka T, Nakagawa T 2011 Endoplasmic reticulum stress enhances $\gamma$-secretase activity. Biochem Biophys Res Commun 416:362-366

105. Muellenbach EA, Diehl CJ, Teachey MK, Lindborg KA, Archuleta TL, Harrell NB, Andersen G, Somoza V, Hasselwander O, Matuschek M, Henriksen EJ 2008 Interactions of the advanced glycation end product inhibitor pyridoxamine and the antioxidant $\alpha$-lipoic acid on insulin resistance in the obese Zucker rat. Metabolism 57:1465-1472

106. Lee SJ, Kim SH, Kang JG, Kim CS, Ihm SH, Choi MG, Yoo HJ 2011 $\alpha$-Lipoic acid inhibits endoplasmic reticulum stress-induced cell death through PI3K/Akt signaling pathway in FRTL5 thyroid cells. Horm Metab Res 43:445-451

107. Takizawa S, Izuhara Y, Kitao Y, Hori O, Ogawa S, Morita Y, Takagi S, van Ypersele de Strihou C, Miyata T 2007 A novel inhibitor of advanced glycation and endoplasmic reticulum stress reduces infarct volume in rat focal cerebral ischemia. Brain Res 1183:124-137

108. Izuhara Y, Nangaku M, Takizawa S, Takahashi S, Shao J, Oishi H, Kobayashi H, van Ypersele de Strihou C, Miyata T 2008 A novel class of advanced glycation inhibitors ameliorates renal and cardiovascular damage in experimental rat models. Nephrol Dial Transplant 23:497-509

109. Ozcan U, Yilmaz E, Ozcan L, Furuhashi M, Vaillancourt E, Smith RO, Görgün CZ, Hotamisligil GS 2006 Chemical chaperones reduce ER stress and restore glucose homeostasis in a mouse model of type 2 diabetes. Science 313:1137-1140

110. Harding HP, Zeng H, Zhang Y, Jungries R, Chung P, Plesken H, Sabatini DD, Ron D 2001 Diabetes mellitus and exocrine pancre- atic dysfunction in perk-/- mice reveals a role for translational control in secretory cell survival. Mol Cell 7:1153-1163

111. Ladiges WC, Knoblaugh SE, Morton JF, Korth MJ, Sopher BL, Baskin CR, MacAuley A, Goodman AG, LeBoeuf RC, Katze MG 2005 Pancreatic $\beta$-cell failure and diabetes in mice with a deletion mutation of the endoplasmic reticulum molecular chaperone gene P58IPK. Diabetes 54:1074-1081

112. Fonseca SG, Fukuma M, Lipson KL, Nguyen LX, Allen JR, Oka Y, Urano F 2005 WFS1 is a novel component of the unfolded protein response and maintains homeostasis of the endoplasmic reticulum in pancreatic $\beta$-cells. J Biol Chem 280:39609-39615

113. Oyadomari S, Koizumi A, Takeda K, Gotoh T, Akira S, Araki E, Mori M 2002 Targeted disruption of the Chop gene delays endoplasmic reticulum stress-mediated diabetes. J Clin Invest 109:525532

114. Song B, Scheuner D, Ron D, Pennathur S, Kaufman RJ 2008 Chop deletion reduces oxidative stress, improves $\beta$ cell function, and promotes cell survival in multiple mouse models of diabetes. J Clin Invest 118:3378-3389

115. Bobrovnikova-Marjon E, Hatzivassiliou G, Grigoriadou C, Romero M, Cavener DR, Thompson CB, Diehl JA 2008 PERK-dependent regulation of lipogenesis during mouse mammary gland development and adipocyte differentiation. Proc Natl Acad Sci USA 105:16314-16319

116. Kammoun HL, Chabanon H, Hainault I, Luquet S, Magnan C, Koike T, Ferré P, Foufelle F 2009 GRP78 expression inhibits insulin and ER stress-induced SREBP-1c activation and reduces hepatic steatosis in mice. J Clin Invest 119:1201-1215

117. Vecchi C, Montosi G, Zhang K, Lamberti I, Duncan SA, Kaufman RJ, Pietrangelo A 2009 ER stress controls iron metabolism through induction of hepcidin. Science 325:877-880 